FIRST MEASUREMENT OF THE MUON ANTI-NEUTRINO CHARGED CURRENT QUASIELASTIC DOUBLE-DIFFERENTIAL CROSS SECTION

By

JOSEPH M. GRANGE

DISSERTATION PRESENTED TO THE GRADUATE SCHOOL OF THE UNIVERSITY OF FLORIDA IN PARTIAL FULFILLMENT OF THE REQUIREMENTS FOR THE DEGREE OF DOCTOR OF PHILOSOPHY UNIVERSITY OF FLORIDA 
(c) 2013 Joseph M. Grange 
To all the lovely people who have helped me along the way 


\section{ACKNOWLEDGEMENTS}

So many folks had a hand in guiding my progress that it would be disingenuous to attempt a complete list here. But I certainly could not have accomplished what I did without the guidance, insight, and contagious enthusiasm of my advisor Heather Ray and those at Fermilab most influential to my analysis: Sam Zeller, Teppei Katori, Richard Imlay and Chris Polly. I am so lucky to call you my mentors, and I hope to live up to your expectations in my postgraduate work. 


\section{TABLE OF CONTENTS}

page

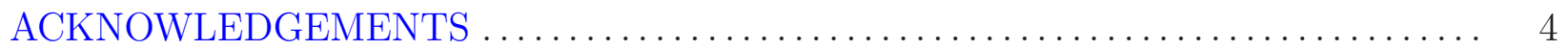

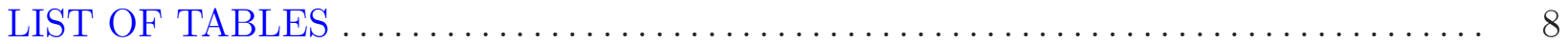

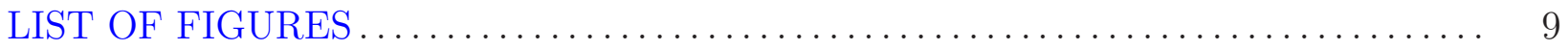

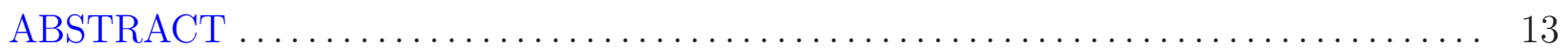

CHAPTER

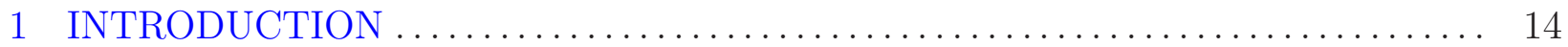

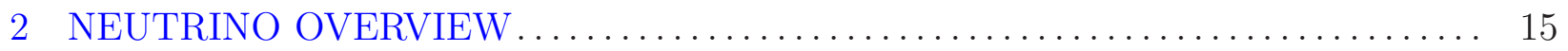

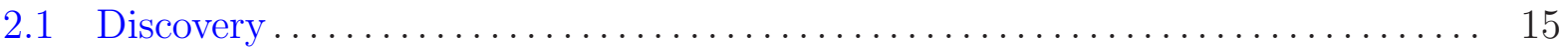

2.2 Interaction and Propagation States ........................... 17

2.3 Flavors of Neutrinos ... . . . . . . . . . . . . . . . . . . . . . . . 19

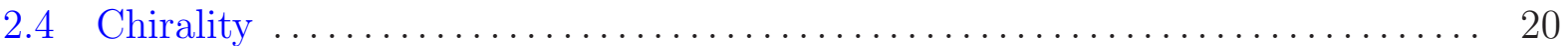

2.5 Magnetic Moment .................................... 22

2.6 Absolute Mass . . . . . . . . . . . . . . . . . . . . . . . . . . . . . . 23

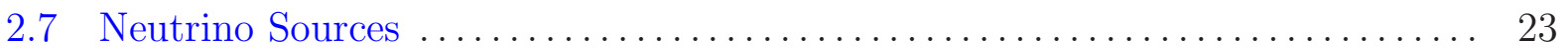

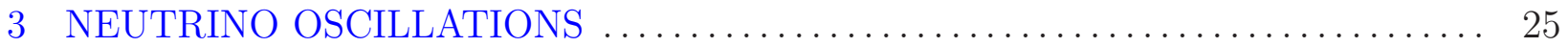

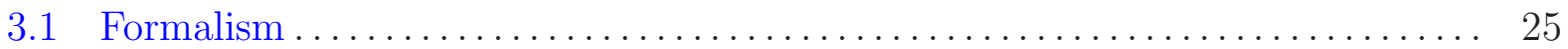

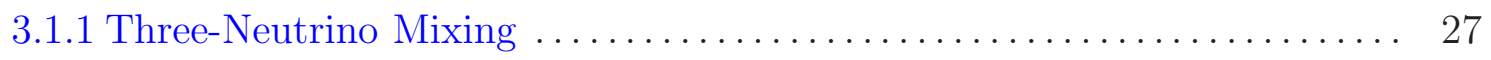

3.1 .2 Two-Neutrino Mixing ............................... 28

3.2 Experimental Evidence for Neutrino Oscillations . . . . . . . . . . . . . . . 30

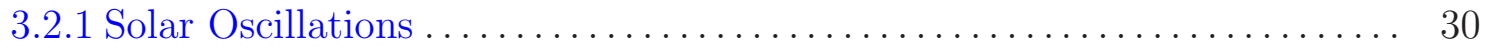

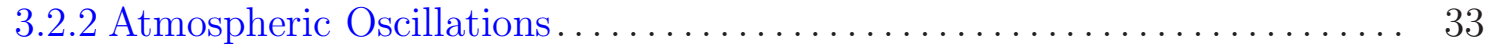

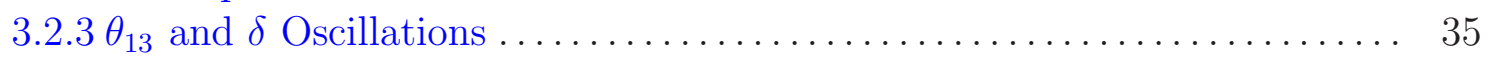

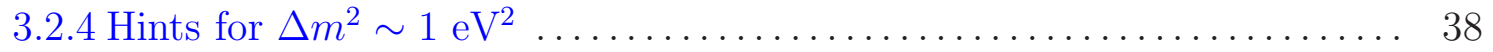

3.3 Summary and Outstanding Questions ......................... 39

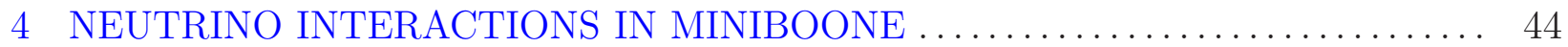

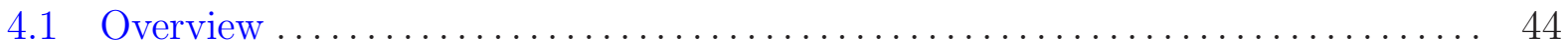

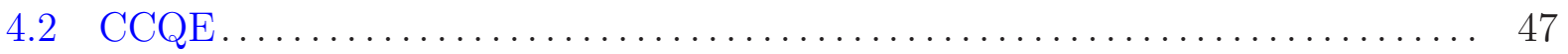

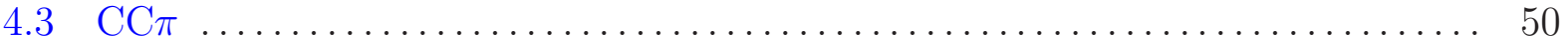

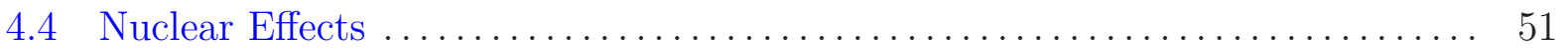

4.4 .1 Nuclear Modeling................................... 51 


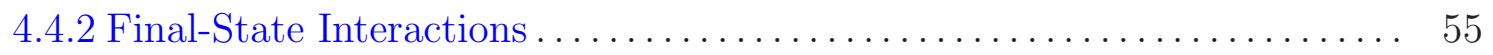

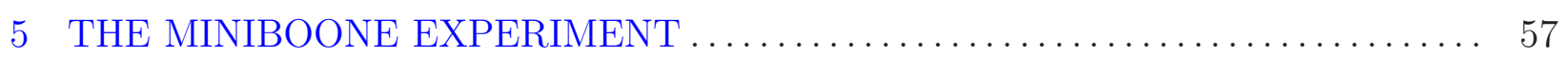

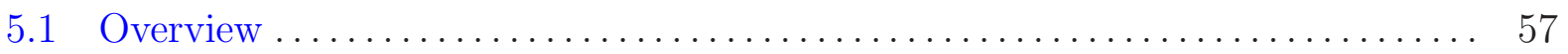

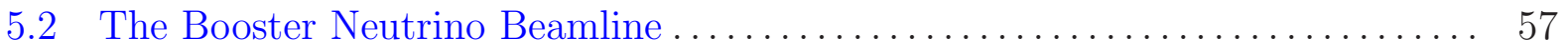

5.2 .1 The Primary Proton Beam............................. 57

5.2 .2 Beryllium Target and Magnetic Focusing Horn .................. 61

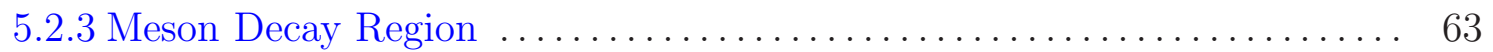

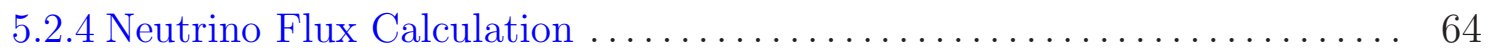

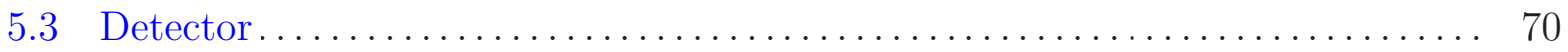

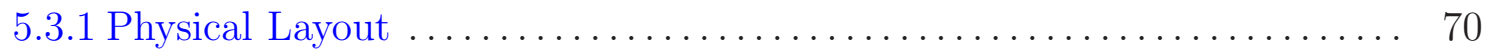

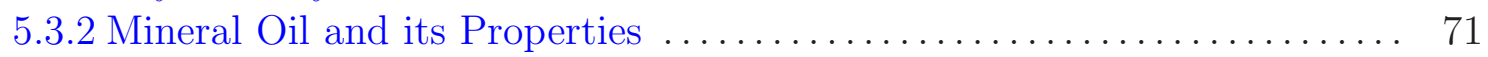

5.3 .3 Photomultiplier Tubes ................................. 74

5.3.4 Calibration Systems ..................................... 74

5.3 .5 Analysis Tools ....................................... 76

6 INTRODUCTION TO THE CROSS-SECTION MEASUREMENT . . . . . . . . . . 82

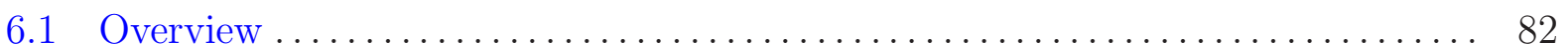

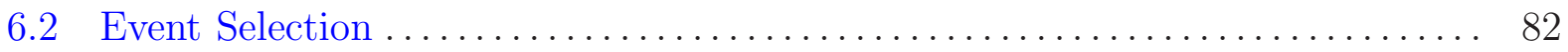

7 BACKGROUND MEASUREMENTS AND CONSTRAINTS $\ldots \ldots \ldots \ldots \ldots \ldots . \ldots 2$

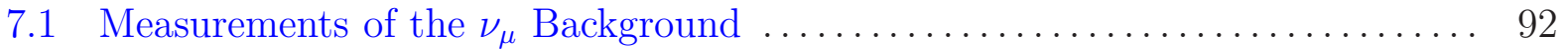

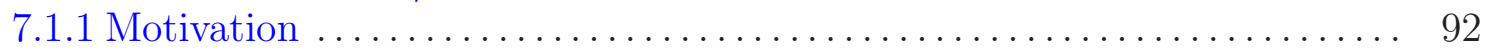

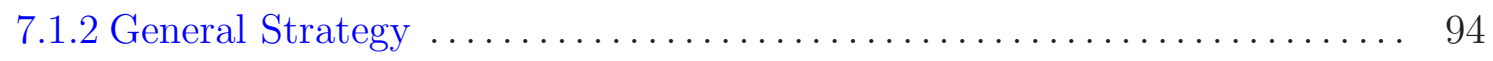

$7.1 .3 \nu_{\mu}$ Flux Measurement Using CC $\pi^{+}$Events $\ldots \ldots \ldots \ldots \ldots \ldots \ldots \ldots . \ldots 9$

Implementation of the $\nu_{\mu} \mathrm{CC} \pi^{+}$Cross Section ................... 98

The Selected Sample .................................. 100

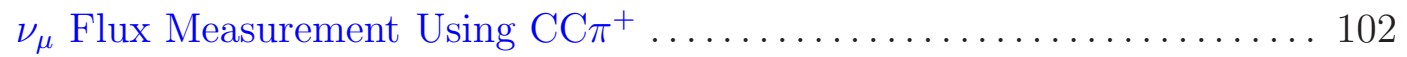

7.1.4 $\nu_{\mu}$ Flux Measurement Using $\mu^{-}$Nuclear Capture .................... 104

Implementation of the $\nu_{\mu}$ CC Cross Sections ..................... 105

Muon Capture Model and Event Selection ..................... 107

Calibrations and Stability Checks Using the Neutrino-Mode Data ...... 114

$\nu_{\mu}$ Flux Measurement Using $\mu^{-}$Capture......................... 118

Systematic Errors...................................... 120

7.1.5 $\nu_{\mu}$ Flux Measurement Using the $\cos \theta_{\mu}$ Distribution $\ldots \ldots \ldots \ldots \ldots \ldots 123$

Overview ............................................ 123

Sample Selection....................................... 124

Measurement Execution.................................. 125

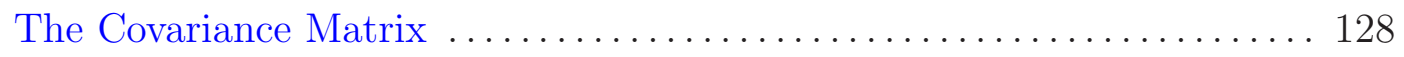

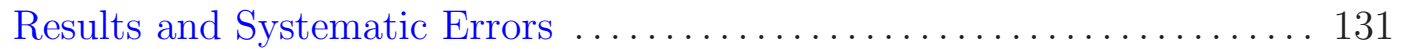

7.1.6 Summary of $\nu_{\mu}$ Flux Measurements ........................ 137

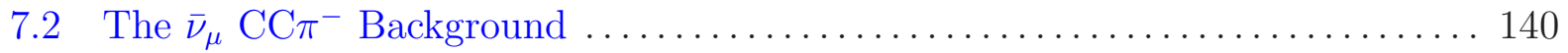


7.3 All Other Backgrounds ..................................... 144

7.4 Background Constraint Summary ............................... 144

$8 \bar{\nu}_{\mu}$ CCQE CROSS-SECTION MEASUREMENT $\ldots \ldots \ldots \ldots \ldots \ldots \ldots \ldots \ldots \ldots \ldots \ldots$

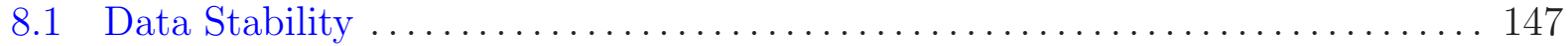

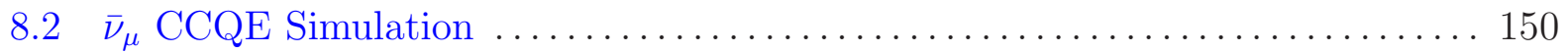

8.3 Cross-Section Calculation ...................................... 153

8.3.1 Unsmearing to True Quantities ............................ 155

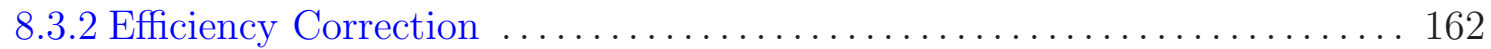

8.3.3 Flux and Interaction Targets............................. 164

8.3 .4 Statistical Uncertainty ................................... 165

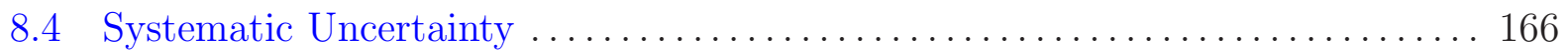

8.4.1 Background Uncertainties................................ 166

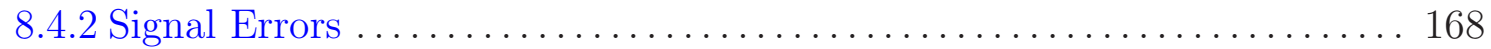

8.4.3 Uncertainty Summary for $\bar{\nu}_{\mu}$ CCQE on Mineral Oil................ 171

8.4.4 Uncertainty Summary for $\bar{\nu}_{\mu}$ CCQE on Carbon.................... 174

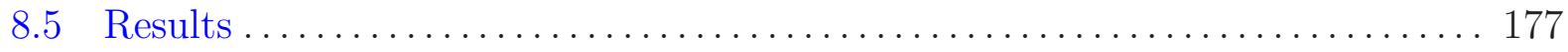

8.5.1 Results Incident on Mineral Oil ............................ 177

8.5.2 Results Incident on Carbon ............................... 183

9 COMBINED $\nu_{\mu}$ AND $\bar{\nu}_{\mu}$ CCQE MEASUREMENTS $\ldots \ldots \ldots \ldots \ldots \ldots \ldots \ldots \ldots \ldots$

9.1 Correlated Measurements ...................................... 191

9.2 Combined $\nu_{\mu}$ and $\bar{\nu}_{\mu}$ CCQE Measurements ........................ 193

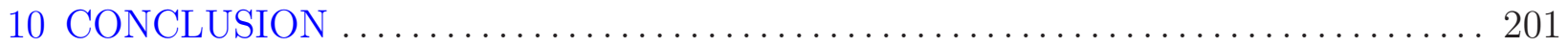

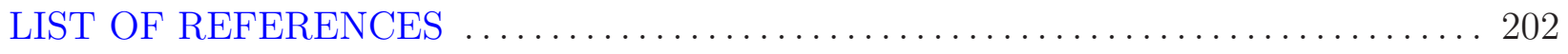

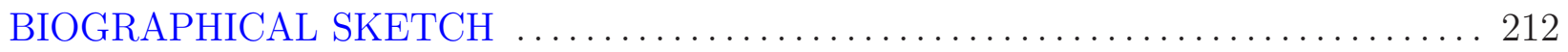




\section{LIST OF TABLES}

$\underline{\text { Table }}$

$\underline{\text { page }}$

5-1 Particle Čerenkov thresholds in the MiniBooNE detector oil $\ldots \ldots \ldots \ldots \ldots .72$

6-1 Purity and detection efficiency for $\bar{\nu}_{\mu}$ CCQE $\ldots \ldots \ldots \ldots \ldots \ldots \ldots \ldots \ldots$

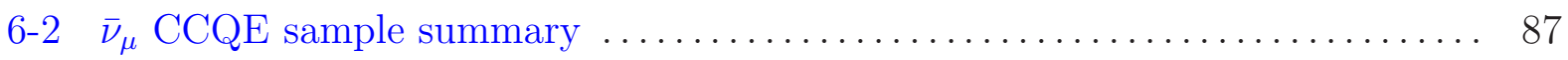

7-1 Data set by absorber period used in the $\mathrm{CC} \pi^{+}$and $\cos \theta_{\mu}$ analyses $\ldots \ldots \ldots 98$

7-2 Selection efficiency and purity for the $\mathrm{CC} \pi^{+}$sample $\ldots \ldots \ldots \ldots \ldots \ldots \ldots \ldots$

7-3 Predicted interaction composition of the antineutrino-mode $\mathrm{CC} \pi^{+}$sample ... 103

7-4 Summary of the $\mathrm{CC} \pi^{+} \nu_{\mu}$ flux measurement $\ldots \ldots \ldots \ldots \ldots \ldots \ldots \ldots \ldots \ldots$

7-5 $\nu_{\mu}$ and $\bar{\nu}_{\mu}$ CC purity for the $\mu^{-}$capture samples $\ldots \ldots \ldots \ldots \ldots \ldots \ldots \ldots \ldots$

7-6 Predicted interaction contributions to the $\mu^{-}$capture samples $\ldots \ldots \ldots \ldots 114$

7-7 Summary of the neutrino-mode $\mu^{-}$capture samples $\ldots \ldots \ldots \ldots \ldots \ldots \ldots \ldots$

7-8 Calibration summary for the $\mu^{-}$capture and Michel detection models ...... 116

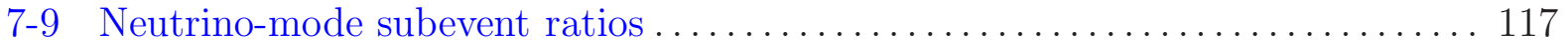

7-10 Calibration effects on the $\nu_{\mu}$ flux measurement using $\mu^{-}$capture $\ldots \ldots \ldots \ldots 120$

7-11 Uncertainty summary for the $\mu^{-}$capture-based measurement of the $\nu_{\mu}$ flux . 122

7-12 Summary of the $\nu_{\mu}$ flux measurement using $\mu^{-}$capture $\ldots \ldots \ldots \ldots \ldots \ldots \ldots$

7-13 Summary of uncertainties for the $\nu_{\mu}$ flux measurement using $\mu^{-}$capture $\ldots . .131$

7-14 Summary of cross-section uncertainties for the analysis of $\cos \theta_{\mu}$ events $\ldots \ldots 132$

7-15 Summary of the $\nu_{\mu}$ flux measurement using the $\cos \theta_{\mu}$ distribution $\ldots \ldots \ldots 136$

7-16 $\nu_{\mu}$ and $\bar{\nu}_{\mu}$ contributions to the CCQE sample before and after the $\cos \theta_{\mu}$ fit $\ldots 137$

7-17 Summary of backgrounds in the $\bar{\nu}_{\mu}$ CCQE sample $\ldots \ldots \ldots \ldots \ldots \ldots \ldots \ldots 146$

8-1 Summary of data groups input to stability tests $\ldots \ldots \ldots \ldots \ldots \ldots \ldots \ldots \ldots$

8-2 Normalization uncertainties for the cross sections on mineral oil ......... 177

8-3 Normalization uncertainties for cross sections on carbon $\ldots \ldots \ldots \ldots \ldots \ldots \ldots 178$ 


\section{LIST OF FIGURES}

$\underline{\text { Figure }} \quad \underline{\text { page }}$

2-1 Detection schematic for the first observation of the neutrino $\ldots \ldots \ldots \ldots \ldots .17$

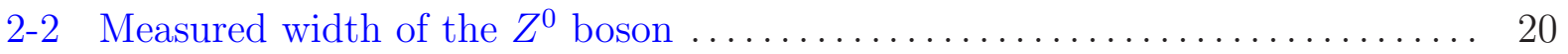

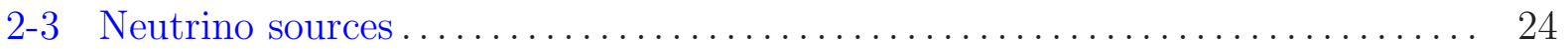

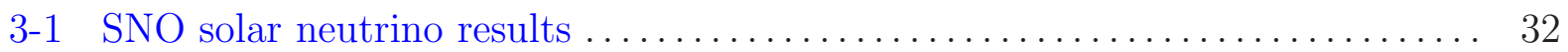

3-2 KamLAND $\bar{\nu}_{e}$ disappearance results ............................ 33

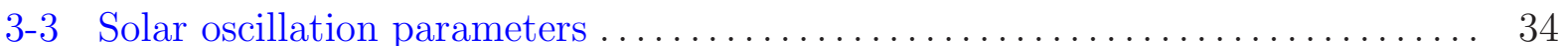

3-4 Evidence of atmospheric oscillations from Super-Kamiokande . . . . . . . . . 35

3-5 Atmospheric oscillation parameters ....................... 36

3-6 Observation of $\bar{\nu}_{e}$ disappearance at Daya Bay $\ldots \ldots \ldots \ldots \ldots \ldots \ldots \ldots \ldots \ldots \ldots$

3-7 Current contraints on $\delta$ and the mass hierarchy $\ldots \ldots \ldots \ldots \ldots \ldots \ldots \ldots \ldots .38$

3-8 Combined $\nu_{e}$ and $\bar{\nu}_{e}$ appearance results from MiniBooNE $\ldots \ldots \ldots \ldots \ldots \ldots .39$

3-9 Degeneracy in the ordering of neutrino mass states $\ldots \ldots \ldots \ldots \ldots \ldots \ldots \ldots .42$

4-1 Comparison of experimental data in $\nu_{\mu}$ and $\bar{\nu}_{\mu} \mathrm{CC}$ interactions........... 46

4-2 Decomposition of the CCQE cross section $\ldots \ldots \ldots \ldots \ldots \ldots \ldots \ldots \ldots \ldots \ldots$

4-3 Binding energy and Fermi momentum in electron scattering data $\ldots \ldots \ldots .53$

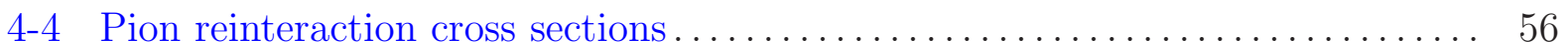

5-1 Cartoon of the first stage of the Fermilab accelerator network $\ldots \ldots \ldots \ldots .58$

$5-2 \quad$ Injection into the Booster synchrotron $\ldots \ldots \ldots \ldots \ldots \ldots \ldots \ldots \ldots \ldots \ldots \ldots$

5-3 Timing structure of the BNB proton spills $\ldots \ldots \ldots \ldots \ldots \ldots \ldots \ldots \ldots \ldots$

5-4 The Booster Neutrino Beamline beryllium target $\ldots \ldots \ldots \ldots \ldots \ldots \ldots \ldots \ldots .62$

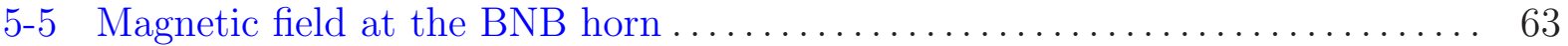

5-6 Primary hadroproduction models at MiniBooNE $\ldots \ldots \ldots \ldots \ldots \ldots \ldots \ldots \ldots$

5-7 Double-differential $\pi^{-}$production at the BNB $\ldots \ldots \ldots \ldots \ldots \ldots \ldots \ldots \ldots .67$

5-8 Contributions to neutrino beams from hadronic reinteractions . . . . . . . . 68

5-9 BNB flux at MiniBooNE for neutrino and antineutrino modes $\ldots \ldots \ldots \ldots .69$ 
5-10 Overview of the MiniBooNE detector housing $\ldots \ldots \ldots \ldots \ldots \ldots \ldots \ldots \ldots$

5-11 MiniBooNE detector cutaway and veto region photo $\ldots \ldots \ldots \ldots \ldots \ldots \ldots .71$

$5-12$ Extinction rate spectrum in the MiniBooNE oil ................... 73

5-13 Quantum efficiency for MiniBooNE PMTs.................... 74

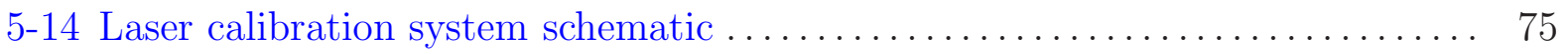

5-15 Cartoon of the muon calibration system and calibration summary ....... 77

5-16 Typical $\bar{\nu}_{\mu}$ CCQE PMT signature $\ldots \ldots \ldots \ldots \ldots \ldots \ldots \ldots \ldots \ldots \ldots \ldots \ldots \ldots$

5-17 Topology and timing of typical electron and muon events in MiniBooNE . . . 79

5-18 Energy and angular resolutions of the MiniBooNE reconstruction $\ldots \ldots \ldots \ldots 81$

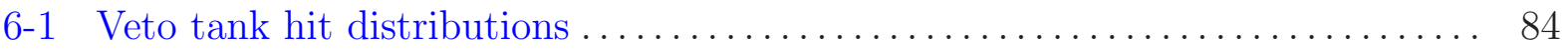

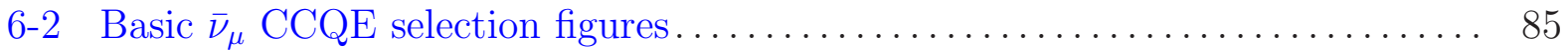

6-3 Spatial correlation between the subevents $\bar{\nu}_{\mu}$ CCQE sample ............ 90

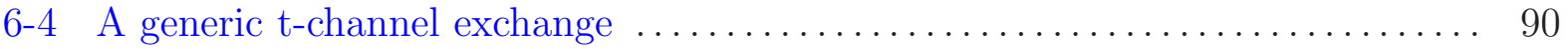

6-5 Kinematic distributions of the $\bar{\nu}_{\mu}$ CCQE sample $\ldots \ldots \ldots \ldots \ldots \ldots \ldots \ldots \ldots$

7-1 Pion production phase space at the BNB $\ldots \ldots \ldots \ldots \ldots \ldots \ldots \ldots \ldots \ldots . . \ldots 3$

7-2 Accepted $\nu_{\mu}$ spectra in neutrino and antineutrino run modes $\ldots \ldots \ldots \ldots \ldots$

7-3 MiniBooNE $\nu_{\mu} \mathrm{CC} \pi^{+}$total cross section $\ldots \ldots \ldots \ldots \ldots \ldots \ldots \ldots \ldots \ldots \ldots$

7-4 $\mathrm{CC} \pi^{+}$fractional uncertainty conversion $\ldots \ldots \ldots \ldots \ldots \ldots \ldots \ldots \ldots \ldots \ldots \ldots \ldots \ldots \ldots \ldots$

7-5 Cross-section correction to the simulated $\nu_{\mu}$ CCQE process $\ldots \ldots \ldots \ldots \ldots$

7-6 Log-likelihood $\mu / e$ particle-ID in the single-subevent sample $\ldots \ldots \ldots \ldots \ldots$.

7-7 Four-momentum transfer cut on for 1 and 2 subevents . . . . . . . . . . 110

7-8 Predicted muon stopping radius for 1 and 2 subevents $\ldots \ldots \ldots \ldots \ldots \ldots \ldots 11$

7-9 Timing difference between the first and second subevents $\ldots \ldots \ldots \ldots \ldots \ldots 112$

7-10 Tank hit distribution for the Michel subevent in antineutrino mode ....... 112

7-11 Tank hit distribution for the Michel subevent in neutrino mode $\ldots \ldots \ldots \ldots 116$

7-12 Stability check of the timing systems with the neutrino-mode data $\ldots \ldots \ldots 118$

7-13 Before-fit $\nu_{\mu}$ and $\bar{\nu}_{\mu} \cos \theta_{\mu}$ distributions $\ldots \ldots \ldots \ldots \ldots \ldots \ldots \ldots \ldots \ldots \ldots \ldots \ldots \ldots$ 
7-14 Two options for spatially correlating the muon and its Michel .......... 126

7-15 Variations in the $\cos \theta_{\mu}$ distribution to due cross-section uncertainties . . . . 128

7-16 Correlation matrix between $\nu_{\mu}$ and $\bar{\nu}_{\mu}$ events in the $\cos \theta_{\mu}$ distribution $\ldots . . .130$

7-17 Angular fit results in the CCQE sample ......................... 135

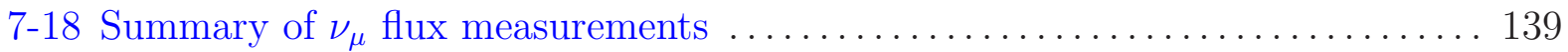

7-19 $\mathrm{CC} \pi^{+}$correction based on MiniBooNE neutrino-mode data $\ldots \ldots \ldots \ldots \ldots 14$

7-20 Comparison of various models to MiniBooNE $\mathrm{CC} \pi^{+}$data ............. 142

7-21 Comparison of various models to the prediction of $\mathrm{CC} \pi^{-}$events ......... 143

8-1 Kolmogorov-Smirnoff test for the $\bar{\nu}_{\mu}$ CCQE sample $\ldots \ldots \ldots \ldots \ldots \ldots \ldots \ldots \ldots$

8-2 Data stability for $E_{\nu}^{Q E}$ in the $\bar{\nu}_{\mu}$ CCQE sample $\ldots \ldots \ldots \ldots \ldots \ldots \ldots \ldots \ldots$

8-3 Normalization stability for the $\bar{\nu}_{\mu}$ CCQE sample................... 149

8-4 Generator-level comparison of the simulate $\bar{\nu}_{\mu}$ CCQE cross section on carbon. 151

8-5 Generator-level cross-section correction for $\bar{\nu}_{\mu}$ CCQE interactions on carbon . . 152

8-6 Generator-level correction for $\bar{\nu}_{\mu}$ CCQE on hydrogen $\ldots \ldots \ldots \ldots \ldots \ldots \ldots \ldots$

8-7 Generator-level comparisons of the simulated $\mathrm{CC} \pi$ processes $\ldots \ldots \ldots \ldots \ldots 154$

8-8 Reconstructed-truth level comparisons for the $\bar{\nu}_{\mu}$ CCQE distributions ...... 155

8-9 Unsmearing matrices for the $\bar{\nu}_{\mu}$ CCQE distributions . . . . . . . . . . . . 159

8-10 Comparison of before and after the unsmearing process $\ldots \ldots \ldots \ldots \ldots \ldots \ldots$

8-11 Comparison of the Bayesian and matrix inversion methods of unsmearing ... 161

8-12 Detection efficiency for the $\bar{\nu}_{\mu}$ CCQE distributions $\ldots \ldots \ldots \ldots \ldots \ldots \ldots \ldots \ldots$

8-13 $\bar{\nu}_{\mu}$ parent $\pi^{-}$phase space at the BNB in antineutrino mode $\ldots \ldots \ldots \ldots \ldots .164$

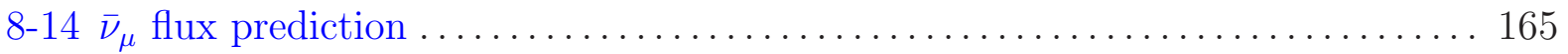

8-15 Fractional uncertainty for the $\bar{\nu}_{\mu}$ CCQE cross sections on mineral oil $\ldots \ldots \ldots 172$

8-16 More fractional uncertainties for the $\bar{\nu}_{\mu}$ CCQE cross sections on mineral oil . 173

8-17 Fractional uncertainty for the $\bar{\nu}_{\mu}$ CCQE cross sections on carbon .......... 175

8-18 More fractional uncertainties for the $\bar{\nu}_{\mu}$ CCQE cross sections on mineral oil .. 176

8-19 $\bar{\nu}_{\mu}$ CCQE cross sections on mineral oil $\ldots \ldots \ldots \ldots \ldots \ldots \ldots \ldots \ldots \ldots \ldots \ldots$ 
8-20 Projections of the $\bar{\nu}_{\mu}$ CCQE $\frac{d^{2} \sigma}{d T_{\mu} d \cos \theta_{\mu}}$ cross section on mineral oil . . . . . . 181 8-21 Shape comparison of the cross section on mineral oil with MC ......... 182

8-22 $\bar{\nu}_{\mu}$ CCQE cross sections on carbon $\ldots \ldots \ldots \ldots \ldots \ldots \ldots \ldots \ldots \ldots \ldots \ldots \ldots \ldots \ldots \ldots \ldots \ldots \ldots \ldots \ldots$

8-23 One-dimensional views of the double-differential cross section on carbon.... 186

8-24 Shape comparison of the $\bar{\nu}_{\mu}$ CCQE cross section on carbon to MC $\ldots \ldots \ldots 187$

8-25 Shape comparison of the single-differential cross section on mineral oil ..... 188

8-26 Comparison between the $\nu_{\mu}$ and $\bar{\nu}_{\mu}$ data from MiniBooNE and NOMAD ... 188

8-27 Total cross section for $\bar{\nu}_{\mu}$ CCQE on carbon $\ldots \ldots \ldots \ldots \ldots \ldots \ldots \ldots \ldots \ldots$

8-28 Total cross section for $\nu_{\mu}$ and $\bar{\nu}_{\mu}$ CCQE on carbon $\ldots \ldots \ldots \ldots \ldots \ldots \ldots \ldots$

9-1 $\nu_{\mu}$ and $\bar{\nu}_{\mu}$ CCQE cross sections used in the correlation analysis $\ldots \ldots \ldots \ldots 6$

9-2 Overall correlation coefficients between the $\nu_{\mu}$ and $\bar{\nu}_{\mu}$ CCQE cross sections . . 197

9-3 Correlation coefficients by uncertainty type $\ldots \ldots \ldots \ldots \ldots \ldots \ldots \ldots \ldots \ldots \ldots$

9-4 Difference measurements of the $\nu_{\mu}$ and $\bar{\nu}_{\mu}$ CCQE cross sections $\ldots \ldots \ldots \ldots$

9-5 Ratio measurements of the $\nu_{\mu}$ and $\bar{\nu}_{\mu}$ CCQE cross sections $\ldots \ldots \ldots \ldots \ldots \ldots$ 


\section{Abstract of Dissertation Presented to the Graduate School of the University Of Florida in Partial Fulfillment of the Requirements for the Degree of Doctor of Philosophy \\ FIRST MEASUREMENT OF THE MUON ANTI-NEUTRINO CHARGED CURRENT QUASIELASTIC DOUBLE-DIFFERENTIAL CROSS SECTION}

By

\section{Joseph M. Grange}

May 2013

\section{Chair: Heather Ray}

Major: Physics

This dissertation presents the first measurement of the muon antineutrino charged current quasi-elastic double-differential cross section. These data significantly extend the knowledge of neutrino and antineutrino interactions in the $\mathrm{GeV}$ range, a region that has recently come under scrutiny due to a number of conflicting experimental results. To maximize the precision of this measurement, three novel techniques were employed to measure the neutrino background component of the data set. Representing the first measurements of the neutrino contribution to an accelerator-based antineutrino beam in the absence of a magnetic field, the successful execution of these techniques carry implications for current and future neutrino experiments.

Finally, combined measurements of these antineutrino and the previously-published neutrino cross section data using the same apparatus maximize the extracted information from these results by exploiting correlated systematic uncertainties. The results of this analysis will help to understand signal and background processes in present and future long-baseline neutrino experiments, the principle goal of which is to measure the ordering of the neutrino masses and a process that may ultimately explain the origin of our matter-dominated universe. 


\section{CHAPTER 1 \\ INTRODUCTION}

The work presented here represents a major step forward in experimentally understanding the behavior of muon neutrinos and antineutrinos. Apart from providing a world's-first measurement of these interactions in a mostly-unexplored energy region, these data advance the neutrino community's preparedness to search for an asymmetry between matter and anti-matter that may well provide the physical mechanism for the existence of our universe.

The details of these measurements are preceded by brief summaries of the history of the neutrino (Chapter 2), the phenomenon of neutrino oscillations (Chapter 3), and a description of their interactions (Chapter 4). Details of the experimental setup for the measurements are given in Chapter 5. Chapter 6 introduces the muon antineutrino cross-section measurement and motivates the need for dedicated, in situ background constraints. The world's first measurements of the neutrino component of an antineutrino beam using a non-magnetized detector, as well as other crucial background constraints, are presented in Chapter 7. The muon antineutrino cross-section measurement is given in Chapter 8. By exploiting correlated systematic uncertainties, combined measurements of the muon neutrino and antineutrino cross sections described in Chapter 9 maximize the precision of the extracted information from both results. Finally, the results are summarized in Chapter 10. 


\section{CHAPTER 2}

\section{NEUTRINO OVERVIEW}

This chapter touches on the various milestone measurements in the history of the neutrino and outlines many of their basic properties. As perhaps the most important development in its young history, a more complete development and review of neutrino oscillations is saved for Chapter 3.

\subsection{Discovery}

At the beginning of the twentieth century, much of the physics community were content to believe the universe was fundamentally composed of electrons, photons and, in the literal sense, atoms. An example of historically bad advice given by an advisor to their student came when the supervisor of Max Planck suggested that "in this field, almost everything is already discovered, and all that remains is to fill a few unimportant holes" [1]. Planck went on to revolutionize the field with his description of quantum mechanics and ultimately helped expose human knowledge to a litany of new particles and phenomena.

And so, like many of the particles discovered in the twentieth century, the neutrino came as a surprise. The first hints came through observations of so-called $\beta$ decay in the 1920's, where a neutron inside a nucleus spontaneously decays. Though the picture of the proton and neutron structure of the nucleus was not yet clear, by energy and spin conservation the decay was believed to be a two-body process:

$$
n \rightarrow p+e^{-}, E_{e}=\frac{m_{n}^{2}+m_{e}^{2}-m_{p}^{2}}{2 m_{n}},
$$

where $E_{e}$ is the energy of the ejected electron in the rest frame of the neutron and $m_{n}, m_{p}$, and $m_{e}$ are the neutron, proton, and electron masses, respectively. As the neutrons housed in nuclei are on average at rest, the observed electron spectrum ought to be nearly monoenergetic. Multiple experiments using a variety of $\beta$-decay sources conclusively rejected this hypothesis [2]. 
Of the many alternate explanations for the observed electron spectrum, Wolfgang Pauli proposed in 1931 the ultimately proven hypothesis: the products of $\beta$ decay include a third, electrically neutral particle of mass far less than the electron and whose interactions are rare enough to have escaped direct detection. The $\beta$ decay reaction is now described as:

$$
n \rightarrow p+e^{-}+\nu, E_{e} \in\left\{m_{e}, \frac{m_{n}^{2}+m_{e}^{2}-\left(m_{p}^{2}+m_{\nu}^{2}\right)}{2 m_{n}}\right\}
$$

where the presence of the neutrino kinematically allows the electron to have a continuous energy spectrum. Regarding the proposed feeble interaction rate of the neutrino, Pauli famously quipped "I have done a terrible thing. I have postulated a particle that cannot be detected" [3].

Fortunately, it took only a few decades for experimental technology and techniques to reach the level of precision necessary to directly observe the neutrino. If Pauli's interpretation of the $\beta$ decay spectrum and Enrico Fermi's extended description of the particle $[4,5]$ were correct, two prolific sources of neutrinos in the 1950's were available in atomic explosions and nuclear reactors. One of the early plans to detect the neutrino involved a retrospectively comical proposal to detonate a dedicated atomic bomb while a neutrino detector was simultaneously dropped down a nearby mineshaft [6]. They eventually proceeded with a more pacific design, aiming to observe neutrinos produced in a nuclear reactor.

If Eq. 2.2 is the correct description of $\beta$ decay, the inverted process induced by a neutrino should also be allowed: $\nu+p \rightarrow n+e^{+}$. In a tank of liquid scintillator doped with cadmium, the positron produced in this inverted $\beta$ decay reaction will annihilate with an in-medium electron, producing two prompt gamma rays emitted in opposing directions $\left(e^{+}+e^{-} \rightarrow 2 \gamma, \theta_{\gamma \gamma}=\pi\right)$. The neutron has a large probability for being captured on the cadmium nuclei, and the characteristic de-excitation photons following this process 


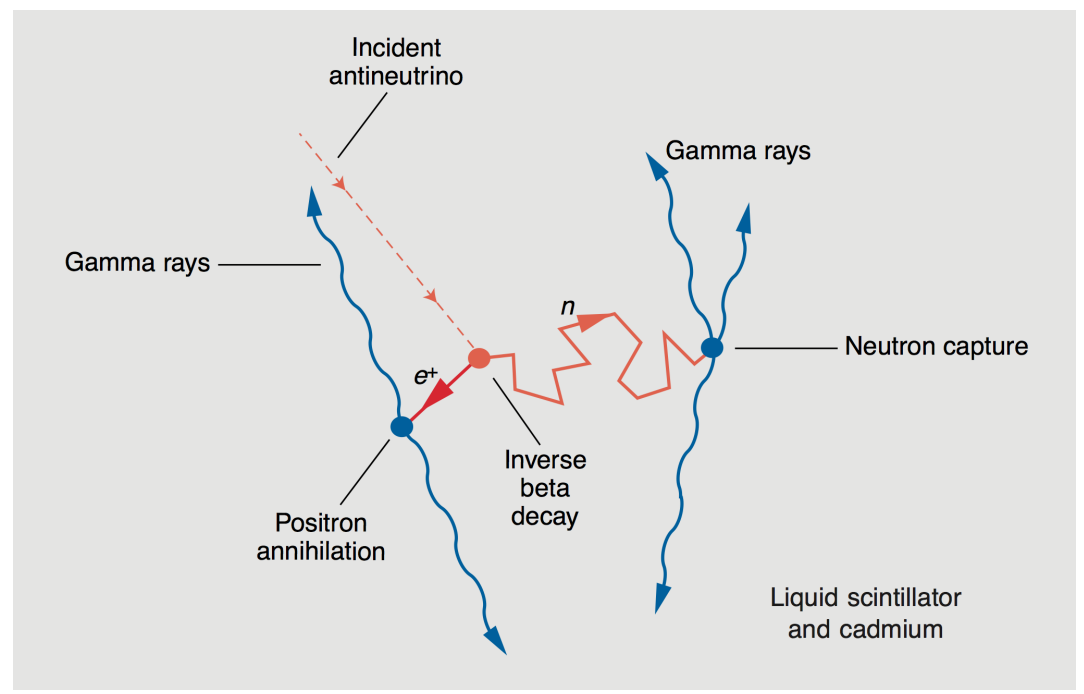

Figure 2-1. The detection scheme for the first conclusive demonstration of the existence of the neutrino. Image from Ref. [7].

provides a clean neutron signature. The detection schematic is shown in Figure 2-1.

Photomultiplier tubes collect the photons from the annihilation and capture reactions, and data from a detector using these principles yielded the first definitive detection of the neutrino in 1956 [8].

\subsection{Interaction and Propagation States}

Any intelligent discussion of the nature of the neutrino must be built on an understanding of its peculiarly misaligned interaction and propagation eigenstates. Generically, an eigenstate is a vector returned by the action of a particular operator. Neutrinos only interact through the weak force, so the eigenstates available upon action with the weak operator (or, more intuitively, upon an interaction with a $W$ or $Z$ boson) are the weakly-participating leptonic "flavors" $e, \mu$, and $\tau$. The other eigenstate that governs the behavior of the neutrino is its Hamiltonian state. This describes the physical propagation of the neutrino in time with a definite value of mass. Throughout this work, the interaction eigenstate is often referred to as the weak, or flavor, state, just as the Hamiltonian state is equated with the propagation, or mass, eigenstate.

For most fundamental particles, their interaction and Hamiltonian eigenstates are 
indistinguishable. However, nothing demands this be the case, and a divergence between the states has been observed in two leptonic sectors: quarks and neutrinos. A helpful way to describe the relationship between the flavor and Hamiltonian eigenstates is a unitary mixing matrix $U$ that connects the arbitrary flavor state $\left|\psi_{\alpha}\right\rangle$ to the mass states $\left|\psi_{k}\right\rangle$ : $\left|\psi_{\alpha}\right\rangle=\sum_{\alpha} U_{\alpha k}^{*}\left|\psi_{k}\right\rangle$. Precision measurements of a variety of baryonic weak interactions yield the following approximate relationship between the quark Hamiltonian and flavor states [9]:

$$
\left(\begin{array}{l}
\psi_{d^{\prime}} \\
\psi_{s^{\prime}} \\
\psi_{b^{\prime}}
\end{array}\right)=\left(\begin{array}{lll}
0.974 & 0.225 & 0.003 \\
0.225 & 0.973 & 0.041 \\
0.009 & 0.040 & 0.999
\end{array}\right)\left(\begin{array}{l}
\psi_{d} \\
\psi_{s} \\
\psi_{b}
\end{array}\right)
$$

where $d^{\prime}, s^{\prime}$, and $b^{\prime}$ refer to the quarks of flavor down, strange, and bottom, respectively, and the convention of using $d, s$, and $b$ for the Hamiltonian states is used. Note that, due to the unitary nature of $U$, this description of the mixing in terms of the down-type quarks instead of the up-type quarks is arbitrary.

The same relationship for neutrino mixing, the formalism for which and whose measurements are described in Chapter 3, is given by:

$$
\left(\begin{array}{l}
\psi_{e} \\
\psi_{\mu} \\
\psi_{\tau}
\end{array}\right)=\left(\begin{array}{ccc}
0.822 & 0.547 & -0.150 \\
-0.356 & 0.704 & 0.614 \\
0.442 & -0.452 & 0.774
\end{array}\right)\left(\begin{array}{l}
\psi_{1} \\
\psi_{2} \\
\psi_{3}
\end{array}\right)
$$

where the values shown assume the mass hierarchy to be normal and the CP-violating phase $\delta$ to be zero (see Chapter 3 for details of both quantities). Eqs. 2.3 and 2.4 clearly show the flavor and mass eigenstates to be distinct for neutrinos and quarks, but also that the details of this mixing differ greatly between the two species. The origin of these mixing 
parameters is not understood, and is one of the outstanding issues preventing a fundamental understanding of the weak interaction.

\subsection{Flavors of Neutrinos}

We now know that the creation or annihilation of a charged lepton $(e, \mu, \tau)$ must also involve either its own charged antiparticle or a neutrino (or antineutrino) of the same flavor $\left(\nu_{e}, \nu_{\mu}, \nu_{\tau}\right)$. This certainly did not need to be the case, and the prediction of this lepton conservation symmetry in the Standard Model (SM) did not arrive until decades after the discovery of the neutrino.

Following the discovery of the neutrino, many experiments contributed to the quickly-growing body of knowledge regarding its properties. Likely the most significant in this period, both in terms of the engineering that would become the future of the field and the milestone it represented in the emerging picture of the neutrino, came from the first observation of neutrinos from an accelerator-based beam [10]. The experiments, led by L. Lederman, observed an off-axis flux of neutrinos dominantly created from $\pi^{+}$decay, the reaction of which was known to proceed via $\pi^{+} \rightarrow \mu^{+}+\nu$. However, unlike in the first observation of the neutrino using a nuclear reactor source, the charged particles that emerged in the detector were negatively charged muons, not positrons. This was particularly compelling because it could have been the case that muons were not created in reactor neutrino interactions simply because because their production would not be energetically allowed: the energy of the source $(<10 \mathrm{MeV})$ is much less than the muon mass $(\sim 105 \mathrm{MeV})$. That no positrons or electrons were observed in the accelerator-based experiment conclusively demonstrated the reactor neutrinos to be distinct from those created in $\pi^{+}$decays. Through the development of the SM, these soon became to be known as electron and muon flavored neutrinos $\nu_{e}$ and $\nu_{\mu}$.

With the discovery of the $\tau$ particle in 1975 [11], a third fundamental neutrino was presumed to accompany it. However, the large mass of the $\tau\left(m_{\tau} \sim 1.8 \mathrm{GeV}\right)$ and its rapid decay (with a lifetime of $\mathcal{O}\left(10^{-13}\right)$ s) with a number of both hadronic and leptonic modes 


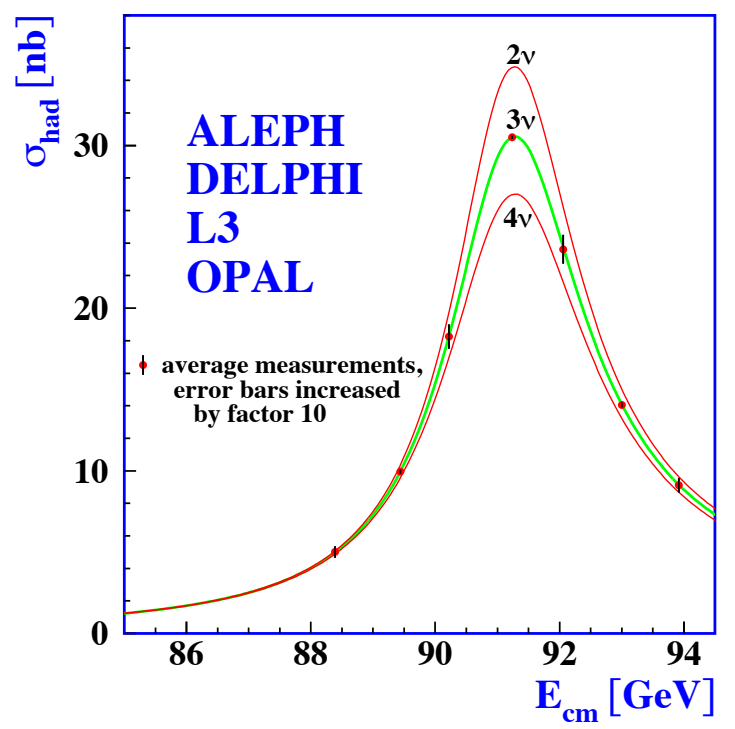

Figure 2-2. Results from a combined analysis of precision measurements of the $Z^{0}$ width. The number of light, active neutrino species is determined to be $2.9840 \pm 0.0082$, consistent with direct observations of the $\nu_{e}, \nu_{\mu}$, and $\nu_{\tau}$ neutrinos. Figure from Ref. [13].

available made the direct observation of the $\nu_{\tau}$ particularly challenging. It wasn't until the year 2000 that the particle was experimentally confirmed, when a team observed four candidate $\nu_{\tau}$ events on a calculated background of 0.34 from an accelerator-based beam of $\sim 100 \mathrm{GeV} \nu_{\tau}$ 's $[12]$. Since then, not more than twenty total $\nu_{\tau}$ events have been observed, making it one of the least experimentally-probed SM members.

Finally, consistent with the direct observations discussed in this section, precision measurements of the $Z^{0}$ boson width has definitively concluded that there exist exactly three neutrino flavors with effective mass less than half the $Z^{0}$ mass and whom also participate in the weak interaction [13]. Figure 2-2 presents the measurement of the $Z^{0}$ width.

\subsection{Chirality}

In the same year the neutrino was discovered, another paramount experimental result revealed an unexpected aspect of the weak interaction. Using a magnetic field to polarize the spin of a collection of unstable ${ }^{60} \mathrm{Co}$ atoms, the observed direction of the emitted electron in the $\beta$-decay reaction was nearly always opposite to the direction of the 
aligned spin of the parent nuclei [14]. The nearly-perfect anti-correlation between the nuclei spins and the electron direction indicated that the mirror-symmetry of parity is violated in weak interactions. It had been suggested previously that the parity symmetry in the weak interaction need not be strictly conserved [15], but the experimental evidence concluded that parity violation was maximal. The implication for the behavior of observable neutrinos was clear: to balance the spin and momentum of the reaction, the observed kinematics of the electron dictate that it must be accompanied by an antineutrino with a definite alignment between its momentum and spin vectors. These data were ambiguous between the two vectors being aligned or anti-aligned for the neutrino, but a few years later a clever technique was executed to measure this correlation: observations of the polarization of de-excitation photons following orbital electron capture on nuclei determined the spin and momentum vectors for the neutrino to be anti-aligned [16]. The inner product between a particle's spin and momentum at any instant is known as it's helicity, and the natural preference for helicity values (if any) is the more fundamental quantity of chirality. Thus, using the mechanical analogy of ordinary screws, the chirality of leptons in the weak interaction is left-handed, while (via the CPT theorem) anti-leptons are right-handed.

One of the direct consequences of neutrino oscillations is the implication of non-zero neutrino mass. With non-zero neutrino mass, it is in principle possible to boost to a frame with velocity $v$ such that $v_{\nu}<v<c$, in which a neutrino (antineutrino) would have positive (negative) helicity.

Worth noting, the correlation between chirality and helicity is perfect for massless particles and decreases sharply for those of non-zero mass, and so the production of positive helicity $e^{-}$is much more allowed relative the emission of negative-helicity antineutrinos. A consequence of this suppression that is crucial to the experimental neutrino program is that the electronic decay of pions $\left(\pi \rightarrow e+\nu_{e}\right)$ relative to the muonic decay $\left(\pi \rightarrow \mu+\nu_{\mu}\right)$ is suppressed by approximately $\left(m_{e} / m_{\mu}\right)^{2}\left(\frac{m_{\pi}^{2}-m_{e}^{2}}{m_{\pi}^{2}-m_{\mu}^{2}}\right)^{2} \sim 1.2 \times 10^{-4}$. This allows for high-purity sources of $\nu_{\mu}$ and $\bar{\nu}_{\mu}$ from the decay of pions in accelerator 
environments, and the control of the beam energy and propagation distance afforded by these artificial sources has made this the standard for probing neutrino oscillation physics.

\subsection{Magnetic Moment}

Non-zero neutrino mass allows for the possibility of a magnetic moment. Though electrically neutral, electromagnetic properties of neutrinos may be accessed through magnetic couplings with photons in loop diagrams. Much like neutrino mass, the magnetic moment would be a property instrinsic to the Hamiltonian eigenstate, and therefore observations of magnetic moments through weak interactions probe superpositions of the true quantities. This relationship can be described as [17]:

$$
\mu_{\alpha}^{2}=\sum_{j}\left|\sum_{k} U_{\alpha k} e^{-i E_{k} L} \mu_{j k}\right|^{2}
$$

where $j$ and $k$ index the Hamiltonian eigenstates, $U$ connects the Hamiltonian eigenstates to the flavor state $\alpha, E$ and $L$ are the energy and travel distance of the neutrino, respectively, and $\mu_{j k}$ describes the coupling of the mass eigenstates to the electromagnetic field.

As a calculable SM process, the neutrino-electron elastic scattering channel $\nu_{\alpha}+e \rightarrow \nu_{\alpha}+e$ is typically used to search for the neutrino's magnetic moment. Evidence of an electromagnetic coupling between the neutrino and electron would present itself as events in excess of the predicted cross section or a distortion in the recoiling electron spectrum.

Many searches for the neutrino magnetic moment have been executed using astrophysical data $[18,19,20]$ and more direct observations of neutrinos from solar [21], accelerator [22], reactor [23], and supernova [24] sources. The current best limit on the effective magnetic moment for any neutrino species comes from observations of reactor $\bar{\nu}_{e}$ 's, 
where it was found $\mu_{\bar{\nu}_{e}}<7.4 \times 10^{-11} \mu_{B}$, where $\mu_{B}=e / 2 m_{e}$ (using natural units) is the Bohr magneton, at $90 \%$ confidence level (C.L.) [25].

\subsection{Absolute Mass}

From neutrino oscillation observations, the mass of at least two of the neutrino states is known to be non-zero, but the sensitivity of various experimental tests have not yet reached the level of precision required to measure their values. Also known from neutrino oscillations, the most massive state is greater or equal to $\sqrt{\Delta m_{32}^{2}} \sim 0.05 \mathrm{eV}$. Meanwhile, observations of cosmological radiation set an upper limit on the sum of active neutrino masses of $\sum m_{\nu}<0.2-0.4 \mathrm{eV}$ at $95 \%$ C.L., where the limit depends on assumptions used to analyze the Lyman- $\alpha$ data [26]. One of the current prospects in probing lower mass regions involves the very interaction that led to the neutrino's discovery: $\beta$ decay. As suggested by Eq. 2.2, the endpoint of the $\beta$ decay spectrum is sensitive to the mass of the $\bar{\nu}_{e}$. This measurement would give the effective mass of the $\bar{\nu}_{e}$, which is a superposition of the true mass states according to their coupling with the electron-flavor neutrino. Currently the best limit based on observations of the endpoint of the $\beta$ decay spectrum is $m_{\nu_{e}}<2.3 \mathrm{eV}$ (95\% C.L.) [27], while the next-generation experiments aim to achieve sub-eV precision [28, 29].

\subsection{Neutrino Sources}

Though the neutrino remains rather poorly understood, many natural and artificial sources spanning an immense energy range are available to continue to probe its nature. Figure 2-3 shows the various neutrino sources and their approximate spectral contributions.

Generally, technology for direct neutrino detection is most effective for neutrinos of energy $\sim 10^{6}-10^{11} \mathrm{eV}$. While we have learned a great deal from observations of this energy range, Figure 2-3 suggests there are many opportunities to expand this knowledge using freely available neutrinos. It may be possible to learn more not only about the neutrino, but there may be rich physics in their production mechanisms as well. 


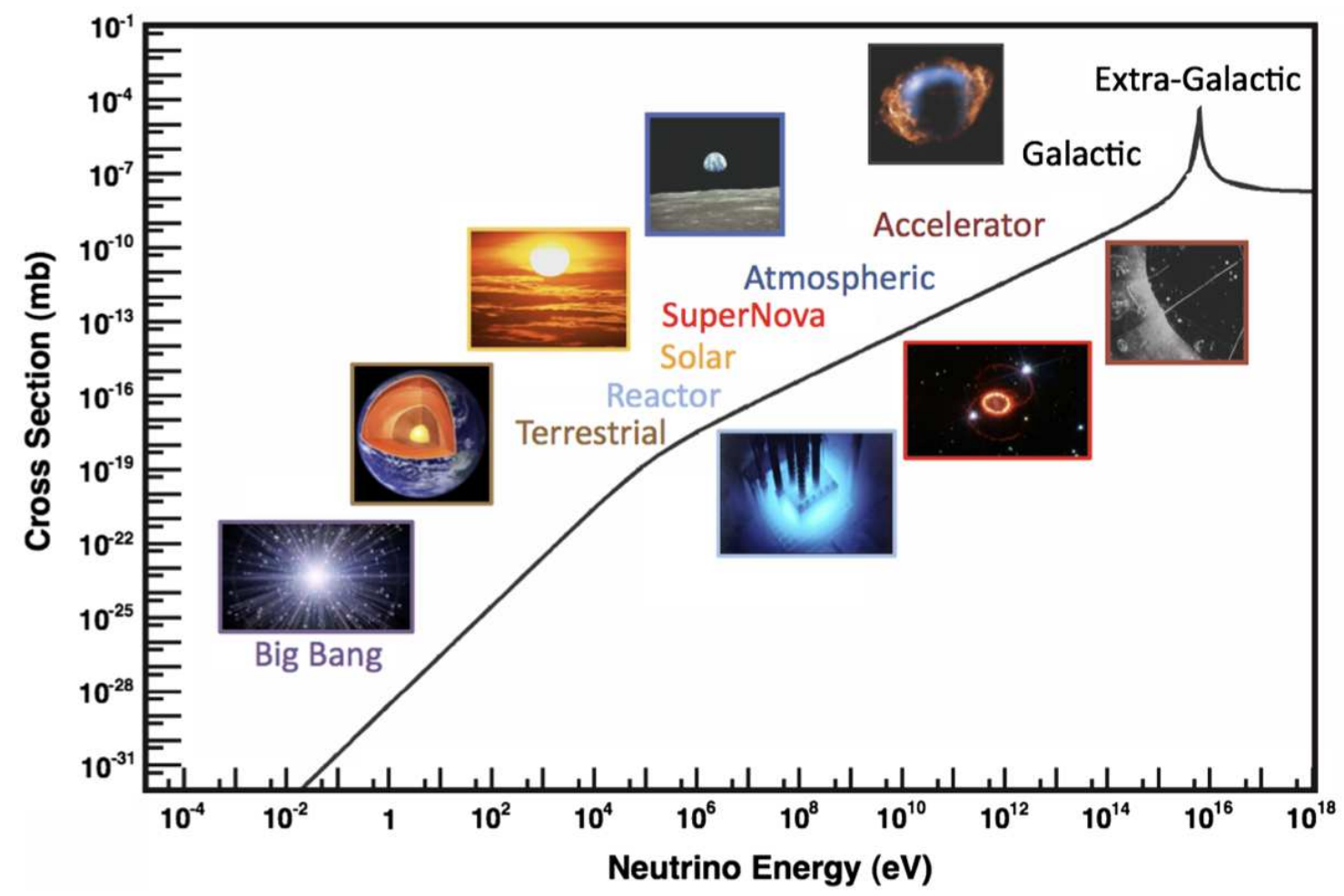

Figure 2-3. Summary of the various prolific neutrino sources in the universe. The cross section for $\bar{\nu}_{e}+e^{-} \rightarrow \bar{\nu}_{e}+e^{-}$is shown for comparison. Figure from Ref. [30].

As an example, a preliminary analysis of two neutrino events of energy $>10^{12} \mathrm{eV}$ was recently reported [31]. These neutrinos have the highest energy ever recorded, and their origin is not clear [32,33]. At the low end of the spectrum, neutrino remnants from the Big Bang are predicted to still permeate the universe at a density of $\sim 100 \mathrm{~cm}^{-3}$. Figure 2-3 shows the cross section for these neutrinos are many orders of magnitude below the currently-accessible range; however, if these neutrinos could be observed, it would be a fantastic addition to the body of evidence for the birth of our universe. 


\section{CHAPTER 3 \\ NEUTRINO OSCILLATIONS}

The phenomenon of neutrino oscillations and its immediate consequence of non-zero neutrino mass is one of the very few particle physics results not predicted by the SM. This chapter describes the discovery of this process, the state of knowledge as of today, and it's phenomenological implications.

\subsection{Formalism}

Central to any discussion of neutrino oscillations is the divergence between its interaction and Hamiltonian eigenstates, an introduction to which is given in Section 2.2. The state of the neutrino accessible by experiments is the interaction eigenstate, which is typically determined by the flavor of charged lepton produced as a result of charged current (CC) interactions $\left(\nu_{l}+X \rightarrow l+X^{\prime}, l=e, \mu, \tau\right)$. If the mechanism through which the neutrino is created is known precisely, then the weak eigenstate at the time of creation is also known. To characterize neutrino oscillations we must dynamically describe the connection between creation and detection. Since the neutrino propagates in its Hamiltonian eigenstate, we begin there. The neutrino with mass eigenstate $i$ will evolve in time according to the time-dependent Schröedinger equation:

$$
\begin{aligned}
i \frac{\partial}{\partial t}\left|\psi_{i}(t)\right\rangle & =\hat{H}\left|\psi_{i}(t)\right\rangle=\sqrt{\left(p_{i}^{2}+m_{i}^{2}\right)}\left|\psi_{i}(t)\right\rangle=p_{i} \sqrt{1+\frac{m_{i}^{2}}{p_{i}^{2}}}\left|\psi_{i}(t)\right\rangle \\
& \approx\left(E+\frac{m_{i}^{2}}{2 E}\right)\left|\psi_{i}(t)\right\rangle
\end{aligned}
$$

where natural units of $\hbar=c=1$ are used, $p$ is the neutrino momentum and $m$ its mass. The neutrino is assumed to travel through free space, and as its mass is much smaller than its momentum for all practical applications, terms of order two and higher in $\left(m_{i}^{2} / p_{i}^{2}\right)$ in the expansion are ignored. This also implies the neutrino energy $E \approx p_{i}$ for each mass state $i$. A solution to this first-order differential equation is immediately apparent: 


$$
\left|\psi_{i}(t)\right\rangle=e^{-i\left(E+m_{i}^{2} / 2 E\right) t}\left|\psi_{i}(0)\right\rangle
$$

This form is particularly convenient because the only time a neutrino's propagation eigenstate corresponds exactly to a single flavor eigenstate occurs in coincidence with its creation. Choosing $t=0$ as the time of a weak interaction to create a neutrino of flavor eigenstate $\alpha$, the propagation state $i$ can be written as:

$$
\left|\psi_{i}(0)\right\rangle=\left|\psi_{\alpha}\right\rangle=\sum_{k} U_{\alpha k}^{*}\left|\psi_{k}\right\rangle
$$

where the arbitrary unitary matrix $U$ describes the coupling between the propagation and interaction eigenstates. If the propagation and interaction eigenstates were identically equal, $U$ would simply be the identity matrix and neutrinos would not oscillate. Substituting Equation 3.3 into Equation 3.2 and again exploiting the assumption of negligible neutrino mass compared to its energy so that $t \approx L$, where $L$ is the distance propagated in time $t$, we find the probability density of a neutrino created in weak eigenstate $\alpha$ after traveling a distance $L$ to be:

$$
\left|\psi_{\alpha}(t=L)\right\rangle=\sum_{\beta}\left(\sum_{k} U_{\alpha k}^{*} e^{-i\left(E+m_{i}^{2} / 2 E\right) L}\left|\psi_{k}\right\rangle U_{\beta k}\right)\left|\psi_{\beta}\right\rangle,
$$

where $\beta$ also indexes the weak eigenstates. Now we can write the probability for a neutrino created in weak eigenstate $\alpha$ to be detected in state $\beta$ as a function of only its energy and the distance traversed: 


$$
\begin{aligned}
P\left(\psi_{\alpha} \rightarrow \psi_{\beta}\right)= & \left|\left\langle\psi_{\beta} \mid \psi_{\alpha}(L)\right\rangle\right|^{2}=\left|\sum_{k} U_{\alpha k}^{*} U_{\beta k} e^{-i\left(E+m_{i}^{2} / 2 E\right) L}\right|^{2} \\
= & \sum_{k j} U_{\alpha k}^{*} U_{\beta k} U_{\alpha j} U_{\beta j}^{*} \exp \left(-i \frac{\Delta m_{k j}^{2} L}{2 E}\right) \\
= & \delta_{\alpha \beta}-4 \sum_{k>j} \operatorname{Re}\left[U_{\alpha k}^{*} U_{\alpha j} U_{\beta k} U_{\beta j}^{*}\right] \sin ^{2}\left(\frac{\Delta m_{k j}^{2} L}{4 E}\right) \\
& +2 \sum_{k>j} \operatorname{Im}\left[U_{\alpha k}^{*} U_{\alpha j} U_{\beta k} U_{\beta j}^{*}\right] \sin \left(\frac{\Delta m_{k j}^{2} L}{2 E}\right)
\end{aligned}
$$

where $\Delta m_{k j}^{2} \equiv m_{k}^{2}-m_{j}^{2}$ is referred to as the "mass splitting" between the Hamiltonian eigenstates $\psi_{k}$ and $\psi_{j}$, and the unitary nature of the matrix $U$ is used in the last step.

Eq. 3.5 is valid for any number of neutrino species; in the next two sections we consider the case of three and two species. Three neutrino species is consistent with direct observations of neutrino flavors (Section 2.3), and the study of two species is particularly instructive to understand oscillatory behavior and also gives an excellent approximation for most experimental probes of the phenomenon.

\subsubsection{Three-Neutrino Mixing}

With three observed flavors of neutrinos $\left(\psi_{\alpha}=\psi_{e}, \psi_{\mu}, \psi_{\tau}\right)$, it is natural to assume there also exist three Hamiltonian eigenstates $\left(\psi_{i}=\psi_{1}, \psi_{2}, \psi_{3}\right)$. This is analogous to the observed mixing in the quark sector. Though many parametrizations of the mixing matrix $U$ are possible, the canonical choice follows the form of the quark-mixing matrix. Under this choice it is referred to as the Pontecorvo-Maki-Nakagawa-Sakata (PMNS) matrix. Using the PMNS matrix, the flavor states are related to the Hamiltonian states by:

$$
\left(\begin{array}{l}
\psi_{e} \\
\psi_{\mu} \\
\psi_{\tau}
\end{array}\right)=\left(\begin{array}{ccc}
c_{12} c_{13} & s_{12} c_{13} & s_{13} e^{-i \delta} \\
-s_{12} c_{23}-c_{12} s_{23} s_{13} e^{i \delta} & c_{12} c_{23}-s_{12} s_{23} s_{13} e^{i \delta} & s_{23} c_{13} \\
s_{12} s_{23}-c_{12} c_{23} s_{13} e^{i \delta} & -c_{12} s_{23}-s_{12} c_{23} s_{13} e^{i \delta} & c_{23} c_{13}
\end{array}\right)\left(\begin{array}{c}
\psi_{1} \\
\psi_{2} \\
\psi_{3}
\end{array}\right)
$$


where $c_{i j} \equiv \cos \left(\theta_{i j}\right)$ and $s_{i j} \equiv \sin \left(\theta_{i j}\right)$ are trigonometric functions of the amplitude for mixing between the Hamiltonian eigenstates $i$ and $j$, and $\delta$ is an arbitrary phase that allows for neutrinos to oscillate differently than antineutrinos [34]. Under the assumptions of $m_{3}>m_{1}$ and $\delta=0$, the values of the PMNS matrix are given in Eq. 2.4. An advantage of the $U^{\text {PMNS }}$ matrix is that it may be factored to isolate the effects of each mixing angle $\theta_{i j}$ :

$$
\begin{aligned}
U^{\mathrm{PMNS}}=\left(\begin{array}{ccc}
1 & 0 & 0 \\
0 & \cos \left(\theta_{23}\right) & \sin \left(\theta_{23}\right) \\
0 & -\sin \left(\theta_{23}\right) & \cos \left(\theta_{23}\right)
\end{array}\right) \times\left(\begin{array}{ccc}
\cos \left(\theta_{13}\right) & 0 & \sin \left(\theta_{13}\right) e^{-i \delta} \\
0 & 1 & 0 \\
-\sin \left(\theta_{13}\right) e^{i \delta} & 0 & \cos \left(\theta_{13}\right)
\end{array}\right) \\
\times\left(\begin{array}{ccc}
\cos \left(\theta_{12}\right) & \sin \left(\theta_{12}\right) & 0 \\
-\sin \left(\theta_{12}\right) & \cos \left(\theta_{12}\right) & 0 \\
0 & 0 & 1
\end{array}\right)
\end{aligned}
$$

As will be described further, most experimental data are consistent with the existence of exactly three Hamiltonian eigenstates and three weakly-interacting neutrinos whose effective mass state lies well below the $Z^{0}$ boson mass of $\sim 91 \mathrm{GeV}$. Under these conditions, the PMNS matrix fully describes the phenomenon of neutrino oscillations. However, some experimental evidence supports the existence of additional Hamiltonian eigenstates. The strongest hints come from the LSND [35] and MiniBooNE [36] experiments. If these signals are confirmed, the PMNS matrix would have to be significantly extended to accommodate the additional degrees of freedom [37]. Data from these experiments and their implications are further discussed in Section 3.2.4.

\subsubsection{Two-Neutrino Mixing}

In the case of only two neutrino flavor $(\alpha, \beta)$ and Hamiltonian $(1,2)$ eigenstates, the matrix $U$ can be expressed in terms of a single mixing angle $\theta$ : 


$$
\left(\begin{array}{l}
\psi_{\alpha} \\
\psi_{\beta}
\end{array}\right)=\left(\begin{array}{cc}
\cos \theta & \sin \theta \\
-\sin \theta & \cos \theta
\end{array}\right)\left(\begin{array}{l}
\psi_{1} \\
\psi_{2}
\end{array}\right),
$$

With this simplified mixing matrix, the arbitrary oscillation probability (Eq. 3.5) reduces to:

$$
P\left(\psi_{\alpha} \rightarrow \psi_{\beta}\right)=\delta_{\alpha \beta}-\sin ^{2} 2 \theta \sin ^{2}\left(1.267 \frac{\Delta m^{2} L}{E}\right),
$$

where the units of $\Delta m^{2}=m_{1}^{2}-m_{2}^{2}, L$ and $E$ are $\mathrm{eV}^{2}, \mathrm{~km}$, and $\mathrm{GeV}$, respectively, and the factor of 1.267 incorporates numerical constants including the factors of $\hbar$ and $c$ ignored previously. Even a cursory examination of Eq. 3.9 shows how the oscillation parameters $\Delta m^{2}$ and $\theta$ affect the experimental signature $L$ and $E$ : the amplitude of the oscillation probability is proportional to $\theta$, while $\Delta m^{2}$ sets the frequency for oscillation as a function of the ratio $L / E$.

The consequences of Eq. 3.9 are worth a few more remarks:

- for a given mixing amplitude $\theta$, the oscillation probability is maximized for $L / E \sim\left(\Delta m^{2}\right)^{-1}$. This informs experimentalists how to choose the parameters $L$ and $E$ to gain sensitivity to a certain region of mass splitting $\Delta m^{2}$. It follows that if the employed $L$ and $E$ are such that $\Delta m^{2} L / E \ll 1$, the effect of $\Delta m^{2}$ on observables will be minimal.

- as $\sin ^{2} \theta$ is an even function in its argument, neutrino oscillations are only sensitive to the absolute value of $\Delta m^{2}$ - that is, the more massive state between the two participating Hamiltonian eigenstates cannot be determined from oscillation observations alone.

- if $\Delta m^{2}=0, P\left(\psi_{\alpha} \rightarrow \psi_{\beta}\right)=\delta_{\alpha \beta}$ and neutrinos do not oscillate. This would imply the 
Hamiltonian eigenstates probed have the same mass, whether zero or non-zero.

- if $\theta=0$, again $P\left(\psi_{\alpha} \rightarrow \psi_{\beta}\right)=\delta_{\alpha \beta}$ and neutrinos do not oscillate. This would imply $U$ reduces to the identity matrix and the neutrino interaction and Hamiltonian eigenstates are identically equal.

It follows from the last two observations that an immediate consequence of the confirmation of neutrino oscillations is that there exist at least as many neutrino Hamiltonian states with non-zero mass as the observed number of mass splittings $\Delta m^{2}$, and the weak and Hamiltonian eigenstates mix. Though the above conditions are most readily recognized with the neutrino oscillation probability under the assumption of only two participating species, they apply equally to the arbitrary case of Eq. 3.5.

As mentioned previously, neutrino oscillations were not predicted in the SM and so the scales of $\Delta m^{2}$ values were completely unconstrained. Fortunately, nature has provided us with two sources of organic neutrinos whose energy $E$ and distance from creation to Earthly detection $L$ is such that their ratio $L / E$ probes two independent neutrino mass splittings. Experiments using artificial neutrino sources such as accelerator-based beams and neutrinos emitted from nuclear reactors have confirmed and refined measurements of these oscillation parameters. Neutrinos from nuclear reactors have very recently also provided measurements of the mixing angle $\theta_{13}$. The experimental evidence for each follows.

\subsection{Experimental Evidence for Neutrino Oscillations}

Assuming three Hamiltonian eigenstates $\left(\psi_{1}, \psi_{2}\right.$, and $\left.\psi_{3}\right)$ and three flavor eigenstates $\left(\psi_{e}, \psi_{\mu}\right.$ and $\left.\psi_{\tau}\right)$, there exist two independent mass splittings $\Delta m_{12}^{2}$ and $\Delta m_{23}^{2}$ (since $\left|\Delta m_{13}^{2}\right|=\left|\Delta m_{12}^{2} \pm \Delta m_{23}^{2}\right|$ ) that mix with the weak eigenstates through three independent mixing angles and one CP-violating phase. The following presents their current measurements or constraints.

\subsubsection{Solar Oscillations}

Often referred to colloquially as solar neutrino oscillations, the first experimental hints of any oscillation signature were caused by the $\Delta m_{12}^{2}$ mass splitting and were 
observed in 1968 [38]. These hints remained a puzzle for more than three decades, when the SNO collaboration [39] provided observations of the entire flux of neutrinos created in solar processes having transmuted into a different flavor composition from creation to detection.

Solar neutrinos are dominantly produced as $\nu_{e}$ 's in the nuclear fusion reaction $p+p \rightarrow{ }^{2} \mathrm{H}+e^{+}+\nu_{e}$. Because of the low energy of these neutrinos $(<10 \mathrm{MeV})$, only electrons are energetically allowed to be produced in CC interactions. Therefore, if solar neutrinos were oscillating into the $\nu_{\mu}$ and $\nu_{\tau}$ weak eigenstates, their entire flux as seen on Earth could only be observed using neutral current (NC) interactions. SNO used the novel idea of employing heavy water $\left({ }^{2} \mathrm{H}_{2} \mathrm{O}\right)$ as the detection medium to exploit neutron capture on deuterium and observe both $\mathrm{NC}$ and $\mathrm{CC}$ events:

$$
\begin{array}{ccc}
\nu_{e}+{ }^{2} \mathrm{H} & \rightarrow & p+p+\mathbf{e}^{-} \\
\nu_{\alpha}+{ }^{2} \mathrm{H} & \rightarrow & p+n+\nu_{\alpha} \\
& \hookrightarrow+{ }^{2} \mathrm{H} \rightarrow{ }^{3} \mathrm{H}+\gamma \\
\nu_{\alpha}+e^{-} & \rightarrow & \nu_{\alpha}+\mathbf{e}^{-}
\end{array}
$$

where the particles observed to determine the reaction are in bold. The analysis of these three reactions is summarized in Figure 3-1. A global fit to these data show they are compatible with $\nu_{e} \rightarrow \nu_{\mu}, \nu_{\tau}$ oscillations with parameters $\Delta m_{\text {solar }}^{2} \sim 10^{-4} \mathrm{eV}^{2}$ and $\theta_{\text {solar }} \sim 34^{\circ}[40]$.

The KamLAND experiment provided an invaluable confirmation of the $\left(\Delta m_{12}^{2}, \theta_{12}\right)$ values reported by SNO using an artificial neutrino source [41]. Perhaps more compelling than the confirmation of the oscillation parameters, their data provided the first clear observation of the sinusoidal nature of neutrino oscillations as a function of $L / E$.

Observing a flux of $\bar{\nu}_{e}$ from 53 nuclear power reactors in Japan, KamLAND measured the probability for $\bar{\nu}_{e}$ disappearance using the inverse $\beta$ decay reaction 


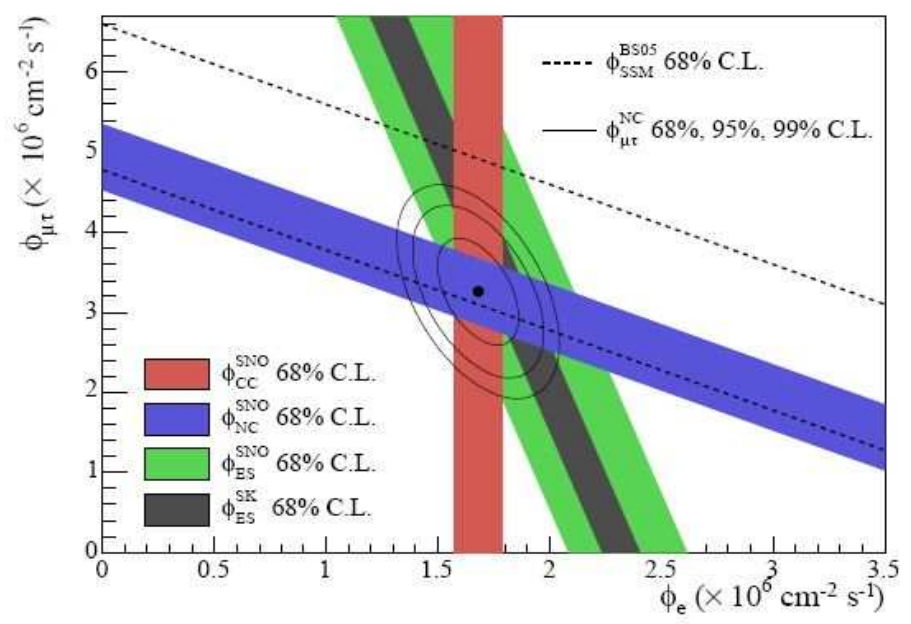

Figure 3-1. Solar $\nu_{\mu}$ and $\nu_{\tau}$ flux versus $\nu_{e}$ flux observed by the SNO detector. The Standard Solar Model (SSM) expectation is shown by the band between the dashed lines and shows good agreement with the best-fit of the SNO data, represented by the solid point. Surrounding the best fit mark are 68\%, 95\% and 99\% C.L. contours. Figure taken from Ref. [39].

$\bar{\nu}_{e}+p \rightarrow e^{+}+n$. The strong correlation between the positron and the incident antineutrino energy and direction allowed for a measurement of the oscillation probability with the ratio $L / E$, clearly supporting the trigonometric form of Eq. 3.9. This is shown in Figure 3-2, and the results of a fit for the oscillation parameters including these and the SNO data are presented in Figure 3-3. Consistency in the observed oscillatory behavior between the $\bar{\nu}_{e}$ 's at KamLAND and the solar $\nu_{e}$ 's observed at SNO supports the CPT theorem. The fit finds $\Delta m_{\text {solar }}^{2}=7.9_{-0.5}^{+0.6} \times 10^{-5} \mathrm{eV}^{2}$ and $\theta_{\text {solar }}=32.3_{-2.4}^{+3.0 \circ}[41]$. A update to this analysis using additional data from both experiments yields the most sensitive measurements of the solar oscillation parameters to date: $\Delta m_{\text {solar }}^{2}=7.59_{-0.21}^{+0.20} \times 10^{-5} \mathrm{eV}^{2}$ and $\theta_{\text {solar }}=34.06_{-0.84}^{+1.16}{ }^{\circ}[42]$.

Notice we are being careful to refer to these oscillation parameters as "solar" instead of as the mixing between two mass eigenstates. In principle, every observed oscillation is affected by all oscillation modes, and so a single set of observed oscillation parameters are highly degenerate in interpretations of the mass splittings and mixing angles chosen by nature. However, we will see in the following sections that the confirmed 


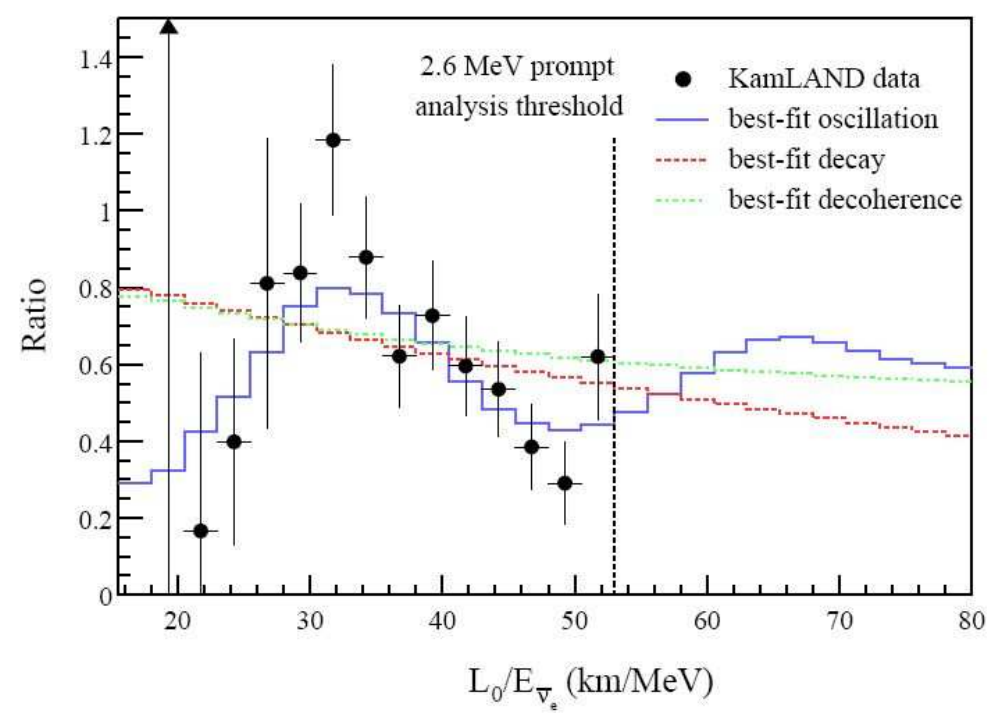

Figure 3-2. KamLAND $\bar{\nu}_{e}$ disappearance results. The ratio shown is the observed data relative to the no-oscillation hypothesis, and the distribution clearly favors the sinusoidal form of neutrino oscillation over alternatives. Figure taken from Ref. [41].

mass splittings are sufficiently separated to eventually refer to these solar parameters as the mixing between only two Hamiltonian states to excellent approximation.

\subsubsection{Atmospheric Oscillations}

Cosmically-produced high energy protons, electrons and stable nuclei collide with Earth's upper atmosphere and produce a flux of neutrinos through pion and muon decay sufficiently intense to be studied on Earth. The Kamiokande detector in Japan [43] was originally designed to search for proton decay, but the secondary physics goal of atmospheric neutrino studies proved much more interesting. As in the puzzle of solar neutrino flux discussed in the previous section, expectations of the neutrino content were not met: the observed ratio $\nu_{\mu} / \nu_{e}$ was significantly lower than predicted [43].

Super-Kamiokande succeeded Kamiokande and featured upgrades that allowed for CC measurements of $\nu_{\mu}$ and $\nu_{e}$ interactions as a function of neutrino travel distance.

Knowledge of the neutrino propagation length was possible through the strong correlation between the direction of the observed charged lepton in $\mathrm{CC}$ interactions and the origin of the incident neutrino. 


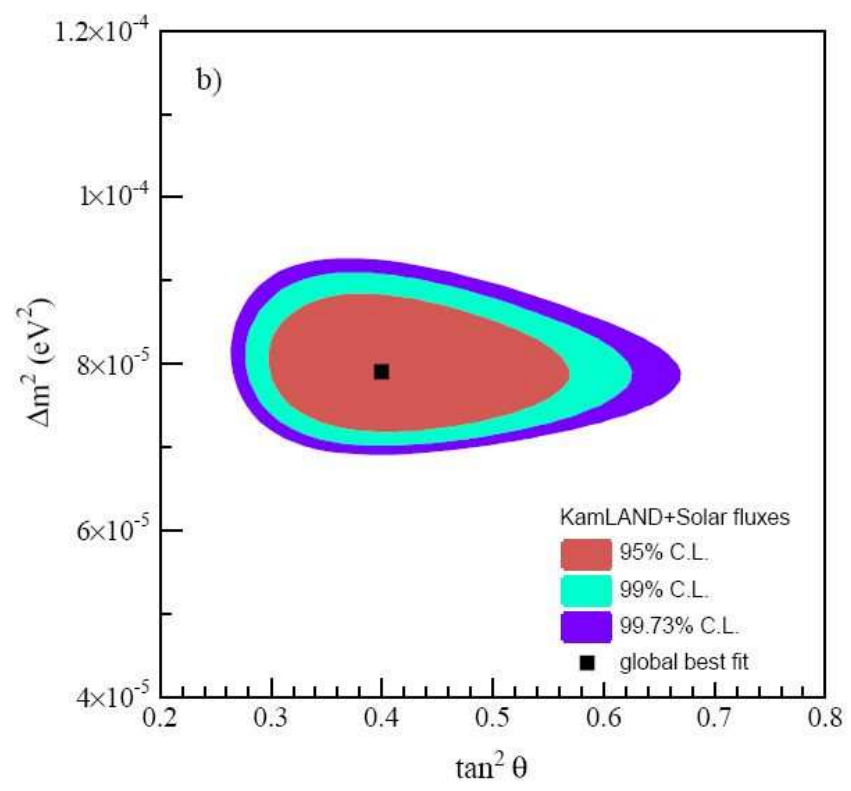

Figure 3-3. Results from a fit to the KamLAND and solar neutrino data to the oscillation parameters $\Delta m_{\text {solar }}^{2}$ and $\theta_{\text {solar. }}$ Figure taken from [41].

The ratio of observed $\nu_{\mu} \mathrm{CC}$ events relative to the no-oscillation hypothesis from Super-Kamiokande detector as a function of $L / E$ is shown in Figure 3-4. A fit to the two-neutrino oscillation hypothesis using these data yields $1.9 \times 10^{-3}<\Delta m_{\mathrm{atm}}^{2}<3.0 \times 10^{-3}$ and $\sin ^{2} 2 \theta_{\mathrm{atm}}>0.90$ at $90 \%$ C.L [44].

Independent confirmation of these oscillation parameters come from the K2K [45] and MINOS [46] experiments, by observing fluxes of accelerator-based neutrino beams at multiple positions along the line of neutrino travel. Motivated by the Super-Kamiokande observations, the neutrino beam energies and detector positions were chosen such that $L$ and $E$ were distinct between the two experiments but the ratio $L / E$ for both $\mathrm{K} 2 \mathrm{~K}$ and MINOS afforded sensitivity to $\Delta m^{2} \sim 10^{-3} \mathrm{eV}^{2}$. With such control over the oscillation region explored by this experimental setup, this method for searching for neutrino oscillations has since become the community standard. Both experiments observed deficits in the observed flux of $\nu_{\mu}$ consistent with $\Delta m^{2} \sim 10^{-3} \mathrm{eV}^{2}$, and the C.L. regions from K2K, MINOS, and Super-Kamiokande are shown in Figure 3-5. A more recent fit to world 


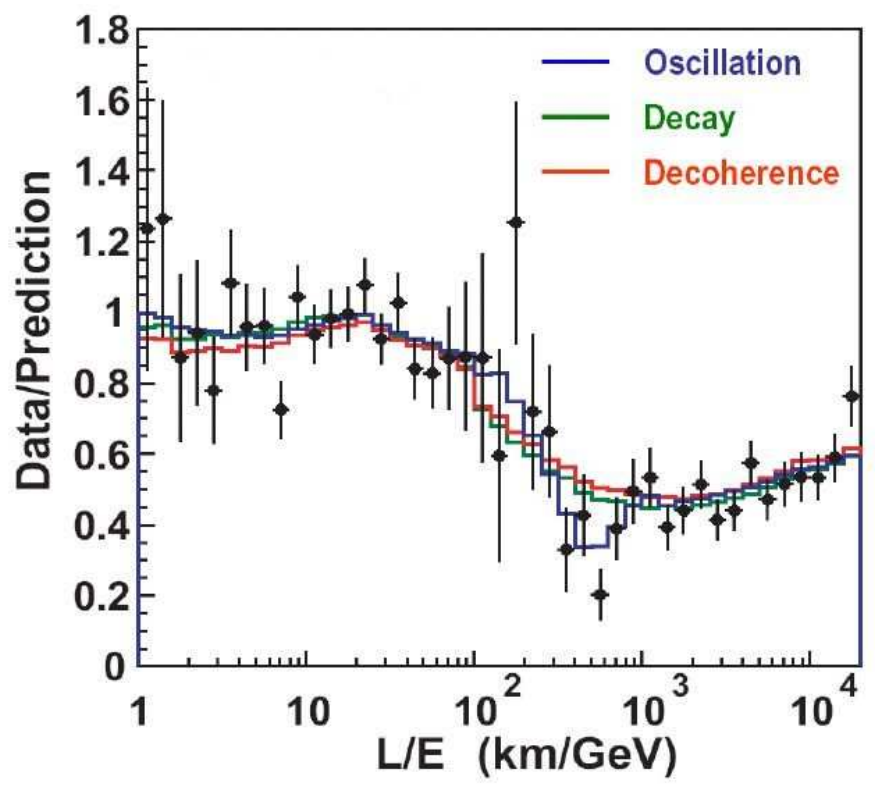

Figure 3-4. Data from the Super-Kamiokande experiment clearly showing an $L / E$ dependence of the observed $\nu_{\mu}$ flux relative to the prediction assuming no oscillations. Figure taken from Ref. [44].

data sensitive to this mass splitting gives $\left|\Delta m_{\text {atm }}^{2}\right|=2.43_{-0.10}^{+0.06} \times 10^{-3} \mathrm{eV}^{2}$ and $\sin ^{2} \theta_{\text {atm }}=0.386_{-0.014}^{+0.024}[47]$.

\subsection{3 $\theta_{13}$ and $\delta$ Oscillations}

As mentioned previously, the magnitude of the mass splitting $\Delta m_{13}^{2}$ is constrained by $\left|\Delta m_{13}^{2}\right|=\left|\Delta m_{12}^{2} \pm \Delta m_{23}^{2}\right|$, where the states either add or subtract depending on the unknown mass hierarchy (discussed in Section 3.3). Considering the separation in the values $\Delta m_{\mathrm{sol}}^{2} \sim 10^{-5} \mathrm{eV}^{2}$ and $\Delta m_{\mathrm{atm}}^{2} \sim 10^{-3} \mathrm{eV}^{2},\left|\Delta m_{13}^{2}\right| \sim \Delta m_{\mathrm{atm}}^{2}$ to good approximation. However, the mixing angle between the $(1,3)$ Hamiltonian eigenstates is unconstrained by the other oscillation parameters and must be independently determined.

The $\theta_{13}$ mixing angle is the most recently measured and confirmed oscillation parameter, its measurement coming last mostly because its diminutive size leads to more subtle effects compared to the other mixing amplitudes. While the accelerator-based experiments MINOS [48] and T2K [49] provided indications that its value is non-zero through $\nu_{\mu} \rightarrow \nu_{e}$ conversions, it was observations of reactor $\bar{\nu}_{e}$ disappearance with the Daya 


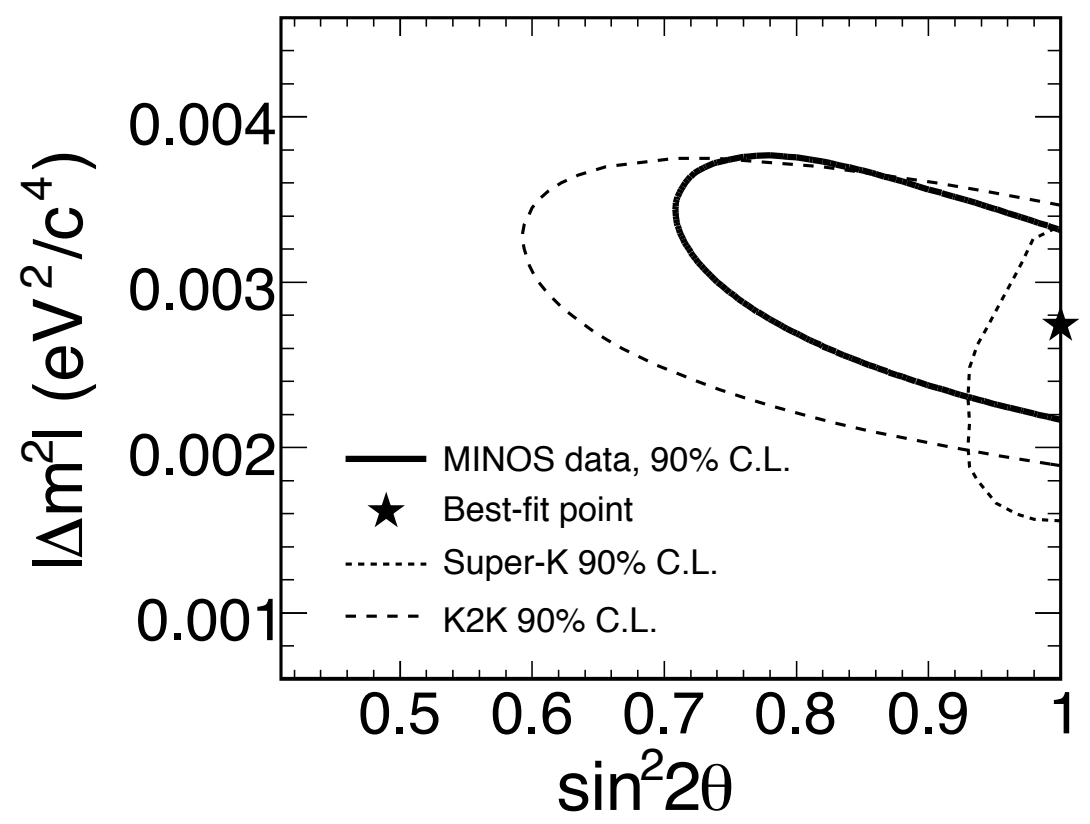

Figure 3-5. Summary of oscillation fits to data sensitive to $\Delta m^{2} \sim 10^{-3} \mathrm{eV}^{2}$. Figure taken from Ref. [46].

Bay [50] and Reno [51] experiments that provided the first measurements of $\theta_{13}$. The Daya Bay experiment uses an impressive number of nearly-identical detectors to measure the reactor $\bar{\nu}_{e}$ flux at a variety of distances, and as shown in Figure 3-6, clearly observes $\bar{\nu}_{e}$ disappearance. This measurement is the most precise to date and finds $\sin ^{2} 2 \theta_{13}=0.089 \pm 0.011[52]$.

The neutrino transitions affected by $\delta$ and the sign of $\Delta m_{13}^{2}$ are most readily experimentally accessible through a comparison of $P\left(\nu_{\mu} \rightarrow \nu_{e}\right)$ with $P\left(\bar{\nu}_{\mu} \rightarrow \bar{\nu}_{e}\right)$. These probabilities cannot be reasonably approximated by the two-neutrino case, as all three mixing angles and mass splittings contribute significantly to the process [53]. Though this indicates Eq. 3.9 is less helpful here, the experimental sensitivity to these transitions are still governed principally by the appropriate ratio of $L / E$, and in this case is $\mathcal{O}\left(\frac{10 \mathrm{~m}}{10^{3} \mathrm{eV}}\right)$. To allow for reasonable production phase-space for the observation of the muon in $\nu_{\mu} \mathrm{CC}$ interactions, $E$ must be $\mathcal{O}(1 \mathrm{GeV})$, setting $L$ of $\mathcal{O}\left(10^{9} \mathrm{~m}\right)$. This distance is roughly an 


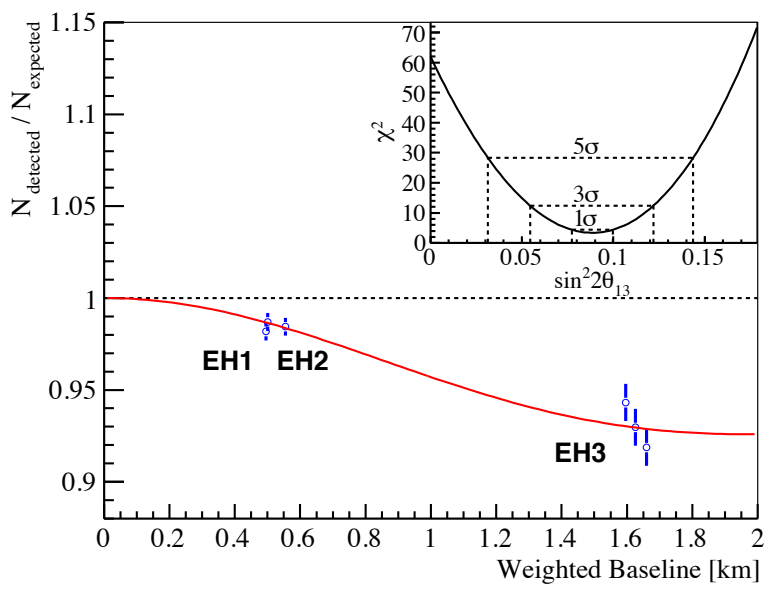

Figure 3-6. Observed $\bar{\nu}_{e}$ flux at the various Daya Bay experimental halls (EH) as a function of distance from the $\bar{\nu}_{e}$ source relative to the prediction assuming no oscillations. The inset shows the compatibility of the data for various values of $\sin ^{2} 2 \theta_{13}$, clearly ruling out $\theta_{13}=0$ at greater than $5 \sigma$ C.L.. Figure taken from Ref. [52].

order of magnitude longer than any previous observations of articificial sources. As the neutrino flux is roughly proportional to $1 / L^{2}$ at large distances from the source, it will be enormously challenging to achieve the beam power and detection precision required to probe values of $\delta$.

Currently the only constraints on $\delta$ come from the MINOS [48] experiment, and are shown in Figure 3-7. It can be seen that no value of $\delta$ for either sign of $\Delta m_{13}^{2}$ (referred to in the figure simply as $\left.\Delta m^{2}\right)$ is strongly preferred over others.

The determination of the sign of $\Delta m_{13}^{2}$ and precision measurements of $\delta$ is currently at the forefront of today's experimental neutrino program, and may dominate the

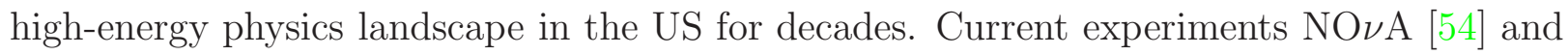
T2K, and later LBNE [55], will lead the search by comparing $P\left(\nu_{\mu} \rightarrow \nu_{e}\right)$ with $P\left(\bar{\nu}_{\mu} \rightarrow \bar{\nu}_{e}\right)$ using few-GeV beams of $\nu_{\mu}$ and $\bar{\nu}_{\mu}$. One of the challenges that must be met before a clean measurement of $\delta$ is possible is a high-precision understanding of the fundamental contributing $\nu_{\mu}$ and $\bar{\nu}_{\mu}$ interactions at this energy range. The work presented in this dissertation provides a first measurement of $\bar{\nu}_{\mu}$ cross sections below $1 \mathrm{GeV}$ and thus significantly advances the community's preparedness to search for CP violation with 


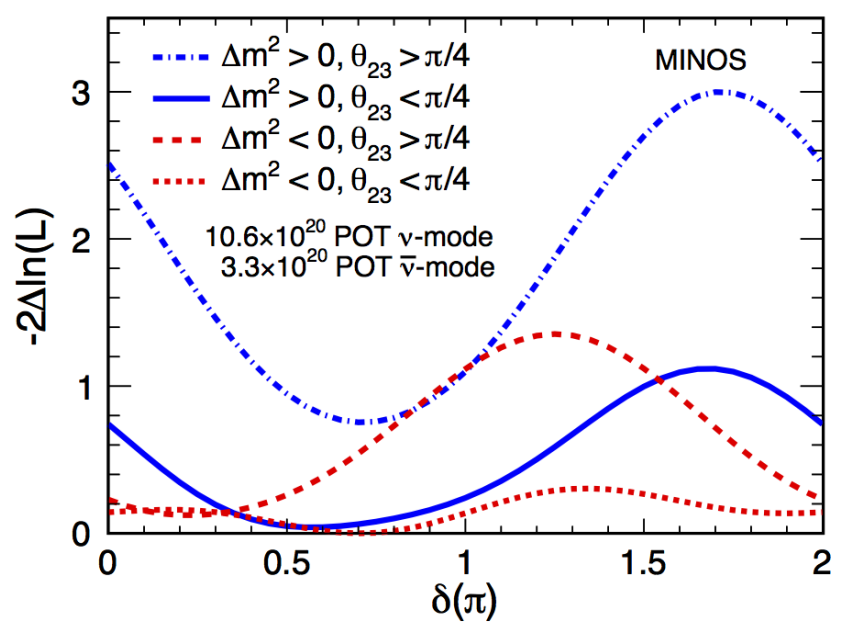

Figure 3-7. Constraints on $\delta$ for various assumptions on the sign of $\Delta m^{2}$ and the value of $\theta_{23}$ from the MINOS experiment. It will be shown that the $\theta_{23}$ is nearly identical to $\theta_{\text {atm }}$. Figure taken from Ref. [48].

neutrinos.

\subsubsection{Hints for $\Delta m^{2} \sim 1 \mathbf{e V}^{2}$}

One of the major outstanding questions in neutrino physics is the existence of another mass splitting in the range of $\Delta m^{2} \sim 1 \mathrm{eV}^{2}$. As with the other mixing parameters, this hypothesis is entirely experimentally-driven. The first indication came from the LSND experiment, where an excess of $\bar{\nu}_{e}$ events were observed from a stopped-pion $\bar{\nu}_{\mu}$ source [35]. As with the accelerator-based confirmations of the solar mixing described in Section 3.2.1, the MiniBooNE experiment was designed to provide an independent check of this splitting by probing the same ratio $L / E$ while $L$ and $E$ were themselves distinct from the values used at LSND. An indication of both $\nu_{\mu} \rightarrow \nu_{e}$ and $\bar{\nu}_{\mu} \rightarrow \bar{\nu}_{e}$ oscillations were observed in the MiniBooNE data as well [36], however neither of the signals from the two experiments exclude the no-oscillation hypothesis at greater than $4 \sigma$. The allowed $\left(\Delta m^{2}, \theta\right)$ regions from both experiments are shown in Figure 3-8. 


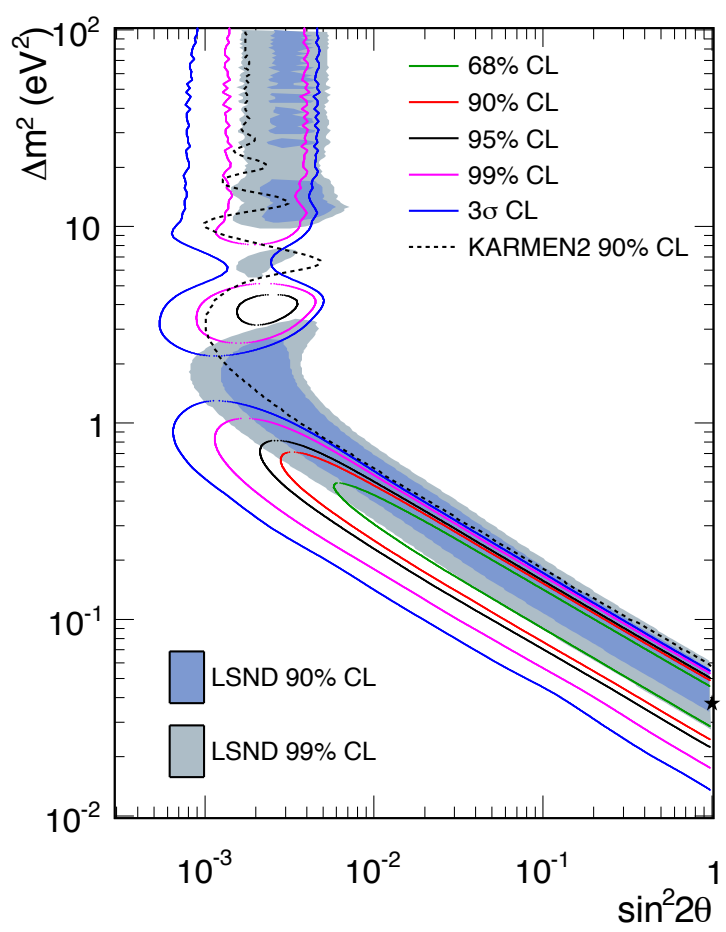

Figure 3-8. Results of a two-neutrino oscillation fit to the combined $\nu_{e}$ and $\bar{\nu}_{e}$ appearance data from MiniBooNE. Also shown are the LNSD allowed regions and limits from the KARMEN experiment [56]. Figure taken from Ref. [36].

Less significant signals indicating $\Delta m^{2} \sim 1 \mathrm{eV}^{2}$ come from cosmological observations [57], radioactive source experiments [58], and from reactor antineutrino data [59]. Particularly in light of the implications of such a mass splitting as discussed in the next section, this signal must be rigorously tested in the near future. Proposed experiments to do so include OscSNS [60], nuSTORM [61], and a search using decay-at-rest kaons [62].

\subsection{Summary and Outstanding Questions}

Noting that the $\Delta m^{2}$ scales discussed in the previous section differ by orders of magnitude, we can drop their conservative solar and atmospheric labels and refer to them as the genuine splitting between neutrino Hamiltonian eigenstates to excellent approximation $^{1}$. Under the suspicion of symmetry between the ordering of the neutrino

\footnotetext{
${ }^{1}$ Note also this is also true if there exists a mass splitting near $1 \mathrm{eV}^{2}$.
} 
mass states and that of the other leptons, we refer to the smaller mass splitting as $\Delta m_{12}^{2}$ and the larger splitting as $\Delta m_{23}^{2}$. The confirmed values for the neutrino mixing parameters are:

$$
\begin{array}{cc}
\Delta m_{12}^{2}=-7.59_{-0.21}^{+0.20} \times 10^{-5} \mathrm{eV}^{2} & \theta_{12}=34.06_{-0.84}^{+1.16 \circ} \\
\left|\Delta m_{32}^{2}\right|=2.43_{-0.10}^{+0.06} \times 10^{-3} \mathrm{eV}^{2} & \sin ^{2} \theta_{23}=0.386_{-0.014}^{+0.024} \\
\Delta m_{13}^{2} \sim \Delta m_{32}^{2} & \theta_{13}=9.0_{-0.5}^{+0.4} \circ
\end{array}
$$

With two independent mass splittings, as least two neutrino mass eigenstates must be non-zero. One of the most important questions about the nature of the neutrino concerns how these masses may be integrated into the SM. This issue is fundamentally tied to whether the neutrino is its own anti-particle, indicating a Majorana nature; if the neutrino and antineutrino are distinct, neutrinos are Dirac particles. For Dirac particles the extension of neutrino mass into the SM is quite simple in that, like other massive particles, the masses are generated by the Higgs field and both left- and right-handed neutrinos and antineutrinos exist. Neutrinos with opposing chirality to the observed states are then not experimentally accessible not becuase of the nature of the neutrino, but because they only interact through the maximally parity-violating weak interaction.

A popular model of the alternative of Majorana neutrinos is equally viable and offers an explanation of the diminutive scale of the neutrino mass compared to the other fermions. In this model the masses of the light and active neutrinos are accompanied by some number of possibly non-weakly interacting neutrinos $N$ such that the product of the two neutrino family masses are related to the scale of the quark $q$ or charged lepton $l$ families: $m_{\nu} m_{N} \sim m_{q, l}^{2}$ [63]. In this way, the large mass of the $N$ neutrino provides a counter-balance for the observed neutrinos to be arbitrarily light, and this model is therefore referred to as the See-Saw mechanism. 
Currently, the best experimental probe to determine whether the neutrino is Majorana or Dirac involves the neutrino-less double-beta decay reaction $\left(n+n \rightarrow p+p+e^{-}+e^{-}\right)$. The decay would involve the emission of an $\bar{\nu}_{e}$ at one vertex of ordinary $\beta$ decay and it's immediate absorption at a second $\beta$ decay vertex playing the role of $\nu_{e}$. Consequently, this process is allowed for Majorana neutrinos but is forbidden if neutrinos are Dirac particles. One experiment has claimed to have observed evidence of this process [64], but this remains unconfirmed.

Another currently-degenerate fundamental property related to the neutrino mass is their hierarchical ordering. From the Mikheyev-Smirnov-Wolfenstein (MSW) effect in solar oscillations, it is known that $m_{2}>m_{1}$, while the ordinal label between the third mass state and the others is arbitrary. As shown in Section 3.1, observations of splittings sensitive to only two mass eigenstates reveal only the absolute value of the splitting, and the current neutrino oscillation data are degenerate between the smaller mass splitting separating the two lightest states and the same splitting separating the most massive states. Figure 3-9 pictorially shows this degeneracy in the mass hierarchy along with the approximate mixing amplitudes between each flavor and mass state. As mentioned in Section 3.2.3, the mass ordering will be addressed in the current and next round of experiments simultaneously searching for the CP-violating phase $\delta$.

It can be seen in Eq. 3.7 that the CP-violating phase $\delta$ is inextricably tied to the mixing angle $\theta_{13}$ and therefore the sign of $\Delta m_{13}^{2}$. The observation of a non-zero value for $\theta_{13}$ offers the opportunity to search for CP-violation in the lepton sector, which is currently one of the best hypotheses for explaining the baryon asymmetry in the universe [65]. It is worth noting that a sufficiently large value of $\delta$ must be accompanied by at least one more species of neutrino, much more massive than the known types, to explain the observed baryon asymmetry. Nevertheless, as the observed CP-violation in the quark sector is far too meager to account for the evolution of our matter-dominated universe [9], searches for the origin of the asymmetry using neutrinos are well-motivated and compelling. 


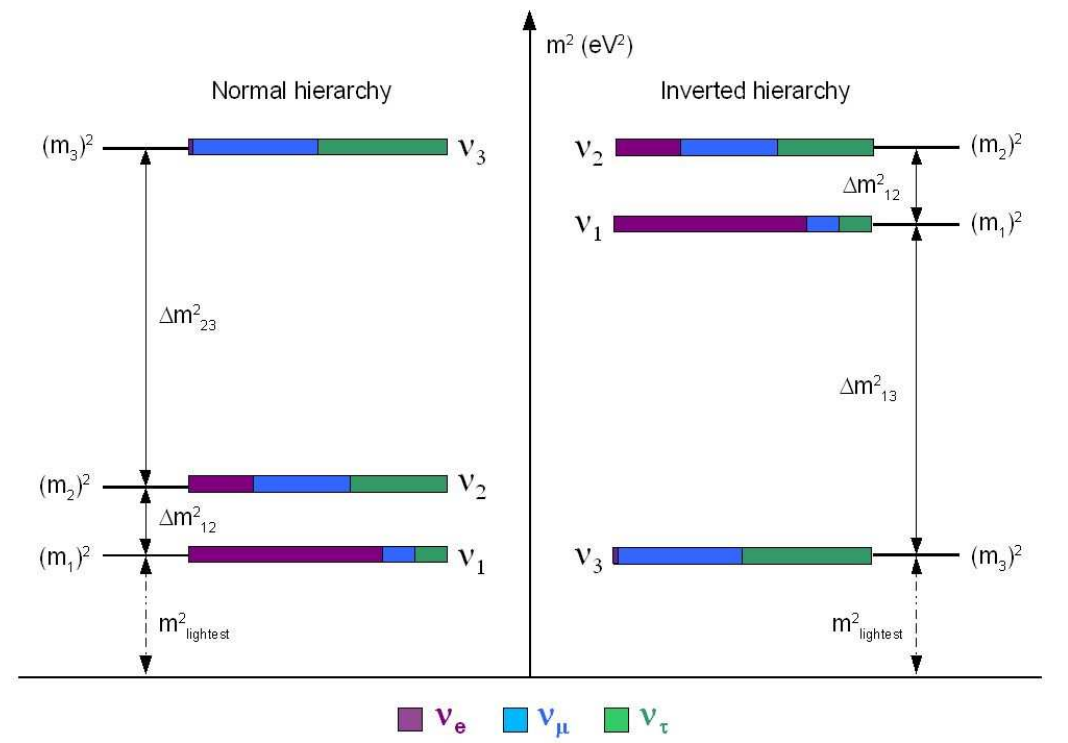

Figure 3-9. The two possible neutrino mass orderings, shown with their approximate couplings to the flavor states.

Finally, if confirmed, the experimental hints of another mass splitting presented in Section 3.2.4 would imply a fourth neutrino mass state, with a fundamentally different coupling to the weak interaction. If there exists a mass splitting near $1 \mathrm{eV}^{2}$, from the precision constraint of the $Z^{0}$ width (shown in Figure 2-2), it must not directly couple to the weak interaction. From the disconnect between its mass and the interaction states, this hypothesized extra neutrino is known as sterile. Furthermore, arguments based on symmetry between neutrinos and the other fermions would suggest these ought to exist in sets of three, if any, and analyses to global data do mildly prefer the addition of more than a single sterile mass state [37]. This would introduce a litany of extra degrees of freedom in neutrinos oscillations in the form of mass splittings and mixing angles, the signals from which are almost entirely degenerate in current experiments.

It is clear by now a quantum mechanical process not predicted by the standard model is real and may be a consequence of some deeper laws of physics we do not yet appreciate. Though there are many unknowns in neutrino physics that will presumably lead to a more fundamental understanding of the weak interaction and how it fits into 
nature, the concrete observation of neutrino oscillations reveal two pieces of information crucial to this quest: that the neutrino mass is non-zero, and that lepton number is not a strictly conserved quantity.

As a final note, it was entirely fortuitous that the community realized the phenomenon of neutrino oscillations. If the nature of oscillations were such that solar and atmospheric neutrinos were unaffected, our ignorance of this process would have persisted for at least many more decades. Therefore, though it appears the community may fully populate the PMNS matrix (Eq. 3.6) with precision measurements in the coming decades, it seems unlikely that this will complete our fundamental understanding of neutrino oscillations. 


\section{CHAPTER 4 \\ NEUTRINO INTERACTIONS IN MINIBOONE}

\subsection{Overview}

MiniBooNE uses the NUANCE neutrino event generator [66] to predict and simulate neutrino interactions in the detector. NUANCE includes a comprehensive cross-section model which considers known interactions in the neutrino energy range from $\sim 100 \mathrm{MeV}$ to $1 \mathrm{TeV}$. Ninety-nine reactions are modeled separately and combined with nuclear models describing bound nucleon states and final-state interactions to predict event rates and kinematics.

Figure 4-1 shows the expectation and experimental data for $\nu_{\mu}$ and $\bar{\nu}_{\mu} \mathrm{CC}$ interactions across a wide range of energies. As the MiniBooNE fluxes of $\nu_{\mu}$ and $\bar{\nu}_{\mu}$ are peaked near $700 \mathrm{MeV}$ (Figure 5-9), the charged-current quasi-elastic (CCQE) and charged-current single pion ( $\mathrm{CC} \pi$ ) interactions are the most abundant interactions in the MiniBooNE data sets. For this reason, in this chapter we concentrate on the expectations and experimental evidence associated with these processes.

A wealth of information is summarized in Figure 4-1, and it is important to point out the overall structure of the cross sections and the features most relevant to the measurements executed in this dissertation. When the neutrino energy is large enough to resolve individual quarks, the CC cross section is approximately linear with energy. This behavior is a confirmation of the quark parton model [67], where higher energy probes gain sensitivity to more scattering interactions through the quark sea. This approximation, of course, breaks down at lower energies where elastic interactions are dominant.

Experimentally, Figure 4-1 shows that these interactions at lower energies feature total error on the order of tens of percent. This is mostly due to experimental difficulties in separating the various contributing processes, a challenge that is unique to the various detector technologies and usually includes dependence on assumptions about the contributing signal and background processes. Finally, the antineutrino cross sections are 
experimentally known less accurately compared to the analogous neutrino processes, and in particular there are no antineutrino CC cross-section measurements below $1 \mathrm{GeV}$. The lower precision and more sparse antineutrino data is due in general to a number of effects, most notably relatively larger backgrounds and low statistics. The measurements in this dissertation break significant ground on both experimental challenges: Chapter 7 presents a first demonstration of a set of techniques to statistically measure a background typical of artificial beams of antineutrinos, and Chapter 8 presents an analysis of antineutrino interactions with more than an order of magnitude of higher statistics compared to all other previously-published antineutrino cross-section measurements combined. In addition, the average antineutrino energy for these measurements is $650 \mathrm{MeV}$, and so these data are sensitive to an almost entirely unprobed energy region. 

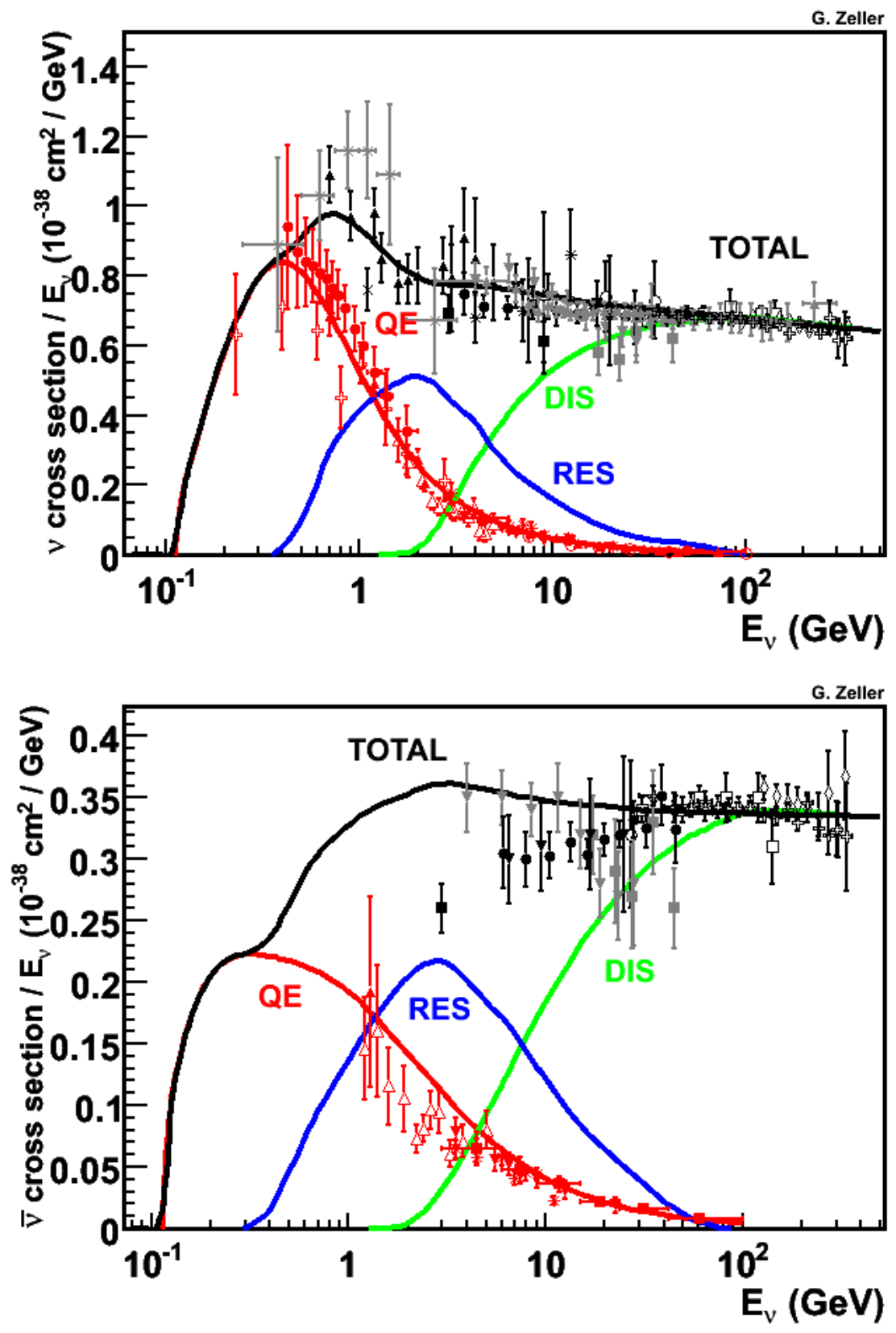

Figure 4-1. Comparison of expectations and experimental data across $\nu_{\mu}$ (top) and $\bar{\nu}_{\mu}$ (bottom) CC interactions. The "QE" and "RES" labels here are referred to as CCQE and CC $\pi$ interactions in the text. Figure from Ref. [30]. 


\subsection{CCQE}

The CCQE process $\left(\nu_{l}+n \rightarrow l^{-}+p\right.$ for neutrinos, and $\bar{\nu}_{l}+p \rightarrow l^{+}+n$ for antineutrinos) is the most abundant interaction at the MiniBooNE energy range, accounting for $\sim 40 \%$ of interactions in the detector. It is typically used as the signal process in neutrino oscillation measurements due to its simple multiplicity, and also the ability to reconstruct the incident neutrino energy, under a few important assumptions, based solely on observations of the charged lepton (Chapter 6). Typically credited to Llewellyn-Smith [68], the differential cross section for this process assuming the exchange of a single $W$ boson as a function of the momentum transfer $Q^{2}$ is:

$$
\frac{d \sigma}{d Q^{2}}=\frac{M^{2} G_{F}^{2}\left|V_{u d}\right|^{2}}{8 \pi E_{\nu}^{2}}\left[A\left(Q^{2}\right) \pm B\left(Q^{2}\right) \times\left(\frac{s-u}{M^{2}}\right)+C\left(Q^{2}\right) \times\left(\frac{s-u}{M^{2}}\right)^{2}\right]
$$

where the positive (negative) sign refers to neutrino (antineutrino) scattering, $G_{F}$ is the Fermi coupling constant, $V_{u d}$ is the Cabbibo coupling between down and up quarks, $m$ is the mass of the charged lepton, $M$ the mass of the target nucleon, and $s, u$ are the usual Mandelstam variables. For a derivation of this expression, see Ref. [69]. Note the terms are

organized in powers of $\frac{s-u}{M^{2}}=\frac{4 M E_{\nu}-Q^{2}-m}{M^{2}}$ and the interference between the axial and vector currents that governs the difference in scattering amplitudes between neutrinos and antineutrinos is entirely contained in the $B\left(Q^{2}\right)$ term. This interference is a consequence of the $\mathrm{V}$-A nature of the weak interaction. The auxiliary functions $A\left(Q^{2}\right), B\left(Q^{2}\right), C\left(Q^{2}\right)$ are parameterized in terms of vector, axial and pseudoscalar form factors: 


$$
\begin{aligned}
A\left(Q^{2}\right)= & \frac{\left(m^{2}+Q^{2}\right)}{M^{2}}\left[(1+\tau) F_{A}^{2}-(1-\tau) F_{1}^{2}+\tau(1-\tau) F_{2}^{2}+4 \tau F_{1} F_{2}-\frac{m^{2}}{4 M^{2}}\left(F_{1}+F_{2}\right)^{2}\right. \\
& \left.+\left(F_{A}+2 F_{P}\right)^{2}-4 F_{P}^{2}(1+\tau)\right] \\
B\left(Q^{2}\right)= & \frac{Q^{2}}{M^{2}} F_{A}\left(F_{1}+F_{2}\right) \\
C\left(Q^{2}\right)= & \frac{1}{4}\left(F_{A}^{2}+F_{1}^{2}+\tau F_{2}^{2}\right),
\end{aligned}
$$

where $\tau=\frac{Q^{2}}{4 M^{2}}, F_{1}$ and $F_{2}$ are vector form factors, $F_{A}$ is the axial form factor, and $F_{P}$ is the pseudoscalar form factor. The vector form factors are:

$$
\begin{aligned}
& F_{1}=\frac{1+\tau\left(1+\mu_{p}-\mu_{n}\right)}{(1+\tau)\left(1+\frac{Q^{2}}{m_{V}^{2}}\right)^{2}} \\
& F_{2}=\frac{\mu_{p}-\mu_{n}}{(1+\tau)\left(1+\frac{Q^{2}}{m_{V}^{2}}\right)^{2}},
\end{aligned}
$$

where $\mu_{p}\left(\mu_{n}\right)=2.793(-1.913) \times \mu_{N}$ is the proton (neutron) anomalous magnetic moment [70], and $m_{V}$ is the empirically-determined "vector mass". Using the conserved vector current (CVC) hypothesis, the results of the plentiful and high-quality elastic electron scattering $\left(e^{-}+N \rightarrow e^{-}+N\right)$ data can be used to constrain these form factors. The dipole forms of Eqs. 4.5 and 4.6 are adequately described with a vector mass of $m_{V}^{2}=0.71 \mathrm{GeV}^{2}$, but recent fits to these data show a clear preference for a non-dipole form [71].

The pseudoscalar form factor is given by:

$$
F_{P}=\frac{2 M^{2}}{m_{\pi}^{2}+Q^{2}} F_{A}
$$


where $m_{\pi}$ is the pion mass. Notice the contribution of $F_{P}$ to the CCQE is suppressed by $\frac{m^{2}}{M^{2}}$, and so its effect relative to the other terms is small.

Finally, and most importantly for the measurements of this dissertation, the axial form factor is:

$$
F_{A}=\frac{g_{A}}{\left(1+\frac{Q^{2}}{M_{A}^{2}}\right)^{2}}
$$

where $g_{A}$ and $M_{A}$ are empirical inputs, and the dipole form is again assumed. Like the vector form factors taken from electron-scattering data, $g_{A}$ is also constrained by external information: $\beta$ decay measurements give $g_{A}=F_{A}\left(Q^{2}=0\right)=-1.267 \pm 0.002$ [72]. This leaves the axial mass $M_{A}$ as the only free parameter in the CCQE cross section. For decades, this parameter was measured with observations of both the total observed CCQE cross section and its shape as a function of the momentum transfer. A combined analysis of the world data through the twentieth century yields $M_{A}=1.026 \pm 0.021 \mathrm{GeV}$ [73] Important to point out, most of these measurements were performed with bubble-chamber detectors housing mostly hydrogen and deuterium media. More recent results from experiments employing larger nuclei in order to more easily gain the statistics needed for oscillation experiments have found tension with these data, and as a result the model for nuclear effects typically used by experiment has come under scrutiny. Further discussion of this model and its implications is found in Section 4.4.

As a final remark, the interference term proportional to $B\left(Q^{2}\right)$ in Eq. 4.2 gives rise to stark kinematic differences in the behavior of neutrinos compared to antineutrinos in CCQE interactions. In Figure 4-2, the differential cross section for $\nu_{\mu}$ CCQE scattering is separated into terms arising from the vector and axial currents, as well as the interference between the two. As the interference term is constructive for neutrino scattering and destructive for antineutrinos, is clear that the divergence of their amplitudes grows with 


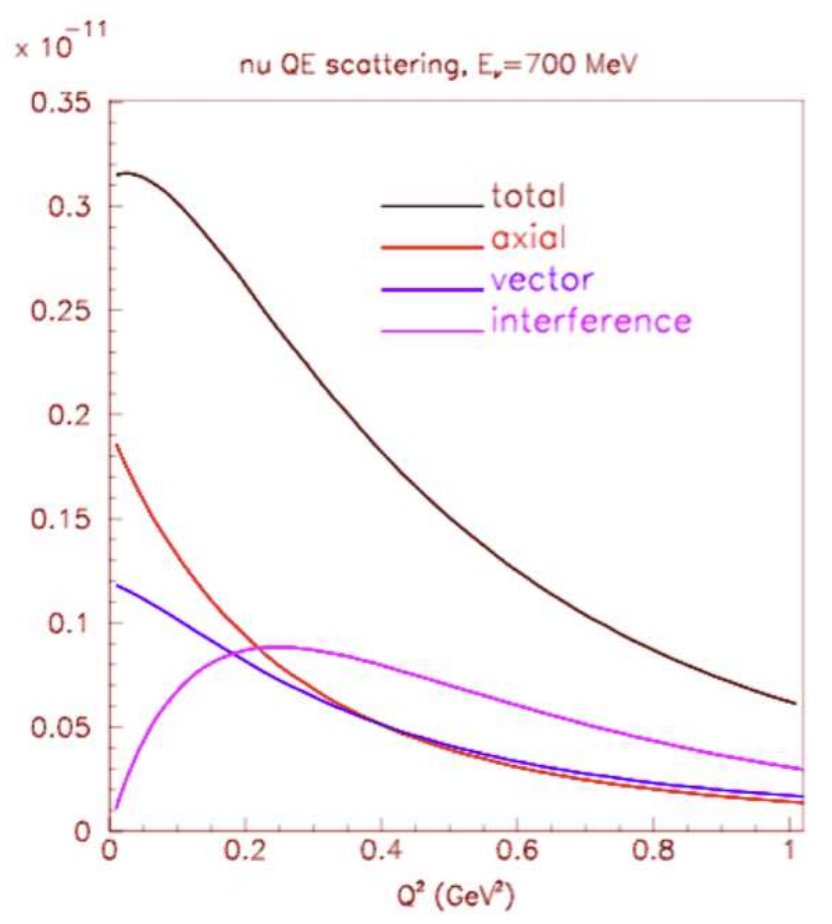

Figure 4-2. Decomposition of the differential CCQE cross section for $700 \mathrm{MeV} \nu_{\mu}$ 's. The ordinate axis is proportional to $d \sigma / d Q^{2}$. Figure from Ref. [74].

momentum transfer. Momentum transfer of the interaction is closely related to the production angle of the charged lepton relative to the neutrino direction, and this difference is exploited in Section 7.1.5 to measure the $\nu_{\mu}$ and $\bar{\nu}_{\mu}$ content of the MiniBooNE antineutrino-mode data.

\section{3 $\mathrm{CC} \pi$}

Neutrinos with energy $\sim 400 \mathrm{MeV}$ and above can produce pions through the excitation and subsequent decay of baryonic resonances. Resonances of Delta $(\Delta)$ particles are most important for the neutrinos observed by MiniBooNE, and their decays are dominated by $\Delta \rightarrow N \pi$ [72]. The formalism to describe these interactions is taken from the Rein-Sehgal model [75], where the relativistic harmonic oscillator quark model is assumed [76] and the pion angular distribution due to the spin structure of the resonances is considered. Eighteen resonances are modeled, though the $\Delta(1232)$ is dominant in the energy range spanned by MiniBooNE. Multi-pion production mechanisms are also 
modeled, though their contribution is predicted to be small.

As the primary interaction for the $\mathrm{CC} \pi$ processes $\left(\nu_{l}+N \rightarrow l+\Delta\right)$ is closely related to CCQE interactions, the formalism also includes a single tunable axial mass parameter $M_{A}^{1 \pi}$. The axial masses in the resonance channels are set simultaneously to reproduce inclusive non-MiniBooNE charged-current data [77]. The extracted values are

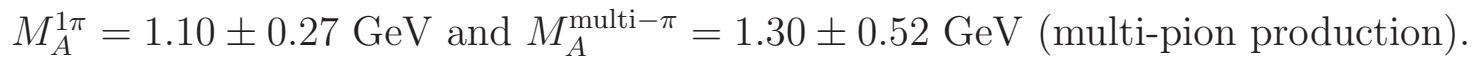

Various levels of discrepancy between this model and the MiniBooNE single $\pi$ production results spanning normalization differences of up to $60 \%$ have been observed [78, 79, 80], and these differences continue to persist in more modern single- $\pi$

production calculations [81]. For these reasons, whenever possible, the various MiniBooNE cross-section and oscillations measurements rely on direct constraints from the various MiniBooNE single- $\pi$ production samples.

\subsection{Nuclear Effects}

\subsubsection{Nuclear Modeling}

The MiniBooNE detector is filled with mineral oil, and as a hydrocarbon material, the bare neutrino-nucleon interaction amplitudes must be combined with effects arising from the nuclear environment for interactions with material bound in carbon. MiniBooNE uses the Relativistic Fermi Gas (RFG) model [82] to describe this connection. Broadly, it combines the free-nucleon cross sections with a potential well in the form of binding energy as well as Pauli blocking to restrict the available kinematics of struck nucleons. The binding energy $E_{B}$ increases the threshold for the reaction to occur, while the effects of Pauli blocking are more subtle.

The phenomenon of Pauli blocking arises from the exclusion principle, which dictates that no two fermions may share the identical set of quantum eigenstates. The RFG model simulates bound nucleons as a "gas" of particles, with a uniform momentum distribution from the lowest state up to an empirically-determined maximum $k_{F}$. These modifications to the CCQE amplitudes are implemented by integrating, with respect to the 
initial nucleon momentum $\vec{k}$, the free-nucleon cross sections scaled by a factor proportional to:

$$
\Theta\left(k_{F}-|\vec{k}|\right) \Theta\left(|\vec{k}+\vec{q}|-k_{F}\right) \delta\left(E_{\vec{k}}-E_{\vec{k}+\vec{q}}-E_{B}+\omega\right),
$$

where $\omega=E_{\nu}-E_{l}$ is the energy transfer, $\vec{q}=\vec{p}_{\nu}-\vec{p}_{l}$ is the three-momentum transfer, and $E_{\vec{k}}$ and $E_{\vec{k}+\vec{q}}$ are the energies of the initial and struck nucleon, respectively. The first term requires the nucleon participating in the interaction to have momentum below $k_{F}$, the second enforces Pauli blocking, and the third assures energy conservation. The second term is appropriate only to nuclear transitions involving $n \leftrightarrow p$ so that the struck nucleon is required to be above the Fermi momentum of the other, fully-populated nucleon Fermi sea. In the case of carbon-12, where $Z=N=6$, a single momentum $k_{F}$ specifies the maximum of both the proton and neutron Fermi levels.

The energy of the lowest-allowed struck nucleon momentum state is closely related to low values of the squared momentum transfer distribution $Q^{2} \equiv-q^{2}$, where $q^{2}$ here is the four-momentum transfer. This region in the MiniBooNE $\nu_{\mu}$ CCQE data was insufficiently described by this RFG model [83], and even after a more rigorous evaluation of the backgrounds it was found that a mild scaling ( $\kappa$ ") of this energy level:

$$
E_{\mathrm{low}}=\kappa\left(\sqrt{k_{F}^{2}+M^{2}}-\omega+E_{B}\right)
$$

was preferred by the data at the level of a $\sim 1 \%$ modification to $E_{\text {low }}$ [84].

The values for the binding energy and Fermi momentum of carbon are informed by electron scattering data. Shown in Figure 4-3, the peak of quasielastic electron-scattering $\left(e^{-}+N \rightarrow e^{-}+N\right)$ data is well-described by a Fermi gas model and $E_{B}\left(p_{F}\right)=25(221)$ $\mathrm{MeV}$, where natural units are used. 


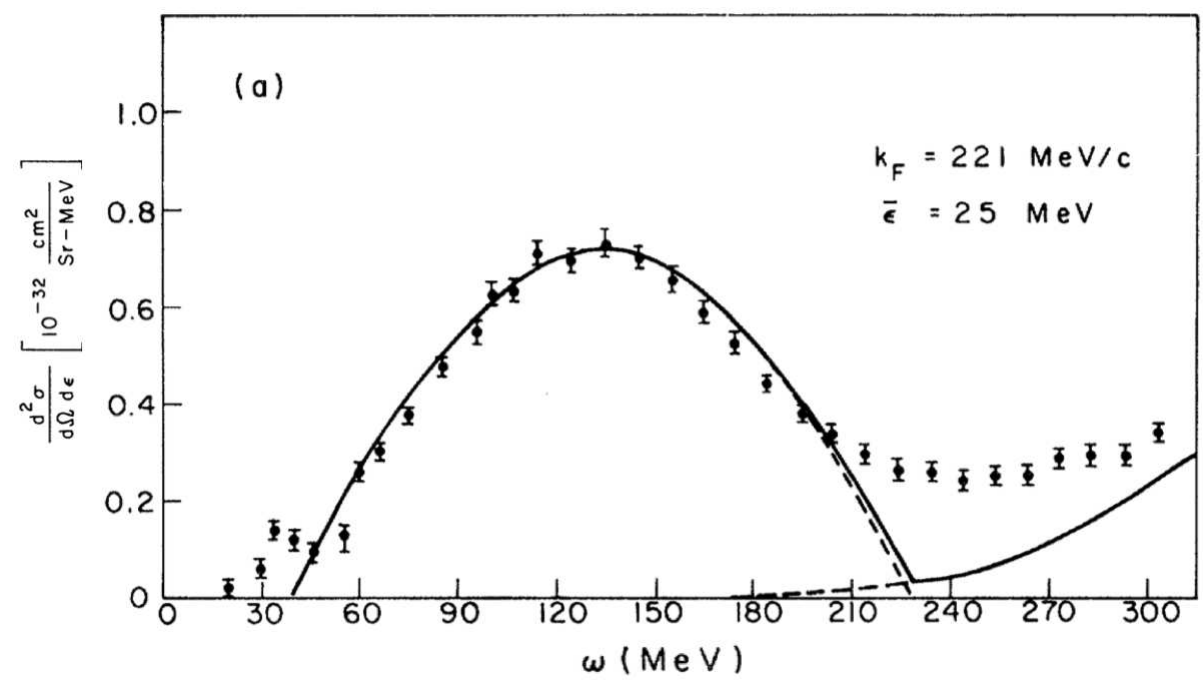

Figure 4-3. The observed differential cross section for electron scattering $e+N \rightarrow e^{\prime}+N$ on carbon-12 in terms of the energy transfer $\omega=E_{e}-E_{e^{\prime}}$. The binding energy is determined by the position of the observed peak, while the Fermi momentum is found from the peak's width. Figure from Ref. [85].

The Fermi momentum $p_{F}=221 \mathrm{MeV}$ is directly implemented into the RFG.

However, for CCQE scattering where $n \leftrightarrow p$, the binding energy must be modified from the determination from electron scattering data, where the initial and outgoing nucleons are of the same type. Additional coulomb repulsion for $n \rightarrow p$ transitions (appropriate to $\nu_{\mu}$ CCQE interactions) adds to the effective binding energy of the system. The asymmetry term in the semi-empirical mass formula [86] estimates this adds $9 \mathrm{MeV}$ to the energy for this transition, resulting in an effective binding energy of $34 \mathrm{MeV}$ for $\nu_{\mu}$ CCQE interactions on carbon-12.

Particularly in the context of this dissertation, it is important to note the RFG assumes all nucleons behave entirely independently of one another. Recent deviations from RFG expectations in the measurements of the CCQE interaction with relatively heavy nuclear targets have cast suspicion on this assumption. While measurements of $M_{A}$ using mostly light nuclear material (discussed in Section 4.2) agree fairly well, data from experiments using relatively heavy nuclei and higher-precision detectors have extracted 
values of $M_{A}$ systematically higher than $1.026 \mathrm{GeV}[84,87,88,89]$. Adding complexity, the modern heavy nuclear target experiment NOMAD has measured values of $M_{A}$ consistent with the light-target analyses [90], while preliminary shape results from the MINER $\nu \mathrm{A}$ experiment seem to also favor $M_{A} \sim 1 \mathrm{GeV}[91]$.

An essential first step to understanding this apparent discrepancy is to recognize the particulars of the model dependence introduced by comparing values of $M_{A}$ between the many experiments. Important experimental differences that may contribute to the discrepancy include disparate neutrino spectra, different neutrino detection technologies and the size of the nuclear media employed. Among the liberties taken to compare $M_{A}$ values across these scattering experiments include the dipole form of $F_{A}$, various expectations of hadronic activity consistent with single-nucleon ejection and the previously-mentioned independent nucleon assumption implicit in both the formalism and in the inference of the $Q^{2}$ distribution. A possible reconciliation between the data sets has been proposed by offering a mechanism resulting in intra-nuclear correlations of greater strength than previously expected [92, 93, 94, 95, 96, 97, 98, 99, 100]. Such a mechanism is consistent with electron scattering data [101, 102]. If this process is confirmed for weak interactions via neutrino scattering, its detailed understanding will significantly expand knowledge of intra-nuclear behavior, and some neutrino oscillation results may need to be revisited $[103,104]$. The best chance to definitively resolve this crucial ambiguity lies in the community's ability and willingness to produce and compare model-independent information in both the leptonic and hadronic interaction sectors between experimental data and theoretical calculations. The results of this dissertation offer a first look at antineutrino CCQE interactions below $1 \mathrm{GeV}$ and thus significantly expand the body of experimental data contributing to this picture. In recognizing the possible deficiencies of the RFG, the main result of this work is the double-differential CCQE cross section $\left(\frac{d^{2} \sigma}{d T_{\mu} d \cos \theta_{\mu}}\right)$ on mineral oil, where no assumptions about the underlying process are necessary. 


\subsubsection{Final-State Interactions}

An important connection between fundamental neutrino-nucleus interactions and what is observed in the detector are the possible strong interactions between the struck baryon and its nuclear environment.

For neutrino interactions with a nucleon bound in carbon, NUANCE propagates the outgoing hadrons including nucleons, mesons and baryonic resonances, and simulates their re-interaction as they exit the nucleus. The initial interaction model employs the impulse approximation which assumes an instantaneous exchange with independent nucleons. Subsequent to the initial neutrino interaction, particles produced inside the nucleus are propagated step-wise in $0.3 \mathrm{fm}$ increments until they emerge from the $\sim 2.5 \mathrm{fm}$ radius sphere. Intermittently, the probability for hadronic re-interaction is calculated using a radially-dependent nucleon density distribution [105] along with external $\pi-N, N-N$ cross-section measurements [106]. For $\Delta$ re-interactions $(\Delta+N \rightarrow N+N)$, an energy-independent probability of $20 \%(10 \%)$ is taken for $\Delta^{+}+N, \Delta^{0}+N$ $\left(\Delta^{++}+N, \Delta^{-}+N\right)$ based on K2K data [77] and is assigned $100 \%$ uncertainty.

Out of all hadronic reinteraction processes, pion absorption $\left(\pi^{ \pm}+X \rightarrow X^{\prime}\right)$ and charge exchange $\left(\pi^{ \pm}+X \leftrightarrow \pi^{0}+X^{\prime}\right)$ are the most relevant in predicting the composition of the samples studied in the analyses of this dissertation. Shown in Figure 4-4, intranuclear fractional uncertainties on pion absorption (charge-exchange) are set to $25 \%$ (30\%) based on comparisons between external data [107] and the NUANCE prediction. 

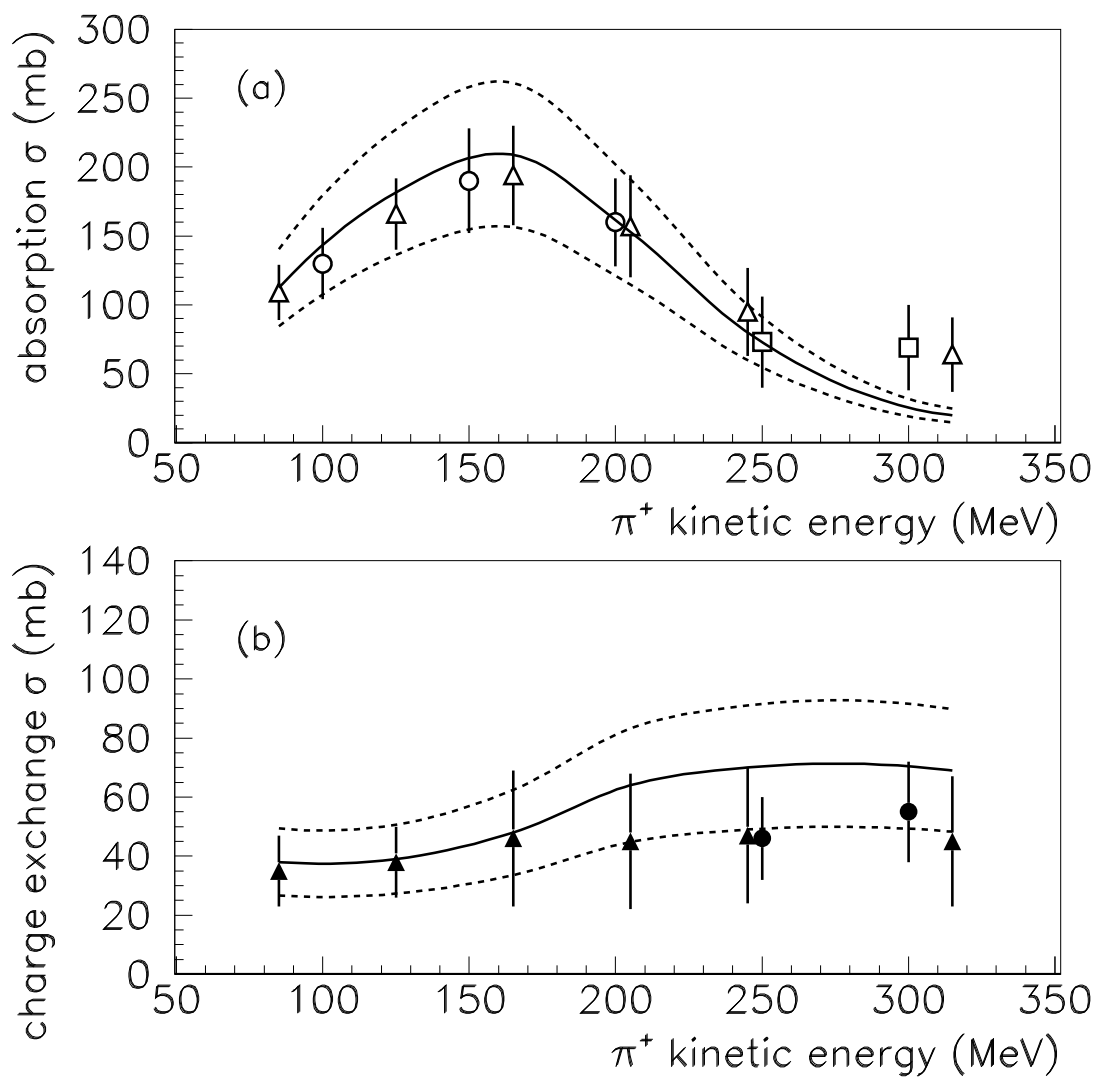

Figure 4-4. Comparison of the NUANCE prediction for $\pi^{+}$absorption (top) and chargeexchange (bottom) to relevant data [107]. Figure from Ref. [84]. 


\section{CHAPTER 5 \\ THE MINIBOONE EXPERIMENT}

\subsection{Overview}

The MiniBooNE experiment was designed to optimize the search for the appearance of $\nu_{e}$ events in a beam of $\nu_{\mu}$. Accordingly, many design choices were made and auxiliary systems implemented to maximize detection efficiency for $\nu_{\mu}$ and $\nu_{e} \mathrm{CC}$ events sensitive to mass splittings of $\Delta m^{2} \sim 1 \mathrm{eV}^{2}$ while maintaining discrimination power between the two neutrino species. This chapter describes the physical layout of the experiment and the detector subsystems most crucial to the measurement of muon kinematics. An expanded description of the beamline and neutrino flux calculation can be found in Ref. [108], while the overall design and performance of the detector is discussed in more detail in Ref. [109].

\subsection{The Booster Neutrino Beamline}

The Booster Neutrino Beamline (BNB) collides $8.9 \mathrm{GeV} / \mathrm{c}$ momentum protons onto a beryllium target, and a magnetic horn is used to sign-select and focus the secondary meson beam in the direction of the detector. Depending on the polarity of the magnetic field, the selected meson decay modes yield an enhanced $\nu_{\mu}$ or $\bar{\nu}_{\mu}$ beam. This section steps through the important instruments in this process, finally arriving at the calculation of the neutrino flux observed by the detector.

\subsubsection{The Primary Proton Beam}

The Fermilab accelerator chain begins with a bottle of hydrogen gas and a voltage multiplier system first demonstrated in 1913 [110]. This Cockroft-Walton system generates a large DC voltage from a small AC input with a ladder network of capacitors and diodes. At each successive stage, the charge on each capacitor is doubled by simultaneously collecting charge stored in the previous capacitor and the $\mathrm{AC}$ input.

The Fermilab Cockroft Walton machine applies a voltage difference of $750 \mathrm{kV}$ across an ionization chamber, the negative potential side of which is coated with cesium metal, and the other wall is partially open in the direction of the Fermilab linear accelerator 


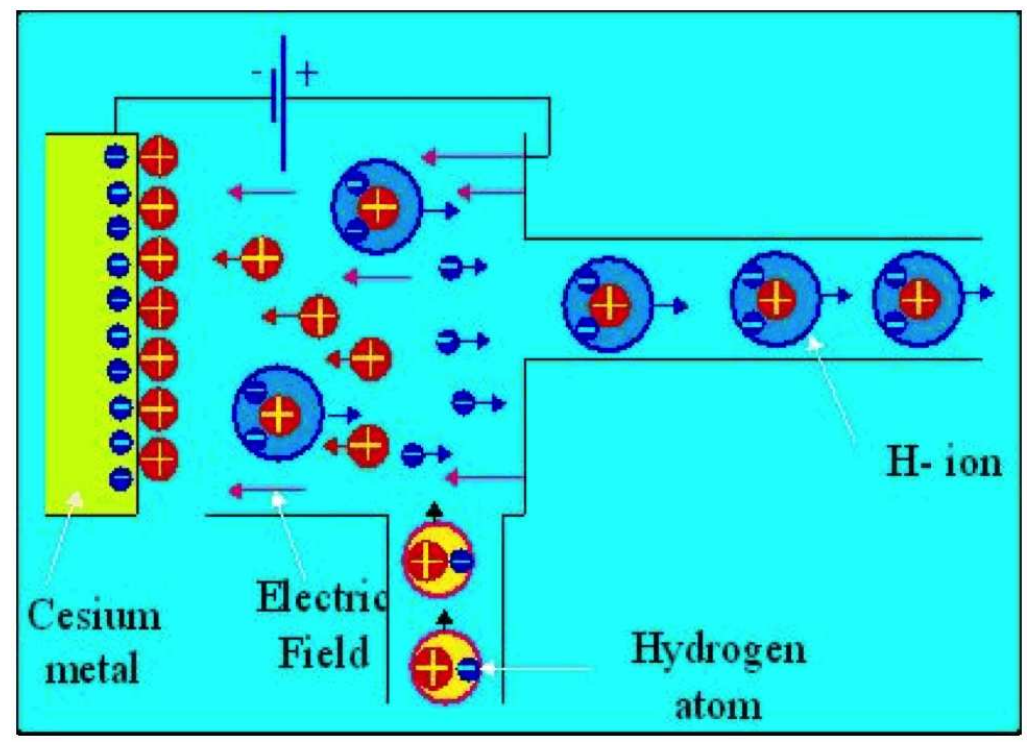

Figure 5-1. Cartoon of the Fermilab pre-accelerator stage. Figure taken from Ref. [111].

(linac). Hydrogen atoms that drift into this chamber will ionize, and the bare protons will collide with the cesium metal. Cesium has a relatively low work function, and some of these collisions result in the transfer of two valence electrons to the proton, forming an $H^{-}$ ion. These $750 \mathrm{keV}$ kinetic energy negatively-charged atoms drift to the wall of positive potential, and may pass through the opening and continue to the next accelerator stage. A cartoon of this process is shown in Figure 5-1.

The $H^{-}$atoms then enter the linac, where an alternately polarized electric field accelerates the ions between gaps of Faraday cage drift tubes. Beam bunches are formed with pulses roughly $5 \mathrm{~ns}$ apart, and the $130 \mathrm{~m}$ long linac terminates with $H^{-}$batches of $400 \mathrm{MeV}$ kinetic energy.

As shown in Figure 5-2, these bunches are injected into the Booster synchrotron via a system featuring a stripping foil placed between a series of dipole magnets in a "dogleg" configuration. The foil strips the $H^{-}$ions of their electrons, and the subsequent magnets steer the bare protons onto the Booster orbit. The dogleg dipole magnet configuration has the effect of a focusing and defocusing (FODO) quadrupole system, where the injected $H^{-}$ atoms and the Booster protons converge to a single beam. 


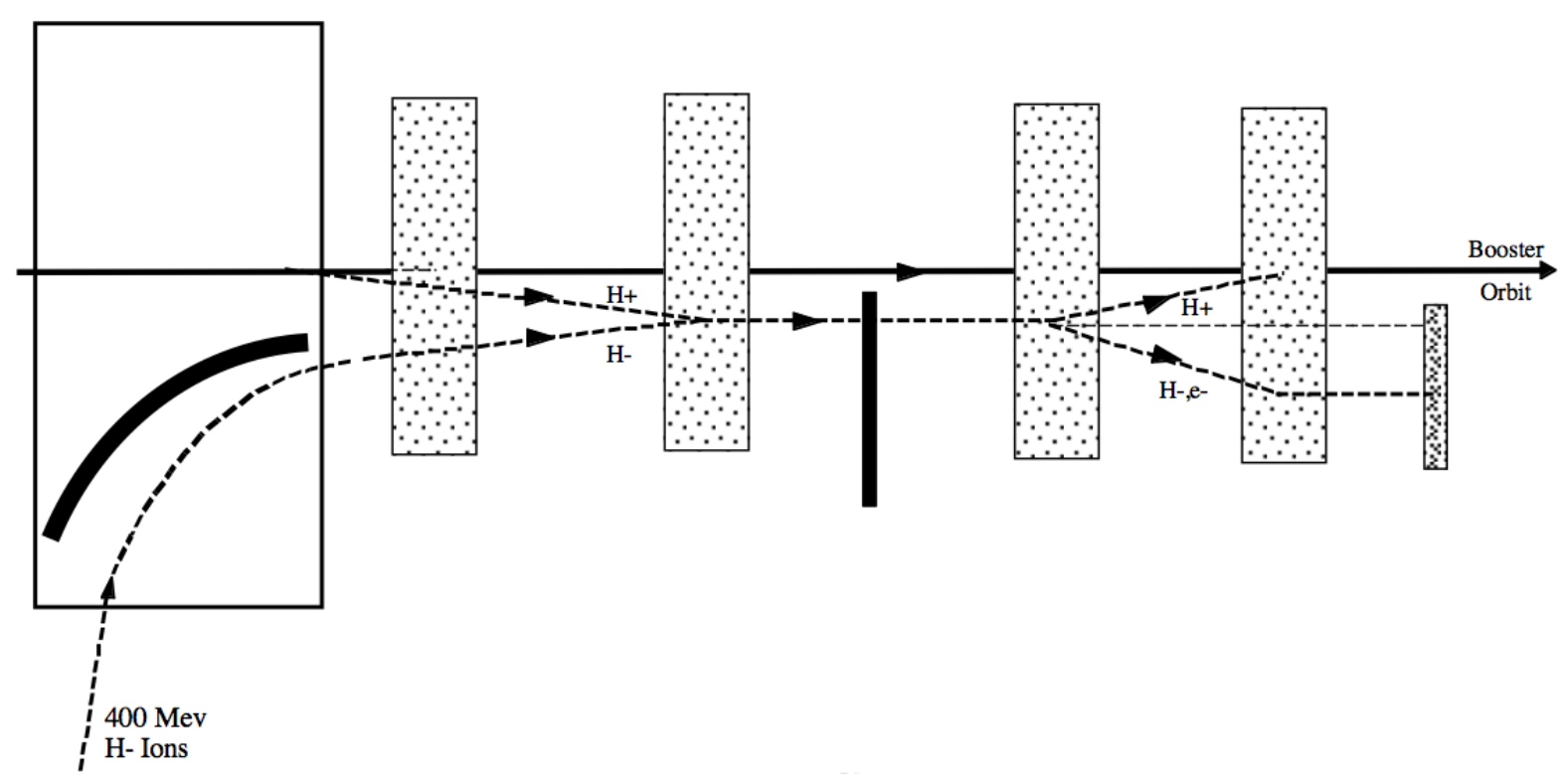

Figure 5-2. Injection diagram for $H^{-}$ions onto the Booster orbit as bare protons. Figure taken from Ref. [112].

To avoid unnecessary beam divergences in the Booster, the dogleg dipoles are only pulsed when beam is injected from the linac. The Booster synchrotron accelerates the $400 \mathrm{MeV}$ kinetic energy protons up to $8 \mathrm{GeV}$ through 17 radio-frequency (RF) stages and is kept on-orbit by 24 periods of FOFDOOD cells. This acceleration takes roughly $33 \mathrm{~ms}$ and 20,000 turns around the $150 \mathrm{~m}$ diameter ring. The harmonic number of the Booster is 84, though typically 3 buckets are not used. These 81 bunches, each separated by $\sim 19$ ns, of $8 \mathrm{GeV}$ kinetic energy protons are extracted from the Booster in a $1.6 \mu$ s spill. Shown in Figure 5-3, this structure is clearly visible in the arrival of neutrino events at the MiniBooNE detector. These spills typically contain $5 \times 10^{12}$ protons and are delivered to the MiniBooNE target and horn system at a maximum rate of $5 \mathrm{~Hz}$. Full details of the Booster synchrotron is available in Ref. [112]. 


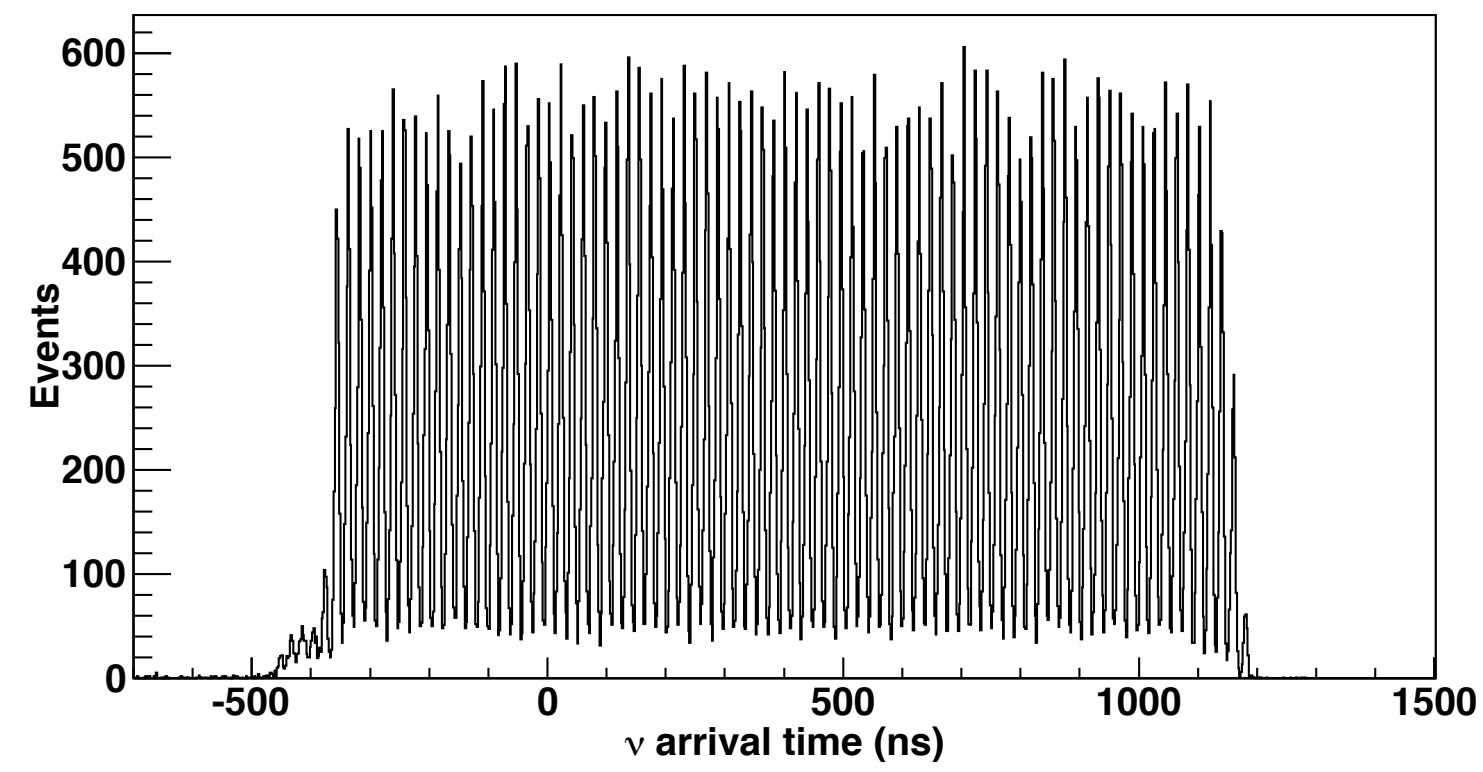

Figure 5-3. Timing structure of the BNB proton spills through the arrival time of neutrino events at MiniBooNE. The represented data is the neutrino-mode CCQE sample, and a time-of-flight correction based on the observed interaction vertex along the beam direction has been applied. The $\sim 500 \mathrm{~ns}$ offset between the arrival and recorded times is due to an offset in the timing instruments. 


\subsubsection{Beryllium Target and Magnetic Focusing Horn}

The next stage in the BNB converts the proton spill into a focused beam of mesons. The proton beam strikes a $71.1 \mathrm{~cm}$ long target, composed of seven $10.2 \mathrm{~cm}$ long and $0.5 \mathrm{~cm}$ radius cylindrical beryllium slugs.

The proton-beryllium interactions deposit $\sim 600 \mathrm{~W}$ under normal running conditions, and so an air-cooling system is implemented to reduce radiation damage to the system and the surrounding environment. The beryllium target is separated from its housing using three supporting "fins", also made of beryllium, and allows for air to be circulated along its entire length. The air flow rate is $\sim 8 \times 10^{-3} \mathrm{~m}^{3} / \mathrm{s}$ and, due to a heat exchanger system, flows continuously during normal running conditions. Engineering designs for the beryllium target and its installation inside the magnetic horn are shown in Figure 5-4.

The proton-beryllium interactions create a spray of secondary particles, including many neutrino-parent mesons. A set of connected inner and outer conductors form a horn system, and an electric current of $\pm 174 \mathrm{kA}$ pulsed through these conductors in time with the BNB proton spill creates a toroidal magnetic field as shown in Figure 5-5. This field simultaneously focuses particles with positive or negative charge, while defocusing the other. In this way, the polarity of this system defines the running mode - focusing positively-charged mesons yields an enhanced $\nu_{\mu}$ beam (dominantly via $\pi^{+} \rightarrow \mu^{+} \nu_{\mu}$ ) while selecting negative mesons creates a $\bar{\nu}_{\mu}$-enhanced beam $\left(\right.$ via $\left.\pi^{-} \rightarrow \mu^{-} \bar{\nu}_{\mu}\right)$.

The magnetic horn simultaneously controls the neutrino composition of the BNB beam and substantially increases the neutrino flux. In neutrino-mode running, the horn increases the observed rate of neutrino interactions by roughly a factor of six. As with the beryllium targets, the magnetic horn must also be cooled to protect against radiation

damage. A closed water system keeps the system exceptionally stable. The first BNB horn pulsed 96 million times before failing due to corrosion, while the second horn is still operational and has been pulsed a world's record 397 million times as of March 2013. 

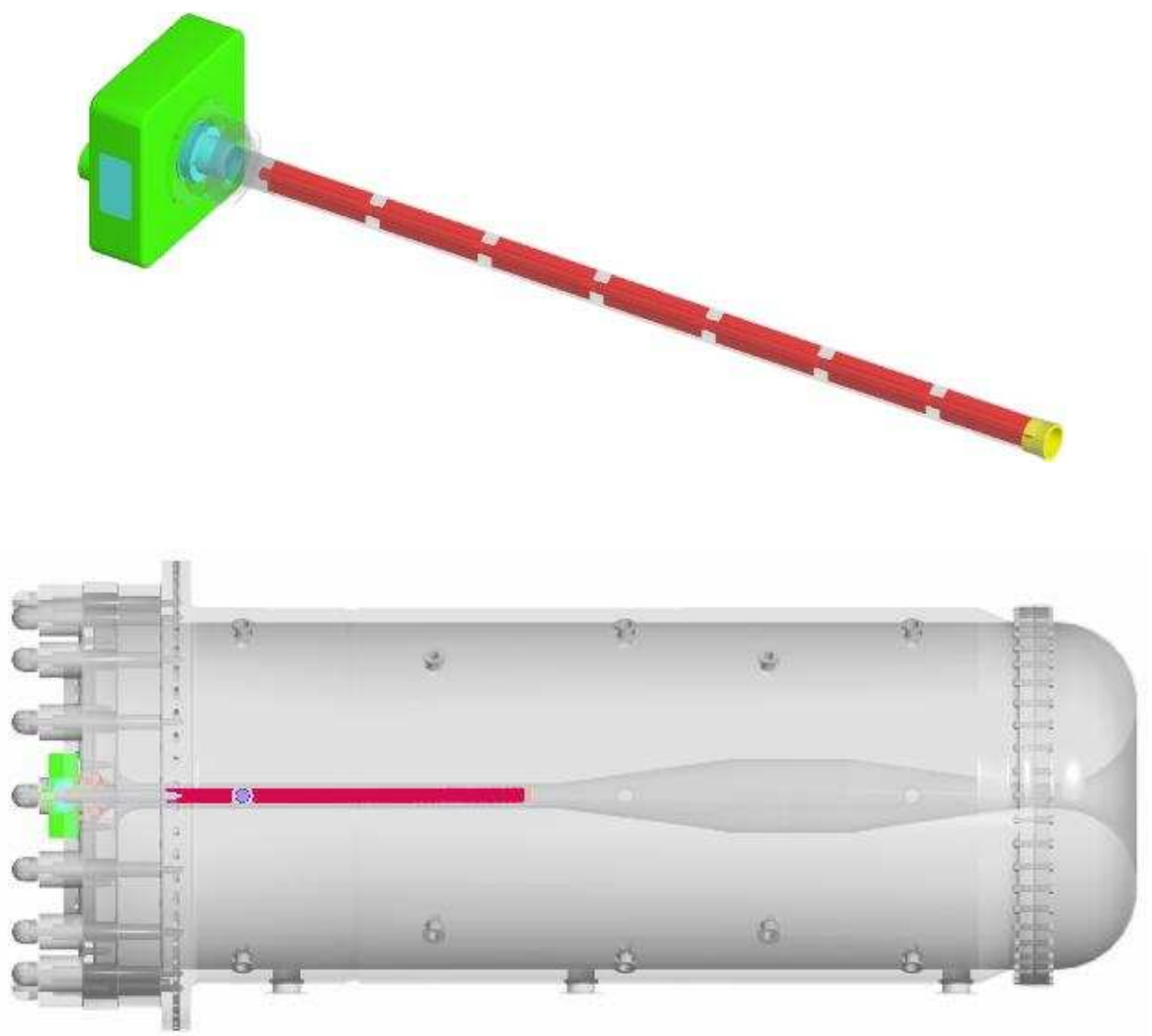

Figure 5-4. The BNB beryllium target. Shown is an expanded view of the segmented target (top) and its place inside the magnetic focusing horn (bottom). The proton beam strikes the target from the left. 


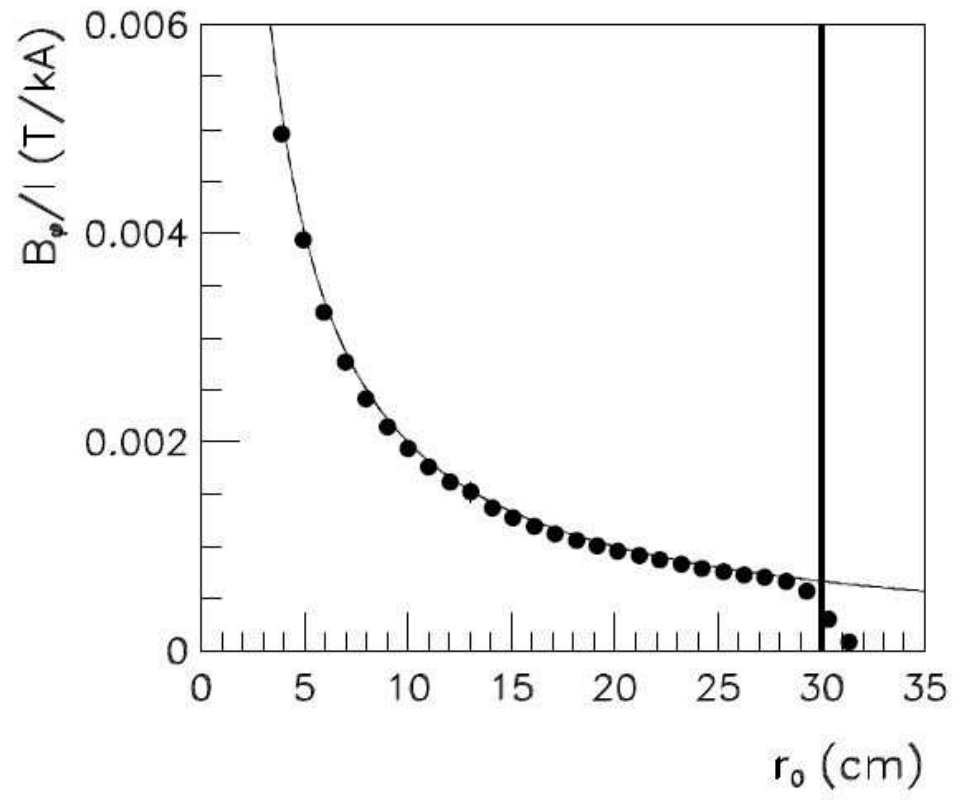

Figure 5-5. A comparison of the azimuthal component of the magnetic field relative to the input current between data (in points) and the expectation (solid curve) of $1 / \mathrm{r}$ dependence. The vertical line identifies the inside edge of the outer conductor. Figure taken from Ref. [113].

\subsubsection{Meson Decay Region}

The mesons accepted into the neutrino beam are collimated immediately downstream through a $60 \mathrm{~cm}$ opening in a concrete slab and subsequently enter a $50 \mathrm{~m}$ long, air-filled decay volume. The mesons decay in flight to produce charged leptons and neutrinos, or are absorbed by a concrete wall at the end of the decay volume. Protons that do not strike the beryllium target may interact with the air molecules in the decay region before terminating at the beam dump. These interactions may also produce mesons boosted towards the detector, and these processes contribute $\sim 5 \%$ of the neutrino flux at MiniBooNE.

Ten 25-ton steel absorber beams are housed above the middle of the decay region, and could be deployed in the hall to systematically alter the normalization and energy spectrum of the neutrino beam. Specifically, the shortened decay region would remove higher-energy neutrinos, including an appreciable amount of the instrinsic $\nu_{e}$ and $\bar{\nu}_{e}$ from 
the $\pi^{ \pm} \rightarrow \mu^{ \pm}$decay chain. Meanwhile, the overall $\nu_{\mu}$ and $\bar{\nu}_{\mu}$ flux would be reduced by roughly $10 \%$ per deployed absorbed. So far, these absorbers have not been intentionally deployed; however, in an early period of antineutrino-mode running, one and then another absorber fell into the beamline. A total of $5.69(6.12) \times 10^{19}$ POT was collected in antineutrino-mode with one (two) absorbers present in the decay hall. Details of the systematic effects caused by these blocks was implemented into simulation, and consistency between the observed and predicted rate and kinematics suggest the modeling is adequate. As the MiniBooNE $\bar{\nu}_{\mu} \rightarrow \bar{\nu}_{e}$ oscillation search is limited by statistics to date [36], these data are included in the oscillation analysis, as is the case with an early determination of the $\nu_{\mu}$ contribution to the antineutrino-mode beam presented in Section 7.1.5. However, the double-differential cross section $\frac{d^{2} \sigma}{d T_{\mu} d \cos \theta_{\mu}}$ for $\bar{\nu}_{\mu}$ CCQE interactions is limited by statistics only in small regions of the distribution tails, and so these absorber-down data are not used in the main result of this dissertation.

\subsubsection{Neutrino Flux Calculation}

The most important piece of an absolute neutrino flux calculation is the production of the neutrino and antineutrino parent $\pi^{+}$and $\pi^{-}$created in proton-beryllium interactions at the target. It is common to rely on a combination of hadroproduction models and data-based extrapolations to meet this goal. However, Figure 5-6 shows modern models $[114,115,116,117]$ for primary hadroproduction $\left(p+\mathrm{Be} \rightarrow \pi^{ \pm}+X\right)$ at $8.9 \mathrm{GeV}$ proton beam momentum dramatically disagree.

Clearly, precision neutrino and antineutrino cross section measurements cannot be made with information from hadroproduction models alone. A much more clean and direct method for constraining the neutrino flux was fortunately available to MiniBooNE: the HARP hadroproduction experiment at CERN collected dedicated data using the same proton momentum and target material as in the BNB. Double-differential cross sections in terms of pion kinematics was measured for both $\pi^{+}$[118] and $\pi^{-}$[119], allowing for a 

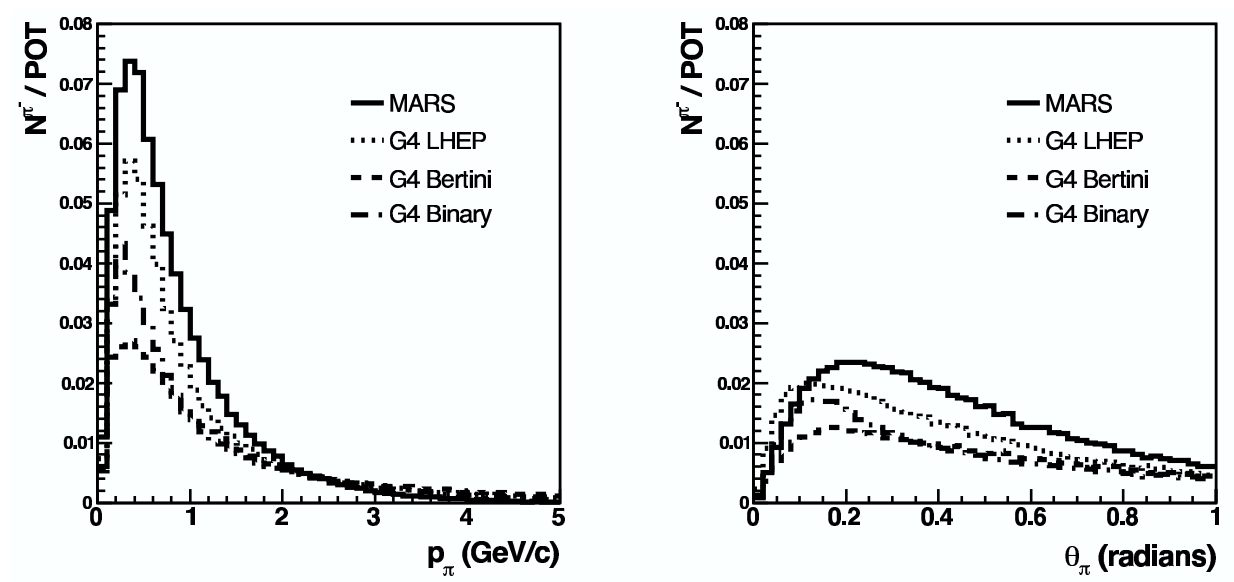

Figure 5-6. Production of primary $\pi^{-}$per POT for $8.9 \mathrm{GeV}$ momentum protons incident on beryllium for various hadroproduction models as a function of $\pi^{-}$momentum (left) and opening angle with respect to the incoming proton beam (right). Figure taken from Ref. [119].

minimally model-dependent determination of primary $\pi$ production at the BNB for both the neutrino-mode and antineutrino mode run configurations.

However, even with dedicated data appropriate to the experimental setup of MiniBooNE, there remain small regions of phase space relevant to the antineutrino-mode beam not covered by the HARP measurements. As will be expanded and directly addressed in Chapter 7, of particular importance to this work is the production of very forward pions with respect to the direction of the incoming proton beam. In the HARP experiment, this same angular region suffers from re-interactions in the target and a severe proton background, preventing a clean measurement of the pion production cross section. For these reasons, pion cross sections in the $\theta_{\pi}<30 \mathrm{mrad}$ region, where $\theta_{\pi}$ is the angle the outgoing pion makes with respect to incoming protons, are not covered by the HARP data. Instead, the nominal primary $\pi$ production cross section for this region in the MiniBooNE flux calculation is extrapolated from the existing HARP data using a Sanford-Wang [120] paramaterization. More suitable for extrapolating uncertainties, errors on primary $\pi$ production come from the piecewise polynomial spline interpolation [121]. This extrapolation is only one of many possible choices, and is therefore subject to large 
uncertainties. Figure 5-7 shows the HARP data, the Sanford-Wang parametrization, and the production uncertainty from the spline procedure for primary $\pi^{-}$production.

The HARP data was taken on a thin version ( $5 \%$ proton interaction length) of the full-sized (170\%) MiniBooNE beryllium target, and so these data do not include possible hadronic re-interactions inside the target. The total cross section for these secondary interactions are calculated with the Glauber model [122], and this calculation is verified with comparisons to data wherever possible. Based on the agreement between this model and the available data, uncertainties on the most important processes contributing pions to the beam are set around $20 \%$ and higher [108, 123]. Fortunately, while some details of this calculation are model-dependent, Figure 5-8 shows the overall contribution of these processes to the overall neutrino flux is rather mild, at the level of $\sim 10 \%$. Moreover, the same figure also suggests the contribution from tertiary pions present in the long MiniBooNE target but not in the thin target data from HARP is small. Therefore, with the exception of the very forward-going angular region, the HARP data allows for a minimally model-dependent determination of the production of neutrino and antineutrino parent pions at the BNB.

A GEANT4-based package [124] is used to calculate the neutrino flux observed at MiniBooNE. The simulation takes as input the previously-described meson production and considers the beamline geometry, proton travel to the target, p-Be interactions in the target, magnetic horn focusing, particle propagation, meson decay, and finally neutrino and antineutrino transport to the detector. For both neutrino and antineutrino mode run configurations, the uncertainty on pion production and the set of all other beamline uncertainties contribute roughly equally to the $\sim 9 \%$ total uncertainty on the absolute flux prediction for the selected neutrino species. Figure 5-9 shows the predicted flux of $\nu_{\mu}, \bar{\nu}_{\mu}$, $\nu_{e}$, and $\bar{\nu}_{e}$ observed by the MiniBooNE detector for both neutrino and antineutrino run modes. 

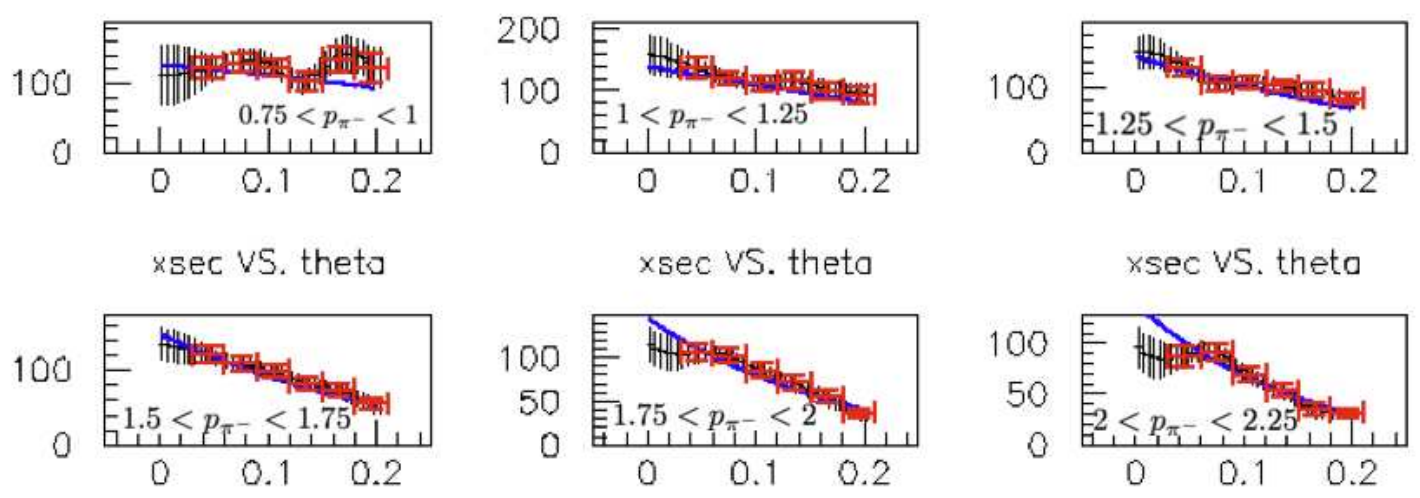

xsec VS, theta

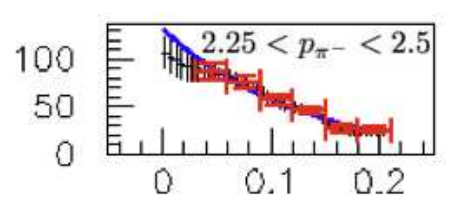

$x \sec V$ s. theta

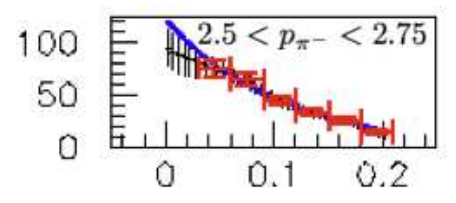

$x \sec$ VS, theta

xsec VS, theta
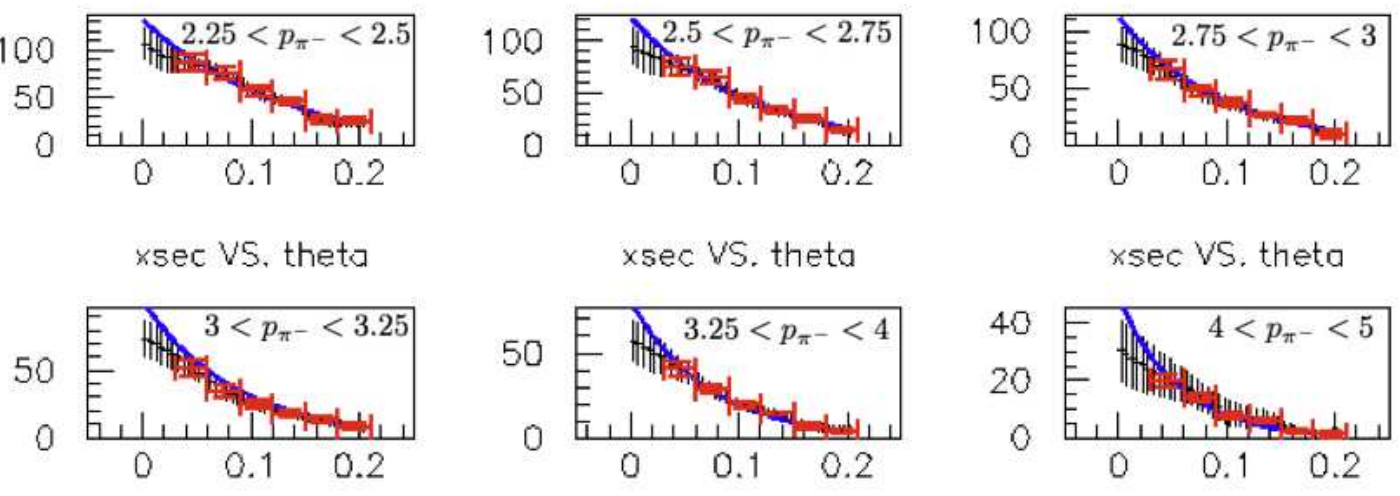

xsec VS, theta

xsec VS. theta

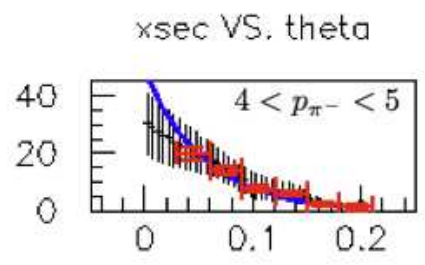

xsec VS, theta

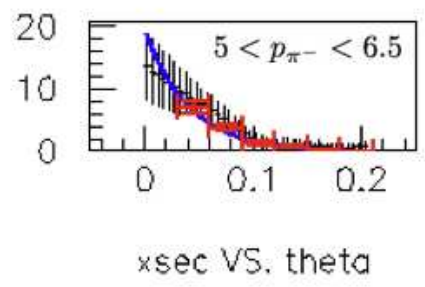

Figure 5-7. Double-differential cross section $d^{2} \sigma / d p_{\pi} d \Omega_{\pi}$ for $p+\mathrm{Be} \rightarrow \pi^{-}+X$ in units of mb / ( $\mathrm{GeV} \mathrm{sr})$. The angular axes have units of radians, and the momentum projections are in units of GeV. The blue curve is the Sanford-Wang parametrization based on the red HARP data points, and the black histogram with uncertainties is the spline interpolation. Figure taken from Ref. [125]. 


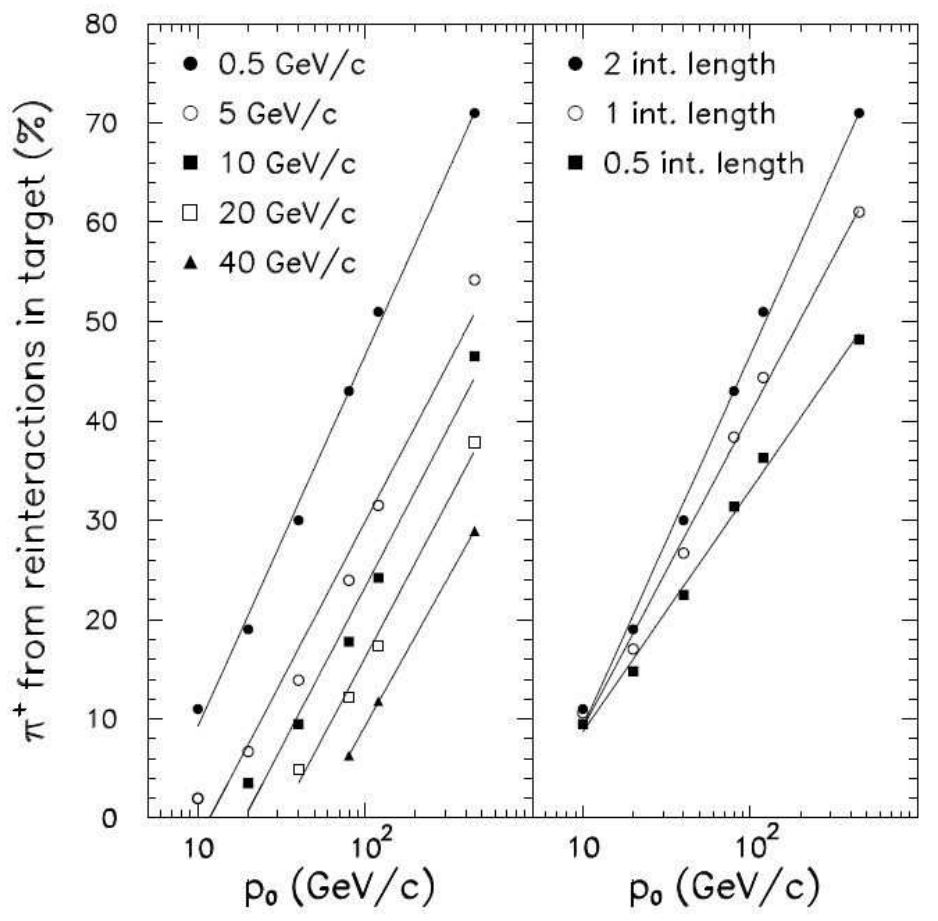

Figure 5-8. Fluka [126] calculations of the tertiary $\pi^{+}$yield from reinteractions in a graphite target. Given as a function of incident proton beam momentum $p_{0}$, the $\pi^{+}$fraction is given for the indicated thresholds on the longitudinal component of the $\pi^{+}$momentum (left), and also for targets of $0.5,1.0$, and 2.0 interaction lengths (right). The primary proton beam at the BNB has momentum $8.9 \mathrm{GeV} / \mathrm{c}$. Figure taken from Ref. [127]. 

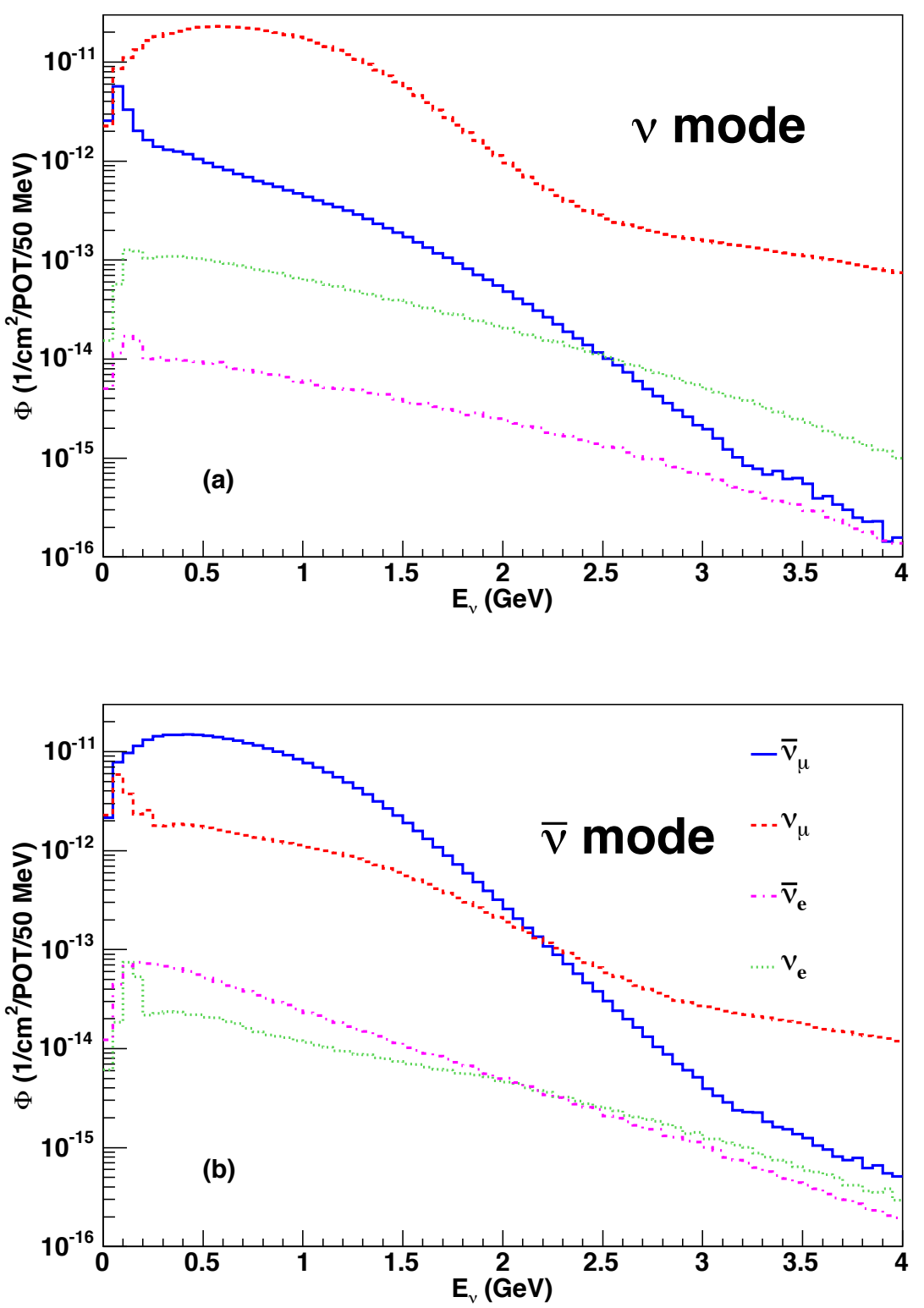

Figure 5-9. The MiniBooNE flux prediction for (a) neutrino mode and (b) antineutrino mode. Data taken from Ref. [108]. 


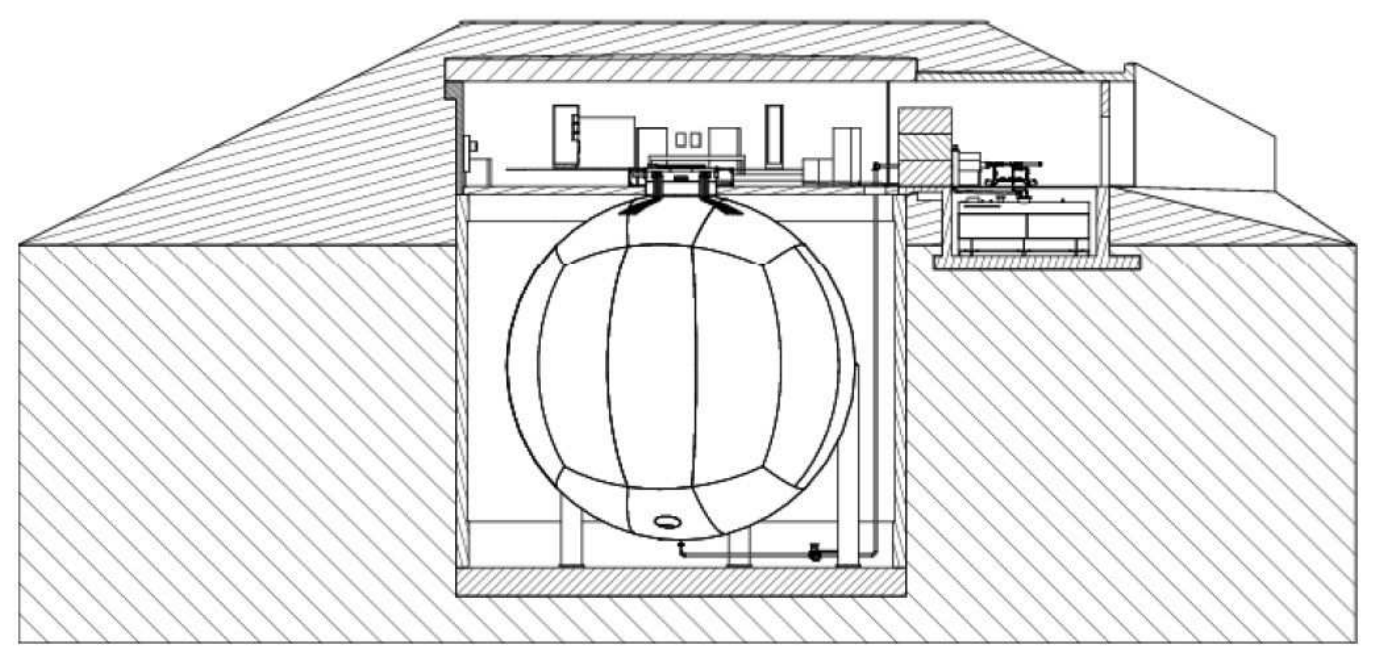

Figure 5-10. Overview of the MiniBooNE detector housing. Image taken from Ref. [128].

\subsection{Detector}

\subsubsection{Physical Layout}

Shown schematically in Figure 5-10, the detector is a $12.2 \mathrm{~m}$ diameter sphere housed in a $13.7 \mathrm{~m}$ underground cylindrical vault such that the top of the tank sits roughly at ground level. The detector shape was motivated by maximizing the volume to surface area ratio, affording greater photocathode coverage for the same number of PMTs. The simple spherical geometry also allows for globally symmetric reconstruction algorithms and thus equal sensitivity to particle kinematics across all scattering angles. An earth overburden of $\sim 3 \mathrm{~m}$ reduces the rate of cosmic-ray muons entering the detector to $\sim 10 \mathrm{kHz}$. Between the detector and the overburden is an access room housing the main electronics, including the muon calibration system crucial to the measurement of this dissertation discussed in Section 5.3.4.

The tank is filled with 818 tons of undoped mineral oil, optically segregated into an inner signal region of radius $575 \mathrm{~m}$ and an outer veto shell of $35 \mathrm{~cm}$ thickness. Light produced in the detector is collected by 1520 8-inch Hamamatsu photomultiplier tubes (PMTs), 1280 of which face into the signal region (11.3\% coverage) while 240 are inside the outer shell. Figure 5-11 shows a cartoon of the MiniBooNE detector partially cut away to 

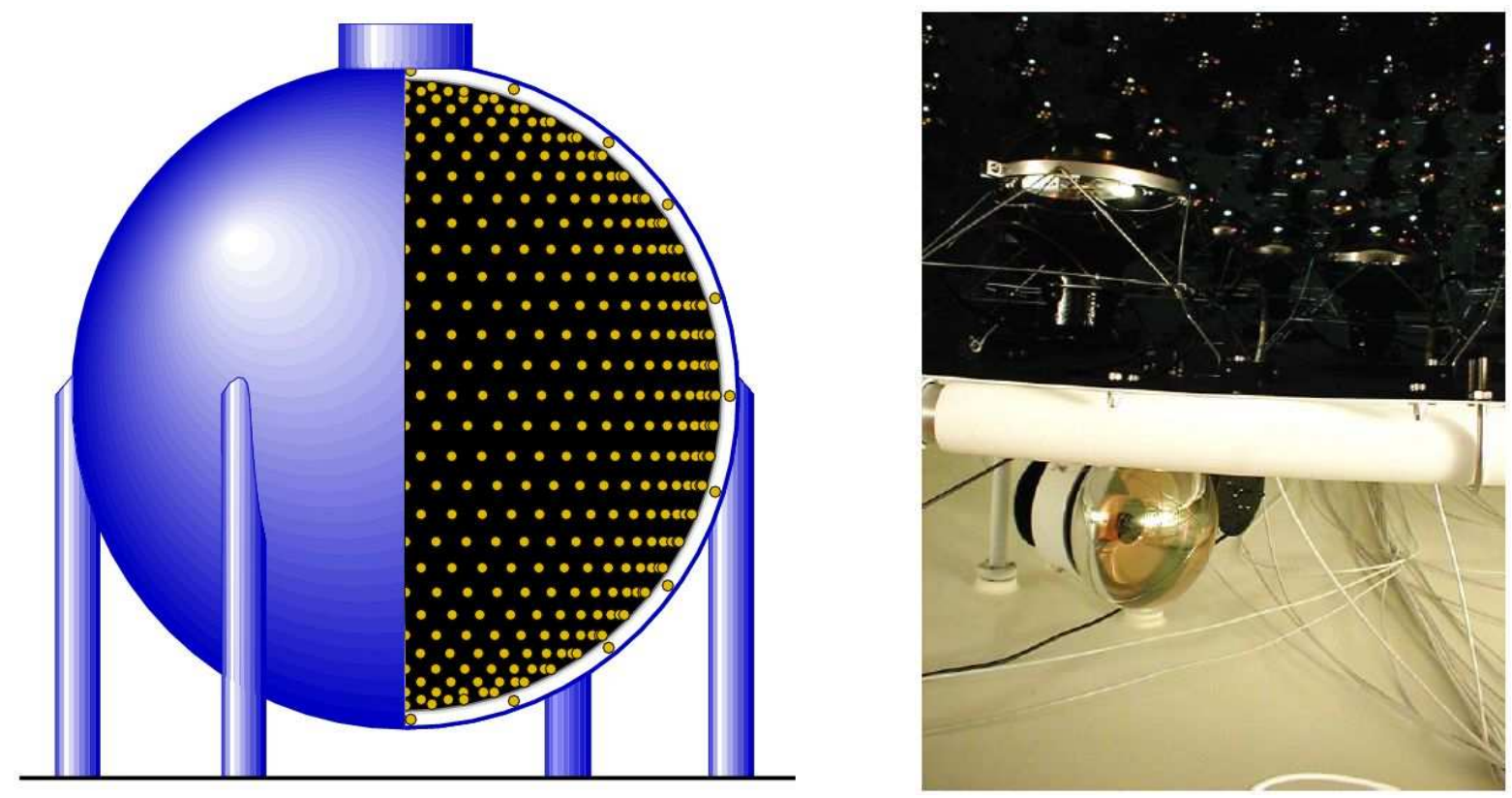

Figure 5-11. On the left, cutaway drawing of the MiniBooNE detector showing PMTs dispersed in the inner and outer regions. The optical barrier separating the outer veto region (painted white) from the inner signal region (painted black) is shown on the right.

show the inner components as well as a photo of the optical barrier separating the two regions. Low activity in the veto region is required in physics analyses to ensure containment of charged particles produced by beam-induced neutrinos while also eliminating contamination from charged particles entering the tank. To encourage photon rescattering and thus maximize detection efficiency for charged particles traversing the veto region, the surfaces are painted white. In contrast, to improve the kinematical resolution of signal events, photon rescattering is minimized with a black surface for the inner region.

\subsubsection{Mineral Oil and its Properties}

A common choice for the detection medium in Čerenkov-based experiments is water. In the case of MiniBooNE, mineral oil was selected over water for a variety of reasons:

- the increased index of refraction yields a lower momentum threshold on Cerenkov light production for all particles, globally improving detection efficiency

- nuclear capture of stopped $\mu^{-}$is $\sim 8 \%$ in mineral oil, compared to $\sim 20 \%$ in water. 
Table 5-1. Momentum threshold for production of Čerenkov radiation for four important particle types in mineral oil compared to water.

\begin{tabular}{ccc}
\hline Particle & \multicolumn{2}{c}{ Cerenkov threshold } \\
& mineral oil, $\mathrm{n}=1.47$ & water, $\mathrm{n}=1.33$ \\
\hline electron & $0.7 \mathrm{MeV} / \mathrm{c}$ & $0.8 \mathrm{MeV} / \mathrm{c}$ \\
muon & $144 \mathrm{MeV} / \mathrm{c}$ & $160 \mathrm{MeV} / \mathrm{c}$ \\
pion & $190 \mathrm{MeV} / \mathrm{c}$ & $212 \mathrm{MeV} / \mathrm{c}$ \\
proton & $1280 \mathrm{MeV} / \mathrm{c}$ & $1423 \mathrm{MeV} / \mathrm{c}$ \\
\hline
\end{tabular}

This allows a cleaner tagging of $\nu_{\mu} \mathrm{CC}$ events, again improving detection efficiency while simultaneously reducing its background contribution to the $\nu_{e} \mathrm{CC}$ sample.

- by exploiting PMT activity timing information, the lowered speed of light in the medium improves interaction vertex resolutions

Kept at $\sim 20^{\circ} \mathrm{C}$, the mineral oil has a density of $0.845 \mathrm{~g} / \mathrm{cm}^{3}$ and an index of refraction of 1.47. Under these conditions, charged particles with velocity $\beta>0.68$ produce Čerenkov radiation. The momentum thresholds for production of Čerenkov radiation for relevant particle species in mineral oil and water are compared in Table 5-1.

The above benefits come at the cost of significantly more complex mechanisms for light production and propagation through the detector. Due to impurities in the oil, molecular excitations produce delayed photons with an isotropic direction and of longer wavelength than the absorbed particle. These are known as fluorophores, or fluors, and four distinct modes were observed in table-top measurements of the MiniBooNE oil [109]. The measured and extrapolated extinction rates of these fluors are shown in Figure 5-12.

The presence of fluors obfuscate the topology of the Čerenkov signature and bias the correlation between the collected Čerenkov light and the true energy of the particle. Fortunately, calibration samples and systems discussed in the next section exist to measure these biases so that their effect on most analyses (including the topic of this dissertation) are minimal. Though the fluors complicate the understanding of the detector, without 


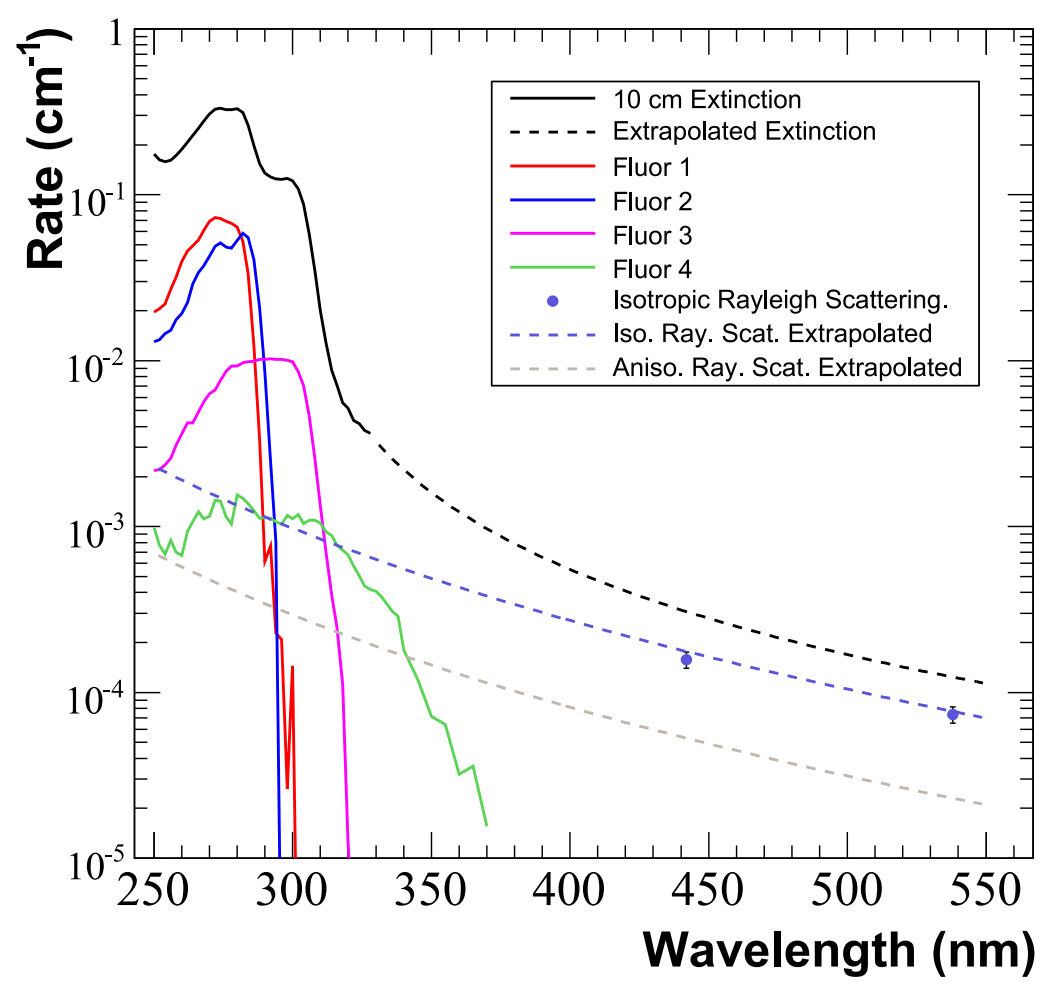

Figure 5-12. Photon extinction rate spectrum in the MiniBooNE oil. As indicated, the solid curves correspond to measurements, while the dashed lines are based on an extrapolation of these data and are tuned to various in situ calibration data samples. Figure taken from Ref. [109].

their presence scintillation-based measurements such as the neutral current elastic cross sections $[129,130]$ would not be possible. 


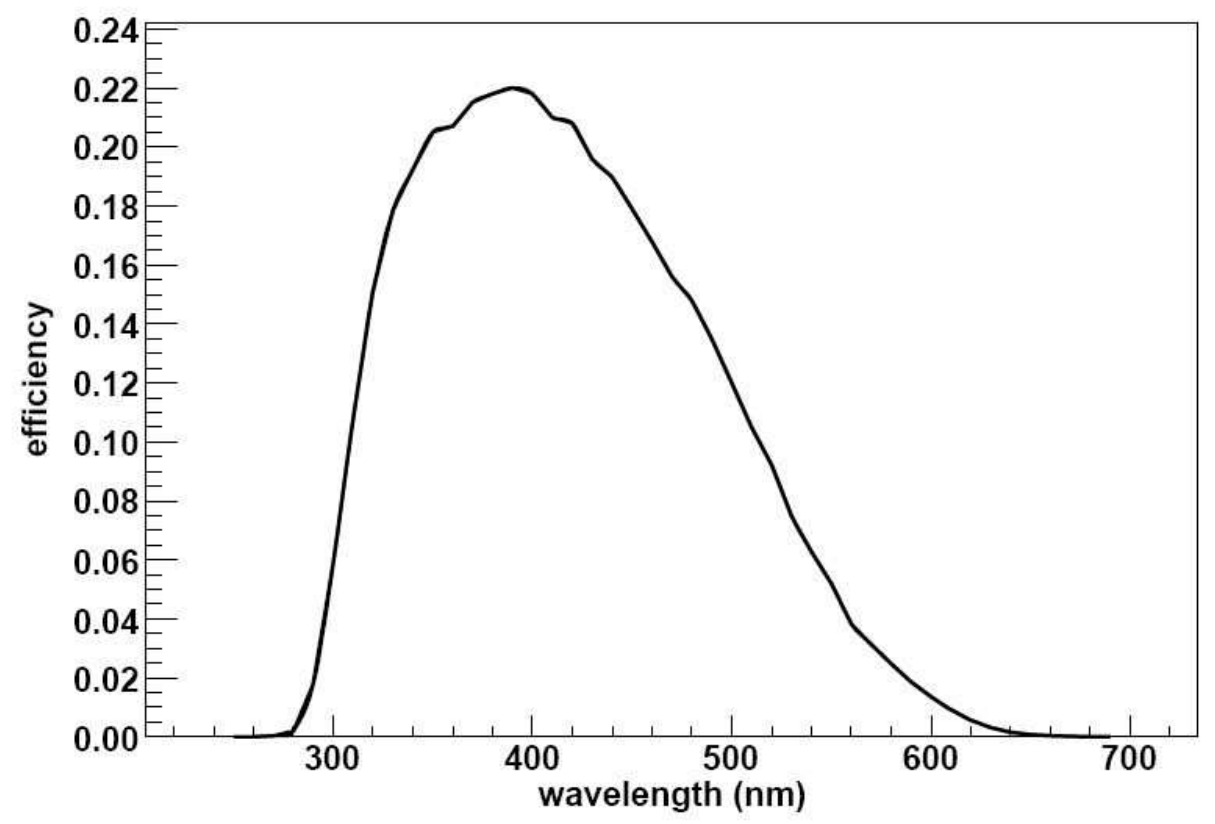

Figure 5-13. Quantum efficiency for the newer MiniBooNE PMTs. Figure taken from Ref. [132].

\subsubsection{Photomultiplier Tubes}

Of the 1520 PMTs, 1198 are 9-stage and have been repurposed following their use in the LSND experiment, while the remainder are 10-stage tubes purchased for MiniBooNE. Tests for charge and time resolution, the voltage level required to meet the desired gain, and the dark current were performed for all PMTs installed into MiniBooNE. The newer tubes feature average timing (charge at one photoelectron) resolution of $\sim 1.1 \mathrm{~ns}(40 \%)$, while the older tubes resolve the same quantities at $\sim 1.7 \mathrm{~ns}(130 \%)$ [131]. The average dark current for the new (old) tubes was found to be 1.0 (1.4) kHz at their operating voltage. Due to their superior performance, the newer PMTs are distributed uniformly in the signal region, while the LSND tubes with higher amounts of dark noise are used in the veto region. The quantum efficiency for the new PMTs is given in Figure 5-13.

\subsubsection{Calibration Systems}

In situ measurements of the PMT performance and the oil attenuation length over the lifetime of the experiment is afforded by a pulsed laser calibration system, shown 


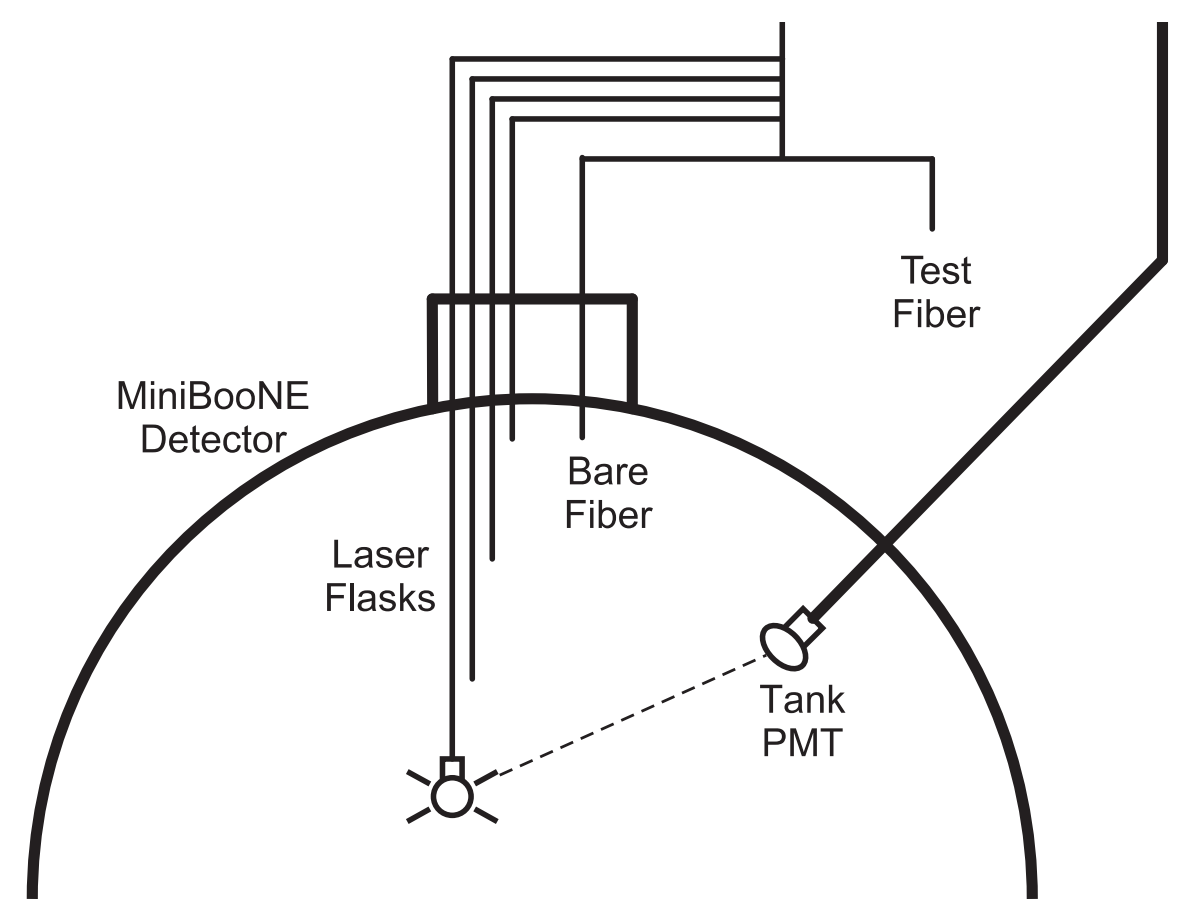

Figure 5-14. Schematic of the laser calibration system. Image taken from Ref. [109].

schematically in Figure 5-14. Four laser dispersion flasks and a single bare optical fiber are distributed throughout the detector and are pulsed at $3.33 \mathrm{~Hz}$ during normal data taking. Given the peak efficiency for the MiniBooNE PMTs at $\sim 400 \mathrm{~nm}$, these lasers are pulsed at $397 \mathrm{~nm}$ and signals from the dispersion flasks illuminate all PMTs with roughly equal intensities. Interpretations of the signals from the dispersion flasks are somewhat degenerate between effects arising from degrading oil properties and changing PMT performance. This degeneracy is partially broken by the signal from the bare optical fiber, which illuminates a small circle of PMTs near the bottom of the detector and is used to more directly study any evolution of the oil properties.

The most important aspects of the PMT performance probed at $3.33 \mathrm{~Hz}$ under normal running conditions (though the system is vetoed in case of coincidence with a beam spill from the BNB) by the laser calibration system are PMT time offsets and gain calibrations. Time offsets due to differing transit times for each readout system are obtained by a simple comparison of the observed laser signal arrival time to the known 
laser pulse, while also considering travel time for the laser light. The gain of individual PMTs can vary in subtle but important ways, and these effects are calibrated by normalizing the response of each PMT to a single value, based on the input intensity of the laser light. The calibrated time offsets are critical to the detector's ability to separate the Čerenkov signatures from different particles, most notably those connected by decay processes, while the gain corrections allow for precise measurements of particle energy uniform in production position and direction.

Even more crucial to the study of $\bar{\nu}_{\mu}$ CCQE interactions, cosmic-ray muons and a dedicated calibration system allow the muon reconstruction algorithm to be verified against data. The detector response to muons is independently measured by observation of the energy and direction of cosmic-ray muons up to $800 \mathrm{MeV}$. A scintillator hodoscope directly above the detector and seven scintillator cubes at various depths within the detector are used to track these particles. Figure 5-15 shows the layout of this system in the MiniBooNE detector.

Each cube is connected by an optical fiber to a PMT for readout. The direction of cosmic-ray muons are measured in the hodoscope, and they may be identified as stopping in one of the scintillation cubes by the observation of a decay electron produced inside the cube. With knowledge of the cube's position and the muon's incident position and angle, it's energy can be calculated based on how much oil it crossed and the Bethe-Block formula for energy loss. In this way, the muon reconstruction algorithm can be verified against data for a variety of muon energies. After all calibration studies, the energy (angle) resolution

for muons improves from 12\% (5.4 deg) at $100 \mathrm{MeV}$ to 3.4\% (1.0 deg) at $800 \mathrm{MeV}$. More details of this reconstruction are given in the next section.

\subsubsection{Analysis Tools}

This section describes the connection between the PMT signals and the analysis of $\bar{\nu}_{\mu}$ CCQE interactions.

A total of 16 triggers may activate the data acquisition (DAQ) system for a total 

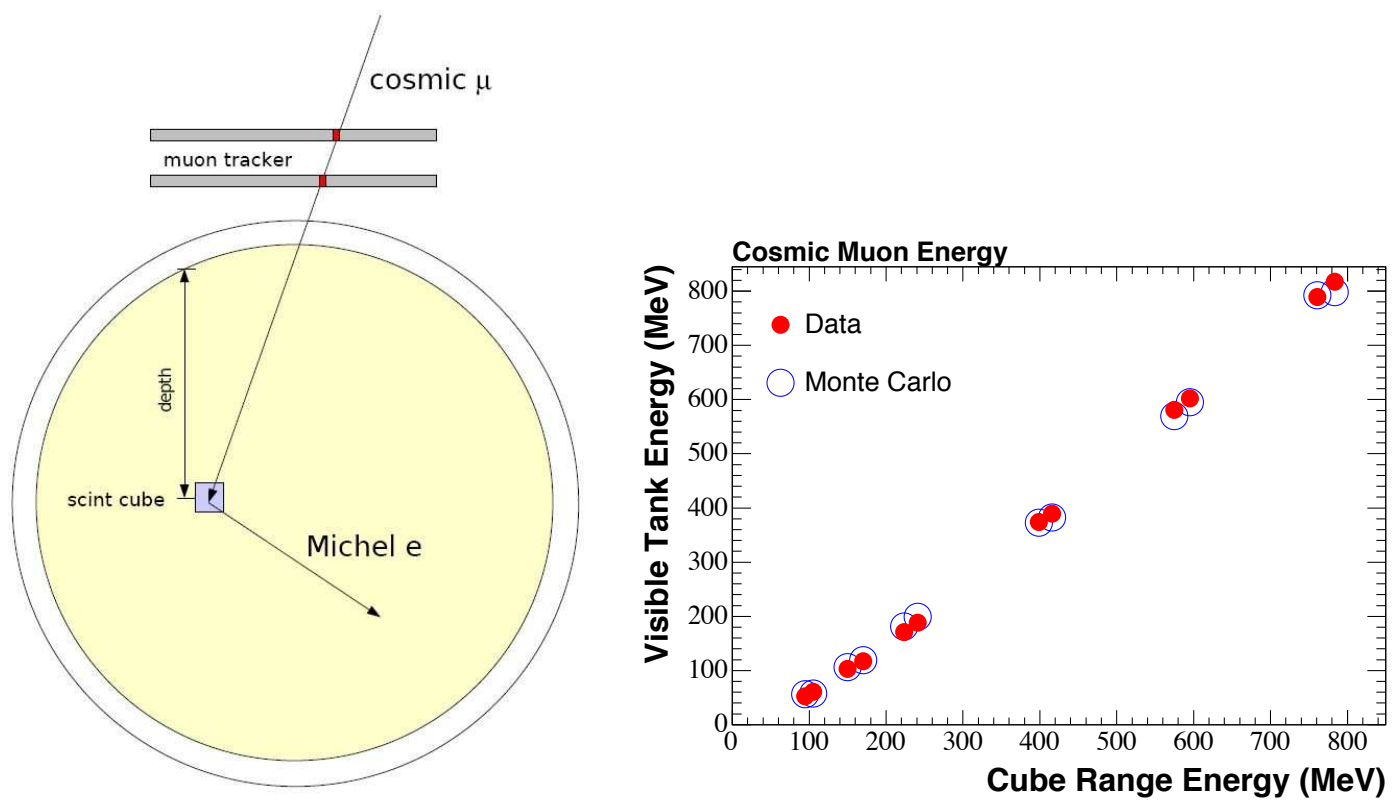

Figure 5-15. Cartoon of the muon calibration system (left) and the relationship between muon energy and range in data and simulation subsequent to calibrations (right). Only one of the seven scintillator cubes are shown in the left figure, and the image is taken from Ref. [109].

rate of $\sim 26 \mathrm{~Hz}$ under normal running conditions, and are used a variety of calibration purposes and physics analyses $[133,134]$. Up to $5 \mathrm{~Hz}$ are due to the primary BNB trigger, and in this case the DAQ records PMT charge and time information from all 1520 phototubes for a total of $19.2 \mu \mathrm{s}$ beginning $\sim 5 \mu$ s before the $1.6 \mu$ s long proton spill. Cosmic-ray muons stopped in the signal region prior to the start of the DAQ window may decay in time with the BNB spill, so PMT activity $5 \mu$ s before proton delivery is monitored and used to minimize this contamination. Activity is recorded subsequent to the beam window for more than $10 \mu$ s to observe electrons from the at-rest decay of muons (hereafter referred to as "Michel" electrons) produced either directly or indirectly through the primary neutrino interaction.

The PMT timing information is used to associate clusters of activity with the signature of a single particle using PMT "hits"; temporal groups of hits form "subevents". A PMT pulse passing the discriminator threshold of $\sim 0.1$ photoelectrons is defined as a 


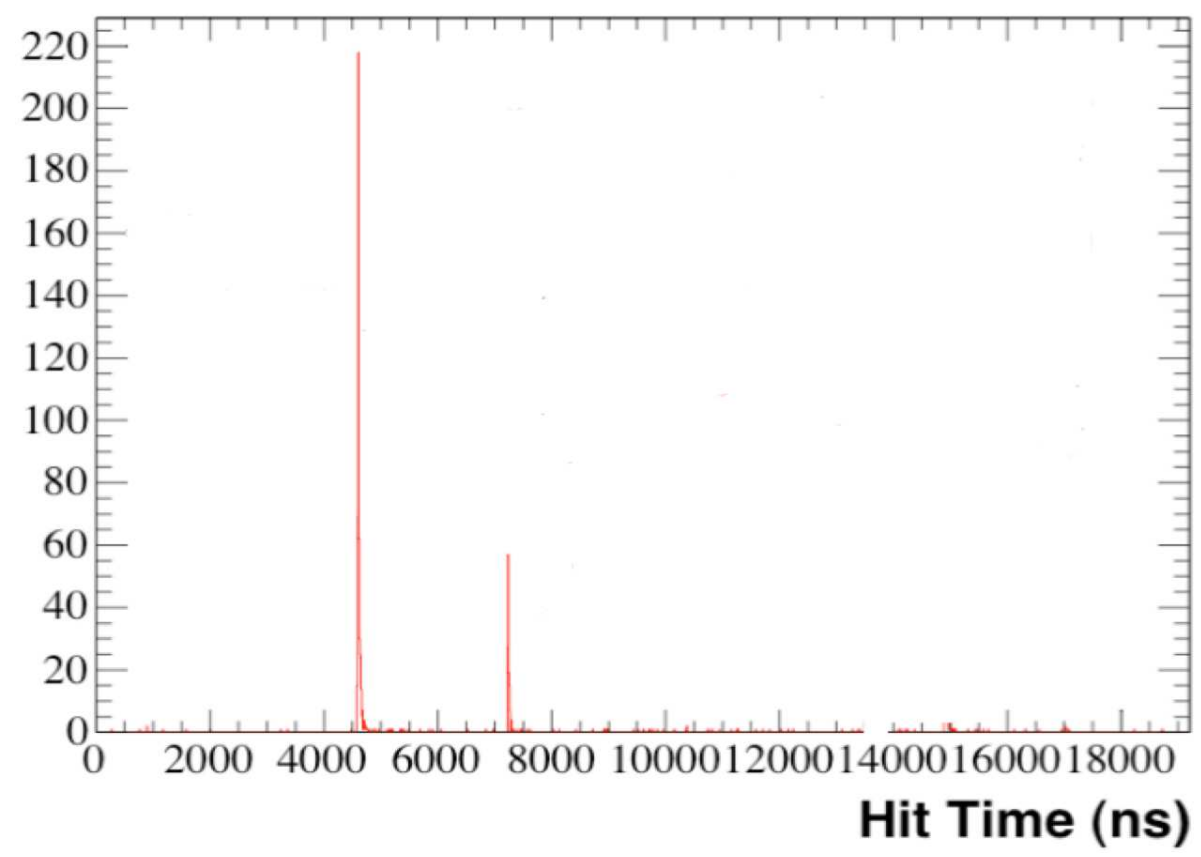

Figure 5-16. Typical PMT hit (ordinate axis) and timing signature of a $\bar{\nu}_{\mu}$ CCQE event. The prompt $\mu^{+}$arrives in time with the BNB spill (from 4600 - 6200 ns relative to the beginning of the DAQ clock) with 100's of MeV in kinetic energy, while the Michel is observed a time characteristic of the muon lifetime later with an energetic endpoint of $\sim 53 \mathrm{MeV}$. Their signatures are easily separated with the subevent definition.

hit, and forms the basic unit of the observed signal intensity. A group of PMT activity with at least 10 hits within a 200 ns window and individual hit times less than 10 ns apart, while allowing for at most two spacings of 10 - $20 \mathrm{~ns}$, defines a subevent. These subevents separate particles whose transit emits significant amounts of Čerenkov light with high efficiency, and so are primarily used isolate the signatures and topologies of muons and electrons. Interactions of $\bar{\nu}_{\mu}$ CCQE typically yield two subevents, the first from the prompt $\mu^{+}$, and the second from its decay positron. Figure 5-16 shows the timing and PMT hit signature of a typical $\bar{\nu}_{\mu} \mathrm{CCQE}$ event.

The pattern, timing, and total charge of prompt Čerenkov radiation collected by the PMTs in the first subevent are used to identify muon kinematics, the quantity most important to the main result of this dissertation. A likelihood function is compared to the topology and timing of the observed PMT hits: 

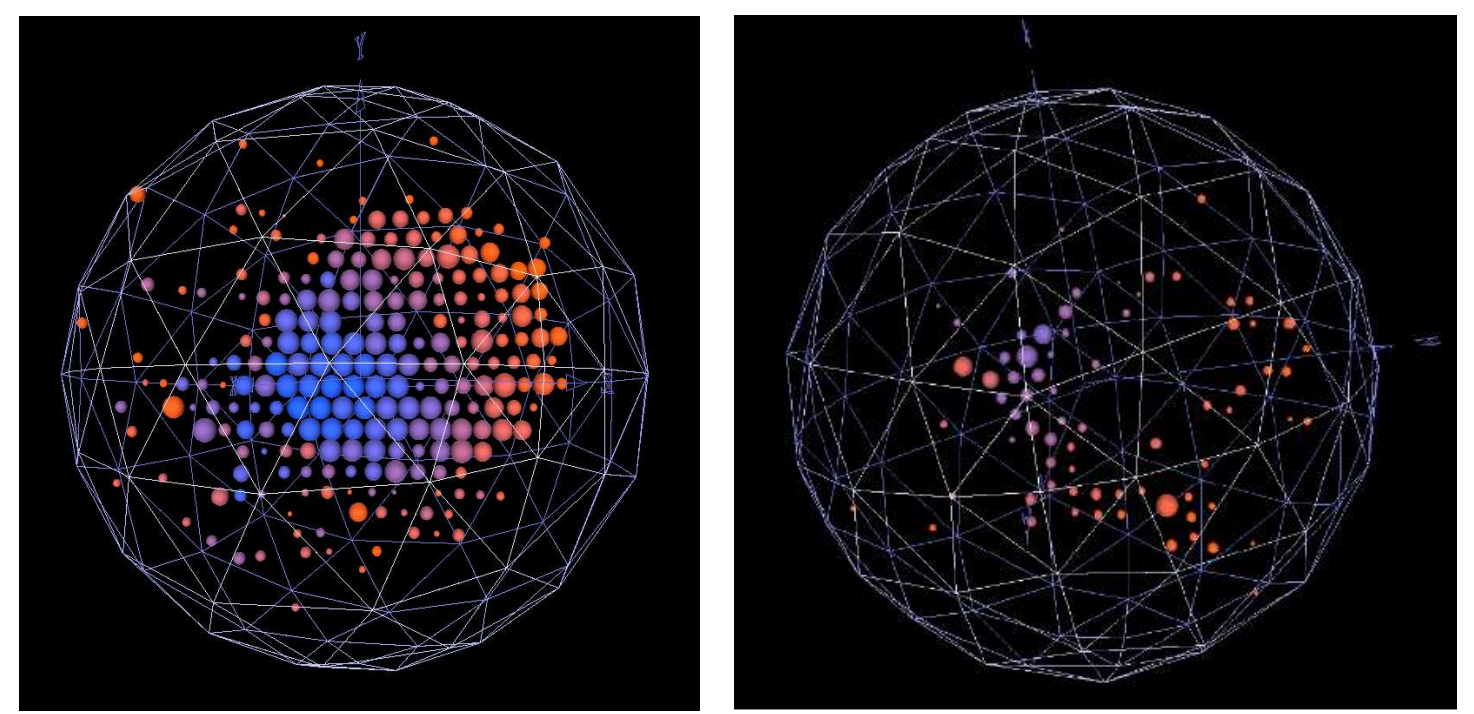

Figure 5-17. Typical PMT hit topology and timing for muon (left) and electron (right) candidate events in MiniBooNE data. PMT charge is correlated to the size of the displayed hits, while timing is given by the color spectrum, where blue hits arrived earliest and red hits arrived last.

$$
\mathcal{L}(\mathbf{x})=\prod_{\text {unhit PMTs } i}(1-P(i \text { hit } ; \mathbf{x})) \times \prod_{\text {hit PMTs } i} P(i \text { hit; } \mathbf{x}) f_{q}\left(q_{i} ; \mathbf{x}\right) f_{t}\left(t_{i} ; \mathbf{x}\right)
$$

where $P(i$ hit; $\mathbf{x})$ is the probability for PMT $i$ to register a hit given the muon vertex and kinematic vector $\mathbf{x}$, and $f_{q}\left(f_{t}\right)$ is a probability distribution function (PDF) for the hit to return the measured charge (time) $q_{i}\left(t_{i}\right)$. As the energy range of particles observed by MiniBooNE is sensitive to the mass difference between muons and electrons, an electron's path of travel in the MiniBooNE detector is more likely to be deflected compared to a muon's via the Bremstrahlung and multiple scattering processes. Electrons may also create electromagnetic showers, and this leads to distinct Čerenkov topologies and therefore different $f_{q}$ and $f_{t}$ PDFs for the two charged leptons. Figure 5-17 compares typical electron and muon timing and charge signatures in MiniBooNE.

The vector $\mathbf{x}$ is composed of the particle's time, energy and position at creation, as well as its momentum projections along the azimuthal and polar angles in spherical 
coordinates. The negative logarithm of the likelihood function in Eq. 5.1 simultaneously varies these seven parameters while comparing to the observed PMT hits. The parameters from the maximized likelihood function yield the reconstructed lepton kinematics. A two-track version of this reconstruction was also developed to identify $\pi^{0}$ candidate events, and the angular and energy resolutions of this reconstruction to all three particle species, operating Eq. 5.1 under the appropriate hypothesis, are given in Figure 5-18. Further details on this reconstruction can be found in Ref. [135].

The direct and high-resolution observation of muon properties using this reconstruction further motivates the choice of emphasizing the $\bar{\nu}_{\mu}$ CCQE cross section as a function of muon kinematics as the main result of this work, while the statistics of the data set also yield unprecedented sensitivity to the behavior of the $\mu^{+}$in $\bar{\nu}_{\mu}$ CCQE interactions. 

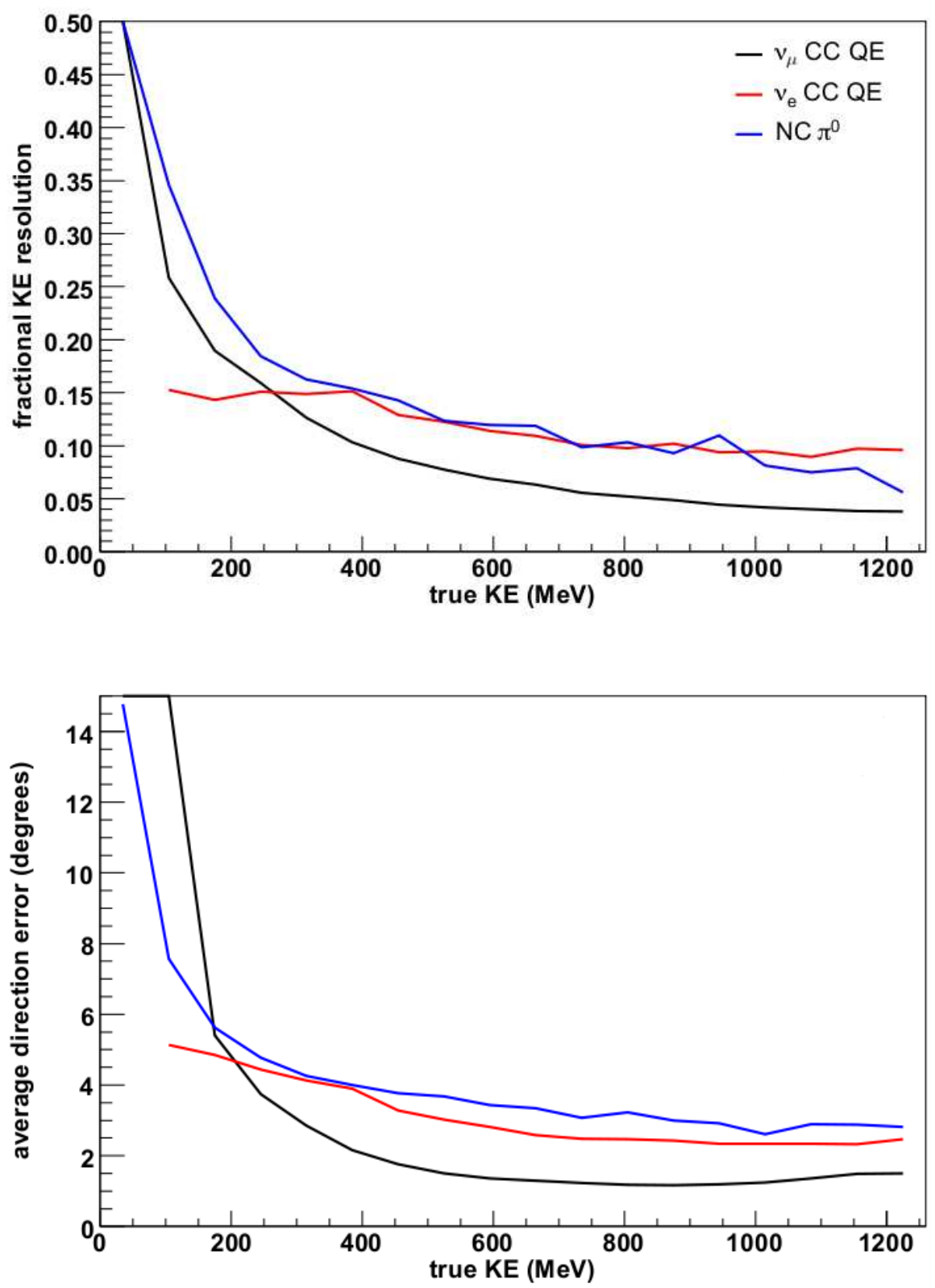

Figure 5-18. Kinetic energy and angular resolution of the MiniBooNE reconstruction to electrons, muons, and neutral pions. Figure from Ref. [135]. 
CHAPTER 6

INTRODUCTION TO THE CROSS-SECTION MEASUREMENT

\subsection{Overview}

This dissertation describes the first measurement of the muon antineutrino CCQE cross section with $\left\langle E_{\bar{\nu}}\right\rangle<1 \mathrm{GeV}$. Before exploring the details, it is helpful to first describe the overall strategy and identify the areas of the calculation deserving of the most attention.

Generically, for one to measure a differential cross section in the distribution $X$, given a data set $d$, total background $b$, using a detector housing a number of interaction targets $N$ with detection efficiency $\epsilon$ and a total $\bar{\nu}_{\mu}$ exposure $\Phi$, the formula is rather simple:

$$
\frac{d \sigma}{d X_{i}}=\frac{\sum_{j} U_{i j}\left(d_{j}-b_{j}\right)}{\Delta X_{i} \epsilon_{i} \Phi N},
$$

where $i$ indexes the region of measurement in the absence of detector effects, $\Delta X_{i}$ is the

width of this region, $j$ labels the same region as observed by the detector, and the matrix $U_{i j}$ connects the two. The other cross-section configurations measured in this work are simple extensions of Eq. 6.1 and will be discussed later. Before we proceed with a cross-section calculation, an analysis sample must be identified. In describing this in the next section, it will become clear that a major complication of this analysis is the presence of large and nominally uncertain backgrounds.

\subsection{Event Selection}

Optimizing the sample to study a particular type of interaction always involves a balance between retaining as many high-quality signal events as possible while minimizing the contamination from background interactions. The selection and its efficacy for the antineutrino-mode CCQE sample follows:

1. Veto hits $<6$, all subevents 
2. First subevent in beam window: $4000<\mathrm{T}(\mathrm{ns})<7000$, where $\mathrm{T}$ is the average PMT hit time

3. Two subevents

4. Reconstructed vertex $<500 \mathrm{~cm}$ from tank center, first subevent

5. $\mathrm{T}_{\mu}>200 \mathrm{MeV}$ (kinetic energy of first subevent)

6. $\ln (\mu / \mathrm{e})>0.0$, first subevent

7. Distance between 1 st and 2 nd subevent vertices $>500 \mathrm{~cm} / \mathrm{GeV} \times T_{\mu}-100 \mathrm{~cm}$

8. Distance between 1 st and 2 nd subevent vertices $>100 \mathrm{~cm}$

Cut 1 simultaneously rejects incoming charged particles and enforces containment of charged particles created in the tank. The upper bound on the acceptable number of veto hits is motivated in Figure 6-1, where six veto hits accepts low-level PMT noise but rejects most exiting and entering activity. Cut 2 requires the first subevent be in time with the proton beam spill.

To motivate and isolate the effects of selections 3-8, the distribution under examination is presented with all other requirements applied. To avoid placing requirements on subevents that may not exist, the subevent distribution is the lone exception.

Figure 6-2 shows the impact of cuts 3-6. Cut 3 simultaneously ensures there are no final-state pions and the event is consistent with the production of a contained muon. The selection of the sample represented in the subevent figure is cuts 1 and 2, where the veto hit requirement is applied to each subevent present. The large excess in the single subevent bin is dominated by Michel electrons produced in time with the beam from cosmic-ray muons, which are not simulated, entering prior to the start of the DAQ window. Cut 4 avoids a class of events that may be reconstructed poorly due to greater sensitivity to PMT coverage. The spike at high radius is due to the relatively dense material in the optical barrier. The requirement of cut 5 also improves reconstruction reliability while avoiding a 
double-coincidence of the kind of Michel electrons mentioned earlier. Cut 6 enhances the purity of the sample by rejecting many $\mathrm{CC} \pi$ events where the pion is energetic enough to produce some Čerenkov light and cause the muon ring to receive a more electron-like score. The $\ln (\mu / e)$ variable is found by comparing the muon-like to the electron-like score of the reconstruction described in Section 5.3.5.
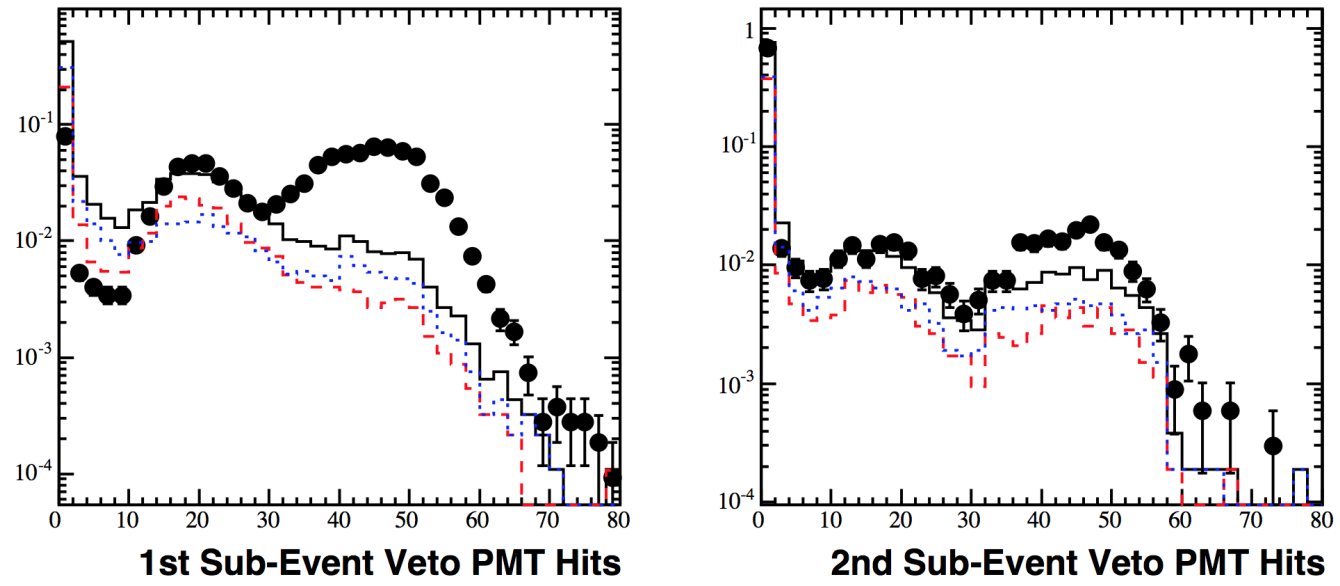

Figure 6-1. Veto hits for early neutrino-mode data and MC for the first two subevents. Points are data, the dotted (dashed) blue (red) histogram is $\nu_{\mu}$ CCQE (all non- $\nu_{\mu}$ CCQE) and the solid line is total MC. All distributions are normalized to unit area. Cosmic rays are not simulated, and this is the origin of the shape discrepancy between data and $\mathrm{MC}$ in the first subevent. Figure from Ref. [136].

Cut 7 enhances the sample purity by requiring the distance between the vertices of the two subevents be consistent with the production and subsequent decay of a minimum ionizing particle (MIP). Cut 8 further reduces the small neutral current $\pi$ background. Cuts 7 and 8 are shown in Figure 6-3.

Stepwise purity and detection efficiency for the resultant sample is presented in Table 6-1, where values are given for both bound (from the carbon contribution to mineral oil) and free (from the hydrogen content) $\bar{\nu}_{\mu} \mathrm{CCQE}$ scattering. A breakdown of the predicted sample composition is given in Table 6-2. 

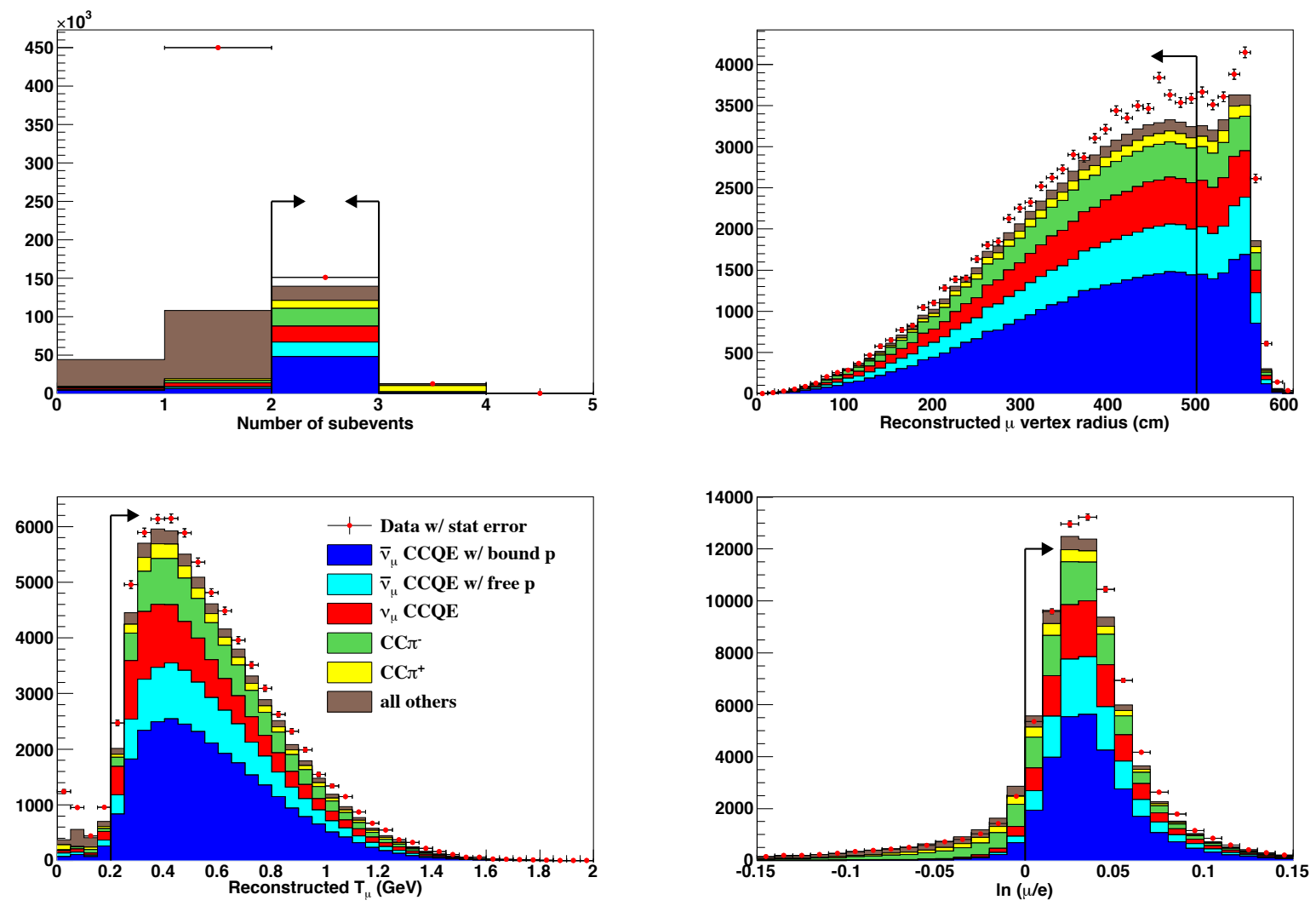

Figure 6-2. Selection requirements 3-6. Descriptions are given in the text, and distributions are normalized to flux. With the exception of the subevent figure, all distributions have every cut applied except the one indicated in the figure. 
Table 6-1. Purity and detection efficiency for the sample described in this section. Signal $\bar{\nu}_{\mu}$ CCQE interactions are presented in their bound (" $12 \mathrm{C}$ ") and free (" $\mathrm{H}_{2}$ ") components and also as the sum. A pre-cut of generated vertex radius $<550 \mathrm{~cm}$ for the primary subevent has been applied, and these estimates reflect the measurements and constraints described in Chapter 7.

\begin{tabular}{ccccccc}
\hline Cut Description & \multicolumn{3}{c}{ Purity (\%) } & \multicolumn{3}{c}{ Efficiency (\%) } \\
& ${ }^{12} \mathrm{C}$ & $\mathrm{H}_{2}$ & Total & ${ }^{12} \mathrm{C}$ & $\mathrm{H}_{2}$ & Total \\
\hline No cuts & 22.6 & 9.0 & 32.3 & 100 & 100 & 100 \\
Veto hits $<$, all subevents & 19.5 & 7.7 & 27.6 & 50.9 & 50.4 & 50.8 \\
First subevent in beam window & 19.6 & 7.7 & 27.7 & 50.5 & 49.9 & 50.3 \\
$T_{\mu}>200 \mathrm{MeV}$ & 25.9 & 10.1 & 36.9 & 44.3 & 43.4 & 44.0 \\
Two subevents & 33.8 & 13.2 & 48.4 & 39.1 & 38.3 & 38.8 \\
Reconstructed radius within $500 \mathrm{~cm}$ & 34.4 & 13.5 & 49.2 & 32.8 & 32.1 & 32.6 \\
$\mu-e$ dist. $>500 \mathrm{~cm} / \mathrm{GeV} \times T_{\mu}-100 \mathrm{~cm}$ & 38.2 & 15.0 & 54.3 & 30.8 & 30.3 & 30.6 \\
$\mu-e$ dist. $>100 \mathrm{~cm}$ & 43.2 & 17.1 & 61.0 & 29.6 & 29.3 & 29.5 \\
$\ln (\mu / e)>0$ & & & & &
\end{tabular}

From these data we will extract three main cross sections: as a function of neutrino energy, with respect to $Q_{Q E}^{2}$ (reconstructed four-momentum transfer under the assumption of CCQE interactions), and the minimally model-dependent double-differential cross section as a function of $\mu$ kinematics. Reconstructing the incident neutrino energy and the squared four momentum transfer on an event-by-event basis is possible only by assuming the observed interaction is $\bar{\nu}_{\mu} \mathrm{CCQE}$ on carbon, and also that it occurs on a single independent nucleon at rest. To begin these calculations, we start with an arbitrary t-channel exchange between two particles $1+2 \rightarrow 3+4$ shown in Figure 6-4. The momentum transfer $q^{2}$ in this interaction is given by:

$$
q^{2}=\left(p_{1}-p_{3}\right)^{2}=\left(p_{2}-p_{4}\right)^{2},
$$

where $p$ is the particle's four-momentum. Using the notation of four-vectors and focusing on the momentum transfer between the 1 and 3 particles, 
Table 6-2. Predicted composition of the sample described in this section. These estimates reflect the measurements and constraints described in the following chapters.

\begin{tabular}{|c|c|}
\hline $\begin{array}{c}\text { integrated POT } \\
\text { mean } \bar{\nu}_{\mu} \text { energy } \\
\text { energy-integrated } \bar{\nu}_{\mu} \text { flux } \\
\bar{\nu}_{\mu} \text { CCQE candidate events } \\
\bar{\nu}_{\mu} \text { CCQE efficiency }(R<550 \mathrm{~cm})\end{array}$ & $\begin{array}{c}10.1 \times 10^{20} \\
665 \mathrm{MeV} \\
2.93 \times 10^{11} \bar{\nu}_{\mu} / \mathrm{cm}^{2} \\
71176 \\
29.5 \% \\
\end{array}$ \\
\hline Interaction & Contribution (\%) \\
\hline $\bar{\nu}_{\mu}+p($ bnd $) \rightarrow \mu^{+}+n$ & 43.2 \\
\hline $\bar{\nu}_{\mu}+p($ free $) \rightarrow \mu^{+}+n$ & 17.1 \\
\hline$\nu_{\mu}+n \rightarrow \mu^{-}+p$ & 16.6 \\
\hline $\bar{\nu}_{\mu}+n \rightarrow \mu^{+}+n+\pi^{-}$ & 7.9 \\
\hline $\bar{\nu}_{\mu}+A \rightarrow \mu^{+}+A+\pi^{-}$ & 3.3 \\
\hline$\nu_{\mu}+p \rightarrow \mu^{-}+p+\pi^{+}$ & 3.1 \\
\hline $\bar{\nu}_{\mu}+p \rightarrow \mu^{+}+p+\pi^{-}$ & 2.5 \\
\hline $\begin{aligned} \bar{\nu}_{\mu}+p & \rightarrow \mu^{+}+\Lambda^{0} \\
\bar{\nu}_{\mu}+n & \rightarrow \mu^{+}+\Sigma^{-} \\
\bar{\nu}_{\mu}+p & \rightarrow \mu^{+}+\Sigma^{0}\end{aligned}$ & 2.0 \\
\hline $\bar{\nu}_{\mu}+p \rightarrow \mu^{+}+n+\pi^{0}$ & 2.0 \\
\hline$\nu_{\mu}+n \rightarrow \mu^{-}+n+\pi^{+}$ & 0.7 \\
\hline$\nu_{\mu}+n \rightarrow \mu^{-}+p+\pi^{0}$ & 0.8 \\
\hline$\nu_{\mu}+A \rightarrow \mu^{-}+A+\pi^{+}$ & 0.2 \\
\hline All other & 0.5 \\
\hline
\end{tabular}

$$
\begin{aligned}
q^{2} & =\left(E_{1}-E_{3}, \vec{p}_{1}-\vec{p}_{3}\right)=\left(E_{1}-E_{3}\right)^{2}-\left|\vec{p}_{1}-\vec{p}_{3}\right|^{2} \\
& =E_{1}^{2}+E_{3}^{2}-2 E_{1} E_{3}-\left(\left|\vec{p}_{1}\right|^{2}+\left|\vec{p}_{3}\right|^{2}-2\left|\vec{p}_{1}\right|\left|\vec{p}_{3}\right| \cos \theta_{1-3}\right)
\end{aligned}
$$

where $\vec{p}$ is the three-momentum vector, and $\theta_{1-3}$ is the scattering angle of particle 3 with respect to the direction of particle 1 . In the case of a neutrino for particle $1,\left|\vec{p}_{1}\right|=E_{1}$ to excellent approximation. For a $\nu_{\mu}$ or $\bar{\nu}_{\mu} \mathrm{CC}$ interaction, particle 3 is a muon, and 


$$
Q^{2}=-q^{2}=2 E_{\nu}^{Q E}\left(E_{\mu}-\left|\vec{p}_{\mu}\right| \cos \theta_{\nu-\mu}\right)-m_{\mu}^{2}
$$

where, for convenience, $Q^{2}$ is defined to be a positive quantity. The form of Eq. 6.4 is particularly useful for accelerator-based neutrino measurements, as the scattering angle $\theta_{\nu-\mu}$ is simply the observed angle of the muon relative to the beam direction, hereafter simply referred to as $\theta_{\mu}$. However, these neutrino sources typically feature a broad range of neutrino energies, and so additional information is needed to find $Q^{2}$. To reconstruct the incident neutrino energy, we employ the same assumptions as before and also introduce an at-rest proton target and an outgoing neutron in the hadronic vertex, appropriate to $\bar{\nu}_{\mu}$ CCQE. Note that the kinematic assumption on the proton is wildly inaccurate; however, as the momentum distribution about any spatial direction must be centered around zero, with enough statistics the bias is small and acceptable. Neglecting the small binding energy for interactions with bound nucleons, four-momentum conservation gives

$$
\left(E_{\nu}^{Q E}+m_{p}, \vec{p}_{\nu}\right)=\left(E_{\mu}+E_{n}, \vec{p}_{\mu}+\vec{p}_{n}\right)
$$

Note the neutrino energy is labeled to explicitly recognize its assumption of a CCQE interaction. Eliminating the neutron kinematics and again neglecting the neutrino mass gives:

$$
m_{p}^{2}+E_{\mu}^{2}+2\left(m_{p} E_{\nu}^{Q E}-E_{\mu} E_{\nu}^{Q E}-m_{p} E_{\mu}\right)=m_{n}^{2}+\left|\vec{p}_{\mu}\right|^{2}-2 E_{\nu}^{Q E}\left|\vec{p}_{\mu}\right| \cos \theta_{\mu} .
$$

Rearranging Eq. 6.6 yields a determination of the neutrino energy solely in terms of muon kinematics: 


$$
E_{\nu}^{Q E}=\frac{m_{n}^{2}-m_{p}^{2}-m_{\mu}^{2}-2 m_{p} E_{\mu}}{2\left(m_{p}-E_{\mu}+\left|\vec{p}_{\mu}\right| \cos \theta_{\mu}\right)} .
$$

We can use this quantity in finding the four-momentum transfer, carrying over the "QE" label to again recognize the propagated CCQE assumption:

$$
Q_{Q E}^{2}=2 E_{\nu}^{Q E}\left(E_{\mu}-p_{\mu} \cos \theta_{\mu}\right)-m_{\mu}^{2}
$$

As many theoretical groups predict a sizable contribution from an unexpected background to the MiniBooNE CCQE sample (Section 4.4.1), the assumption of CCQE interactions embedded in $E_{\nu}^{Q E}$ and $Q_{Q E}^{2}$ is particularly troubling. This is the primary motivation for highlighting the double-differential cross section $\frac{d^{2} \sigma}{d T_{\mu} d \cos \theta_{\mu}}$ as the main result of this work. Nevertheless, producing cross sections in $E_{\nu}^{Q E}$ and $Q_{Q E}^{2}$ can be helpful to facilitate historical comparisons.

The full MiniBooNE $\bar{\nu}_{\mu}$ CCQE sample in $E_{\nu}^{Q E}, Q_{Q E}^{2}$ and the kinematics of the muon is shown in Figure 6-5, as well as the two-dimensional muon kinematical ratio of data to the prediction.

Table 6-2 estimates the $\bar{\nu}_{\mu}$ CCQE sample features a purity of $\sim 60 \%$. With a signal:background rate approaching $1: 1$, it is crucial to evaluate how well these backgrounds are understood before they can be reliably subtracted from the data to produce $\bar{\nu}_{\mu}$ CCQE cross sections. The next chapter is dedicated to the various measurements and constraints obtained for the dominant backgrounds. 

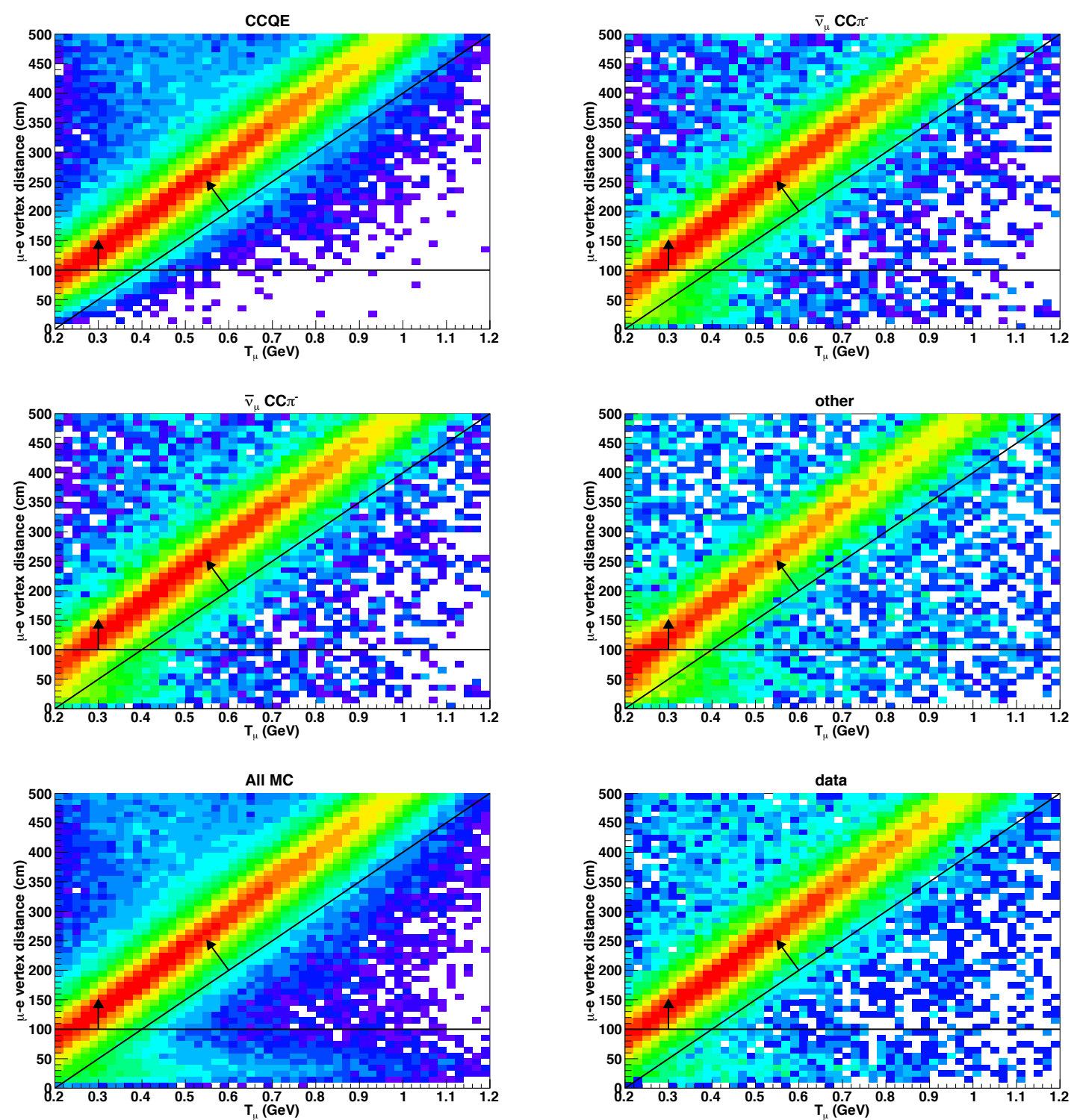

Figure 6-3. $\mathrm{T}_{\mu}$ dependent range cut for different channels and samples, as labeled. Events are plotted on a logarithmic scale.

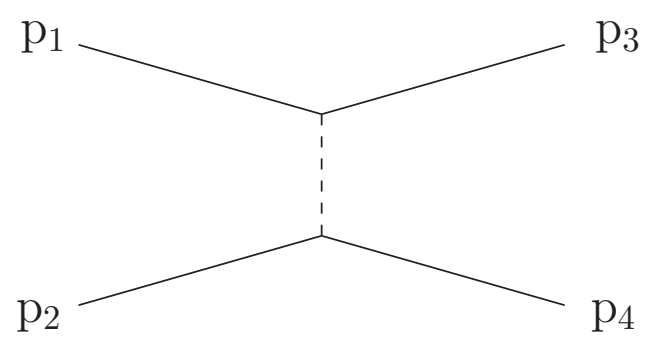

Figure 6-4. An arbitrary t-channel exchange. 

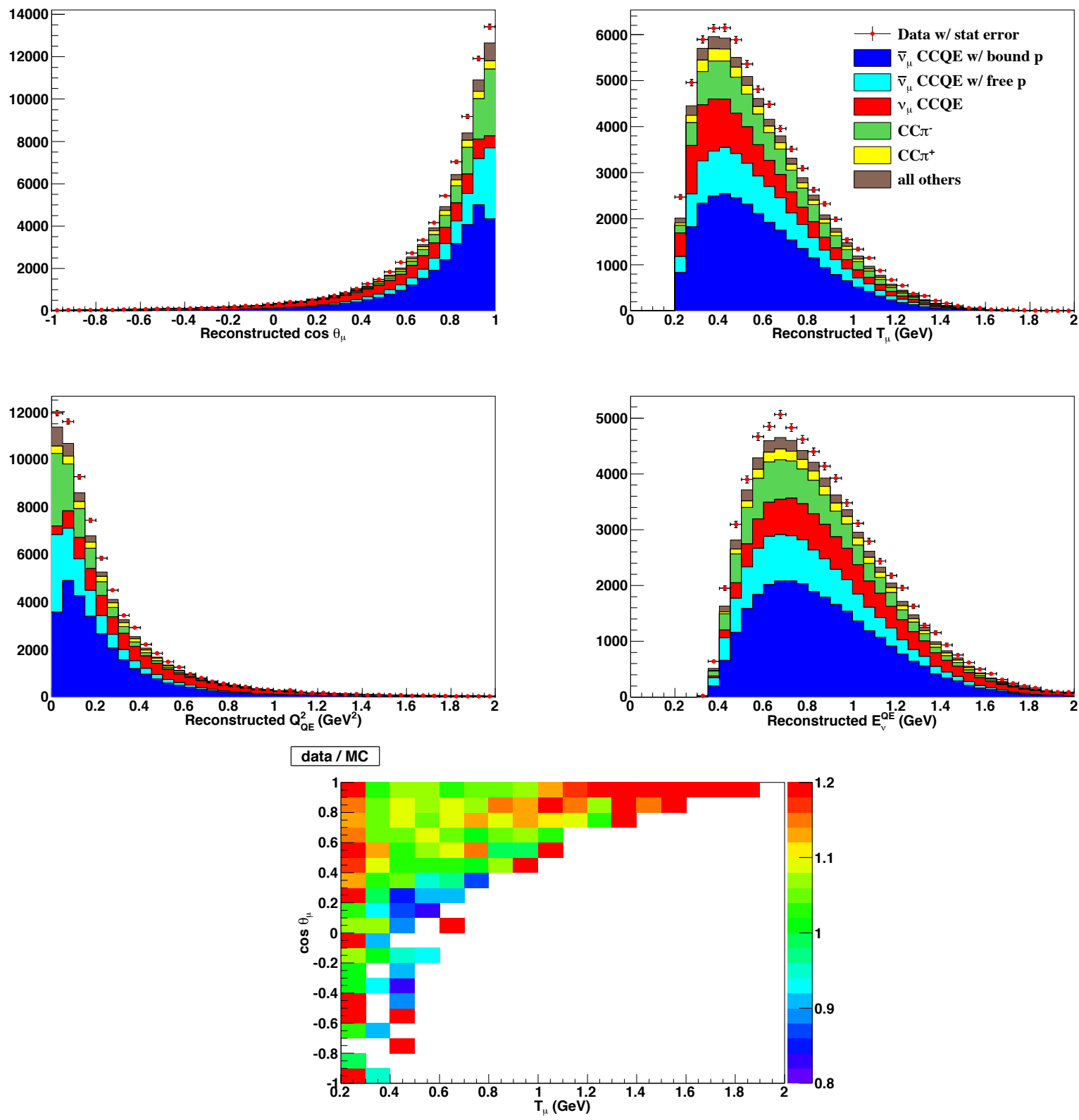

Figure 6-5. The four variables cross sections will be reported in. Distributions are normalized to flux. 


\section{CHAPTER 7 \\ BACKGROUND MEASUREMENTS AND CONSTRAINTS}

With backgrounds accounting for nearly half of the total sample studied, their precise contribution and kinematics must be rigorously verified before a reliable

background subtraction can be made. This section presents various measurements and constraints on these processes. As $\nu_{\mu}$ and $\mathrm{CC} \pi^{-}$interactions are dominant, particular attention is paid to understanding their contribution.

\subsection{Measurements of the $\nu_{\mu}$ Background}

\subsubsection{Motivation}

Interactions induced by $\nu_{\mu}$ events form the largest single background to the $\bar{\nu}_{\mu}$ CCQE sample, accounting for $\sim 20 \%$ of the selected events, and half of the total background. Given the high-quality pion production data from the HARP experiment and the litany of $\nu_{\mu}$ cross-section measurements from the MiniBooNE neutrino-mode data $[84,78,137,79,138,80,139]$, one might assume the $\nu_{\mu}$ contribution to the antineutrino-mode data is well-constrained. However, as Figure 7-1 shows, the majority of the $\nu_{\mu}$ events contributing to the antineutrino-mode data are produced in a kinematic region of the parent $\pi^{+}$that is not constrained by the HARP data. The particulars of Figure 7-1 warrant a few more remarks:

- both parent pion distributions leading to the "wrong-sign" contribution (neutrinos in antineutrino mode and vice versa) peak at the lowest opening angles. This shows how these events contribute to the beam: their transverse momentum is insufficient to be significantly altered by the magnetic field, and so their path is much less deflected compared to pions created at larger $\theta_{\pi}$.

- the antineutrino contribution to the neutrino-mode data is minuscule in comparison to the converse. This is due to a convolution of flux and cross-section effects that simultaneously serve to enhance the neutrino component while the antineutrino contribution is suppressed: the leading-particle effect at the beryllium target (the 

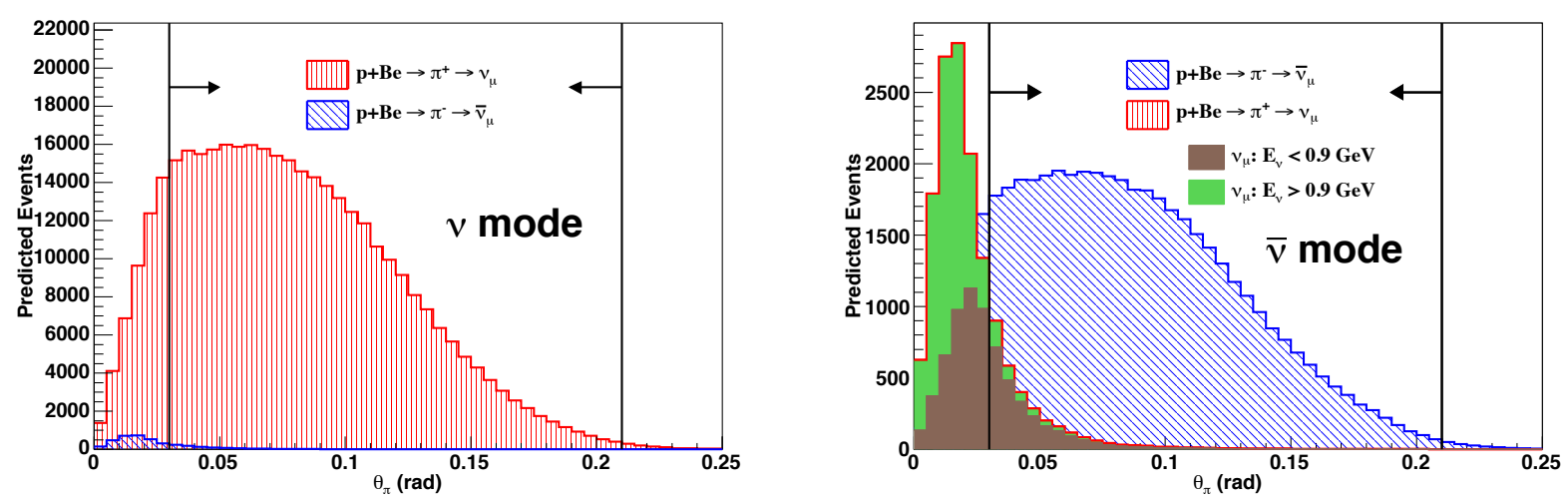

Figure 7-1. Predicted angular distributions of pions with respect to the incident proton beam $\left(\theta_{\pi}\right)$ producing $\nu_{\mu}$ and $\bar{\nu}_{\mu}$ in neutrino (left) and antineutrino (right) modes. Only pions leading to $\nu_{\mu}$ and $\bar{\nu}_{\mu}$ events in the detector are shown, and all distributions are normalized to $10.1 \times 10^{20}$ protons on target. Arrows indicate the region where HARP data [118] are available.

$p+$ Be initial state has a net positive charge) naturally leads to the creation of roughly twice as many $\pi^{+}$as $\pi^{-}$, and neutrino cross sections are typically around three times as large as antineutrino cross sections around $1 \mathrm{GeV}$.

- the above observation explains why this is a complication unique to antineutrino mode: the wrong-sign component in neutrino-mode data is small enough so that even for large fractional uncertainty on this background, the resultant error on the $\nu_{\mu}$ cross-section measurements are negligible compared to other systematic uncertainties.

- as seen in the antineutrino-mode distribution, high-energy $\nu_{\mu}$ 's are strongly correlated with the decay of $\pi^{+}$created at very small opening angles. This indicates their flux is more poorly constrained by the HARP data compared to lower-energy $\nu_{\mu}$ 's. So, not only is the overall $\nu_{\mu}$ flux in antineutrino mode highly uncertain, the accuracy of the extrapolated $\nu_{\mu}$ flux prediction may be a function of neutrino energy.

The above observations motivate dedicated studies of the $\nu_{\mu}$ contribution to the antineutrino-mode beam, and in as many exclusive regions of neutrino energy as is allowed by statistics to examine the flux spectrum. 
Many other experiments deal with this background in a much more direct way: they employ a magnetic field to determine the sign of the outgoing lepton. This provides $\nu / \bar{\nu}$ discrimination for CC interactions on an event-by-event basis. Modern examples of magnetized neutrino oscillation experiments include MINOS [46], NOMAD [140], and the T2K near detector [141].

The analyses in this section provide a first demonstration that, in the absence of a magnetic field, $\nu_{\mu}$ and $\bar{\nu}_{\mu}$ content of any mixed neutrino flux can be modestly separated using statistical methods. These methods could also aid current and future neutrino experiments that will test for CP violation in the lepton sector using large unmagnetized

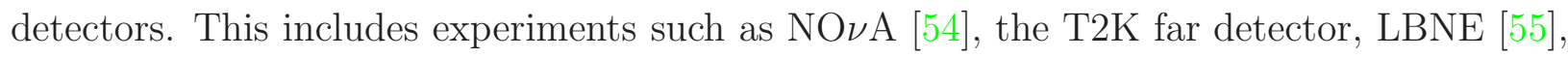
LAGUNA [142], and Hyper-K [143]. Also, it has been argued that the separation of charged-current neutrino and antineutrino events afforded by these kinds of analyses may be sufficient to meet the lofty physics goals of neutrino factories [144]. Finally, the MINER $\nu \mathrm{A}$ [145] neutrino cross-section experiment could gain crucial kinematic and statistical sensitivity by using these kinds of techniques to analyze CC events not accepted into their magnetized muon calorimeter.

The following sections present the first measurement of the $\nu_{\mu}$ component of an antineutrino-mode beam observed by a non-magnetized detector. Three statistical techniques are used to constrain this $\nu_{\mu}$ background to a sub-dominant uncertainty in the extraction of the $\bar{\nu}_{\mu}$ CCQE cross sections. Two of these techniques are published in Physical Review D [146], while the third is currently under review [147].

\subsubsection{General Strategy}

To statistically measure the wrong-sign background, we must exploit asymmetries in the way neutrinos, antineutrinos, and their byproducts interact in the detector. Analyzing the various samples gives a direct handle on their contribution to the data, which is the only knowledge necessary for performing the background subtraction. However, with the valuable cross-section data from MiniBooNE's neutrino-mode run, we can extract 
information about the $\nu_{\mu}$ flux as well, which can be used to test the accuracy of the extrapolation of the HARP data (described in Section 5.2.4) into the low-angle region. The $\nu_{\mu}$ channels contributing to the physics samples we will analyze are dominated by CCQE and $\mathrm{CC} \pi^{+}$interactions, and results from their cross-section analyses in the neutrino-mode data $[84,78]$ are applied to the antineutrino-mode simulation. With accurate cross sections implemented into the Monte Carlo (MC) simulation, differences of the observed rates and the expectation from simulation reflect the accuracy of the model-dependent $\nu_{\mu}$ flux prediction.

The relevance of the measured $\nu_{\mu}$ cross sections to the antineutrino-mode beam depends on the relative overlap in the $\nu_{\mu}$ spectra between the two running modes. As the $\nu_{\mu} \pi^{+}$-parent particles are sign-selected in neutrino mode and feature a focusing peak, while their acceptance in antineutrino mode is mostly due to low-angle and high energy production, it is reasonable to expect the relative $\nu_{\mu}$ spectra to be drastically different. Figure 5-9 shows this to be the case, where the produced $\nu_{\mu}$ flux spectrum in antineutrino mode is significantly harder compared to the $\nu_{\mu}$ 's in neutrino-mode running. If the antineutrino-mode data were sensitive to the details of the differing spectra, this would indicate the relevance of the observed $\nu_{\mu}$ cross sections is only marginal, and interpretations of these analyses as flux measurements would be inaccurate. Fortunately, Figure 7-2 shows that the accepted spectrum of $\nu_{\mu}$ 's in the CCQE samples across both run modes is very similar. This is mostly due to the rejection of high-energy $\nu_{\mu}$ 's via the muon containment requirement. The large overlap between these spectra allows the observed $\nu_{\mu}$ interactions in the antineutrino-mode beam to be tightly constrained by the neutrino-mode measurements.

In principle, the extracted flux information from these analyses could be used to re-analyze the neutrino-mode data with much stronger constraints on the low-angle region of the $\nu_{\mu}$ flux prediction. Figure 7-1 shows this region contributes roughly $10 \%$ of the $\nu_{\mu}$ flux in neutrino-mode running. Notice that, due to the small overlap between the parent $\pi^{+}$phase space across the two running modes, some circularity would be present in such an 


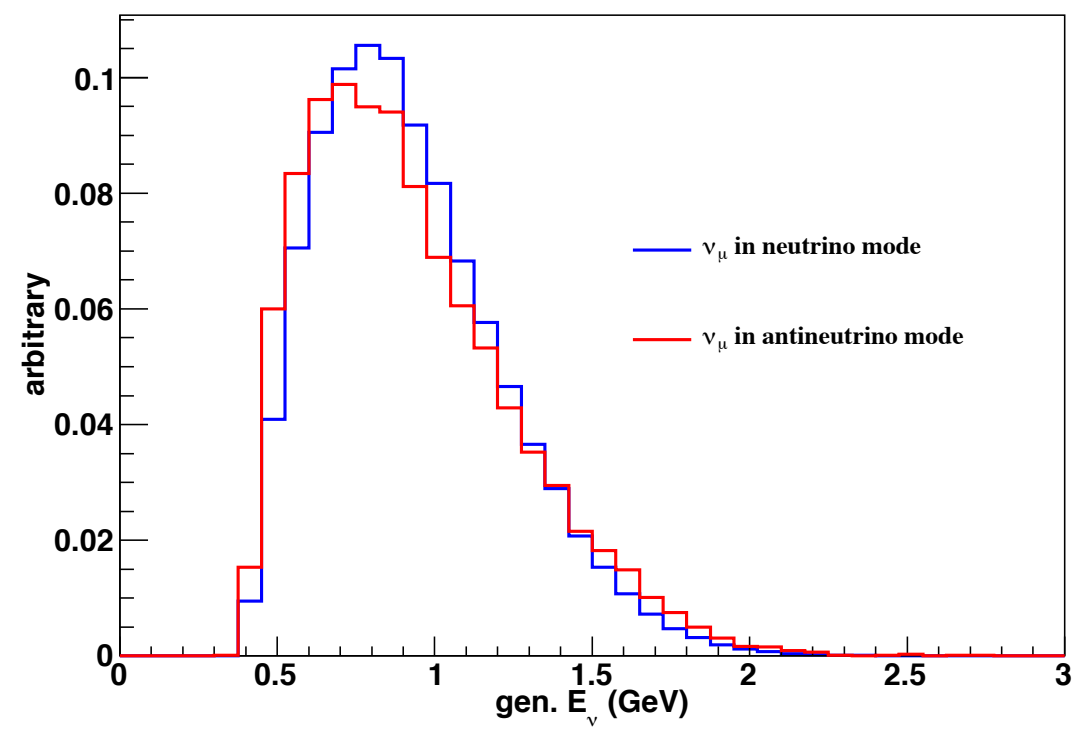

Figure 7-2. Generated $\nu_{\mu}$ energy distributions accepted into the neutrino and antineutrino CCQE samples. The substantial overlap indicates the $\nu_{\mu}$ 's background to the $\bar{\nu}_{\mu}$ CCQE sample are kinematically covered by the $\nu_{\mu}$ cross sections observed in the neutrino-mode data.

analysis. Nonetheless, this last advantage exposes a unique feature of this technique: it will be shown that the $\nu_{\mu}$ flux measurement in antineutrino mode is dominated by uncertainties on the MiniBooNE $\nu_{\mu}$ cross-section measurements, which are in turn dominated by the $\pi^{+}$ HARP errors through the $\nu_{\mu}$ flux uncertainty. So the techniques presented here effectively constrain regions of hadroproduction phase-space not covered by the HARP data to the level of precision of the regions that are covered.

A final advantage of determining a flux with this strategy is the cancellation of systematic uncertainties that affect the $\nu_{\mu}$ processes in the same way across both run mode configurations. These fully-correlated errors are mostly detector-related; in particular, a unique feature of this measurement of the $\nu_{\mu}$ flux is its independence of many final-state interaction processes. 


\subsection{3 $\nu_{\mu}$ Flux Measurement Using $\mathbf{C C} \pi^{+}$Events}

The first and most direct measurement of the $\nu_{\mu}$ background is a simple rate analysis of the three subevent sample. In the neutrino-mode data, this sample is dominated by $\mathrm{CC} \pi^{+}$production, mostly through the $\Delta(1232)$ resonance. The three subevents arise from the prompt $\mu^{-}$and two decay electrons, one each from the $\mu^{-}$and $\pi^{+}$:

$$
\begin{array}{ccc}
1: & \nu_{\mu}+p(n) \rightarrow \mu^{-}+p(n)+ & \\
& \hookrightarrow \mu^{+}+\nu_{\mu} \\
2 / 3: & \hookrightarrow e^{-}+\bar{\nu}_{e}+\nu_{\mu} & \\
2 / 3: & & \hookrightarrow e^{+}+\nu_{e}+\bar{\nu}_{\mu} .
\end{array}
$$

The mono-energetic $\mu^{+}$from decay-at-rest $\pi^{+}$is below Čerenkov threshold, and regardless the quick decay of the $\pi^{+}$would make the $\mu^{+}$not separable from the prompt $\mu^{-}$ using timing alone. Also, due to the fast decay of the $\pi^{+}$, it is effectively random which decay electron yields the second or third subevent. Few other processes in the MiniBooNE detector yield this signal, and the neutrino-mode $\mathrm{CC} \pi^{+}$sample has a purity of $\sim 90 \%$ [78].

From simple electric charge and lepton number conservation, the analogous charged-current single pion mechanism induced by antineutrinos yields a $\pi^{-}$. As stopped- $\pi^{-}$experiences nuclear capture on ${ }^{12} \mathrm{C}$ at nearly $100 \%$ [148], its decay is not observed and therefore it mostly yields two subevents:

$$
\begin{aligned}
& \text { 1: } \quad \bar{\nu}_{\mu}+p(n) \rightarrow \mu^{+}+p(n)+\pi^{-} \\
& \pi^{-}+{ }^{12} \mathrm{C} \rightarrow X \\
& 2: \quad \hookrightarrow e^{+}+\nu_{e}+\bar{\nu}_{\mu}
\end{aligned}
$$

where the remnants of $\pi^{-}$nuclear capture $X$ typically do not yield observable light in the detector. While the $\pi^{-}$nuclear capture mechanism vacates $\mathrm{CC} \pi^{-}$events from the three subevent sample and so allows for the present measurement of the the $\nu_{\mu}$ flux, one can 
readily recognize the sample $\mathrm{CC} \pi^{-}$events do populate is the main study of this dissertation, that of $\bar{\nu}_{\mu}$ CCQE. This background is addressed in Section 7.2.

Some $\mathrm{CC} \pi^{-}$events do yield a third subevent, mostly when the $\pi^{-}$decays in flight. Even with this additional background, the simple requirements outlined in the next section give a high-purity sample of $\nu_{\mu} \mathrm{CC} \pi^{+}$events with which we can use to make a powerful measurement of the $\nu_{\mu}$ flux in the antineutrino-mode beam.

At the time of the analysis of $\mathrm{CC} \pi^{+}$events, only a subset of the full $1.0 \times 10^{20} \mathrm{POT}$ taken in antineutrino mode was available. Since fewer data were available at this time, some less reliable runs were used in which absorber blocks accidentally fell into the decay tunnel at the BNB (described in Section 5.2.3). These blocks preferentially absorb high-energy $\pi$ 's and $\mu$ 's, reducing the contribution of high-energy $\nu_{\mu}$ and $\bar{\nu}_{\mu}$ to the beam. Since this measurement is not limited by statistics, the analysis was not updated as more POT became available. This is also the case for the analysis of the $\cos \theta_{\mu}$ distribution (Section 7.1.5). Table 7-1 shows the contribution of these absorber-down runs to the total amount analyzed.

Table 7-1. Summary of data periods used in the analyses of $\mathrm{CC} \pi^{+}$events and the $\cos \theta_{\mu}$ distribution (Section 7.1.5).

\begin{tabular}{cc}
\hline Period & POT (e20) \\
\hline 0 absorbers & 4.480 \\
1 absorber & 0.569 \\
2 absorbers & 0.612 \\
Total & 5.661 \\
\hline
\end{tabular}

\section{Implementation of the $\nu_{\mu} \mathrm{CC} \pi^{+}$Cross Section}

An important distinction in this analysis is the definition of $\mathrm{CC} \pi^{+}$events treated as signal. To avoid dependence on final-state interactions, the MiniBooNE neutrino-mode $\nu_{\mu}$ $\mathrm{CC} \pi^{+}$cross section was reported as an observable quantity: specifically, the final state studied consisted of one $\mu^{-}$, one $\pi^{+}$, and any number of nucleons [78]. This final state was 


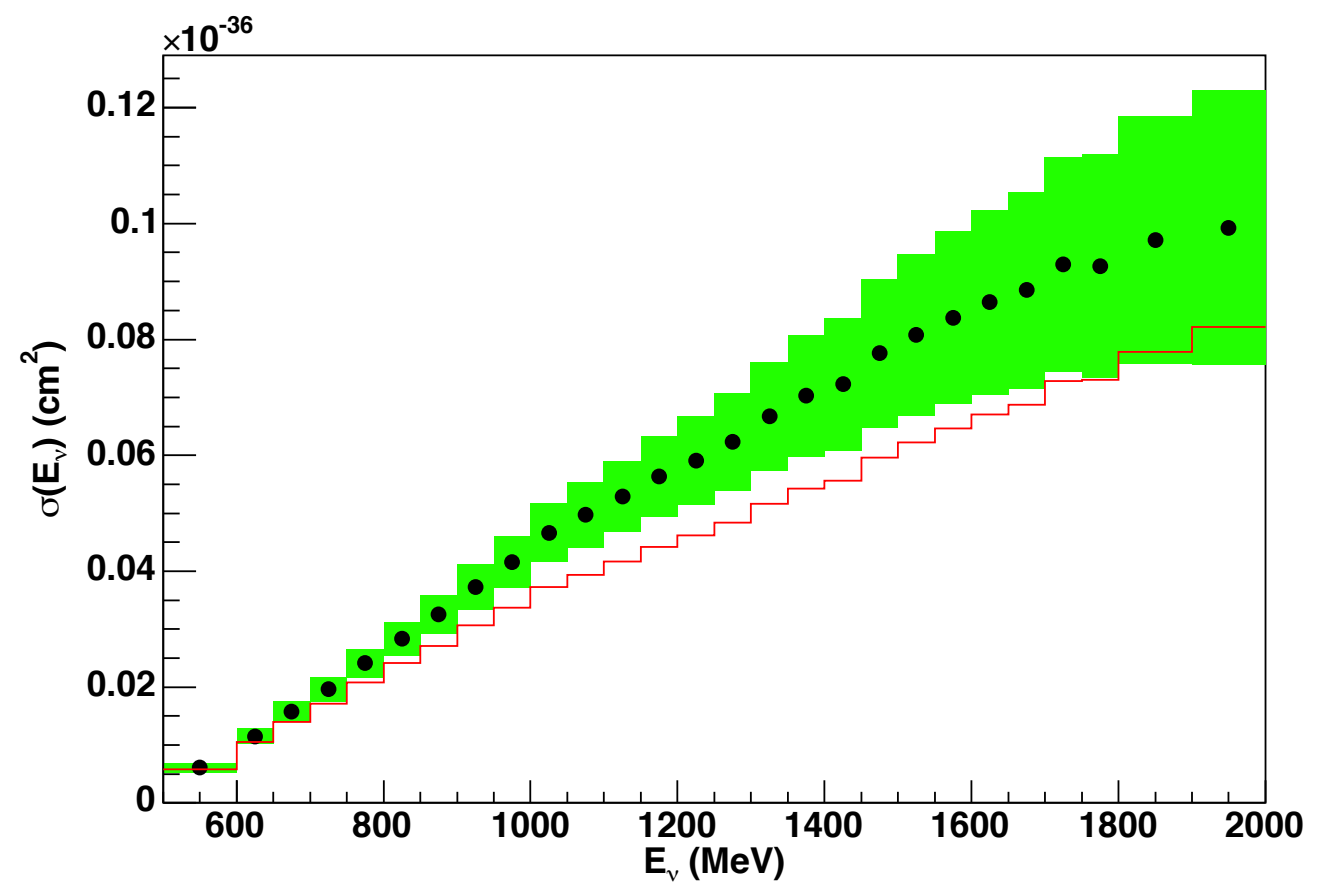

Figure 7-3. The $\nu_{\mu} \mathrm{CC} \pi^{+}$total cross section. Black points with green error bands are MiniBooNE data, and red is the MC expectation. Figure reproduced from Ref. [78].

not corrected for final-state interactions. Some of the more important implications are that some amount of nucleon-level $\mathrm{CC} \pi^{0}\left(\nu_{\mu}+N \rightarrow \mu^{-}+\pi^{0}+N^{\prime}\right)$ contribute to this sample, while some $\mathrm{CC} \pi^{+}$events are not present due to the pion charge-exchange $\left(\pi^{ \pm}+X \leftrightarrow \pi^{0}+X^{\prime}\right)$ and absorption $\left(\pi^{ \pm}+X \rightarrow X^{\prime}\right)$ processes. While this introduces a level of ambiguity in interpretations between the nucleon-level $\mathrm{CC} \pi^{+}$process and final-state interactions, it is an experimentally clean signature and may be used to rigorously test the $\mathrm{CC} \pi^{+}$process when final-state interactions are better understood. As we use this neutrino-mode $\mathrm{CC} \pi^{+}$result in this work, observable $\mathrm{CC} \pi^{+}$events are also treated as signal here.

A number of single and double-differential MiniBooNE $\nu_{\mu} \mathrm{CC} \pi^{+}$cross sections are published in kinematics of the $\mu^{-}$and $\pi^{+}$. However, since the present study is a simple rate measurement, it is sufficient to simply implement the total cross section as a function of neutrino energy. Figure 7-3 compares the data to the simulation expectation. 
Functionally, the $\nu_{\mu} \mathrm{CC} \pi^{+}$cross section data is implemented into this analysis through correcting the antineutrino-mode expectation of observable $\mathrm{CC} \pi^{+}$. The ratio data/MC is measured in regions of generated neutrino energy, according to the bin delimitations. Note this is only possible because the exact set of underlying physics parameters, most importantly the single-pion axial masses and the final-state interaction model, are identical between the neutrino-mode simulation used to calculate the expectation shown in Figure 7-3 and the MC used in the present antineutrino-mode analysis. With the observed $\nu_{\mu} \mathrm{CC} \pi^{+}$cross-section data implemented in this analysis, the rate measurement presented in this section is also a measurement of the $\nu_{\mu}$ flux contribution to the antineutrino-mode beam.

As will be shown, the uncertainty on this measurement is dominated by error on the $\nu_{\mu} \mathrm{CC} \pi^{+}$cross section. To convert the uncertainty from the original measurement to the binning optimized for this analysis, a polynomial of order 4 is fit to the fractional $\mathrm{CC} \pi^{+}$ systematic uncertainty, and the values of this function evaluated in the center for the bins chosen in this analysis are taken as the $\mathrm{CC} \pi^{+}$uncertainty. Figure 7-4 shows the polynomial function and the two fractional error distributions.

The largest contribution to the uncertainty on the $\mathrm{CC} \pi^{+}$cross section comes from the neutrino-mode flux uncertainty, which is the only systematic error associated with the cross-section measurement that is also independent of the measurement made here. Because the other $\mathrm{CC} \pi^{+}$uncertainties are treated as uncorrelated between the neutrino-mode and the antineutrino-mode data, a partial cancellation of errors is ignored in the present $\nu_{\mu}$ flux measurement.

\section{The Selected Sample}

As mentioned in the previous section, the main requirement to select a $\mathrm{CC} \pi^{+}$ sample is the observation of 3 subevents. The full selection set is:

1. Three subevents

2. First subevent in beam window: $4000<\mathrm{T}(\mathrm{ns})<7000$, where $\mathrm{T}$ is the average PMT 


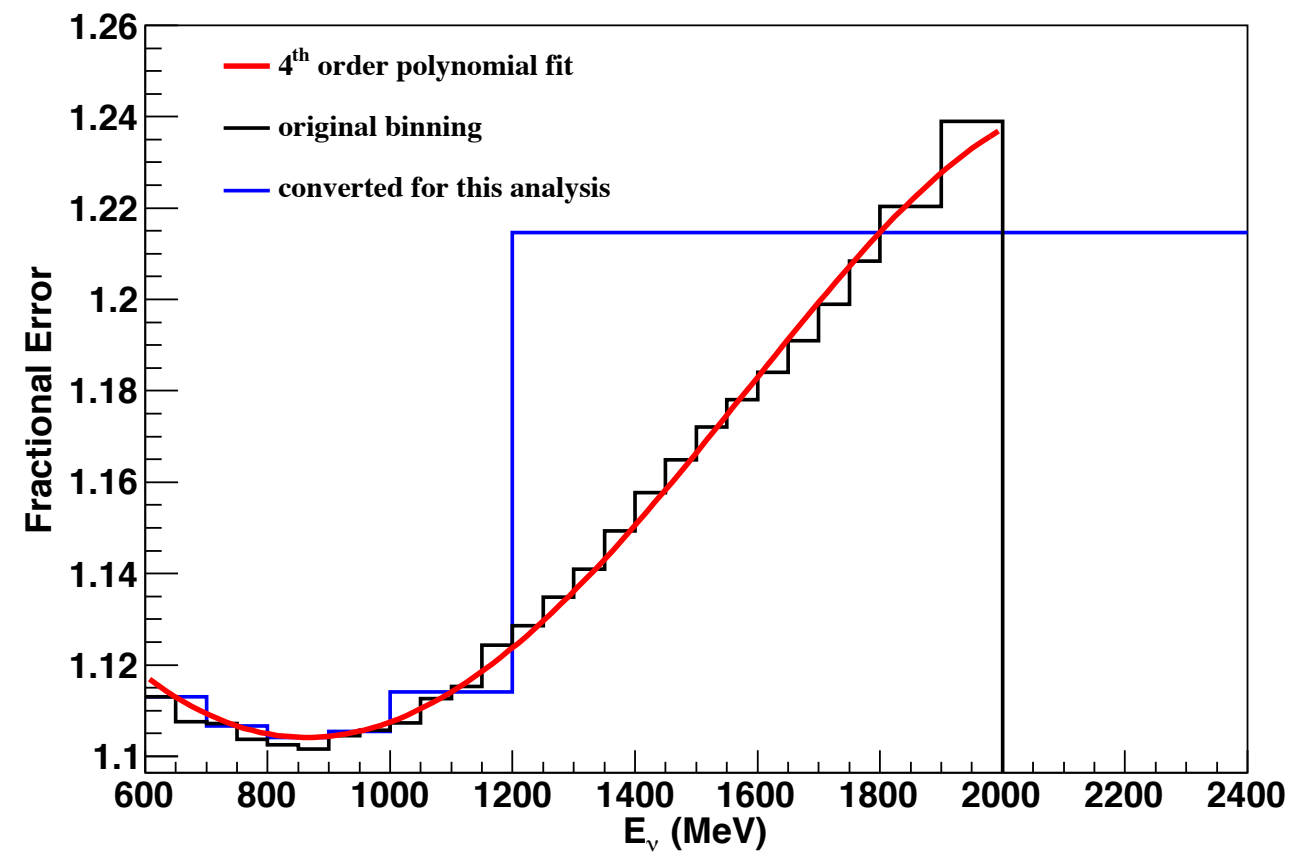

Figure 7-4. The $\mathrm{CC} \pi^{+}$fractional uncertainty conversion.

hit time

3. All subevents: reconstructed vertex $<500 \mathrm{~cm}$ from tank center

4. 1st subevent: tank hits $>200$

5. 2nd and 3rd subevents: tank hits $<200$

6. All subevents: veto hits $<6$

7. Distance between calculated end of 1 st subevent and nearest decay electron vertex < $150 \mathrm{~cm}$

Cut 1 requires the event be consistent with the production of three leptons above Čerenkov threshold, and cut 2 assures it be associated with the proton beam spill. Cuts 3 and 4 enhance the reliability of the reconstruction used, and cut 5 requires the final two subevents be consistent with a Michel electron, whose energetic endpoint of $\sim 53 \mathrm{MeV}$ leads to roughly 175 tank hits. Cut 6 ensures the leptons be contained and that no charged 
Table 7-2. Summary of selection cuts in the $\mathrm{CC} \pi^{+}$sample. Purity and efficiency numbers are sequential and are calculated for the "observable $\mathrm{CC} \pi^{+}$" event signature: $1 \mu^{-}, 1 \pi^{+}$.

\begin{tabular}{|c|c|c|c|}
\hline Cut \# & Description & $\begin{array}{l}\text { Efficiency } \\
\quad(\%)\end{array}$ & $\begin{array}{l}\text { Purity } \\
(\%)\end{array}$ \\
\hline 0 & No cuts & 100 & 10 \\
\hline 1 & Three subevents & 30 & 29 \\
\hline 2 & $\begin{array}{l}\text { 1st subevent in event time window } \\
\qquad 4000<\mathrm{T}(\mathrm{ns})<7000\end{array}$ & 28 & 34 \\
\hline 3 & $\begin{array}{c}\text { All subevents: reconstructed } \\
\text { vertex }<500 \mathrm{~cm} \text { from tank center }\end{array}$ & 23 & 36 \\
\hline 4 & 1st subevent: tank hits $>200$ & 22 & 39 \\
\hline 5 & $\begin{array}{c}\text { 2nd and 3rd subevents: } \\
\text { tank hits }<200\end{array}$ & 19 & 65 \\
\hline 6 & All subevents: veto hits $<6$ & 16 & 78 \\
\hline 7 & $\begin{array}{l}\text { Distance between reconstructed } \\
\text { end of } 1 \text { st subevent and nearest } \\
\text { Michel electron vertex }<150 \mathrm{~cm}\end{array}$ & 12 & 82 \\
\hline
\end{tabular}

particles entered the detector. Finally, cut 7 enforces spatial correlation between one of the Michel electrons and the end of the calculated muon path. As mentioned previously, due to the fast decay of the $\pi^{+}$, timing alone cannot determine the origin of the decay electrons. Using this selection, Table 7-2 presents the detection efficiency and purity for $\mathrm{CC} \pi^{+}$events, and Table 7-3 summarizes the sample composition.

\section{$\nu_{\mu}$ Flux Measurement Using $\mathrm{CC} \pi^{+}$}

The purity of the $\mathrm{CC} \pi^{+}$sample is sufficiently high to perform a simple background-subtracted rate measurement to test the $\nu_{\mu}$ flux. With the notation $A$ for data, $B$ for the expected $\bar{\nu}_{\mu}$ contributions, $C$ for signal $\mathrm{CC} \pi^{+}$, and $D$ for non-CC $\pi^{+} \nu_{\mu}$ events, we calculate the flux measurement $\alpha_{\nu}$ as

$$
\alpha_{\nu}=\frac{A-B}{C+D}
$$

The assigned uncertainties on these quantities are as follows:

- A: statistical uncertainty on the data. Following gaussian statistics, the uncertainty is 
Table 7-3. Summary of the $\mathrm{CC} \pi^{+}$sample in antineutrino mode, including the nucleon-level composition.

\begin{tabular}{cc}
\hline integrated POT & $5.66 \times 10^{20}$ \\
$\mathrm{CC} \pi^{+}$candidate events & 3268 \\
observable CC $\pi^{+}$efficiency $(R<550 \mathrm{~cm})$ & $12.0 \%$ \\
\hline Interaction & Contribution (\%) \\
\hline$\nu_{\mu} N \rightarrow \mu^{-} \pi^{+} N$ (resonant) & 64 \\
$\nu_{\mu} A \rightarrow \mu^{-} \pi^{+} A$ (coherent) & 7 \\
$\bar{\nu}_{\mu} N \rightarrow \mu^{+} \pi^{-} N^{\prime}$ (resonant) & 6 \\
$\nu_{\mu} n \rightarrow \mu^{-} p$ & 2 \\
$\nu_{\mu} n \rightarrow \mu^{-} \pi^{0} p$ & 1 \\
$\bar{\nu}_{\mu} p \rightarrow \mu^{+} \pi^{0} n$ & 14 \\
Other $(\operatorname{mostly} \mathrm{DIS})$ & 82 \\
\hline "Observable CC $\pi^{+"}$ & \\
$\left(1 \mu^{-}, 1 \pi^{+}\right)$ &
\end{tabular}

taken as $\sqrt{N}$, where $\mathrm{N}$ is the number of observed events.

- B: $\bar{\nu}_{\mu}$ background. This accounts for $14 \%$ of the sample, mostly $\bar{\nu}_{\mu}$ deep inelastic scattering (DIS) and $\mathrm{CC} \pi^{-}$events in which the $\pi^{-}$decayed in flight. An overall uncertainty of $30 \%$ is assigned.

- C: signal observable $\mathrm{CC} \pi^{+}$. Per Figure 7-3, fractional uncertainty on this process varies with neutrino energy and is at a minimum of $\sim 10 \%$ around $800 \mathrm{MeV}$.

- D: non-signal $\nu_{\mu}$ events. This accounts for $6 \%$ of the sample, and is dominated by $\nu_{\mu}$ DIS. An overall uncertainty of $30 \%$ is assigned.

These fractional uncertainties are propagated onto the $\nu_{\mu}$ flux measurement $\alpha_{\nu}$ with a simple quadrature sum of the uncorrelated uncertainties due to the processes $A, B, C$ and $D:$ 


$$
\begin{aligned}
\frac{\delta \alpha_{\nu}}{\alpha_{\nu}} & =\sqrt{\left(\frac{\delta A}{A}\right)^{2}+\left(\frac{\delta B}{B}\right)^{2}+\left(\frac{\delta C}{C}\right)^{2}+\left(\frac{\delta D}{D}\right)^{2}} \\
& =\frac{1}{C+D} \sqrt{(\delta A)^{2}+(\delta B)^{2}+\left(\frac{A-B}{C+D}\right)^{2}\left[(\delta C)^{2}+(\delta D)^{2}\right]}
\end{aligned}
$$

To test the accuracy of the simulated flux spectrum, the $\nu_{\mu}$ flux measurement is

performed in exclusive regions of reconstructed energy $E_{\nu}^{\Delta}$, where $E_{\nu}^{\Delta}$ follows the derivation of Eq. 6.7 appropriate to $\mathrm{CC} \pi^{+}$events:

$$
E_{\nu}^{\Delta}=\frac{m_{\Delta}^{2}-m_{p}^{2}-m_{\mu}^{2}-2 m_{p} E_{\mu}}{2\left(m_{p}-E_{\mu}+\left|\vec{p}_{\mu}\right| \cos \theta_{\mu}\right)}
$$

This reconstruction assumes a quasi-elastic interaction $\nu_{\mu}+N \rightarrow \Delta+N$ for all events. While this is a model-dependent valuation of the neutrino energy, separating the sample into exclusive regions of $E_{\nu}^{\Delta}$ nevertheless affords statistical sensitivity to the accuracy of the simulated flux spectrum. The $\nu_{\mu}$ flux measurement in the antineutrino-mode beam using $\mathrm{CC} \pi^{+}$events is summarized in Table 7-4.

\subsection{4 $\nu_{\mu}$ Flux Measurement Using $\mu^{-}$Nuclear Capture}

Another opportunity to measure the $\nu_{\mu}$ flux using nuclear capture is available through the $\mu^{-}$. Any $\mathrm{CC}$ event from $\nu_{\mu}$ and $\bar{\nu}_{\mu}$ will produce a muon, the $\mu^{-}$'s of which will produce fewer Michel electrons due to $\sim 8 \%$ nuclear capture on carbon [148]. An advantage of this analysis over the determination of the $\nu_{\mu}$ flux in antineutrino mode using the $\mathrm{CC} \pi^{+}$sample is the natural sensitivity to lower $\nu_{\mu}$ energies. The dominant mechanism for $\mathrm{CC} \pi^{+}$production involves the $\Delta(1232)$ resonance, and the examination of the $\nu_{\mu}$ flux using these interactions tests $\nu_{\mu}$ energies greater than $900 \mathrm{MeV}$. Fortunately, the present 
Table 7-4. Antineutrino-mode $\mathrm{CC} 1 \pi^{+}$sample details and $\nu_{\mu}$ flux component measurement. "Mean Gen. $\mathrm{E}_{\nu}$ " is the average generated neutrino energy in each reconstructed energy bin.

\begin{tabular}{cccccc}
\hline $\begin{array}{c}\mathrm{E}_{\nu}^{\Delta} \text { Range } \\
(\mathrm{MeV})\end{array}$ & $\begin{array}{c}\text { Mean Gen. } \\
\mathrm{E}_{\nu}(\mathrm{MeV})\end{array}$ & $\begin{array}{c}\text { Events } \\
\text { in Data }\end{array}$ & $\nu_{\mu}$ & $\bar{\nu}_{\mu}$ & $\nu_{\mu}$ Flux \\
\hline $600-700$ & 961 & 465 & 556 & 104 & $0.65 \pm 0.10$ \\
$700-800$ & 1072 & 643 & 666 & 118 & $0.79 \pm 0.10$ \\
$800-900$ & 1181 & 573 & 586 & 97 & $0.81 \pm 0.10$ \\
$900-1000$ & 1285 & 495 & 474 & 78 & $0.88 \pm 0.11$ \\
$1000-1200$ & 1426 & 571 & 646 & 92 & $0.74 \pm 0.10$ \\
$1200-2400$ & 1685 & 521 & 614 & 74 & $0.73 \pm 0.15$ \\
Inclusive & 1266 & 3268 & 3542 & 563 & $0.76 \pm 0.11$ \\
\hline
\end{tabular}

analysis can reach further down in neutrino energy to directly test the flux spectrum of the $\nu_{\mu}$ 's that are background to the main analysis of $\bar{\nu}_{\mu}$ CCQE interactions.

A complication of this measurement is the substantial component of $\bar{\nu}_{\mu}$ CCQE events present in the analysis samples, and so it is critical to evaluate the bias caused by the assumptions used to predict their contribution. If this bias were significant and the measurement were used to subtract the $\nu_{\mu}$ background from the data, the final $\bar{\nu}_{\mu}$ CCQE cross section would have an appreciable dependence on the CCQE interaction model. It will be shown that this is the case for the angular analysis of CCQE events presented in Section 7.1.5, and so its results are ignored in subtracting the $\nu_{\mu}$ background from the $\bar{\nu}_{\mu}$ CCQE sample. Meanwhile, it will be shown that the bias caused by assumptions on the $\bar{\nu}_{\mu}$ CCQE cross section in the present $\mu^{-}$capture analysis is small and negligible compared to other uncertainties.

\section{Implementation of the $\nu_{\mu} \mathrm{CC}$ Cross Sections}

The MiniBooNE $\nu_{\mu}$ CCQE analysis found the shape of the kinematics in data to be described well by the RFG assuming a few empirical parameter adjustments: $M_{A}=1.35 \pm 0.17 \mathrm{GeV}$ and $\kappa=1.007 \pm 0.012$ [84]. While the observed normalization is also consistent with this model within uncertainties, the data lies $\sim 8 \%$ high. Therefore, to implement the $\nu_{\mu} \mathrm{CCQE}$ cross section into the present $\nu_{\mu}$ flux measurement, the RFG 


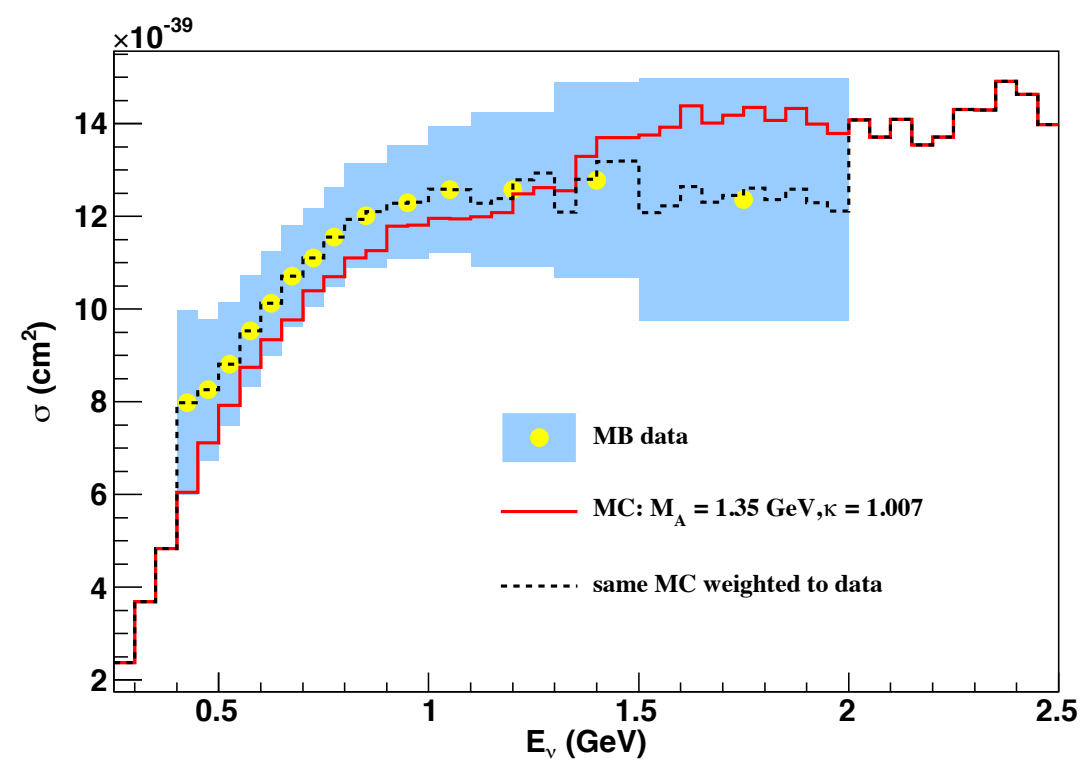

Figure 7-5. Summary of the normalization correction to the $\nu_{\mu}$ CCQE cross section in the antineutrino-mode simulation. The ratio of the data to the red $\mathrm{MC}$ histogram is applied binby-bin to the simulated $\nu_{\mu} \mathrm{CCQE}$ events. Also visible is the effect of low-precision sampling with the NUANCE generator at high energies. This is discussed further in Section 8.2.

model with $M_{A}=1.35 \mathrm{GeV}$ and $\kappa=1.007$ is assumed by simulation and the mild normalization discrepancy is accounted for by reweighting events. The reweighting values are found by a generator-level comparison between the observed MiniBooNE $\nu_{\mu} \mathrm{CCQE}$ total cross section and the RFG with the previously-mentioned parameter adjustments. Figure 7-5 compares the unfolded MC distributions before and after the correction.

The $\mathrm{CC} \pi^{+}$interactions also contribute significantly to the selected $\mu^{-}$capture samples. Their interaction rate and kinematics are implemented through the $Q_{Q E}^{2}$-based measurement in the neutrino-mode data [84]. This function and its origin are explained further in Section 7.2.

It will be shown that the $\nu_{\mu} \mathrm{CCQE}$ and $\mathrm{CC} \pi^{+}$interactions represent more than $94 \%$ of the $\nu_{\mu}$ channels contributing to the $\mu^{-}$capture samples. With both the kinematics and the normalization of these interactions implemented into the antineutrino-mode $\mathrm{MC}$, the rate measurement of the $\nu_{\mu}$ contribution to the $\mu^{-}$capture analysis samples may be cleanly 
interpreted as measurement of the $\nu_{\mu}$ flux component of the antineutrino-mode beam.

\section{Muon Capture Model and Event Selection}

In mineral oil, stopped $\mu^{-}$are captured on carbon nuclei with a probability of $7.78 \pm 0.07 \%$ [148]. For such capture events in MiniBooNE, typically little or no extra activity is observed in the detector. However, the low-energy neutron and photons from the primary capture reaction as well as de-excitations of the boron isotope may be energetic enough to produce a Michel-like event. The simulated production of these particles is based on the measurements of Refs. [149, 150, 151, 152, 153, 154], and the model that propagates these particles and possible re-interactions through the MiniBooNE detector estimates $6.60 \%$ of $\mu^{-}$capture events lead to activity similar to a low-energy Michel. Thus, the apparent $\mu^{-}$nuclear capture probability in the detector is predicted to be $7.78 \times(1$ $6.60 \%)=7.26 \pm 0.20 \%$, where the uncertainty is substantially increased to recognize the model dependence of the rate to regain Michel-like events following $\mu^{-}$capture. This rate is partially constrained by the calibration procedure described in Section 7.1.4, and it will be shown that the assigned uncertainty on effective $\mu^{-}$nuclear capture has a negligible impact on the final measurements.

Sensitivity to the $\mu^{-}$content of the data is obtained by simultaneously analyzing two samples: those with only a muon candidate event, and events consistent with the observation of a muon and its decay electron. Therefore, this analysis takes as signal $\nu_{\mu}$ and $\bar{\nu}_{\mu} \mathrm{CC}$ events. Apart from the requirement of either one or two subevents, the event selection for this analysis closely follows that described in Section 6.2 with a few changes appropriate to different backgrounds and a higher sensitivity to Michel detection efficiency. Table 7-5 details the $\nu_{\mu}$ and $\bar{\nu}_{\mu}$ charged-current purity of the two samples after each cut.

The primary samples of this analysis are separated by cut 1 , where $\nu_{\mu} \mathrm{CC}$ events have an enhanced contribution in the single subevent sample due to $\mu^{-}$capture. Cuts 2-5 are common to the analysis presented in the main body of this work and are motivated in Section 6.2. Cuts 6 and 8 reduce the NC background in the single-subevent sample. 
Figure 7-6 shows NC single $\pi$ events are largely rejected by the requirement on the $\mu / e$ $\log$-likelihood variable, while Figure 7-7 shows cut 8 further reduces their contribution.

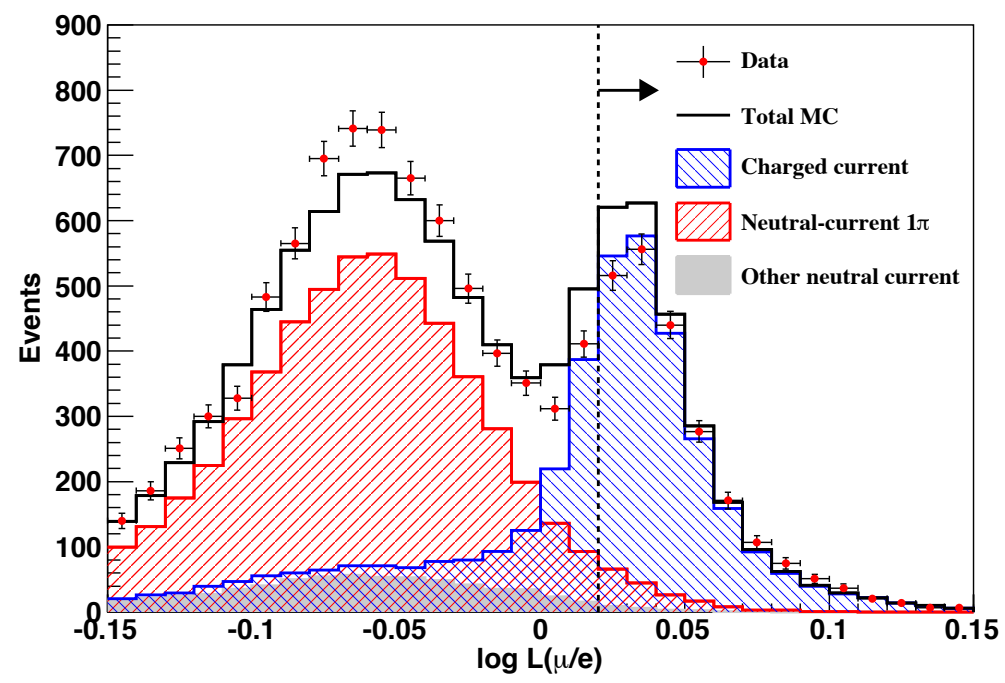

Figure 7-6. The log-likelihood $\mu / e$ particle-ID variable in the single-subevent sample. All other selection requirements have been applied. The simulation is normalized to flux, and errors shown on data are statistical only.

Cut 7 eliminates events in which the Michel electron is produced near the optical barrier, where modeling of the electron detection efficiency may be less reliable. In this region many more Michels are lost due to the minimum requirement of 10 PMT tank hits to form a subevent, while some are missed due to Michels entering the veto region. To explore these effects, we begin with a prediction of where the Michel ought to be produced, assuming it is the decay product of the prompt muon. Calculating the stopping radius of the muon based on its observed vertex, direction and energy, we find:

$$
\mu \text { stopping radius }=\sqrt{\left(V_{x}+\overline{d E / d X}^{-1} \times T_{\mu} \times U_{x}\right)^{2}+\text { [same for y and z directions] }},
$$

where $V_{x}$ and $U_{x}$ are the reconstructed muon vertex and direction in the x-direction respectively, and $T_{\mu}$ is the muon kinetic energy. $\overline{d E / d x}$ is the average energy deposited per 
unit of distance traveled for muons in mineral oil at MiniBooNE energies. Fitting $\mathrm{dE} / \mathrm{dX}$ data for stopping power of mineral oil to a linear function finds $\overline{d E / d x}=1.9 \mathrm{MeV} / \mathrm{cm}$.

Figure 7-8 shows adequate agreement between data and simulation at high radius in the 2SE sample, where a Michel is both produced and detected, while the agreement is worse in the single-subevent sample. Regardless, the ratio of single/two subevent events as a function of the muon endpoint presented in the same figure shows this ratio clearly increases with radius at large values and so is quite sensitive to the details of Michel detection near the optical barrier.

Away from this barrier, where Michel detection is not a function of position, most Michel electrons not detected are missed due to the timing cut used to separate subevents and not the requirement of at least 10 tank PMT hits within the temporal window. Figure 7-9 shows the difference in the timing distributions for the 2SE sample, while Figure 7-10 presents the tank hit distribution for the second subevent. Less than $0.5 \%$ of events are rejected by the 10 PMT hit requirement, while $\sim 8 \%$ of Michels are produced too close in time with the muon to be temporally separated.

Cut 7 also enhances $\nu_{\mu}$ purity due to kinematic differences between $\nu_{\mu}$ and $\bar{\nu}_{\mu}$ CCQE, where the more forward-going nature of the $\mu^{+}$from $\bar{\nu}_{\mu}$ interactions preferentially stop at high radius in the downstream region of the detector.

With the full selection, nucleon-level interaction contributions to the subevent samples are given in Table 7-6. 

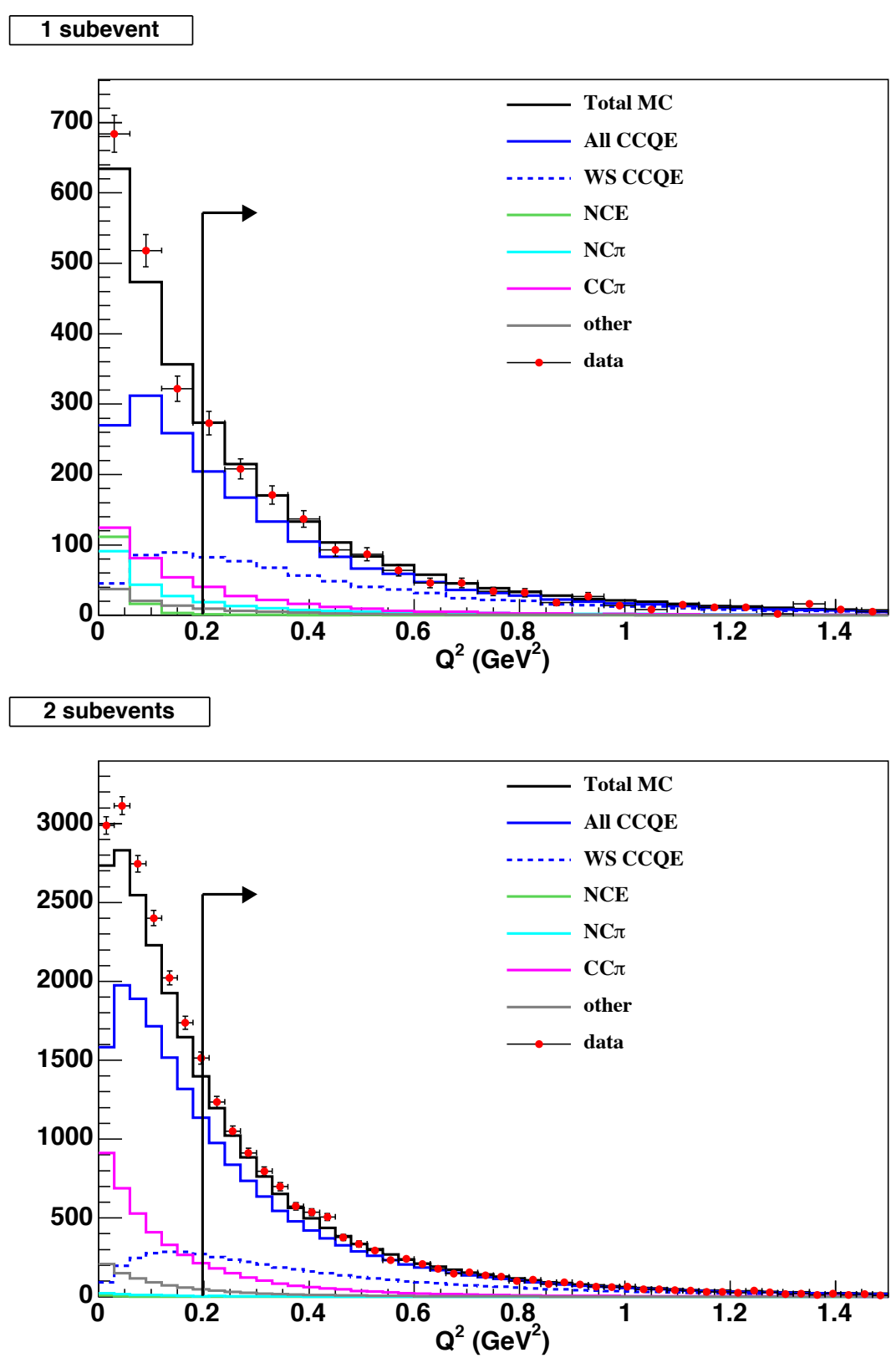

Figure 7-7. $\mathrm{Q}_{Q E}^{2}$ for the single-subevent (top) and two-subevent (bottom) samples. All other selection cuts have been applied. Events with $\mathrm{Q}^{2}>0.2 \mathrm{GeV}^{2}$ are selected to reject some NC events, particularly in the single-subevent sample. Distributions are normalized to flux. 

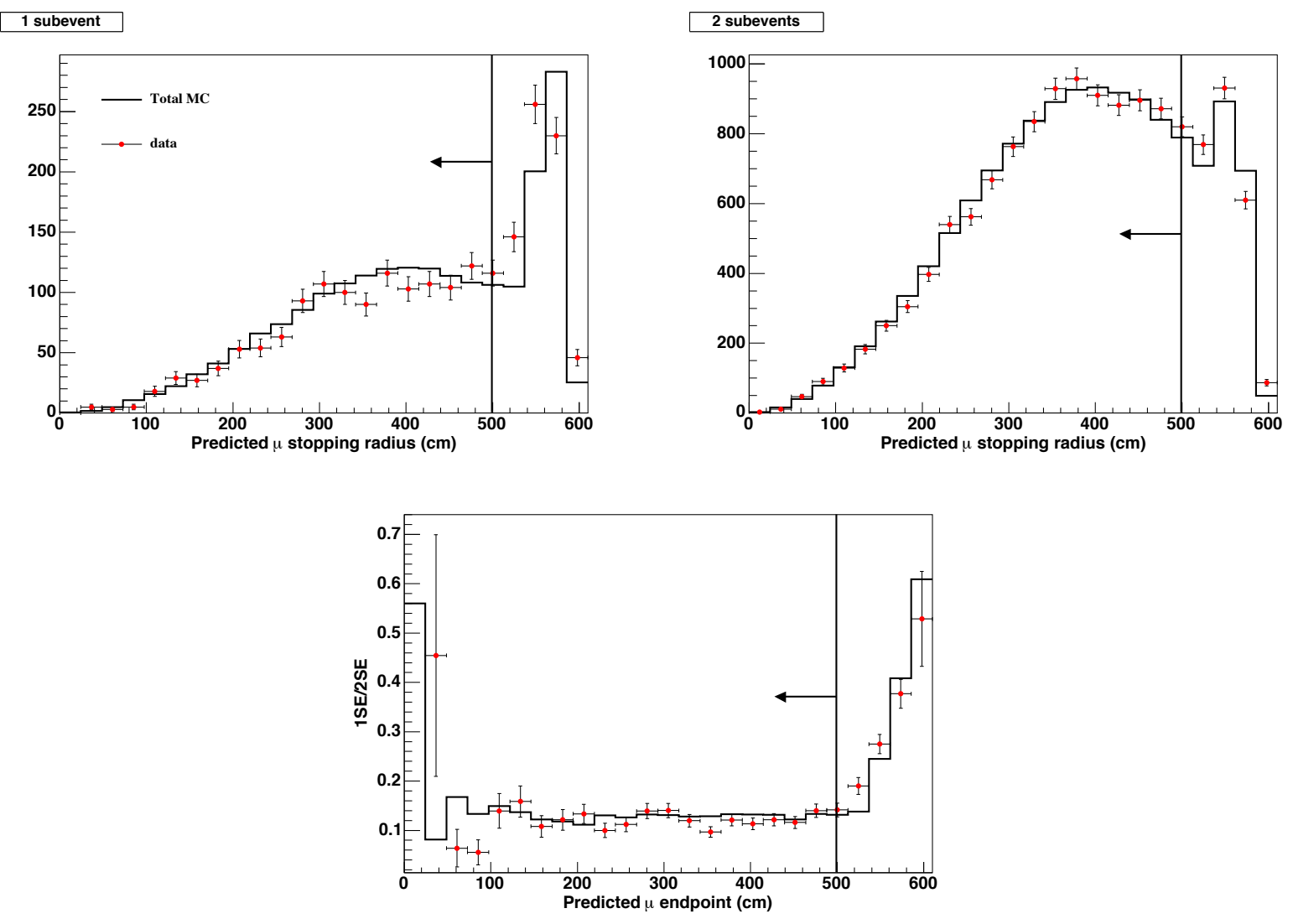

Figure 7-8. Predicted muon endpoint radius for the single-subevent (top left), two-subevent (top right) samples and the ratio single/two subevents (bottom). All other selection cuts have been applied. Data-MC discrepancy is only present at high radius, presumably due to difficulties in modeling Michel detection close to the optical barrier. The peak above 500 $\mathrm{cm}$ in the single-subevent and the ratio distribution is due to the lower Michel detection efficiency in this region. There are zero events in the first bin of the single-subevent data. Distributions are relatively normalized to data. 


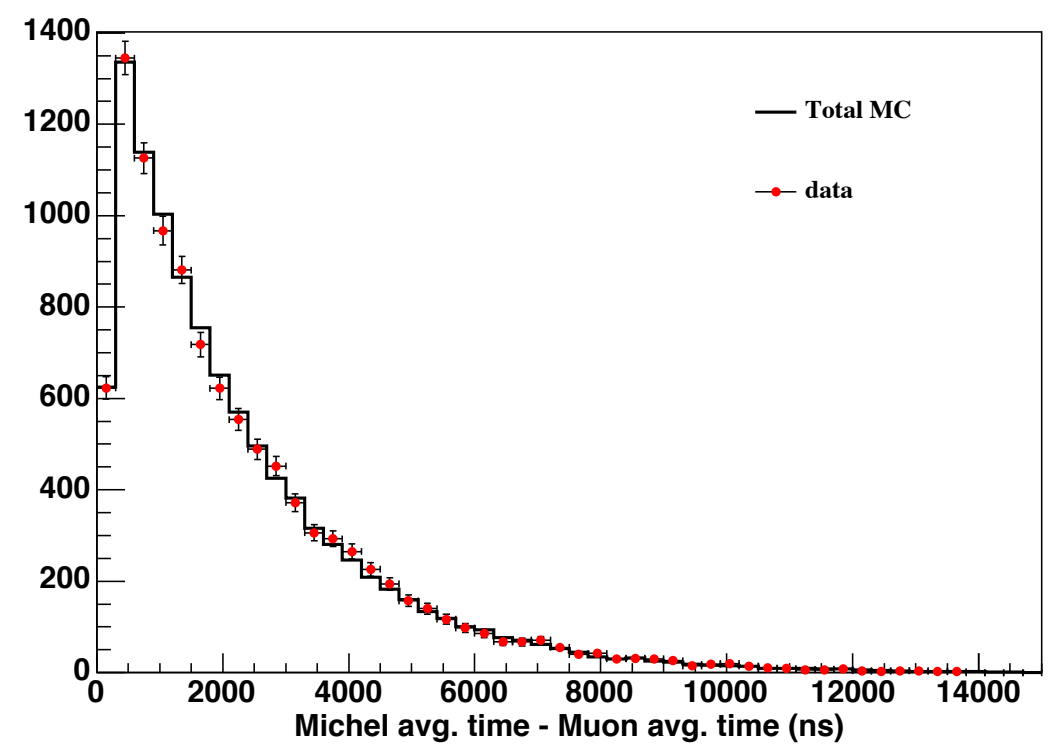

Figure 7-9. Difference between average hit times for the Michel-like subevent and the muonlike subevent in antineutrino mode. The distributions deviate from an exponential form at low timing differences due to the temporal definition of a subevent. Simulation is relatively normalized to data.

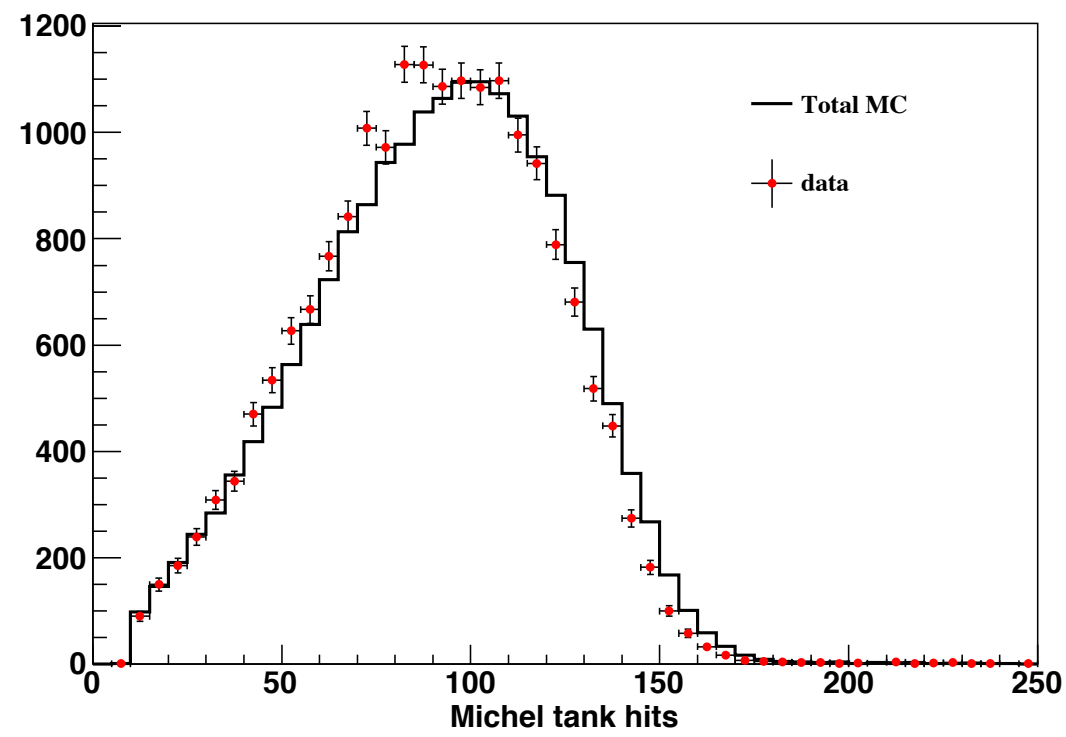

Figure 7-10. Tank hit distributions for the second subevent in antineutrino mode. Simulation is normalized to data. 


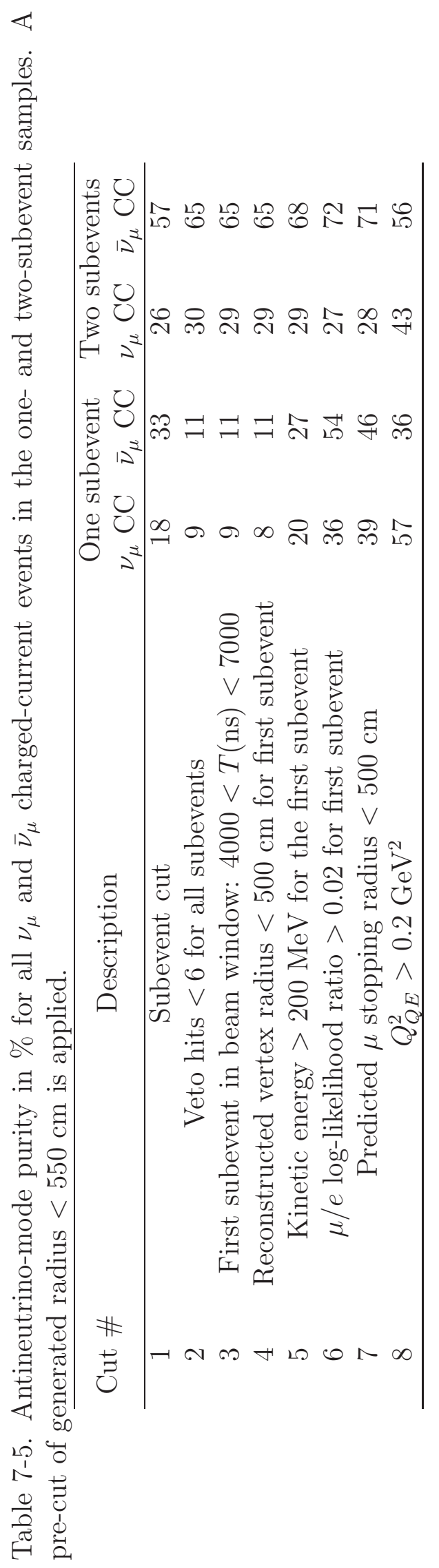


Table 7-6. Summary of predicted nucleon-level interactions in the antineutrino-mode subevent samples. The small contribution from neutral current processes are presented as the sum of the $\nu_{\mu}$ and $\bar{\nu}_{\mu}$ interactions.

\begin{tabular}{ccc}
\hline Process & \multicolumn{2}{c}{ Contribution (\%) } \\
& One subevent & Two subevents \\
\hline $\bar{\nu}_{\mu}+p \rightarrow \mu^{+}+n$ & 31 & 49 \\
$\nu_{\mu}+n \rightarrow \mu^{-}+p$ & 48 & 36 \\
$\bar{\nu}_{\mu}+N \rightarrow \mu^{+}+N+\pi^{-}$ & 3 & 5 \\
$\nu_{\mu}+N \rightarrow \mu^{-}+N+\pi^{+}$ & 7 & 7 \\
$\nu_{\mu}\left(\bar{\nu}_{\mu}\right)+N \rightarrow \nu_{\mu}\left(\bar{\nu}_{\mu}\right)+N$ & 1 & 0 \\
$\nu_{\mu}\left(\bar{\nu}_{\mu}\right)+N \rightarrow \nu_{\mu}\left(\bar{\nu}_{\mu}\right)+N+\pi^{0}$ & 3 & 0 \\
$\nu_{\mu}\left(\bar{\nu}_{\mu}\right)+N \rightarrow$ & 4 & 0 \\
$\nu_{\mu}\left(\bar{\nu}_{\mu}\right)+N+\pi^{ \pm}$ & & \\
other & 3 & 3 \\
\hline All $\nu_{\mu}$ & 58 & 43 \\
All $\bar{\nu}_{\mu}$ & 42 & 57 \\
\hline
\end{tabular}

\section{Calibrations and Stability Checks Using the Neutrino-Mode Data}

The success of this analysis is dependent upon being able to interpret differences between the one- and two-subevent antineutrino-mode data and MC samples as being due to the amount of $\nu_{\mu}$ in the beam. In principle, any difference discovered between data and the simulation is ambiguous between the $\nu_{\mu}$ content and inadequate modeling of the total migration rate between the subevent samples.

Fortunately, the neutrino-mode data offers an opportunity to calibrate the migration rate between the subevent samples for $\nu_{\mu} \mathrm{CC}$ events. Due to the convolution of flux and cross-section effects discussed in Section 7.1.1, the neutrino-mode subevent samples are dominantly due to $\mathrm{CC} \nu_{\mu}$ interactions. Table $7-7$ shows the predicted neutrino species and interaction type contributions to the neutrino-mode subevent samples with the same selection described in the previous section. With a high-purity $\nu_{\mu} \mathrm{CC}$ sample, the accuracy of Michel detection and effective $\mu^{-}$capture in simulation can be tested. For CC $\nu_{\mu}$ events without final-state pions ( $\nu_{\mu} \mathrm{CC}$ "), the number of events in the neutrino-mode one-subevent ("1SE" ) and two-subevent ("2SE" ) samples are given by: 
Table 7-7. A brief description of the neutrino-mode subevent samples for the same selection described in the previous section.

\begin{tabular}{ccc}
\hline \multirow{2}{*}{ Process } & \multicolumn{2}{c}{ Contribution (\%) to } \\
& One subevent & Two subevents \\
\hline All $\nu_{\mu}$ CC & 95.4 & 99.0 \\
All $\bar{\nu}_{\mu}$ & 0.4 & 0.7 \\
All NC & 4.3 & 0.3 \\
\hline
\end{tabular}

$$
\begin{aligned}
1 \mathrm{SE}^{\nu} & =\nu_{\mu} \mathrm{CC} \times(\delta+\beta(1-\delta))+\mathrm{N}_{1}^{\nu} \\
2 \mathrm{SE}^{\nu} & =\nu_{\mu} \mathrm{CC} \times(1-\delta-\beta(1-\delta))+\mathrm{N}_{2}^{\nu}
\end{aligned}
$$

where $\mathrm{N}_{1}^{\nu}\left(\mathrm{N}_{2}^{\nu}\right)$ is the $\mathrm{NC}$ contribution to the $1 \mathrm{SE}(2 \mathrm{SE})$ sample, $\delta$ is the Michel detection inefficiency and $\beta$ is the effective $\mu^{-}$capture rate described previously. The rate for Michel non-detection can be solved in terms of the effective $\mu^{-}$capture rate and the small NC contribution:

$$
\delta=\frac{\frac{1 \mathrm{SE}^{\nu}-\mathrm{N}_{1}^{\nu}}{1 \mathrm{SE}^{\nu}+2 \mathrm{SE}^{\nu}-\left(\mathrm{N}_{1}^{\nu}+\mathrm{N}_{2}^{\nu}\right)}-\beta}{1-\beta}
$$

Noting the symmetry in Equations 7.8 and 7.9 between $\delta$ and $\beta$, Equation 7.10 can also express the effective $\mu^{-}$capture rate in terms of Michel detection with $\delta \leftrightarrow \beta$. Table 7-8 gives values of $\delta$ and $\beta$ from simulation and data based on the observed or predicted event rates in the $1 \mathrm{SE}^{\nu}$ and $2 \mathrm{SE}^{\nu}$ samples.

As the $\nu_{\mu}$ charged-current migration rate to the single-subevent sample is due to a convolution of Michel detection and effective $\mu^{-}$capture, the processes cannot be simultaneously calibrated with the neutrino-mode data - that is, for example, the calibration of $\delta$ assumes the MC valuation of $\beta$ is correct. Future experiments may be able 
Table 7-8. Calibration summary for Michel detection inefficiency $(\delta)$ and the rate of effective $\mu^{-}$nuclear capture $(\beta)$. Note that both processes cannot be simultaneously constrained.

\begin{tabular}{cccc}
\hline Process & data & MC & data/MC \\
\hline$\delta$ & 0.073 & 0.074 & 0.98 \\
$\beta$ & 0.071 & 0.073 & 0.98 \\
\hline
\end{tabular}

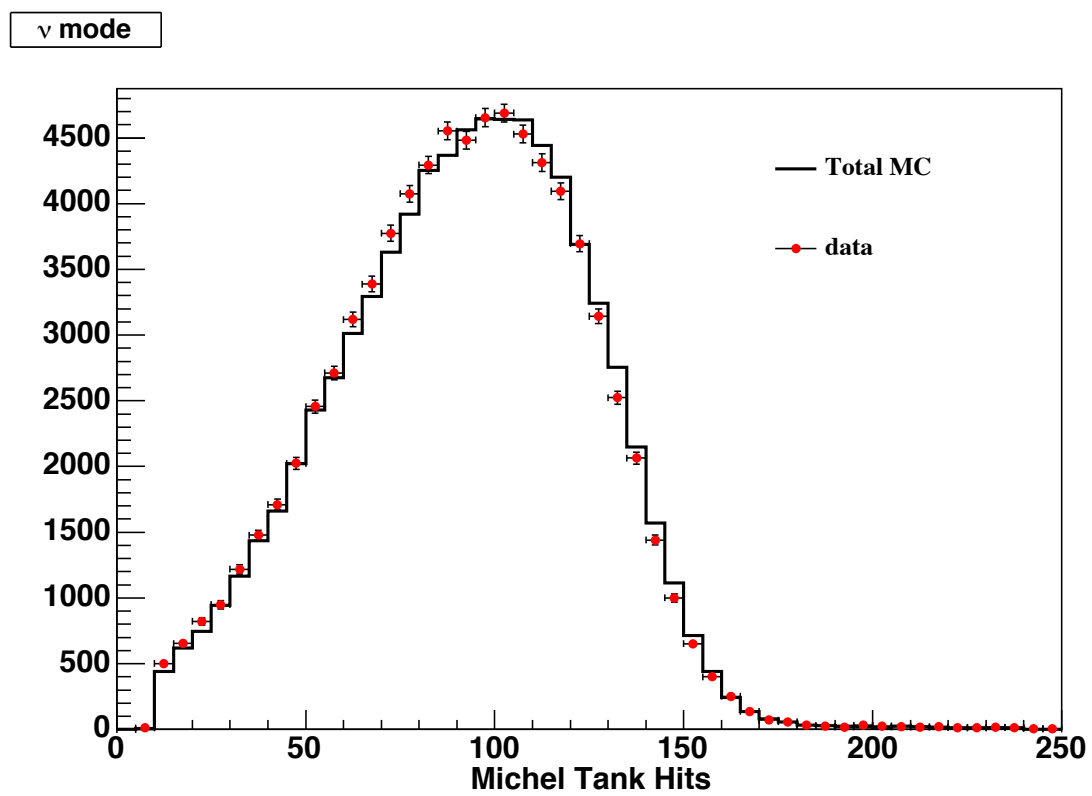

Figure 7-11. Tank hit distributions for the second subevent in neutrino mode. Events from $\mu^{-}$capture are expected to only contribute in the lowest bins, and the consistency between data and simulation gives confidence that the $\mu^{-}$capture model is adequate. MC is relatively normalized to data.

to break this degeneracy by examining the low-energy region of the Michel spectrum across both neutrino and antineutrino modes, where the contribution from activity following $\mu^{-}$ capture is enhanced. In the case of MiniBooNE, the Michel spectrum in antineutrino-mode is given in Figure 7-10, and the neutrino-mode analogue is shown in Figure 7-11. While consistency in the low-energy region between data and simulation indicate the $\mu^{-}$capture model is not grossly wrong, the statistics of the antineutrino sample prevent a rigorous test of the Michel-like contributions following $\mu^{-}$capture.

As the calibration results shown in Table 7-8 are quite mild and within systematic 
uncertainties, this procedure gives confidence in the ability to unambiguously measure the $\nu_{\mu}$ content of the antineutrino-mode data using $\mu^{-}$capture.

The substantially higher event rate in neutrino-mode compared to antineutrino-mode also offers the opportunity for a robust stability check of the Michel detection efficiency. If there were some variation or degradation of the electronics during neutrino-mode running that would affect the detection of Michels, it should appear as differences in some suitable variables between temporal bins.

The neutrino-mode data is separated into four chronologically sequential groups of data with roughly equal POT contributions. The first variable to look at is the ratio 1SE/2SE. Table 7-9 offers event counts in the 1 and 2SE samples and their ratio with statistical error for the four data groups. Within statistical uncertainty, the subevent ratios are consistent and we find no evidence of systematic variations affecting Michel detection.

Table 7-9. 1SE and 2SE event details in four sequential and roughly equally sized neutrinomode data groups. The $1 \mathrm{SE} / 2 \mathrm{SE}$ ratios are consistent within one standard deviation.

\begin{tabular}{cccc}
\hline Run numbers & 1SE events & 2SE events & 1SE/2SE \\
\hline $3539-7999$ & 3658 & 21318 & $0.172 \pm 0.003$ \\
$8000-10999$ & 4413 & 26380 & $0.167 \pm 0.003$ \\
$11000-11999$ & 2355 & 13933 & $0.169 \pm 0.003$ \\
$12000-12842$, & 3112 & 18576 & $0.168 \pm 0.003$ \\
$15833-17160$ & & & \\
\hline
\end{tabular}

A final check on Michel detection stability can be made by looking at the very early timing distribution of the 2SE sample. Figure 7-12 presents a 0 - 800 ns window of the average time separating the two subevents for the four sets of neutrino-mode data. No evidence of a time-dependent shift between the data runs is observed.

We conclude that in the sample most sensitive to any pathological evolution of Michel detection in time, none are observed. The statistics of the single-subevent sample in antineutrino mode prohibit the execution of the same tests using the primary analysis samples. 


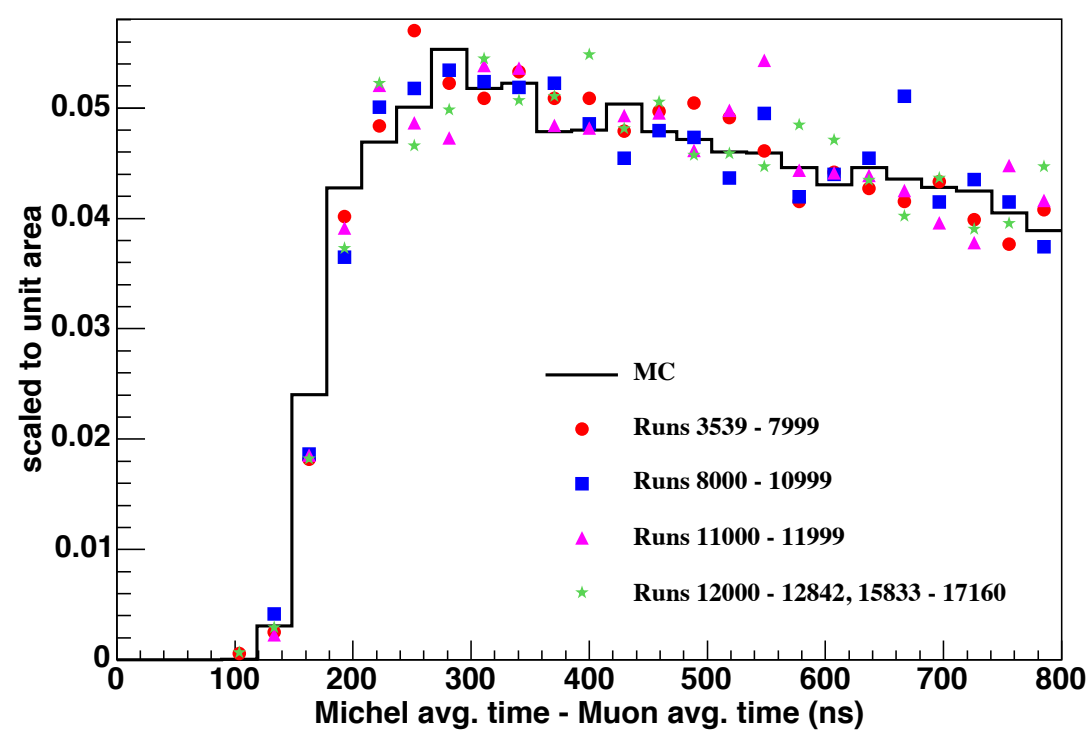

Figure 7-12. Early separation between the two subevents in neutrino mode. No significant shape difference is observed between the four chronologically sequential groups of data. Distributions are scaled to unit area.

\section{$\nu_{\mu}$ Flux Measurement Using $\mu^{-}$Capture}

The $\nu_{\mu}$ flux in the antineutrino-mode beam is measured by adjusting the MC prediction of the $\nu_{\mu}$ and $\bar{\nu}_{\mu}$ content to match the data in regions of reconstructed energy for the subevent samples. Following the conventions of Eqs. 7.8 and 7.9 and introducing $\bar{\nu}_{\mu} \mathrm{CC}$ for the $\bar{\nu}_{\mu}$ charged-current content, the predicted $\nu_{\mu}$ and $\bar{\nu}_{\mu}$ contributions to the subevent samples in antineutrino mode are defined as

$$
\begin{aligned}
\nu_{\mathrm{MC}}^{1 \mathrm{SE}} & =\nu_{\mu} \mathrm{CC} \times(\delta+\beta(1-\delta)) \\
\nu_{\mathrm{MC}}^{2 \mathrm{SE}} & =\nu_{\mu} \mathrm{CC} \times(1-\delta-\beta(1-\delta)) \\
\bar{\nu}_{\mathrm{MC}}^{1 \mathrm{SE}} & =\bar{\nu}_{\mu} \mathrm{CC} \times \delta \\
\bar{\nu}_{\mathrm{MC}}^{2 \mathrm{SE}} & =\bar{\nu}_{\mu} \mathrm{CC} \times(1-\delta)
\end{aligned}
$$


Then the single- ("1SE ${ }^{\bar{\nu} ")}$ ) and two-subevent ("2SE" mode are given by

$$
\begin{aligned}
1 \mathrm{SE}^{\bar{\nu}} & =\alpha_{\nu} \times \nu_{\mathrm{MC}}^{1 \mathrm{SE}}+\alpha_{\bar{\nu}} \times \bar{\nu}_{\mathrm{MC}}^{1 \mathrm{SE}}+\mathrm{N}_{1}^{\bar{\nu}} \\
2 \mathrm{SE}^{\bar{\nu}} & =\alpha_{\nu} \times \nu_{\mathrm{MC}}^{2 \mathrm{SE}}+\alpha_{\bar{\nu}} \times \bar{\nu}_{\mathrm{MC}}^{2 \mathrm{SE}}+\mathrm{N}_{2}^{\bar{\nu}}
\end{aligned}
$$

where $\alpha_{\nu}$ and $\alpha_{\bar{\nu}}$ are scale factors for the $\nu_{\mu}$ and $\bar{\nu}_{\mu}$ charged-current content, respectively, to be measured in this analysis. The $\mathrm{NC}$ content $\left(\mathrm{N}_{2}^{\bar{\nu}}\right.$ and $\left.\mathrm{N}_{1}^{\bar{\nu}}\right)$ include contributions from both $\nu_{\mu}$ and $\bar{\nu}_{\mu}$. Equations 7.15 and 7.16 can be solved for $\alpha_{\nu}$ and $\alpha_{\bar{\nu}}$ :

$$
\begin{aligned}
& \alpha_{\nu}=\frac{\left(1 \mathrm{SE}^{\bar{\nu}}-\mathrm{N}_{1}^{\bar{\nu}}\right) \bar{\nu}_{\mathrm{MC}}^{2 \mathrm{SE}}-\left(2 \mathrm{SE}^{\bar{\nu}}-\mathrm{N}_{2}^{\bar{\nu}}\right) \bar{\nu}_{\mathrm{MC}}^{1 \mathrm{SE}}}{\bar{\nu}_{\mathrm{MC}}^{2 \mathrm{SE}} \nu_{\mathrm{MC}}^{1 \mathrm{SE}}-\bar{\nu}_{\mathrm{MC}}^{1 \mathrm{SE}} \nu_{\mathrm{MC}}^{2 \mathrm{SE}}} \\
& \alpha_{\bar{\nu}}=\frac{\left(1 \mathrm{SE}^{\bar{\nu}}-\mathrm{N}_{1}^{\bar{\nu}}\right) \nu_{\mathrm{MC}}^{2 \mathrm{SE}}-(2 \mathrm{SE}}{\left.\nu_{\mathrm{MC}}^{2 \mathrm{SE}} \bar{\nu}_{\mathrm{MC}}^{1 \mathrm{SE}}-\nu_{\mathrm{MC}}^{1 \mathrm{SE}} \bar{\nu}_{\mathrm{MC}}^{2 \mathrm{SE}}\right) \nu_{\mathrm{MC}}^{1 \mathrm{SE}}}
\end{aligned}
$$

To check the modeling of the $\nu_{\mu}$ flux spectrum, this measurement is performed in three regions of reconstructed energy $E_{\nu}^{Q E}$ (Eq. 6.7): above and below $900 \mathrm{MeV}$, and an inclusive energy sample.

As described in the previous section, the calibration from the neutrino-mode data is ambiguous between Michel detection and the effective $\mu^{-}$capture model. As these effects change the expectations for $\bar{\nu}_{\mathrm{MC}}^{1 \mathrm{SE}}, \bar{\nu}_{\mathrm{MC}}^{2 \mathrm{SE}}, \nu_{\mathrm{MC}}^{1 \mathrm{SE}}$ and $\nu_{\mathrm{MC}}^{2 \mathrm{SE}}$ in different ways, the measurement of $\alpha_{\nu}$ and $\alpha_{\bar{\nu}}$ is, in principle, sensitive to which rate is calibrated. In the absence of a compelling reason to choose one over the other, the final evaluations for $\alpha_{\nu}$ and $\alpha_{\bar{\nu}}$ are taken to be the average of the two calculations assuming each rate is calibrated. A calibration uncertainty spanning the difference in the two measurements is added to the systematic errors discussed next. The central values for $\alpha_{\nu}$ and $\alpha_{\bar{\nu}}$ are presented in Table 7-10. 
Table 7-10. Results for scale factors relative to the expectation for the $\nu_{\mu}$ and $\bar{\nu}_{\mu}$ chargedcurrent content of the antineutrino-mode data.

\begin{tabular}{ccccc}
\hline \multirow{2}{*}{ Parameter } & Calibrated & \multicolumn{3}{c}{$E_{\nu}^{Q E}$ range $(\mathrm{GeV})$} \\
& process & $<0.9$ & $\geq 0.9$ & All \\
\hline \multirow{3}{*}{$\alpha_{\nu}$} & $\delta$ & 0.78 & 0.79 & 0.78 \\
& $\beta$ & 0.78 & 0.79 & 0.78 \\
$\alpha_{\bar{\nu}}$ & Average & $\mathbf{0 . 7 8}$ & $\mathbf{0 . 7 9}$ & $\mathbf{0 . 7 8}$ \\
\hline & $\beta$ & 1.16 & 1.15 & 1.16 \\
& Average & 1.16 & 1.15 & 1.16 \\
& & $\mathbf{1 . 1 6}$ & $\mathbf{1 . 1 5}$ & $\mathbf{1 . 1 6}$ \\
\hline
\end{tabular}

\section{Systematic Errors}

Systematic uncertainties on $\alpha_{\nu}$ and $\alpha_{\bar{\nu}}$ are evaluated by assigning relevant errors to the physics processes contributing to the subevent samples and observing how the measurement changes as the channels are varied within their uncertainty. These uncertainties are treated as uncorrelated, so the uncertainty on $\alpha_{\nu}$, for example, due to physics processes $P_{1}, \cdots, P_{N}$ is simply

$$
\delta \alpha_{\nu}^{2}=\sum_{i=1}^{N}\left(\frac{\partial \alpha_{\nu}}{\partial P_{i}} \delta P_{i}\right)^{2}
$$

Table 7-11 shows the errors assigned to the various contributing processes and their propagated uncertainty onto $\alpha_{\nu}$ and $\alpha_{\bar{\nu}}$. The most important process for extracting the $\nu_{\mu}$ flux measurement is the $\nu_{\mu}$ CCQE interaction, and its cross section and assigned uncertainty reflect the measurement and accuracy of the MiniBooNE result [84]. The same is true for the $\nu_{\mu}$ and $\bar{\nu}_{\mu}$ neutral-current single $\pi^{0}$ channels [155]; however the error is increased to recognize a possible rate difference in these interactions between the cross-section measurements and this analysis due to using the opposite side of the log-likelihood variable shown in Figure 7-6. The $\nu_{\mu}$ and $\bar{\nu}_{\mu}$ charged-current single charged $\pi$ channels are adjusted to reflect the $\nu_{\mu}$ measurement [84] and their uncertainty is increased 
to recognize the extrapolation to the $\bar{\nu}_{\mu}$ processes. Treating the uncertainties on the $\nu_{\mu}$ processes constrained by MiniBooNE data as uncorrelated ignores a common dependence on the neutrino-mode flux uncertainties, and a small cancellation of errors that could be propagated onto $\alpha_{\nu}$ and $\alpha_{\bar{\nu}}$ is ignored. The $\nu_{\mu}$ neutral-current elastic process is also constrained by MiniBooNE data [156], while the neutral-current charged-pion production processes are completely unconstrained and so the assigned uncertainty is large. Preliminary results for the $\bar{\nu}_{\mu}$ CCQE process [157] informs the choice of a $20 \%$ uncertainty relative to the RFG model with $M_{A}=1.35 \mathrm{GeV}$. With these systematic uncertainty assumptions, as seen in Table 7-11, the uncertainty on the main result of this work $\alpha_{\nu}$ is dominated by statistics and the $\nu_{\mu} \mathrm{CCQE}$ cross section. As the $\nu_{\mu}$ CCQE process is directly constrained by MiniBooNE data, the measurement of the $\nu_{\mu}$ flux scale $\alpha_{\nu}$ features negligible model dependence. Table 7-12 summarizes the measurements of $\alpha_{\nu}$ and $\alpha_{\bar{\nu}}$.

As the cross sections for the dominant $\nu_{\mu}$ processes have been applied to simulation, the deviation from unity for $\alpha_{\nu}$ represents the accuracy of the $\nu_{\mu}$ flux prediction in antineutrino mode. As the bulk of the $\bar{\nu}_{\mu}$ flux prediction is constrained by the HARP data, the $\alpha_{\bar{\nu}}$ scale factor is representative of the level of cross-section agreement between data and the RFG with $M_{A}=1.35 \mathrm{GeV}$ for the $\bar{\nu}_{\mu}$ CCQE process. 


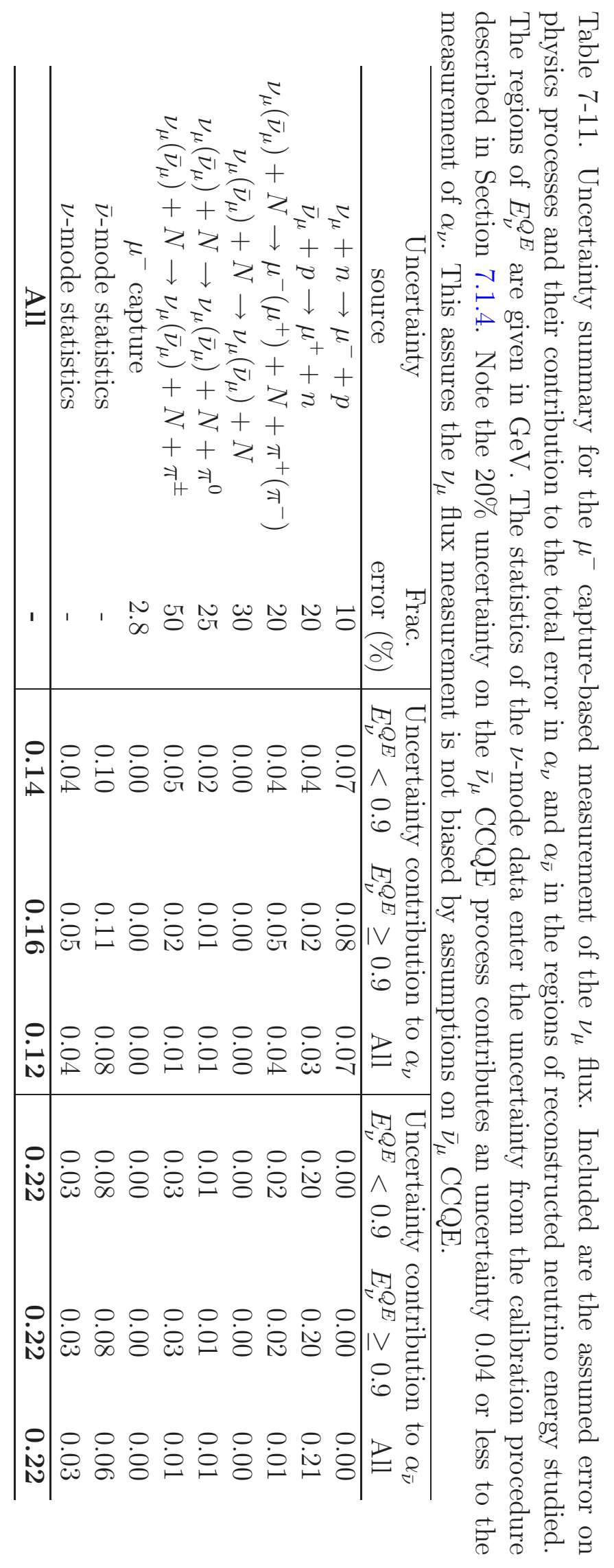




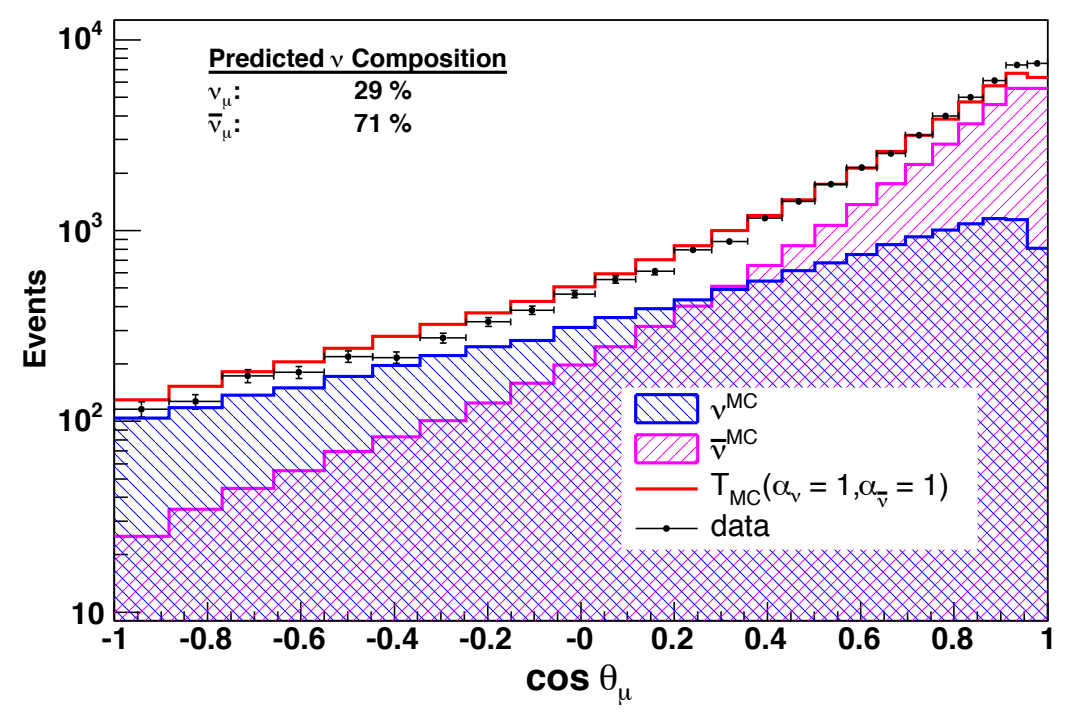

Figure 7-13. The $\cos \theta_{\mu}$ distribution of the CCQE sample by neutrino type before fitting. The nominal MC prediction is normalized to flux, and notations used in the legend are used in the execution of the fit.

Table 7-12. Summary of measurements for the $\nu_{\mu}$ flux scale $\alpha_{\nu}$ and the $\bar{\nu}_{\mu}$ rate scale $\alpha_{\bar{\nu}}$.

\begin{tabular}{cccc}
\hline \multirow{2}{*}{ Parameter } & \multicolumn{3}{c}{$E_{\nu}^{Q E}$ range $(\mathrm{GeV})$} \\
& $<0.9$ & $\geq 0.9$ & All \\
\hline$\alpha_{\nu}$ & $0.78 \pm 0.14$ & $0.79 \pm 0.16$ & $0.78 \pm 0.12$ \\
$\alpha_{\bar{\nu}}$ & $1.16 \pm 0.22$ & $1.15 \pm 0.22$ & $1.16 \pm 0.22$ \\
\hline
\end{tabular}

\subsection{5 $\nu_{\mu}$ Flux Measurement Using the $\cos \theta_{\mu}$ Distribution}

\section{Overview}

The final constraint on $\nu_{\mu}$ events comes from the observed muon angular distribution $\cos \theta_{\mu}$, where $\theta_{\mu}$ is the muon scattering direction relative to the incoming neutrino beam. Due to the axial-vector interference term (Section 4.2), the contribution from $\bar{\nu}_{\mu}$ events to backward-scattering muons is predicted to be heavily suppressed. Figure 7-13 compares the predicted $\nu_{\mu}$ and $\bar{\nu}_{\mu}$ contributions to the muon scattering angle with data.

This large asymmetry offers the opportunity to fit a combination of the $\nu_{\mu}$ and $\bar{\nu}_{\mu}$ 
content to the observed data. However, this asymmetry is model-dependent, as the details of $\bar{\nu}_{\mu}$ CCQE scattering are not well known, and in fact the $\bar{\nu}_{\mu}$ processes contributing to the MiniBooNE CCQE sample may be much more isotropic than suggested by Figure 7-13. Of course, detailed measurements of $\bar{\nu}_{\mu}$ CCQE scattering is the main focus of this dissertation. Therefore, any dependence of the background $\nu_{\mu}$ estimation on assumptions of $\bar{\nu}_{\mu} \mathrm{CCQE}$ must be strictly avoided. For this reason, the results of this analysis are not used to subtract the $\nu_{\mu}$ background to the $\bar{\nu}_{\mu}$ CCQE sample. However, in the future, when the processes contributing to samples like these are better understood, this technique could prove to be powerful.

As mentioned in Section 7.1.3, the data used in this analysis does include the small absorber-down antineutrino-mode runs.

\section{Sample Selection}

The only difference between the CCQE sample selected here and that used in the main analysis of $\bar{\nu}_{\mu}$ CCQE interactions (detailed in Section 6.2) is the replacement of the $\mu$ range-based cut with the requirement that the reconstructed vertex of the second subevent be within a $100 \mathrm{~cm}$ radius of the predicted $\mu$ stopping point. The radius between the predicted muon $\mu$ stopping point and the Michel vertex is hereafter referred to as the "Michel distance."

To directly see the difference between these cuts, the slope of the range cut versus

the Michel distance can be examined. The slope of the range cut is $\frac{\text { Range }+100 \mathrm{~cm}}{T_{\mu}}(\mathrm{cm} / \mathrm{GeV})$, and $500 \mathrm{~cm} / \mathrm{GeV}$ is the cut used in the neutrino CCQE analysis. The $500 \mathrm{~cm} / \mathrm{GeV}$ cut is simply the inverse of the standard MIP energy loss $2 \mathrm{MeV} / \mathrm{cm}$. The range cut slope versus Michel distance for is plotted for data, MC, and the significant interaction channels in Figure 7-14. Note the effect of the $100 \mathrm{~cm} \mu$ range cut (cut 8 in Section 6.2) is not included in this comparison. It can be seen that the $\mu$ range-based cut keeps more signal events while rejecting around the same amount of background. However, either choice of spatial correlation requirement between the muon and its decay electron result in mostly the same 
purity and efficiency for $\nu_{\mu}$ and $\bar{\nu}_{\mu}$ events.

\section{Measurement Execution}

To measure the neutrino content using the muon angular distribution, the MC sample is separated into two $\cos \theta_{\mu}$ templates, one arising from all $\nu_{\mu}$ interactions and the other from $\bar{\nu}_{\mu}$, regardless of interaction channel. A linear combination of these two templates is then formed,

$$
T_{M C}\left(\alpha_{\nu}, \alpha_{\bar{\nu}}\right) \equiv \alpha_{\nu} \nu^{M C}+\alpha_{\bar{\nu}} \bar{\nu}^{M C}
$$

where $T_{M C}$ is the total predicted $\cos \theta_{\mu}$ distribution to be fit to data, $\alpha_{\nu}$ and $\alpha_{\bar{\nu}}$ are neutrino and antineutrino rate scales, and $\nu^{M C}$ and $\bar{\nu}^{M C}$ are the MC neutrino and antineutrino scattering angular predictions, respectively.

Many backgrounds to the CCQE sample peak in the most forward scattering region of the $\cos \theta_{\mu}$ distribution. This includes pion production and hydrogen CCQE scattering while the latter is technically not a background, the proper handling of the difference in nuclear effects between bound and free targets is not straightforward. Additionally, the forward scattering region is dominated by $\bar{\nu}_{\mu}$ interactions, while the present analysis is principally interested in $\nu_{\mu}$-dominated backwards scattering region. For these reasons, events with $\cos \theta_{\mu}>0.91$ are not included in the fit to data, where $\theta_{\mu}$ is the outgoing muon angle relative to the incoming neutrino beam.

Ignoring this forward-scattering region, the modified simulation sample in Eq. 7.20 is compared to data by forming a goodness-of-fit $\chi^{2}$ test as a function of the rate scales:

$$
\chi^{2}\left(\alpha_{\nu}, \alpha_{\bar{\nu}}\right)=\sum_{i, j}\left(T_{M C}\left(\alpha_{\nu}, \alpha_{\bar{\nu}}\right)_{i}-d_{i}\right) M_{i j, \mathrm{FIT}}^{-1}\left(T_{M C}\left(\alpha_{\nu}, \alpha_{\bar{\nu}}\right)_{j}-d_{j}\right)
$$

where $i$ and $j$ label bins of $\cos \theta_{\mu}, d$ is data and $M_{\mathrm{FIT}}$ is the covariance matrix described in the next section. 

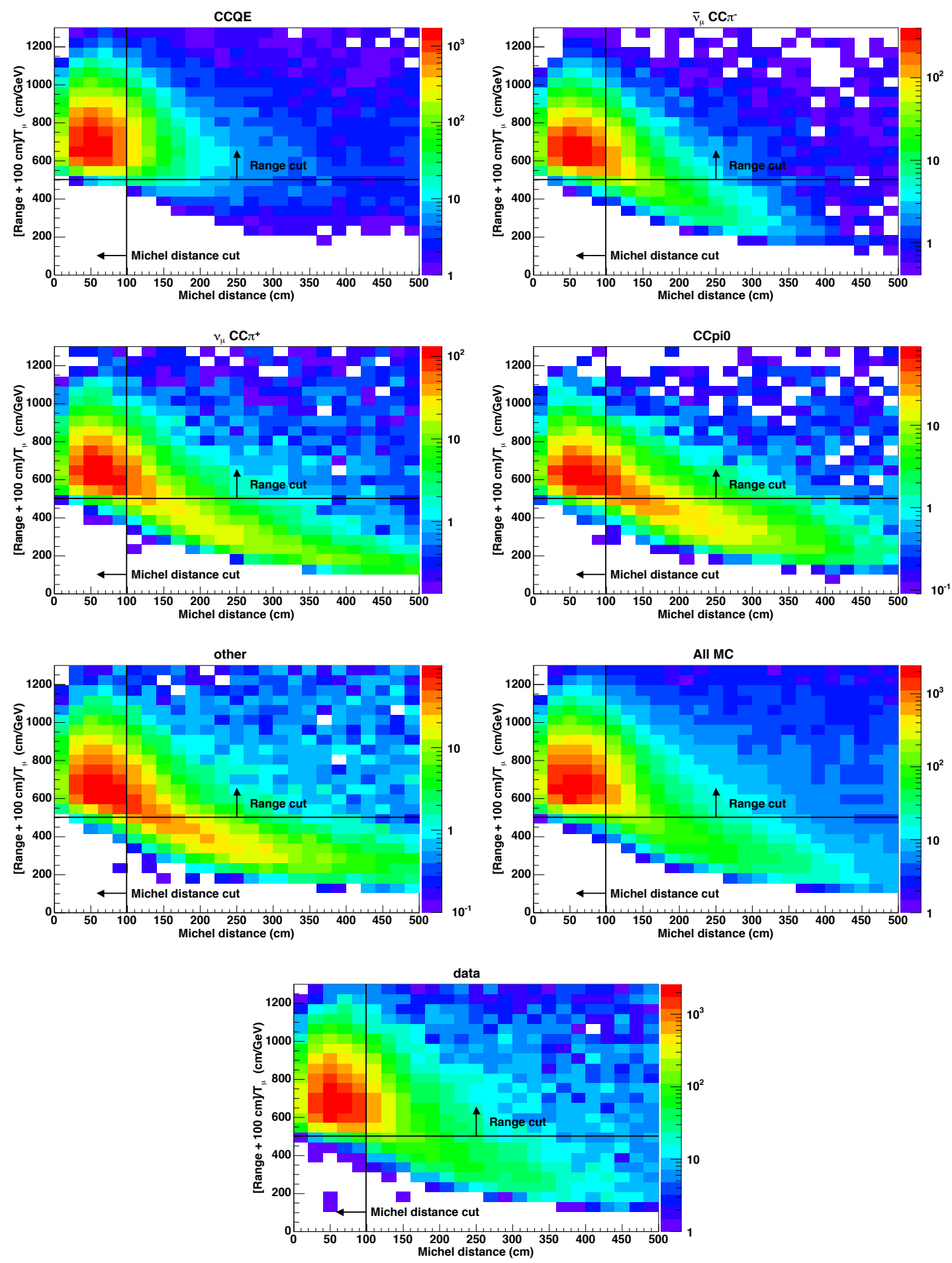

Figure 7-14. Michel distance versus the range slope as described in the text. Distributions are absolutely normalized. 
The linearity of this fit allows for an analytic solution. The scales $\alpha_{\nu}$ and $\alpha_{\bar{\nu}}$ describe the data best when the $\chi^{2}$ function in Eq. 7.21 is minimized simultaneously with respect to both parameters:

$$
\frac{\partial \chi^{2}}{\partial \alpha_{\nu}}=\frac{\partial \chi^{2}}{\partial \alpha_{\bar{\nu}}}=0 .
$$

By exploiting the symmetric nature of the error matrix, we can simplify and arrive at a unique solution for the fit parameters $\alpha_{\nu}$ and $\alpha_{\bar{\nu}}$ in terms of the data $d$ and MC distributions of $\nu_{\mu}\left(" \nu^{M C} "\right)$ and $\bar{\nu}_{\mu}\left(" \bar{\nu}^{M C "}\right)$ :

$$
\left[\begin{array}{c}
\alpha_{\bar{\nu}}^{\mathrm{BF}} \\
\alpha_{\nu}^{\mathrm{BF}}
\end{array}\right]=\left[\begin{array}{ll}
\sum_{i, j} \bar{\nu}_{i}^{M C} \bar{\nu}_{j}^{M C} M_{i j, \mathrm{FIT}}^{-1} & \sum_{i, j} \bar{\nu}_{i}^{M C} \nu_{j}^{M C} M_{i j, \mathrm{FIT}}^{-1} \\
\sum_{i, j} \nu_{i}^{M C} \bar{\nu}_{j}^{M C} M_{i j, \mathrm{FIT}}^{-1} & \sum_{i, j} \nu_{i}^{M C} \nu_{j}^{M C} M_{i j, \mathrm{FIT}}^{-1}
\end{array}\right]^{-1}\left[\begin{array}{l}
\sum_{i, j} \bar{\nu}_{i}^{M C} d_{j} M_{i j, \mathrm{FIT}}^{-1} \\
\sum_{i, j} \nu_{i}^{M C} d_{j} M_{i j, \mathrm{FIT}}^{-1}
\end{array}\right],
$$

where $\alpha_{\nu}^{\mathrm{BF}}$ and $\alpha_{\bar{\nu}}^{\mathrm{BF}}$ are the best-fit scales for the neutrino and antineutrino distributions, respectively. The uncertainty on $\alpha_{\nu}$ and $\alpha_{\bar{\nu}}$ is determined by the projections of the $\chi^{2}$ function (Eq. 7.21) for each parameter while holding the other fixed at its best-fit value. The uncertainty on the parameters is:

$$
\begin{aligned}
\delta \alpha_{\nu} & =\left|\alpha_{\nu}^{\mathrm{BF}}-\alpha_{\nu}\left[\chi^{2}\left(\alpha_{\nu}^{\mathrm{BF}}, \alpha_{\bar{\nu}}^{\mathrm{BF}}\right) \pm \Delta \chi^{2}\right]\right| \\
\delta \alpha_{\bar{\nu}} & =\left|\alpha_{\bar{\nu}}^{\mathrm{BF}}-\alpha_{\bar{\nu}}\left[\chi^{2}\left(\alpha_{\nu}^{\mathrm{BF}}, \alpha_{\bar{\nu}}^{\mathrm{BF}}\right) \pm \Delta \chi^{2}\right]\right|,
\end{aligned}
$$

where $\Delta \chi^{2}$ for the $68 \%$ C.L. in a two-parameter fit is 2.30 [158]. Note the uncertainties symmetric in the fit parameters assumed by Eqs. 7.24 and 7.25 are not general, and is the case here due to the linearity of the fit. 


\section{The Covariance Matrix}

The covariance matrix is used to propagate correlated uncertainties on parameters and processes to the quantities reported in the analysis while accounting for correlations between $\nu_{\mu}$ and $\bar{\nu}_{\mu}$ events. It is made by first forming weights corresponding to simulation excursions set by Gaussian variations of parameters within their associated error. The difference of these weighted events from the simulated central value forms the error matrix. Correlations between $\nu_{\mu}$ and $\bar{\nu}_{\mu}$ are not considered in the generation of these excursions, and so must be explicitly addressed in this analysis.

The $\cos \theta_{\mu}$ correlations between $\nu_{\mu}$ and $\bar{\nu}_{\mu}$ are treated by first expanding the distributions input to the covariance matrix to include both $\nu_{\mu}$ and $\bar{\nu}_{\mu} \cos \theta_{\mu}$ templates, side-by-side. An example of the central value distribution and 100 instances of cross section uncertainties related to the various contributing interactions is shown in Figure 7-15.

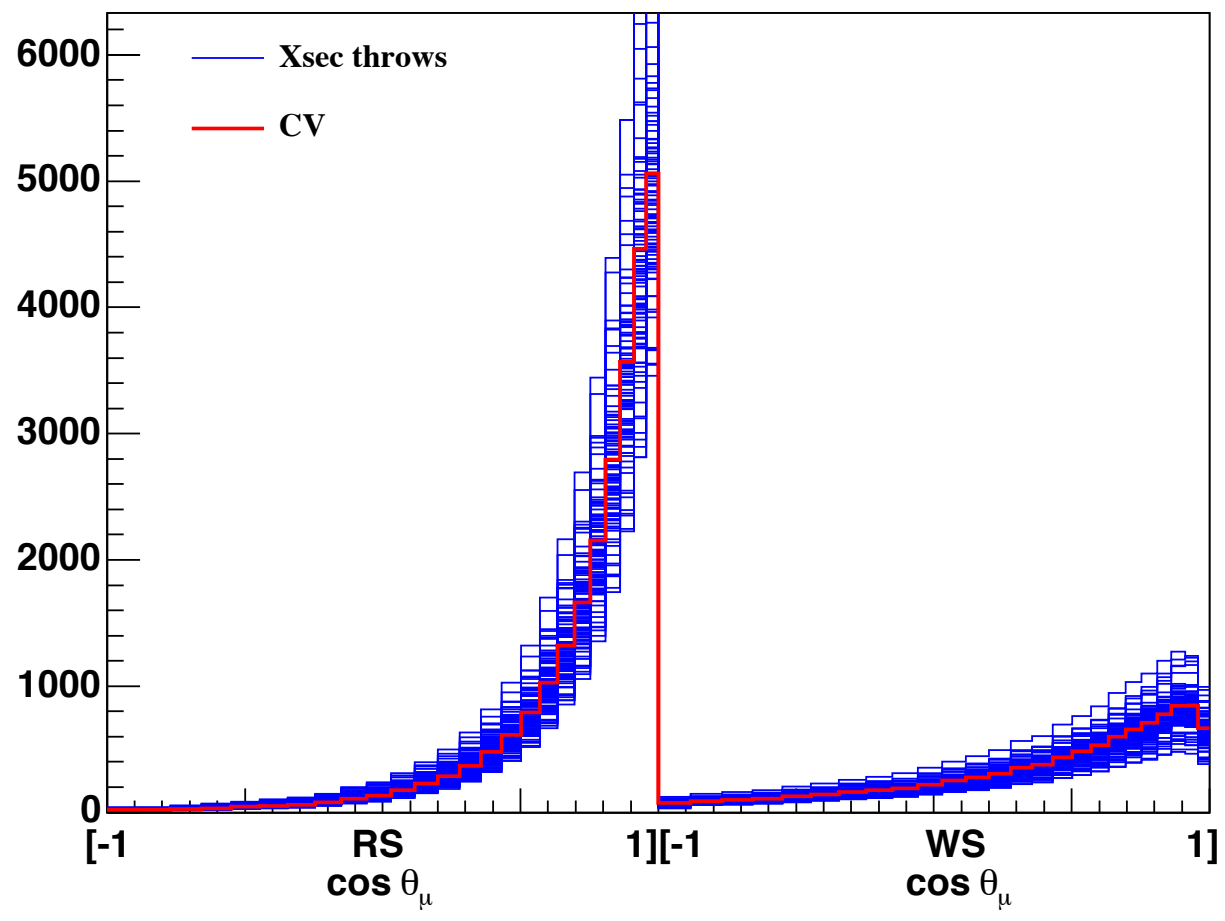

Figure 7-15. Central-value (CV) $\cos \theta_{\mu}$ prediction versus 100 distributions created by cross section throws for right-sign (RS) $\bar{\nu}_{\mu}$ and wrong-sign (WS) $\nu_{\mu}$ events. 
Using these distributions, the covariance matrix is calculated as:

$$
M_{i j}=\frac{1}{K} \sum_{s=1}^{K}\left(N_{i}^{s}-N_{i}^{C V}\right) \times\left(N_{j}^{s}-N_{j}^{C V}\right)=\rho_{i j} \sigma_{i} \sigma_{j}
$$

where $K$ simulation excursions are used ( $K=100$ in this analysis), $N^{s}$ is the re-weighted number of entries corresponding to the $s^{t h}$ simulation set and $N^{C V}$ represents the simulation central value. The total uncertainty in each bin $i$ is $\sigma_{i}$, and the correlation between bins $i$ and $j$ is given by $\rho_{i j}$. In this analysis, for uncertainties on processes with correlated errors, $K=100$ while $K=1$ is sufficient for uncorrelated errors. This technique is further described in Ref. [159]. Systematic uncertainties requiring correlated errors include the production of $\pi^{-}$in the proton beam target, the connection between $\pi^{-}$ production and the focused $\bar{\nu}_{\mu}$ beam, optical transport in the detector, and final-state interactions.

With the $\nu_{\mu}$ and $\bar{\nu}_{\mu}$ input distributions separated as in Figure 7-15, this matrix contains $\nu_{\mu^{-}}$and $\bar{\nu}_{\mu}$-only covariance information on the block-diagonals, while the off-diagonal pieces contain the level of correlation between $\nu_{\mu}$ and $\bar{\nu}_{\mu}$ events. A simple rearranging of Eq. 7.26 gives the correlation values:

$$
\rho_{i j}=M_{i j} /\left(\sigma_{i} \sigma_{j}\right)
$$

where the individual bin uncertainties $\sigma_{i}$ are trivially found from $M_{i i}=\sigma_{i}^{2}$, since $\rho_{i i}=1$. Figure 7-16 shows the level of correlation between all bins in the $\nu_{\mu}$ and $\bar{\nu}_{\mu} \cos \theta_{\mu}$ distributions. It will be shown that the overall positive correlation is mostly due to the dominant uncertainties related to highly-correlated $\nu_{\mu}$ and $\bar{\nu}_{\mu}$ cross sections.

To use the covariance matrix in the context of a fit, its size must first be reduced to the dimension of a single $\cos \theta_{\mu}$ distribution. Since the total sample $T_{M C}$ in each bin $i$ is a simple sum of $\nu_{\mu}$ and $\bar{\nu}_{\mu}$ events, 


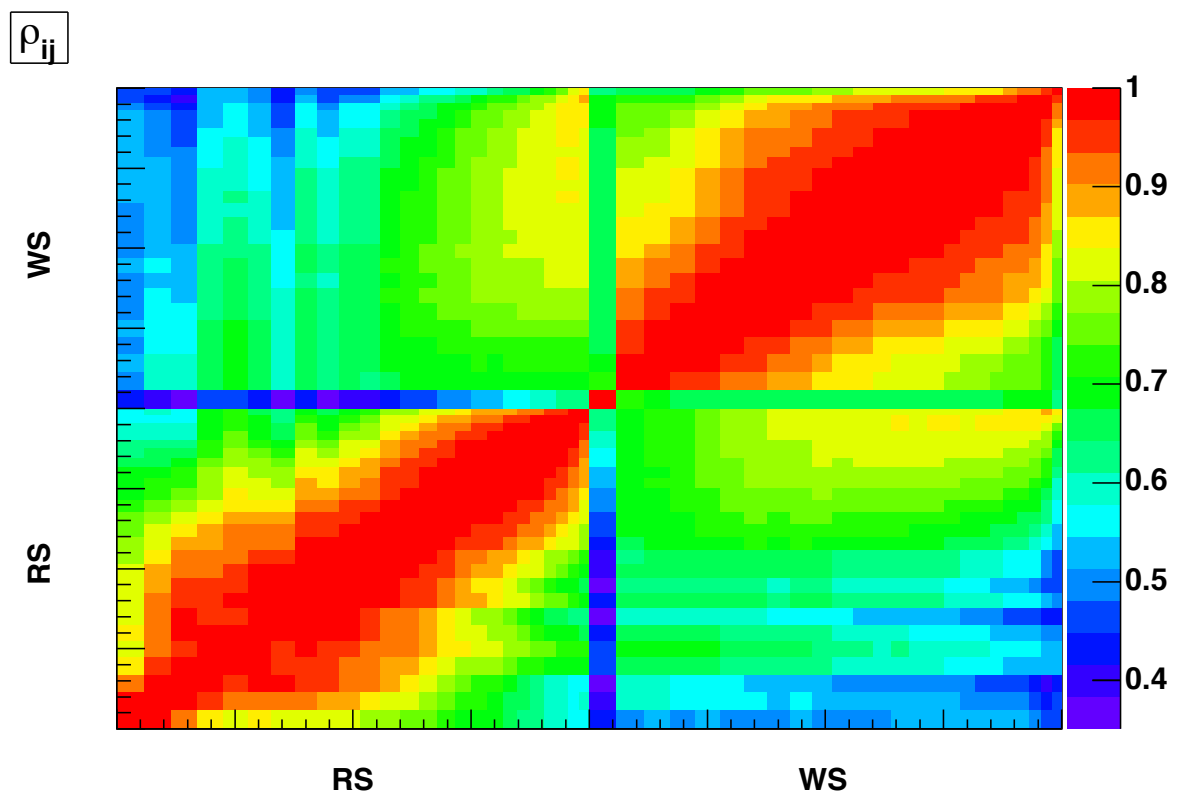

Figure 7-16. Correlation matrix for $\nu_{\mu}$ and $\bar{\nu}_{\mu}$ events in the $\cos \theta_{\mu}$ distribution. From left to right and bottom to top, the domain is $[-1,1]$ for the RS and WS distributions. The block-diagonals give bin correlation information between the $\nu_{\mu}$ events (top right) and $\bar{\nu}_{\mu}$ (bottom left) events, while the off-diagonals contain the $\nu_{\mu}-\bar{\nu}_{\mu}$ correlations. All bins are positively correlated to each other due to the dominant cross-section uncertainties, many of which affect the generation of $\nu_{\mu}$ and $\bar{\nu}_{\mu}$ events in the same way.

$$
T_{i, M C}(1,1)=\nu_{i}^{M C}+\bar{\nu}_{i}^{M C}
$$

Using $j$ for another arbitrary $\cos \theta_{\mu}$ bin, the covariance for this distribution is:

$$
\begin{aligned}
\sigma_{T_{i, M C}} \sigma_{T_{j, M C}} \rho_{\left(T_{i, M C}-T_{j, M C}\right)}= & \sigma_{\nu_{i}^{M C}} \sigma_{\nu_{j}^{M C}} \rho_{\left(\nu_{i}^{M C}-\nu_{j}^{M C}\right)}+\sigma_{\bar{\nu}_{i}^{M C}} \sigma_{\bar{\nu}_{j}^{M C}} \rho_{\left(\bar{\nu}_{i}^{M C}-\bar{\nu}_{j}^{M C}\right)} \\
& \left.+2 \sigma_{\nu_{i}^{M C}} \sigma_{\bar{\nu}_{j}^{M C}} \rho_{\left(\nu_{i}^{M C}-\bar{\nu}_{j}^{M C}\right.}\right)
\end{aligned}
$$

The terms on the right side of Eq. 7.29 can be recognized as entries of the full covariance matrix in Eq. 7.26. Finally, if the dimension of a single $\cos \theta_{\mu}$ distribution is $N_{d}$, 
Table 7-13. Summary of systematic error contribution to the scale parameter $\alpha_{\nu}$ in the inclusive energy fit. Individual error contributions are found for the $i$ th systematic error by first repeating the fits with only independent systematics considered. The fractional error contributions are then found by $\sqrt{\left(\Delta \alpha_{\nu} / \alpha_{\nu}\right)_{\text {syst }}^{2}+\text { stat }}-\left(\Delta \alpha_{\nu} / \alpha_{\nu}\right)_{\text {stat }}^{2}$, where $\Delta \alpha_{\nu}$ is the onesigma error reported in Table 7-15. The statistical error is found by considering the second term only. This method does not account for small changes in the $\alpha_{\nu}$ best fit parameter between the fits considering various errors, and so the individual fractional errors do not add in quadrature to produce the total fractional error reported in Table 7-15 and in the final column.

\begin{tabular}{cc}
\hline Source of Error & Fractional Uncertainty (\%) \\
\hline Statistical & 8 \\
Detector Modeling & 11 \\
$\mathrm{CC} \pi^{+}$Constraint & 4 \\
Cross Section & 26 \\
Total Fractional Error & 35 \\
\hline
\end{tabular}

the final error matrix to be used in this analysis is:

$$
M_{i, j}^{\mathrm{FIT}}=M_{i, j}+M_{i+N_{d}, j}+M_{i, j+N_{d}}+M_{i+N_{d}, j+N_{d}}
$$

where $i$ and $j \in\left[0, N_{d}\right]$.

\section{Results and Systematic Errors}

As the present analysis directly measures the neutrino component in the antineutrino-mode beam, systematic errors relating to beam geometry and meson production at the target are not considered. The remaining systematic errors include those arising from detector modeling, the single pion production background, and the cross section parameters in the underlying CCQE model. Contributions propagated from these errors to the uncertainty on the parameter $\alpha_{\nu}$ in the inclusive energy sample are given in Table 7-13.

Apart from final-state interaction uncertainties leading to errors on the cross section, the error on the $\mathrm{CC} \pi^{+}$background contributes to the systematic error through the 
Table 7-14. Summary of cross-section errors used in this analysis. The bottom portion presents fractional uncertainties assigned to processes in addition to parameter errors. Errors given on pion absorption and charge exchange are relevant to pion propagation in the detector medium.

\begin{tabular}{|c|c|}
\hline Parameter & Value with Error \\
\hline$M_{A}^{C}$ (carbon target) & $1.35 \pm 0.07 \mathrm{GeV}$ \\
\hline$M_{A}^{H}$ (hydrogen target) & $1.03 \pm 0.02 \mathrm{GeV}$ \\
\hline$\kappa$ & $1.007 \pm 0.005$ \\
\hline$E_{B}$ & $34 \pm 9 \mathrm{MeV}$ \\
\hline$\Delta \mathrm{s}$ & $0.0 \pm 0.1$ \\
\hline $\mathrm{M}_{A}^{1 \pi}$ & $1.10 \pm 0.28 \mathrm{GeV}$ \\
\hline $\mathrm{M}_{A}^{\mathrm{multi}-\pi}$ & $1.30 \pm 0.52 \mathrm{GeV}$ \\
\hline $\mathrm{p}_{F}$ & $220 \pm 30 \mathrm{MeV}$ \\
\hline Process & $\begin{array}{c}\text { Fractional } \\
\text { Uncertainty }(\%)\end{array}$ \\
\hline$\pi^{+}$Charge Exchange & 50 \\
\hline$\pi^{+}$Absorption & 35 \\
\hline CCQE $\sigma$ Normalization & 10 \\
\hline All $\bar{\nu}_{\mu} \sigma$ Normalization & 10 \\
\hline$\Delta+\mathrm{N} \rightarrow \mathrm{N}+\mathrm{N}$ & 100 \\
\hline
\end{tabular}

error labeled "CC $\pi^{+}$Constraint" in Table 7-13. This measurement uncertainty is based on a $Q^{2}$-dependent shape-only scale factor to improve data-simulation agreement in the neutrino-mode $\mathrm{CC} \pi^{+}$sample [160]. The cross section (both CCQE and $\mathrm{CC} \pi^{+}$) uncertainty is dominant in these fits and warrants further discussion. Table 7-14 offers a breakdown of cross section parameters and associated errors. The error on carbon $M_{A}^{C}$ has been reduced from that reported in Ref. [84] to avoid double-counting MiniBooNE systematic errors applicable to both the measurement of $M_{A}$ and the measurement reported here. The $26 \%$ uncertainty due to cross-section errors reported in Table 7-13 can be expanded as the quadrature sum of $16 \%$ from the $10 \%$ normalization errors on $\bar{\nu}_{\mu}$ and CCQE processes, $14 \%$ from the error on $\mathrm{M}_{A}$ and $\kappa$, and $15 \%$ from the remaining processes.

The fit is performed analytically in three bins of reconstructed energy and also in an inclusive energy sample. Results including statistical and systematic uncertainties are presented in Table 7-15, and the fits to data are shown in Figure 7-17. As the main 
contributions to the dominant cross section systematic error apply to both $\nu_{\mu}$ and $\bar{\nu}_{\mu}$ scattering, $\alpha_{\nu}$ and $\alpha_{\bar{\nu}}$ are positively correlated as reported in Table $7-15$. The adjusted contributions of $\nu_{\mu}$ and $\bar{\nu}_{\mu}$ to the CCQE sample are compared to the prediction in Table 7-16.

The $\chi^{2}$ value for the angular fit in the reconstructed energy range $E_{\nu}^{\mathrm{QE}}>900 \mathrm{MeV}$ is unusually low at $\chi^{2}=7$ for 21 degrees of freedom. This is likely to be simply due to chance, as the statistical error only fit agrees with the data exceptionally well within the error, returning $\chi^{2}=13$ for 21 degrees of freedom.

As the $\nu_{\mu}$ angular template has been corrected for the observed cross section per Ref. [84], $\alpha_{\nu}$ may be interpreted as a flux scale factor, and significant deviations from unity would imply a flux mismodeling. Consistent with the results reported in the previous sections using $\mathrm{CC} \pi^{+}$and $\mu^{-}$capture events, fits in the antineutrino-mode CCQE sample indicate the true neutrino flux to be somewhat lower than the simulation predicts. Over all reconstructed energies, the neutrino flux component of the antineutrino-mode beam should be scaled by 0.65 to match the observed data. Fits in individual reconstructed energy bins indicate that the neutrino flux component shape is well-modeled. The rate scale $\alpha_{\bar{\nu}}$ is ambiguous in interpretation, as the cross section is yet unmeasured.

The results from this technique depend on knowing the angular distributions of neutrino and antineutrino CCQE interactions in the detector. While the procedure relies on exploiting the effect of the interference term in the CCQE cross section, the angular distributions may be somewhat altered by nuclear effects. In this analysis the measured angular distribution of neutrino interactions on carbon [84] is employed, but the measurement relies on the scattering model to predict antineutrino interactions. This model does not include two-body current effects which may be larger than previously expected [161, 93, 95, 98, 99, 100] and may introduce additional neutrino and antineutrino angular differences. Despite this inherent model dependence, the results present a demonstration of a technique aimed at informing future experiments looking to separately 
constrain neutrino and antineutrino events in an unmagnetized environment. By that time, the effect of additional nuclear processes on the angular dependence of antineutrino CCQE scattering should be better known. 

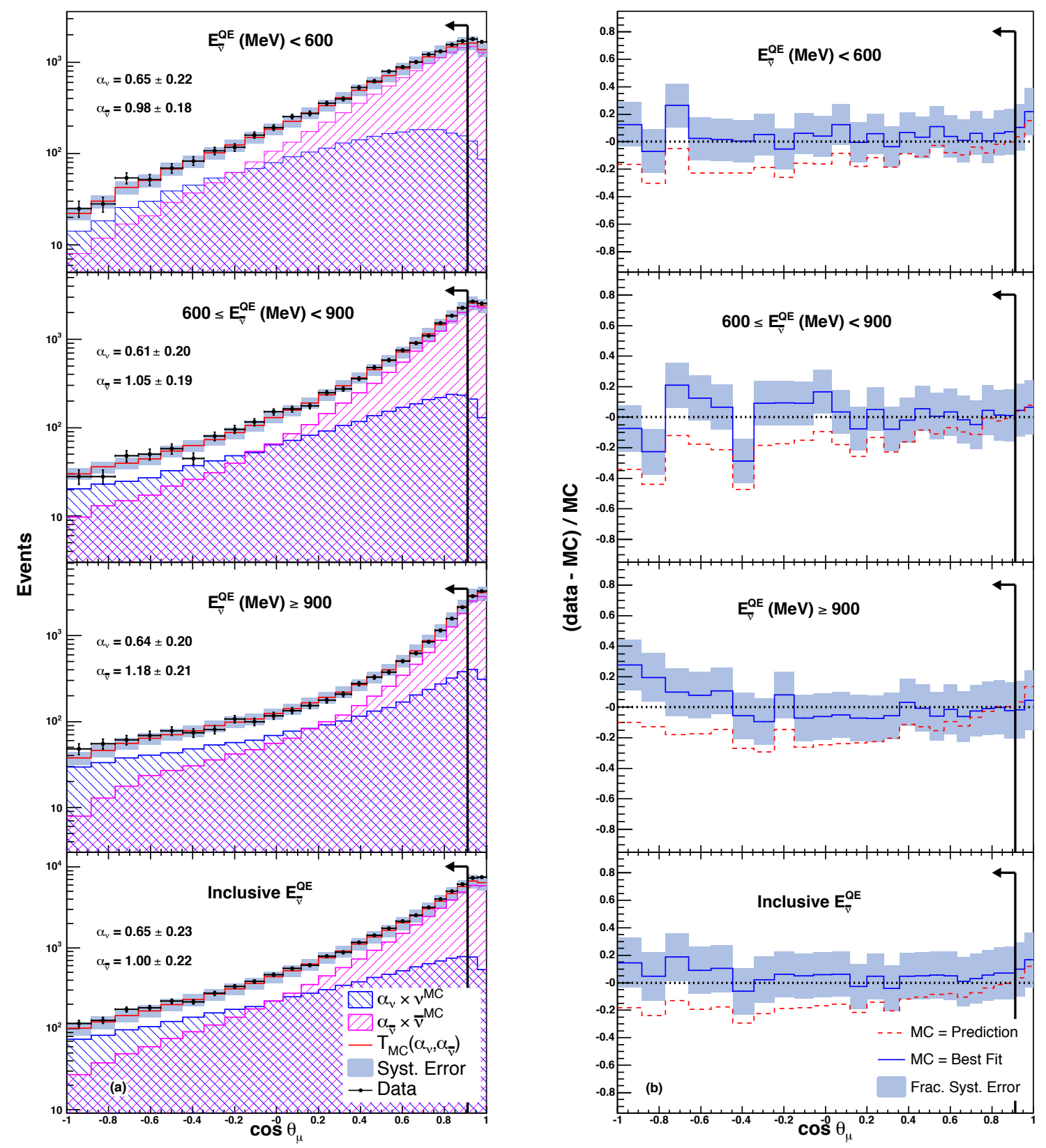

Figure 7-17. Results of the muon angular fits to the CCQE data. Shown are (a) the fits and (b) fractional differences (data - simulation) / simulation for both the unmodified prediction and the best fit. Along with an inclusive sample, three reconstructed energy bins are considered. The before-fit simulation is absolutely normalized to $5.66 \times 10^{20}$ protons on target. As indicated, only events with $\cos \theta_{\mu}<0.91$ participate in the fit. 


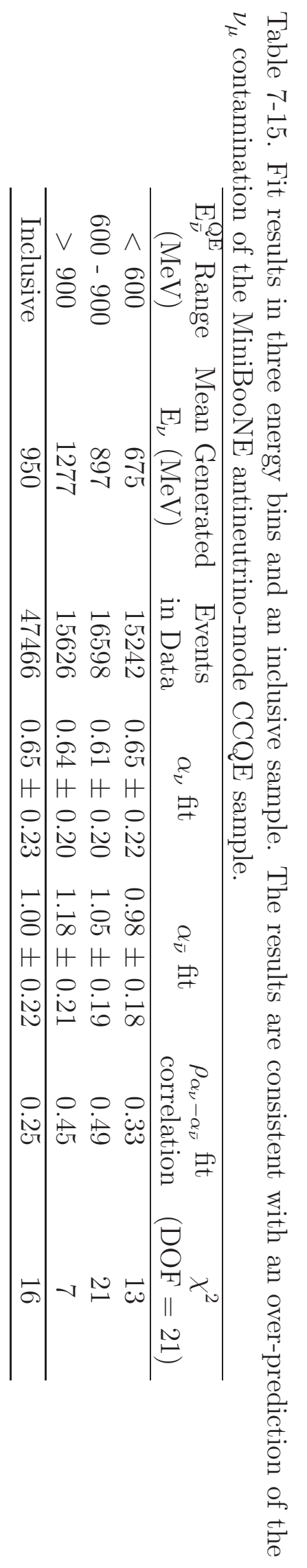


Table 7-16. Fractional composition of the antineutrino-mode CCQE sample before and after angular fits.

\begin{tabular}{ccccc}
\hline $\mathrm{E}_{\bar{\nu}}^{\mathrm{QE}}$ Range & \multicolumn{2}{c}{ Before Fit (\%) } & \multicolumn{2}{c}{ After Fit (\%) } \\
$(\mathrm{MeV})$ & $\nu_{\mu}$ & $\bar{\nu}_{\mu}$ & $\nu_{\mu}$ & $\bar{\nu}_{\mu}$ \\
\hline$<600$ & 25 & 75 & $18 \pm 6$ & $82 \pm 16$ \\
$600-900$ & 26 & 74 & $17 \pm 6$ & $83 \pm 15$ \\
$>900$ & 35 & 65 & $23 \pm 7$ & $77 \pm 15$ \\
\hline Inclusive & 29 & 71 & $21 \pm 8$ & $79 \pm 18$ \\
\hline
\end{tabular}

\subsubsection{Summary of $\nu_{\mu}$ Flux Measurements}

The results from Sections 7.1.3 - 7.1.5 provide the first demonstration of a set of statistical techniques used to measure the $\nu_{\mu}$ component of an antineutrino-mode beam. Their results are summarized in Figure 7-18, where measurements performed in exclusive reconstructed neutrino energy bins are given as a function of the mean generated neutrino energy for that region.

Results from all three measurements indicate the normalization of the nominal $\nu_{\mu}$ flux prediction using a Sanford-Wang-based [120] extrapolation of the HARP data (discussed in Section 5.2.4) requires a uniform reduction of 20-30\%. This indicates the simulated shape of the flux spectrum appears to be adequate. Interesting to note, given the results in Figure 7-18 along with the comparison of two possible $\pi^{-}$extrapolations into the low-angle region shown in Figure 5-7, the spline-based prediction appears to more accurately describe the data.

It is helpful to mention again that the analysis of the $\cos \theta_{\mu}$ distribution is somewhat dependent on the model for $\bar{\nu}_{\mu}$ CCQE interactions assumed by the simulation, and so its results are not used in the background subtraction of $\nu_{\mu}$ events from the $\bar{\nu}_{\mu}$ CCQE sample. Conversely, it has been shown that the analyses based on $\mathrm{CC} \pi^{+}$and $\mu^{-}$ capture events are almost entirely free from model dependence. Moreover, that the analyzed samples are dominated by different physics processes indicates a level of independence between the two measurements. The results of these two analyses can 
therefore be used to find a combined measurement of the $\nu_{\mu}$ flux in the antineutrino-mode beam featuring a reduced uncertainty compared to either measurement alone. For two measurements $x_{1}, x_{2}$, along with their associated uncertainties $\sigma_{1}, \sigma_{2}$ and correlation $\rho$, the combined measurement and uncertainty can be expressed as [162]:

$$
\begin{aligned}
\langle x\rangle & =x_{1}+\frac{1-a \rho}{1-2 a \rho+a^{2}}\left(x_{2}-x_{1}\right) \\
\langle\sigma\rangle^{2} & =\frac{\left(1-\rho^{2}\right) a^{2} \sigma_{1}^{2}}{1-2 a \rho+a^{2}}
\end{aligned}
$$

where $a=\sigma_{2} / \sigma_{1}$ and $\sigma_{2} \geq \sigma_{1}$. Consistency of the $\mu^{-}$capture and $\mathrm{CC} \pi^{+}$measurements across the observed energy range (Figure 7-18) indicates the simulated $\nu_{\mu}$ flux shape to be well-modeled, and so a combined measurement applied universally to the $\nu_{\mu}$ background events is adequate. The measurement from the $\mu^{-}$capture measurement gives $0.78 \pm 0.12$, while the $\mathrm{CC} \pi^{+}$measurement yields $0.76 \pm 0.11$. The uncertainty in the $\mu^{-}$capture measurement is in roughly equal parts due to statistics and the neutrino-mode flux errors, while the error in the $\mathrm{CC} \pi^{+}$measurement is dominated by the neutrino-mode flux uncertainty. Based on this, the correlation coefficient $\rho$ is estimated to be 0.5 . With these values implemented into Eqs. 7.31 and 7.32, the combined measurement of the $\nu_{\mu}$ flux in the antineutrino-mode beam is $0.77 \pm 0.10$ relative to the extrapolated and highly-uncertain prediction. This will be the data-based constraint of the $\nu_{\mu}$ uncertainty assumed in the background subtraction process in finding the $\bar{\nu}_{\mu}$ CCQE cross sections presented in Chapter 8. Notice the level of knowledge necessary for background subtraction is how many events are present in the analysis sample, which is directly measured through the $\mu^{-}$capture-based measurement. Therefore, using the uncertainty on the $\nu_{\mu}$ flux results in a mild overestimate of the uncertainty of the $\nu_{\mu}$ background. 


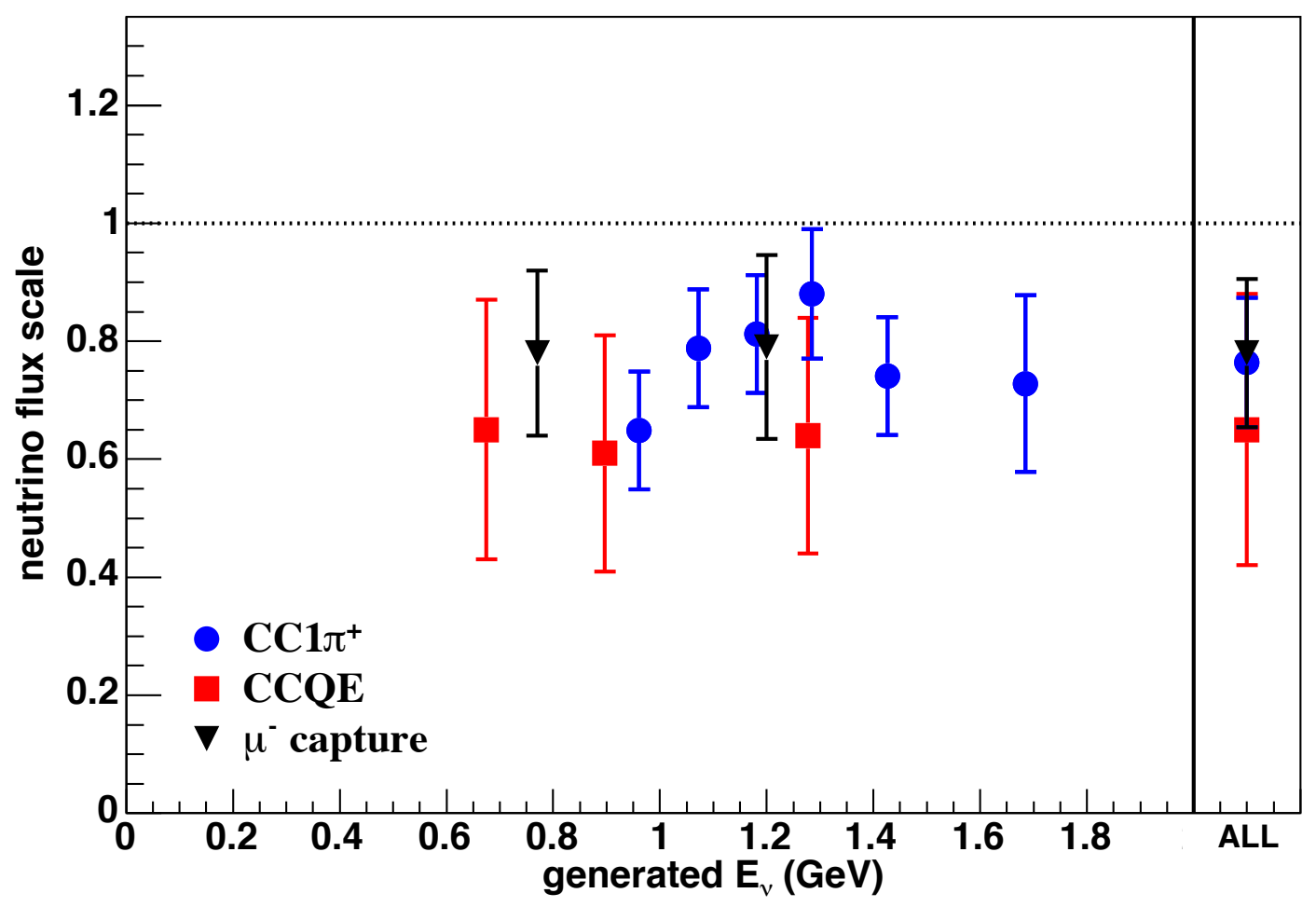

Figure 7-18. Summary of the results from three techniques used to measure the $\nu_{\mu}$ flux in the antineutrino-mode beam. Measurements performed in exclusive regions of reconstructed energy are placed here at the mean of their associated distribution of generated energy. Shown as a dotted line at unity, the measurements are made relative to an extrapolation of HARP data into a region where no relevant hadroproduction data exists. 


\subsection{The $\bar{\nu}_{\mu} \mathrm{CC} \pi^{-}$Background}

While the high rate of stopped- $\pi^{-}$nuclear capture allows for the powerful measurement of the $\nu_{\mu}$ flux using $\mathrm{CC} \pi^{+}$events presented in Section 7.1.3, $\mathrm{CC} \pi^{-}$events migrate into the $\bar{\nu}_{\mu}$ CCQE sample and form an irreducible background to the main analysis of this dissertation. Following the discussion of $\bar{\nu}_{\mu}$ CCQE selection efficacy given in Section 6.2, Table 6-2 reports that these $\mathrm{CC} \pi^{-}$events are predicted to account for $\sim 15 \%$ of the sample. Considering the level of agreement between various calculations and the experimental data for single-pion interactions discussed in Section 4.3, constraints and uncertainties for $\mathrm{CC} \pi^{-}$events are based entirely on direct comparisons with MiniBooNE data.

An indirect constraint of $\mathrm{CC} \pi^{-}$events is obtained through an extrapolation of a MiniBooNE CC $\pi^{+}$-based measurement, and a discussion of the origin of this correction is warranted. As suggested in Section 7.1.3, single-pion events induced by $\nu_{\mu}$ typically give rise to Michel electrons through the decay chain $\pi^{+} \rightarrow \mu^{+} \rightarrow e^{+}$of stopped pions, which can be observed and used to reject these events. However, an appreciable number of $\pi^{+}$are destroyed in flight through the nuclear absorption process $\left(\pi^{+}+X \rightarrow X^{\prime}\right)$ and therefore formed a significant background to the neutrino-mode $\nu_{\mu}$ CCQE sample. Measurements of $\mathrm{CC} \pi^{+}$events tagged through the observation of an additional Michel allowed a direct constraint of the rate and kinematics of the $\mathrm{CC} \pi^{+}$background to the $\nu_{\mu} \mathrm{CCQE}$ analysis.

Figure 7-19 shows the MiniBooNE neutrino-mode $\mathrm{CC} \pi^{+}$data, prediction, and the obtained constraint. To guarantee the selected sample probes the same kinematics of $\mathrm{CC} \pi^{+}$events that enter as backgrounds to the CCQE selection, sample formation was identical to that for CCQE described in Section 6.2, with the replacement of the two subevent requirement with three subevents. Along the same lines, the measurement is based on $Q_{Q E}^{2}$ (Eq. 6.8 with $n \leftrightarrow p$, appropriate to $\nu_{\mu}$ CCQE scattering), which assumes the underlying interaction to be CCQE. Clearly this assumption is incorrect for this

physics sample, and so the comparison in Figure 7-19 cannot be rigorously used to identify 

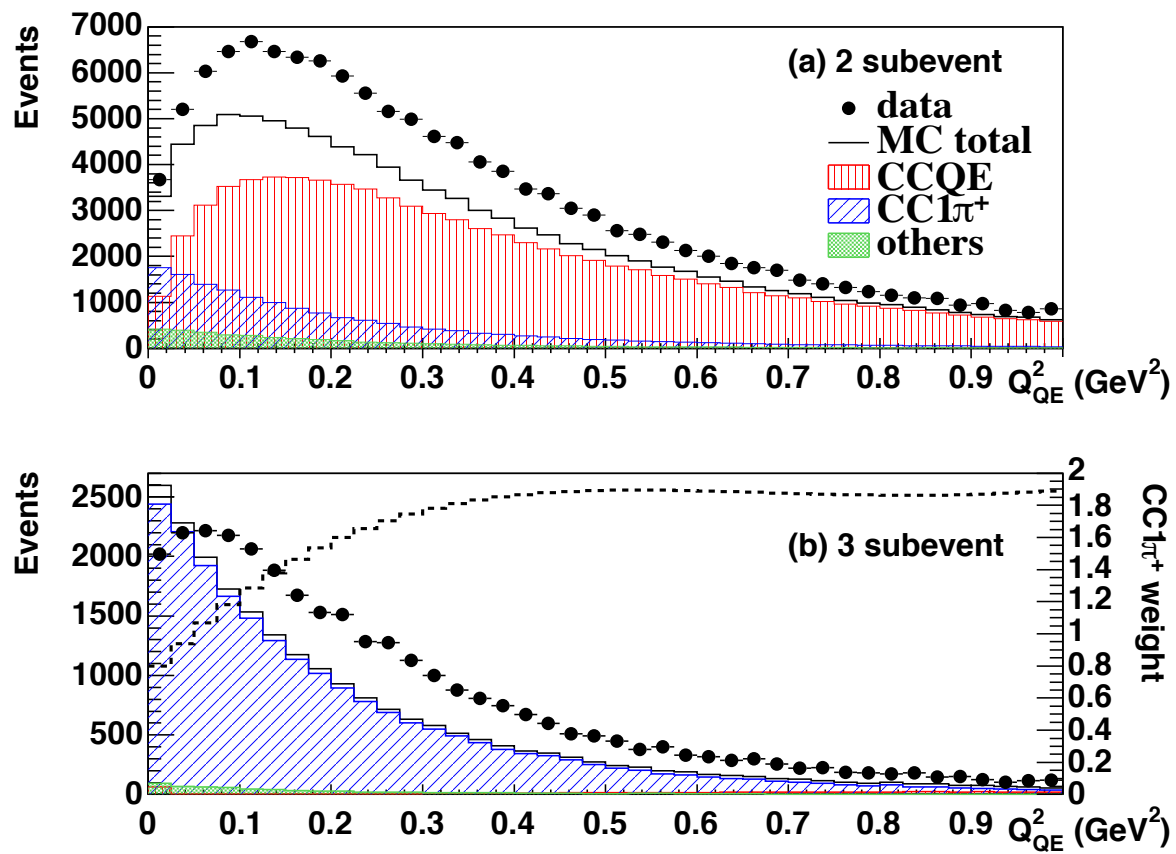

Figure 7-19. Summary of the MiniBooNE $\mathrm{CC} \pi^{+}$analysis for the background measurement to the $\nu_{\mu}$ CCQE sample. Shown is (a) the neutrino-mode CCQE sample and (b) the CC $\pi^{+}$ sample before the application of constraints and parameter fits. The dashed line in (b) shows the ratio of prediction to data in the $\mathrm{CC} \pi^{+}$sample, and its value is given by the right ordinate axis. This measurement is used to indirectly constrain the rate and kinematics of the $\mathrm{CC} \pi^{-}$contribution to the $\bar{\nu}_{\mu}$ CCQE sample. Figure taken from Ref. [84].

the level of agreement between data and the underlying model for $\mathrm{CC} \pi^{+}$interactions. However, obtaining the constraint in this variable does allow its direct application to $\mathrm{CC} \pi^{+}$ events background to the $\nu_{\mu}$ CCQE sample.

Due to $\pi^{-}$nuclear capture, using an analogous procedure to measure the rate and kinematics of $\mathrm{CC} \pi^{-}$events is not possible. In the absence of such a measurement, the constraint obtained in neutrino mode for $\nu_{\mu} \mathrm{CC} \pi^{+}$is applied to the $\mathrm{CC} \pi^{-}$Rein-Sehgal prediction. This assumes the underlying effects observed in the MiniBooNE $\mathrm{CC} \pi^{+}$data not predicted by the Rein-Sehgal model are identical for the $\mathrm{CC} \pi^{-}$process. In the absence of additional information, a large extrapolation uncertainty would be warranted.

Fortunately, a more modern external calculation with success in describing world single-pion production data is available to use in predicting the contribution of $\mathrm{CC} \pi^{-}$ 


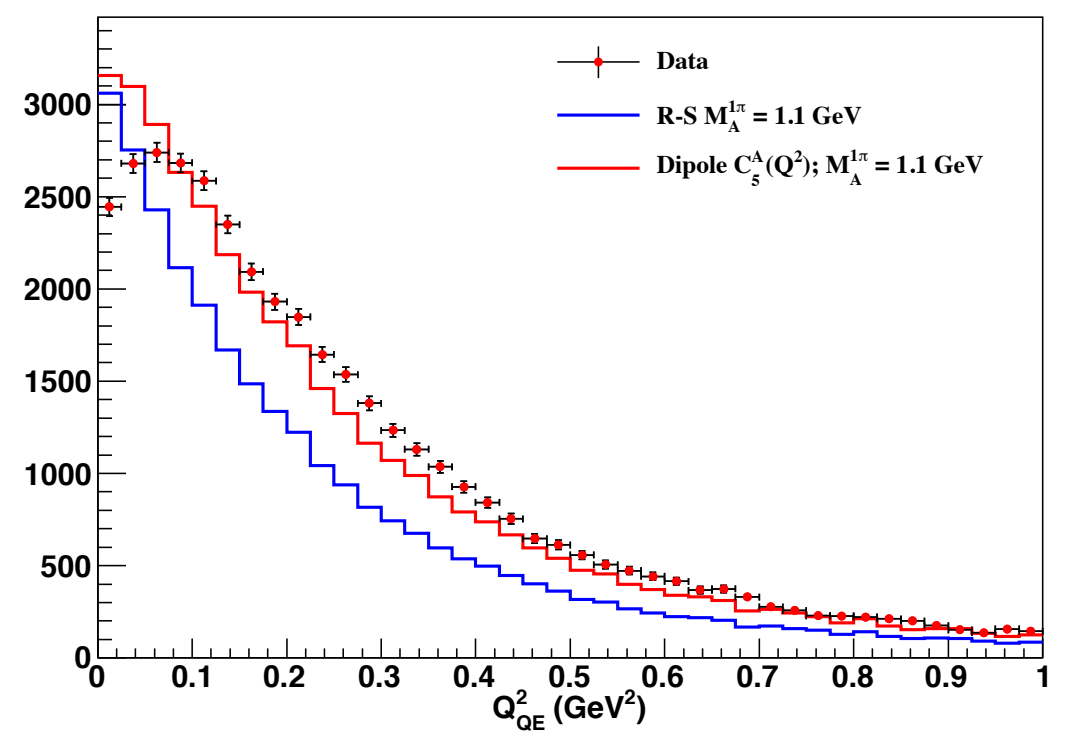

Figure 7-20. Comparison of the nominal Rein-Sehgal model to a more modern model that also describes world pion-production data more accurately.

events to the $\bar{\nu}_{\mu}$ CCQE sample [163]. This alternate model is implemented in NUANCE and is based on improvements to the Rein-Sehgal model originally developed in Refs. [164, 165, 166]. This updated calculation includes muon mass terms and a modified vector form factor to yield better agreement with world pion production data [167]. Figure 7-20 shows this model also offers excellent agreement with the MiniBooNE $\mathrm{CC} \pi^{+}$ data for $Q^{2} \gtrsim 0.1 \mathrm{GeV}^{2}$.

This model is used as a second constraint on the prediction for the contribution of $\mathrm{CC} \pi^{-}$events to the $\bar{\nu}_{\mu}$ CCQE sample. The level of agreement between this calculation and the indirect constraint based on the observed MiniBooNE $\mathrm{CC} \pi^{+}$data is shown in Figure 7-21. Consistency between these two predictions for $\mathrm{CC} \pi^{-}$production suggests an uncertainty of $20 \%$ is sufficient for the $\mathrm{CC} \pi^{-}$background. 


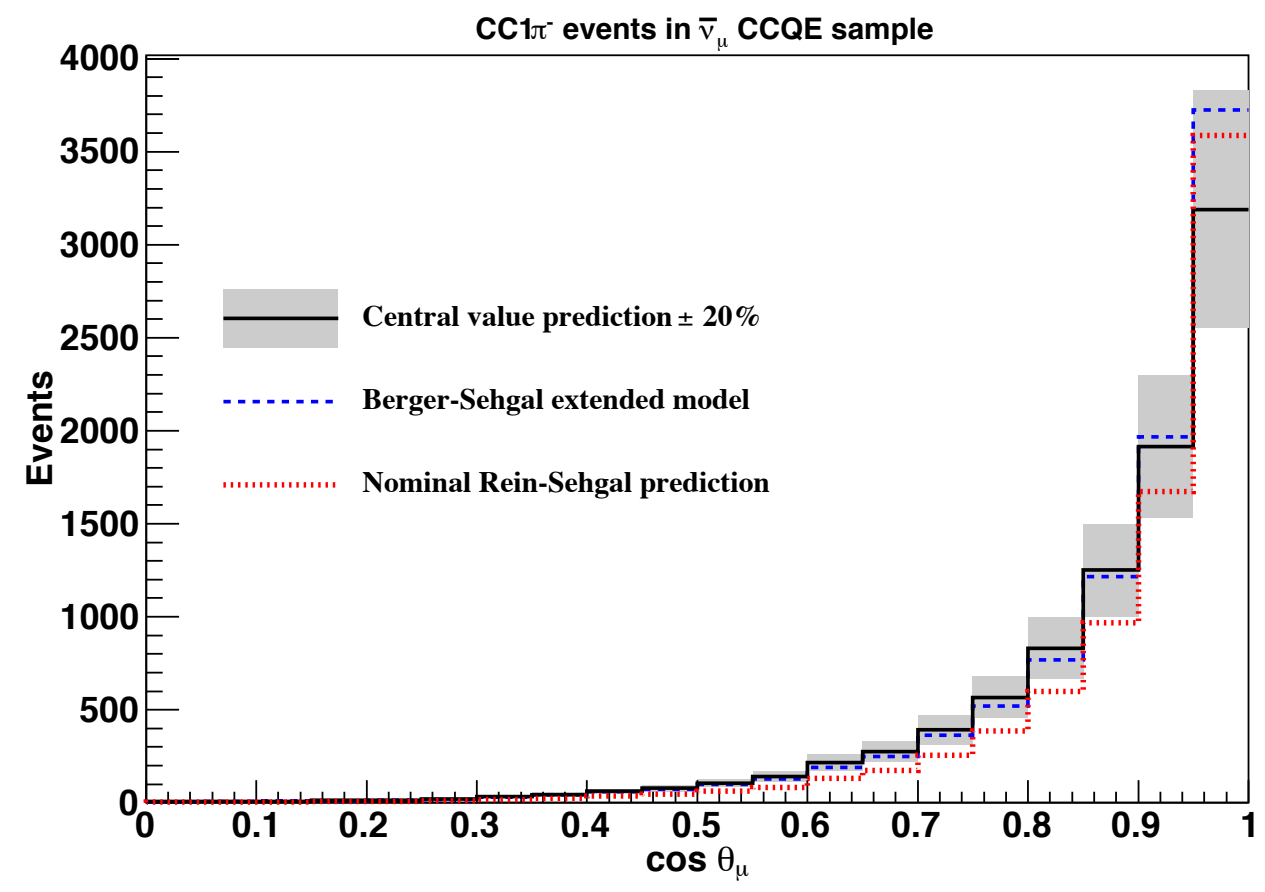

Figure 7-21. Three calculations for the $\mathrm{CC} \pi^{-}$background contribution to the $\bar{\nu}_{\mu} \mathrm{CCQE}$ sample as a function of $\cos \theta_{\mu}$. The "central value" distribution corresponds to the nominal Rein-Sehgal prediction for $\mathrm{CC} \pi^{-}$events in MiniBooNE constrained by the observed kinematics in the neutrino-mode $\nu_{\mu} \mathrm{CC} \pi^{+}$sample. This agrees well with a more recent calculation ("Berger-Sehgal extended model") that is based on an improved version of the Rein-Sehgal model. For comparison, the nominal Rein-Sehgal prediction for $\mathrm{CC} \pi^{-}$events is also shown. Distributions are normalized to flux. 


\subsection{All Other Backgrounds}

The analyses in Sections 7.1 and 7.2 constrain the contribution of $\nu_{\mu}$ CCQE and $\mathrm{CC} \pi^{ \pm}$interactions to the $\bar{\nu}_{\mu}$ CCQE sample. According to Table 6-2, the remaining channels account for $\sim 6 \%$ of the analysis sample. About half of these interactions are from $\nu_{\mu}$ and $\bar{\nu}_{\mu} \mathrm{CC} \pi^{0}$ production, and following the normalization discrepancy found in the MiniBooNE $\nu_{\mu} \mathrm{CC} \pi^{0}$ cross-section analysis [80], the contribution from both $\nu_{\mu}$ and $\bar{\nu}_{\mu}$ $\mathrm{CC} \pi^{0}$ events is increased by $60 \%$ of the prediction. The remaining half is dominated by $\bar{\nu}_{\mu}$ Cabibbo-suppressed quasi-elastic hyperon production, which ought to be closely related to the $\bar{\nu}_{\mu}$ CCQE results, but is experimentally poorly understood.

Two configurations of $\bar{\nu}_{\mu}$ CCQE cross sections are produced in this dissertation: incident on mineral oil (with atomic composition $\sim \mathrm{CH}_{2}$ ) and incident on carbon only. Many measurements of $\nu_{\mu}$ and $\bar{\nu}_{\mu}$ CCQE on free or quasi-free nucleons at a variety of energies have produced results consistent with the RFG model and $M_{A} \sim 1 \mathrm{GeV}$ [168], and so the carbon-only configuration is attained by treating the $\bar{\nu}_{\mu}$ CCQE events on hydrogen as background. Its contribution is predicted with the RFG and $M_{A}=1.026 \pm 0.021 \mathrm{GeV}$ following the analysis of relevant global data in Ref. [73].

\subsection{Background Constraint Summary}

The largest backgrounds in the $\bar{\nu}_{\mu}$ CCQE sample are those from $\nu_{\mu}$ and from the $\bar{\nu}_{\mu}$ $\mathrm{CC} \pi^{-}$contributions. The dominant $\nu_{\mu}$ interactions are from CCQE and $\mathrm{CC} \pi^{+}$channels, and their fundamental cross sections have been measured in the MiniBooNE data [84, 78]. The implementation of these direct constraints is explained in Section 7.1.4. The $\nu_{\mu}$ flux accepted into the antineutrino-mode data is constrained by the novel and unique measurements presented in Section 7.1, and per Section 7.1.6, the combined constraint on the $\nu_{\mu}$ flux relative to the extrapolated and highly-uncertain prediction (described in Section 5.2.4) is $0.77 \pm 0.10$. No additional error is taken on the $\nu_{\mu} \mathrm{CCQE}$ and $\mathrm{CC} \pi^{+}$ interactions, as the $\nu_{\mu}$ flux uncertainty is nearly fully correlated with the CCQE and CC $\pi^{+}$ cross section errors. 
As discussed in Section 7.2, the $\mathrm{CC} \pi^{-}$interaction cross sections are only indirectly constrained through the measurement of the rate and kinematics of $\nu_{\mu} \mathrm{CC} \pi^{+}$events extrapolated to the $\bar{\nu}_{\mu}$ processes. Consistency between this prediction for the $\mathrm{CC} \pi^{-}$ contribution to the $\bar{\nu}_{\mu}$ CCQE analysis sample with an external model for resonance events capable of describing world CC $\pi$ production data provides confidence in our description of $\mathrm{CC} \pi^{-}$events. Following these studies, a $20 \%$ normalization uncertainty is assigned to the $\mathrm{CC} \pi^{-}$interactions.

Finally, the small contribution from $\nu_{\mu}$ and $\bar{\nu}_{\mu} \mathrm{CC} \pi^{0}$ events are increased by $60 \%$ of the NUANCE-based prediction to reflect the MiniBooNE $\nu_{\mu} \mathrm{CC} \pi^{0}$ results [80]. The uncertainty on interactions not induced by $\nu_{\mu}$ and are non-CC $\pi^{-}$interactions is $30 \%$ of their prediction.

A summary of the various backgrounds in the $\bar{\nu}_{\mu}$ CCQE sample, including their uncertainties and constraints, if any, is provided in Table 7-17. 


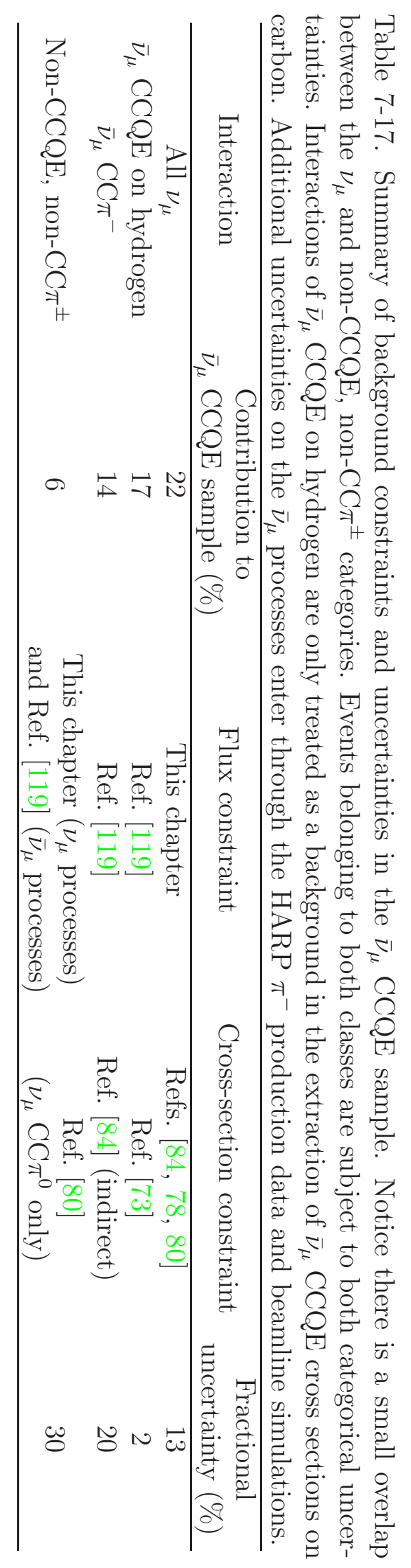




\section{CHAPTER 8 \\ $\bar{\nu}_{\mu}$ CCQE CROSS-SECTION MEASUREMENT}

Following the introduction to this measurement given in Chapter 6 and the opportunistic background measurements and constraints presented in Chapter 7, we now turn to the cross-section calculation and its various ingredients. This measurement is also described in Ref. [147].

\subsection{Data Stability}

Certainly the most important quantity to have confidence in is the data itself. A total of $10.09 \times 10^{20}$ POT of antineutrino-mode data are used in this analysis. This corresponds to the full MiniBooNE antineutrino-mode data set through April 2012, excluding the brief period in 2006 when absorber blocks fell into the meson decay tunnel. Many stability checks have been performed on the CCQE sample over the years and they've typically shown consistency within $1 \%$. Those most directly related to this analysis are presented here. For historical reasons, the data selection used in these stability checks differ slightly from that described in the Section 6.2, however the two selection sets result in roughly the same purity and efficiency. To test for any effective time dependence in the distributions, perhaps due to a systematic change in the experimental setup, the data is separated chronologically into groups described in Table 8-1. The shape compatibility of the four distributions we will turn into cross sections, $Q_{Q E}^{2}$ (Eq. 6.8), $E_{\nu}^{Q E}$ (Eq. 6.7) and the muon kinematic properties $T_{\mu}$ and $\cos \theta_{\mu}$ are assessed over different run periods are assessed through the Kolmogorov-Smirnoff (K-S) test [169] and are presented in Figure 8-1.

Not independent from Figure 8-1 but perhaps more accessible is the shape of the $E_{\nu}^{Q E}$ distribution shown for the same run periods on Figure 8-2.

A direct test of the normalization of the primary analysis sample is presented in Figure 8-3, where the events passing selection are given per POT for each period. 
Table 8-1. Summary of data groups input to the stability tests.

\begin{tabular}{ccc}
\hline Label & Run dates & POT $\left(\times 10^{20}\right)$ \\
\hline "jul07" & Jan. 2006 - Sep. 2008 & 2.205 \\
"sep09" & Sep. 2008 - Jun. 2009 & 1.477 \\
"mar10" & Aug. 2009 - Mar. 2010 & 0.798 \\
"oct10" & Mar. 2010 - Oct. 2010 & 1.160 \\
"may11" & Oct. 2010 - May 2011 & 1.763 \\
"mar12" & May 2011 - Apr. 2012 & 2.688 \\
\hline
\end{tabular}

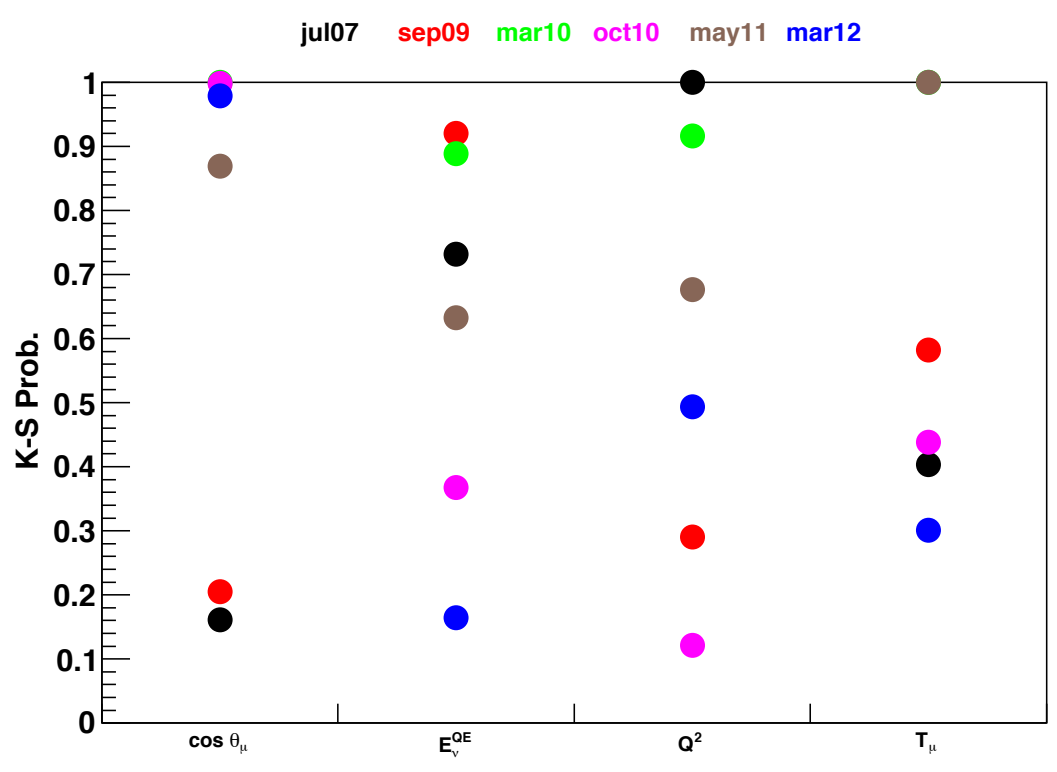

Figure 8-1. Kolmogorov-Smirnoff test for different antineutrino run periods. Each data point is the result of the K-S test of that run against the sum of the other subsamples. The data are consistent with a uniform distribution between null and unity. Important to note is that $E_{\nu}^{Q E}$ and $Q_{Q E}^{2}$ are derived quantities from $T_{\mu}$ and $\cos \theta_{\mu}$, so these tests are not independent. 


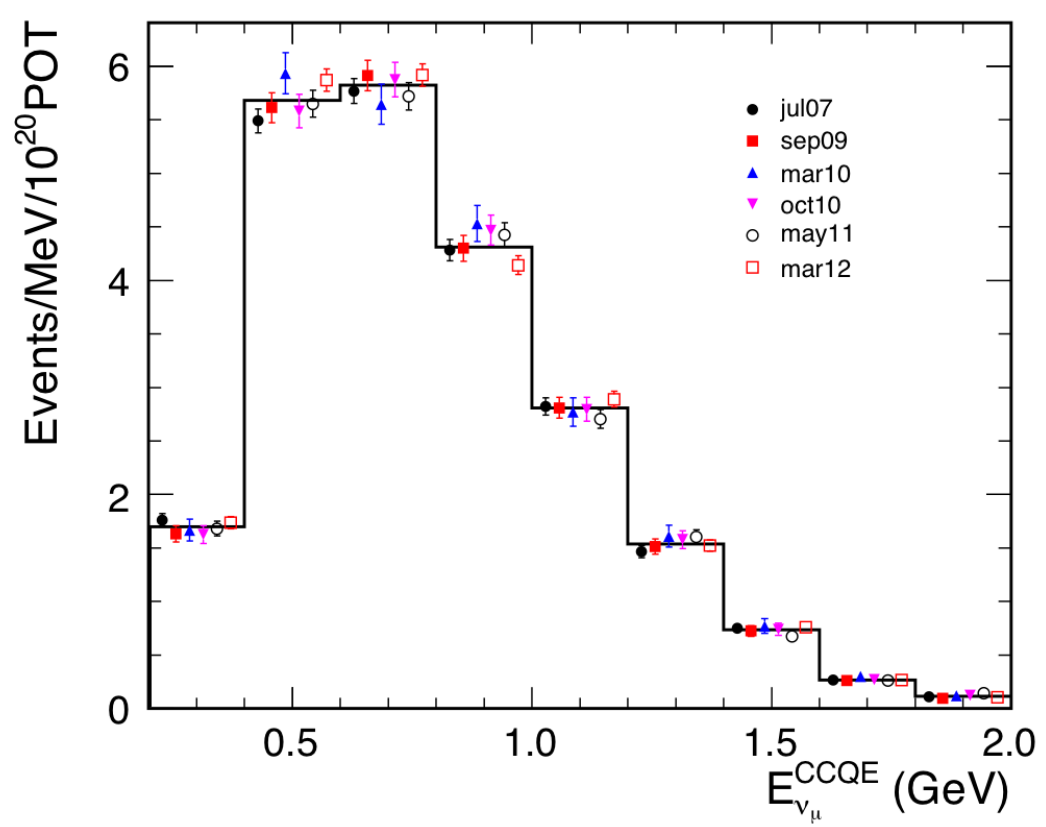

Figure 8-2. Reconstructed antineutrino data for various run periods. Good agreement within statistical errors indicate stable running. Included here in "jul07" and not in the main analysis is the small absorber down sample.

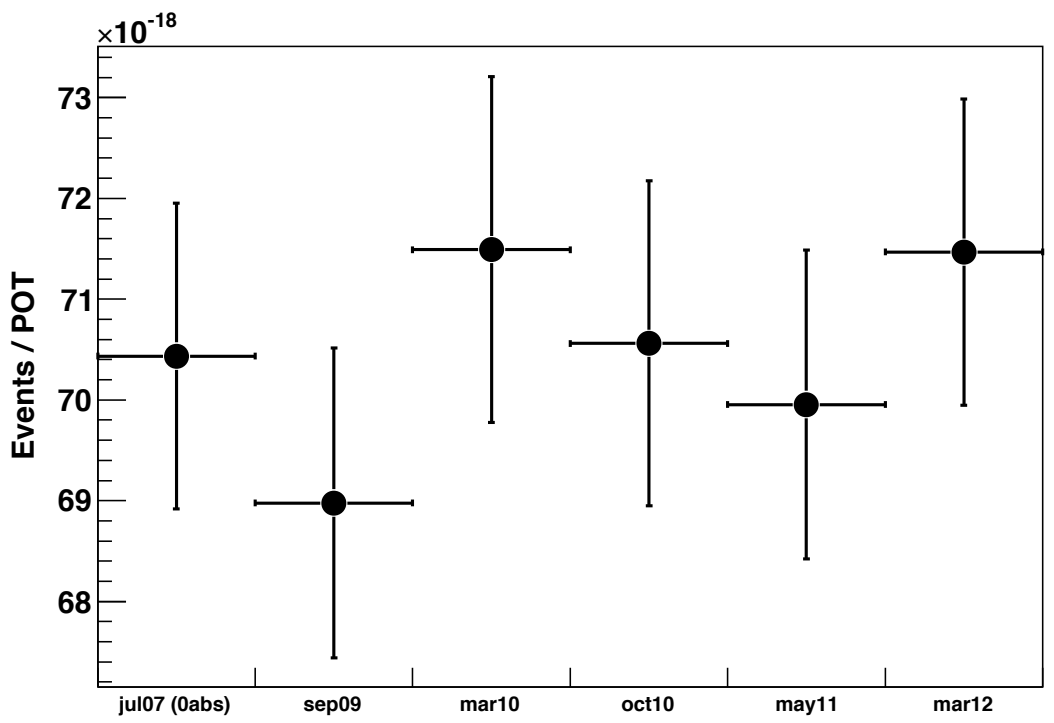

Figure 8-3. Normalization stability over various run periods. Included uncertainties are statistics and a $2 \%$ error on delivered POT. 


\section{$8.2 \bar{\nu}_{\mu}$ CCQE Simulation}

Data extraction is mostly insensitive to assumptions on the signal processes, but it is important to qualify their event generation to better understand the final cross-section comparisons between data and simulation.

The RFG simulation of $\bar{\nu}_{\mu}$ CCQE interactions in this analysis assumes an axial mass for $\bar{\nu}_{\mu}$ CCQE events on protons bound in carbon atoms (hereafter referred to as " $M_{A}^{C}$ ") of $1.35 \mathrm{GeV}$, and for events on free protons (" $M_{A}^{H}$ ") of $1.02 \mathrm{GeV}$. Signal events involving bound nucleons also receive the mild Pauli blocking modification $\kappa=1.007$ (Section 4.4.1). These parameters are chosen because they adequately reproduce the shape of the data in the reconstructed quantities (Figure 6-5) while maintaining consistency with the MiniBooNE $\nu_{\mu}$ CCQE analysis [84] and the light-target CCQE data [73]. If not for a few issues in generating the MiniBooNE MC, this description of our signal assumptions would be sufficient.

The first issue originates in the reweighting scheme for finding $\kappa=1.007$. Following the $2008 \nu_{\mu}$ CCQE analysis [83], the MC files were generated with $M_{A}^{C}=1.23 \mathrm{GeV}$ and $\kappa$ $=1.022$. As $\kappa$ is a lower bound on the available outgoing nucleon phase space, we cannot produce a lower value of $\kappa$ compared to the generated value using traditional reweighting. In other words, events that do not exist cannot be recovered by reweighting.

For our simulation to reflect $\kappa=1.007$, we first calculate the absolute cross section per nucleon for signal events involving bound protons from the baseline MC. We exclude hydrogen events here because they are unaffected by $\kappa$. This cross section is compared to the NUANCE-generated rate for $M_{A}^{C}=1.35 \mathrm{GeV}$ and $\kappa=1.007$ in Figure 8-4. The deficit in MC due to lowering $\kappa$ is clear at lower energies, while an independent problem shows itself above $E_{\nu} \sim 1.5 \mathrm{GeV}$. When the same baseline $\mathrm{MC}$ was generated, NUANCE was run in the logarithmic low precision flux sampling mode, meaning the flux spectrum was sampled increasingly sporadically at higher energies. As the $\bar{\nu}_{\mu}$ flux rapidly decreases with $E_{\nu}>1$ $\mathrm{GeV}$ or so (Figure 5-9), this level of sensitivity to the $\bar{\nu}_{\mu}$ flux shape is sufficient for the all 


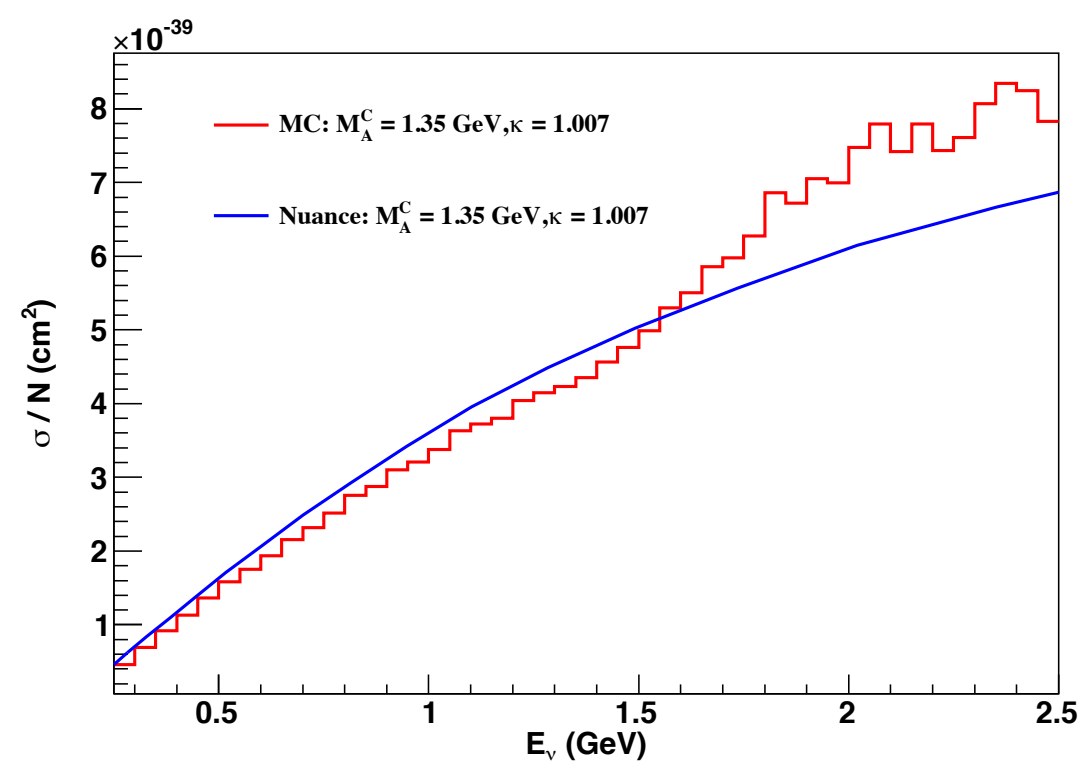

Figure 8-4. Comparison between generator-level total cross section for bound signal events to the unfolded MC. The files input to the MC distribution were generated with $\kappa=1.022$, and the failed attempt to recover $\kappa=1.007$ through traditional reweighting is clear at lower energies, while the flux sampling issue dominates the discrepancy at high energy.

non-cross section MiniBooNE analyses. In the present analysis, the $\bar{\nu}_{\mu}$ flux was sufficiently sampled for the bulk of the distribution but the high energy tail was not accurately explored by NUANCE. If not corrected, the details of the issue would lead to a too-low detection efficiency and a spuriously high cross section.

As $\kappa$ was introduced to improve kinematic agreement with $\nu_{\mu}$ CCQE data, it would be insufficient to recover it by reweighting MC to NUANCE based on the total cross section. Therefore we scale MC to the generator-level distribution in bins of the double-differential cross section in muon kinematics $\frac{d^{2} \sigma}{d T_{\mu} d \cos \theta_{\mu}}$, and the resultant weights are shown in Figure 8-5.

As the double-differential cross section is flux-integrated, it is only mildly sensitive to the high-energy flux tail. A final set of weights in the absolute cross section is applied to MC to achieve generator-level agreement at the few-\% level even at high energies.

As mentioned, the $\kappa$ issue is irrelevant for signal scattering off hydrogen, but of 


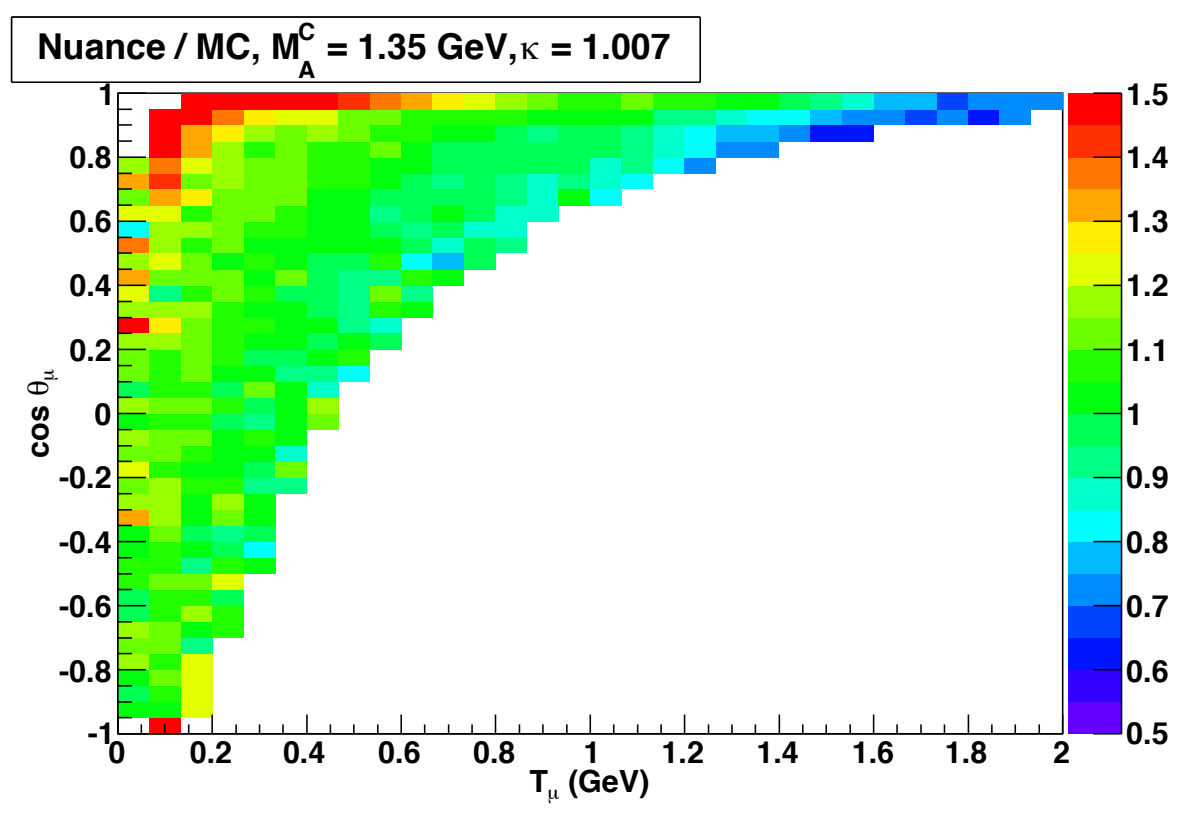

Figure 8-5. Weights as a function of muon kinematics applied to MC to recover $\kappa=1.007$. As expected, the weights are strongest in regions of forward-going, low-energy muons.

course the high energy issue is present. Figure 8-6 shows MC before and after correcting for the flux sampling problem.

It is important to note that, with the exception of the high-energy issue affecting the efficiency calculation, these signal assumptions hardly affect our extracted cross sections. The normalization of the MC signal events does not enter the cross-section calculation, while sensitivity to the simulated true shape of signal events is quite small. It will be shown later that a conservatively large span of $M_{A}^{C}$ and $\kappa$ signal values lead to negligible differences on the final cross sections. However, this procedure of reweighting our simulation to the NUANCE cross sections with $\kappa=1.007$ was an important step to have a reliable efficiency calculation and be able to faithfully report the model used in data extraction and comparisons.

A final note on the high-energy flux sampling problem involves its effect on the $\mathrm{CC} \pi$ interactions. Since the $Q_{Q E^{-}}^{2}$ based correction (described in Section 7.2) to $\mathrm{CC} \pi^{+}$and $\mathrm{CC} \pi^{-}$ events was measured in the neutrino-mode $\mathrm{CC} \pi^{+}$sample, the constraint could be sensitive 


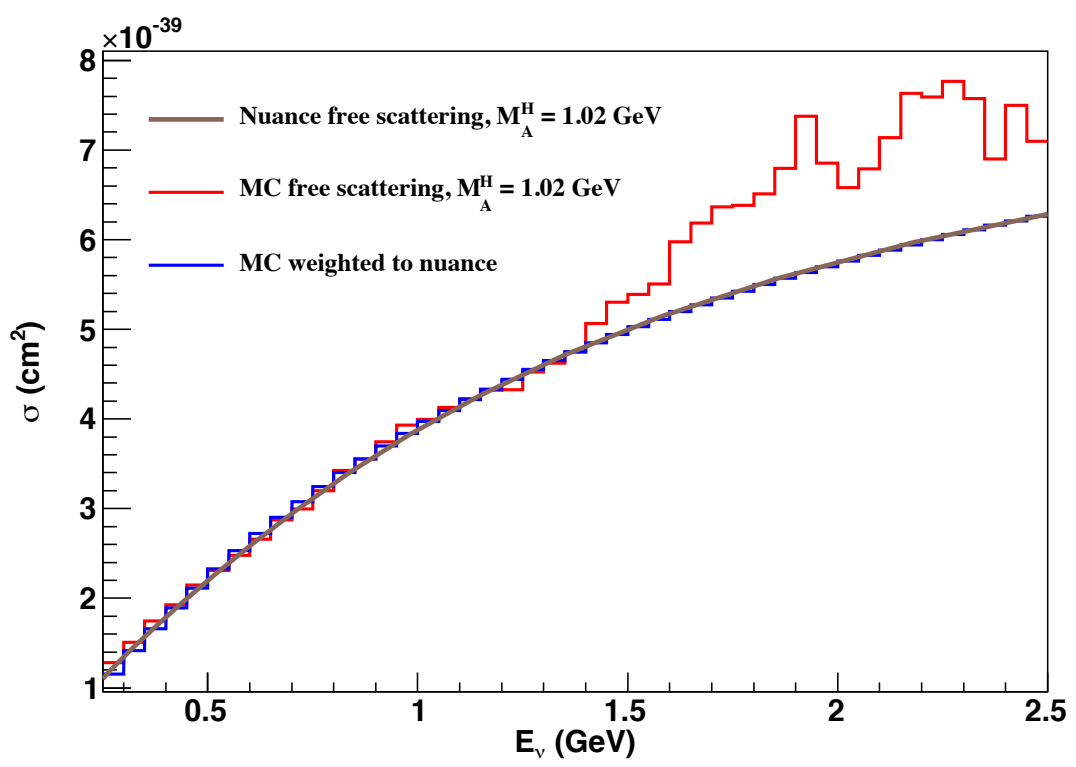

Figure 8-6. Summary of the high energy rate correction for $\bar{\nu}_{\mu}$ CCQE interactions on hydrogen.

to the details of the high-energy problem and its implementation into the

antineutrino-mode analyses could be erroneous through the mildly different accepted $\nu_{\mu}$ spectra between the two run configurations. However, Figure 8-7 shows that the flux sampling problem has a much more mild effect on $\mathrm{CC} \pi$ interactions compared to CCQE, presumably due to the different shape of the total cross section around the MiniBooNE energy range.

\subsection{Cross-Section Calculation}

The total cross section per nucleon in the $i$ th bin is given by

$$
\sigma\left(E_{\nu}\right)_{i}=\frac{\sum_{j} U_{i j}\left(d_{j}-b_{j}\right)}{\epsilon_{i} \Phi_{i} N},
$$

where $d_{j}\left(b_{j}\right)$ is the data (background) reconstructed in the $j$ th bin, $U_{i j}$ is the probability for an event of true quantity within bin $i$ to be reconstructed in bin $j, \epsilon$ is the detection 


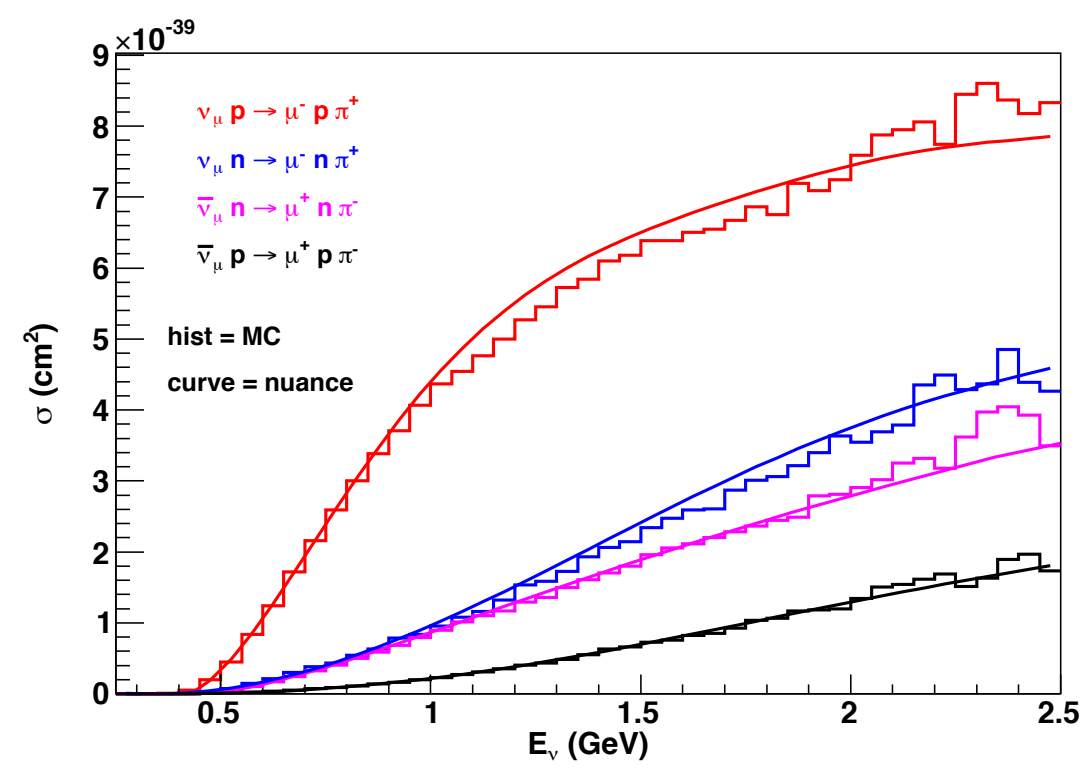

Figure 8-7. Comparison of NUANCE-generated single pion cross sections to the unfolded MC. Unlike in the CCQE interactions, the high energy flux sampling problem is small enough to ignore here.

efficiency, $\Phi$ is the $\bar{\nu}_{\mu}$ flux corresponding to the delivered protons on target, and $N$ is the number of nuclear targets in the volume considered. The differential expressions are similar: for $Q_{Q E}^{2}$ we have

$$
\frac{d \sigma}{d Q_{Q E_{i}}^{2}}=\frac{\sum_{j} U_{i j}\left(d_{j}-b_{j}\right)}{\Delta Q_{Q E}^{2} \epsilon_{i} \Phi N},
$$

where $\Delta Q_{Q E}^{2}$ is the width of the $i$ th bin and $\Phi$ is now the integrated flux. The double-differential calculation is a trivial extension:

$$
\frac{d^{2} \sigma}{d T_{\mu} d\left(\cos \theta_{\mu}\right)_{i}}=\frac{\sum_{j} U_{i j}\left(d_{j}-b_{j}\right)}{\Delta T_{\mu} \Delta \cos \theta_{\mu} \epsilon_{i} \Phi N}
$$

The following subsections expands on each of these quantities. 

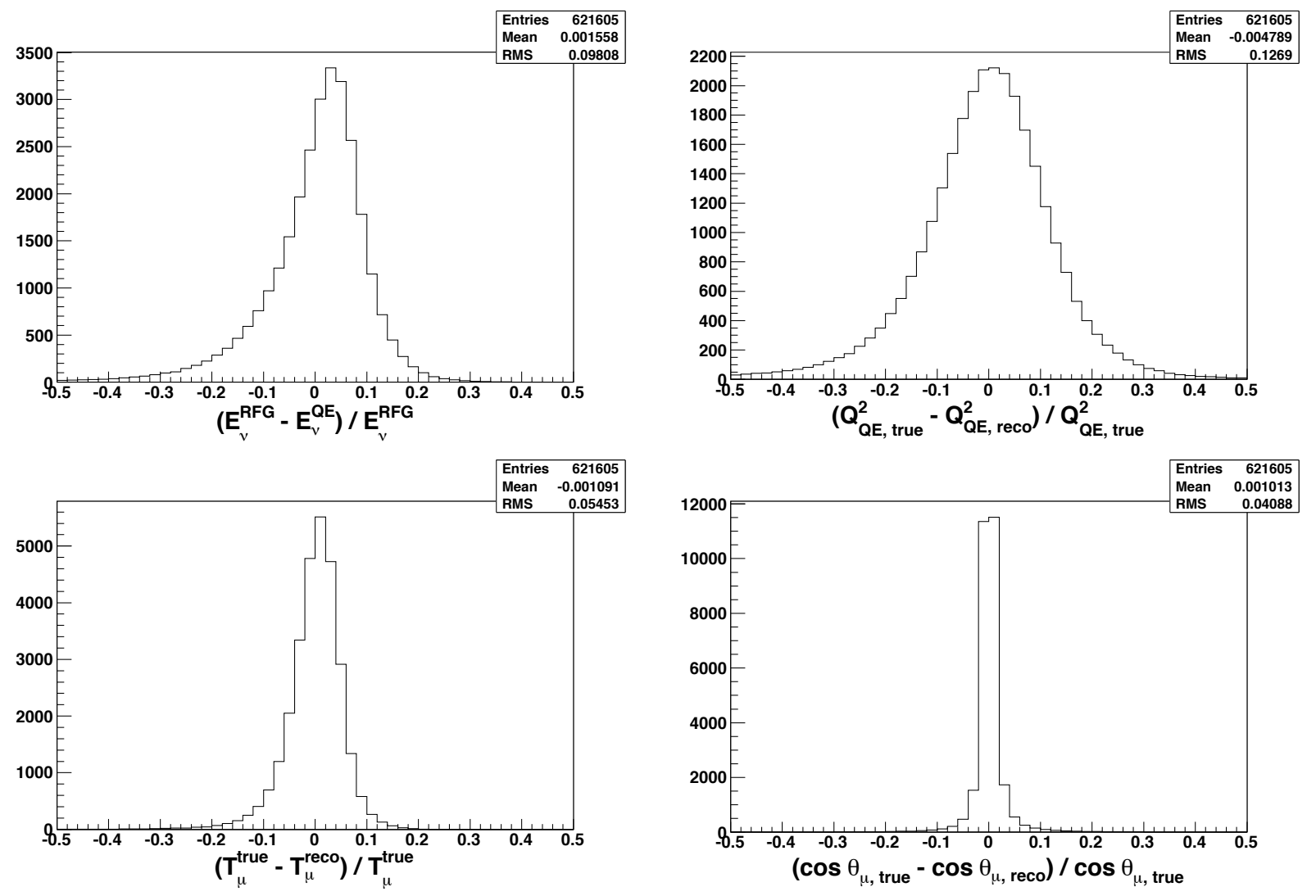

Figure 8-8. Fractional differences between truth and reconstructed quantities in the four relevant variables. As in all distributions peaked near null, the average fractional difference in $Q_{Q E}^{2}$ is higher than it would be otherwise.

\subsubsection{Unsmearing to True Quantities}

All measurements are biased at some level by detectors and analysis tools. The unsmearing process removes this bias so that the underlying quantities may be reported. First we should understand the overall relationship between the quantities we are looking to connect. Figure 8-8 shows the fractional difference between the reconstructed and truth-level variables relevant to this analysis.

It is tempting to refer to the RMS values printed in Figure 8-8 as the resolution of the MiniBooNE detector to these quantities, but this is not the case for $Q_{Q E}^{2}$ and, in particular, for the neutrino energy. To avoid dependence on the nuclear model of the RFG, reconstructed $Q_{Q E}^{2}$ is unsmeared to "true" $Q_{Q E}^{2}$, that is using the truth-level $\mu$ quantities 
in Eq. 6.8 instead of the generator-level squared four-momentum transfer. Of course this is not a perfect solution, as any theoretical calculation of the underlying $Q^{2}$ will have to first be translated into $Q_{Q E}^{2}$ before a rigorous comparison with these data can be made. In contrast, reconstructed $E_{\nu}^{Q E}$ is corrected to the generator-level neutrino energy, referred to here as $E_{\nu}^{R F G} . E_{\nu}^{Q E}$ and $E_{\nu}^{R F G}$ are entirely different quantities, and the RMS printed on Figure 8-8 is simply the average fractional difference between the two. The choice of correcting the neutrino energy back to the generated value is made to be able to compare with historical data produced with the same assumptions.

It is partially for these reasons that the double-differential cross section $\frac{d^{2} \sigma}{d T_{\mu} d \cos \theta_{\mu}}$ is the main result of this work, as it does not rely on a physics model to connect the reconstructed and true quantities. Of course the other advantage is that no assumptions (as are implicit in Equations 6.7 and 6.8) about the primary interaction need be made to reconstruct muon kinematics.

Following the other MiniBooNE cross-section analyses [129, 84, 78, 79, 80], the primary method for unsmearing detector effects employs the Bayesian approach [170]. This method is biased by MC assumptions on the underlying distribution, but we will show this prejudice is small. An unbiased estimator will be used to cross-check the results (albeit in less compelling binning), and ultimately the bias is assessed by evaluating the results under a conservatively wide range of signal assumptions. Fortunately error due to this bias is negligible compared to flux and background uncertainties. Another motivation to use the Bayesian method is the aim to follow as closely as reasonable the analysis choices of the $\nu_{\mu}$ CCQE cross section to better facilitate the combined analysis of Chapter 9.

If we refer to the underlying true data distribution as $\vec{\alpha}$ and to the same distribution under the influence of detector and reconstruction biases as $\vec{\beta}$, the two are connected by the unsmearing matrix $U$ present in Equations 8.1 - 8.3 as simply: 


$$
\vec{\alpha}=\check{U} \vec{\beta}
$$

Each entry $U_{i j}$ represents the probability for an event drawn from the underlying distribution in bin $i$ to be reconstructed in the $j$ th bin. In a perfect detector, $\check{U}$ would be the identity matrix. The reconstructed vector $\vec{\beta}$ is readily recognized as $(\vec{d}-\vec{b})$ in the cross section formulae. To build $\check{U}$ we first populate a matrix with signal MC events in the reconstructed vs. true variables, referred to here as $\check{M}$. The entries of $\check{M}$ are simply connected to $\check{U}$ by normalization factors

$$
U_{i j}=\frac{M_{i j}}{\sum_{k} M_{k j}}
$$

This naturally conserves the number of reconstructed events, i.e. $\sum_{i} U_{i j}=1$ for all $j$. The probability matrix $\breve{U}$ is shown in Figure 8-9 for the four standard distributions in the binning chosen for this analysis, as well as the diagonal entries for muon kinematic plane.

In the application of this method to histograms whose domain may exclude part of the sample, underflow and overflow bins are included. A simple consistency check, passed for all distributions, is that this unsmearing procedure applied to reconstructed MC signal events exactly returns the generated distribution.

Figure 8-10 shows the effect of the $\check{U}$ matrix to the vector $\vec{d}-\vec{b}$, where $\vec{d}$ and $\vec{b}$ are the reconstructed data and background, respectively. The distributions in the same figure represent the numerator in cross-section Eqs. 8.1 - 8.3.

The so-called "inversion method" of connecting the reconstructed to true distributions is unbiased by a priori assumptions about the underlying interactions. In this procedure the matrix (referred to as $\check{R}$ ) that describes unsmearing operates on the true distribution $\vec{\alpha}$ : 


$$
\vec{\beta}=\check{R} \vec{\alpha}
$$

A quick comparison with Equation 8.4 shows $\check{R}=\check{U}^{-1}$. The matrix $\check{R}$ is also formed with $\check{M}$, but this time by normalizing over the reconstructed index:

$$
R_{i j}=\frac{R_{i j}}{\sum_{k} M_{i k}}
$$

Since $\check{R}$ must be inverted in order to find the true distribution, the matrix inversion method of unsmearing is exceptionally unstable. In particular, too-fine binning gives rise to the "Gibb's phenomenon", where the calculated true distribution oscillates wildly bin-to-bin. Anecdotally, this can be avoided by choosing the binning such that on-diagonal elements of $\check{R}$ are no lower than 0.8 . This requirement constrains the bins to be quite modest. This is particularly true in the case of the neutrino energy, where the correlation between the $E_{\nu}^{Q E}$ and $E_{\nu}^{R F G}$ variables is relatively weak (Figure 8-8).

Figure 8-11 compares the results of the matrix inversion method to the Bayesian procedure described previously. The binning has been optimized such that the entries $R_{i i}$ are close to 0.8 , because binning finer than those shown might be subject to the Gibb's phenomenon. That the Bayesian method gives results consistent with the unbiased matrix inversion method gives a qualitative upper limit to its bias. However, since unsmearing is a shape-only procedure and the binning in the unbiased method is relatively conservative, this is not an especially powerful test. More compelling evidence that the Bayesian method does not significantly bias the results is shown in Section 8.4, where the prejudice is evaluated to be negligible in the presence of other systematic uncertainties. 

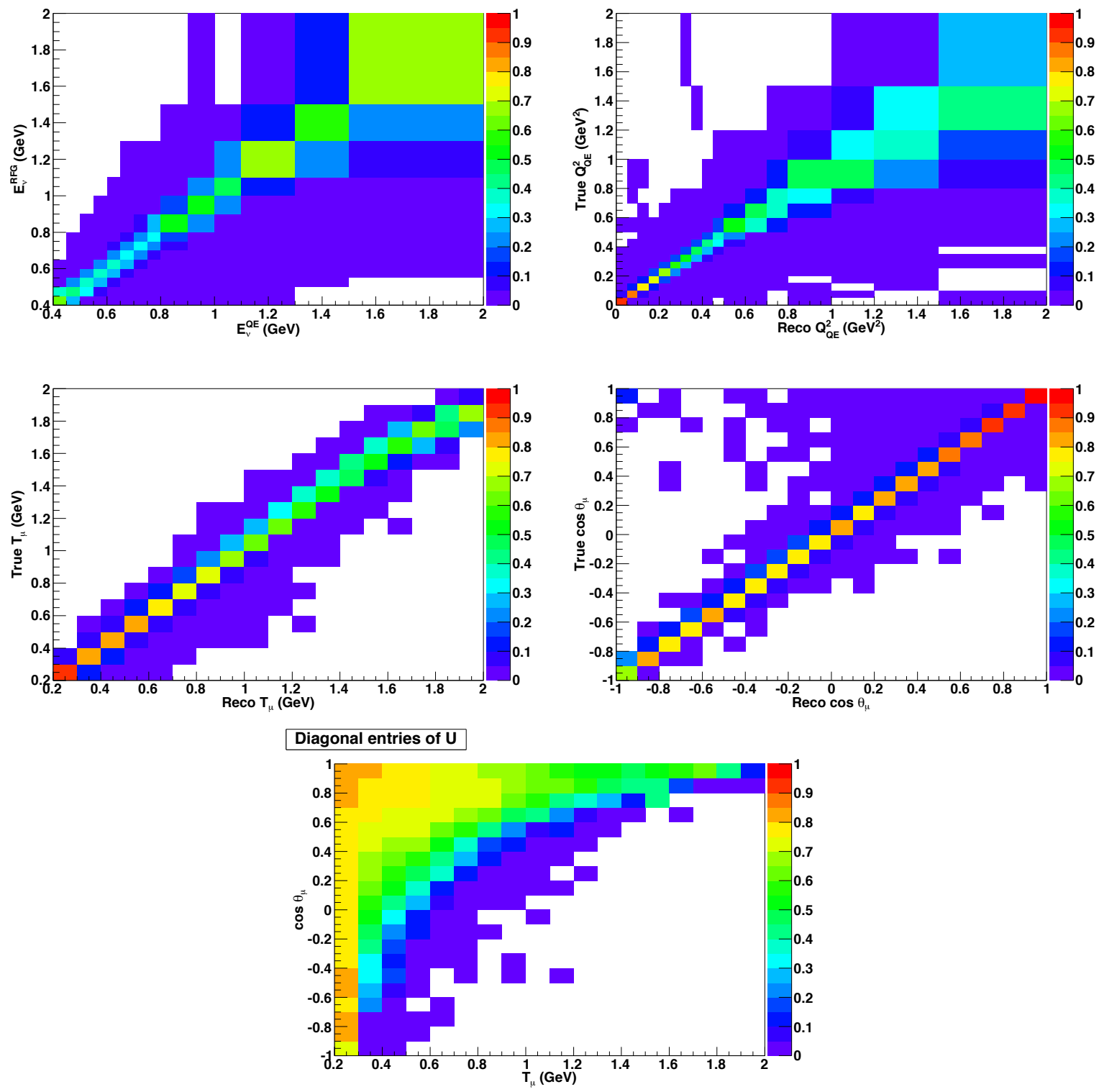

Figure 8-9. The matrix $\check{U}$ connecting the true and reconstructed quantities for the four variables of interest. For the two-dimensional distribution, in principle $\check{U}$ is four dimensional and only its diagonal entries are shown here. 

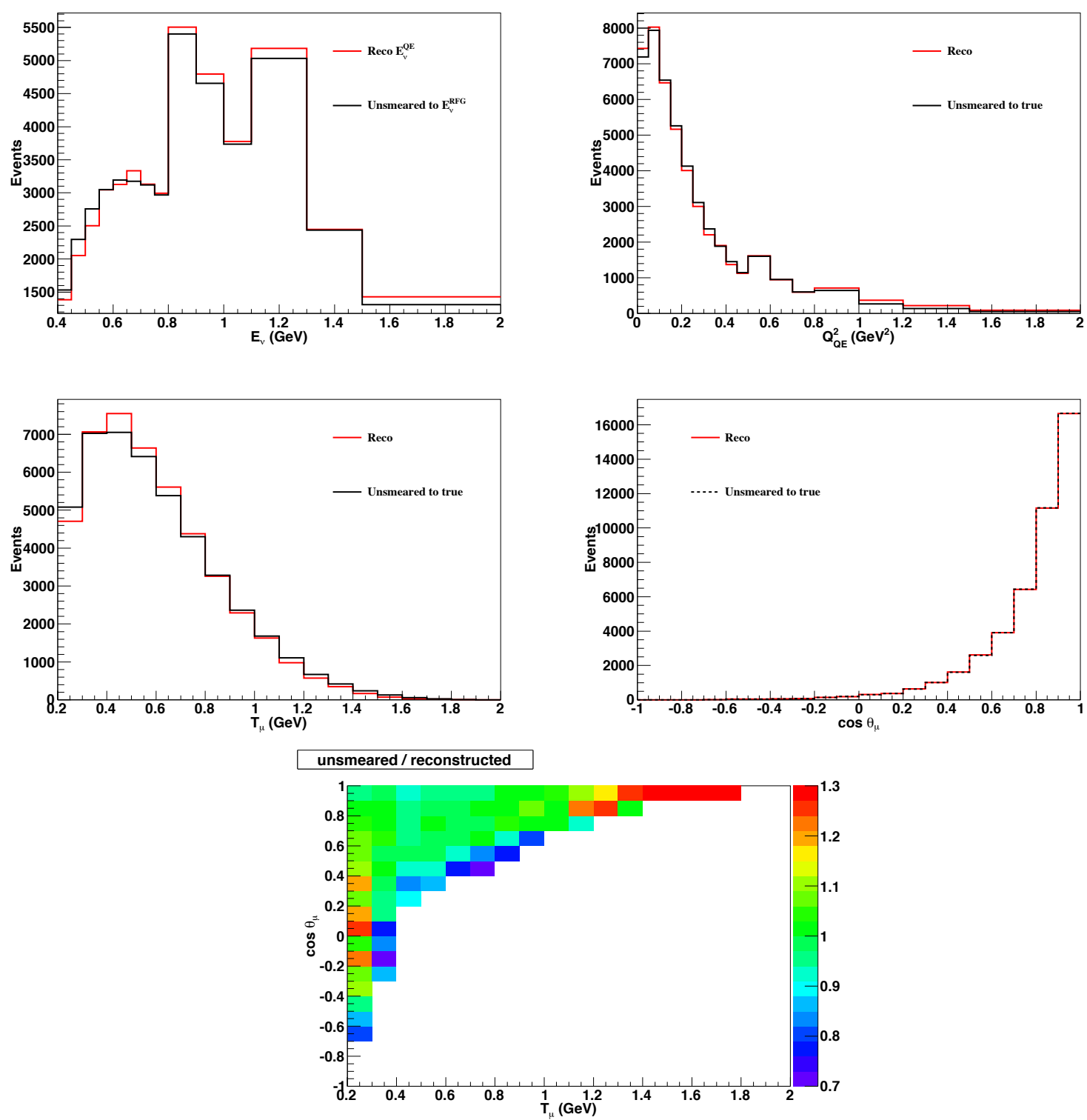

Figure 8-10. Comparison of before and after the unsmearing procedure for signal events. The two-dimensional ratio includes a requirement that there be at least 10 events in each bin of both the reconstructed and unsmeared distributions. 

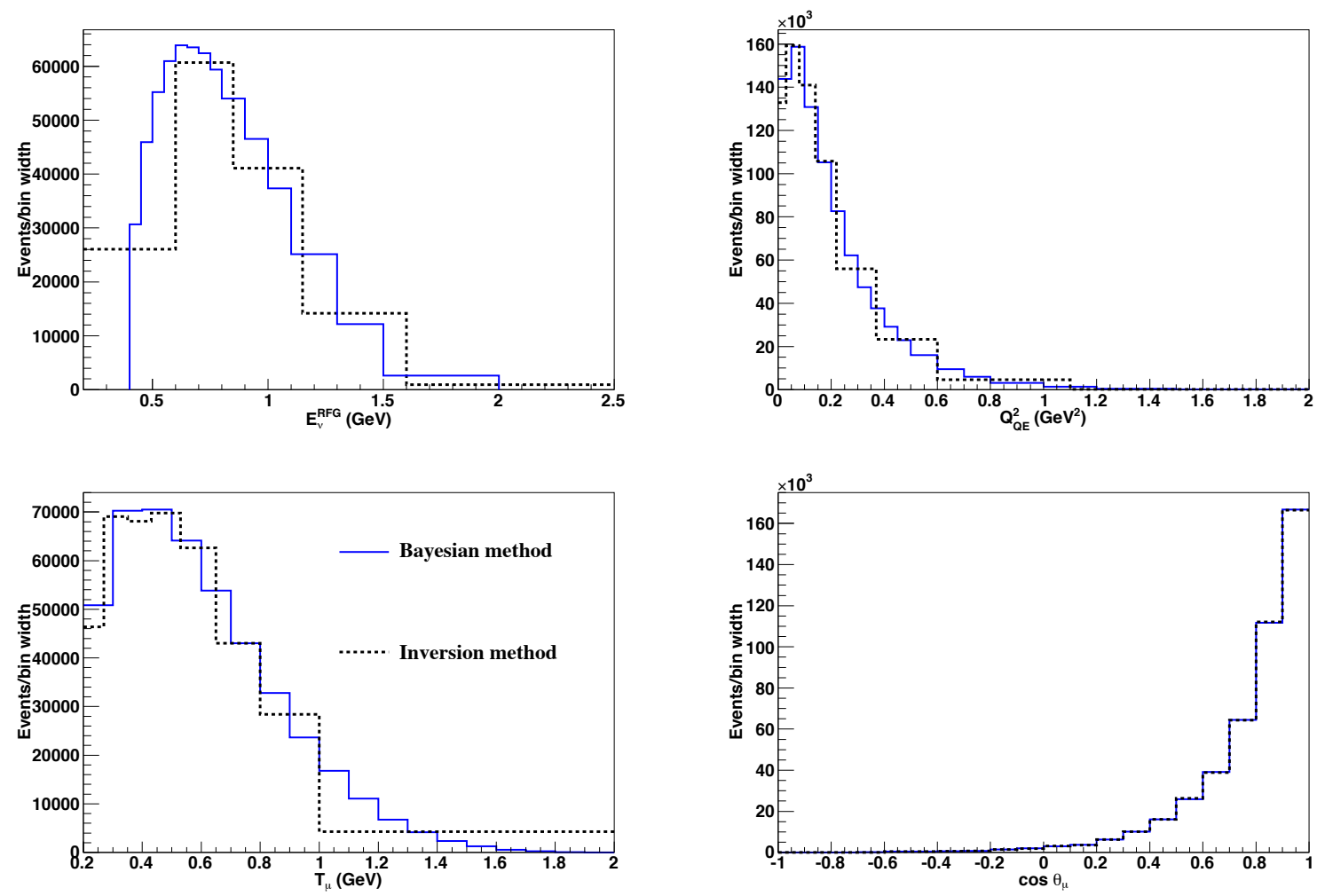

Figure 8-11. Comparison of the two unsmearing methods studied in this section. 


\subsubsection{Efficiency Correction}

To correct for the signal events lost due to sample selection, the detection efficiency is calculated and applied to data bin-by-bin in each distribution. Since the unsmearing procedure described previously has (up to some uncertainty) returned the observed data to the true distribution, efficiencies are measured as a function of the true variable. The efficiency is evaluated in a simulated sample of signal events in a spherical volume of radius $550 \mathrm{~cm}$, the value of which is chosen to avoid a potential rate bias due to the iron optical barrier at $575 \mathrm{~cm}$ (this effect is visible in Figure 6-2), while a negligible number of signal events $(<0.2 \%)$ that pass selection criteria have a generated radius greater than $550 \mathrm{~cm}$.

Figure 8-12 shows sequential efficiency for each analysis cut in the four standard variables, as well as the total efficiency for the two-dimensional distribution. The majority of the loss of events is caused by requirements on the kinematics of the muon, the simulation for which has been vetted most rigorously against cosmic ray muon data (Section 5.3.4). 

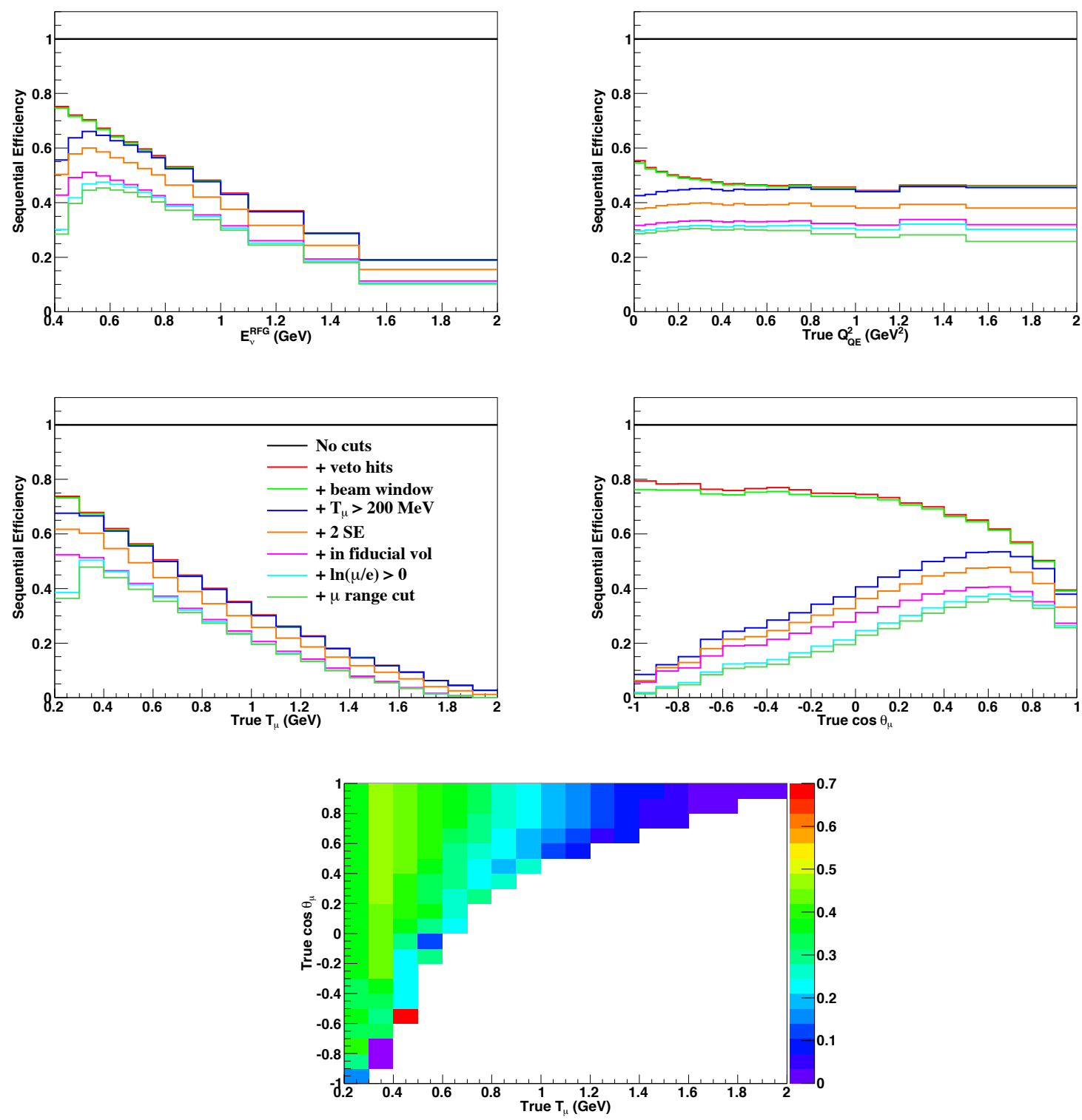

Figure 8-12. Detection efficiency for each cut in the relevant distributions. Only the total efficiency is shown for two-dimensional muon kinematics. 


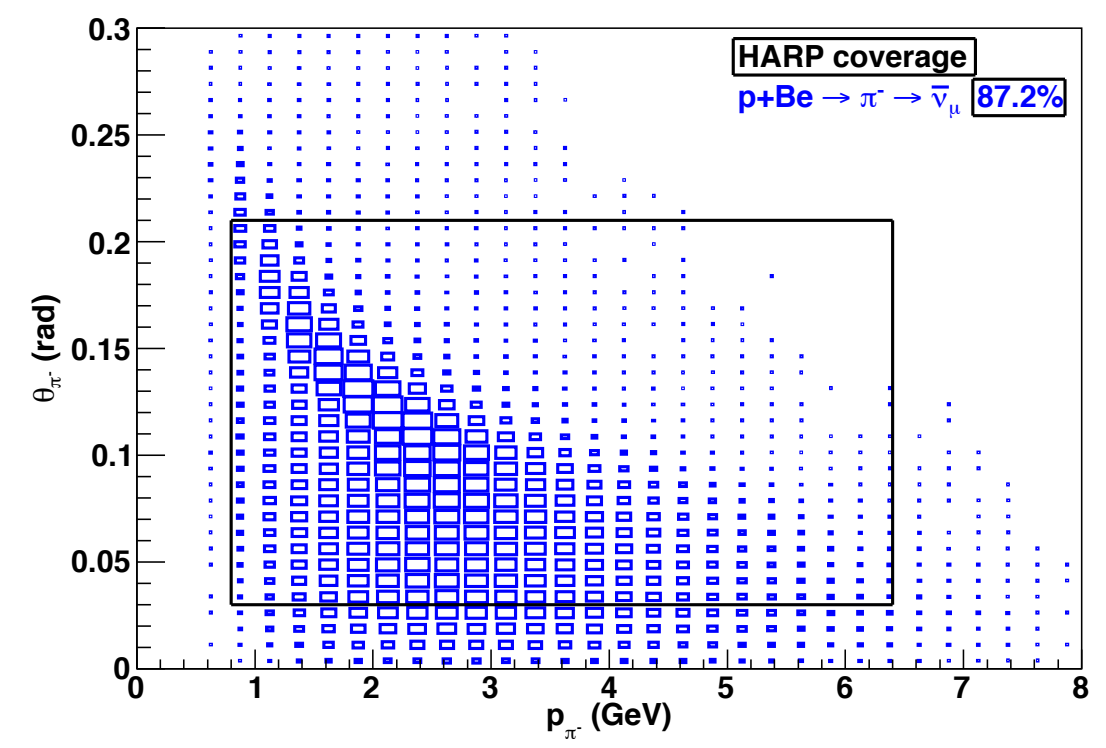

Figure 8-13. Predicted outgoing phase space for $\pi^{-}$before horn focusing. Only $\pi^{-}$'s focused by the horn and that subsequently lead to an interaction in the detector are shown. As printed on the figure, roughly $90 \%$ of the flux is covered by the HARP $\pi^{-}$cross-section data.

\subsubsection{Flux and Interaction Targets}

As described in Section 5.2.4, the prediction for the absolute $\bar{\nu}_{\mu}$ flux in antineutrino mode is nearly model independent. Figure 8-13 shows the outgoing $\pi^{-}$phase space at the BNB target contributing to the present data set is well-constrained by the HARP data.

Combining the HARP production data with detailed Geant4 target, horn and beamline geometry gives the absolute $\bar{\nu}_{\mu}$ flux prediction shown in Figure 8-14.

Since the total cross section and the flux are both functions of the neutrino energy, the flux histogram in Figure 8-14 is rebinned to match that used in the analysis. In the case of the differential measurements, the integrated flux is used excluding the region $E_{\nu}$ $<100 \mathrm{MeV}$ due to the interaction requirement of muon production.

The final element in the cross-section calculations is the number of nucleon targets for the signal. This involves the detector volume corresponding to that assumed by the efficiency correction in Section 8.3.2, the mineral oil density, the mass density of relevant protons per molecule, and Avogadro's number. This is calculated for all protons (bound 


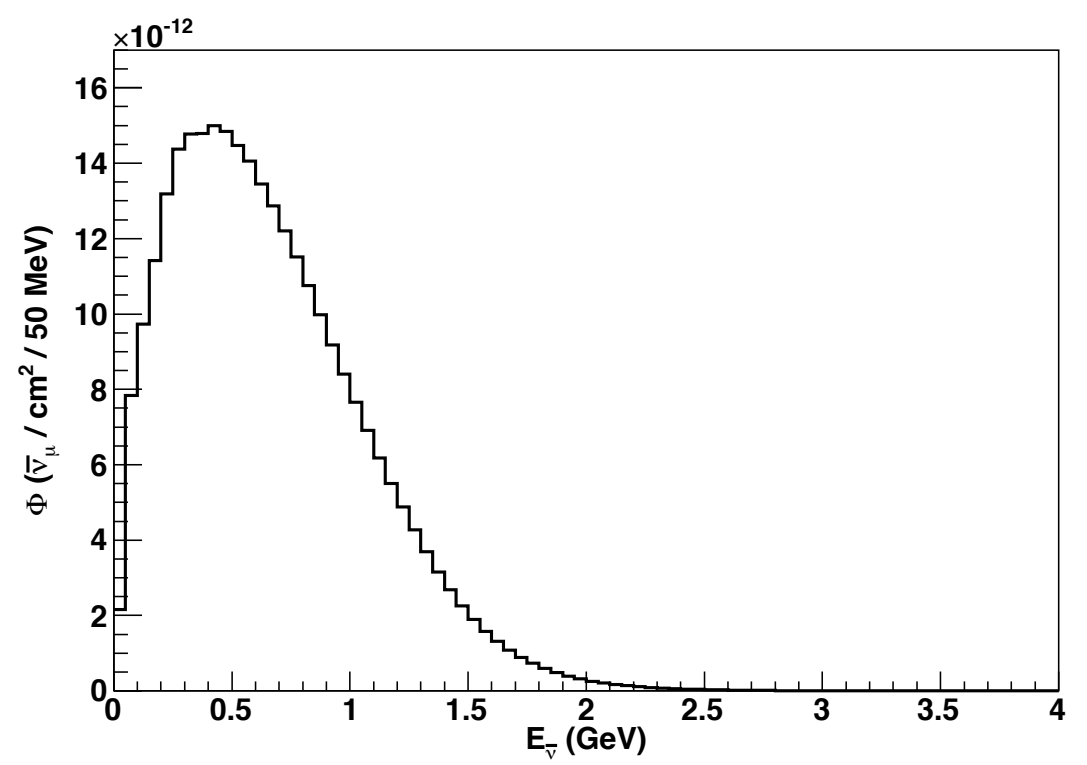

Figure 8-14. Flux prediction for $\bar{\nu}_{\mu}$ in antineutrino mode for $10.1 \times 10^{20}$ POT.

protons only) by:

$$
N=\frac{4}{3} \pi[550 \mathrm{~cm}]^{3} \times 0.845 \frac{\mathrm{g}}{\mathrm{cm}^{3}} \times 6.02214 \cdot 10^{23} \times \frac{6.0(8.06)}{14.06}=1.5134(2.0330) \times 10^{32}
$$

The density of the oil is measured from a sample extracted from the detector, and the composition of the oil is determined to be $\mathrm{C}_{n} \mathrm{H}_{2 n+2}, n \sim 30$.

\subsubsection{Statistical Uncertainty}

To avoid regions of statistics that would be incorrectly analyzed with a Gaussian treatment, at least 25 events are required to appear in each reconstructed bin $(\vec{d}-\vec{b})$. The statistical error in the $i$ th bin is calculated by

Stat $\operatorname{error}_{i}=\frac{\sqrt{T_{i} \times \frac{\mathrm{data}}{\mathrm{MC}}}}{S_{i}} \times \sigma_{i}$ 
where $T_{i}$ and $S_{i}$ are the predicted number of events in the total and signal samples respectively, "data" and "MC" are the respective normalizations and $\sigma$ is the (total or differential) cross section in that bin. The data / MC ratio is included to correct for the observed statistics. It might be tempting to not include the normalization ratio and instead use the number of data events for $T_{i}$, but then the statistical error itself would be subject to statistical fluctuations. As with all MiniBooNE cross-section measurements, the statistical error will prove to be negligible with the exception of the tails of the double-differential cross section.

\subsection{Systematic Uncertainty}

Broadly, systematic errors are evaluated by recalculating the cross sections under appropriate excursions from the assumptions input to the MC regarding parameters and processes that might affect the extraction of the $\bar{\nu}_{\mu}$ CCQE cross sections. The implementation of this idea varies among the systematic errors and the details are presented in this section. The differences between these alternate cross sections and the one described in the previous section are then used to form covariance matrices, and the on-diagonal elements of the quadrature addition of all error matrices sets the total uncertainty on the data. The formation of this covariance matrix is developed in Section 7.1 .5 and is not repeated here.

\subsubsection{Background Uncertainties}

Uncertainty on the background is evaluated by the "unisim" method, where a single excursion from the central value prediction is sufficient to propagate uncertainty onto data. Appropriate to the description in Section 7.4, backgrounds are separated into three categories: $\nu_{\mu}$ events from $\pi^{+}$decay in the beam, $\mathrm{CC} \pi^{-}$, and non-CCQE, non-CC $\pi^{ \pm}$ interactions. As reported in Table 6-2, these backgrounds comprise 17\%, 14\%, and 6\% of the sample, respectively. Note there is some overlap in the $\nu_{\mu}$ and the non-CCQE, non-CC $\pi^{ \pm}$categories. Section 7.4 summarizes the background constraints and assumed uncertainties on their contributions to the $\bar{\nu}_{\mu}$ CCQE sample. 
With the uncertainties on the background interactions set, we re-calculate the cross sections from data with the various backgrounds one standard deviation from their nominal prediction:

$$
\begin{aligned}
\sigma\left(E_{\nu}\right)_{i}^{\mathrm{bkg} \text { err }} & =\frac{\sum_{j} U_{i j}\left(d_{j}-\left[b_{j} \pm \delta b_{j}\right]\right)}{\epsilon_{i} \Phi_{i} N}, \\
\frac{d \sigma}{d X}_{i}^{\text {bkg err }} & =\frac{\sum_{j} U_{i j}\left(d_{j}-\left[b_{j} \pm \delta b_{j}\right]\right)}{\Delta X \epsilon_{i} \Phi N}, \\
{\frac{d^{2} \sigma}{d T_{\mu} d\left(\cos \theta_{\mu}\right)_{i}}}^{\text {bkg err }} & =\frac{\sum_{j} U_{i j}\left(d_{j}-\left[b_{j} \pm \delta b_{j}\right]\right)}{\Delta T_{\mu} \Delta \cos \theta_{\mu} \epsilon_{i} \Phi N} .
\end{aligned}
$$

where $X$ is a single-differential cross sections and $\delta b$ is the uncertainty on each background. Since the error matrix formed by these uncertainties involves squaring differences between these alternate calculations and the central value cross sections, it is irrelevant whether the uncertainty on the background is added or subtracted in Equation 8.10. A separate error matrix is formed for each background by

$$
\mathrm{EM}_{i j}^{\mathrm{bkg}}=\left(\sigma_{i}^{\mathrm{CV}}-\sigma_{i}^{\mathrm{bkg}}\right) \times\left(\sigma_{j}^{\mathrm{CV}}-\sigma_{j}^{\mathrm{bkg}}\right)
$$

where $\sigma$ refers to any of the cross section measurements. The total error in a given bin $i$ for these error matrices is simply $\sqrt{\mathrm{EM}_{i i}}$.

The gross deviation of the cross sections calculated in Equation 8.10 compared to the central value is summarized in Section 8.4.3.

As mentioned in Section 8.4.2, uncertainties on processes affecting signal rates like flux and the optical model also affect background levels and so a small part of these errors are due to backgrounds. 


\subsubsection{Signal Errors}

Uncertainties affecting signal rates are handled in subtly different ways, according to how the excursions from the central value are practically generated.

Flux, inter-medium pion interactions, and model dependence errors are evaluated by the MultisimMatrix package. It takes as input a covariance matrix for a set of parameters and generates a set of weights corresponding to individual throws against a Gaussian distribution for each parameter, according to its specified uncertainty, and is constrained by correlations. Each set of weights $k$ are then used to calculate the range of cross sections due to the input covariance matrix:

$$
\begin{aligned}
& \sigma\left(E_{\nu}\right)_{i}^{k}=\frac{\sum_{j} U_{i j}^{k}\left(d_{j}-b_{j}^{k}\right)}{\left[\frac{N^{\text {acc, } \mathrm{k}}}{N^{\text {gen }, \mathrm{CV}}}\right]_{i} \Phi_{i} N}, \\
& {\frac{d \sigma^{k}}{d X}}_{i}=\frac{\sum_{j} U_{i j}^{k}\left(d_{j}-b_{j}^{k}\right)}{\left[\frac{N^{\text {acc, } \mathrm{k}}}{N^{\text {gen }, \mathrm{CV}}}\right]_{i} \Delta X \Phi N}, \\
& \frac{d^{2} \sigma}{d T_{\mu} d\left(\cos \theta_{\mu}\right)_{i}}=\frac{\sum_{j} U_{i j}^{k}\left(d_{j}-b_{j}^{k}\right)}{\left[\frac{N^{\text {acc, } \mathrm{k}}}{N^{\text {gen }, \mathrm{CV}}}\right]_{i} \Delta T_{\mu} \Delta \cos \theta_{\mu} \Phi N} \text {. }
\end{aligned}
$$

The set of weights appear in three terms: the unsmearing matrix $U_{i j}^{k}$, the background prediction $b_{j}^{k}$ and in an alternate efficiency calculation $\left[\frac{N^{\text {acc, } \mathrm{k}}}{N^{\text {gen,CV }}}\right]_{i}$. The alternate unsmearing matrix incorporates shape uncertainties in the generated signal distribution. Perhaps most intuitively, for example, this is where uncertainties on the flux spectrum will result in shape errors on the total cross section.

The final term $\frac{N^{\text {acc, }}}{N^{\text {gen,CV }}}$ incorporates uncertainties on the signal process due to the $k$ th throw, if any. $N^{\text {acc,k }}$ refers to the number of signal events passing selection for the $k$ th excursion from the central value, while $N^{\text {gen, } \mathrm{CV}}$ is the distribution of signal events before cuts for the central value predition. It may be non-intuitive to account for flux uncertainties through the effeciency term, but it is trivial to see how a flux excursion from 
the nominal prediction would affect the calculated error on the cross section in an identical fashion if the normalization difference originated in the $\epsilon$ term rather than the $\Phi$ term. Note that, in principle, this could lead to a calculated efficiency greater than 1 , but of course these factors are related to normalization uncertainties and not true detection rates. Since the errors discussed in this section are generated by reweighting the central value prediction, it is crucial that the denominator in this alternate efficiency calculation refer to the generated central value prediction, and not that from the $k$ th generated distribution. If the weighted generated distribution were taken, the weights intended to be propagated as uncertainties onto the data would be suppressed.

This is also the method for measuring the cross-section uncertainty due to the model for light propagation in the detector (described in Section 5.3.2), where 35 possibly correlated parameters are varied within their uncertainty according to external measurements and calibration data. In this case, $k$ in Equation 8.12 refers to the $k$ th optical model. Additionally, to remove the statistical error the optical models were generated with, for the neutrino energy and $Q_{Q E}^{2}$ variables the ratio of each optical model to the central value prediction is smoothed to a fourth order polynomial. Such attempts at smoothing for the two-dimensional distribution would be untenable, so to minimize the intrinsic statistical error of the optical models, the size of each sample used is increased to a little more than twice that of the data statistics. As statistical error in this analysis is negligible, this mild overestimate negligibly affects the extracted cross section.

For the optical model and each systematic uncertainty evaluated with MultisimMatrix, the error matrix is calculated by:

$$
\mathrm{EM}_{i j}=\frac{\sum_{k=1}^{N_{k}}\left(\sigma_{i}^{\mathrm{CV}}-\sigma_{i}^{k}\right) \times\left(\sigma_{j}^{\mathrm{CV}}-\sigma_{j}^{k}\right)}{N_{k}-1}
$$


where $N_{k}$ refers to the number of variations from the central value used, and again $\sigma$ refers to the various cross sections calculated. $N_{k}=100$ for uncertainties on the $\pi^{-}$flux, the $\bar{\nu}_{\mu}$ CCQE model dependence, processes entering the sample due to $\pi$ charge exchange or absorption, and, in the case of calculating the carbon-only $\bar{\nu}_{\mu}$ CCQE cross sections, the $\bar{\nu}_{\mu}$ CCQE hydrogen background. $N_{k}=70$ for the optical model variations.

The flux errors due to $\pi^{-}$production at the target are taken from a spline fit to the $\operatorname{HARP} \pi^{-}$double-differential cross-section uncertainties. All other flux uncertainties not directly related to secondary $\pi^{-}$production are referred to as beam unisim errors, and the most important of these include reinteractions in the target and uncertainties due to magnetic focusing and are further described in Section 5.2.4. The uncertainties on the inter-medium pion interactions of charge exchange (absorption) are set at 50\% (35\%) based on comparisons between simulation and external data [107].

The model dependence of the unsmearing procedure is evaluated by forming variations of the unsmearing matrix $\check{U}$ with conservative errors on the underlying model parameters $M_{A}^{C}=1.35 \pm 0.35 \mathrm{GeV}, M_{A}^{H}=1.02 \pm 0.35 \mathrm{GeV}$, and $\kappa=1.007 \pm 0.007$. In this case, only the matrix $\check{U}$ is varied in Equation 8.12, as it is the only term sensitive to the underlying physics model. Finally, applicable only when the hydrogen CCQE component is treated as background, its rates are varied according to a global fit to the light-target data, where $M_{A}^{H}=1.020 \pm 0.014 \mathrm{GeV}$.

The final systematic errors are related to uncertainties on the PMT discriminator threshold (labeled in figures and tables as "disc") and changes in rates due to charge-time correlation ("QT corr") effects. An independent MC sample is available for each uncertainty, and so the alternate cross sections extracted using these samples are entirely based on their distributions: 


$$
\begin{array}{r}
\sigma\left(E_{\nu}\right)_{i}^{p}=\frac{\sum_{j} U_{i j}^{p}\left(d_{j}-b_{j}^{p}\right)}{\epsilon_{i}^{p} \Phi_{i} N}, \\
\frac{d \sigma^{p}}{d X_{i}}=\frac{\sum_{j} U_{i j}^{p}\left(d_{j}-b_{j}^{p}\right)}{\epsilon_{i}^{p} \Delta X \Phi N}, \\
\frac{d^{2} \sigma \quad{ }^{p}}{d T_{\mu} d\left(\cos \theta_{\mu}\right)_{i}}=\frac{\sum_{j} U_{i j}^{p}\left(d_{j}-b_{j}^{p}\right)}{\epsilon_{i}^{p} \Delta T_{\mu} \Delta \cos \theta_{\mu} \Phi N} .
\end{array}
$$

where $p$ refers to each of the two distributions with alternate assumptions on the PMT behavior. Note that this is identical to Equation 8.12 with the replacement of the MC central value generated distribution in the effective efficiency calculation by the same quantity in the independent samples. The error matrices associated with these detector uncertainties are calculated by:

$$
\mathrm{EM}_{i j}^{p}=\left(\sigma_{i}^{\mathrm{CV}}-\sigma_{i}^{p}\right) \times\left(\sigma_{j}^{\mathrm{CV}}-\sigma_{j}^{p}\right)
$$

The various contributions to the total uncertainty for the $\bar{\nu}_{\mu}$ CCQE cross sections incident on mineral oil and on carbon only are summarized in Sections 8.4.3 and 8.4.4.

\subsubsection{Uncertainty Summary for $\bar{\nu}_{\mu}$ CCQE on Mineral Oil}

The total error matrix is formed by simply adding together each error matrix calculated in the previous subsections. As the entries of the error matrix represent bin-by-bin variances and covariances, the linear addition of the entries is equivalent to addition in quadrature. Then, the total uncertainty in bin $i$ is simply $\sqrt{E M_{i i}^{\text {tot }}}$. The overall effect and relative importance of each error can be evaluated with bin-by-bin fractional error $\left(\frac{\sqrt{E M_{i i}^{\text {tot }}}}{\sigma_{i}^{\mathrm{CV}}}\right)$, where $\sigma^{\mathrm{CV}}$ is the central value cross section for each uncertainty. Figure 8-15 shows fractional errors for each source of uncertainty for the four one-dimensional distributions and their sum for the two-dimensional cross section. Figure 8-16 shows the same for those with large maximum uncertainties. 

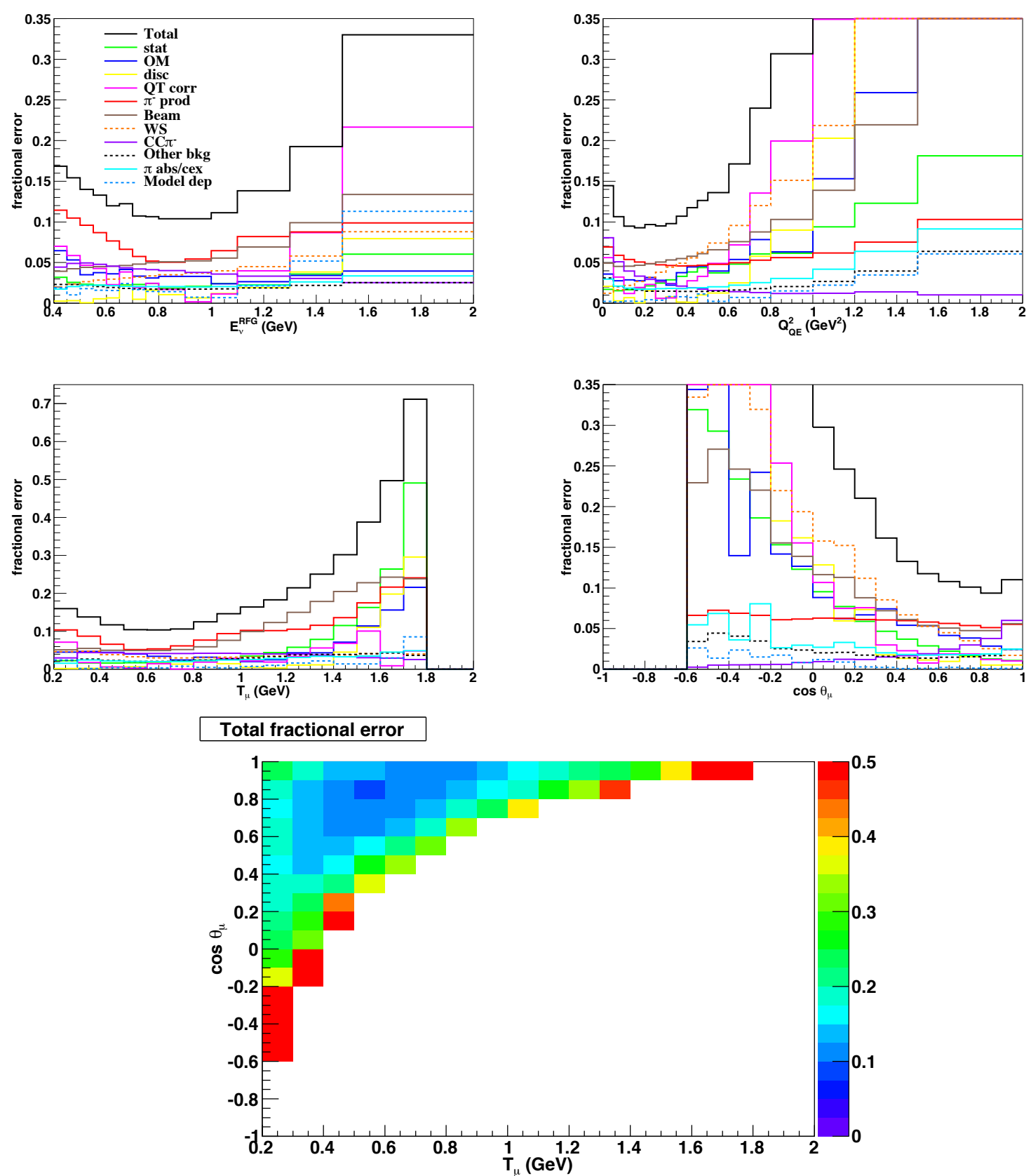

Figure 8-15. Fractional uncertainty contributions to the total and differential cross sections including the hydrogen content as signal. The total uncertainty is the quadrature sum of the error sources shown. 

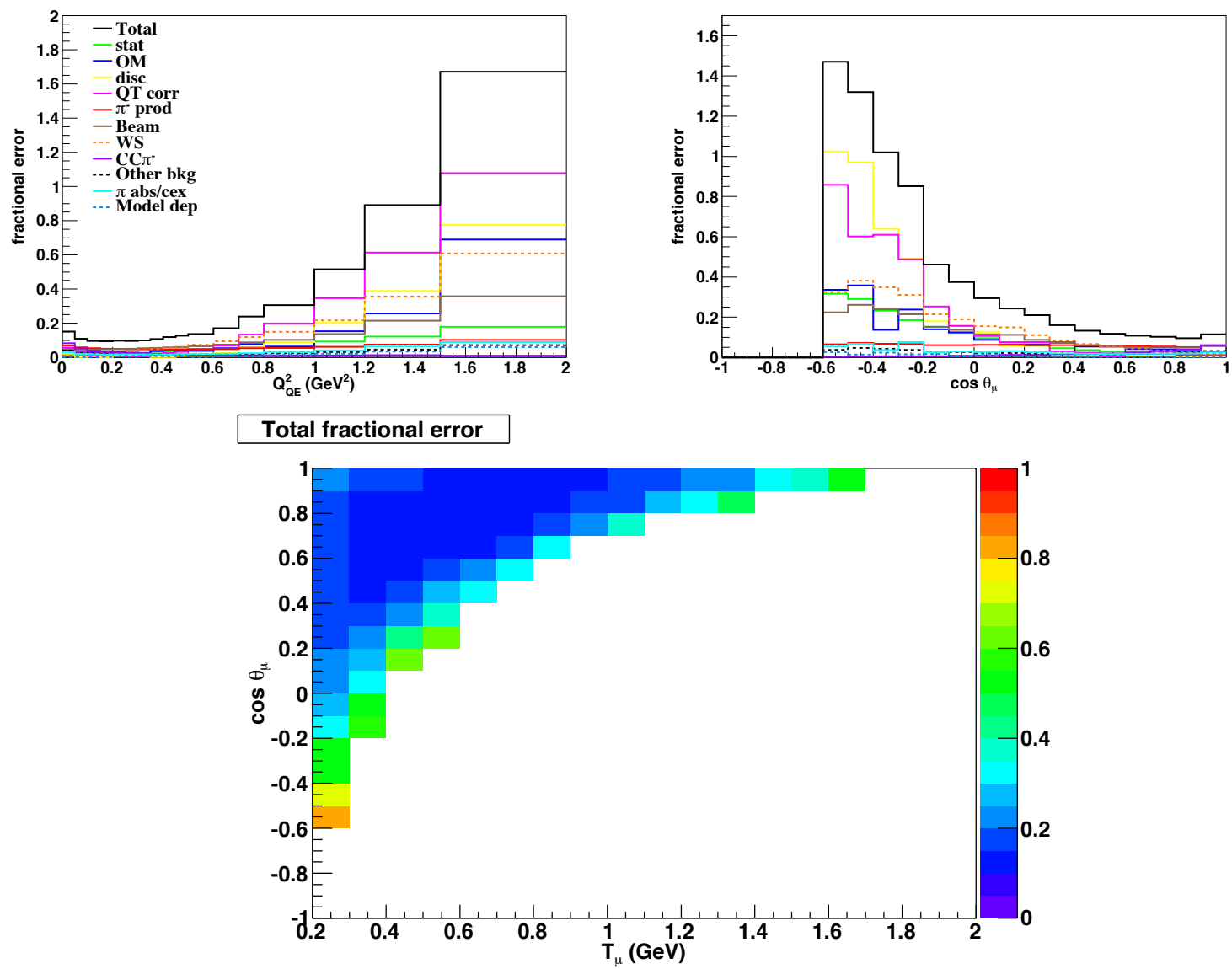

Figure 8-16. The full range of fractional uncertainty for $Q_{Q E}^{2}, \cos \theta_{\mu}$ and the doubledifferential cross sections including the hydrogen content as signal. 
The overall uncertainty for each error source can be reported numerically with the total normalization error. This quantity is equivalent to the total uncertainty if the measurement were a single number (e.g., a distribution with a single bin). Using the sum rule for variances and covariances $\left(\sigma_{i+j}^{2}=\sigma_{i}^{2}+\sigma_{j}^{2}+2 \rho_{i j} \sigma_{i} \sigma_{j}\right.$, where $\sigma[\rho]$ refers to a total error [correlation]), the total normalization error for a given uncertainty in terms of its error matrix is simply

$$
\text { Norm. error }=\frac{\sqrt{\sum_{i j} \mathrm{EM}_{i j}}}{\sum_{i} \sigma_{i}^{\mathrm{CV}}}
$$

These values are given in Table 8-2 for each uncertainty and each result. Due to the exclusion of some events whose value in the distribution may be excluded from the choices in binning, care must be taken in comparing normalization uncertainties across the different distributions. Events generated with $\mathrm{T}_{\mu}<0.2 \mathrm{GeV}$ and $T_{\mu}>2 \mathrm{GeV}$ are not

recovered through the efficiency calculation in the $\frac{d \sigma}{d T_{\mu}}$ and $\frac{d^{2} \sigma}{d T_{\mu} d \cos \theta_{\mu}}$ cross sections, while only events whose true energy would lie in $0.4>E_{\nu}^{R F G}>2 \mathrm{GeV}$ are included for the total cross section. For $\cos \theta_{\mu}$ the entire range of kinematics is included, while the same is almost true for $Q_{Q E}^{2}$, where the effective cut of $Q_{Q E}^{2}>2 \mathrm{GeV}^{2}$ excludes very few events.

\subsubsection{Uncertainty Summary for $\bar{\nu}_{\mu}$ CCQE on Carbon}

Fractional uncertainty levels for the results treating the free scattering component of $\bar{\nu}_{\mu}$ CCQE interactions as background are given in Table 8-3 and Figure 8-17. Figure 8-18 shows the full range of fractional uncertainty for those with some values greater than unity. 

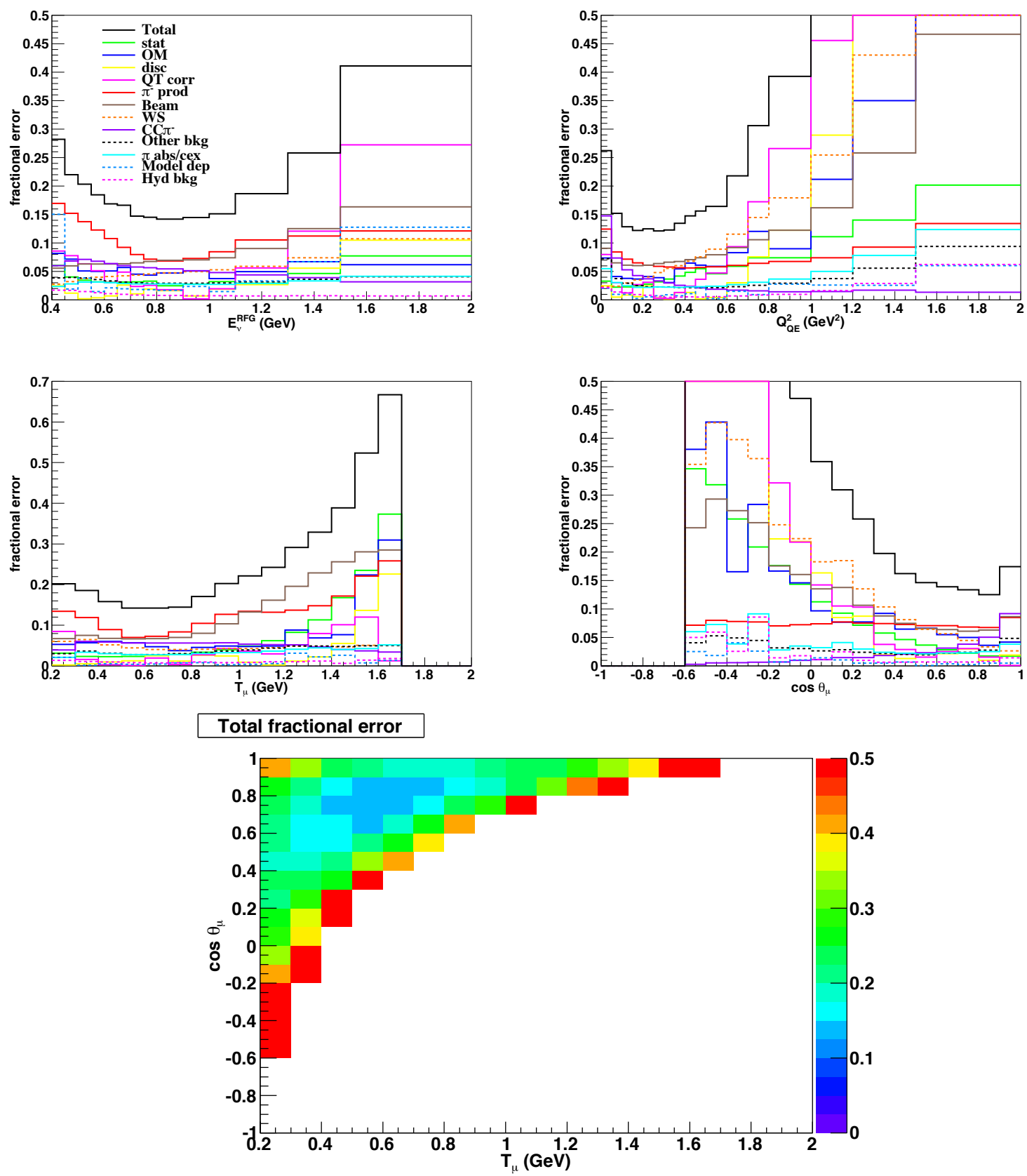

Figure 8-17. Fractional uncertainty contributions to the total and differential cross sections taking $\bar{\nu}_{\mu}$ CCQE interaction on hydrogen as background. The total uncertainty is the quadrature sum of the error sources shown. 

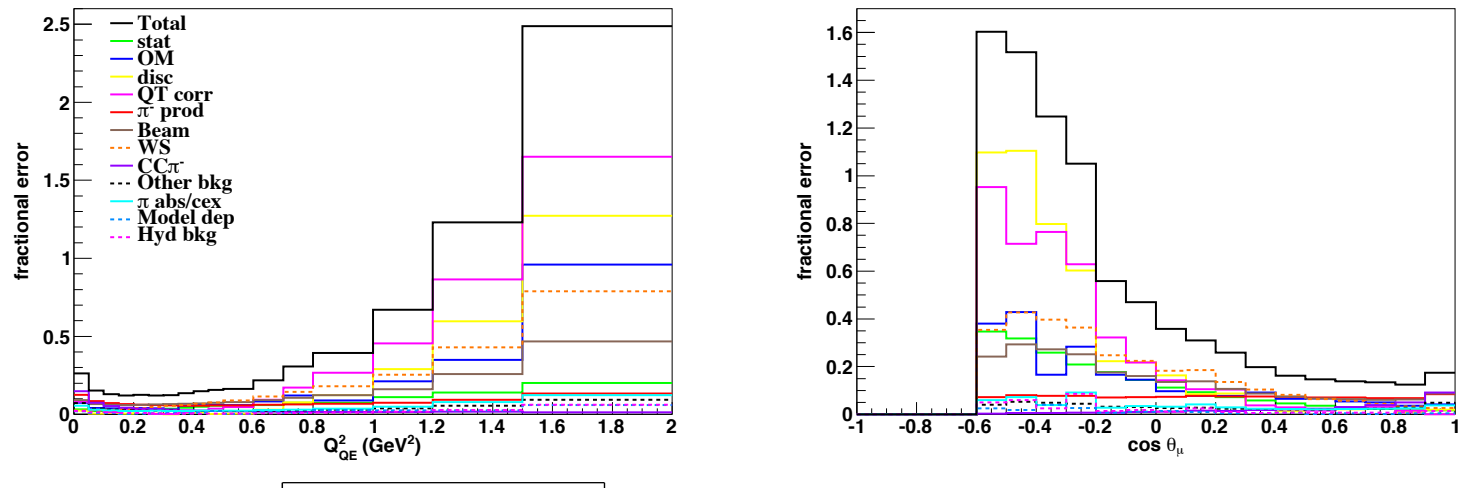

Total fractional error

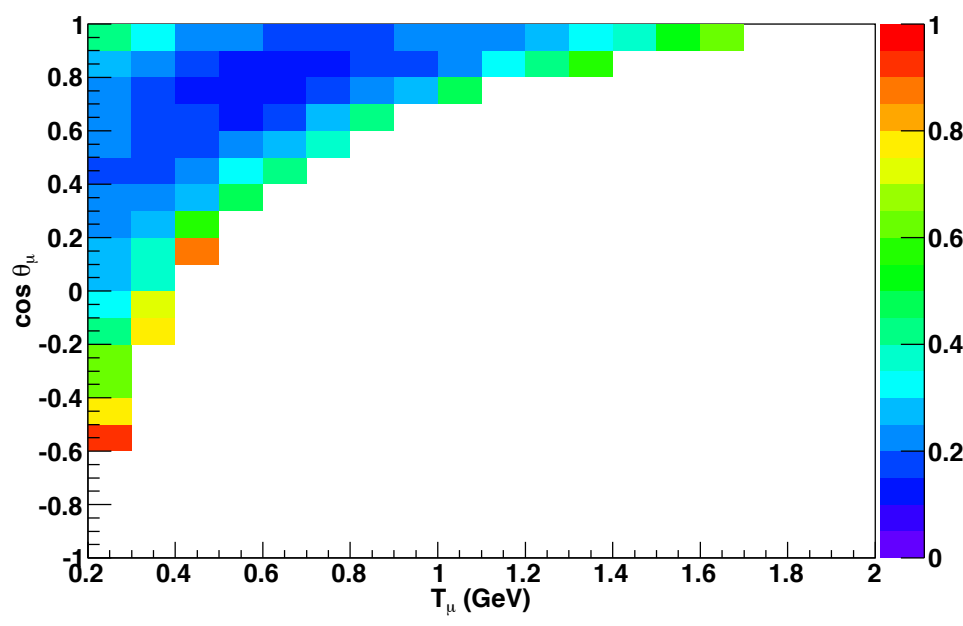

Figure 8-18. The full range of fractional uncertainty for $Q_{Q E}^{2}, \cos \theta_{\mu}$ and the doubledifferential cross sections. 
Table 8-2. Normalization errors for each cross section and each source of error for $\bar{\nu}_{\mu}$ CCQE events on mineral oil. Due to differences in cross-section shapes and relative regional sensitivity to each uncertainty, the normalization errors vary by a few percent across the distributions. The hydrogen content is included as signal here. $T_{\mu}-\cos \theta_{\mu}$ refers to the doubledifferential cross section.

\begin{tabular}{clcccc}
\hline Uncertainty source & \multicolumn{5}{c}{ Normalization error $(\%)$} \\
& $E_{\nu}^{R F G}$ & $Q_{Q E}^{2}$ & $T_{\mu}$ & $\cos \theta_{\mu}$ & $T_{\mu^{-}}$ \\
& & & & & $\cos \theta_{\mu}$ \\
\hline Statistics & 1.1 & 0.7 & 1.0 & 0.7 & 0.8 \\
Optical model & 2.8 & 3.1 & 2.9 & 3.8 & 3.8 \\
PMT discriminator thresh. & 1.5 & 0.1 & 0.7 & 1.7 & 0.1 \\
PMT charge-time corr. & 2.9 & 1.4 & 0.1 & 0.7 & 0.9 \\
$\pi^{-}$production & 5.1 & 5.3 & 5.4 & 5.2 & 6.4 \\
Beam unisims & 6.4 & 5.0 & 6.5 & 5.5 & 7.2 \\
All $\nu_{\mu}$ background & 4.4 & 3.1 & 3.6 & 4.6 & 3.5 \\
CC $\pi^{-}$background & 3.8 & 4.3 & 4.0 & 4.4 & 4.0 \\
Non-CCQE, non-CC $\pi^{ \pm}$background & 2.6 & 2.5 & 2.6 & 2.7 & 2.6 \\
Unsmearing model dependence & 1.7 & 0.0 & 0.2 & 0.2 & 0.8 \\
$\pi$ charge exchange + absorption & 2.3 & 2.1 & 2.3 & 2.2 & 2.3 \\
Total & $\mathbf{1 1 . 7}$ & $\mathbf{1 0 . 2}$ & $\mathbf{1 1 . 1}$ & $\mathbf{1 1 . 4}$ & $\mathbf{1 2 . 9}$ \\
\hline
\end{tabular}

\subsection{Results}

Results for defining the analysis signal as either all $\bar{\nu}_{\mu}$ CCQE interactions or only those bound in carbon atoms are presented in this section. While the more inclusive measurement is a more precise and less model-dependent measurement, the assumption that the free scattering interaction is well-known is motivated both by consistency among the previous light-target data sets and by theoretical calculations predicting enhancements only for bound nucleon targets.

\subsubsection{Results Incident on Mineral Oil}

Results including the hydrogen CCQE component are presented in Figure 8-19.

The agreement between data and the RFG model under various assumptions in the double-differential cross section is shown in Figure 8-20.

It is clear that the MC lies somewhat low from data in normalization, and the level of agreement in the shape can be evaluated by forming the shape-only error matrix. The 
Table 8-3. Normalization errors for each cross section and each source of error, treating $\bar{\nu}_{\mu}$ CCQE events on hydrogen as background. Fractional uncertainties are generally higher compared to those reported in Table 8-2 due to the significantly lower purity of the sample.

\begin{tabular}{clcccc}
\hline Uncertainty source & \multicolumn{5}{c}{ Normalization error (\%) } \\
& $E_{\nu}^{R F G}$ & $Q_{Q E}^{2}$ & $T_{\mu}$ & $\cos \theta_{\mu}$ & $T_{\mu^{-}}$ \\
& & & & & $\cos \theta_{\mu}$ \\
\hline Statistics & 1.4 & 0.9 & 1.3 & 1.0 & 1.2 \\
Optical model & 3.9 & 4.1 & 4.6 & 5.0 & 4.2 \\
PMT discriminator thresh. & 2.3 & 0.3 & 0.4 & 2.3 & 1.1 \\
PMT charge-time corr. & 4.2 & 1.5 & 1.9 & 1.4 & 2.6 \\
$\pi^{-}$production & 6.8 & 7.2 & 6.7 & 7.1 & 8.0 \\
Beam unisims & 8.3 & 6.8 & 9.0 & 7.4 & 9.2 \\
All $\nu_{\mu}$ background & 5.8 & 4.1 & 6.2 & 5.4 & 6.8 \\
CC $\pi^{-}$background & 5.1 & 5.8 & 6.7 & 5.9 & 7.4 \\
Non-CCQE, non-CC $\pi^{ \pm}$background & 3.4 & 3.3 & 4.8 & 3.6 & 5.5 \\
Unsmearing model dependence & 2.4 & 0.0 & 1.4 & 0.2 & 2.2 \\
Hydrogen background & 0.8 & 1.0 & 1.6 & 0.9 & 2.2 \\
$\pi$ charge exchange + absorption & 3.0 & 2.9 & 3.3 & 3.0 & 3.6 \\
Total & $\mathbf{1 5 . 5}$ & $\mathbf{1 3 . 8}$ & $\mathbf{1 7 . 2}$ & $\mathbf{1 5 . 0}$ & $\mathbf{1 8 . 6}$ \\
\hline
\end{tabular}

covariance matrix can be used to separate the correlated normalization uncertainties from the total error, leaving information related to how much the shape of the observed data may vary within the systematic errors [69]. These uncertainties are identified by first defining a data vector $V$ with entries corresponding to the observed relative normalization of each bin: $V_{i}=\left\{D_{1} / D_{T}, D_{2} / D_{T}, \cdots, D_{n} / D_{T}, D_{T}\right\}$. Notice this vector has dimension $n+1$, where $n$ is the number of bins measured. The covariance matrix $Q$ for this new vector $V$ involves the Jacobian matrix of partial derivatives $J$ and is given by:

$$
Q_{k l}=\sum_{i j}^{n} J_{k i} M_{i j} J_{l j}=\sum_{i j}^{n} \frac{\partial V_{k}}{\partial D_{i}} M_{i j} \frac{\partial V_{l}}{\partial D_{j}} .
$$

The diagonals of the matrix $Q$ are related to the shape uncertainty in each kinematic bin. For entries $\{1,2, \cdots, n\}$, 


$$
\begin{aligned}
Q_{k k} & =\frac{1}{D_{T}^{2}}\left[M_{k k}-2 \frac{D_{k}}{D_{T}} \sum_{i}^{n} M_{i k}+\frac{N_{k}^{2}}{N_{T}^{2}} \sum_{i j}^{n} M_{i j}\right] \\
& =\left(\delta D_{k, \text { shape }}\right)^{2}
\end{aligned}
$$

The shape and total error of the cross sections is compared to MC normalized to data in Figure 8-21. As values for $M_{A}$ are typically extracted from the $Q^{2}$ distribution, it's helpful to at least calculate the compatibility between data and MC. Printed on the $Q_{Q E}^{2}$ distribution is the $\chi^{2}$ between MC and data using shape-only uncertainties. 

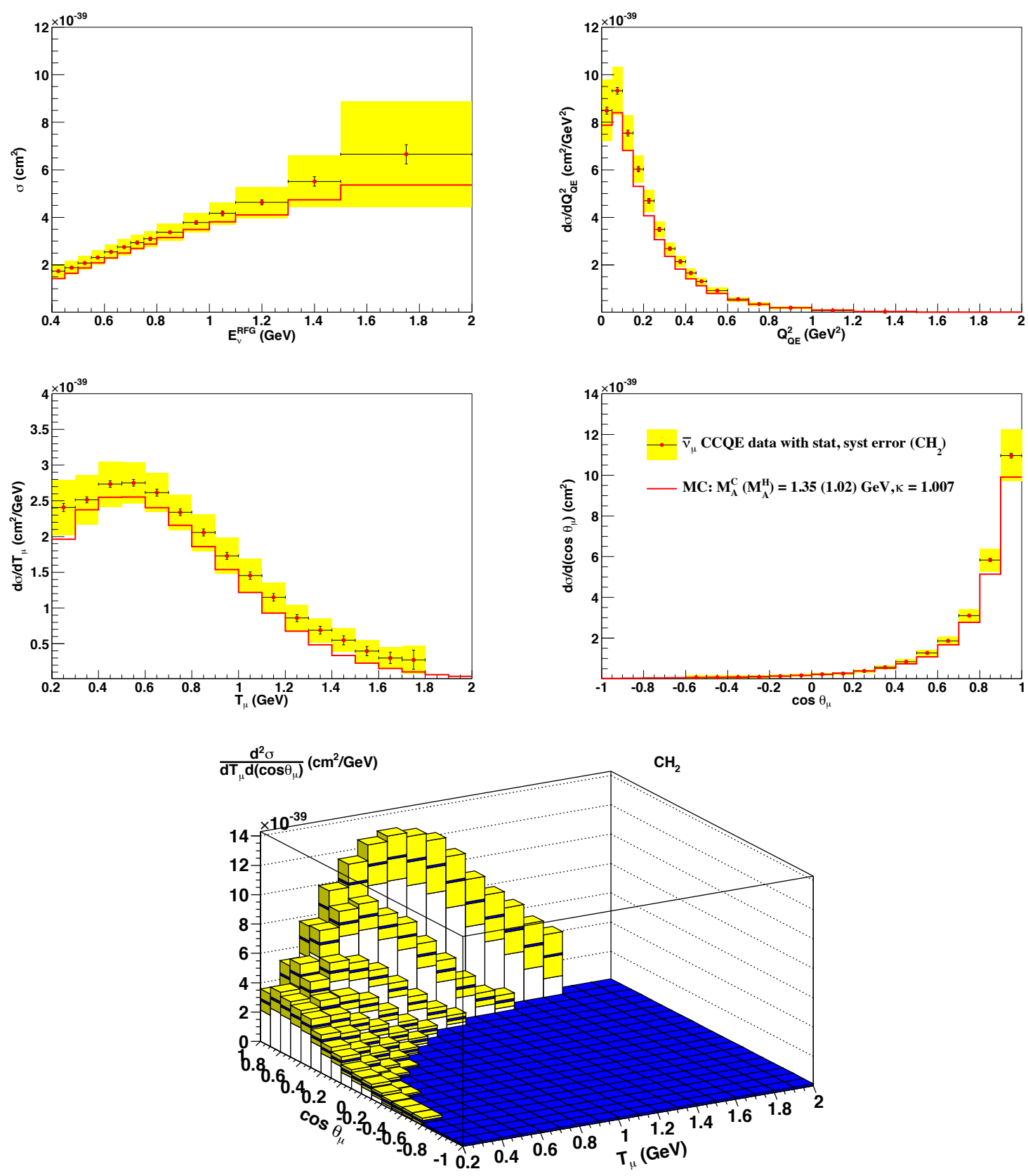

Figure 8-19. Extracted $\bar{\nu}_{\mu}$ CCQE cross sections with total uncertainty compared to central value MC with the hydrogen component not subtracted. 


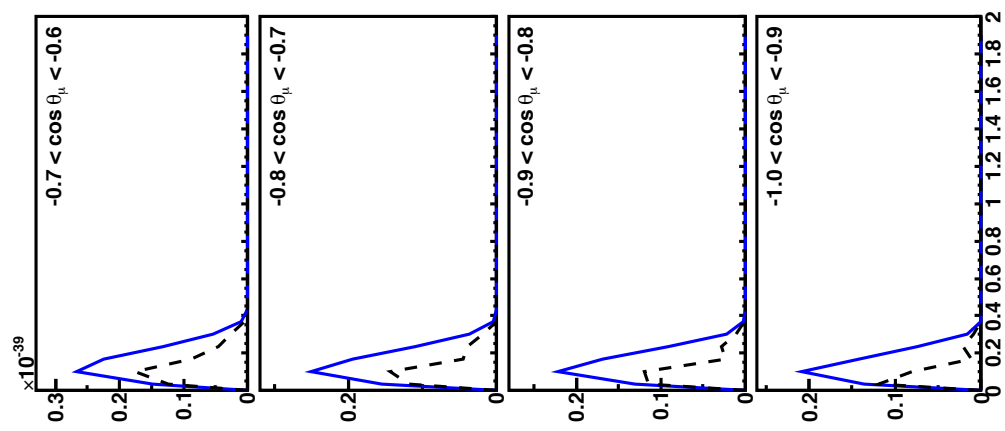

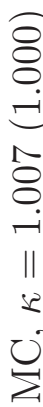
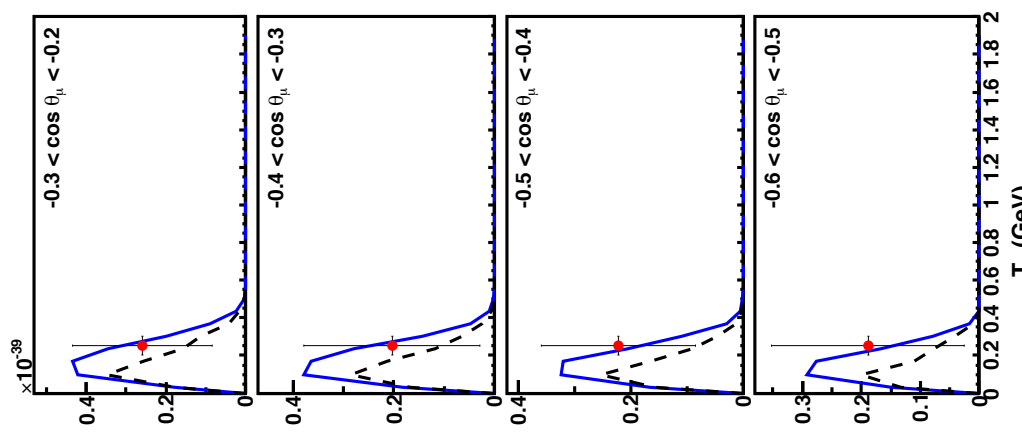

oิ
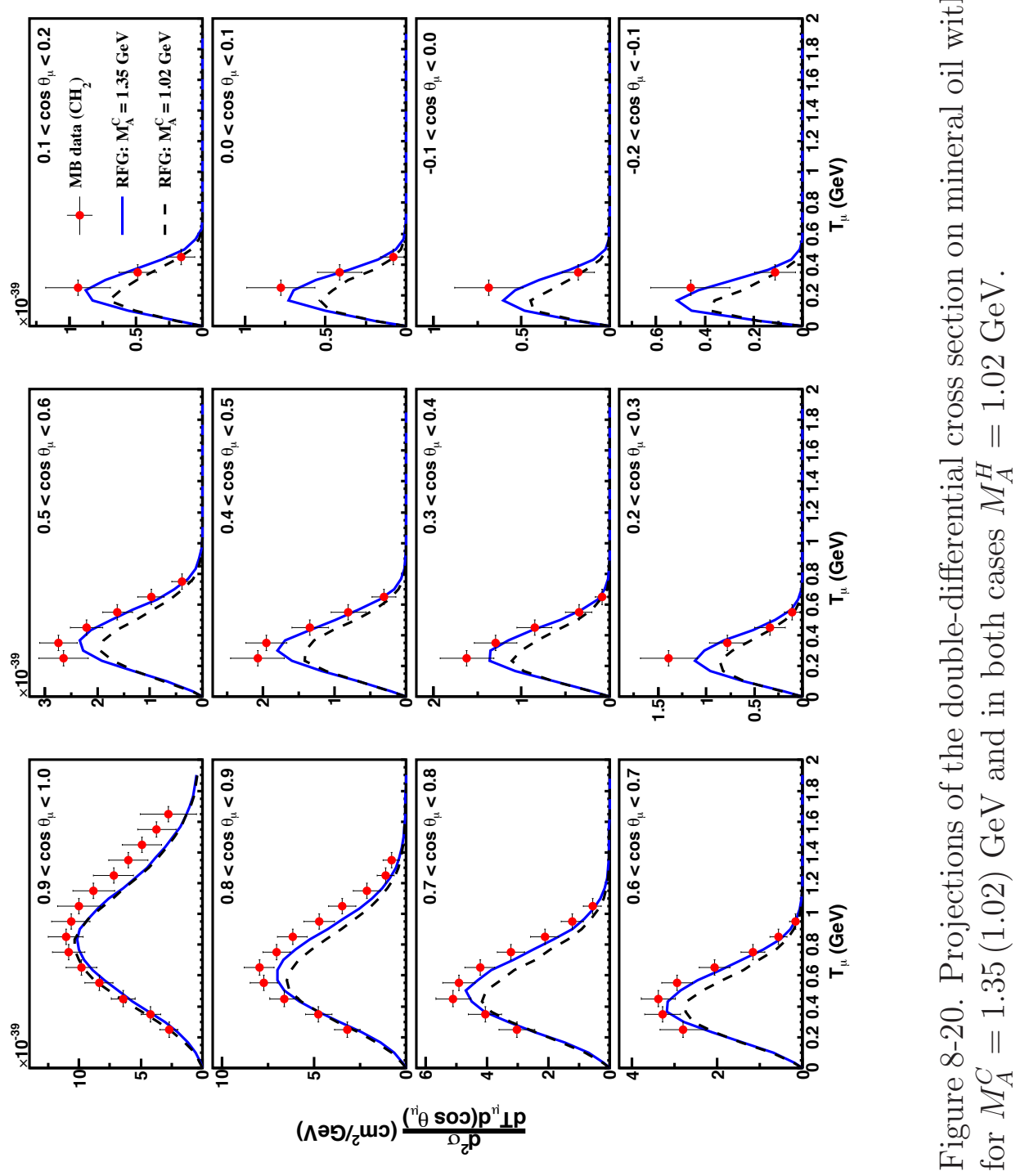

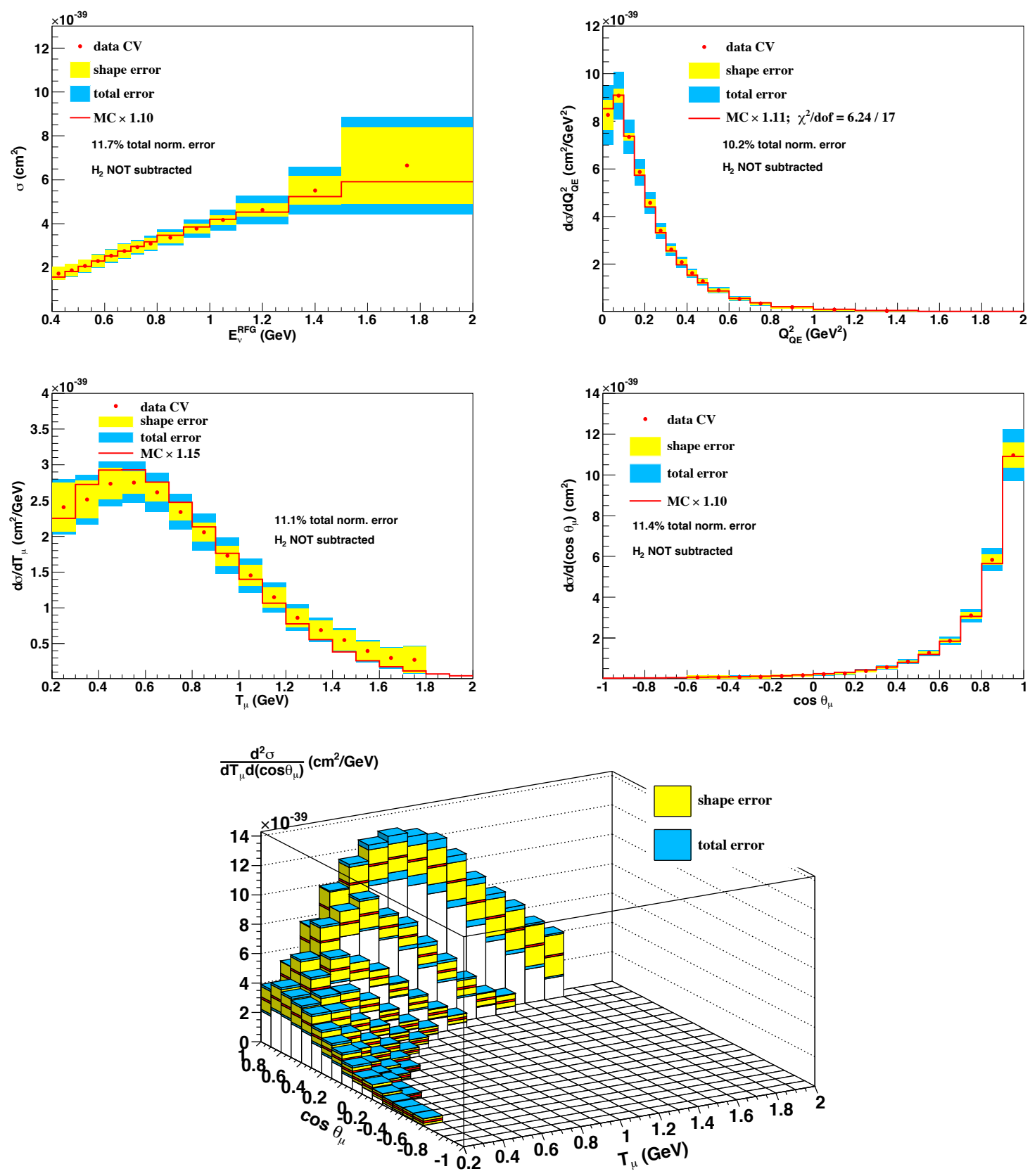

Figure 8-21. Extracted $\bar{\nu}_{\mu}$ CCQE cross sections with total and shape uncertainty compared to central value $\mathrm{MC}$ with the hydrogen component not subtracted. For the differential cross sections, $\mathrm{MC}$ is scaled to the integrated cross section from data $\left(\int \frac{d \sigma}{d X} d X\right)$, while for the total cross section $\mathrm{MC}$ is scaled to data based on the discrepancy in the bin at the interaction peak $(0.65-0.7 \mathrm{GeV})$. 


\subsubsection{Results Incident on Carbon}

Results for all distributions treating the free scattering $\bar{\nu}_{\mu}$ CCQE component as background are shown in Figure 8-22, and Figure 8-23 presents a detailed view of the double-differential cross section with comparisons to the RFG and three external predictions [98, 99, 96].

Analogous to Figure 8-21, Figure 8-24 compares shape and total errors on data to relatively normalized MC.

As CCQE model parameters are typically extracted from the $Q^{2}$ distribution, it's interesting to see how these data compare to the historically-accepted values. Figure 8-25 compares the shape of the RFG with various choices of $M_{A}$ and $\kappa$ to the data. To give a feel numerically for the shape compatibility of each distribution with the data, printed on the figure legend is the $\chi^{2}$ for each parameter choice using the shape-only uncertainties. The $\chi^{2}$ for the RFG with $M_{A}=1.35 \mathrm{GeV}, \kappa=1.007$ is surprisingly low at just 3.7 for 17 degrees of freedom.

Along the same lines, one of the only recent experiments using nuclear targets to measure $M_{A} \sim 1 \mathrm{GeV}$ is the NOMAD experiment. Much speculation revolving around the disparate energy regimes and detector types has been made in attempts to reconcile the discrepancy in $\nu_{\mu}$ CCQE cross section between MiniBooNE and NOMAD [140], and Figure 8-26 compares the $\nu_{\mu}$ and $\bar{\nu}_{\mu}$ CCQE data sets from both experiments.

Figure 8-27 compares the total cross sections to the available theoretical predictions [93, 95, 96, 97, 98, 99, 100], and Figure 8-28 also includes the level of agreement between the same models and the $\nu_{\mu}$ data.

It is clear that the RFG model with canonical assumptions does not adequately describe these data neither in shape nor in normalization. Consistent with other recent CCQE measurements on nuclear material [84, 87, 88, 125], a significant enhancement in the normalization that grows with decreasing muon scattering angle is observed compared to the expectation with $M_{A}=1 \mathrm{GeV}$. As discussed in Section 4.4, these observations are 
consistent with the presence of an intra-nuclear mechanism of greater importance than previously expected, and contributions from such a source are tested in comparisons between various predictions and the data analyzed here in Figures 8-23 and 8-27.

However, these data find tension with the NOMAD $\bar{\nu}_{\mu}$ CCQE results, which are described both in shape and normalization by $M_{A} \sim 1 \mathrm{GeV}$ [90]. This tension is also common to the $\nu_{\mu}$ CCQE analyses from the two experiments. However, care should be taken in comparing model-dependent results among experiments with such different neutrino fluxes and detector technologies. A definitive unification of these apparently discrepant data sets will require the continued increase of both experimental and theoretical activity surrounding this topic. Fortunately, many experiments at a variety of neutrino energies capable of making high-resolution, model-independent neutrino and antineutrino CCQE measurements with different detector technologies and nuclear media currently have data or will soon. These include MINER $\nu$ A [145], SciBooNE [172], MicroBooNE [173], ArgoNeuT [174], ICARUS [175] and the T2K [141] and NO $\nu \mathrm{A}$ [54] near detectors. 

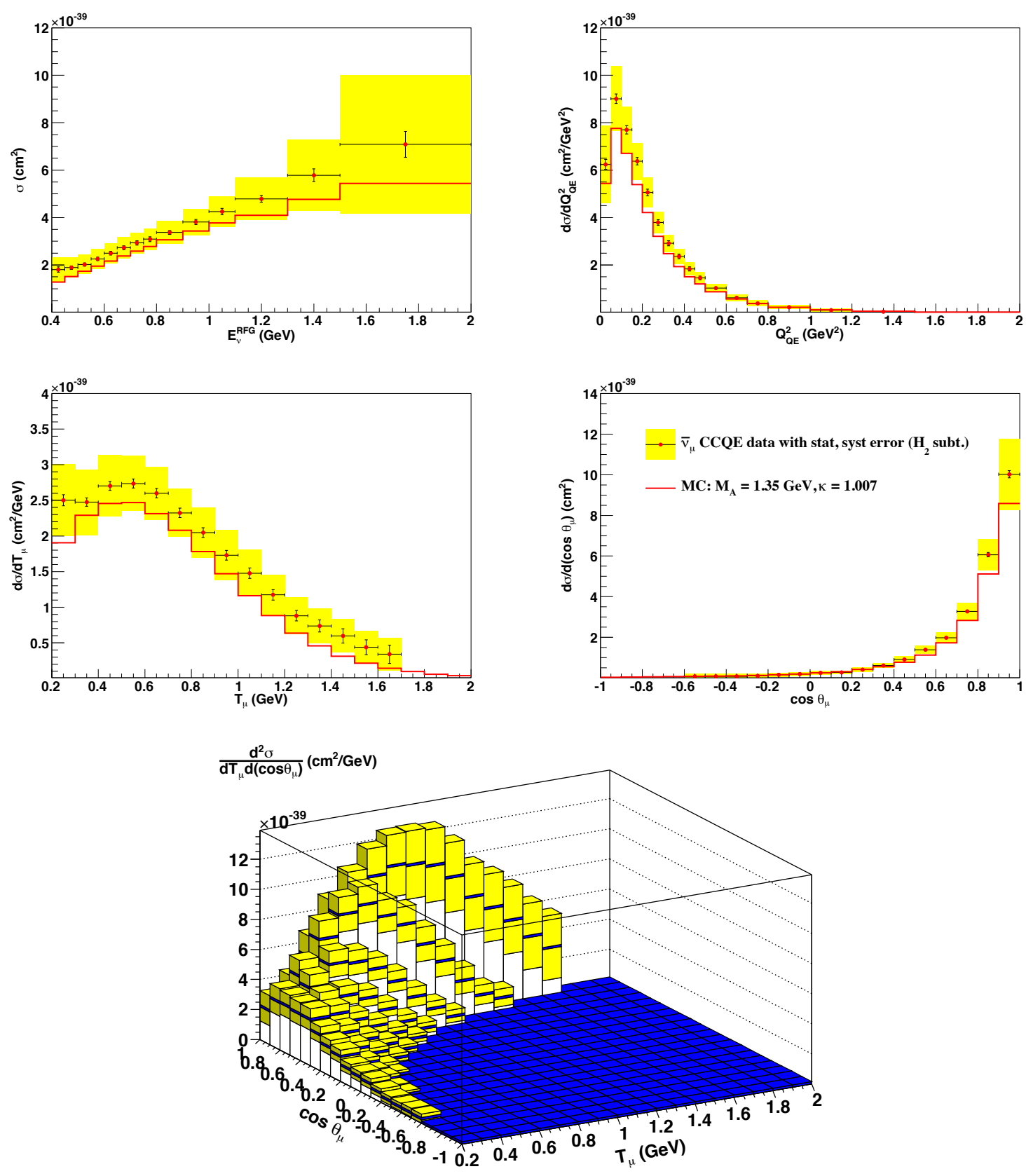

Figure 8-22. Extracted $\bar{\nu}_{\mu}$ CCQE cross sections with total uncertainty compared to central value $\mathrm{MC}$ with the hydrogen component subtracted. 


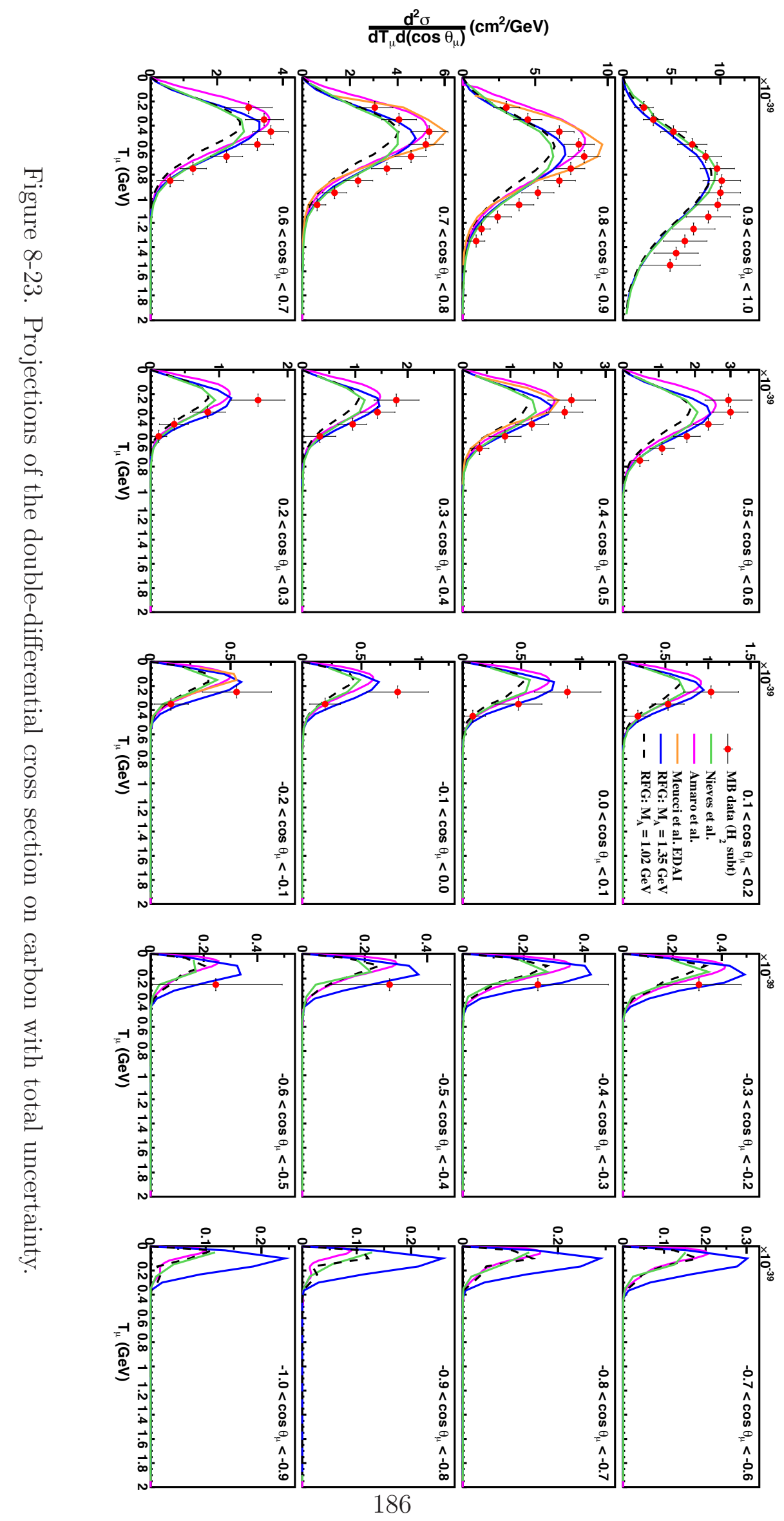



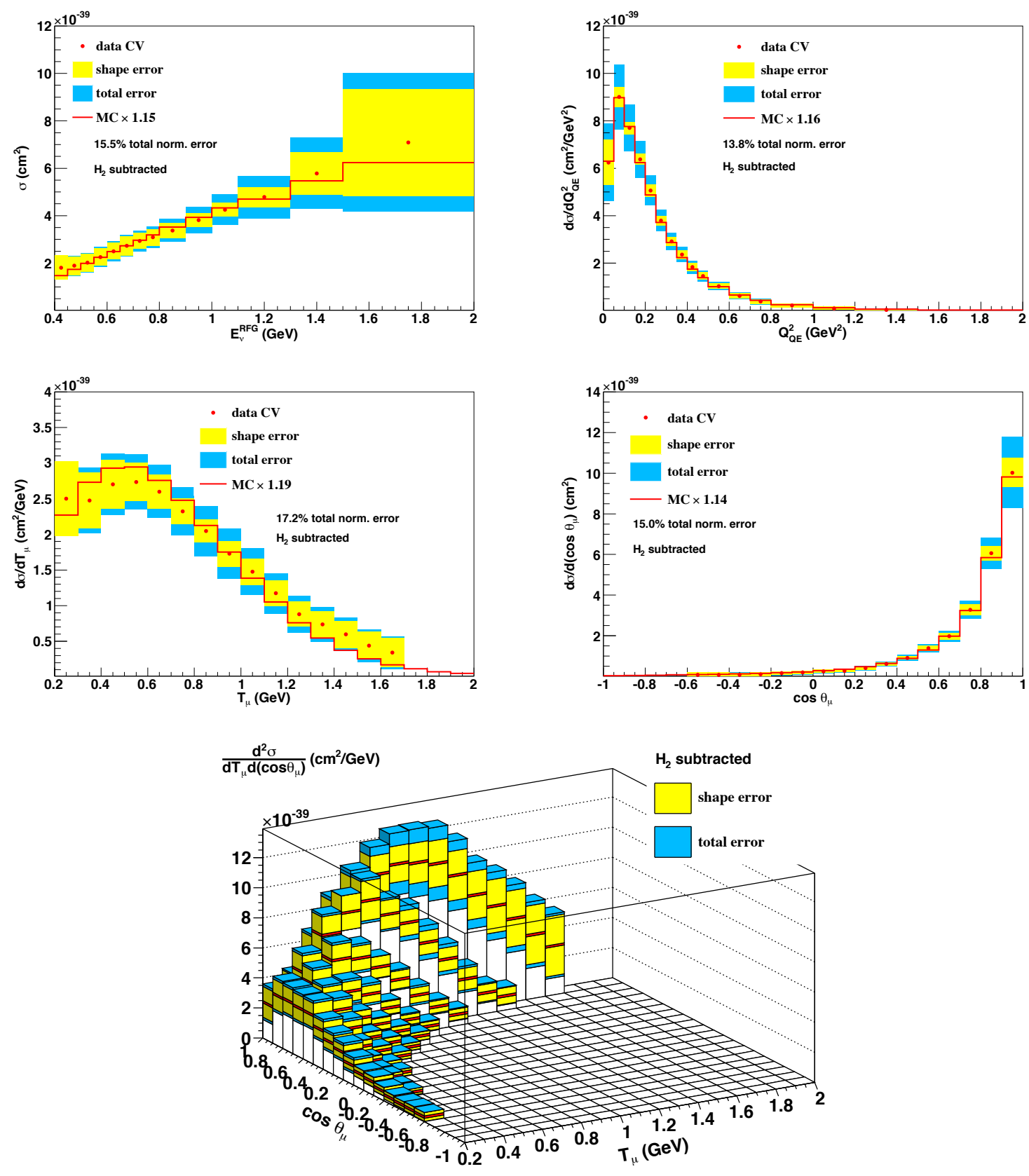

Figure 8-24. Extracted $\bar{\nu}_{\mu}$ CCQE cross sections with total and shape uncertainty compared to central value $\mathrm{MC}$ with the hydrogen component subtracted. For the differential cross sections, $\mathrm{MC}$ is scaled to the integrated cross section from data $\left(\int \frac{d \sigma}{d X} d X\right)$, while for the total cross section $\mathrm{MC}$ is scaled to data based on the discrepancy in the bin at the interaction peak $(0.65-0.7 \mathrm{GeV})$. 


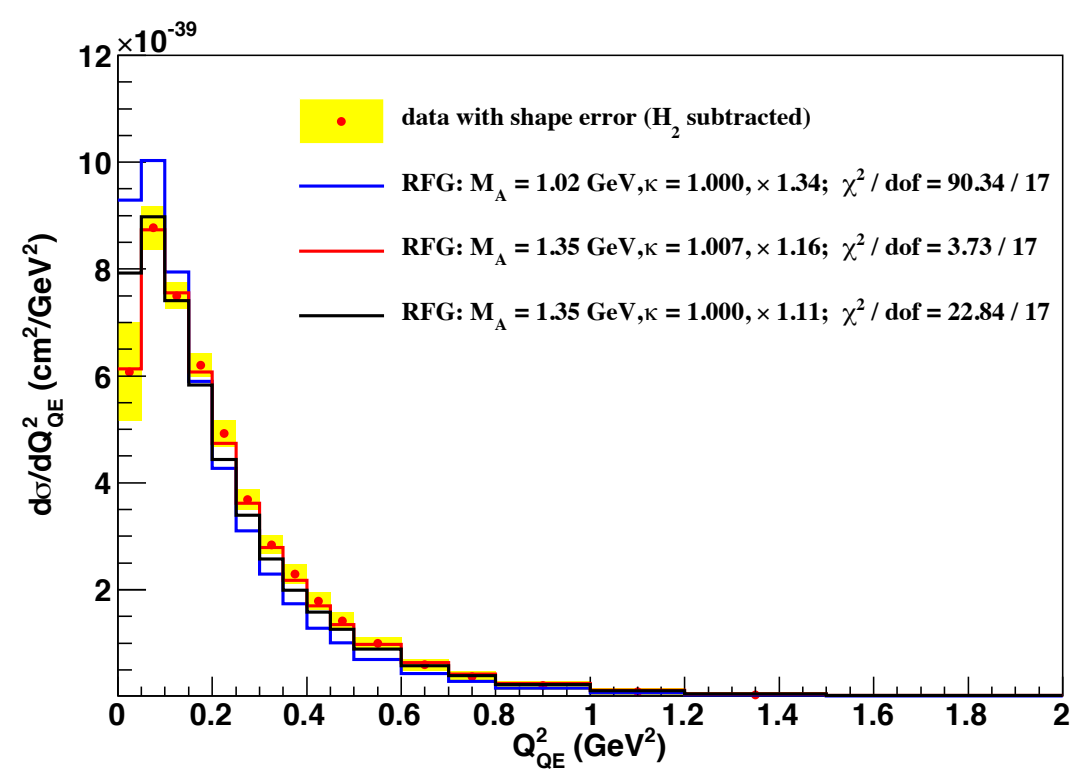

Figure 8-25. Shape comparison for hydrogen-subtracted data and the RFG under various parameter choices. The shape of central value $\mathrm{MC}$ with $M_{A}=1.35 \mathrm{GeV}$ and $\kappa=1.007$ agrees with data remarkably well. In particular, the mild change in $\kappa(1.000 \rightarrow 1.007)$ determined from the $\nu_{\mu} \mathrm{CCQE}$ analysis seems to be preferred by the data. Also printed on the figure is the scale for each prediction to match the data in normalization.

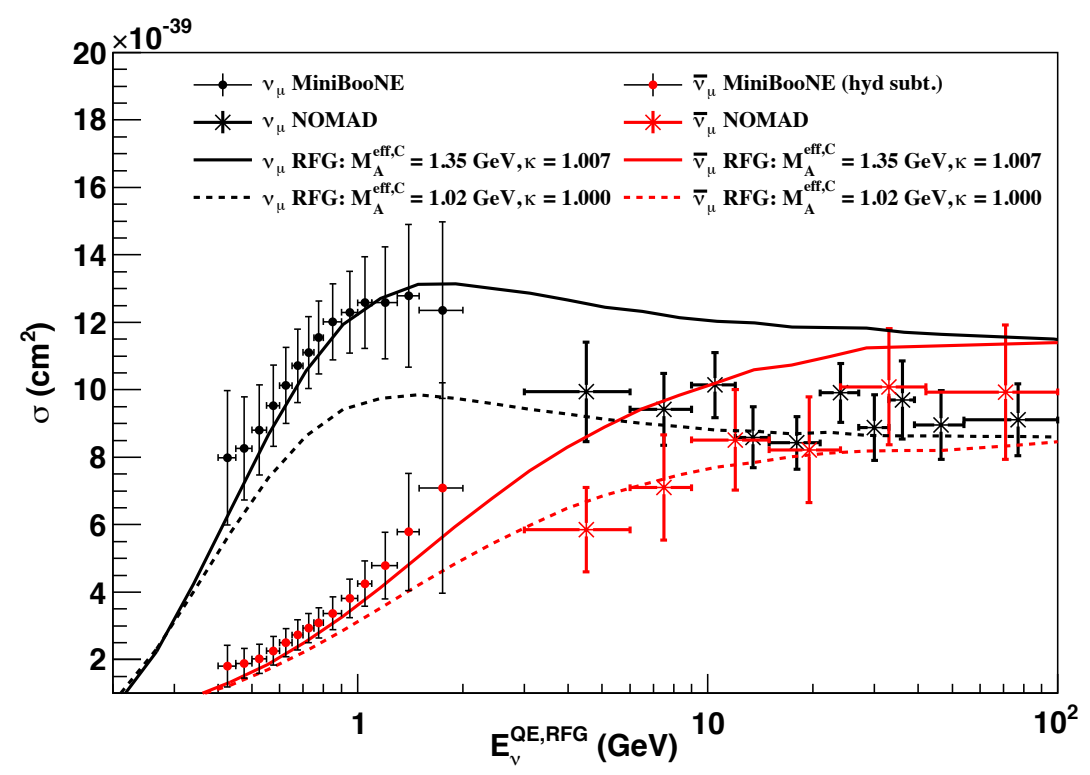

Figure 8-26. Comparison between MiniBooNE and NOMAD $\nu_{\mu}$ and $\bar{\nu}_{\mu}$ data, as well as some predictions from the RFG. Tension exists across both $\nu_{\mu}$ and $\bar{\nu}_{\mu}$ data from the two experiments under the assumptions of CCQE with the RFG. NOMAD data from Ref. [171]. 


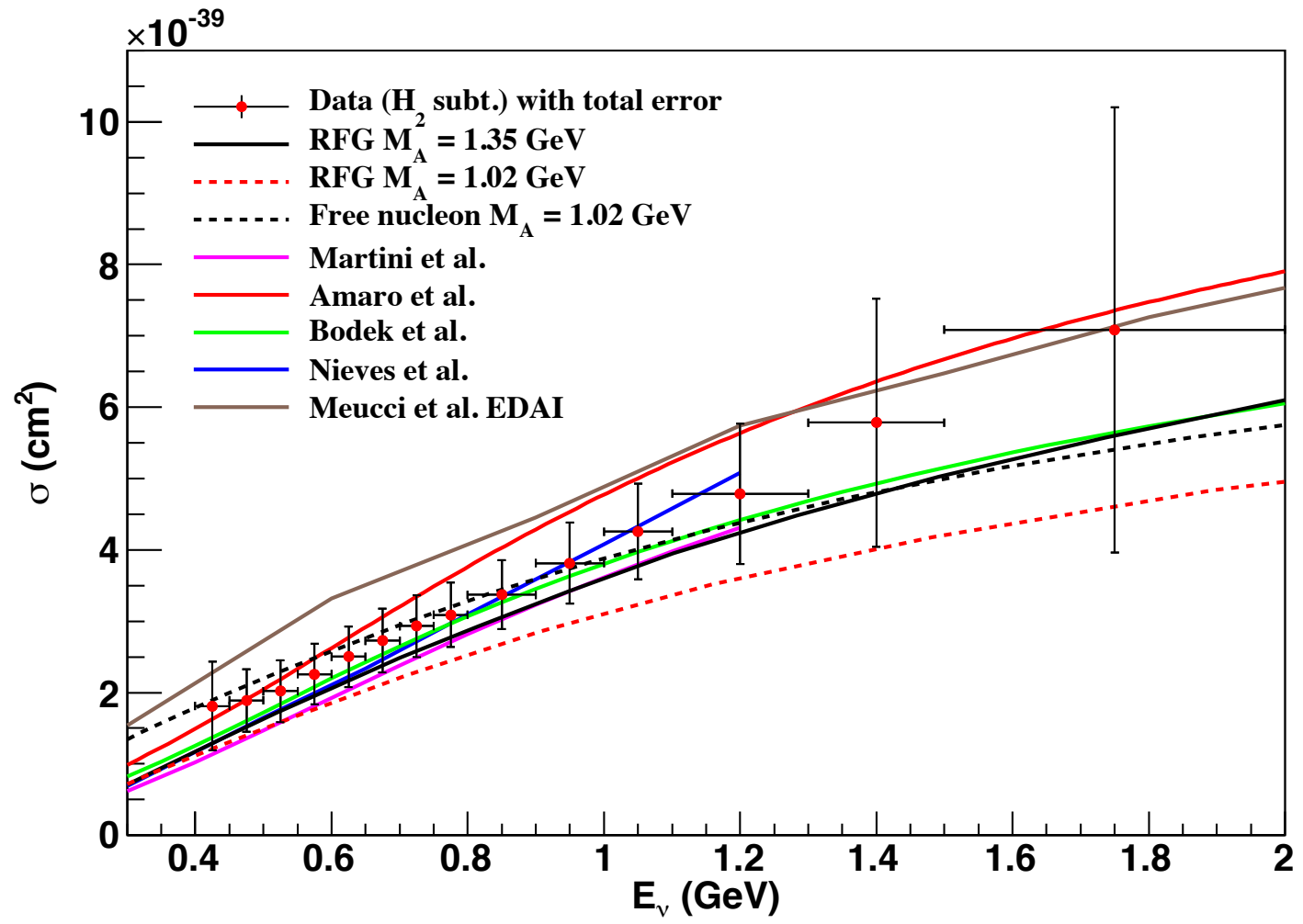

Figure 8-27. Total cross section per nucleon for $\bar{\nu}_{\mu}$ CCQE data with the hydrogen content removed compared to various predictions. 


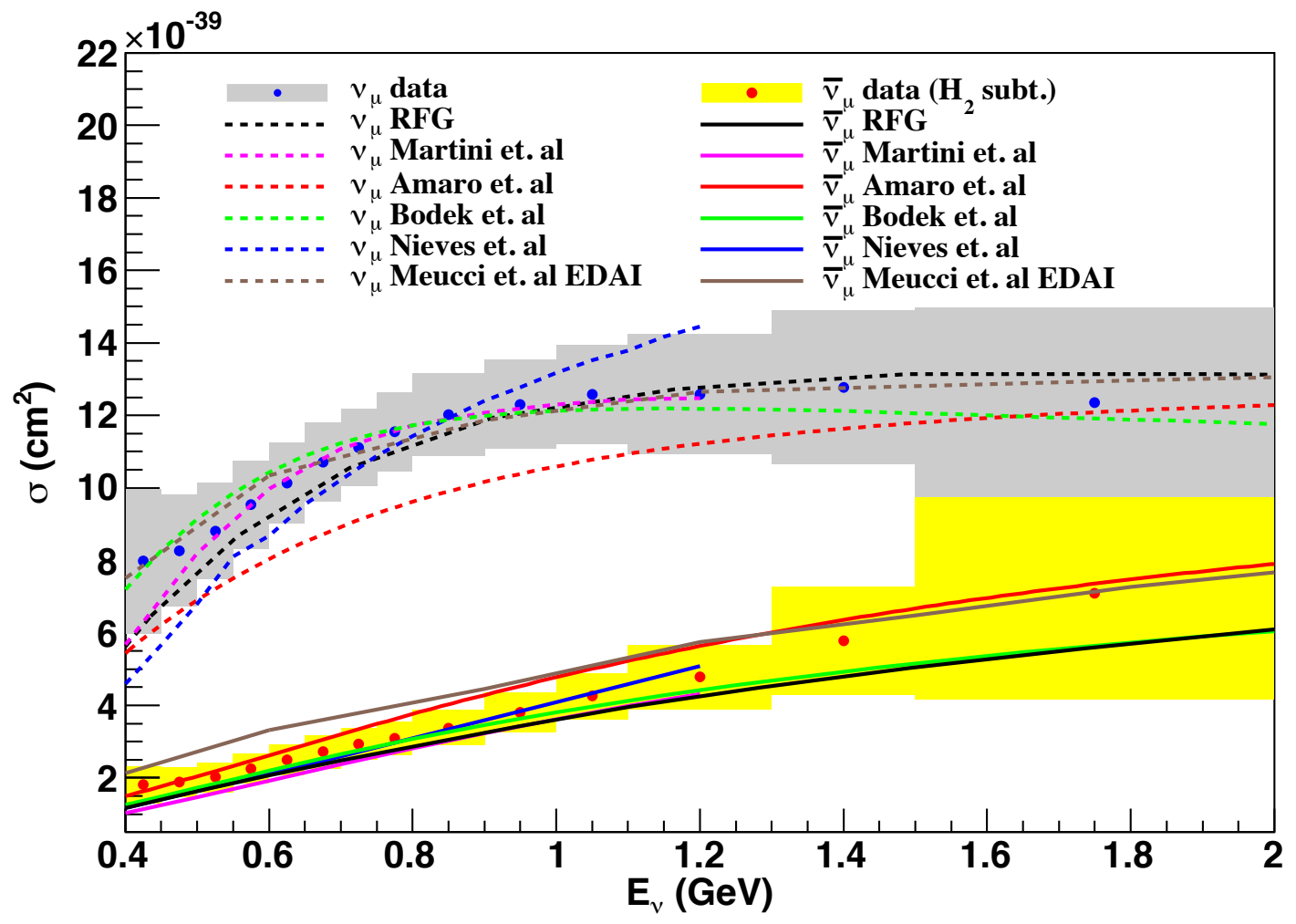

Figure 8-28. Total cross section per nucleon for $\nu_{\mu}$ and $\bar{\nu}_{\mu}$ CCQE data compared to various predictions. The "RFG" distribution assumes $M_{A}=1.35 \mathrm{GeV}$ and $\kappa=1.007$. Total uncertainty is shown with both MiniBooNE data sets. 


\section{CHAPTER 9 \\ COMBINED $\nu_{\mu}$ AND $\bar{\nu}_{\mu}$ CCQE MEASUREMENTS}

With the high-statistics MiniBooNE $\nu_{\mu}$ (Ref. [84]) and $\bar{\nu}_{\mu}$ (Chapter 8 and Ref. [147]) CCQE cross sections, an opportunity exists to extract even more information out of these data sets by exploiting correlated systematic uncertainties between the two measurements. Simple difference and ratio analyses between the two results will more stringently test the various models for CCQE-like interactions around $1 \mathrm{GeV}$. We begin with a brief discussion of how to use correlated information to reduce uncertainties in combined measurements.

\subsection{Correlated Measurements}

This treatment of systematic correlations follows Ref [176]. Consider two arbitrary results $x$ and $y$ that are used to calculate some combined measurement $q(x, y)$. As in any quantity, given the set of $N$ measurements of the quantity $q$, its uncertainty $\sigma_{q}$ is:

$$
\sigma_{q}^{2}=\frac{1}{N} \sum_{i}^{N}\left(q_{i}-\bar{q}\right)^{2}
$$

where $\bar{q}$ is the CV measurement of $q$. We are interested in how the object $q$ changes under variations in $x$ and $y$, so we begin by examining how individual excursions $x_{i}$ and $y_{i}$ from their respective results $(\bar{x}, \bar{y})$ propagate onto $q$ :

$$
\begin{aligned}
q_{i} & =q\left(x_{i}, y_{i}\right) \\
& =\sum_{n_{1}=0}^{\infty} \sum_{n_{2}=0}^{\infty} \frac{\left(x_{i}-\bar{x}\right)^{n_{1}}\left(y_{i}-\bar{y}\right)^{n_{2}}}{n_{1} ! n_{2} !} \frac{\partial^{n_{1}+n_{2}}}{\partial x^{n_{1}} \partial y^{n_{2}}} q(\bar{x}, \bar{y}) \\
& \approx q(\bar{x}, \bar{y})+\left(x_{i}-\bar{x}\right) \frac{\partial}{\partial x} q(\bar{x}, \bar{y})+\left(y_{i}-\bar{y}\right) \frac{\partial}{\partial y} q(\bar{x}, \bar{y}),
\end{aligned}
$$

where disregarding the higher order terms in the last step assumes the deviations $\left(x_{i}-\bar{x}\right)$ and $\left(y_{i}-\bar{y}\right)$ to be small. Recognizing $q(\bar{x}, \bar{y})=\bar{q}$ and substituting Eq. 9.4 into Eq. 9.1, we 
get:

$$
\begin{aligned}
\sigma_{q}^{2}= & \frac{1}{N} \sum_{i}^{N}\left[\frac{\partial q}{\partial x}\left(x_{i}-\bar{x}\right)+\frac{\partial q}{\partial y}\left(y_{i}-\bar{y}\right)\right]^{2} \\
= & \left(\frac{\partial q}{\partial x}\right)^{2} \frac{1}{N} \sum_{i}^{N}\left(x_{i}-\bar{x}\right)^{2}+\left(\frac{\partial q}{\partial y}\right)^{2} \frac{1}{N} \sum_{i}^{N}\left(y_{i}-\bar{y}\right)^{2} \\
& +2 \frac{\partial q}{\partial x} \frac{\partial q}{\partial y} \frac{1}{N} \sum_{i}^{N}\left(x_{i}-\bar{x}\right)\left(y_{i}-\bar{y}\right)
\end{aligned}
$$

where the partial derivaties of $q$ are still evaluated at the point $(\bar{x}, \bar{y})$. The first two terms in Eq. 9.6 are readily recognized as the standard deviations $\sigma_{x}$ and $\sigma_{y}$, while the last term gives information about the correlation between $x$ and $y$. It is easy to see that if the measurements of $x$ and $y$ were independent of one another, the last term will approach zero as $N \rightarrow \infty$. It is convenient to define a correlation coefficient $\rho_{x y}$ in terms of this information and the standard deviations of $x$ and $y$ :

$$
\rho_{x y}=\frac{\sum_{i}^{N}\left(x_{i}-\bar{x}\right)\left(y_{i}-\bar{y}\right)}{\sqrt{\sum_{i}^{N}\left(x_{i}-\bar{x}\right)^{2} \sum_{i}^{N}\left(y_{i}-\bar{y}\right)^{2}}}=\frac{\frac{1}{N} \sum_{i}^{N}\left(x_{i}-\bar{x}\right)\left(y_{i}-\bar{y}\right)}{\sigma_{x} \sigma_{y}} .
$$

Notice that $\rho_{x y} \in\{-1,1\}$. Then Eq. 9.6 becomes:

$$
\sigma_{q}^{2}=\left(\frac{\partial q}{\partial x}\right)^{2} \sigma_{x}^{2}+\left(\frac{\partial q}{\partial y}\right)^{2} \sigma_{y}^{2}+2 \frac{\partial q}{\partial x} \frac{\partial q}{\partial y} \rho_{x y} \sigma_{x} \sigma_{y}
$$

Depending on the sign of the product $\frac{\partial q}{\partial y} \frac{\partial q}{\partial y} \rho_{x y}$, the uncertainty on the measurement of $q$ will either be increased or reduced by including the correlation information. 


\subsection{Combined $\nu_{\mu}$ and $\bar{\nu}_{\mu}$ CCQE Measurements}

Many systematic uncertainties of the MiniBooNE $\nu_{\mu}$ and $\bar{\nu}_{\mu}$ CCQE cross-section results are related to the resolution of $\mu^{-}$and $\mu^{+}$kinematics in the detector, and are therefore expected to affect the two measurements in a similar way. Upon examination of Eq. 9.7, such an expectation would predict the correlation coefficient $\rho_{\nu \bar{\nu}}$ to be a positive quantity in most regions. Then, to form a combined measurement for the $\nu_{\mu}$ and $\bar{\nu}_{\mu}$ CCQE cross sections that features greater precision by including the information about their correlation, the sign of $\frac{\partial q}{\partial \nu} \frac{\partial q}{\partial \bar{\nu}}$ ought to be negative. Two simple cases of this are difference

$\left(\nu_{\mu}-\bar{\nu}_{\mu}\right)$ and ratio $\left(\frac{\nu_{\mu}}{\bar{\nu}_{\mu}}\right)$ measurements of the cross sections in the various distributions, most importantly the double-differential cross section $\frac{d^{2} \sigma}{d T_{\mu} d \cos \theta_{\mu}}$.

It is important to mention that this study is incomplete: while all systematic uncertainties inherent to the MiniBooNE instruments are included here, possible correlations between the $\pi^{+}$and $\pi^{-}$production data from the HARP experiment (Section 5.2.4) are unknown. In this study, the correlation between the $\nu_{\mu}$ and $\bar{\nu}_{\mu}$ parent $\pi^{+}$and $\pi^{-}$primary production cross sections are assumed to be uncorrelated. As the uncertainties on these quantities significantly contribute to the $\nu_{\mu}$ and $\bar{\nu}_{\mu}$ CCQE cross-section errors, it will be important to eventually include this information.

The goal of this study is to simply evaluate the level of correlation between each bin in the various $\nu_{\mu}$ and $\bar{\nu}_{\mu}$ CCQE cross sections in order to use Eq. 9.7 to extract the most information possible from the MiniBooNE data sets. To more easily interpret the results of this study as measurements of nuclear effects in carbon, we use the $\bar{\nu}_{\mu}$ CCQE cross section configurations in which the hydrogen CCQE component is treated as background. We begin by forming the covariance matrix to be used in the calculation of an arbitrary combined measurement $q$ in the same way as presented in Section 7.1.5: the various $\nu_{\mu}$ and $\bar{\nu}_{\mu}$ CCQE cross sections and the systematic uncertainty histograms are combined into a single distribution, side-by-side. Then, as in Eq. 7.26, a covariance matrix is formed that now includes the correlation information between each $\nu_{\mu}$ and $\bar{\nu}_{\mu}$ CCQE bin. 
The details of the calculated covariance matrix offer a few important consistency checks: the normalization uncertainty (Eq. 8.16) of each systematic error when only considering the $\nu_{\mu}$ or $\bar{\nu}_{\mu}$ region of the covariance matrix must be compared to the original normalization uncertainty findings, and the calculated cross sections from data must of course match the results of the original analyses. In this analysis, the relevant quantities match the original $\nu_{\mu}$ and $\bar{\nu}_{\mu}$ CCQE results within a few percent of their value. Also important to note when cross-checking these results, mild statistical differences are expected between the obtained neutrino-mode $\nu_{\mu}$ CCQE cross sections compared to those in Ref. [84] due to the inclusion of additional data. The analysis in Ref. [84] includes a total of $5.6 \times 10^{20} \mathrm{POT}$, while we use the additional neutrino-mode data collected since then in this analysis for a total of $6.4 \times 10^{20}$ POT. Figure 9-1 shows the resultant cross sections, along with the recovered total uncertainty from the diagonal entries of the covariance matrix.

The overall correlation coefficients between each bin in the $\nu_{\mu}$ and $\bar{\nu}_{\mu}$ CCQE cross sections are evaluated through Eq. 9.7, where the term $\frac{1}{N} \sum_{i}^{N}\left(x_{i}-\bar{x}\right)\left(y_{i}-\bar{y}\right)$ can be recognized as a given entry in the covariance matrix between two arbitrary bins $x$ and $y$. Figure 9-2 shows the overall correlation between each bin in the $\nu_{\mu}$ and $\bar{\nu}_{\mu}$ CCQE total and single-differential cross sections. Recall correlations in the HARP data are not taken into account, so the correlations presented here are generally expected to be a mild underestimate. Also shown in the same figure is the total correlation between a given bin in the $\nu_{\mu}$ and $\bar{\nu}_{\mu}$ CCQE double-differential cross sections.

Figure 9-2 shows the correlations between the $\nu_{\mu}$ and $\bar{\nu}_{\mu}$ CCQE cross sections to be rather mild. This is mostly due to the presence of large $\nu_{\mu}$ and $\mathrm{CC} \pi^{-}$backgrounds unique to the $\bar{\nu}_{\mu}$ CCQE analysis. Figure 9-3 compares the coefficients $\rho_{\nu, \bar{\nu}}$ for the most important correlated systematic uncertainties.

From this correlation information, we will extract two quantities: the difference between the $\nu_{\mu}$ ("A") and $\bar{\nu}_{\mu}$ ("B") CCQE measurements $q_{\text {diff }}=A-B$ and their ratio 
$q_{\text {ratio }}=\frac{A}{B}$. The application of Eq. 9.8 to $q_{\text {diff }}$ is straightforward:

$$
\sigma_{q_{\mathrm{diff}}}^{2}=\sigma_{A}^{2}+\sigma_{B}^{2}-2 \rho_{A B} \sigma_{A} \sigma_{B}
$$

while the uncertainty for $q_{\text {ratio }}$ :

$$
\sigma_{q_{\mathrm{ratio}}}^{2}=\left(\frac{\sigma_{A}}{B}\right)^{2}+\left(\frac{A \sigma_{A}}{B^{2}}\right)^{2}-\frac{2 A}{B^{3}} \rho_{A B} \sigma_{A} \sigma_{B}
$$

can be written more coherently as a combination of fractional uncertainties:

$$
\left(\frac{\sigma_{q_{\text {ratio }}}}{q_{\text {ratio }}}\right)^{2}=\left(\frac{\sigma_{A}}{A}\right)^{2}+\left(\frac{\sigma_{B}}{B}\right)^{2}-2 \rho_{A B}\left(\frac{\sigma_{A}}{A}\right)\left(\frac{\sigma_{B}}{B}\right) .
$$

Notice Eqs. 9.9 and 9.11 are symmetric under $A \leftrightarrow B$. Using these expressions for the uncertainty and the level of correlation between each bin in the $\nu_{\mu}$ and $\bar{\nu}_{\mu}$ CCQE cross section distributions, the combined measurements may be executed. Figure 9-4 shows the difference measurements, while Figure 9-5 presents results from the ratio analysis.

There is some independent information gleaned when comparing various predictions to the data across both the difference and the ratio measurements: the ratio $q_{\text {ratio }}$ is sensitive only to the absolute normalization of the $\nu_{\mu}$ and $\bar{\nu}_{\mu}$ CCQE cross sections, while the difference $q_{\text {diff }}$ is also sensitive to the relative normalization between the two cross sections. Up to the inclusion of the correlation of the HARP $\pi^{+}$and $\pi^{-}$production data, these measurements represent the extraction of the most CCQE information possible with the MiniBooNE detector using only observations of the muon. 

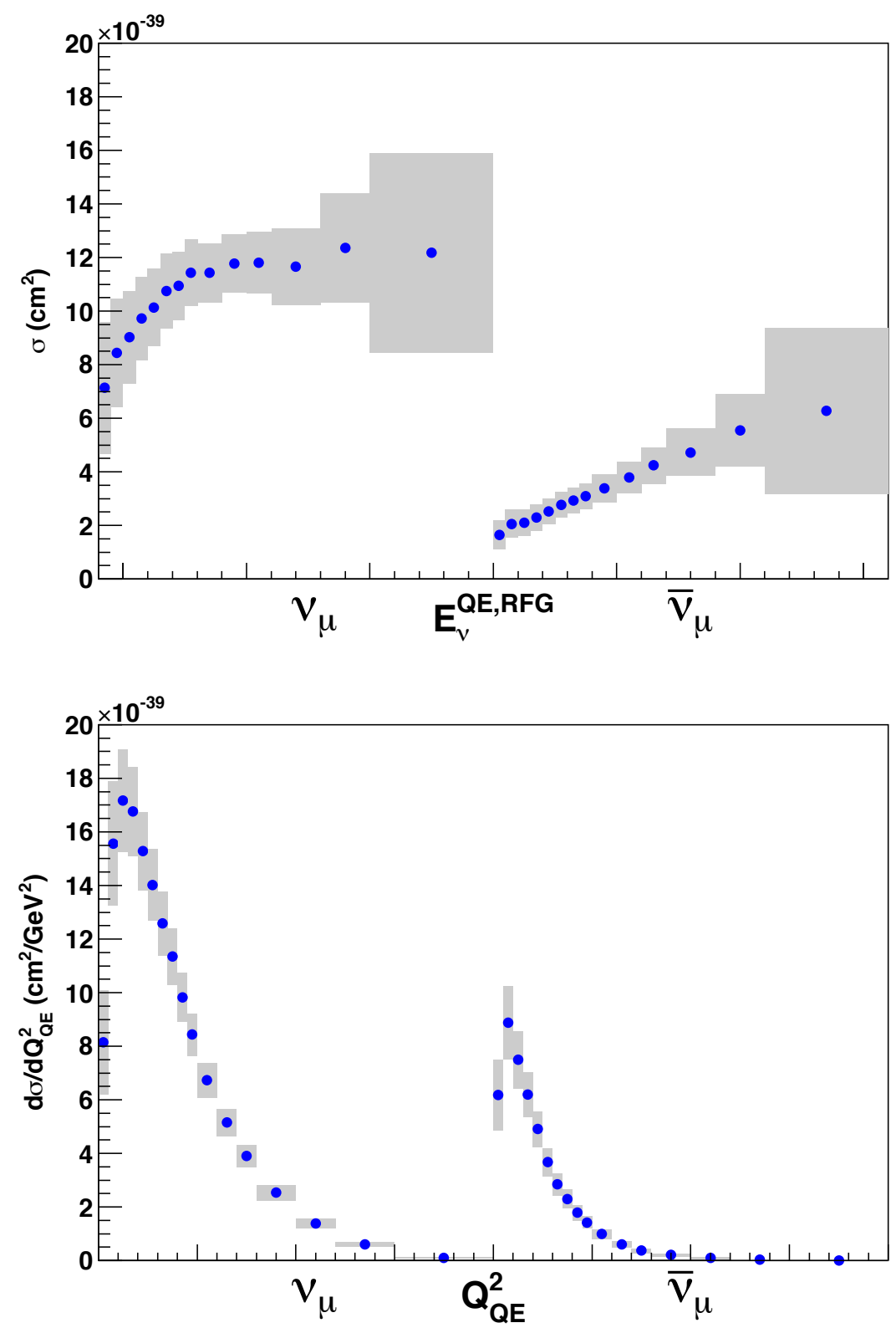

Figure 9-1. Recovered $\nu_{\mu}$ (left half of each distribution) and $\bar{\nu}_{\mu}$ (right half of each distribution) CCQE cross sections for the correlation analysis. Each bin in the $E_{\nu}^{Q E, R F G}$ distribution (left) is $\in\{0.4,2.0\} \mathrm{GeV}$ and is identical to the bins delimited in Figure 8-27, while the $Q_{Q E}^{2}$ distribution (right) is $\in\{0.0,2.0\} \mathrm{GeV}^{2}$ and corresponds to the binning in Figure 8-25. 


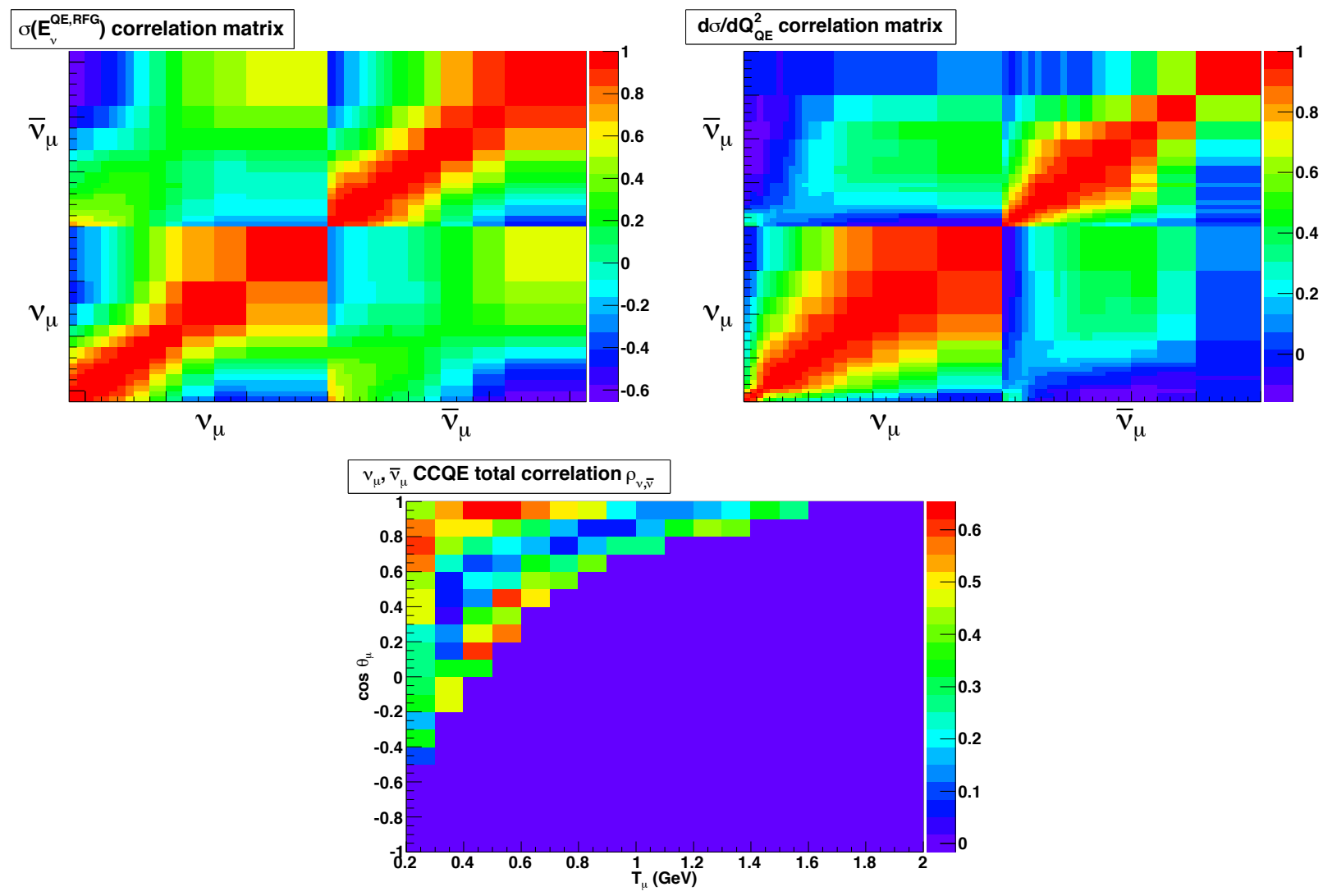

Figure 9-2. Correlation matrices for the various $\nu_{\mu}$ and $\bar{\nu}_{\mu}$ cross section results. To highlight the important information, only the $\rho_{\nu, \bar{\nu}}$ coefficients are shown for each bin in the $\frac{d^{2} \sigma}{d T_{\mu} d \cos \theta_{\mu}}$ distribution (bottom). The analogous entries correspond to the on-diagonal coefficients of the $\nu_{\mu}-\bar{\nu}_{\mu}$ off-diagonal blocks in the $\sigma\left(E_{\nu}^{Q E, R F G}\right)$ (top left) and $d \sigma / d Q_{Q E}^{2}$ (top right) matrices. 


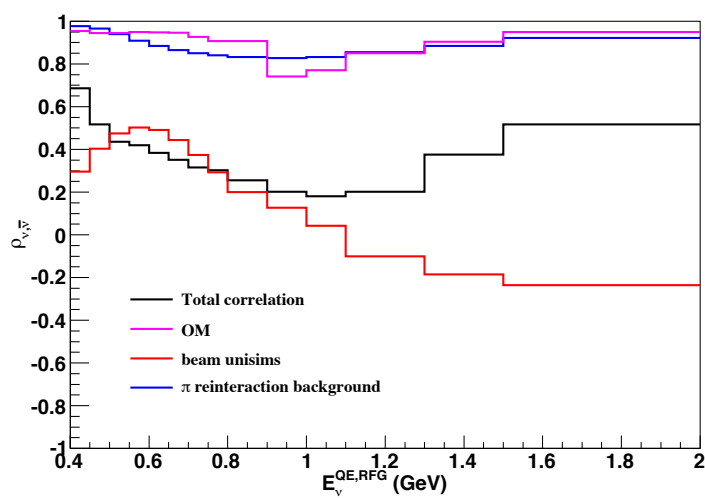

$\pi$ reinteration background $\rho_{v, \bar{v}}$
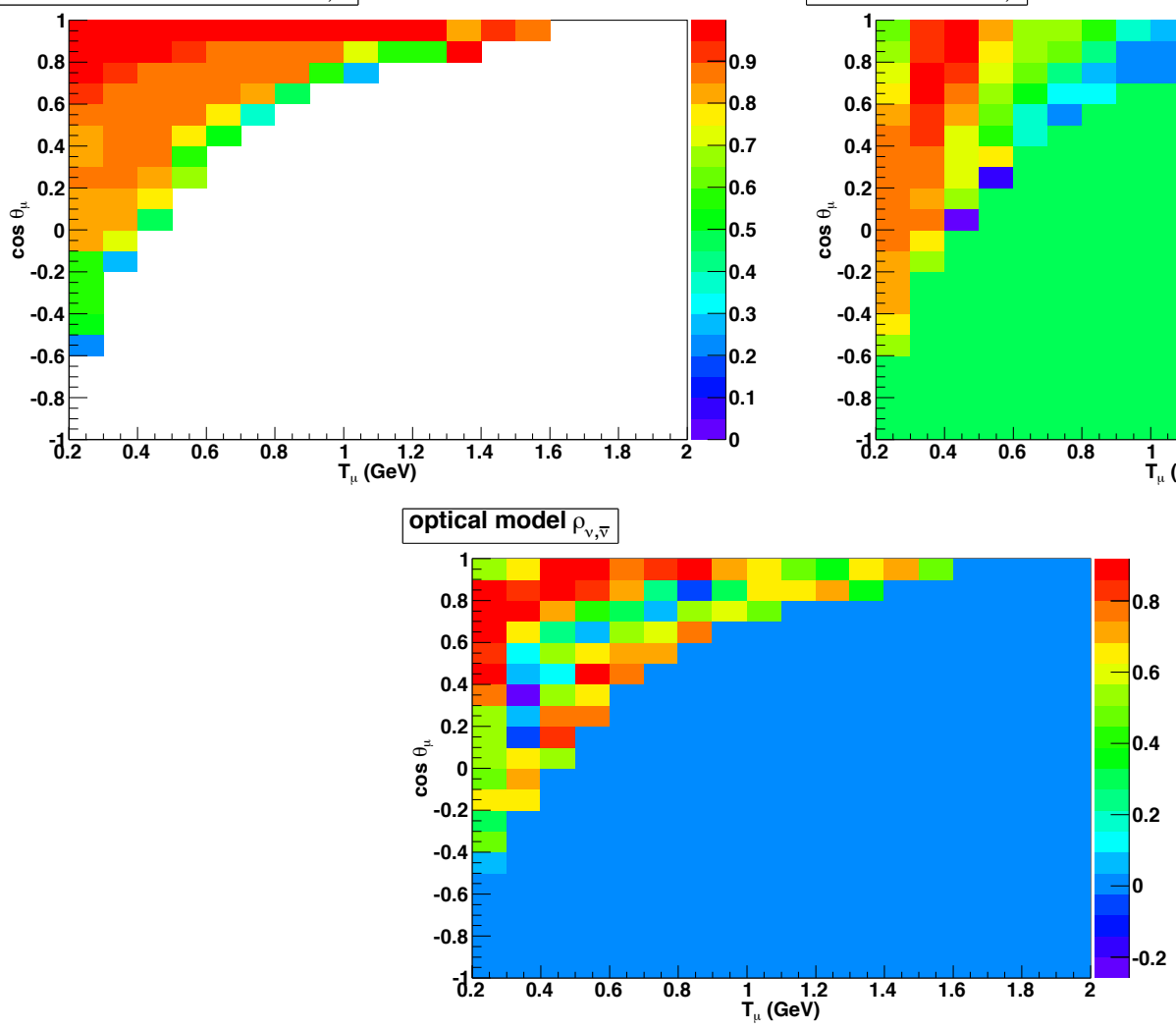

Figure 9-3. Correlation coefficients by uncertainty type for the various distributions. As expected, the correlations are mostly positive between the $\nu_{\mu}$ and $\bar{\nu}_{\mu}$ CCQE measurements. 

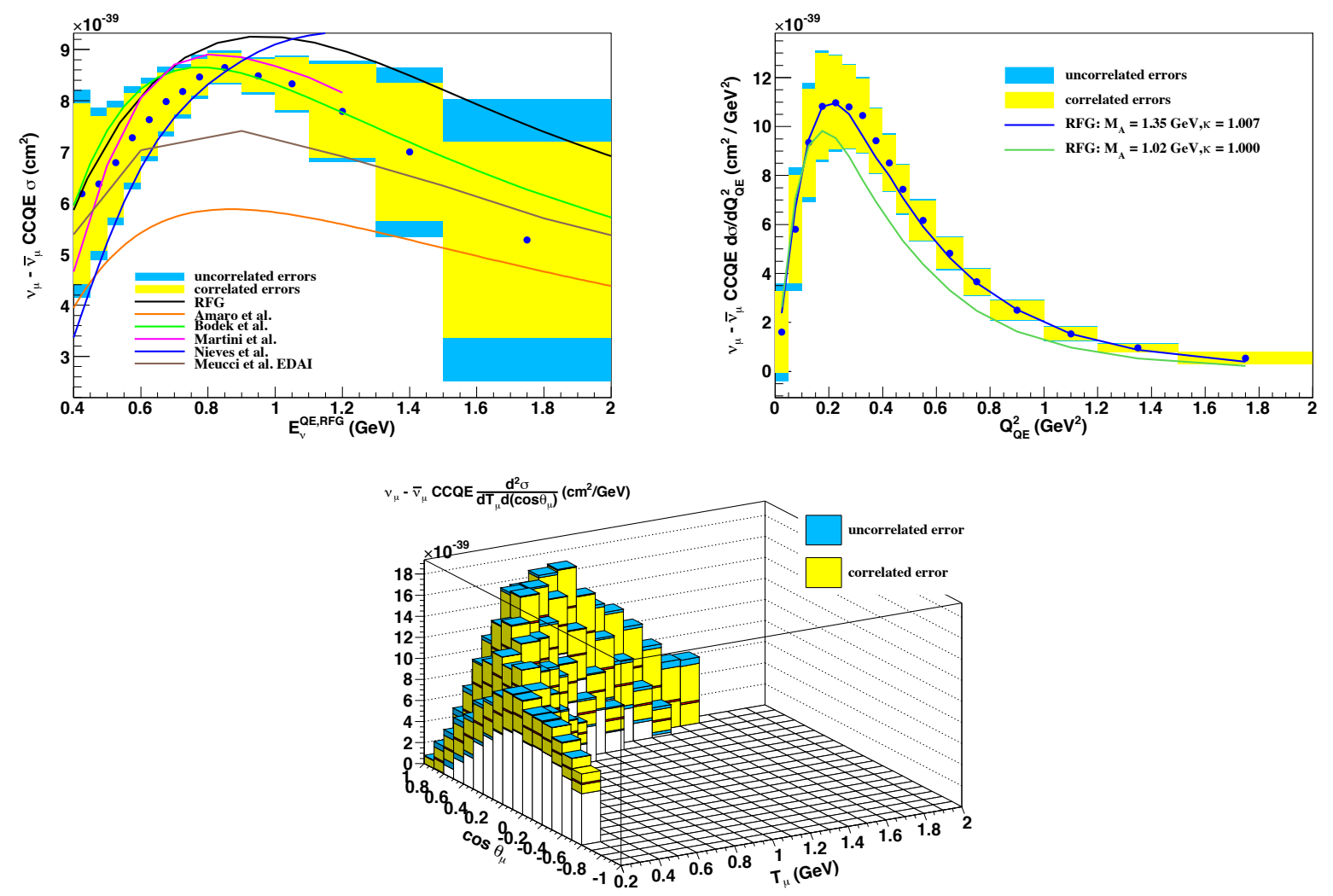

Figure 9-4. Difference measurements of the $\nu_{\mu}$ and $\bar{\nu}_{\mu}$ CCQE cross sections compared to various predictions. The "RFG" curve in the top left figure assumes $M_{A}=1.35 \mathrm{GeV}$ and $\kappa=1.007$. The "uncorrelated uncertainty", found by setting $\rho_{\nu, \bar{\nu}}=0$ in the uncertainty determination, is included to appreciate the level of sensitivity gained by using the correlation information. 

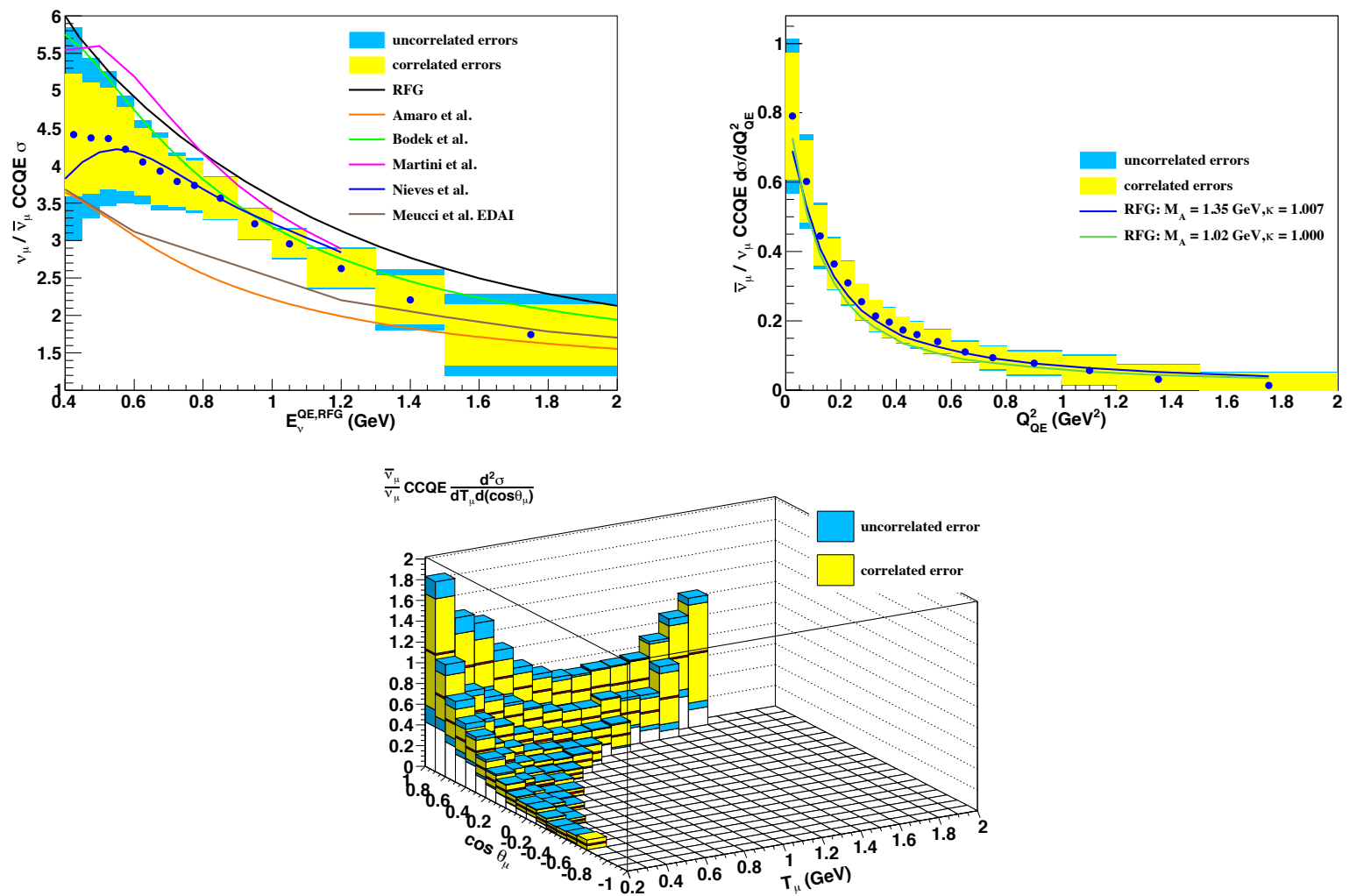

Figure 9-5. Ratio measurements of the $\nu_{\mu}$ and $\bar{\nu}_{\mu}$ CCQE cross sections compared to various predictions. The "RFG" curve in the top left figure assumes $M_{A}=1.35 \mathrm{GeV}$ and $\kappa=1.007$. The "uncorrelated uncertainty", found by setting $\rho_{\nu, \bar{\nu}}=0$ in the uncertainty determination, is included to appreciate the level of sensitivity gained by using the correlation information. 


\section{CHAPTER 10}

CONCLUSION

This dissertation presents a variety of antineutrino CCQE cross sections, including the minimally model-dependent double-differential cross section. While the cross section configurations including the free scattering component of $\bar{\nu}_{\mu}$ CCQE interactions in the detector features the least model dependence and is the main result of this work, the results evoking an axial mass $M_{A} \sim 1 \mathrm{GeV}$ to exclude the hydrogen component are also given to facilitate historical comparisons for neutrino and antineutrino interactions on carbon. This result is also used to test modern nuclear models that predict how a background arising from intra-nuclear correlations of greater size than expected might contribute to the analysis sample. These data are the first antineutrino cross-section results below $1 \mathrm{GeV}$, a crucial energy region for present and future neutrino oscillation experiments looking to measure CP violation.

To facilitate this measurement, novel and crucial evaluations of the $\nu_{\mu}$ background to the $\bar{\nu}_{\mu}$ CCQE sample were developed and executed. In the absence of a magnetic field, the analyses described in Chapter 7 measure the $\nu_{\mu}$ flux of the antineutrino mode beam with $\sim 15 \%$ fractional uncertainty. These techniques could be used in current and future neutrino oscillation programs, particularly when modest charge identification is sufficient to meet the physics goals [144].

The combined measurements of the MiniBooNE $\nu_{\mu}$ and $\bar{\nu}_{\mu}$ CCQE cross sections extract the most information of the CCQE processes possible with the MiniBooNE detector using only observations of the muon. While these measurements are entirely ignorant of hadronic activity, these analyses avoid model dependence typically associated with quantities such as momentum tracking thresholds and nucleon reinteractions used to identify CCQE interactions. 


\section{LIST OF REFERENCES}

[1] Lightman, Alan P. (2005). The discoveries: great breakthroughs in twentieth-century science, including the original papers. Toronto: Alfred A. Knopf Canada. p. 8.

[2] C. D. Ellis and W. A. Wooster, "The Continuous Spectrum of $\beta$-Rays," Nature 119 563 (1927).

[3] As quoted by F. Reines in the Foreward: C. Sutton, Spaceship Neutrino, Cambridge University Press, (1992).

[4] E. Fermi, "Versuch einer Theorie der $\beta$-Strahlen," Z. Phys. 88, 161 (1934).

[5] E. Fermi, "Tentativo di una Teoria Dei Raggi $\beta$," Nuovo Cimento 11, 1 (1934).

[6] F. Reines and C. Cowan, "The Reines-Cowan Experiments: Detecting the Poltergeist", Los Alamos Science 25, 3 (1997).

[7] "Celebrating the Neutrino", Los Alamos Science 25, 1 (1997).

[8] C. L. Cowan, Jr. et al., "Detection of the Free Neutrino: a Confirmation", Science 124103 (1956).

[9] K. Nakamura et al., "Review of Particles Physics: The CKM Quark-Mixing Matrix", Journal of Physics G37, 75021 (2010).

[10] G. Danby et al., "Observation of High-Energy Neutrino Reactions and the Existence of Two Kinds of Neutrinos", Phys. Rev. Lett. 9, 36 (1962).

[11] M. L. Perl et al., "Evidence for Anomalous Lepton Production in $\mathrm{e}^{+} \mathrm{e}^{-}$ Annihilation", Physical Review Letters 35, 1489 (1975).

[12] K. Kodama et al. [DONUT Collaboration], "Observation of Tau Neutrino Interactions", Phys. Lett. B504 218 (2001).

[13] [ADELPH et al. Collaborations], "Precision Electroweak Measurements on the Z Resonance," Phys. Rept. 427, 257 (2006).

[14] C. S. Wu et al., "Experimental Test of Parity Conservation in Beta Decay" Phys. Rev. 105, 1413 (1957).

[15] T. D. Lee and C. N. Yang, "Question Of Parity Conservation In Weak Interactions," Phys. Rev. 104, 254 (1956).

[16] M. Goldhaber, L. Grodzins, and A. W. Sunyar, "Helicity of Neutrinos", Phys. Rev. 109, 1015 (1958).

[17] J. F. Beacom and P. Vogel, Phys. Rev. Lett. 83, 5222 (1999).

[18] S. Dodelson, G. Gyuk, and M. S. Turner, "Primordial Nucleosynthesis with a Decaying Tau Neutrino," Phys. Rev. D49, 5068 (1994). 
[19] G. G. Raffelt, "New bound on neutrino dipole moments from globular-cluster stars," Phys. Rev. Lett. 64, 2856 (1990).

[20] J. M. Lattimer and J. Cooperstein, Phys. Rev. Lett. 61, 23 (1988).

[21] D. W. Liu et al. [Super-Kamiokande Collaboration], "Limits on the Neutrino Magnetic Moment using 1496 Days of Super-Kamiokande-I Solar Neutrino Data," Phys. Rev. Lett. 93, 021802 (2004).

[22] L. B. Auerbach et al., "Measurement of electron-neutrino electron elastic scattering," Phys. Rev. D63, 112001 (2001).

[23] F. Reines, H. S. Gurr, and H. W. Sobel, "Detection of $\bar{\nu}_{e}$-e Scattering," Phys. Rev. Lett. 37, 315 (1976).

[24] R. Barbieri and R. N. Mohapatra, "Limit on the Magnetic Moment of the Neutrino from Supernova 1987A Observations," Phys. Rev. Lett. 61, 27 (1988).

[25] H. T. Wong et al., "Search of Neutrino Magnetic Moments with a High-Purity Germanium Detector at the Kuo-Sheng Nuclear Power Station," Phys. Rev. D75, 012001 (2007).

[26] Ariel Goobar et al., "The neutrino mass bound from WMAP 3 year data, the baryon acoustic peak, the SNLS supernovae and the Lyman- $\alpha$ forest," JCAP 06, 019 (2006).

[27] C. Kraus et al. [Mainz Collaboration], Eur. Phys. J. C40, 447 (2005).

[28] L. Bornschein et al. [KATRIN Collaboration], Nucl. Phys. A752, 14 (2005).

[29] S. Kraft-Bermuth et al. [MARE Collaboration], J. Phys. Conf. Ser. 136, 042055 (2008).

[30] J. A. Formaggio and G. P. Zeller, "From eV to EeV: Neutrino cross sections across energy scales," Rev. Mod. Phys. 84, 1307 (2012).

[31] A. Ishihara [for the IceCube Collaboration], "Ice Cube: Ultra High Energy Neutrinos," A presentation at the Neutrino 2012 conference, Kyoto Japan, June 2012 [http://neu2012.kek.jp/index.html].

[32] I. Cholis and D. Hooper, "On The Origin of IceCube's PeV Neutrinos," [arXiv:1211.1974].

[33] V. Barger, J. Learned and S. Pakvasa, "IceCube PeV Cascade Events Initiated by Electron-Antineutrinos at Glashow Resonance," [arXiv:1207.4571].

[34] F. Englert and R. Brout, Phys. Rev. Lett. 13, 321 (1964).

[35] A. Aguilar et al. [LSND Collaboration], "Evidence for neutrino oscillations from the observation of $\bar{\nu}_{e}$ appearance in a $\bar{\nu}_{\mu}$ beam", Phys. Rev. D 64, 112007 (2001), [arXiv:0104049]. 
[36] A. Aguilar et al. [MiniBooNE Collaboration], "An improved search for $\bar{\nu}_{\mu} \rightarrow \bar{\nu}_{e}$ oscillations in the MiniBooNE experiment", [arXiv:1303.2588].

[37] J. M. Conrad et al., "Sterile neutrino fits to short baseline neutrino oscillation measurements" [arXiv:1207.4765].

[38] R. J. Davis, D. S. Harmer and K. C. Hoffman, "Search for neutrinos from the sun," Phys. Rev. Lett. 20, 1205 (1968).

[39] B. Aharmim et al. [SNO Collaboration], "Electron energy spectra, fluxes, and day-night asymmetries of B-8 solar neutrinos from the 391-day salt phase SNO data set," Phys. Rev. C 72, 055502 (2005) [arXiv:nucl-ex/0502021].

[40] M.C. Gonzalez-Garcia and M. Maltoni, "Phenomenology of Massive Neutrinos, [arXiv:hep-ph/0704.1800v2].

[41] T. Araki et al. [KamLAND Collaboration], "Measurement of neutrino oscillation with KamLAND: Evidence of spectral distortion," Phys. Rev. Lett. 94, 081801 (2005) [arXiv:hep-ex/0406035].

[42] B. Aharmim et al. [SNO Collaboration], "Low energy threshold analysis of the phase I and phase II data sets of the Sudbury Neutrino Observatory", Phys. Rev. C81, 055504 (2010).

[43] Y. Fukuda et al. [Kamiokande Collaboration], "Atmospheric muon-neutrino / electron-neutrino ratio in the multi-GeV energy range," Phys. Lett.B335, 237 (1994).

[44] Y. Ashie et al. [Super-Kamiokande Collaboration], "Evidence for an oscillatory signature in atmospheric neutrino oscillations", Phys. Rev. Lett. 93,, 101801 (2004). [arXiv:hep-ex/0404034].

[45] E. Aliu et al. [K2K Collaboration], "Evidence for muon neutrino oscillation in an accelerator-based experiment," Phys. Rev. Lett. 94, 081802 (2005). [arXiv:hep-ex/0411038].

[46] P. Adamson et al. [MINOS Collaboration], "Study of muon neutrino disappearance using the Fermilab Main Injector neutrino beam", Phys. Rev. D77, 072002 (2008).

[47] , "Global analysis of neutrino masses, mixings and phases: entering the era of leptonic CP violation searches", [arXiv:hep-ex/1205.5254].

[48] P. Adamson et al. [MINOS Collaboration], "Improved search for muon-neutrino to electron-neutrino oscillations in MINOS", Phys. Rev. Lett. 107, 181802 (2011).

[49] K. Abe et al. [T2K Collaboration], "Indication of electron neutrino appearance from an accelerator-produced off-axis muon neutrino beam", Phys. Rev. Lett. 107, 041801 (2011).

[50] F. P. An et al. [Daya Bay Collaboration], Phys. Rev. Lett. 108, 171803 (2012). 
[51] J. K Ahn et al. [Reno Collaboration], Phys. Rev. Lett. 108, 191802 (2012).

[52] F. P. An et al. [Daya Bay Collaboration], "Improved measurement of electron antineutrino disappearance at Daya Bay," Chin. Phys. C37, 011001 (2013). [arXiv:hep-ex/1210.6327].

[53] M. C. Gonzalez-Garcia and M. Maltoni, "Phenomenology with Massive Neutrinos", Phys. Rept. 460, 1 (2008).

[54] D. S. Ayres et al. [NOvA Collaboration], "The NOvA Technical Design Report," FERMILAB-DESIGN-2007-01.

[55] V. Barger et al. [LBNE Collaboration], "Report of the US Long Baseline Meutrino Experiment Study", [arXiv:0705.4396].

[56] B. Armbruster et al. [KARMEN Collaboration], Phys. Rev. D65, 112001 (2002).

[57] J. Hamann et al., "Cosmology favoring extra radiation and sub-eV mass sterile neutrinos as an option", Phys. Rev. Lett. 105, 181301 (2010).

[58] C. Giunti and M. Laveder, "Short-baseline electron neutrino disappearance, tritium beta decay and neutrinoless Double-Beta Decay", Phys. Rev. D82, 053005 (2010).

[59] G. Mention et al., "The reactor antineutrino anomaly", Phys. Rev. D83, 073006 (2011). [arXiv:1101.2755].

[60] H. Ray for the OscSNS Collaboration, [arXiv:0810.3175].

[61] P. Kyberd et al. [nuSTORM Collaboration], "nuSTORM: Neutrinos from STORed Muons" [arXiv:1206.0294].

[62] J. Spitz, "Sterile neutrino search with kaon decay at rest", Phys. Rev. D85, 093020 (2012).

[63] M. Gell-Mann, P. Ramond and R. Slansky, PRINT-80-0576-CERN (1980).

[64] H.V. Klapdor-Kleingrothaus et al. [The Heidelberg-Moscow Collaboration], "Evidence for Neutrinoless Double Beta Decay", Mod. Phys. Lett. A16, 2409 (2001).

[65] S. Davidson, E. Nardi and Y. Nir, Phys. Rep. 466, 105 (2008).

[66] D. Casper, Nucl. Phys. Proc. Suppl. 112, 161 (2002).

[67] R. Feynman, "Very High-Energy Collisions of Hadrons," Phys. Rev. Lett. 23, 1415 (1969).

[68] C. H. Llwellyn-Smith, Phys. Rep. 3, 261 (1972).

[69] T. Katori, PhD Thesis, Indiana University (2008).

[70] W.-M. Yao et al., Journal of Physics G33, 1 (2006). 
[71] H. Budd, A. Bodek, and J. Arrington, "Modeling Quasi-Elastic Form Factors for Electron and Neutrino Scattering," [arXiv:hepex/0308005].

[72] Amsler C et al. (Particle Data Group), Phys. Lett. 667B, 1 (2008).

[73] V. Bernard et al., J. Phys. G28, R1 (2002).

[74] G. P. Zeller, private communication.

[75] D. Rein and L. M. Sehgal, Annals Phys. 133, 79 (1981).

[76] R. P. Feynman, M. Kislinger, and F. Ravndal, Phys. Rev. D3, 2706 (1971).

[77] D. Casper, private communication.

[78] A. A. Aguilar-Arevalo et al. [MiniBooNE Collaboration], Phys. Rev. D83, 052007 (2011).

[79] A. A. Aguilar-Arevalo et al. [MiniBooNE Collaboration], Phys. Rev. D81, 013005 (2010).

[80] A. A. Aguilar-Arevalo et al. [MiniBooNE Collaboration], Phys. Rev. D83, 052009 (2011).

[81] O. Lalakulich and U. Mosel, "Pion Production in the MiniBooNE Experiment," [arXiv:1210.4717].

[82] R. A. Smith and E. J. Moniz, Nucl. Phys. B43, 605 (1972); erratum: ibid. B101, 547 (1975).

[83] A. A. Aguilar-Arevalo [MiniBooNE Collaboration], "Measurement of Muon Neutrino Quasi-Elastic Scattering on Carbon" Phys. Rev. Lett. 100, 032301 (2008).

[84] A. A. Aguilar-Arevalo et al. [MiniBooNE Collaboration], Phys. Rev. D81, 092005 (2010).

[85] E. J. Moniz et al., "Nuclear Fermi Momenta from Quasielastic Electron Scattering," Phys. Rev. Lett. 26, 445 (1971).

[86] C. F. Weizscker et al., "On the theory of nuclear masses," Journal of Physics 96, 431 (1935).

[87] R. Gran et al., Phys. Rev. D74, 052002 (2006).

[88] M. Dorman for the MINOS Collaboration, AIP Conf. Proc. 1189, 133 (2009).

[89] J. L. Alcaraz-Aunion and J. Walding, AIP Conf. Proc. 1189, 145 (2009).

[90] K. S. Kuzmin, V. V. Lyubushkin, and V. A. Naumov, Eur. Phys. J. C 54, 517 (2008); V. V. Lyubushkin et al. [NOMAD Collaboration], arXiv:0812.4543 [hep-ex]. 


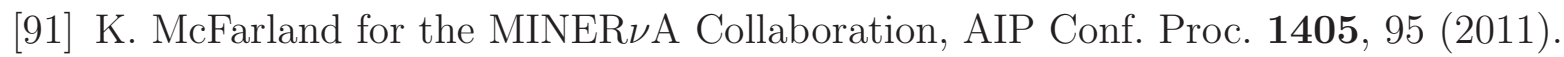

[92] M. Martini et al., "A unified approach for nucleon knock-out, coherent and incoherent pion production in neutrino interactions with nuclei," Phys. Rev. C80, 065501 (2009). [arXiv:0910.2622].

[93] M. Martini et al., "Neutrino and antineutrino quasielastic interactions with nuclei," Phys. Rev. C81, 045502 (2010).

[94] M. Martini et al., "Quasielastic and multinucleon excitations in antineutrino-nucleus interactions" [arXiv:1303.7199].

[95] J. Nieves et al., "Inclusive Charged-Current Neutrino-Nucleus Reactions," Phys. Rev. C83,045501 (2011). [arXiv:1102.2777].

[96] J. Nieves et al., "Two Particle-Hole Excitations in Charged Current Quasielastic Antineutrino-Nucleus Scattering," [arXiv:1302.0703].

[97] J. Amaro et al., "Relativistic analyses of quasielastic neutrino cross sections at MiniBooNE kinematics." Phys. Rev. D84, 033004 (2011). [arXiv:1104.5446].

[98] J. Amaro et al., "Meson-exchange currents and quasielastic antineutrino cross sections in the SuperScaling Approximation," [arXiv:1112.2123].

[99] A. Meucci and C. Giusti, Phys. Rev. D85, 093002 (2012).

[100] A. Bodek et al., Eur. Phys. J. C71, 1726 (2011).

[101] W. M. Alberico et al., Phys. Rev. C38, 1801 (1988).

[102] T. W. Donnelly and I. Sick, Phys. Rev. C60, 065502 (1999).

[103] M. Martini et al., arXiv:1202.4745 [hep-ph].

[104] O. Lalakulich and U. Mosel, Phys. Rev. C86, 054606 (2012).

[105] W. Reuter et al., Phys. Rev. C 26, 806 (1982).

[106] V. Flaminino et al., CERN-HERA-83-01 (1983); CERN-HERA-83-02 (1983); CERN-HERA-84-01 (1984).

[107] D. Ashery et al., Phys. Rev. C23, 2173 (1981); M. K. Jones et al., Phys. Rev. C48, 2800 (1993); R. D. Ransome et al., Phys. Rev. C45, R509 (1992).

[108] A. A. Aguilar-Arevalo et al. [MiniBooNE Collaboration], "The neutrino flux prediction at MiniBooNE" Phys. Rev. D79, 072002 (2009).

[109] A. A. Aguilar-Arevalo et al. [MiniBooNE Collaboration], Nucl. Instr. Meth. A599, 28 (2009). 
[110] H. Greinacher, "The Ionometer and its Application to the Measurement of Radium and Rntgen Rays", Physikal. Zeitsch. 15, 410 (1914).

[111] See http://nagysandor.eu/AsimovTeka/tevatron_hu/multiturn.html

[112] B. Worthel et al., Booster Rookie Book, http://www-bdnew.fnal.gov/operations/rookie_books/.

[113] M. Sorel, "Search for Sterile Neutrinos Using the MiniBooNE Beam," PhD Thesis, Columbia University, 2005.

[114] N. V. Mokhov and S. I. Striganov, "MARS15 overview, AIP Conf. Proc. 896, 50 (2007).

[115] See

http://geant4.web.cern.ch/geant4/UserDocumentation/UsersGuides/PhysicsReferenceManual/html

[116] A. Heikkinen, N. Stepanov and J. P. Wellisch, "Bertini intra-nuclear cascade implementation in Geant4," [arXiv:nucl-th/0306008].

[117] G. Folger, V. N. Ivanchenko and J. P. Wellisch, "The Binary cascade model," Eur. Phys. Jour. A21, 407 (2004).

[118] M. G. Catanesi et al. [HARP Collaboration], Eur. Phys. J. C52, 29 (2007).

[119] D. Schmitz, "A measurement of hadron production cross-sections for the simulation of accelerator neutrino beams and a search for muon neutrino to electron neutrino oscillations in the $\Delta m^{2} \sim 1 \mathrm{eV}^{2}$ region", PhD Thesis, Columbia University, 2008.

[120] J. R. Sanford and C. L. Wang, Brookhaven National Laboratory Note 11299 (1967).

[121] The CERN Program Library (CERNLIB), see cernlib.web.cern.ch/cernlib.

[122] R. J. Glauber (1959), in Lectures in Theoretical Physics, Volume 1, edited by W. E. Britten et al.

[123] M. Wilking, "Measurement of Neutrino Induced, Charged Current, Charged Pion Production", PhD Thesis, University of Colorado, 2009.

[124] S. Agostinelli et al., Nucl. Instrum. Meth. A506, 250 (2003).

[125] S. Brice, MiniBooNE Note 257.

[126] G. Collazuol et al., Nucl. Instr. Meth. 449, 609 (2000).

[127] S. Kopp, “Accelerator Neutrino Beams," Phys. Rept. 439, 101 (2007). [arXiv:0609129]

[128] I. Stancu et al., "The MiniBooNE Detector Technical Design Report", http://www-boone.fnal.gov/about_boone/ . 
[129] A. A. Aguilar-Arevalo et al. [MiniBooNE Collaboration], "Measurement of the neutrino neutral-current elastic differential cross section", Phys. Rev. D82, 092005 (2010).

[130] A. A. Aguilar-Arevalo et al. [MiniBooNE Collaboration], "Neutrino and antineutrino cross sections at MiniBooNE", AIP Conf. Proc. 1382, 271 (2011).

[131] S. Brice et al., "Photomultiplier Tubes in the MiniBooNE Experiment", Nucl. Instrum. Meth. A562, 97 (2006).

[132] R. B. Patterson, "A search for muon neutrino to electron neutrino oscillations at $\Delta m^{2}>0.1 \mathrm{eV}^{2}, " \mathrm{PhD}$ Thesis, Princeton University (2009).

[133] A. A. Aguilar-Arevalo et al. [MiniBooNE Collaboration], "A search for core-collapse supernovae using the MiniBooNE neutrino detector" Phys. Rev. D81, 032001 (2010).

[134] A. A. Aguilar-Arevalo et al. [MiniBooNE Collaboration], "First measurement of $\nu_{\mu}$ and $\nu_{e}$ events in an off-axis horn-focused neutrino beam", Phys. Rev. Lett. 102, 211801 (2009).

[135] R. B. Patterson et al., "The Extended-Track Event Reconstruction for MiniBooNE", Nucl. Instrum. Meth. A608, 206 (2009).

[136] J.R. Monroe, "A combined $\nu_{\mu}$ and $\nu_{e}$ oscillation search at MiniBooNE", PhD Thesis, Columbia University (2006).

[137] A. A. Aguilar-Arevalo et al. [MiniBooNE Collaboration], Phys. Rev. Lett. 103, 081801 (2009).

[138] A. A. Aguilar-Arevalo et al. [MiniBooNE Collaboration], Phys. Rev. D82, 092005 (2010).

[139] A. A. Aguilar-Arevalo et al. [MiniBooNE Collaboration], Phys. Lett. B664, 41 (2008).

[140] P. Astier et al. [NOMAD Collaboration], "Search for $\nu_{\mu} \rightarrow \nu_{e}$ oscillations in the NOMAD experiment", Phys. Lett. B570, 19-31 (2003).

[141] P.-A. Amaudruza [T2K ND280 FGD Collaboration], "The T2K fine-grained detectors", Nucl. Instrum. Meth. A696, 1 (2012).

[142] A. de Bellefon et al., "MEMPHYS: a large water Čerenkov detector at Fréjus", [arXiv:hep-ex/0607026].

[143] K. Nakamura, "Hyper-Kamiokande - a next generation water Cherenkov detector", Int. J. Mod. Phys. A 18, 4053 (2003).

[144] P. Huber and T. Schwetz, Phys. Lett. B669, 294 (2008), [arXiv:0805.2019]. 
[145] D. D. Stancil et al. [MINER $\nu$ A Collaboration], "Demostration of communication using neutrinos", Mod. Phys. Lett. A27,1250077 (2012).

[146] A. A. Aguilar-Arevalo et al. [MiniBooNE Collaboration], "Measurement of the neutrino component of an anti-neutrino beam observed by a non-magnetized detector," Phys. Rev. D84, 072005 (2011). [arxiv:1102.1964].

[147] A. A. Aguilar-Arevalo et al. [MiniBooNE Collaboration], "First measurement of the muon anti-neutrino double-differential charged current quasi-elastic cross section," [arxiv:1301.7067].

[148] A. Shinohara et al., Phys. Rev. A53, 130 (1996).

[149] E. J. Maier, R. M. Edelstein and R. T. Siegel, Phys. Rev. 133, B663 (1964).

[150] L. Ph. Roesch et al., Phys. Lett. 107B, 31 (1981).

[151] Yu. G. Budyashov et al., Sov. Phys. JETP 31, 651 (1970).

[152] M. E. Plett and S.E. Sobottka, Phys. Rev. C3, 1003 (1971).

[153] B. Macdonald et al., Phys. Rev. 139, B1253 (1965).

[154] G. H. Miller et al., Phys. Lett 41B, 50 (1972).

[155] A. A. Aguilar-Arevalo et al. [MiniBooNE Collaboration], Phys. Rev. D81, 013005 (2010).

[156] A. A. Aguilar-Arevalo et al. [MiniBooNE Collaboration], Phys. Rev. D82, 092005 (2010).

[157] J. Grange for the MiniBooNE Collaboration, AIP Conf. Proc. 1405, 83 (2011).

[158] See http://root.cern.ch/root/html/TMinuit.html.

[159] B. P. Roe, Nucl. Instrum. Meth. A 570, 159 (2007).

[160] A. A. Aguilar-Arevalo et al. [MiniBooNE Collaboration], Phys. Rev. Lett. 103, 061802 (2009).

[161] M. S. Athar, S. Ahmad, and S. K. Singh, "Charged current antineutrino reactions from ${ }^{12} \mathrm{C}$ at MiniBooNE energies", Phys. Rev. D75, 093003 (2007).

[162] M. G. Cox et al., "The generalized weighted mean of correlated values", Metrologia 43, S268 (2006).

[163] J. Nowak, AIP Conf. Proc. 1189, 243 (2009).

[164] Ch. Berger and L. M. Sehgal, Phys. Rev. D76, 113004 (2007). 
[165] K.S. Kuzmin, V.V. Lyubushkin and V.A. Naumov, "Polarization of tau leptons produced in quasielastic neutrino-nucleon scattering", Mod. Phys. Lett. A19, 2919 (2004).

[166] K.S. Kuzmin, V.V. Lyubushkin and V.A. Naumov, "Extended Rein-Sehgal model for tau lepton production", Nucl. Phys. B (Proc. Suppl.) 139, 154 (2005).

[167] K. M. Graczyk and J. T. Sobczyk, Phys. Rev. D77, 053001 (2008).

[168] A. Bodek et al., J. Phys. Conf. Ser. 110, 082004 (2008).

[169] Kolmogorov A, "Sulla determinazione empirica di una legge di distribuzione", G. Inst. Ital. Attuari 4, 83 (1933). Smirnov N. V., "Tables for estimating the goodness of fit of empirical distributions", Annals of Mathematical Statistics 19, 279 (1948).

[170] G. D'Agostini, Nucl. Instrum. Meth A362, 487 (1995).

[171] K. S. Kuzmin, V. V. Lyubushkin, and V. A. Naumov, Eur. Phys. J. C 54, 517 (2008); V. V. Lyubushkin et al. [NOMAD Collaboration], [arXiv:0812.4543].

[172] A. A. Aguilar-Arevalo et al. [SciBooNE Collaboration], arXiv:0601022 [hep-ex].

[173] M. Soderberg for the MicroBooNE Collaboration, AIP Conf. Proc. 1189, 83 (2009).

[174] C. Anderson et al. [ArgoNeuT Collaboration], Phys. Rev. Lett. 108, 161802 (2012).

[175] S. Amoruso et al. [ICARUS Collaboration], Eur. Phys. J. C33, 233 (2004).

[176] Taylor, John R. Error Analysis. Sausalito, CA: University Science Books, 1997. 


\section{BIOGRAPHICAL SKETCH}

Joseph Grange received his bachelor's degree from the University of Puget Sound in 2006. His degrees from the University of Florida include a Master of Science in 2009 and a Doctor of Philosophy in 2013. 


\title{
Measurement of the neutrino component of an antineutrino beam observed by a nonmagnetized detector
}

A. A. Aguilar-Arevalo, ${ }^{12}$ C. E. Anderson, ${ }^{15}$ S. J. Brice, ${ }^{6}$ B. C. Brown, ${ }^{6}$ L. Bugel,${ }^{11}$ J. M. Conrad, ${ }^{11}$ R. Dharmapalan, ${ }^{1}$ Z. Djurcic, ${ }^{2}$ B. T. Fleming, ${ }^{15}$ R. Ford ${ }^{6}$ F. G. Garcia, ${ }^{6}$ G. T. Garvey,${ }^{9}$ J. Grange, ${ }^{7}$ J. A. Green, ${ }^{8,9}$ R. Imlay, ${ }^{10}$ R. A. Johnson, ${ }^{3}$ G. Karagiorgi, ${ }^{11}$ T. Katori, ${ }^{8,11}$ T. Kobilarcik, ${ }^{6}$ S. K. Linden, ${ }^{15}$ W. C. Louis, ${ }^{9}$ K. B. M. Mahn, ${ }^{5}$ W. Marsh, ${ }^{6}$ C. Mauger, ${ }^{9}$ W. Metcalf, ${ }^{10}$ G. B. Mills, ${ }^{9}$ J. Mirabal,${ }^{9}$ C. D. Moore, ${ }^{6}$ J. Mousseau, ${ }^{7}$ R. H. Nelson, ${ }^{4}$ V. Nguyen,,${ }^{11}$ P. Nienaber, ${ }^{14}$ J. A. Nowak, ${ }^{10}$ B. Osmanov, ${ }^{7}$ A. Patch,${ }^{9}{ }^{11}$ Z. Pavlovic, ${ }^{9}$ D. Perevalov, ${ }^{1}$ C. C. Polly, ${ }^{6}$ H. Ray, ${ }^{7}$ B. P. Roe, ${ }^{13}$ A. D. Russell, ${ }^{6}$ M. H. Shaevitz, ${ }^{5}$ M. Sorel,${ }^{5, *}$ J. Spitz ${ }^{15}$ I. Stancu, ${ }^{1}$ R. J. Stefanski, ${ }^{6}$ R. Tayloe, ${ }^{8}$ M. Tzanov, ${ }^{4}$ R. G. Van de Water, ${ }^{9}$ M. O. Wascko, ${ }^{10, \dagger}$ D. H. White, ${ }^{9}$ M. J. Wilking, ${ }^{4}$ G. P. Zeller, ${ }^{6}$ and E. D. Zimmerman ${ }^{4}$

(The MiniBooNE Collaboration)

\author{
${ }^{1}$ University of Alabama, Tuscaloosa, Alabama 35487, USA \\ ${ }^{2}$ Argonne National Laboratory, Argonne, Illinois 60439, USA \\ ${ }^{3}$ University of Cincinnati, Cincinnati, Ohio 45221, USA \\ ${ }^{4}$ University of Colorado, Boulder, Colorado 80309, USA \\ ${ }^{5}$ Columbia University, New York, New York 10027, USA \\ ${ }^{6}$ Fermi National Accelerator Laboratory, Batavia, Illinois 60510, USA \\ ${ }^{7}$ University of Florida, Gainesville, Florida 32611, USA \\ ${ }^{8}$ Indiana University, Bloomington, Indiana 47405, USA \\ ${ }^{9}$ Los Alamos National Laboratory, Los Alamos, New Mexico 87545, USA \\ ${ }^{10}$ Louisiana State University, Baton Rouge, Louisiana 70803, USA \\ ${ }^{11}$ Massachusetts Institute of Technology, Cambridge, Massachusetts 02139, USA \\ ${ }^{12}$ Instituto de Ciencias Nucleares, Universidad Nacional Autónoma de México, D.F. 04510, México \\ ${ }^{13}$ University of Michigan, Ann Arbor, Michigan 48109, USA \\ ${ }^{14}$ Saint Mary's University of Minnesota, Winona, Minnesota 55987, USA \\ ${ }^{15}$ Yale University, New Haven, Connecticut 06520, USA \\ (Received 20 March 2011; published 26 October 2011)
}

Two methods are employed to measure the neutrino flux of the antineutrino-mode beam observed by the MiniBooNE detector. The first method compares data to simulated event rates in a high-purity $\nu_{\mu}$-induced charged-current single $\pi^{+}\left(\mathrm{CC} 1 \pi^{+}\right)$sample while the second exploits the difference between the angular distributions of muons created in $\nu_{\mu}$ and $\bar{\nu}_{\mu}$ charged-current quasielastic (CCQE) interactions. The results from both analyses indicate the prediction of the neutrino flux component of the predominately antineutrino beam is overestimated - the $\mathrm{CC} 1 \pi^{+}$analysis indicates the predicted $\nu_{\mu}$ flux should be scaled by $0.76 \pm 0.11$, while the $\mathrm{CCQE}$ angular fit yields $0.65 \pm 0.23$. The energy spectrum of the flux prediction is checked by repeating the analyses in bins of reconstructed neutrino energy, and the results show that the spectral shape is well-modeled. These analyses are a demonstration of techniques for measuring the neutrino contamination of antineutrino beams observed by future nonmagnetized detectors.

DOI: 10.1103/PhysRevD.84.072005

PACS numbers: $14.60 . \mathrm{Lm}, 14.60 . \mathrm{Pq}, 14.60 . \mathrm{St}$

\section{INTRODUCTION}

If $\theta_{13}$ is nonzero, next-generation neutrino oscillation experiments will embark on a program to measure the neutrino mass ordering and look for evidence of $C P$ violation in the neutrino sector. This effort will require precise oscillation measurements with both neutrino and antineutrino beams in order to isolate these effects. Since beams

\footnotetext{
*Present address: IFIC, Universidad de Valencia and CSIC, Valencia 46071, Spain.

${ }^{\dagger}$ Present address: Imperial College; London SW7 2AZ, United Kingdom., USA
}

produced in an accelerator environment are never purely neutrino nor antineutrino in content, detectors must be able to separate the two contributions. Most commonly, this is achieved by employing a magnetic field to identify the final-state $\mu^{-}$(or $\mu^{+}$) produced in charged-current $\nu_{\mu}$ (or $\bar{\nu}_{\mu}$ ) interactions. A handle on the overall level and energy dependence of $\nu_{\mu}$ versus $\bar{\nu}_{\mu}$ induced events, however, is also possible in unmagnetized detectors with a suitable choice of reaction channels.

Accelerator-based neutrino beams are typically created by colliding proton beams with thick nuclear targets. Mesons produced at a variety of energies and angles are focused by a magnetic horn before entering a decay tunnel. 
Meson decays can be calculated sufficiently well for a given beam geometry that the neutrino flux uncertainties arise mainly from uncertainties in the meson production cross sections. In particular, to avoid extrapolating data taken with diverse nuclear target materials or proton energies, neutrino experiments require dedicated hadron production cross section measurements taken with the same beam energy and target to obtain a reliable flux prediction. If an accelerator-based neutrino experiment lacks such hadron production data, it may be able to meet its oscillation analysis goals using calibrations from a near detector; however, the secondary physics goal of measuring neutrino-nucleon absolute cross sections will still be limited by flux uncertainties.

To avoid ambiguity, in this paper references to "neutrinos" are not meant to also refer to antineutrinos, and "mode" refers to the polarity of the magnetic focusing horn used in the beamline. In this way, for example, "antineutrino events" refers to antineutrino-induced events exclusively while "antineutrino-mode events" refers to data obtained when the horn polarity focuses negatively charged particles, which is a mix of neutrino and antineutrino-induced events.

The Mini Booster Neutrino Experiment (MiniBooNE) is located at Fermilab in Batavia, Illinois, and has made many oscillation [1-5] and cross section [6-13] measurements. For MiniBooNE, the pion production data crucial to the flux model come from proton-beryllium cross sections on a $5 \%$ interaction length target reported by the HARP experiment [14]. However, even with dedicated data appropriate to the experimental setup of MiniBooNE, there remain small regions of phase space relevant to MiniBooNE not covered by these HARP measurements. Of particular importance is the production of very forward pions with respect to the direction of the incoming proton beam. This is the dominant production region of parent particles contributing neutrinos to the antineutrino-mode beam, or vice versa. MiniBooNE uses a magnetic horn to defocus the majority of these background parent particles, but as Fig. 1 suggests, the very forward pions can escape magnetic deflection. This same angular region suffers from a sizable beam-related proton background and would also require a model-dependent acceptance correction [15]. For these reasons, pion cross sections in the $\theta_{\pi}<30 \mathrm{mrad}$ region, where $\theta_{\pi}$ is the angle the outgoing pion makes with respect to the incoming proton beam, are not reported by HARP and the majority of the MiniBooNE flux prediction arising from $\pi^{+}\left(\pi^{-}\right)$decay while focusing $\pi^{-}$ $\left(\pi^{+}\right)$is extrapolated from the available hadron production data. The hadron production data cover $\sim 90 \%$ of signselected pions, while less than $25 \%$ of oppositely charged pions in the same beam are constrained. Some of these acceptance limitations could be reduced by use of the longtarget data taken by HARP, which are actively being analyzed.
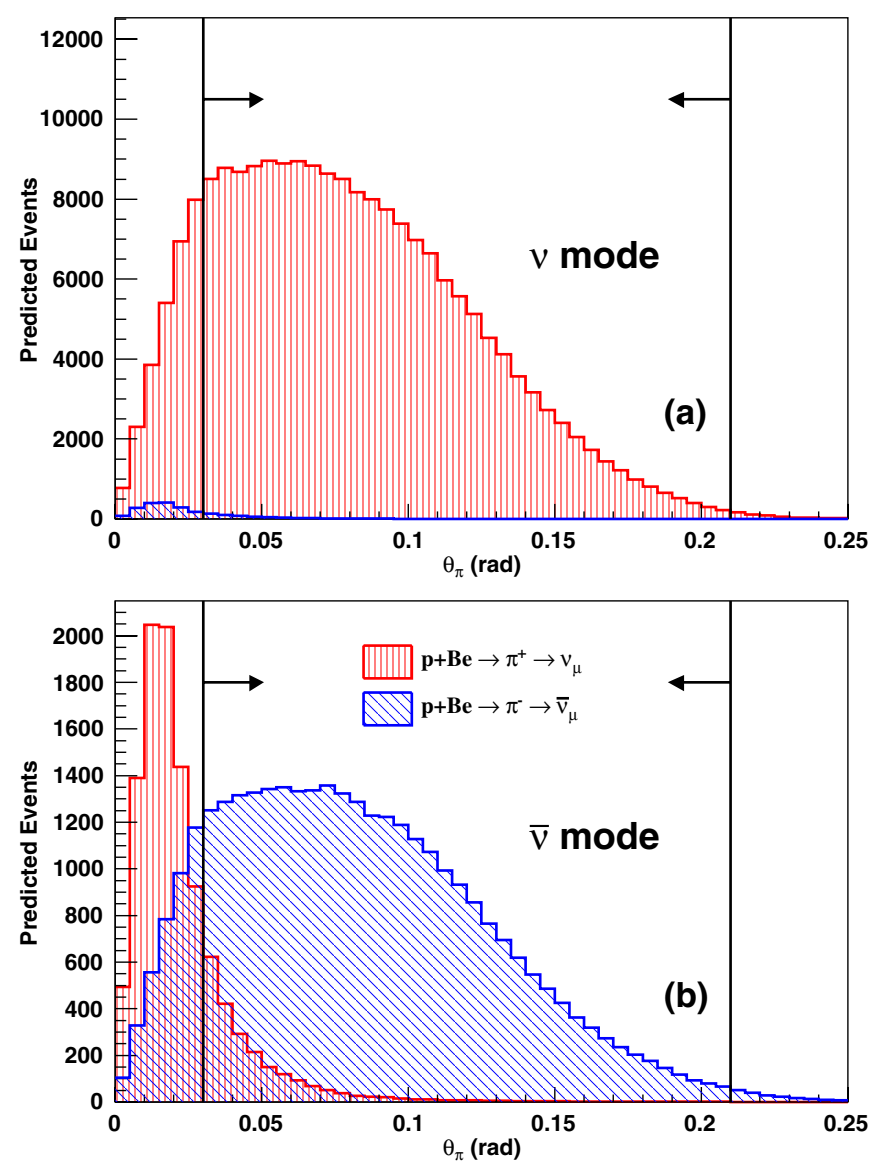

FIG. 1 (color online). Predicted angular distributions of pions with respect to the incident proton beam $\left(\theta_{\pi}\right)$ producing $\nu_{\mu}$ and $\bar{\nu}_{\mu}$ in (a) neutrino mode and (b) antineutrino mode. Only pions leading to $\nu_{\mu}$ and $\bar{\nu}_{\mu}$ events in the detector are shown, and all distributions are normalized to $5.66 \times 10^{20}$ protons on target. Arrows indicate the region where HARP data [14] are available.

The overall contamination rate is more significant in antineutrino mode due to effects from both flux and cross section: the leading-particle effect at the target preferentially produces about twice as many $\pi^{+}$as $\pi^{-}$, and the neutrino cross section is about 3 times higher than the antineutrino cross section in the MiniBooNE energy range $(\sim 1 \mathrm{GeV})$ [16]. For these reasons antineutrino-induced events are not a serious complication for neutrino-mode running, as these flux and cross section effects conspire to suppress their contribution, while the same effects amplify the neutrino contamination in antineutrino-mode data. Simulation predicts antineutrino events account for $\sim 1 \%$ of neutrino-mode data while neutrinos are responsible for $\sim 30 \%$ of antineutrino-mode data. This motivates a dedicated study of the neutrino flux contribution to the antineutrino-mode data. A data set corresponding to $5.66 \times 10^{20}$ protons on target is analyzed here and is important for both the ongoing MiniBooNE antineutrino oscillation search [4,5] and antineutrino cross section measurements. 
Two approaches for measuring the neutrino flux in antineutrino mode are taken. In the first method, a high-purity sample of charged-current single $\pi^{+}\left(\mathrm{CC} 1 \pi^{+}\right)$events isolate the $\nu_{\mu}$ contribution in the beam. The second method exploits the interference term in the chargedcurrent quasielastic (CCQE) cross section, where the angular distribution of final-state muons are predicted to be distinct for $\nu_{\mu}$ compared to $\bar{\nu}_{\mu}$ interactions. Both techniques were introduced in the MiniBooNE antineutrinomode run proposal [17]. These two approaches offer complementary means of measuring the neutrino flux component in antineutrino-mode data, with the CCQE sample providing a constraint at lower neutrino energies while the $\mathrm{CC} 1 \pi^{+}$measurement covers higher energies. They provide both a check of the MiniBooNE beam simulation in a region not covered by external data and demonstrate a set of techniques for measuring the $\nu_{\mu}$ contamination in an antineutrino-mode beam in the absence of a magnetized detector. It has been argued elsewhere that even modest statistical separation of charged-current neutrino and antineutrino events, afforded by the kind of analyses presented here, may be sufficient to meet the physics goals in proposed future experiments such as neutrino factories [18].

This paper is organized as follows: the MiniBooNE experiment is described in Sec. II while Sec. III details the neutrino and antineutrino scattering models. Two techniques to measure the neutrino contribution to the antineutrino flux are presented in Secs. IV and V. The results are compared in Sec. VI, implications for other neutrino experiments are discussed in Sec. VII and this work is summarized in Sec. VIII.

\section{THE MINIBOONE EXPERIMENT}

\section{A. Beamline and flux}

The Booster Neutrino Beamline (BNB) provides the neutrino and antineutrino flux to MiniBooNE. A beam of $8 \mathrm{GeV}$-kinetic-energy protons is extracted from the Booster synchrotron in "spills" of $5 \times 10^{12}$ protons over $1.6 \mu \mathrm{s}$ at a maximum rate of $5 \mathrm{~Hz}$. A lattice of alternatively focusing and defocusing quadrupole magnets steer the proton bunches to a beryllium target $71 \mathrm{~cm}$ (1.75 interaction lengths) long. The protons collide with the target to create a spray of secondary particles. An aluminum electromagnetic horn surrounding the target is pulsed to coincide with the p-Be collisions, creating a toroidal magnetic field to focus mesons of the desired charge. The horn pulses are such that the magnetic field is constant for the duration of the proton spill. In neutrino mode, the magnetic horn focuses positively charged secondary particles while defocusing those with negative charge; the horn effects are reversed in antineutrino mode. The focused mesons are allowed to decay in a $50 \mathrm{~m}$ air-filled decay region which terminates at a steel beam dump. The dominant decay modes of the mesons, mostly pions, produce muon neutrinos and antineutrinos.

A GEANT4-based model [19] is used to predict the neutrino and antineutrino flux at the detector. The simulation considers proton travel to the target, $\mathrm{p}$-Be interactions in the target including meson production, magnetic horn focusing, particle propagation, meson decay, and finally neutrino and antineutrino transport to the detector. As mentioned earlier, measurement of pion cross sections from $\mathrm{p}$-Be interactions are obtained from the HARP experiment. The HARP double differential cross section error matrix is used to set pion production uncertainties [20]. Even with valuable data constraints, meson production at the target contributes the largest systematic error to the flux prediction. The fractional uncertainty on pion production is $\sim 8 \%$ around the flux peak, while the uncertainty grows significantly in regions dominated by pions unconstrained by HARP data. The flux prediction in neutrino and antineutrino modes is presented in Fig. 2. Details of the beamline and flux prediction are given in Ref. [20].
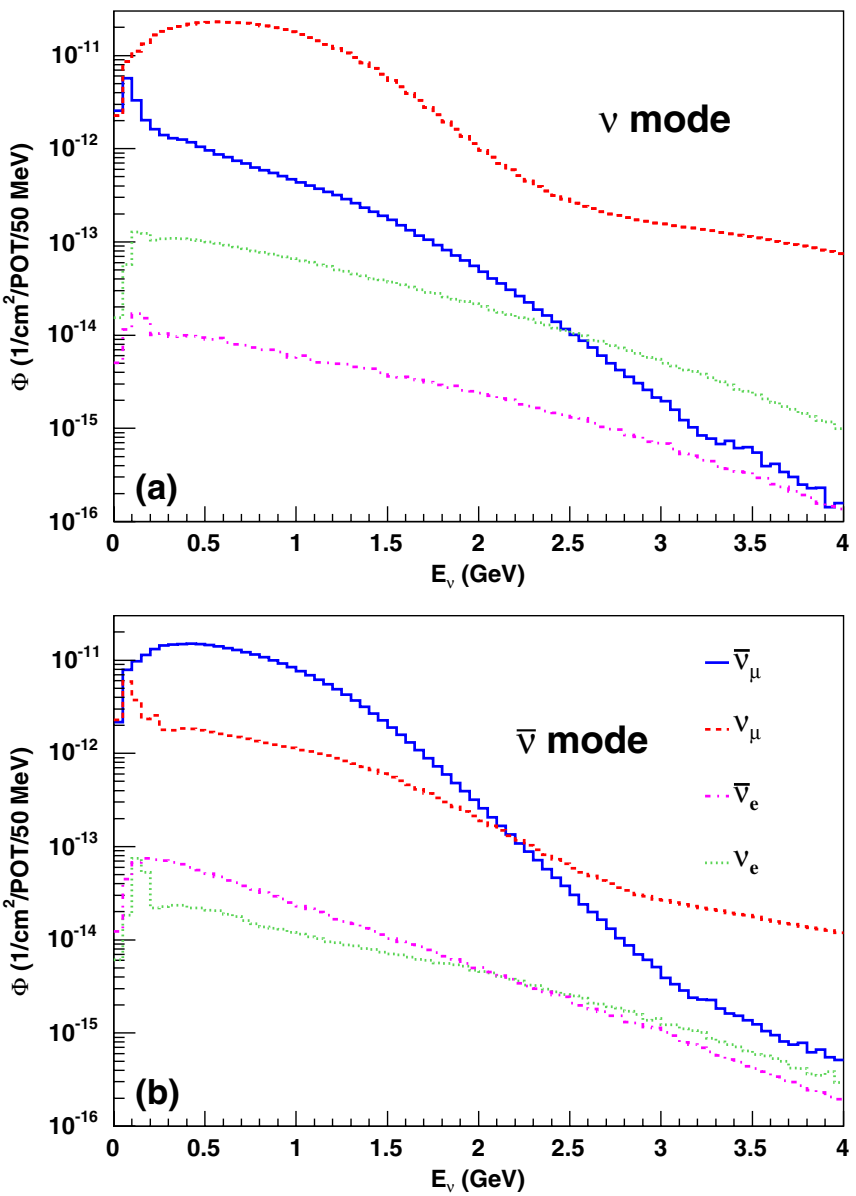

FIG. 2 (color online). The MiniBooNE flux prediction for (a) neutrino mode and (b) antineutrino mode. Because of the leading-particle effect, the neutrino contribution to the antineutrino-mode flux is more significant compared to the antineutrino component of the neutrino-mode beam. Plots taken from Ref. [20]. 


\section{B. Detector}

The MiniBooNE detector is a $6.1 \mathrm{~m}$-radius sphere filled with 818 tons of pure Marcol7 mineral oil. It houses 1520 8-inch Hamamatsu photomultiplier tubes (PMTs) segregated into two optically isolated regions: an inner signal region of $575 \mathrm{~cm}$ radius and an outer veto shell of thickness $35 \mathrm{~cm}$. The former contains 1280 PMTs (11.3\% coverage) while the latter holds 240 PMTs. The veto region is used to enforce containment of charged particles produced by neutrinos and antineutrinos from the beam and reject charged particles entering the tank.

The mineral oil has a density of $0.845 \mathrm{~g} / \mathrm{cm}^{3}$ with an index of refraction of 1.47 at $20^{\circ} \mathrm{C}$. Charged particles with velocity $\beta>0.68$ produce Cherenkov radiation. Particle identification and reconstruction is principally obtained through the pattern and timing of this prompt Cherenkov light; however, delayed scintillation light present due to fluorescent components in the oil has also been used effectively to provide energy information for charged particles produced below Cherenkov threshold [10].

MiniBooNE electronics record PMT charge and time information beginning about $5 \mu \mathrm{s}$ before the $1.6 \mu \mathrm{s}$ BNB proton delivery. Data are recorded for a total of $19.2 \mu \mathrm{s}$. The $5 \mu$ s interval before the beam spill is primarily present to minimize data contamination caused by cosmic ray muons stopping in the signal region prior to the start of the DAQ window. PMT activity is recorded for more than $10 \mu$ s after beam delivery to observe electrons from the atrest decay of muons (hereafter referred to as "Michel" electrons) subsequent to the initial neutrino or antineutrino-induced interaction.

The detector response to muons is measured using a dedicated muon tagging system that independently measures the energy and direction of cosmic ray muons up to $800 \mathrm{MeV}$. MiniBooNE employs a scintillator hodoscope directly above the detector and seven internal scintillator cubes at different depths, each connected to a dedicated 1-inch PMT for readout. The measured ranges and directions of muons traversing the hodoscope and stopping in cubes are used to verify muon reconstruction algorithms. The energy (angle) resolution improves from $12 \%$ (5.4 deg) at $100 \mathrm{MeV}$ to $3.4 \%$ (1.0 deg) at $800 \mathrm{MeV}$. Full detector details and calibrations are available in Ref. [21].

\section{Detector simulation}

The detector response to particle interactions and propagation is simulated using GEANT3 [22]. The entire detector geometry is considered, including the steel tank, external supports and main inner components. In addition, the surrounding environment composed of dirt external to the MiniBooNE enclosure, the concrete cylindrical housing and the air-filled gap between the detector and walls is treated. Of critical importance is the treatment of particle transport in the detector medium. The GEANT3 program takes as input the final-state particles emerging from the nucleus and simulates their propagation in the detector.

With a few exceptions, MiniBooNE uses the standard GEANT3 settings to simulate physics processes. Deviations include a custom model for light propagation in the detector oil and a substitution of the hadronic interaction model. The default GFLUKA hadron model is replaced by the GCALOR [23] package, which better models pion absorption $\left(\pi^{ \pm}+X \rightarrow X^{\prime}\right)$ and charge-exchange $\left(\pi^{ \pm}+\right.$ $\left.X \leftrightarrow \pi^{0}+X^{\prime}\right)$ processes. This is particularly relevant for the present analysis, where the predicted event composition of the two interaction samples studied is dependent on the pion survival model. Based on comparisons with external data [24] and the GCALOR prediction, an uncertainty of $35 \%(50 \%)$ is assigned to the pion absorption (charge exchange) interaction in the detector medium. The uncertainty for the same processes inside the nucleus is discussed in Sec. III C.

The model for light propagation in the oil is formed using a combination of external measurements and calibration data. Photon emission through Cherenkov and scintillation processes is simulated and propagated until the photon either is absorbed or hits a PMT photocathode, possibly leading to photoelectron production. Light emission, attenuation and scattering are included. The optical model of the detector describes the wavelength, time, and angular dependence of these processes [25].

\section{PREDICTED NEUTRINO AND ANTINEUTRINO INTERACTIONS}

MiniBooNE uses the NUANCE [16] event generator to simulate neutrino and antineutrino interactions in the detector. NUANCE includes a comprehensive neutrino and antineutrino cross section model which considers known interactions in the neutrino and antineutrino energy range from $\sim 100 \mathrm{MeV}$ to $1 \mathrm{TeV}$. Ninety-nine reactions are modeled separately and combined with nuclear models describing bound nucleon states and final-state interactions to predict event rates and kinematics.

Bound nucleons in the detector medium are described by the Relativistic Fermi Gas model [26]. This assumes the nucleons to be independent and quasifree. Also specified is a hard cutoff in available struck nucleon energies as dictated by the exclusion principle.

The neutrino and antineutrino interaction types relevant to the analysis presented here are charged-current quasielastic (Sec. III A) and pion production (Sec. III B). The neutrino-induced absolute cross sections for both processes have been measured at MiniBooNE using a flux prediction well determined by HARP data. These cross section measurements are utilized in the antineutrino-mode simulation. 


\section{A. Charged-current quasielastic scattering}

To model CCQE interactions, this analysis uses measured cross sections from the MiniBooNE neutrinomode CCQE data [9] and a model which has been found to reproduce well the kinematics of such events. Specifically, MiniBooNE adopts the CCQE scattering formalism of Smith-Moniz [26]. The vector component of the interaction is measured by electron scattering experiments and is assumed to have a nondipole form [27]. The axial-vector form factor employs a dipole construction, containing an "axial mass," $M_{A}$, taken either from MiniBooNE or external data, depending on the neutrino target.

The MiniBooNE mineral oil is composed of $\mathrm{C}_{n} \mathrm{H}_{2 n+2}$, $n \sim 20$, and the prediction for CCQE scattering is different for the two flavors of target. In the present analysis, $M_{A}^{\text {eff }}=1.35 \pm 0.17 \mathrm{GeV}$, together with a Pauli blocking adjustment, $\kappa=1.007 \pm 0.012$, are assumed for bound nucleon scattering. These values come from a highstatistics analysis of MiniBooNE $\nu_{\mu}$ CCQE events on carbon [9] and are consistent with values recently determined from an independent MiniBooNE neutral-current elastic scattering sample [10]. A previous shape-only study has shown that these CCQE model parameters reproduce the MiniBooNE antineutrino-mode data shape [28], and therefore the same $M_{A}^{\text {eff }}$ and $\kappa$ values are applied to both $\nu_{\mu}$ and $\bar{\nu}_{\mu}$ CCQE scattering events on carbon.

For free scattering of hydrogen, a process accessible to antineutrino and not neutrino CCQE events, a value of $M_{A}=1.03 \pm 0.02 \mathrm{GeV}$ is used based on a global fit to previous light target data [29].

In the case of carbon scattering, the superscript "eff", short for "effective," on $M_{A}$ is introduced to allow for the possibility that nuclear effects are responsible for the apparent discrepancy between the MiniBooNE carbon-based measurements and light target results. This is also theoretically motivated by a possible reconciliation between the measurements through a mechanism resulting in intranuclear correlations of greater importance than previously thought [30-34]. Such a mechanism would indicate a larger CCQE cross section for nuclear targets than for free scattering, which in this case, is reflected in the higher $M_{A}$ choice for carbon versus hydrogen scattering.

\section{B. Pion production}

Baryonic resonances are the dominant source of single pion production at MiniBooNE. The formalism to describe these events is taken from the Rein-Sehgal model [35], where the relativistic harmonic oscillator quark model is assumed [36]. Eighteen resonances are considered, however the $\Delta(1232)$ is dominant in the energy range spanned by MiniBooNE. Multipion production mechanisms are also considered, though their contribution is predicted to be small.
The axial masses in the resonance channels are set simultaneously to reproduce inclusive non-MiniBooNE charged-current data [37]. The extracted values are $M_{A}^{1 \pi}=1.10 \pm 0.27 \mathrm{GeV}$ (single pion production) and $M_{A}^{\text {multi- } \pi}=1.30 \pm 0.52 \mathrm{GeV}$ (multipion production).

In the present analysis the charged-current single $\pi^{+}\left(\mathrm{CC} 1 \pi^{+}\right)$prediction with these assumptions is adjusted to reproduce the kinematic distributions measured in MiniBooNE neutrino-mode data $[9,12]$.

\section{Final-state interactions}

For a neutrino or antineutrino interaction with a nucleon bound in carbon, NUANCE propagates the outgoing hadrons including nucleons, mesons and baryonic resonances, and simulates their reinteraction as they exit the nucleus. The initial interaction model employs the impulse approximation which assumes an instantaneous exchange with independent nucleons. Subsequent to the initial neutrino or antineutrino interaction, particles produced inside the nucleus are propagated stepwise in $0.3 \mathrm{fm}$ increments until they emerge from the $\sim 2.5 \mathrm{fm}$ radius sphere. Intermittently, the probability for hadronic reinteraction is calculated using a radially-dependent nucleon density distribution [38] along with external $\pi-N, N-N$ cross section measurements [39]. For $\Delta$ reinteractions $(\Delta+$ $N \rightarrow N+N$ ), an energy-independent probability of $20 \%$ $(10 \%)$ is taken for $\Delta^{+}+N, \Delta^{0}+N\left(\Delta^{++}+N, \Delta^{-}+N\right)$ based on K2K data [37] and is assigned $100 \%$ uncertainty.

As mentioned earlier, out of all hadronic reinteraction processes, pion absorption and charge exchange $\left(\pi^{ \pm}+\right.$ $X \leftrightarrow \pi^{0}+X^{\prime}$ ) are the most relevant in predicting the composition of the $\mathrm{CC} 1 \pi^{+}$(Sec. IVA) and CCQE (Sec. VA) samples studied in this analysis. Intranuclear fractional uncertainties on pion absorption (charge exchange) are set to $25 \%$ (30\%) based on comparisons between external data [24] and NUANCE. The simulation of these two processes in the detector medium is addressed separately in the detector simulation (Sec. II C).

\section{MEASURING THE NEUTRINO FLUX COMPONENT IN THE CC1 $\pi^{+}$SAMPLE}

\section{A. The CC1 $\pi^{+}$sample}

The events in the $\mathrm{CC} 1 \pi^{+}$sample in antineutrino mode originate almost exclusively from $\nu_{\mu}$ interactions, making it an excellent candidate for measuring the $\nu_{\mu}$ content of the antineutrino-mode beam. In the few-GeV energy range, the dominant charged-current single pion production channels contain a final-state $\pi^{+}\left(\pi^{-}\right)$in the case of $\nu_{\mu}\left(\bar{\nu}_{\mu}\right)$ scattering. MiniBooNE cleanly identifies $\mathrm{CC} 1 \pi^{+}$events by selecting 3 "subevents", attributed to the muon from the primary $\nu_{\mu}$ interaction and two subsequent decay electrons, one each from the $\mu^{-}$and $\pi^{+}$decay chain: 


$$
\begin{aligned}
\text { 1: } & \nu_{\mu}+p(n) \rightarrow & \mu^{-}+p(n)+\pi^{+} \hookrightarrow \mu^{+}+\nu_{\mu} \\
\text { 2: } & & \hookrightarrow e^{-}+\bar{\nu}_{e}+\nu_{\mu} \\
\text { 3: } & & \hookrightarrow e^{+}+\nu_{e}+\bar{\nu}_{\mu} .
\end{aligned}
$$

The monoenergetic $\mu^{+}$from the decay of stopped $\pi^{+}$ does not lead to a separate subevent due to the short lifetime of the $\pi^{+}$. Subevents are defined as clusters in time of PMT activity (or PMT "hits"). A hit is any PMT pulse passing the discriminator threshold of $\sim 0.1$ photoelectrons. A temporal cluster of PMT activity with at least 10 hits within a 200 ns window and individual hit times less than $10 \mathrm{~ns}$ apart, while allowing for at most two spacings of 10-20 ns, defines a subevent. Apart from detection efficiencies, some neutrino-induced $\mathrm{CC} 1 \pi^{+}$ events do not enter the three-subevent sample as $\sim 8 \%$ of $\mu^{-}$are captured in carbon [40] and therefore do not lead to the production of a Michel electron. Other selection cuts made to enhance sample purity and improve reconstruction are given with efficiencies in Table I. Cut 1 is the threesubevent criterion previously detailed. Cut 2 requires that the first subevent occur during a $3 \mu$ s time window centered on the BNB proton spill. Cut 3 rejects events close to the detector edge that are likely to be poorly reconstructed. Selection cuts on the number of tank hits are based primarily on the observation that Michel electrons produce fewer than 200 tank hits. Cut 4 ensures the first subevent is not a Michel electron and rejects low-energy muons that might be reconstructed poorly. Cut 5 requires that the number of hits for the second and third subevents is consistent with a Michel electron. Veto PMT activity is monitored simultaneously with the main tank PMTs, thus Cut 6 ensures no subevent is due to charged particles entering the tank and that all charged particles produced inside the detector are contained. Cut 7 enforces spatial correlation between the end of the muon track and the closest Michel

TABLE I. Summary of selection cuts in the CC1 $\pi^{+}$sample.

\begin{tabular}{|c|c|c|c|}
\hline Cut \# & Description & $\begin{array}{l}\text { Efficiency } \\
\quad(\%)\end{array}$ & $\begin{array}{l}\text { Purity } \\
(\%)\end{array}$ \\
\hline 0 & No cuts & 100 & 10 \\
\hline 1 & Three subevents & 30 & 29 \\
\hline 2 & $\begin{array}{l}\text { 1st subevent in event time window } \\
\qquad 4000<T(\mathrm{~ns})<7000\end{array}$ & 28 & 34 \\
\hline 3 & $\begin{array}{l}\text { All subevents: reconstructed vertex } \\
<500 \mathrm{~cm} \text { from tank center }\end{array}$ & 23 & 36 \\
\hline 4 & 1st subevent: tank hits $>200$ & 22 & 39 \\
\hline 5 & $\begin{array}{l}\text { 2nd, 3rd subevents: } \\
\text { tank hits }<200\end{array}$ & 19 & 65 \\
\hline 6 & All subevents: veto hits $<6$ & 16 & 78 \\
\hline 7 & $\begin{array}{l}\text { Distance between reconstructed } \\
\text { end of } 1 \text { st subevent and nearest } \\
\text { Michel electron vertex }<150 \mathrm{~cm}\end{array}$ & 12 & 82 \\
\hline
\end{tabular}
Purity and efficiency numbers are sequential and are calculated for the "observable $\mathrm{CC} 1 \pi^{+}$" event signature $-1 \mu^{-}, 1 \pi^{+}$. electron vertex. This reduces a class of backgrounds where neither the second nor the third subevent arise from the decay of the muon to a Michel electron. This cut is applied only to the Michel closest to the end of the reconstructed primary muon track as the pion lifetime compared to the muon is short enough that either Michel can occur temporally first.

Charged-current single $\pi^{-}$events induced by $\bar{\nu}_{\mu}$ are largely rejected by the primary requirement of three subevents because most $\pi^{-}$come to rest and are captured by carbon nuclei [41], yielding no decay electron. The predicted event composition after this selection is presented in Table II. The sample is $82 \%$ observable $\mathrm{CC} 1 \pi^{+}$events (i.e., events with a single muon, a single $\pi^{+}$, and any number of nucleons exiting the initial target nucleus). Some $\bar{\nu}_{\mu} \mathrm{CC} 1 \pi^{-}$events do make it into the sample, primarily due to decay-in-flight $\pi^{-}$. Starting from an event population that is $\sim 70 \% \bar{\nu}_{\mu}$, this simple two-decay electron requirement remarkably yields a sample that is $\sim 80 \%$ pure $\nu_{\mu}$.

\section{B. $\mathrm{CC1} \pi^{+}$event reconstruction}

In this analysis, charged-current single $\pi^{+}$event reconstruction relies exclusively on the observation of the outgoing muon. Muon kinematics are obtained by the pattern, timing, and total charge of prompt Cherenkov radiation collected by PMTs in the first subevent of the interaction. The topology and timing of the observed PMT hits are compared to a likelihood function operating under a muon hypothesis. This likelihood function predicts hit patterns and timing based on the interaction vertex and the momentum four-vector of the muon. The likelihood function simultaneously varies these seven parameters while comparing to the observed PMT hits. The parameters from the maximized likelihood function yield the reconstructed muon kinematics.

Under the assumption of $\Delta(1232)$ production by a neutrino scattering off a stationary nucleon target in carbon, the neutrino energy is given by

$$
E_{\nu}^{\Delta}=\frac{2\left(M_{p}-E_{B}\right) E_{\mu}-\left(E_{B}^{2}-2 M_{p} E_{B}+m_{\mu}^{2}+\Delta M^{\prime 2}\right)}{2\left[\left(M_{p}-E_{B}\right)-E_{\mu}+p_{\mu} \cos \theta_{\mu}\right]},
$$

TABLE II. Predicted event composition of the CC1 $\pi^{+}$sample in antineutrino mode.

\begin{tabular}{lc}
\hline \hline Interaction channel & Contribution (\%) \\
\hline$\nu_{\mu} N \rightarrow \mu^{-} \pi^{+} N$ (resonant) & 64 \\
$\nu_{\mu} A \rightarrow \mu^{-} \pi^{+} A$ (coherent) & 7 \\
$\bar{\nu}_{\mu} N \rightarrow \mu^{+} \pi^{-} N$ (resonant) & 6 \\
$\nu_{\mu} n \rightarrow \mu^{-} p$ & 6 \\
$\nu_{\mu} n \rightarrow \mu^{-} \pi^{0} p$ & 2 \\
$\bar{\nu}_{\mu} p \rightarrow \mu^{+} \pi^{0} n$ & 1 \\
Other (mostly DIS) & 14 \\
"Observable CC $1 \pi^{+}$" $\left(1 \mu^{-}, 1 \pi^{+}\right)$ & 82 \\
\hline \hline
\end{tabular}




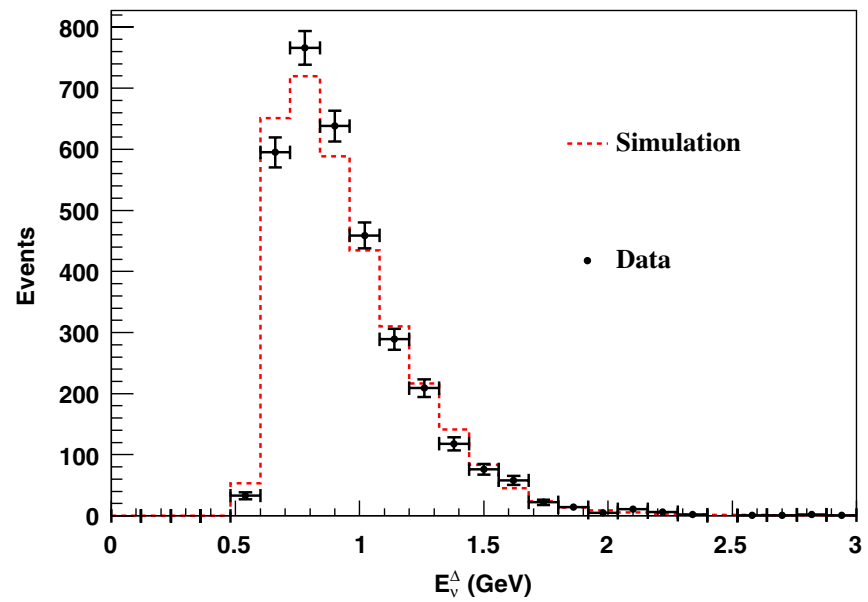

FIG. 3 (color online). The reconstructed energy spectrum for simulation versus data in the antineutrino-mode $\mathrm{CC} 1 \pi^{+}$sample. Simulation is normalized to data, and only statistical errors are shown.

where $E_{B}=34 \mathrm{MeV}$ is the binding energy, $m_{\mu}$ is the muon mass, $\Delta M^{\prime 2}=M_{p}^{2}-M_{\Delta}^{2}$, where $M_{\Delta}\left(M_{p}\right)$ is the $\Delta(1232)$ (proton) mass, $p_{\mu}$ is the muon momentum, and $\theta_{\mu}$ is the outgoing muon angle relative to the incoming neutrino beam. Effects not accounted for in the reconstruction include nonresonant pion production, contributions from higher mass $\Delta$ resonances, and scattering off the quasifree protons in hydrogen instead of carbon. A shape comparison of reconstructed $E_{\nu}^{\Delta}$ in data and simulation is presented in Fig. 3.

\section{Measuring the neutrino flux component in the antineutrino-mode $\mathrm{CC} 1 \pi^{+}$sample}

The simulation sample is separated into two components: observable $\mathrm{CC} 1 \pi^{+}$events and background. All observable $\mathrm{CC} 1 \pi^{+}$events in the simulation are modeled using the $\mathrm{CC} 1 \pi^{+}$cross section that has been measured in MiniBooNE neutrino-mode data [12]. Given that the majority of the $\mathrm{CC} 1 \pi^{+}$sample in antineutrino mode is induced by neutrinos, with this cross section measurement applied any remaining normalization difference between data and simulation is interpreted as a neutrino flux measurement. Results are presented in Table III. Events in the antineutrino-mode $\mathrm{CC} 1 \pi^{+}$sample indicate the neutrino flux in antineutrino mode is lower than the simulation predicts. The extracted calibration is $0.76 \pm 0.11$ of the nominal prediction over all reconstructed energies, while the analysis applied to individual energy ranges does not indicate any significant energy dependence.

\section{Systematic errors}

The systematic error on the neutrino flux measurement using the antineutrino-mode $\mathrm{CC} 1 \pi^{+}$sample comes from two sources that are treated as uncorrelated with each other: the uncertainty on the $\mathrm{CC} 1 \pi^{+}$cross section obtained
TABLE III. Antineutrino-mode CC $1 \pi^{+}$sample details and $\nu_{\mu}$ flux component measurement. The measured cross section has been applied to simulation, and the $\nu_{\mu}$ flux scale is found by calculating (observed events-expected $\bar{\nu}_{\mu}$ events)/expected $\nu_{\mu}$ events. The reported error is discussed in more detail in Sec. IV D. The Monte Carlo sample is generated so that the associated statistical error is negligible compared to the other sources of uncertainty.

\begin{tabular}{lccccc}
\hline \hline $\begin{array}{l}E_{\nu}^{\Delta} \text { Range } \\
(\mathrm{MeV})\end{array}$ & $\begin{array}{c}\text { Mean gen. } E_{\nu} \\
(\mathrm{MeV})\end{array}$ & $\begin{array}{c}\text { Events } \\
\text { in data }\end{array}$ & \multicolumn{2}{c}{ Expected } & \multicolumn{2}{c}{$\nu_{\mu}$ Flux } \\
\hline $600-700$ & 961 & 465 & 556 & 104 & $0.65 \pm 0.10$ \\
$700-800$ & 1072 & 643 & 666 & 118 & $0.79 \pm 0.10$ \\
$800-900$ & 1181 & 573 & 586 & 97 & $0.81 \pm 0.10$ \\
$900-1000$ & 1285 & 495 & 474 & 78 & $0.88 \pm 0.11$ \\
$1000-1200$ & 1426 & 571 & 646 & 92 & $0.74 \pm 0.10$ \\
$1200-2400$ & 1685 & 521 & 614 & 74 & $0.73 \pm 0.15$ \\
Inclusive & 1266 & 3268 & 3542 & 563 & $0.76 \pm 0.11$ \\
\hline \hline
\end{tabular}

from [12] and the uncertainty in the background prediction. The largest contribution to the uncertainty on the $\mathrm{CC} 1 \pi^{+}$ cross section comes from the neutrino-mode flux uncertainty, which is the only systematic error associated with the cross section measurement that is also independent of the measurement made here. Because the other $\mathrm{CC} 1 \pi^{+}$ uncertainties are treated as uncorrelated, a partial cancellation of errors is ignored in the present neutrino flux measurement. This results in a slight overestimate of the neutrino flux uncertainty. Both $\nu_{\mu}$ and $\bar{\nu}_{\mu}$ background events in the sample are assigned $30 \%$ uncertainties to conservatively recognize the model dependence of the sample composition. The fractional uncertainty contributions to the flux measurement are presented in Table IV.

\section{MEASURING THE NEUTRINO FLUX THROUGH MUON ANGULAR DISTRIBUTIONS IN THE CCQE SAMPLE}

\section{A. The CCQE sample}

The CCQE interaction is the dominant channel in MiniBooNE's energy range. CCQE events typically have

TABLE IV. Fractional uncertainty (\%) contributions to the neutrino flux measurement in the $\mathrm{CC} 1 \pi^{+}$sample. The $\nu_{\mu}$ uncertainty is dominated by the $\mathrm{CC} 1 \pi^{+}$crosssection error.

\begin{tabular}{lcccc}
\hline \hline$E_{\nu}^{\mathrm{QE}}$ Range $(\mathrm{MeV})$ & Statistical & $\bar{\nu}_{\mu}$ & $\nu_{\mu}$ & Total fractional error \\
\hline $600-700$ & 6 & 9 & 11 & 15 \\
$700-800$ & 5 & 7 & 10 & 13 \\
$800-900$ & 5 & 6 & 10 & 13 \\
$900-1000$ & 5 & 6 & 10 & 13 \\
$1000-1200$ & 5 & 6 & 11 & 13 \\
$1200-2400$ & 5 & 5 & 19 & 20 \\
Inclusive & 2 & 6 & 13 & 14 \\
\hline \hline
\end{tabular}


two subevents, attributed to the primary muon and the associated decay positron:

$$
\begin{array}{ll}
\text { 1: } \bar{\nu}_{\mu}+p \rightarrow & \mu^{+}+n \\
2: & \hookrightarrow e^{+}+\nu_{e}+\bar{\nu}_{\mu} .
\end{array}
$$

The CCQE sample is therefore similar in formation to the $\mathrm{CC} 1 \pi^{+}$sample with one major divergence: a requirement of two subevents instead of three. As shown in Table V, the CCQE selection cuts closely follow those motivated in Sec. IVA, with a few exceptions appropriate to the inclusion of a single Michel electron. The Michel tank hit and veto PMT hit cuts apply to the second subevent only now (Cuts 5 and 6, respectively), and the muon endpoint-electron vertex cut in Cut 7 is tightened to $100 \mathrm{~cm}$ in light of larger backgrounds. The selection cuts outlined here are identical to those employed in a previous shape-only extraction of CCQE model parameters [6] and closely follow those used in the absolute measurement of the $\nu_{\mu}$ induced CCQE cross section [9], with only minor differences that result in approximately the same sample efficiency and purity.

Despite the selection cuts, there are formidable backgrounds to the antineutrino-mode CCQE sample. Prior to this analysis, simulation estimates the antineutrino-mode CCQE sample has a purity just above $50 \%$ as shown in Table VI. The major backgrounds include $\mathrm{CC} 1 \pi^{+}$and $\mathrm{CC} 1 \pi^{-}$events, which account for a total of $\sim 20 \%$ of the sample, and the $\nu_{\mu}$ processes, predicted to be responsible for $\sim 30 \%$ of the sample. The $30 \%$ predicted $\nu_{\mu}$ contamination is investigated and ultimately constrained in this analysis.

A few additional modifications to the simulation are made to accommodate the backgrounds. The largest nonCCQE background in the sample is single pion production which enters the sample due to nuclear effects, including

TABLE V. Summary of selection cuts with efficiencies in the CCQE sample. "Purity" refers to $\bar{\nu}_{\mu}$ CCQE only, and purity and

\begin{tabular}{|c|c|c|c|}
\hline Cut \# & Description & $\begin{array}{l}\text { Efficiency } \\
\quad(\%)\end{array}$ & $\begin{array}{l}\text { Purity } \\
(\%)\end{array}$ \\
\hline 0 & No cuts & 100 & 32 \\
\hline 1 & Two subevents & 49 & 41 \\
\hline 2 & $\begin{array}{l}\text { 1st subevent in event time window } \\
\qquad 4000<T(\mathrm{~ns})<7000\end{array}$ & 47 & 42 \\
\hline 3 & $\begin{array}{l}\text { 1st subevent: reconstructed vertex } \\
<500 \mathrm{~cm} \text { from tank center }\end{array}$ & 38 & 43 \\
\hline 4 & 1st subevent: tank hits $>200$ & 35 & 45 \\
\hline 5 & 2nd subevent: tank hits $<200$ & 33 & 45 \\
\hline 6 & Both subevents: veto hits $<6$ & 29 & 49 \\
\hline 7 & $\begin{array}{l}\text { Distance between reconstructed } \\
\text { end of } 1 \text { st subevent and } \\
\text { 2nd subevent vertex }<100 \mathrm{~cm}\end{array}$ & 25 & 54 \\
\hline
\end{tabular}
efficiency numbers are sequential.
TABLE VI. Predicted composition of the antineutrino-mode CCQE sample.

\begin{tabular}{lc}
\hline \hline Channel & Contribution $(\%)$ \\
\hline $\bar{\nu}_{\mu} p \rightarrow \mu^{+} n$ & 54 \\
$\nu_{\mu} n \rightarrow \mu^{-} p$ & 20 \\
$\bar{\nu}_{\mu} N \rightarrow \mu^{+} \pi^{-} N$ (resonant) & 8 \\
$\nu_{\mu} N \rightarrow \mu^{-} \pi^{+} N$ (resonant) & 6 \\
$\bar{\nu}_{\mu} A \rightarrow \mu^{+} \pi^{-} A$ (coherent) & 4 \\
$\bar{\nu}_{\mu} N \rightarrow \mu^{+} \Lambda, \Sigma$ & 3 \\
$\bar{\nu}_{\mu} p \rightarrow \mu^{+} \pi^{0} n$ & 2 \\
Other & 3 \\
All $\bar{\nu}_{\mu}$ & 71 \\
All $\nu_{\mu}$ & 29 \\
\hline \hline
\end{tabular}

$\mu^{-}, \pi^{-}$capture and final-state interactions; however, in the case of antineutrino-induced $\mathrm{CC} 1 \pi^{-}$scattering, due to $\pi^{-}$nuclear capture almost $100 \%$ of $\mathrm{CC} 1 \pi^{-}$events have only two subevents and are experimentally indistinguishable from CCQE. This implies a direct background measurement of $\mathrm{CC} 1 \pi^{-}$events (analogous to what was done in Ref. [9]) is impossible. Therefore, though the $\mathrm{CC} 1 \pi^{+}$yield constraint made in Ref. [9] is strictly appropriate to neutrino-induced $\mathrm{CC} 1 \pi^{+}$events only, it is applied to both predicted $\mathrm{CC} 1 \pi^{+}$and $\mathrm{CC} 1 \pi^{-}$background events in the CCQE sample.

Many backgrounds to the CCQE sample peak in the most forward scattering region of the muon angular distribution with respect to the incoming neutrino beam. This includes pion production and hydrogen CCQE scatteringwhile the latter is technically not a background, the proper handling of the difference in nuclear effects between bound and free targets is not straightforward. Additionally, the forward scattering region is strongly correlated with low $Q^{2}$ events, a problematic region both experimentally and theoretically [42]. Such low $Q^{2}$ data are dominated by $\bar{\nu}_{\mu}$ interactions, while the present analysis is principally interested in backwards scattering muons which is dominated by $\nu_{\mu}$. For these reasons, events with $\cos \theta_{\mu}>0.91$ are not included in the fit to data, where $\theta_{\mu}$ is the outgoing muon angle relative to the incoming neutrino beam.

\section{B. CCQE event reconstruction}

Event reconstruction in the antineutrino-mode CCQE sample proceeds similarly as in the $\mathrm{CC} 1 \pi^{+}$sample, described in Sec. IV B. As in the $\mathrm{CC} 1 \pi^{+}$reconstruction, the measurement of muon kinematics from the primary interaction is solely responsible for recreating the incident neutrino energy. No requirement is made on the ejected nucleon; this is an important distinction from the CCQE definitions used by other experiments [43,44], where a single proton track may be required in the case of neutrino-induced CCQE. A similar energy reconstruction as described in Sec. IV B is implemented, but in this sample a $\bar{\nu}_{\mu}$ probe is assumed: 


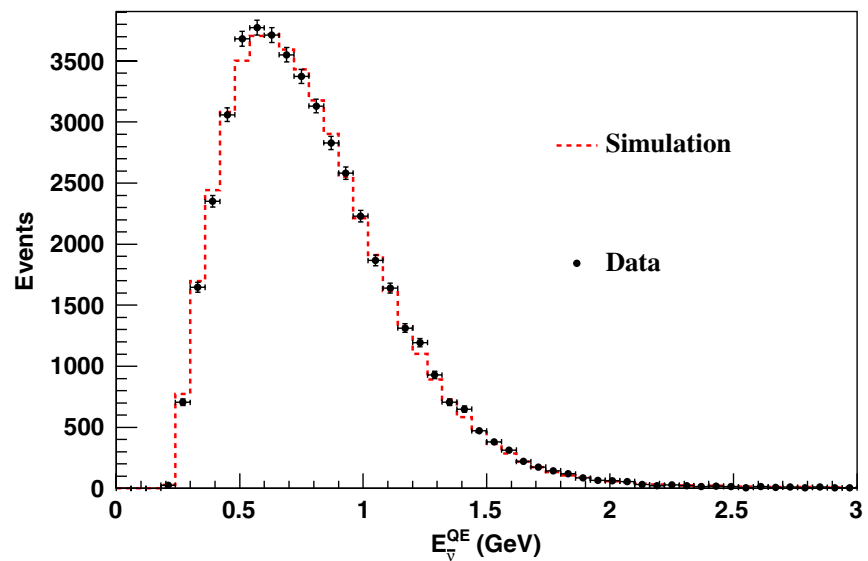

FIG. 4 (color online). The reconstructed energy spectrum for simulation versus data in the antineutrino-mode CCQE sample. Simulation is normalized to data, and only statistical errors are shown.

$$
E_{\bar{\nu}}^{\mathrm{QE}}=\frac{2\left(M_{p}-E_{B}\right) E_{\mu}-\left(E_{B}^{2}-2 M_{p} E_{B}+m_{\mu}^{2}+\Delta M^{2}\right)}{2\left[\left(M_{p}-E_{B}\right)-E_{\mu}+p_{\mu} \cos \theta_{\mu}\right]},
$$

where the same definitions from Eq. (2) apply and $\Delta M^{2}=$ $M_{p}^{2}-M_{n}^{2}$, where $M_{n}$ is the neutron mass. Figure 4 presents the reconstructed energy distributions in simulation and data in the CCQE sample. CCQE scattering with free protons in hydrogen are indistinguishable from those on bound protons in carbon, so all events in data and simulation are reconstructed using the carbon scattering assumption implicit in Eq. (4).

\section{Neutrino flux measurement using CCQE}

Neutrino and antineutrino CCQE cross sections differ exclusively by an axial-vector interference term that amplifies $\nu$ scattering while suppressing $\bar{\nu}$ events. A particularly clean way to exploit this cross section difference is to fit the angular distribution of the primary muon. The contribution from $\bar{\nu}_{\mu}$ is suppressed in the backward scattering region. Figure 5 shows the predicted $\nu_{\mu}$ and $\bar{\nu}_{\mu}$ contributions to the cosine of the outgoing muon angle.

To measure the neutrino content in the antineutrinomode beam, the Monte Carlo sample is separated into two $\cos \theta_{\mu}$ templates, one arising from all $\nu_{\mu}$ interactions and the other from $\bar{\nu}_{\mu}$, regardless of interaction channel and nuclear target. A linear combination of these two templates is then formed

$$
T_{\mathrm{MC}}\left(\alpha_{\nu}, \alpha_{\bar{\nu}}\right) \equiv \alpha_{\nu} \nu^{\mathrm{MC}}+\alpha_{\bar{\nu}} \bar{\nu}^{\mathrm{MC}},
$$

where $T_{\mathrm{MC}}$ is the total predicted $\cos \theta_{\mu}$ distribution to be compared to data, $\alpha_{\nu}$ and $\alpha_{\bar{\nu}}$ are neutrino and antineutrino rate scales, and $\nu^{\mathrm{MC}}$ and $\bar{\nu}^{\mathrm{MC}}$ are the Monte Carlo neutrino and antineutrino scattering angular predictions, respectively. The modified simulation sample is compared to

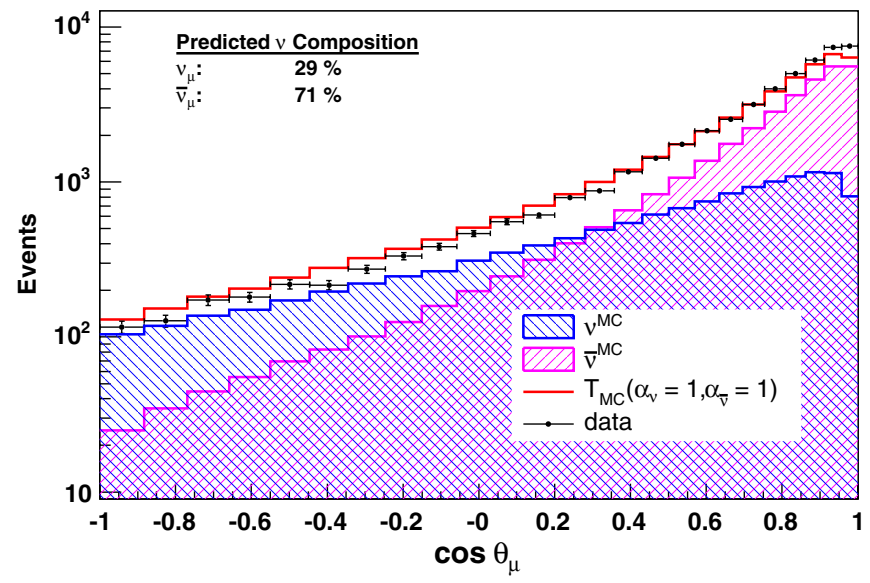

FIG. 5 (color online). The $\cos \theta_{\mu}$ distribution of the CCQE sample by neutrino type before fitting. As printed on the figure, $29 \%$ of the sample is predicted to be induced by neutrinos. The Monte Carlo sample has been normalized to $5.66 \times 10^{20}$ protons on target.

data by forming a goodness-of-fit $\chi^{2}$ test as a function of the rate scales

$\chi^{2}=\sum_{i, j}\left(T_{\mathrm{MC}}\left(\alpha_{\nu}, \alpha_{\bar{\nu}}\right)_{i}-d_{i}\right) M_{i j}^{-1}\left(T_{\mathrm{MC}}\left(\alpha_{\nu}, \alpha_{\bar{\nu}}\right)_{j}-d_{j}\right)$,

where $i$ and $j$ label bins of $\cos \theta_{\mu}, d$ is data and $M$ is the symmetric error matrix given in Eq. (7). The error matrix is used to propagate correlated uncertainties on parameters and processes to the quantities reported in the analysis. It is made by first forming weights corresponding to simulation excursions set by Gaussian variations of parameters within their associated error. The difference of these weighted events from the simulated central value forms the error matrix

$$
M_{i j}=\frac{1}{K} \sum_{s=1}^{K}\left(N_{i}^{s}-N_{i}^{\mathrm{CV}}\right) \times\left(N_{j}^{s}-N_{j}^{\mathrm{CV}}\right) .
$$

Here $K$ simulation excursions are used $(K=100$ in this analysis), $N^{s}$ is the reweighted number of entries corresponding to the sth simulation set, and $N^{\mathrm{CV}}$ represents the MiniBooNE simulation central value. This technique is further described in Ref. [45]. Bin-by-bin $\cos \theta_{\mu}$ correlations between $\nu_{\mu}$ and $\bar{\nu}_{\mu}$ are also treated. The specific systematic errors are discussed in the next section.

The fit is performed analytically in three bins of reconstructed energy and also in an inclusive energy sample. Results including statistical and systematic uncertainties are presented in Table VII. The fits to data are shown in the Appendix, where Fig. 7 contains both the fitted distributions and the fractional differences between the simulation and data before and after the fits. The adjusted contributions of $\nu_{\mu}$ and $\bar{\nu}_{\mu}$ to the CCQE sample are compared to the prediction in Table VIII.

The $\chi^{2}$ value for the angular fit in the reconstructed energy range $E_{\nu}^{\mathrm{QE}}>900 \mathrm{MeV}$ is unusually low at 
TABLE VII. Fit results in three energy bins and an inclusive sample. The results are consistent with an overprediction of the $\nu_{\mu}$ contamination of the MiniBooNE antineutrino-mode CCQE sample.

\begin{tabular}{lcccccr}
\hline \hline $\begin{array}{l}E_{\bar{\nu}}^{\mathrm{QE}} \text { Range } \\
(\mathrm{MeV})\end{array}$ & $\begin{array}{c}\text { Mean generated } \\
E_{\nu}(\mathrm{MeV})\end{array}$ & $\begin{array}{c}\text { Events in } \\
\text { data }\end{array}$ & $\alpha_{\nu}$ fit & $\alpha_{\bar{\nu}}$ fit & $\begin{array}{c}\rho_{\alpha_{\nu}-\alpha_{\bar{\nu}} \text { fit }} \\
\text { correlation }\end{array}$ & $\chi^{2}(\mathrm{DOF}=21)$ \\
\hline$<600$ & 675 & 15242 & $0.65 \pm 0.22$ & $0.98 \pm 0.18$ & 0.33 & 13 \\
$600-900$ & 897 & 16598 & $0.61 \pm 0.20$ & $1.05 \pm 0.19$ & 0.49 & 21 \\
$>900$ & 1277 & 15626 & $0.64 \pm 0.20$ & $1.18 \pm 0.21$ & 0.45 & 7 \\
Inclusive & 950 & 47466 & $0.65 \pm 0.23$ & $1.00 \pm 0.22$ & 0.25 & 16 \\
\hline \hline
\end{tabular}

$\chi^{2}=7$ for 21 degrees of freedom. This is believed due simply to chance, as the statistical error only fit agrees with the data exceptionally well within the error, returning $\chi^{2}=13$ for 21 degrees of freedom.

As the $\nu_{\mu}$ angular template has been corrected for the observed cross section per Ref. [9], $\alpha_{\nu}$ may be interpreted as a flux scale factor, and significant deviations from unity would imply a flux mismodeling. Consistent with the results reported in $\mathrm{Sec}$. IV C, fits in the antineutrinomode CCQE sample indicate the true neutrino flux to be somewhat lower than the simulation predicts. Over all reconstructed energies, the neutrino flux component of the antineutrino-mode beam should be scaled by 0.65 to match the observed data. Fits in individual reconstructed energy bins show that the neutrino flux component shape is well-modeled. Finding the calibration on the neutrino flux component inconsistent with unity is not surprising, as the neutrino parent pions originate primarily in a poorly constrained production region (cf. Fig. 1). The rate scale $\alpha_{\bar{\nu}}$ is ambiguous in interpretation, as the cross section is yet unmeasured.

Care must be taken when comparing these results to the $\mu^{+} / \mu^{-}$yield numbers reported in the MiniBooNE $\bar{\nu}_{\mu} \rightarrow \bar{\nu}_{e}$ oscillation analysis [4,5], since the interaction prediction is different. In the oscillation analysis the cross section parameters measured in Ref. [6] are employed, which includes $M_{A}^{\text {eff }}=1.23(1.13) \mathrm{GeV}$ for bound (free) nucleon CCQE scattering and $\kappa=1.022$. When the muon angular fit technique described in this section is repeated with this prediction, yield rates of $\alpha_{\nu}=0.99 \pm$ 0.23 and $\alpha_{\bar{\nu}}=1.20 \pm 0.23$ are found, as reported in Ref. [5]. With this alternate CCQE scattering model, the angular fit over all reconstructed energies reports a neutrino contamination in the sample of $23 \pm 6 \%$,

TABLE VIII. Fractional composition of the antineutrino-mode CCQE sample before and after angular fits.

\begin{tabular}{llccc}
\hline \hline & \multicolumn{2}{c}{ Before fit $(\%)$} & \multicolumn{2}{c}{ After fit $(\%)$} \\
$E_{\bar{\nu}}^{\mathrm{QE}}$ Range $(\mathrm{MeV})$ & $\nu_{\mu}$ & $\bar{\nu}_{\mu}$ & $\nu_{\mu}$ & $\bar{\nu}_{\mu}$ \\
\hline$<600$ & 25 & 75 & $18 \pm 6$ & $82 \pm 16$ \\
$600-900$ & 26 & 74 & $17 \pm 6$ & $83 \pm 15$ \\
$>900$ & 35 & 65 & $23 \pm 7$ & $77 \pm 15$ \\
Inclusive & 29 & 71 & $21 \pm 8$ & $79 \pm 18$ \\
\hline \hline
\end{tabular}

consistent with the $21 \pm 8 \%$ contamination found with the scattering assumptions described in Sec. III A.

The results from this technique depend on knowing the angular distributions of neutrino and antineutrino CCQE interactions in the detector. While the procedure relies on exploiting the effect of the interference term in the CCQE cross section, the angular distributions may be somewhat altered by nuclear effects. In this analysis the measured angular distribution of neutrino interactions on carbon [9] is employed, but the measurement relies on the scattering model described in Sec. III A to predict antineutrino interactions. This model does not include two-body current effects which may be larger than previously expected [30] and may introduce additional neutrino and antineutrino angular differences. Despite this inherent model dependence, the results present a demonstration of a technique aimed at informing future experiments looking to separately constrain neutrino and antineutrino events in an unmagnetized environment. By that time, the effect of additional nuclear processes on the angular dependence of antineutrino CCQE scattering should be better known.

\section{Systematic errors}

As the present analysis directly measures the neutrino component in the antineutrino-mode beam, systematic errors relating to beam geometry and meson production

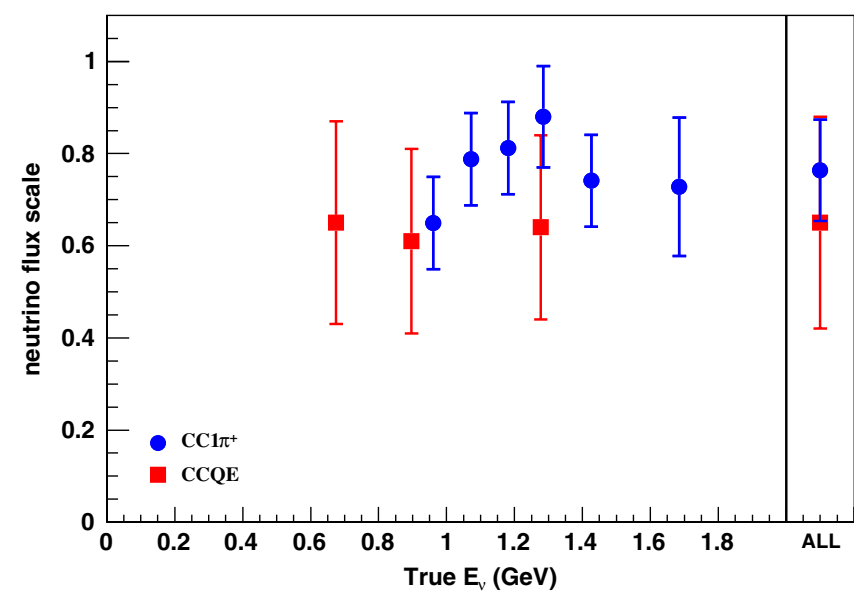

FIG. 6 (color online). Summary of the neutrino flux constraint in the antineutrino-mode beam from the $\mathrm{CC} 1 \pi^{+}$(Sec. IV) and CCQE (Sec. V) measurements. 
TABLE IX. Summary of systematic error contribution to the scale parameter $\alpha_{\nu}$ in the inclusive energy fit. Individual error contributions are found for the $i$ th systematic error by first repeating the fits with only independent systematics considered. The fractional error contributions are then found by $\sqrt{\left(\Delta \alpha_{\nu} / \alpha_{\nu}\right)_{\text {syst }_{i}+\text { stat }}^{2}-\left(\Delta \alpha_{\nu} / \alpha_{\nu}\right)_{\text {stat }}^{2}}$, where $\Delta \alpha_{\nu}$ is the onesigma error reported in Table VII. The statistical error is found by considering the second term only. This method does not account for small changes in the $\alpha_{\nu}$ best fit parameter between the fits considering various errors, and so the individual fractional errors do not add in quadrature to produce the total fractional error reported in Table VII and in the final column.

\begin{tabular}{lc}
\hline Source of error & Fractional uncertainty $(\%)$ \\
\hline Statistical & 8 \\
Detector modeling & 11 \\
CC $1 \pi^{+}$Constraint & 4 \\
Cross section & 26 \\
Total fractional error & 35 \\
\hline \hline
\end{tabular}

at the target are not considered. The remaining systematic errors include those arising from detector modeling, the single pion production background, and the cross section parameters in the underlying model. Contributions propagated from these errors to the uncertainty on the parameter $\alpha_{\nu}$ in the inclusive energy sample are given in Table IX.

Apart from final-state interaction uncertainties leading to errors on the cross section, the error on the $\mathrm{CC} 1 \pi^{+}$ background contributes to the systematic error through the error labeled "CC1 $\pi^{+}$Constraint" in Table IX. This measurement uncertainty is based on a $Q^{2}$-dependent shape-only scale factor to improve data-simulation agreement in the neutrino-mode $\mathrm{CC} 1 \pi^{+}$sample [3]. The cross section (both $\mathrm{CCQE}$ and $\mathrm{CC} 1 \pi^{+}$) uncertainty is dominant in these fits and warrants further discussion. Table X offers a breakdown of cross section parameters and associated errors. The error on carbon $M_{A}^{\text {eff }}$ has been reduced from that reported in Ref. [9] to avoid double-counting MiniBooNE systematic errors applicable to both the measurement of $M_{A}^{\text {eff }}$ and the measurement reported here. The $26 \%$ uncertainty due to cross section errors reported in Table IX can be expanded as the quadrature sum of $16 \%$ from the $10 \%$ normalization errors on $\bar{\nu}_{\mu}$ and CCQE processes, $14 \%$ from the error on $M_{A}$ and $\kappa$, and $15 \%$ from the remaining.

As the main contributions to the dominant cross section systematic error apply to both $\nu_{\mu}$ and $\bar{\nu}_{\mu}$ scattering, $\alpha_{\nu}$ and $\alpha_{\bar{\nu}}$ are positively correlated as reported in Table VII.

\section{RESULT COMPARISON}

Including all reconstructed energies in the $\mathrm{CC} 1 \pi^{+}$sample, a neutrino flux component scale of $0.76 \pm 0.11$ is found, while the CCQE analysis yields $0.65 \pm 0.23$. The measurements are compatible and complementary as each analysis includes energy regions not covered by the other
TABLE X. Summary of cross section errors used in this analysis. The bottom portion presents fractional uncertainties assigned to processes in addition to parameter errors. Errors given on pion absorption and charge exchange are relevant to pion propagation in the detector medium.

\begin{tabular}{lc}
\hline \hline Parameter & Value with error \\
\hline$M_{A}^{\text {eff }}$ carbon target & $1.35 \pm 0.07 \mathrm{GeV}$ \\
$M_{A}^{\text {eff }}$ hydrogen target & $1.03 \pm 0.02 \mathrm{GeV}$ \\
$\kappa$ & $1.007 \pm 0.005$ \\
$E_{B}$ & $34 \pm 9 \mathrm{MeV}$ \\
$\Delta \mathrm{s}$ & $0.0 \pm 0.1$ \\
$M_{A}^{1 \pi}$ & $1.10 \pm 0.28 \mathrm{GeV}$ \\
$M_{A}^{\text {multi- } \pi}$ & $1.30 \pm 0.52 \mathrm{GeV}$ \\
$p F$ & $220 \pm 30 \mathrm{MeV}$ \\
\hline Process & Fractional uncertainty $(\%)$ \\
\hline$\pi^{+}$Charge exchange & 50 \\
$\pi^{+}$Absorption & 35 \\
CCQE $\sigma$ Normalization & 10 \\
All $\bar{\nu}_{\mu} \sigma$ Normalization & 10 \\
$\Delta+N \rightarrow N+N$ & 100 \\
\hline \hline
\end{tabular}

as shown in Fig. 6. The results indicate the simulated neutrino component of the antineutrino-mode flux is overestimated by $\sim 30 \%$. These flux measurements constrain the very forward $\pi^{+}$created at the target, where an external data constraint is not available. Results from both methods are summarized in Fig. 6, where measurements are placed at the mean of the generated energy distribution for each reconstructed energy sample.

\section{IMPLICATIONS FOR OTHER EXPERIMENTS}

The techniques applied here could also aid future neutrino experiments that will test for $C P$ violation in the lepton sector using large unmagnetized detectors. This includes experiments such as $\mathrm{NO} \nu \mathrm{A}$ [46], $\mathrm{T} 2 \mathrm{~K}$ [47], LBNE [48], LAGUNA [49], and Hyper-K [50]. A magnetized near detector can provide a powerful constraint on the neutrino flux and provide precise cross sections. However, a measurement of the neutrino rate at the far detector can still be very useful given that the the beam spreads from an extended source and oscillates while traveling between the detectors.

Additional techniques could offer potentially helpful constraints on the neutrino component in an antineutrinomode beam. This includes taking advantage of the effective lifetime difference between $\mu^{-} / \mu^{+}$due to $\mu^{-}$capture in a nuclear environment. Fitting the lifetime distributions or measuring how often a decay electron is produced could supply constraints that are especially useful as they are independent of the underlying neutrino interaction cross sections. Also, selection of CCQE interactions with and without a proton in the final state may afford additional neutrino versus antineutrino tagging capabilities $[51,52]$. 


\section{CONCLUSIONS}

Two analyses are presented to measure the neutrino flux in the MiniBooNE antineutrino-mode beam. The two measurements have a common dependence on the neutrino flux in the neutrino-mode beam [20] that MiniBooNE obtained from HARP hadroproduction data. At present the CCQE angular distribution method is largely limited by uncertainties in the cross sections, especially the antineutrino cross section which MiniBooNE is in the process of measuring, while the uncertainty of the $\mathrm{CC} 1 \pi$ method is dominated by the neutrino-mode flux uncertainty.

Using two event samples dominated by independent physics processes, compatible and complementary results are found. The results from both analyses indicate the
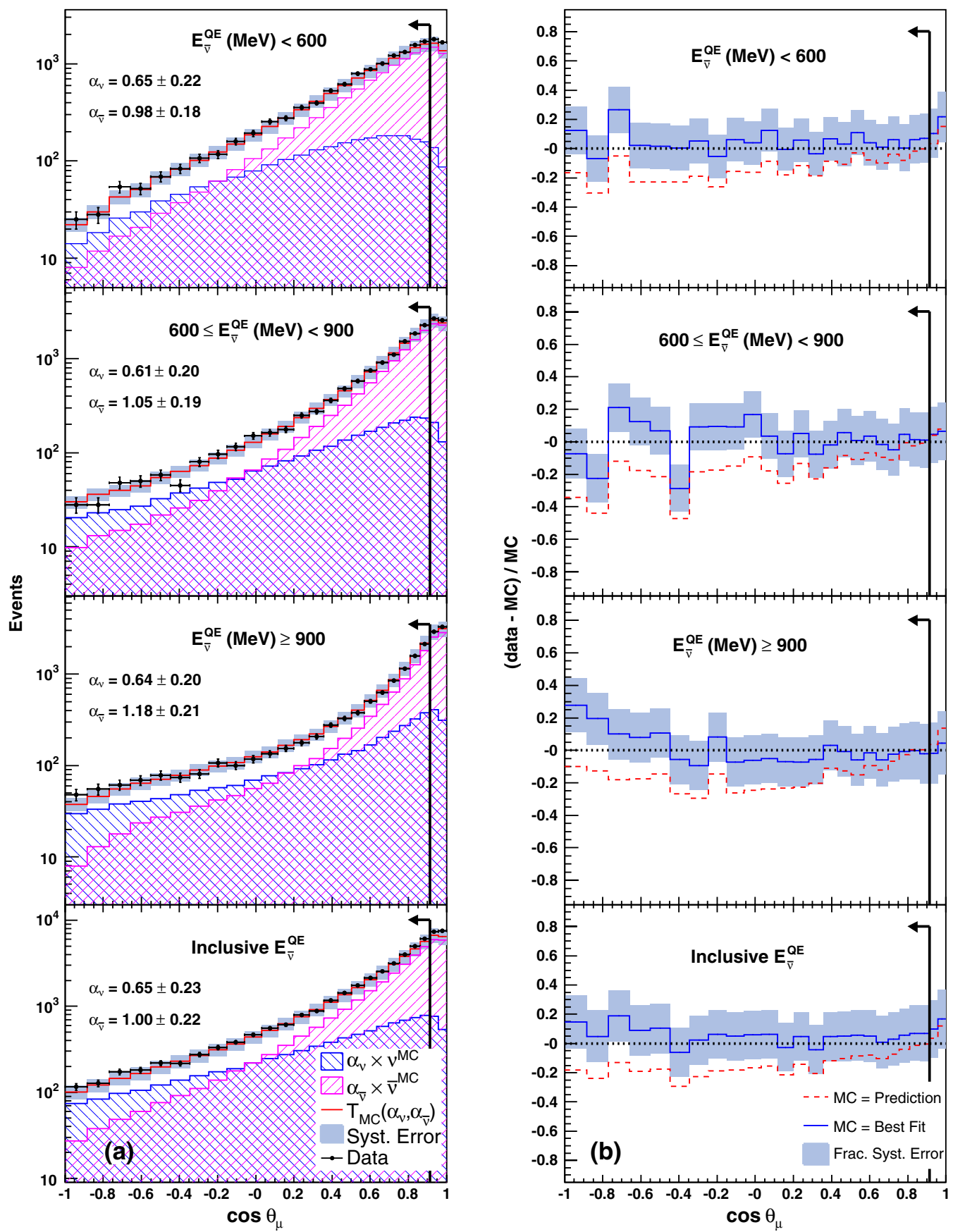

FIG. 7 (color online). Results of the muon angular fits to the CCQE data described in Sec. V. Shown are (a) the fits and (b) fractional differences (data-simulation)/simulation for both the unmodified prediction and the best fit. Along with an inclusive sample, three reconstructed energy bins are considered. The before-fit simulation is absolutely normalized to $5.66 \times 10^{20}$ protons on target. Only events with $\cos \theta_{\mu}<0.91$ are considered. 
prediction of the neutrino flux component of the antineutrino beam is overestimated-the $\mathrm{CC} 1 \pi^{+}$analysis (Sec. IV) indicate the predicted $\nu_{\mu}$ flux should be scaled by $0.76 \pm 0.11$, while the CCQE angular fits (Sec. V) yield $0.65 \pm 0.23$. Results from repeating the analyses in bins of reconstructed neutrino and antineutrino energy indicate that the predicted flux spectrum shape is well-modeled. The results from fitting the muon angular distributions in the CCQE sample has already been employed in the MiniBooNE oscillation analysis [4,5], while the $\mathrm{CC} 1 \pi^{+}$-based measurement will likely be more valuable to MiniBooNE antineutrino cross section extractions, as it is much less model-dependent and carries comparatively smaller uncertainty. These types of analyses, along with others discussed in Sec. VII may be of use to present and future precision neutrino experiments testing $C P$ violation with neutrino and antineutrino beams.

\section{ACKNOWLEDGMENTS}

We wish to acknowledge the support of Fermilab, the National Science Foundation, and the Department of Energy in the construction, operation, and data analysis of the MiniBooNE experiment.

\section{APPENDIX A: CCQE ANGULAR FIT DETAILS}

This appendix presents details on the CCQE angular fit results described in Sec. V. The fits to data are plotted in Fig. 7.
[1] A. A. Aguilar-Arevalo et al. (MiniBooNE Collaboration), Phys. Rev. Lett. 98, 231801 (2007).

[2] A. A. Aguilar-Arevalo et al. (MiniBooNE Collaboration), Phys. Rev. Lett. 102, 101802 (2009).

[3] A. A. Aguilar-Arevalo et al. (MiniBooNE Collaboration), Phys. Rev. Lett. 103, 061802 (2009).

[4] A. A. Aguilar-Arevalo et al. (MiniBooNE Collaboration), Phys. Rev. Lett. 103, 111801 (2009).

[5] A. A. Aguilar-Arevalo et al. (MiniBooNE Collaboration), Phys. Rev. Lett. 105, 181801 (2010).

[6] A. A. Aguilar-Arevalo et al. (MiniBooNE Collaboration), Phys. Rev. Lett. 100, 032301 (2008).

[7] A. A. Aguilar-Arevalo et al. (MiniBooNE Collaboration), Phys. Rev. Lett. 103, 081801 (2009).

[8] A. A. Aguilar-Arevalo et al. (MiniBooNE Collaboration), Phys. Rev. D 81, 013005 (2010).

[9] A. A. Aguilar-Arevalo et al. (MiniBooNE Collaboration), Phys. Rev. D 81, 092005 (2010).

[10] A. A. Aguilar-Arevalo et al. (MiniBooNE Collaboration), Phys. Rev. D 82, 092005 (2010).

[11] A. A. Aguilar-Arevalo et al. (MiniBooNE Collaboration), arXiv:1010.3264.

[12] A. A. Aguilar-Arevalo et al. (MiniBooNE Collaboration), Phys. Rev. D 83, 052007 (2011).

[13] A. A. Aguilar-Arevalo et al. (MiniBooNE Collaboration), Phys. Lett. B 664, 41 (2008).

[14] M. G. Catanesi et al. (HARP Collaboration), Eur. Phys. J. C 52, 29 (2007).

[15] M. G. Catanesi et al. (HARP Collaboration), Nucl. Phys. B732, 1 (2006).

[16] D. Casper, Nucl. Phys. B, Proc. Suppl. 112, 161 (2002).

[17] M.O. Wascko (MiniBooNE Collaboration), arXiv:0602051.

[18] P. Huber and T. Schwetz, arXiv:0805.2019.

[19] S. Agostinelli et al., Nucl. Instrum. Methods Phys. Res., Sect. A 506, 250 (2003).

[20] A. A. Aguilar-Arevalo et al. (MiniBooNE Collaboration), Phys. Rev. D 79, 072002 (2009).
[21] A. A. Aguilar-Arevalo et al. (MiniBooNE Collaboration), Nucl. Instrum. Methods Phys. Res., Sect. A 599, 28 (2009).

[22] CERN Program Library Long Writeup W5013, 1993, http://wwwasdoc.web.cern.ch/wwwasdoc/geant/geantall .html.

[23] C. Zeitnitz and T. A. Gabriel, Nucl. Instrum. Methods Phys. Res., Sect. A 349, 106 (1994).

[24] D. Ashery et al., Phys. Rev. C 23, 2173 (1981); M. K. Jones et al., Phys. Rev. C 48, 2800 (1993); R. D. Ransome et al., Phys. Rev. C 45, R509 (1992).

[25] B.C. Brown et al., in 2004 IEEE Nuclear Science Symposium Conference Record, Vol. 1, p. 652 (2006).

[26] R. A. Smith and E. J. Moniz, Nucl. Phys. B43, 605 (1972); B101, 547(E) (1975).

[27] H. Budd, A. Bodek, and J. Arrington, arXiv:hep-ex/ 0308005 [Nucl. Phys. B, Proc. Suppl. (to be published)].

[28] J. Grange, in 6th International Workshop on NeutrinoNucleus Interactions in the Few-GeV Region (NUINT 2009), edited by F. Sanchez et al., AIP Conf. Proc. No. 1189, (AIP, New York, 2009), 331.

[29] V. Bernard et al., J. Phys. G 28, R1 (2002).

[30] M. Martini et al., Phys. Rev. C 80, 065501 (2009).

[31] M. Martini et al., Phys. Rev. C 81, 045502 (2010).

[32] J. Nieves et al., Phys. Rev. C 83, 045501 (2011).

[33] J. Nieves et al., arXiv:1106.5374.

[34] J. E. Amaro et al., Phys. Rev. D 84, 033004 (2011).

[35] D. Rein and L. M. Sehgal, Ann. Phys. (N.Y.) 133, 79 (1981).

[36] R. P. Feynman, M. Kislinger, and F. Ravndal, Phys. Rev. D 3, 2706 (1971).

[37] D. Casper (private communication).

[38] W. Reuter et al., Phys. Rev. C 26, 806 (1982).

[39] V. Flaminino et al., CERN-HERA-83-01, 1983; CERNHERA-83-02, 1983; CERN-HERA-84-01, 1984.

[40] T. Suzuki et al., Phys. Rev. C 35, 2212 (1987).

[41] A. Shinohara et al., Phys. Rev. A 53, 130 (1996).

[42] A. M. Ankowski et al., arXiv:1001.0481. 
[43] J.L. Alcaraz-Aunion and J. Walding, AIP Conf. Proc. 1189, 145 (2009).

[44] K. S. Kuzmin, V. V. Lyubushkin, and V. A. Naumov, Eur. Phys. J. C 54, 517 (2008); V. V. Lyubushkin et al. (NOMAD Collaboration), Eur. Phys. J. C 63, 355 (2009).

[45] B. P. Roe, Nucl. Instrum. Methods Phys. Res., Sect. A 570, 159 (2007).

[46] D.S. Ayres et al. (NOvA Collaboration), FERMILABDESIGN-2007-01.
[47] Y. Itow et al., arXiv:hep-ex/0106019.

[48] V. Barger et al., Report of the US long baseline neutrino experiment study, http://nwg.phy.bnl.gov/fnal-bnl.

[49] A. de Bellefon et al., arXiv:hep-ex/0607026.

[50] K. Nakamura, Int. J. Mod. Phys. A 18, 4053 (2003).

[51] A. A. Aguilar-Arevalo et al., arXiv:hep-ex/0601022.

[52] M. Fechner and C. W. Walter, J. High Energy Phys. 11 (2009) 040. 


\section{First measurement of the muon antineutrino double-differential charged-current quasielastic cross section}

A. A. Aguilar-Arevalo, ${ }^{12}$ B. C. Brown, ${ }^{6}$ L. Bugel,,${ }^{11}$ G. Cheng, ${ }^{5}$ E. D. Church, ${ }^{15}$ J. M. Conrad,${ }^{11}$ R. Dharmapalan, ${ }^{1}$ Z. Djurcic, ${ }^{2}$ D. A. Finley, ${ }^{6}$ R. Ford, ${ }^{6}$ F. G. Garcia, ${ }^{6}$ G. T. Garvey, ${ }^{9}$ J. Grange, ${ }^{7}$ W. Huelsnitz, ${ }^{9}$ C. Ignarra,${ }^{11}$ R. Imlay, ${ }^{10}$ R. A. Johnson, ${ }^{3}$ G. Karagiorgi, ${ }^{5}$ T. Katori, ${ }^{11}$ T. Kobilarcik, ${ }^{6}$ W. C. Louis, ${ }^{9}$ C. Mariani, ${ }^{16}$ W. Marsh, ${ }^{6}$ G. B. Mills, ${ }^{9}$ J. Mirabal, ${ }^{9}$ C. D. Moore, ${ }^{6}$ J. Mousseau, ${ }^{7}$ P. Nienaber, ${ }^{14}$ B. Osmanov, ${ }^{7}$ Z. Pavlovic, ${ }^{9}$ D. Perevalov, ${ }^{6}$ C. C. Polly, ${ }^{6}$ H. Ray, ${ }^{7}$ B. P. Roe, ${ }^{13}$ A. D. Russell, ${ }^{6}$ M. H. Shaevitz,${ }^{5}$ J. Spitz,${ }^{11}$ I. Stancu, ${ }^{1}$ R. Tayloe, ${ }^{8}$ R. G. Van de Water, ${ }^{9}$ M. O. Wascko, ${ }^{17}$ D. H. White, ${ }^{9}$ D. A. Wickremasinghe, ${ }^{3}$ G. P. Zeller, ${ }^{6}$ and E. D. Zimmerman ${ }^{4}$

(MiniBooNE Collaboration)

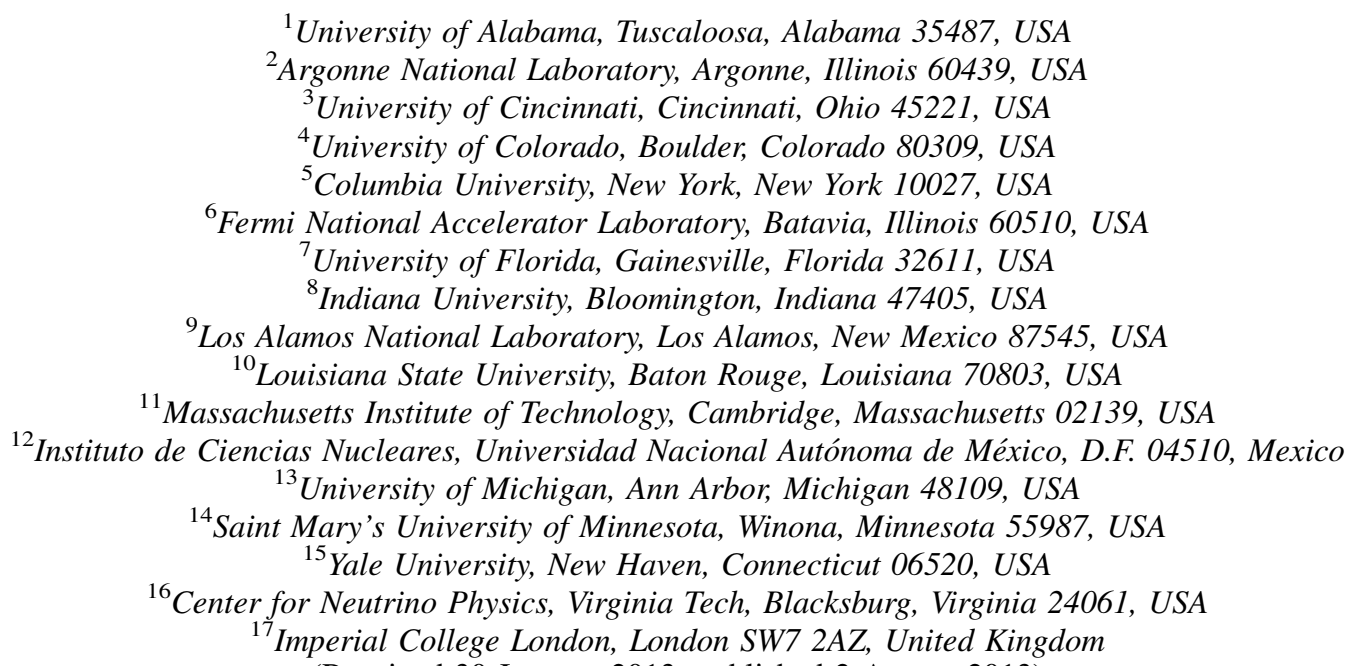

(Received 30 January 2013; published 2 August 2013)

The largest sample ever recorded of $\bar{\nu}_{\mu}$ charged-current quasielastic (CCQE, $\bar{\nu}_{\mu}+p \rightarrow \mu^{+}+n$ ) candidate events is used to produce the minimally model-dependent, flux-integrated double-differential cross section $\frac{d^{2} \sigma}{d T_{\mu} d \cos \theta_{\mu}}$ for $\bar{\nu}_{\mu} \mathrm{CCQE}$ for a mineral oil target. This measurement exploits the large statistics of the MiniBooNE antineutrino mode sample and provides the most complete information of this process to date. In order to facilitate historical comparisons, the flux-unfolded total cross section $\sigma\left(E_{\nu}\right)$ and singledifferential cross section $\frac{d \sigma}{d Q^{2}}$ on both mineral oil and on carbon are also reported. The observed cross section is somewhat higher than the predicted cross section from a model assuming independently acting nucleons in carbon with canonical form factor values. The shape of the data are also discrepant with this model. These results have implications for intranuclear processes and can help constrain signal and background processes for future neutrino oscillation measurements.

DOI: 10.1103/PhysRevD.88.032001

PACS numbers: $14.60 . \mathrm{Lm}, 14.60 . \mathrm{Pq}, 14.60 . \mathrm{St}$

\section{INTRODUCTION}

With the recent determination of the nonzero value of $\theta_{13}$ [1-5], present and future neutrino oscillation experiments will focus on measurements of the neutrino mass ordering and searches for leptonic $C P$ violation. To reach discovery-level sensitivity to each of these effects, GeVrange $\nu_{e}$ and $\bar{\nu}_{e}$ appearance must be observed in a longbaseline program with few-percent precision [6-10]. To facilitate such an ambitious program, the cross section for signal and background $\nu_{\mu}, \nu_{e}, \bar{\nu}_{\mu}$, and $\bar{\nu}_{e}$ charged-current processes must be known to high precision. This goal is commonly met by using a near detector to directly measure the rate and shape of the unoscillated spectrum. However, if the cross sections are not independently and precisely understood, the extracted information may be model dependent and significantly biased from their true value $[11,12]$. In the absence of a near detector, detailed knowledge of the contributing reactions is even more critical to the successful execution of these measurements. While the experimental and theoretical knowledge of GeV-range neutrino interactions on nuclear targets is improving, the 
experimental precision of interactions in this range is not better than $10 \%$ [13]. Of even more concern, as will be discussed in more detail, the fundamental processes contributing to neutrino interactions with nuclear matter are not well understood.

In general, antineutrino cross sections in the few-GeV region are not as well known as their neutrino counterparts, and in particular there are no charged-current antineutrino cross-section measurements below $1 \mathrm{GeV}$. In this work we present the first measurement of the antineutrino chargedcurrent quasielastic (CCQE) double-differential cross section with respect to kinematic properties of the outgoing muon. These data are obtained using a muon antineutrino beam with mean energy $\left\langle E_{\bar{\nu}}\right\rangle=665 \mathrm{MeV}$ and an exposure of $10.1 \times 10^{20}$ protons on target (POT). This measurement represents an important step towards reaching the level of knowledge required for next-generation oscillation measurements.

Apart from the valuable constraint these results provide for future experiments seeking to use antineutrino events to measure the few remaining unknown fundamental properties of neutrinos, the interpretation of the data will offer insight into an emerging puzzle. These results significantly contribute to the body of experimental information that suggest the canonically used model in neutrino generators of the relativistic Fermi gas [14] (RFG) is insufficient for describing neutrino interactions in nuclear media. It has been argued elsewhere that the discrepancy may come from inadequate form factors or a combination of the nuclear model and the relevant form factors [15]. The RFG assumes the impulse approximation, under which nucleons housed in dense material are treated as quasifree, independently acting participants subject to a global binding energy and Fermi motion, while the surrounding environment is entirely passive. In this formalism the interaction is parametrized by a set of tensor, vector, and axial-vector form factors [16]. The vector form factors are measured in electron scattering data [17] while the axial-vector form factor is left to be empirically determined by neutrino experiments and is typically assumed to take a dipole form:

$$
F_{A}=\frac{g_{A}}{\left(1+\frac{Q^{2}}{M_{A}^{2}}\right)^{2}},
$$

where $g_{A}$ is measured from nuclear beta decay [18], $Q^{2}$ is the squared four-momentum transfer and, while constraints exist from pion electroproduction data [19], neutrino experiments usually treat the axial mass $M_{A}$ as a free parameter.

By measuring the total rate of CCQE interactions and fitting the inferred $Q^{2}$ distribution, a variety of experiments employing bubble-chamber detectors housing mostly light nuclear targets typically produced consistent measurements of $M_{A}$. From these data, the averaged value is $M_{A}=1.026 \pm 0.021 \mathrm{GeV}[19,20]$. With the discovery of neutrino oscillations, the use of light nuclear targets for the detection medium became impractical, as the statistics required to make high-precision oscillation measurements are much more easily obtained using dense targets. With these relatively heavy nuclei and higher-precision detectors, more recent experiments have extracted values of $M_{A}$ systematically higher than $1.026 \mathrm{GeV}$ [21-24]. Meanwhile, the modern heavy nuclear target experiment NOMAD has measured a value of $M_{A}$ consistent with the bubble-chamber analyses [25], and preliminary shape results from the MINER $\nu \mathrm{A}$ experiment seem to also favor $M_{A} \sim 1 \mathrm{GeV}$ [26].

An essential first step to understanding this apparent discrepancy is to recognize the particulars of the model dependence introduced by comparing values of $M_{A}$ between the many experiments. Important experimental differences that may contribute to the discrepancy include disparate neutrino spectra, different neutrino detection technologies, and the size of the nuclear media employed. The liberties taken to compare $M_{A}$ values across these scattering experiments include the dipole form of $F_{A}$, various expectations of hadronic activity consistent with single-nucleon ejection, and the previously mentioned independent nucleon assumption implicit in both the formalism and in the inference of the $Q^{2}$ distribution. A possible reconciliation between the data sets has been proposed through a mechanism resulting in intranuclear correlations of greater strength than previously expected (see Ref. [27] and references therein). Such a mechanism is consistent with observations in electron scattering data [28,29]. If this process is confirmed for weak interactions via neutrino scattering, its detailed understanding will significantly expand knowledge of intranuclear behavior, and some neutrino oscillation results may need to be revisited $[11,12]$. The best chance to definitively resolve this crucial ambiguity lies in the community's ability and willingness to produce and compare model-independent information in both the leptonic and hadronic interaction sectors between experimental data and theoretical calculations. For this reason, the main result of this work is the doubledifferential CCQE cross section $\left(\frac{d^{2} \sigma}{d T_{\mu} d \cos \theta_{\mu}}\right)$ on mineral oil, where no assumptions about the underlying process is necessary for its reconstruction. Regardless of the fundamental interactions contributing to the sample studied, this work reports the first cross-section measurements of $\sim \mathrm{GeV}$ antineutrinos and thus significantly advances the community's preparedness to search for $C P$ violation with neutrinos.

This paper is organized as follows: The MiniBooNE experiment is described in Sec. II while Sec. III describes the model for neutrino interactions. The analysis is presented in Sec. IV, and the conclusions are summarized in Sec. V. Appendix A presents a measurement of the $\nu_{\mu}$ charged-current background to the analysis sample, which exploits $\mu^{-}$nuclear capture. Various model-dependent $\bar{\nu}_{\mu}$ CCQE cross sections are provided in Appendices B and C, and Appendix D tabulates all cross-section results. 


\section{THE MINIBOONE EXPERIMENT}

\section{A. Beam line and flux}

MiniBooNE observes an on-axis neutrino flux from the Fermilab Booster neutrino beam line (BNB). A beam of $8.9 \mathrm{GeV} / c$ momentum protons is extracted from the Booster synchrotron in bunches of $5 \times 10^{12}$ protons over $1.6 \mu \mathrm{s}$ at an average rate of up to $5 \mathrm{~Hz}$. A lattice of alternatively focusing and defocusing quadrupole magnets steers the proton spills into a beryllium target $71 \mathrm{~cm}(1.75$ interaction lengths) long. The protons collide with the target to create a spray of secondary particles. An aluminum electromagnetic horn surrounding the target is pulsed to coincide with the $\mathrm{p}$-Be collisions, creating a toroidal magnetic field to focus mesons of the desired charge. For the data used in this analysis, the polarity of the magnetic horn is set such that negatively charged secondary particles are focused while those with positive charge are defocused. The accepted mesons are allowed to decay in a $50 \mathrm{~m}$ long air-filled hall, which terminates at a steel beam dump. The dominant decay modes of these mesons, mostly pions, produce muon neutrinos and antineutrinos.

At MiniBooNE's request, the HARP experiment measured pion production cross sections with a $8.9 \mathrm{GeV} / c$ momentum proton beam on a 5\% interaction length replica MiniBooNE target [30]. The HARP double-differential cross section in pion energy and angle minimizes the model dependence of the BNB neutrino flux calculation [31]. A Geant4-based model [32] takes these data as input and is used to predict the flux of neutrino and antineutrinos observed by the detector. The simulation considers proton transport to the target, $\mathrm{p}$-Be interactions in the target including secondary interactions, meson production, and their propagation through the magnetic field inside the horn, meson decay, and finally neutrino propagation to the detector. The uncertainty of primary $\pi^{-}$production at the target is based exclusively on the HARP $\pi^{-}$doubledifferential cross section [30]. Though the beryllium target used to collect the HARP data is substantially shorter compared to the MiniBooNE target (5\% vs $170 \%$ interaction lengths, respectively), the difference in $\pi$ production arising from the thickness between the two targets is calculated to be small. For the proton energies used by the $\mathrm{BNB}$, roughly $90 \%$ of the neutrino beam is expected to come from the decay of primary $\pi$ 's [33], making the MiniBooNE flux prediction minimally dependent on the model for reinteractions in the long target. The antineutrino-mode beam intersecting the detector is composed of $83.7 \% \bar{\nu}_{\mu}, 15.7 \% \nu_{\mu}, 0.4 \% \bar{\nu}_{e}$, and $0.2 \% \nu_{e}$. The $\nu_{\mu}$ and $\bar{\nu}_{\mu}$ flux predictions are presented in Fig. 1. The electron-type neutrinos are irrelevant to this analysis, but as the MiniBooNE detector is unmagnetized, the $\nu_{\mu}$ contribution represents a significant background. Furthermore, Fig. 2 shows that, while the majority of $\bar{\nu}_{\mu}$ 's produced by $\pi^{-}$decay are constrained by the HARP measurement,

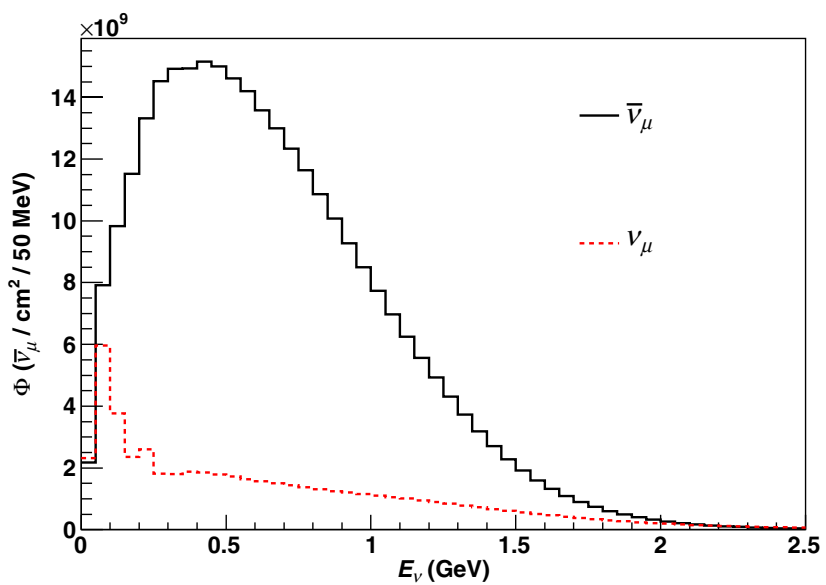

FIG. 1 (color online). The MiniBooNE $\bar{\nu}_{\mu}$ and $\nu_{\mu}$ flux predictions for antineutrino mode for the $10.1 \times 10^{20}$ POT exposure used in this analysis. Numerical values for the $\bar{\nu}_{\mu}$ flux are provided in Table XI.

most of the $\nu_{\mu}$ 's originating from $\pi^{+}$decay arise from a region not reported by HARP. In the analysis, the accepted flux of $\nu_{\mu}$ in the antineutrino-mode beam is thus constrained using the observed rate of $\nu_{\mu}$ events in the MiniBooNE detector, as presented in Ref. [34] and Appendix A. These analyses constrain the knowledge of the $\nu_{\mu}$ flux and the number of neutrino events in the antineutrino-mode sample to less than $15 \%$ for the bulk of the spectrum. The fractional uncertainty of the $\bar{\nu}_{\mu}$ flux prediction is around $7 \%$ at the interaction peak, due in roughly equal amounts to errors on $\pi^{-}$production and the model that connects their production to the $\bar{\nu}_{\mu}$ flux.

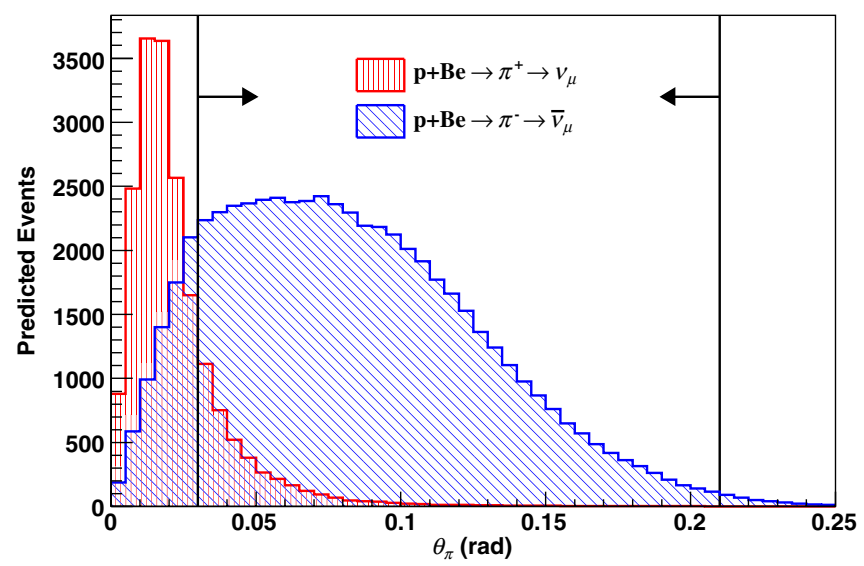

FIG. 2 (color online). Predicted angular distributions of pions with respect to the incident proton beam $\left(\theta_{\pi}\right)$ producing $\nu_{\mu}$ and $\bar{\nu}_{\mu}$ events in the MiniBooNE detector in the antineutrino-mode beam configuration. The $\nu_{\mu} / \bar{\nu}_{\mu}$ event fraction is significantly larger than the flux fraction due to the respective cross sections. Distributions are normalized to $10.1 \times 10^{20}$ POT, and arrows indicate the region of HARP data [30] constraints. Figure taken from Ref. [34]. 
Further details of the beam line and flux prediction are given in Ref. [31].

\section{B. Detector}

The detector is a $12.2 \mathrm{~m}$ diameter sphere filled with 818 tons of undoped mineral oil. The tank is optically segregated into an inner signal region of radius $575 \mathrm{~cm}$ and an outer veto shell of $35 \mathrm{~cm}$ thickness. Light produced in the detector is collected by 15208 in. Hamamatsu photomultiplier tubes (PMTs), 1280 of which face into the signal region ( $11.3 \%$ coverage) while 240 are inside the outer shell. Low activity in the veto region is required in physics analyses to ensure containment of charged particles produced by beam-induced neutrinos while also eliminating contamination from charged particles entering the tank.

Kept at $\sim 20^{\circ} \mathrm{C}$, the mineral oil has a density of $0.845 \mathrm{~g} / \mathrm{cm}^{3}$ with an index of refraction of 1.47 . Under these conditions, charged particles with velocity $\beta>0.68$ produce Cherenkov radiation. Lepton particle identification and reconstruction is principally obtained through the pattern and timing of this prompt Cherenkov light. The PMTs have a quantum efficiency of $\sim 20 \%$ and a timing resolution of $\sim 2 \mathrm{~ns}$, and the prompt Cherenkov component is easily separable from the delayed scintillation light present due to impurities in the oil. Four dispersion flasks at various locations in the detector are used to illuminate the signal-region PMTs with light from a pulsed laser. The laser data provide a calibration of PMT responses and allow an in situ measurement of light scattering properties over time. Throughout more than ten years of MiniBooNE running, the observed energy scale has been stable to within $1 \%$.

PMT charge and time information is collected for a total of $19.2 \mu$ s beginning $\sim 5 \mu$ s before the $1.6 \mu$ s long proton spill from the BNB. Cosmic ray muons stopped in the signal region prior to the start of the DAQ window may decay in time with the BNB spill, so PMT activity $5 \mu \mathrm{s}$ before proton delivery is monitored and used to minimize this contamination. Activity is recorded subsequent to the beam window for more than $10 \mu \mathrm{s}$ to observe electrons from the at-rest decay of muons (hereafter referred to as "Michel" electrons) produced directly or indirectly through the primary neutrino interaction.

The detector response to muons is calibrated using a dedicated system that independently measures the energy and direction of cosmic ray muons up to $800 \mathrm{MeV}$. A scintillator hodoscope directly above the detector and seven scintillator cubes at various depths within the detector are used to track these particles. Each cube is connected by optical fiber to a PMT for readout. Signals generated in the hodoscope and PMTs consistent with a muon stopping in a scintillator cube afford a direct calibration of the detector response to the range of muon kinematics most important to this analysis. These signals are used to verify muon reconstruction algorithms. Full reconstruction details are available in Ref. [35], while the detector is described further in Ref. [36].

\section{PREDICTED NEUTRINO INTERACTIONS}

MiniBooNE uses the NUANCE [37] event generator to simulate neutrino interactions. NUANCE includes a comprehensive neutrino and antineutrino cross-section model which considers known interactions in the neutrino and antineutrino energy range from $\sim 100 \mathrm{MeV}$ to $1 \mathrm{TeV}$. Ninety-nine reactions are modeled separately and combined with nuclear models describing bound nucleon states and final-state interactions to predict event rates and kinematics.

Bound nucleons in the detector medium are described by the RFG [14]. This assumes the nucleons to be independent and quasifree. Also specified is a hard cutoff in available struck nucleon energies as dictated by the exclusion principle.

The neutrino interaction types most relevant to the current analysis are charged-current quasielastic (Sec. III A) and single-pion production (Sec. III B). The neutrinoinduced absolute cross sections for both processes have been measured at MiniBooNE using a flux prediction well determined by HARP data [30]. These cross-section measurements are utilized in the antineutrino-mode simulation. More broadly, to minimize the model dependence of the extracted $\bar{\nu}_{\mu}$ CCQE cross section, each clear opportunity to constrain the backgrounds using MiniBooNE data was exploited.

\section{A. Charged-current quasielastic scattering}

CCQE interactions are the most prevalent channel in MiniBooNE's energy range and are predicted to account for $\sim 40 \%$ of all events. Their simulation in this analysis is chosen based on results from the MiniBooNE $\nu_{\mu}$ and $\bar{\nu}_{\mu}$ CCQE data sets. The formalism is described by a relativistic Fermi gas model [14], and with a few empirical parameter adjustments, this model adequately reproduces the kinematics of both CCQE data sets $[21,38]$. Through the procedure to correct for detector resolution effects, this choice only mildly affects the shape of the extracted true muon kinematics, while the normalization of the distribution is entirely unaffected. It will be shown later that this effect is negligible compared to other systematic uncertainties.

The vector and tensor components of the interaction are constrained by data from electron scattering experiments and a nondipole form is taken based on the results of Ref. [17]. As shown in Eq. (1), the axial-vector form factor assumes a dipole form and contains the empirical "axial mass" parameter $M_{A}$. In this analysis, the value of $M_{A}$ is chosen based on results from neutrino interactions. 
As $\nu_{\mu}$ CCQE interactions exclusively interact with nucleons bound in carbon, $M_{A}^{\text {eff,C }}=1.35 \mathrm{GeV}$ together with a Pauli blocking adjustment, $\kappa=1.007$ is sufficient to describe the kinematics of such events based on a fit to the MiniBooNE data [21]. The parameter $\kappa$ scales the lowest allowed outgoing nucleon energy for interactions with carbon: $E_{\text {low }}=\kappa\left(\sqrt{k_{F}^{2}+M^{2}}-\omega+E_{B}\right)$, where $k_{F}$, $M, \omega$, and $E_{B}$ are the Fermi momentum, nucleon mass, energy transfer, and binding energy, respectively. With the kinematics of $\nu_{\mu} \mathrm{CCQE}$ interactions characterized by this adjusted prediction, the total cross section for the simulated process is subsequently corrected to the observed normalization in the data. In this way, the details of the observed $\nu_{\mu}$ CCQE data are reproduced in the present simulation for this process.

The MiniBooNE mineral oil is composed of $\mathrm{C}_{n} \mathrm{H}_{2 n+2}$, $n \sim 20$, and so CCQE scattering off of both bound and quasifree protons are accessible to $\bar{\nu}_{\mu}$ 's. For the hydrogen scattering component, $M_{A}^{\text {eff,H }}=1.02 \mathrm{GeV}$ is chosen based on the body of experimental results for the CCQE process incident on light nuclear targets $[19,20]$. For interactions with protons bound in a carbon nucleus, the binding energy (Fermi momentum) is set to $30 \mathrm{MeV}(220 \mathrm{MeV})$ based on electron scattering data for the QE process [39]. As electron QE scattering probes all nucleons while QE interactions with neutrinos and antineutrinos are sensitive to a specific nucleon type, the binding energy determined from electron scattering data is adjusted based on estimates of Coulomb and isospin effects [40]. Along with the same CCQE model parameters measured in the $\nu_{\mu}$ data of $M_{A}^{\text {eff,C }}=1.35 \mathrm{GeV}$ and $\kappa=1.007$, these choices are adopted for $\bar{\nu}_{\mu}$ CCQE interactions on carbon. This choice is made exclusively due to observed agreement between this model and the MiniBooNE $\bar{\nu}_{\mu}$ CCQE data [38]. Note that, due to the axial-vector interference term in the formalism, the kinematics of $\bar{\nu}_{\mu}$ CCQE features a softer momentum-transfer spectrum and so, in the RFG, the same value of $\kappa$ has a larger effect on $\bar{\nu}_{\mu}$ CCQE compared to $\nu_{\mu}$ CCQE. More importantly, it will be shown later that the extracted $\bar{\nu}_{\mu}$ CCQE double-differential cross section is only negligibly affected by these choices.

The superscript "eff," short for "effective," on $M_{A}$ is used throughout this work to allow for the possibility that nuclear effects are responsible for the apparent discrepancy between the light target results and those from the more recent experiments using dense nuclear material. As discussed in the Introduction, this is also motivated by theoretical work that predicts an extra class of events whose contribution to the CCQE sample in Cherenkov detectors, such as MiniBooNE, enters due to the lack of requirement on hadronic activity [27]. The letter following the "eff" identifies the relevant nucleon target in MiniBooNE's hydrocarbon medium: $\mathrm{H}$ for the quasifree hydrogen targets and $\mathrm{C}$ for those bound in carbon.

\section{B. Pion production}

The majority of single-pion production $\left(\nu_{l}+N \rightarrow l+\right.$ $\pi+N^{\prime}$ ) events at MiniBooNE energies are mediated by baryonic resonances. The formalism to describe these events is taken from the Rein-Sehgal model [41], where the relativistic harmonic oscillator quark model is assumed [42]. The production of $\Delta(1232)$ is dominant in the energy range spanned by MiniBooNE, but 17 other and highermass resonances are also considered.

The charged-current single-pion channels for $\nu_{\mu}$ $\left(\nu_{\mu}+N \rightarrow \mu^{-}+\pi^{+}+N\right.$, "CC1 $\left.\pi^{+} "\right)$ and $\bar{\nu}_{\mu}\left(\bar{\nu}_{\mu}+\right.$ $N \rightarrow \mu^{+}+\pi^{-}+N$, "CC1 $\pi^{-}$") dominate the pionproducing interactions contributing to the $\bar{\nu}_{\mu} \mathrm{CCQE}$ sample. The $\mathrm{CC} 1 \pi^{+}$events enter from the $\nu_{\mu}$ content of the beam. The $\mathrm{CC} 1 \pi^{-}$background results from $\bar{\nu}_{\mu}$ interactions, and their presence in the CCQE sample is mostly due to stopped $\pi^{-}$capture in the nuclear medium. Stopped $\pi^{-}$capture in the presence of carbon is $\sim 100 \%$, so they are not separable from the $\bar{\nu}_{\mu}$ CCQE sample through observation of $\pi^{-}$decay. In the current analysis, the Rein-Sehgal prediction for both classes of events is adjusted to reproduce the kinematic distributions measured in MiniBooNE neutrino-mode $\mathrm{CC} 1 \pi^{+}$data $[21,43]$.

\section{Final-state interactions}

Subsequent to a neutrino interaction involving a nucleon bound in carbon, NUANCE propagates the outgoing hadrons including nucleons, mesons, and baryonic resonances, and simulates their reinteraction as they exit the nucleus. The initial interaction model employs the impulse approximation which assumes an instantaneous exchange with independent nucleons. Subsequent to the initial neutrino or antineutrino interaction, particles produced inside the nucleus are propagated stepwise in $0.3 \mathrm{fm}$ increments until they emerge from the $\sim 2.5 \mathrm{fm}$ radius sphere. Intermittently, the probability for hadronic reinteraction is calculated using a radially dependent nucleon density distribution [44] along with external $\pi-N, N-N$ cross-section measurements [45]. For $\Delta$ reinteractions $(\Delta+N \rightarrow N+N)$, an energy-independent probability of $20 \%(10 \%)$ is taken for $\Delta^{+}+N, \Delta^{0}+N\left(\Delta^{++}+N\right.$, $\Delta^{-}+N$ ) based on $\mathrm{K} 2 \mathrm{~K}$ data [46] and is assigned $100 \%$ uncertainty. The dominant final-state interactions affecting this analysis are pion charge exchange $\left(\pi^{ \pm}+X \leftrightarrow \pi^{0}+X^{\prime}\right)$ and absorption $\left(\pi^{ \pm}+X \rightarrow X^{\prime}\right)$.

\section{ANALYSIS}

This section describes the extraction of the $\bar{\nu}_{\mu} \mathrm{CCQE}$ double-differential cross section. It is necessary to first identify the experimental complications that distinguish this measurement from the MiniBooNE $\nu_{\mu}$ CCQE result.

Though the same detector, reconstruction, and event selection are used for the $\nu_{\mu}$ [21] and $\bar{\nu}_{\mu}$ CCQE analyses, subtleties related to the detector material and the different 
beam configuration result in substantially different sample content in both the signal and background processes. Due to leading-particle effects at the beryllium target, the mean energy of the $\bar{\nu}_{\mu}$ flux in antineutrino mode (shown in Fig. 1) is appreciably lower $\left(\left\langle E_{\bar{\nu}}\right\rangle=665 \mathrm{MeV}\right)$ compared to the $\nu_{\mu}$ flux in neutrino mode $\left(\left\langle E_{\nu}\right\rangle=788 \mathrm{MeV}\right)$. The content of the two CCQE signal samples is also fundamentally different since $\bar{\nu}_{\mu} \mathrm{CCQE}$ events arise from interactions with protons while $\nu_{\mu} \mathrm{CCQE}$ events involve interactions on neutrons. The hydrocarbon nature of the detection medium provides a mix of bound and quasifree interaction targets for $\bar{\nu}_{\mu} \mathrm{CCQE}$, while $\nu_{\mu} \mathrm{CCQE}$ involves only bound nucleons. The two interaction types for $\bar{\nu}_{\mu}$ CCQE are not separable, and so the sum of all $\bar{\nu}_{\mu} \mathrm{CCQE}$ interactions are treated as the signal for this analysis. However, as historical data on mostly light targets are adequately described with $M_{A} \sim 1 \mathrm{GeV}$, results evoking this model to subtract the quasifree $\bar{\nu}_{\mu} \mathrm{CCQE}$ content are given in Appendix B.

Backgrounds in this analysis also offer unique complications, as mentioned in Sec. II A and expanded in the next section. Broadly, the analysis sample is formed with a simple selection that requires the prompt muon be contained in the detector and that its decay is observed. The dominant backgrounds with this selection are $\nu_{\mu}$ $\mathrm{CC}$, and $\bar{\nu}_{\mu} \mathrm{CC} 1 \pi^{-}$interactions. The $\nu_{\mu} \mathrm{CCQE}$ contribution is indistinguishable event by event from $\bar{\nu}_{\mu} \mathrm{CCQE}$; however, statistical measurements of their overall rate and shape discussed in Sec. IVA constrain the knowledge of this background to $\sim 15 \%$. The $\mathrm{CC} 1 \pi^{-}$contamination enters from the capture process on carbon nuclei and is known less precisely, as it is not separable in the data from $\bar{\nu}_{\mu}$ CCQE. Furthermore, there are no measurements of this process in external data sets at the MiniBooNE energy range. Due to the size of the $\nu_{\mu} \mathrm{CCQE}$ and single-pion backgrounds, the signal purity is only $61 \%$ in this work, compared to $77 \%$ for the $\nu_{\mu}$ CCQE analysis. Multiple dedicated analyses and comparisons were necessary to reduce the uncertainty on these processes to a manageable level, and as a result, the final uncertainty on the extracted $\bar{\nu}_{\mu}$ cross sections are dominated by the level of $\bar{\nu}_{\mu}$ flux uncertainty.

\section{A. Constraints on background processes}

The largest background in the $\bar{\nu}_{\mu}$ CCQE sample is the $\nu_{\mu}$ contamination. Moreover, as Fig. 2 shows, the majority of $\pi^{+}$particles contributing to the beam are produced at small angles with respect to the incoming protons (and so are affected less by the magnetic field), and thus their contribution to the antineutrino-mode beam is mostly unconstrained by the HARP hadroproduction data. As MiniBooNE is nonmagnetized, this motivated a dedicated study of the $\nu_{\mu}$ beam content using statistical methods. Three techniques, described in detail in Ref. [34] and
Appendix A, were used to measure this crucial background for the MiniBooNE data. Two of these measurements are largely model independent, and the final fractional uncertainty on the $\nu_{\mu}$ contribution to the antineutrino-mode beam is $\sim 15 \%$ for the bulk of the observed spectrum. These analyses are the first of their kind and their uncertainty has reduced the error on the $\bar{\nu}_{\mu} \mathrm{CCQE}$ cross section due to $\nu_{\mu}$ interactions to a subdominant uncertainty.

The three measurements of the $\nu_{\mu}$ contribution to the antineutrino-mode data exploit various differences between charged-current $\nu_{\mu}$ and $\bar{\nu}_{\mu}$ processes to statistically measure their respective contributions. Broadly, these measurements are executed by performing rate analyses on samples with the $\nu_{\mu}$ and $\bar{\nu}_{\mu}$ content statistically separated. These techniques include the use of $\mu^{-}$nuclear capture, $\pi^{-}$nuclear capture, and angular differences between $\nu_{\mu}$ and $\bar{\nu}_{\mu}$ in CCQE interactions. The analysis based on $\mu^{-}$ capture is described in Appendix A, and the other analyses are presented in Ref. [34]. The $\mu^{-}$capture analysis exploits the $\sim 8 \%$ of $\nu_{\mu}$-induced CC interactions on carbon that do not lead to a decay electron, while nuclear capture of $\pi^{-}$also affords sensitivity to the $\nu_{\mu}$ beam content. The second most prevalent interaction type in the MiniBooNE detector is CC single-pion production, which produces a $\pi^{+}$in the case of $\nu_{\mu}$ scattering and a $\pi^{-}$for $\bar{\nu}_{\mu}$ reactions. As almost all stopped $\pi^{-}$are absorbed in the hydrocarbon medium [47], the sample consisting of a single muon and two decay electrons (one each from the prompt muon and the pion decay chain) is predominantly due to $\nu_{\mu}$ events. Finally, the observed angular distribution of CCQE events is fit to a combination of $\nu_{\mu}$ and $\bar{\nu}_{\mu}$ events, where $\bar{\nu}_{\mu}$ interactions are predicted to be much more forward going with respect to the beam direction. This last analysis is dependent on details of the RFG prediction for $\bar{\nu}_{\mu} \mathrm{CCQE}$ scattering, and its results are not used in the subtraction of the $\nu_{\mu}$ background. In the future, where CCQE and CCQElike interactions should be better understood, this technique could provide a valuable constraint. The results from these analyses are summarized in Fig. 3, where the nominal and highly uncertain prediction of the $\nu_{\mu}$ flux in the antineutrino-mode beam appears to be roughly $20 \%$ high in normalization, while the energy dependence seems to be well modeled. Based on the results from the $\mu^{-}$and $\pi^{-}$nuclear capture analyses, the $\nu_{\mu}$ flux in the antineutrino-mode beam is corrected by a scale of 0.77 with an uncertainty of 0.10 . These values are obtained using a method for combining correlated measurements [48] with an estimated correlation coefficient of 0.5 based on the common dependence of the HARP $\pi^{+}$production data in the $\mathrm{CC} 1 \pi^{+}$and $\mu^{-}$capture analyses. To recognize possible spectral dependencies in these data, the uncertainty of 0.10 is increased outside the regions directly constrained. This increased uncertainty is particularly important at lower energies, where much of the $\nu_{\mu}$ flux 


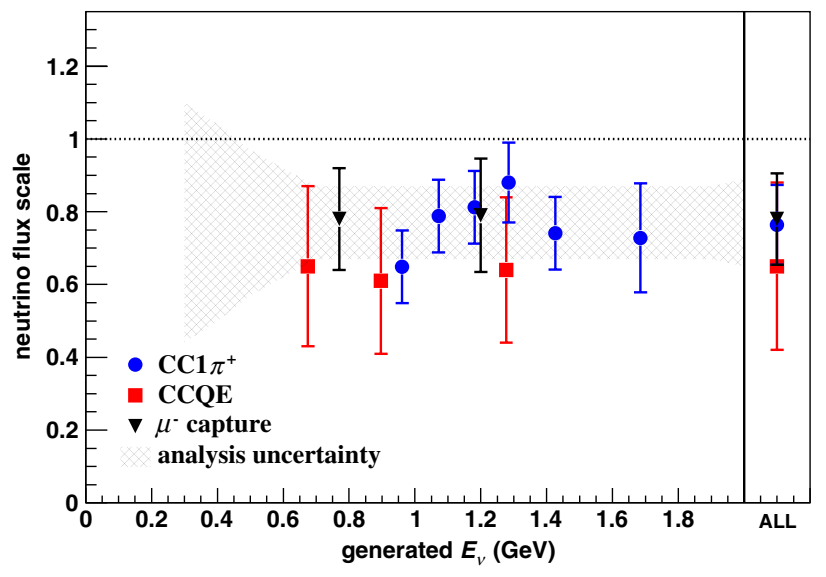

FIG. 3 (color online). Summary of the results from three techniques used to measure the $\nu_{\mu}$ flux in the antineutrinomode beam. The " $\mu^{-}$capture" analysis is described in Appendix A and the others in Ref. [34]. The measurements are given relative to an extrapolation of HARP data into a region where no relevant hadroproduction data exist (shown as a dotted line at unity). Due to dependence on assumptions of $\bar{\nu}_{\mu} \mathrm{CCQE}$ scattering, results from the "CCQE" determination of the $\nu_{\mu}$ flux are not used in the $\bar{\nu}_{\mu}$ CCQE cross-section analysis.

originates in the decay of $\pi^{+}$produced in regions that are constrained by the HARP measurements. The uncertainty on the $\nu_{\mu}$ subtraction in the calculation of the $\bar{\nu}_{\mu}$ doubledifferential cross section $\frac{d^{2} \sigma}{d T_{\mu} d \cos \theta_{\mu}}$ is shown in Fig. 3. Note these corrections calibrate the primary $\pi^{+}$production cross section in $p+\mathrm{Be}$ interactions contributing to the antineutrino-mode beam. Other systematic effects, such as the modeling of the magnetic field and secondary interactions in the target, allow energy-dependent shifts and are evaluated and included in the analysis separately.

The measurements summarized in Fig. 3 calibrate the simulated $\pi^{+}$production at the beryllium target to the level that the cross sections for the $\nu_{\mu}$ processes contributing to the analysis samples are known. The most important interactions are the $\nu_{\mu} \mathrm{CCQE}$ and $\mathrm{CC} 1 \pi^{+}$processes measured in the MiniBooNE neutrino-mode exposure [21,43]. Due to the disparate $\pi^{+}$acceptance to the beam, the $\nu_{\mu}$ flux spectrum in neutrino mode is much harder in energy compared to the $\nu_{\mu}$ 's in antineutrino mode. See Fig. 2 of Ref. [34]. However, as suggested by Fig. 5, high-energy neutrinos are largely rejected by the analysis requirement of contained muons, and the accepted $\nu_{\mu}$ spectrum between neutrino and antineutrino run modes is nearly identical. This shared $\nu_{\mu}$ spectrum allows the cross sections extracted from the neutrino-mode data to be directly applied to the antineutrino-mode simulation without relying on knowledge of the relationship between muon kinematics and incident neutrino energy. As discussed in Refs. [11,12], this connection [Eq. (A12)] may be unreliable in the presence of background interactions that originate from intranuclear processes.
Charged-current single $\pi^{-}$production constitutes the second-largest background to this analysis, accounting for $\sim 15 \%$ of the sample. These events enter through a different and experimentally disadvantageous mechanism compared to the analogous process for the $\nu_{\mu} \mathrm{CCQE}$ sample. Single-pion events induced by $\nu_{\mu}$ typically give rise to Michel electrons through the decay chain $\pi^{+} \rightarrow$ $\mu^{+} \rightarrow e^{+}$of stopped pions, which can be observed and used to reject these events. However, an appreciable number of $\pi^{+}$are destroyed in flight through the nuclear absorption process $\left(\pi^{+}+X \rightarrow X^{\prime}\right)$. In contrast, almost all single-pion events from $\bar{\nu}_{\mu}$ interactions enter the $\bar{\nu}_{\mu}$ CCQE sample since $\pi^{-}$that are not absorbed in flight stop and undergo nuclear capture with $\sim 100 \%$ efficiency. While this fortuitously allows for the $\mathrm{CC} 1 \pi^{+}$-based $\nu_{\mu}$ flux measurement, this also implies $\mathrm{CC} 1 \pi^{-}$events are not separable from the $\bar{\nu}_{\mu}$ CCQE sample. This is in contrast to the MiniBooNE $\nu_{\mu}$ CCQE analysis, where the single-pion events tagged through the observation of an additional Michel electron allow a direct constraint of the rate and kinematics of $\mathrm{CC} 1 \pi^{+}$events. A correction was thus measured and applied to the background prediction for the $\nu_{\mu}$ CCQE sample [21]. This constraint is applied by correcting the $\mathrm{CC} 1 \pi^{+}$events according to the observed reconstructed momentum transfer. The correction is shown in Fig. 7(b) of Ref. [21]. In the absence of such a measurement for $\mathrm{CC} 1 \pi^{-}$interactions, the constraint obtained in neutrino mode for $\nu_{\mu} \mathrm{CC} 1 \pi^{+}$is applied to the $\mathrm{CC} 1 \pi^{-}$Rein-Sehgal prediction described in Sec. IIIB. Figure 4 shows this

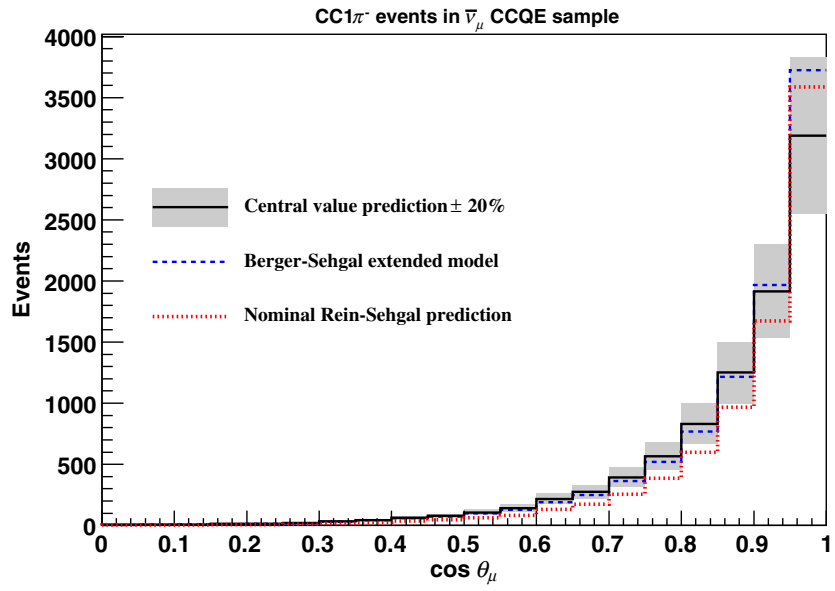

FIG. 4 (color online). Three calculations for the $\mathrm{CC} 1 \pi^{-}$background contribution to the $\bar{\nu}_{\mu}$ CCQE sample as a function of $\cos \theta_{\mu}$, where $\theta_{\mu}$ is the angle of the muon relative to the neutrino direction. The "central-value" distribution corresponds to the nominal Rein-Sehgal [41] prediction for $\mathrm{CC} 1 \pi^{-}$events in MiniBooNE constrained by the observed kinematics in the neutrino-mode $\nu_{\mu} \mathrm{CC} 1 \pi^{+}$sample. This agrees well with a more recent calculation ("Berger-Sehgal extended model") that is based on an improved version of the Rein-Sehgal model. For comparison, the bare Rein-Sehgal prediction for $\mathrm{CC} 1 \pi^{-}$events is also shown. Distributions are normalized to $10.1 \times 10^{20}$ POT. 
TABLE I. Sample purity and detection efficiency for all $\bar{\nu}_{\mu}$ CCQE events, which are due to a mix of scattering on bound and quasifree nuclear targets. Efficiencies are normalized to events with a generated radius $r<550 \mathrm{~cm}$.

\begin{tabular}{lccc}
\hline \hline Cut \# & Description & Purity $(\%)$ & Efficiency $(\%)$ \\
\hline 0 & No cuts & 32.3 & 100 \\
1 & Veto hits $<6$, all subevents & 27.6 & 50.8 \\
2 & First subevent: in beam window $4000<T(\mathrm{~ns})<7000$ & 27.7 & 50.3 \\
3 & First subevent: muon kinetic energy $T_{\mu}>200 \mathrm{MeV}$ & 36.9 & 44.0 \\
4 & Only two subevents & 48.4 & 38.8 \\
5 & First subevent: reconstructed vertex radius $R<500 \mathrm{~cm}$ & 54.3 & 32.6 \\
6 & Distance between subevent reconstructed vertices & & 30.6 \\
& $>500$ cm/GeV $\times T_{\mu}-100$ cm Distance & & \\
7 & between subevent reconstructed vertices $>100 \mathrm{~cm}$ & 61.0 \\
\hline \hline
\end{tabular}

prediction agrees well with an external calculation [49] for such events. This alternate model is implemented in NUANCE and is based on extensions of the Rein-Sehgal model [50-52]. This updated calculation includes muon mass terms and a modified vector form factor to yield better agreement with world pion production data [53]. Consistency between these two predictions for $\mathrm{CC} 1 \pi^{-}$ production, along with the level of agreement between the extended Rein-Sehgal calculation and the MiniBooNE CC1 $\pi^{+}$data (shown in Ref. [49]) suggests an uncertainty of $20 \%$ is sufficient for the $\mathrm{CC} 1 \pi^{-}$background. Future tests of the accuracy of this prediction may be made through comparisons to the subtracted $\mathrm{CC} 1 \pi^{-}$ background, as given in Appendix D.

Based on results from the neutrino-mode $\nu_{\mu} \mathrm{CC} 1 \pi^{0}$ analysis [54], the small contribution from $\mathrm{CC} 1 \pi^{0}$ events induced by both $\nu_{\mu}$ and $\bar{\nu}_{\mu}$ are increased by a factor of 1.6 relative to the NUANCE prediction. The generated predictions for all other interactions, accounting for $<3 \%$ of the sample, are not adjusted.

\section{B. Reconstruction and analysis sample}

The identification of $\bar{\nu}_{\mu}$ CCQE candidate events relies solely on the observation of a single muon and no finalstate $\pi^{+}$. Muon kinematics are obtained by the pattern, timing, and total charge of prompt Cherenkov radiation collected by PMTs. A likelihood function operating under a muon hypothesis is compared to the topology and timing of the observed PMT hits. This likelihood function predicts hit patterns and timing based on the interaction vertex and the momentum four-vector of the muon. The likelihood function simultaneously varies these seven parameters while comparing to the observed PMT hits. The parameters from the maximized likelihood function yield the reconstructed muon kinematics. Integrated over the spectrum of observed muons, the resolution of this reconstruction for muon energy (angle) is roughly 8\% ( $\left.2^{\circ}\right)$ [35]. The direct and high-resolution observation of muon properties motivates the choice to present the $\bar{\nu}_{\mu} \mathrm{CCQE}$ cross section as a function of muon kinematics as the main result of this work, while the large statistics of the data set analyzed yield sensitivity to previously unprobed regions of the interaction.

As in the $\nu_{\mu}$ CCQE work, no requirement is made on hadronic activity. This is an important distinction from the CCQE definitions used by other experiments [23,25], where a single proton track may be required for $\nu_{\mu} \mathrm{CCQE}$ selection. However, note that in the case of $\bar{\nu}_{\mu} \mathrm{CCQE}$ scattering, where a single ejected neutron is expected, the experimental definition used by tracking detectors is largely based on a single muon track. Therefore, in general, the selection used by tracking detectors and Cherenkov-based measurements, such as this one for $\bar{\nu}_{\mu}$ CCQE, follow each other more closely as compared to the $\nu_{\mu}$ case.

In MiniBooNE, final-state neutrons lead to low-energy scintillation light primarily through elastic scattering with the quasifree protons in the hydrogen content of the oil. The prompt PMT signals that define the analysis sample are dominated by Cherenkov light, and so the delayed scintillation light caused by neutron interactions have a negligible effect on the acceptance of $\bar{\nu}_{\mu}$ CCQE events.

The event selection is identical to that used in the MiniBooNE $\nu_{\mu}$ CCQE analysis [21]. Table I provides cumulative purity and efficiency values for the selected sample. Notice the requirement of low veto activity immediately halves the collection efficiency of $\bar{\nu}_{\mu} \mathrm{CCQE}$ interactions. As shown in Fig. 5, this is primarily due to the rejection of high-energy muons not fully contained within the inner detector region. Sample selection is based on requirements of temporally correlated collections of PMT activity (or PMT "hits") referred to as "subevents." A hit is any PMT pulse passing the discriminator threshold of $\sim 0.1$ photoelectrons, and a cluster of at least ten hits within a $200 \mathrm{~ns}$ window with individual hit times less than $10 \mathrm{~ns}$ apart defines a subevent. Two or fewer spacings between 10-20 ns among individual hit times are also allowed. The primary requirement to identify $\bar{\nu}_{\mu} \mathrm{CCQE}$ events is two 


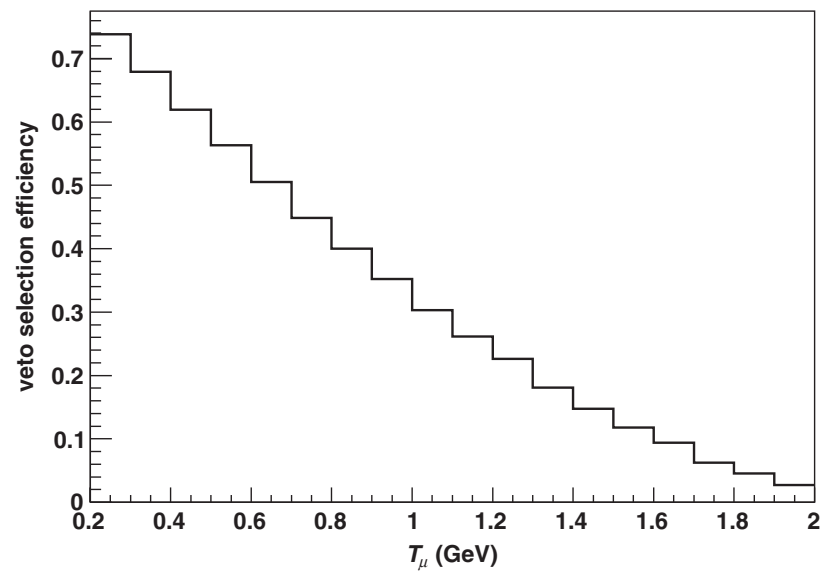

FIG. 5. Selection efficiency as a function of generated muon kinetic energy for $\bar{\nu}_{\mu}$ CCQE events passing the veto requirement. Higher-energy muons are less likely to stop in the inner region and are removed by this selection.

and only two subevents, due dominantly to Cherenkov light from the prompt muon and its decay positron:

$$
\text { 1: } \bar{\nu}_{\mu}+p \rightarrow \mu^{+}+n \quad 2: \hookrightarrow e^{+}+\nu_{e}+\bar{\nu}_{\mu} .
$$

The difference in average PMT hit time between the two subevents is given in Fig. 6 and shows both the characteristic lifetime of muons in the sample and the effect of the subevent definition on CCQE detection for quickly decaying muons. The selection criteria are enumerated in Table I. Cut 1 enforces containment of charged particles produced inside the detector while also rejecting incoming charged particles. Cut 2 requires the muon subevent be correlated with the BNB proton spill. Cut 3 ensures the first subevent is not a Michel electron and avoids a region of muon energy with relatively poor reconstruction. Cut 4 eliminates most neutral-current events and rejects most

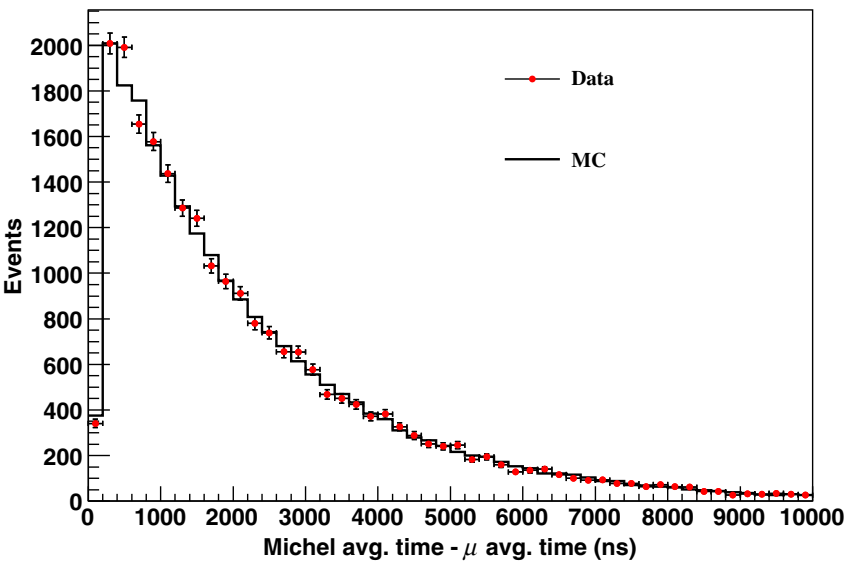

FIG. 6 (color online). Time separation in the average PMT hit time between the two subevents in the CCQE sample. The deviation from an exponential form at low time differences is due to the subevent separation requirement. Simulation is normalized to data, and statistical errors are shown with data. interactions with final-state $\pi^{+}$. Cut 5 further enhances the reliability of the reconstruction by reducing sensitivity to PMT coverage. Cut 6 ensures the measurements of the muon energy and the subevent vertices are consistent with the production and subsequent decay of a minimum ionizing particle. This cut rejects many events where the Michel electron is not associated with the primary muon, mainly $\mathrm{CC} 1 \pi^{+}$and $\mathrm{NC} 1 \pi^{+}$events where the second subevent is a decay positron from the $\pi^{+}$decay chain. Cut 7 requires that the candidate primary muon is better fit as a muon than as an electron. This cut reduces the background from most processes, most notably from $\mathrm{CC} 1 \pi^{+}$and $\mathrm{CC} 1 \pi^{-}$.

With this selection, the neutrino interaction assumptions detailed in Sec. III, the constrained backgrounds described in Sec. IVA and $10.1 \times 10^{20}$ POT, the sample consists of 71176 events with $\bar{\nu}_{\mu} \mathrm{CCQE}$ purity (detection efficiency) of $60.3 \%(29.5 \%)$. Table II presents a summary of the $\bar{\nu}_{\mu}$ CCQE sample, while Fig. 7 shows how these channels contribute to the reconstructed kinematical distributions of the final-state muon.

\section{Cross-section calculation and uncertainties}

The flux-integrated double-differential cross section per nucleon in the $i$ th bin is given by

$$
\left(\frac{d^{2} \sigma}{d T_{\mu} d\left[\cos \theta_{\mu}\right]}\right)_{i}=\frac{\sum_{j} U_{i j}\left(d_{j}-b_{j}\right)}{\left(\Delta T_{\mu}\right)_{i}\left(\Delta\left[\cos \theta_{\mu}\right]\right)_{i} \epsilon_{i} \Phi N},
$$

where $d_{j}$ refers to data, $b_{j}$ the background, $U_{i j}$ is an unfolding matrix connecting the reconstructed variable index $j$ to the true index $i, \epsilon_{i}$ is the detection efficiency, $\Delta T_{\mu}$ and $\Delta\left[\cos \theta_{\mu}\right]$ the respective bin widths, $\Phi$ the

TABLE II. Summary of the $\bar{\nu}_{\mu}$ CCQE sample. Contributions reflect all adjustments to simulation based on constraints from MiniBooNE data.

\begin{tabular}{lc}
\hline \hline Integrated POT & $10.1 \times 10^{20}$ \\
Energy-integrated $\bar{\nu}_{\mu}$ flux & $2.93 \times 10^{11} \bar{\nu}_{\mu} / \mathrm{cm}^{2}$ \\
$\bar{\nu}_{\mu}$ CCQE candidate events & 71176 \\
$\bar{\nu}_{\mu}$ CCQE efficiency $(R<550 \mathrm{~cm})$ & $29.5 \%$ \\
Interaction channel & Contribution $(\%)$ \\
$\bar{\nu}_{\mu}+p \rightarrow \mu^{+}+n$ (bound $p$ ) & 43.2 \\
$\bar{\nu}_{\mu}+p \rightarrow \mu^{+}+n$ (quasifree $p$ ) & 17.1 \\
$\nu_{\mu}+n \rightarrow \mu^{-}+p$ & 16.6 \\
$\bar{\nu}_{\mu}+N \rightarrow \mu^{+}+N+\pi^{-}$(resonant) & 10.4 \\
$\nu_{\mu}+N \rightarrow \mu^{-}+N+\pi^{+}$(resonant) & 3.8 \\
$\bar{\nu}_{\mu}+A \rightarrow \mu^{+}+A+\pi^{-}$(coherent) & 3.3 \\
$\bar{\nu}_{\mu}+N \rightarrow \mu^{+}+N+\pi^{0}$ & 2.8 \\
$\ldots$ & $\ldots$ \\
$\bar{\nu}_{\mu}+p \rightarrow \mu^{+}+\Lambda^{0}$ & \\
$\bar{\nu}_{\mu}+n \rightarrow \mu^{+}+\Sigma^{-}$ & 2.0 \\
$\bar{\nu}_{\mu}+p \rightarrow \mu^{+}+\Sigma^{0}$ & \\
$\ldots$ & $\ldots$ \\
All others & 0.8 \\
\hline
\end{tabular}



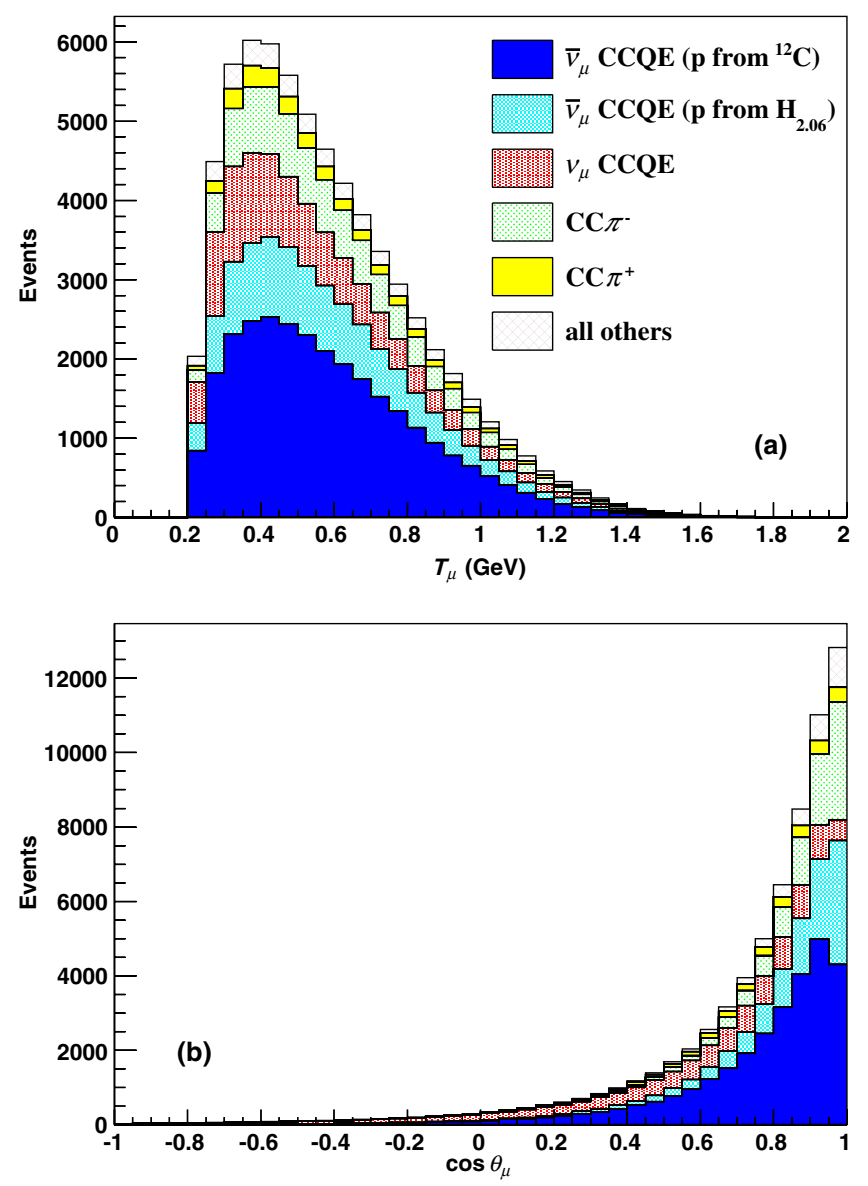

FIG. 7 (color online). Predicted sample composition as a function of reconstructed muon kinematics in the $\bar{\nu}_{\mu}$ CCQE sample. The top figure (a) shows the kinetic energy of the muon, while the bottom (b) shows $\cos \theta_{\mu}$, where $\theta_{\mu}$ is the muon direction relative to the incoming neutrinos. Distributions are normalized to the exposure of $10.1 \times 10^{20}$ POT.

integrated $\bar{\nu}_{\mu}$ exposure, and $N$ the number of proton targets in the volume studied.

The unfolding matrix $U_{i j}$ is based on the Bayesian method proposed in Ref. [55] to account for reconstruction biases. The high-sensitivity resolution of the reconstruction used to identify muon kinematics leads to only mild corrections. However, this procedure does introduce some dependence on the generated muon kinematics of $\bar{\nu}_{\mu}$ CCQE interactions. This bias is evaluated by unfolding the data with 100 different versions of $U_{i j}$ generated using a conservative range of CCQE model parameters. The bias introduced by the Bayesian unfolding method for the cross sections reported here is found to be negligible. Meanwhile, a particular strength of this cross-section configuration is that this unfolding matrix is entirely independent of assumptions regarding the underlying interaction. This is in contrast to, for example, the total cross section $\sigma\left(E_{\nu}\right)$ computed with only observations of muon kinematics.
It is important to note that by directly subtracting the background from data in the reconstructed distribution, this cross-section extraction procedure is entirely independent of the normalization of the generated signal $\bar{\nu}_{\mu} \mathrm{CCQE}$ processes. That is, though the RFG with a large value for the effective axial mass is assumed by simulation, the extracted cross section is not affected by this choice.

Systematic uncertainties are evaluated by forming an error matrix that propagates correlated uncertainties on parameters and processes that affect $\bar{\nu}_{\mu} \mathrm{CCQE}$ interactions onto the calculated cross section. The covariance matrix is constructed by first forming a distribution of weights corresponding to simulated excursions set by Gaussian variations of parameters and measurements within their associated error. These weights are then used to recalculate the double-differential cross section in Eq. (3), replacing the central-value $\mathrm{MC}$ valuations with the excursion values for terms appropriate to each systematic uncertainty. The difference of these alternate cross-section calculations compared to the "best guess" distribution forms the covariance matrix:

$$
M_{i j}=\frac{1}{K} \sum_{s=1}^{K}\left(N_{i}^{s}-N_{i}^{\mathrm{CV}}\right) \times\left(N_{j}^{s}-N_{j}^{\mathrm{CV}}\right) .
$$

Here $K$ simulation excursions are used, $N^{s}$ is the reweighted cross-section value corresponding to the sth simulation set, and $N^{\mathrm{CV}}$ represents the simulation central value. This technique is further described in Ref. [56]. For uncertainties on processes with correlated errors, typically $K=100$ while $K=1$ is sufficient for uncorrelated errors. Systematic uncertainties requiring correlated errors are the production of $\pi^{-}$in the proton beam target, the connection between $\pi^{-}$production and the focused $\bar{\nu}_{\mu}$ beam, optical transport in the detector, final-state interactions, and the bias due to the unfolding procedure.

As mentioned in Sec. II A, the uncertainty on the production of $\bar{\nu}_{\mu}$ parent $\pi^{-}$at the beryllium target is driven by the HARP data [30], and the absolute $\bar{\nu}_{\mu}$ flux prediction is minimally dependent on the hadroproduction model. Subsequent to $\pi^{-}$production, errors on the processes that culminate in the $\bar{\nu}_{\mu}$ beam include the amount of delivered POT, optics of the primary beam, magnetic focusing, and hadronic interactions in the target and the enclosing horn. More details on uncertainties of the flux prediction are available in Ref. [31]. Uncertainties on the model for optical transport in the detector are based on both external and in situ measurements of light attenuation, scintillation strength, and the refractive index of the oil [57]. For this uncertainty, 70 samples generated with variations of 35 parameters that describe the optical model are used to find the uncertainty propagated to the measurement. The most important final-state interactions affecting the composition of the $\bar{\nu}_{\mu}$ CCQE sample are the pion charge-exchange $\left(\pi^{ \pm}+X \leftrightarrow \pi^{0}+X^{\prime}\right)$ and absorption 
$\left(\pi^{ \pm}+X \rightarrow X^{\prime}\right)$ processes. The uncertainty on pion charge exchange (absorption) inside the nucleus is set to $30 \%$ (25\%) based on the difference between the NUANCE prediction and external data [58]. The intermedium processes are evaluated separately with 50\% (35\%) fractional uncertainty based on comparisons with the GCALOR prediction and the same external data. The final correlated systematic error evaluates the bias introduced by the Bayesian unfolding procedure, where 100 different matrices $U_{i j}$ are generated within $M_{A}=1.35 \pm 0.35 \mathrm{GeV}$ and $\kappa=1.007 \pm 0.007$. The negligible bias found when the data are extracted with these alternate matrices assuming a conservative range of CCQE parameter values assures this cross-section measurement is largely independent of the CCQE interaction model.

Uncertainties described by a single excursion from the simulation central value include errors due to detector PMT response and on background processes not due to final-state interactions. Large sets of simulation are generated separately to evaluate rate biases due to uncertainties on phototube discriminator threshold and the correlation between pulse time and delivered charge. The background processes are grouped into three classes: $\mathrm{CC} 1 \pi^{-}$events, those induced by $\nu_{\mu}$, and all non-CCQE, non-CC single-pion events. Note these groups are not mutually exclusive and all constraints are described in Sec. IVA. Based on consistency of the prediction using an extrapolated $\mathrm{CC} 1 \pi$ constraint with a robust external model for the $\mathrm{CC} 1 \pi^{-}$background, these events are assigned 20\% uncertainty. All $\nu_{\mu}$ background events are subject to the measured uncertainty shown in Fig. 3. The cross sections for the $\nu_{\mu} \mathrm{CCQE}$ and $\mathrm{CC} 1 \pi^{+}$processes are directly measured by MiniBooNE data [21,43], and so only their flux is uncertain. The uncertainty on the small contribution from coherent $\pi$ production is set to $60 \%$, while the other non-CCQE, non-CC1 $\pi^{ \pm}$processes are assigned $30 \%$ cross-section uncertainty.

The overall size of these covariance matrices can be expressed with a single number representing the normalization uncertainty of each error. Using the sum rule for variances and covariances, the total normalization uncertainty can be thought of as the error on the cross section if the measurement consisted of a single bin:

$$
\delta D_{T} / D_{T}=\sqrt{\sum_{i j}^{n} M_{i j}} / \sum_{i}^{n} D_{i},
$$

where $D_{T}=\sum_{i}^{n} D_{i}$ represents the double-differential cross-section measurement summed over each kinematic region $i$. This is also commonly referred to as the uncertainty on the scale of the measurement. Table III shows the contributions of various errors to the total normalization uncertainty.

The covariance matrix can also be used to separate the correlated normalization uncertainties from the total error,
TABLE III. Normalization uncertainty for various sources of error for the $\bar{\nu}_{\mu}$ CCQE cross section on mineral oil.

\begin{tabular}{lc}
\hline \hline Uncertainty type & Normalization uncertainty $(\%)$ \\
\hline $\bar{\nu}_{\mu}$ flux & 9.6 \\
Detector & 3.9 \\
Unfolding & 0.5 \\
Statistics & 0.8 \\
$\nu_{\mu}$ background & 3.9 \\
CC1 $\pi^{-}$background & 4.0 \\
All backgrounds & 6.4 \\
Total & $\mathbf{1 3 . 0}$ \\
\hline \hline
\end{tabular}

leaving information related to how much the shape of the observed data may vary within the systematic errors [40]. These uncertainties are identified by first defining a data vector $V$ with entries corresponding to the observed relative normalization of each bin: $V_{i}=$ $\left\{D_{1} / D_{T}, D_{2} / D_{T}, \ldots, D_{n} / D_{T}, D_{T}\right\}$. Notice this vector has dimension $n+1$, where $n$ is the number of bins measured. The covariance matrix $Q$ for this new vector $V$ involves the Jacobian matrix of partial derivatives $J$ and is given by

$$
Q_{k l}=\sum_{i j}^{n} J_{k i} M_{i j} J_{l j}=\sum_{i j}^{n} \frac{\partial V_{k}}{\partial D_{i}} M_{i j} \frac{\partial V_{l}}{\partial D_{j}} .
$$

The diagonals of the matrix $Q$ are related to the shape uncertainty in each kinematic bin. For entries $\{1,2, \ldots, n\}$,

$$
\begin{aligned}
Q_{k k} & =\frac{1}{D_{T}^{2}}\left[M_{k k}-2 \frac{D_{k}}{D_{T}} \sum_{i}^{n} M_{i k}+\frac{N_{k}^{2}}{N_{T}^{2}} \sum_{i j}^{n} M_{i j}\right] \\
& =\left(\delta D_{k, \text { shape }}\right)^{2} .
\end{aligned}
$$

As the full covariance matrix $M$ for the doubledifferential cross section is in principle a four-dimensional object with over 100000 entries, the combination of the total normalization error and the bin-by-bin shape error is the preferable method to report the complete experimental uncertainty. This is argued more completely in Ref. [55], and Ref. [59] provides an example of how to use this information in the context of a fit to these data.

The main result of this work is the $\bar{\nu}_{\mu} \mathrm{CCQE}$ doubledifferential cross section on mineral oil. However, as the majority of the bubble-chamber CCQE analyses using light targets for the interaction medium are adequately described with $M_{A} \sim 1 \mathrm{GeV}[19,20]$, the cross section on carbon only is found by assuming this value to subtract the quasifree hydrogen content of the $\bar{\nu}_{\mu}$ CCQE data. This alternate cross section is calculated by including $\bar{\nu}_{\mu}$ hydrogen CCQE events in the background term $b_{j}$ in Eq. (3), while the other terms in the calculation based on the signal definition now are based on only $\bar{\nu}_{\mu}$ CCQE events involving protons bound in carbon. Most notably, this reduces the number of interaction targets in the fiducial volume. 


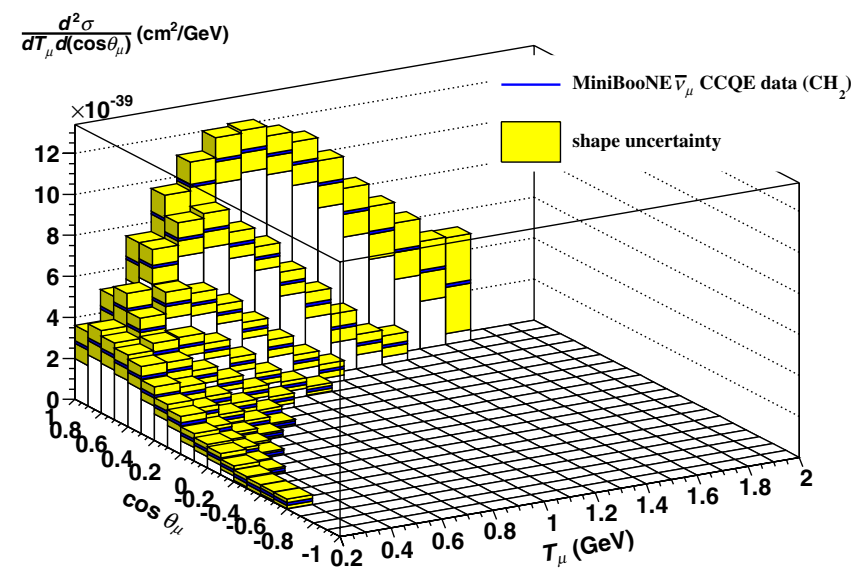

FIG. 8 (color online). The $\bar{\nu}_{\mu} \mathrm{CCQE}$ per-proton doubledifferential cross section with shape uncertainty. The normalization uncertainty of $13.0 \%$ is not shown. Numerical values for this cross section and its uncertainty are provided in Tables XIII and XIV, respectively.

Informed by the results of fits to the light-target CCQE experiments, $M_{A}^{\text {eff,H }}=1.026 \pm 0.021 \mathrm{GeV}[19,20]$ is assumed and subtracted from the data. Systematic error due to this background is evaluated with the method described earlier in this section with $K=100$ throws against the $0.021 \mathrm{GeV}$ uncertainty. Including this additional error and, more importantly, considering the lower sample purity for this alternate definition of signal events, the fractional normalization uncertainty increases to $17.4 \%$.

\section{Results}

The $\bar{\nu}_{\mu}$ CCQE double-differential flux-integrated cross section on mineral oil is shown with shape uncertainty in Fig. 8, and the one-dimensional projections are compared to RFG predictions in Fig. 9. The configuration with the hydrogen content subtracted is given in Appendix D and may be more readily compared to theoretical calculations for $\bar{\nu}_{\mu}$ CCQE interaction on carbon, such as in Refs. [60-65]. Bins in the kinematic region $-1<\cos \theta_{\mu}<$ +1 and $0.2<T_{\mu}(\mathrm{GeV})<2.0$ are reported if they meet the statistical requirement of at least 25 events in the reconstructed and background-subtracted data term $\left(d_{j}-b_{j}\right)$ in Eq. (3). If this threshold is not met, no measurement is reported. As no explicit assumptions about the underlying interaction are necessary to reconstruct muon kinematics, this result is nearly model independent. Since some background processes are not directly constrained by data, most notably $\mathrm{CC} 1 \pi^{-}$, Appendix D tabulates the subtracted data.

\section{CONCLUSION}

This work presents the first measurement of the $\bar{\nu}_{\mu}$ CCQE double-differential cross section in terms of muon angle and energy. This measurement is also the first $\bar{\nu}_{\mu}$ charged-current cross-section measurement with the ma-

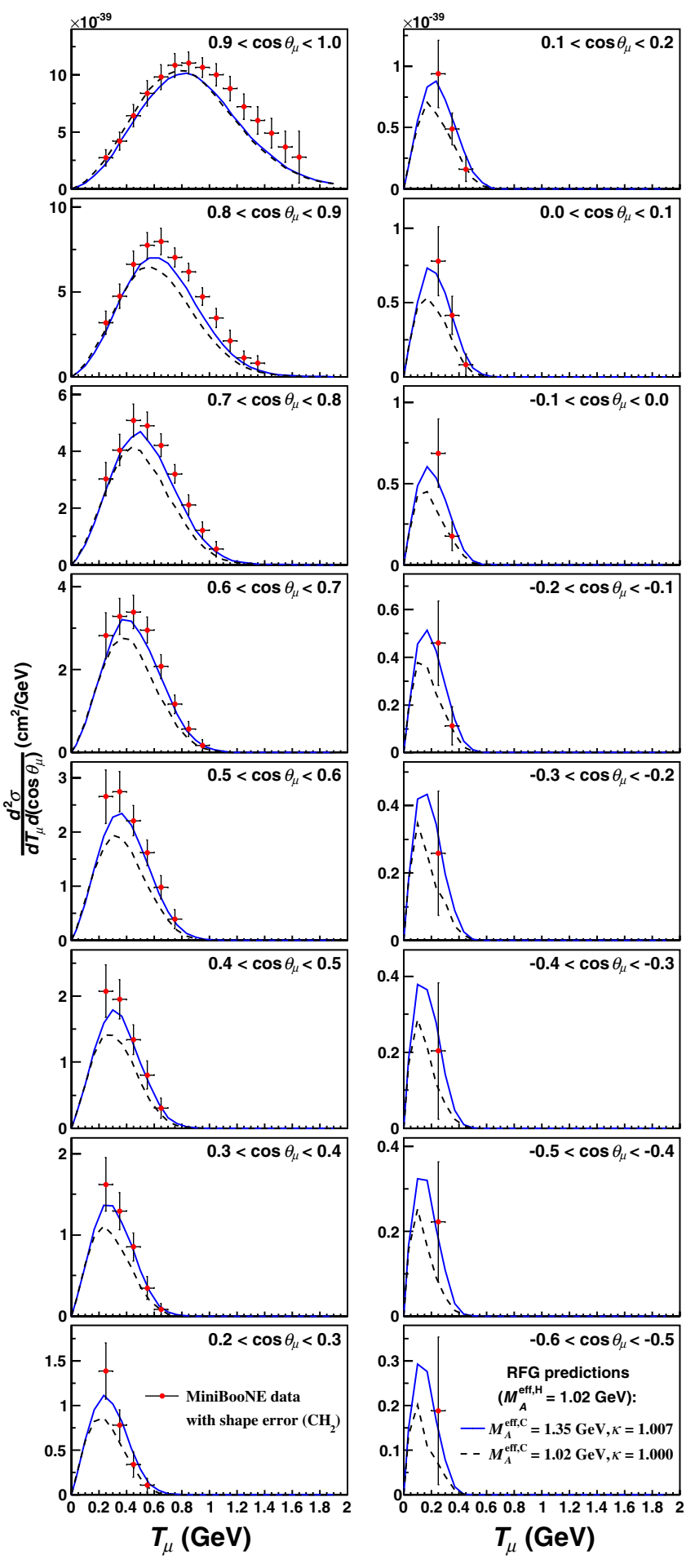

FIG. 9 (color online). Projections of the per-proton doubledifferential cross section in muon kinetic energy $T_{\mu}$ for various scattering angles $\cos \theta_{\mu}$. As indicated, both RFG predictions assume an effective axial mass of $1.02 \mathrm{GeV}$ for the quasifree hydrogen component of the data, while two choices for CCQE model parameters are shown. Data with $\cos \theta_{\mu}<-0.6$ have insufficient statistics and this region is not shown. Shape uncertainties are shown, an additional normalization uncertainty of $13.0 \%$ is not. Numerical values for this cross section and its uncertainty are provided in Tables XIII and XIV, respectively. 
jority of interactions with $E_{\bar{\nu}}<1 \mathrm{GeV}$. This cross section is the least model-dependent measurement possible with the MiniBooNE detector and is thus the main result of this work.

It is clear in Fig. 9 that the RFG model (described in Sec. III) assuming $M_{A}^{\text {eff,C }} \sim 1 \mathrm{GeV}$ does not adequately describe these data in shape or in normalization. Consistent with other recent CCQE measurements on nuclear material [21-24], a significant enhancement in the normalization that grows with decreasing muon scattering angle is observed compared to the expectation with $M_{A}=1.0 \mathrm{GeV}$.

These data find tension with the NOMAD $\bar{\nu}_{\mu}$ CCQE results, which are described both in shape and normalization by $M_{A}=1.06 \pm 0.12$ [25]. This tension is common among the $\nu_{\mu} \mathrm{CCQE}$ analyses from the two experiments. However, care should be taken in comparing modeldependent results among experiments with such different neutrino fluxes and detector technologies. A definitive unification of these apparently discrepant data sets will require the continued increase of both experimental and theoretical activity surrounding this topic. Fortunately, many experiments at a variety of neutrino energies capable of making high-resolution, model-independent neutrino and antineutrino CCQE measurements with different detector technologies and nuclear media using both neutrino and antineutrino beams currently have data or will soon. These include MINER $\nu$ A [66], SciBooNE [67], MicroBooNE [68], ArgoNeuT [69], ICARUS [70], and the $\mathrm{T} 2 \mathrm{~K}[4]$ and $\mathrm{NO} \nu \mathrm{A}[6]$ near detectors.

Finally, a novel and crucial evaluation of the $\nu_{\mu}$ background in this work is presented in Appendix A. In the absence of a magnetic field, this analysis and those described in Ref. [34] measure the $\nu_{\mu}$ flux of the antineutrino-mode beam with $\sim 15 \%$ fractional uncertainty. These techniques could be used in current and future neutrino oscillation programs, particularly when modest charge identification is sufficient to meet the physics goals [71].

\section{ACKNOWLEDGMENTS}

This work was made possible with the support of Fermilab, the National Science Foundation, and the Department of Energy through the construction, operation, and data analysis of the MiniBooNE experiment. Operated by Fermi Research Alliance, LLC under Contract No. DeAC02-07CH11359 with the United States Department of Energy.

\section{APPENDIX A: MEASUREMENT OF $\nu_{\mu}$ FLUX IN ANTINEUTRINO MODE USING $\mu^{-}$CAPTURE}

\section{Introduction}

MiniBooNE uses dedicated hadroproduction measurements from the HARP experiment [30] to predict the $\nu_{\mu}$ and $\bar{\nu}_{\mu}$ fluxes for the antineutrino-mode beam. However, as shown in Fig. 2 in Sec. II A, most of the $\nu_{\mu}$ flux arises from the very forward-going region of $\pi^{+}$production and is not well constrained by the HARP measurements. Fortunately, there are several ways to determine the $\nu_{\mu}$ content of the beam directly from MiniBooNE data. Two such analyses are described in Ref. [34] and a third is presented in this appendix. These analyses show that, in the absence of a magnetic field, the $\nu_{\mu}$ and $\bar{\nu}_{\mu}$ content can still be modestly separated using statistical methods.

The measurement of the $\nu_{\mu}$ flux in the antineutrinomode beam described in this appendix exploits the asymmetry in the production of decay electrons between $\mu^{-}$and $\mu^{+}$in nuclear material. The results are consistent with and complementary to those of Ref. [34].

\section{Muon capture model and event selection}

The model for $\mu^{-}$capture and the processes that can obscure its rate in the MiniBooNE detector are described in this section, followed by details on the analysis samples studied. In mineral oil, stopped $\mu^{-}$are captured on carbon nuclei with a probability of $(7.78 \pm 0.07) \%$ [72]. In such capture events, typically little or no extra activity is observed in the detector. However, the low-energy neutron and photons from the primary capture reaction as well as deexcitations of the boron isotope may be energetic enough to produce a Michel-electron-like event. The simulated production of these particles is based on the measurements of Refs. [73-78], and the model that propagates these particles and possible reinteractions through the MiniBooNE detector estimates $6.60 \%$ of $\mu^{-}$capture events lead to activity similar to a low-energy Michel electron. Thus, the apparent $\mu^{-}$nuclear capture probability in the detector is predicted to be $7.78 \times$ $(1-0.066) \%=7.26 \pm 0.20 \%$, where the uncertainty is substantially increased to recognize the model dependence of the rate to regain Michel-electron-like events following $\mu^{-}$capture. This rate is partially constrained by the calibration procedure described in Appendix A 3, and it will be shown that the assigned uncertainty on effective $\mu^{-}$ nuclear capture has a negligible impact on the final measurements.

Sensitivity to the $\mu^{-}$content of the data is obtained by simultaneously analyzing two samples: those with only a muon candidate event and events consistent with a muon and its decay electron. Therefore, this analysis takes as signal all $\nu_{\mu}$ and $\bar{\nu}_{\mu}$ charged-current events. Apart from the requirement of either one or two subevents, the event selection for this analysis closely follows that described in Sec. IV B with a few changes appropriate to different backgrounds and a higher sensitivity to Michel electron detection efficiency. Table IV details the $\nu_{\mu}$ and $\bar{\nu}_{\mu}$ charged-current purity of the two samples after each cut.

The primary samples of this analysis are separated by cut 1 , where $\nu_{\mu} \mathrm{CC}$ events have an enhanced contribution 
TABLE IV. Antineutrino-mode purity in $\%$ for all $\nu_{\mu}$ and $\bar{\nu}_{\mu}$ charged-current events in the one- and two-subevent samples. A precut of generated radius $<550 \mathrm{~cm}$ is applied.

\begin{tabular}{|c|c|c|c|c|c|}
\hline \multirow[b]{2}{*}{ Cut \# } & \multirow[b]{2}{*}{ Description } & \multicolumn{2}{|c|}{ One subevent } & \multicolumn{2}{|c|}{ Two subevents } \\
\hline & & $\nu_{\mu} \mathrm{CC}$ & $\bar{\nu}_{\mu} \mathrm{CC}$ & $\nu_{\mu} \mathrm{CC}$ & $\bar{\nu}_{\mu} \mathrm{CC}$ \\
\hline 1 & Subevent cut & 18 & 33 & 26 & 57 \\
\hline 2 & Veto hits $<6$ for all subevents & 9 & 11 & 30 & 65 \\
\hline 3 & First subevent in beam window: $4000<T$ (ns) $<7000$ & 9 & 11 & 29 & 65 \\
\hline 4 & Reconstructed vertex radius $<500 \mathrm{~cm}$ for first subevent & 8 & 11 & 29 & 65 \\
\hline 5 & Kinetic energy $>200 \mathrm{MeV}$ for first subevent under $\mu$ hypothesis & 20 & 27 & 29 & 68 \\
\hline 6 & $\mu / e$ log-likelihood ratio $>0.02$ for first subevent & 36 & 54 & 27 & 72 \\
\hline 7 & Predicted $\mu$ stopping radius $<500 \mathrm{~cm}$ & 39 & 46 & 28 & 71 \\
\hline 8 & $Q_{\mathrm{QE}}^{2}>0.2 \mathrm{GeV}^{2}$ & 57 & 36 & 43 & 56 \\
\hline
\end{tabular}

in the single-subevent sample due to $\mu^{-}$capture. Cuts 2-5 are common to the analysis presented in the main body of this work and are motivated in Sec. IV B. Cuts 6 and 8 reduce the neutral-current background in the singlesubevent sample: Fig. 10 shows neutral-current single $\pi$ events are largely rejected by the requirement on the $\mu / e$ log-likelihood variable, while cut 8 further reduces their contribution. Cut 7 uses the observed muon kinematics and the stopping power of mineral oil for minimum-ionizing particles to calculate where the muon will stop. This cut removes Michel electrons produced near the optical barrier where Michel electron detection efficiency decreases rapidly with radius and is thus sensitive to modeling, while Michel electron detection is constant below $500 \mathrm{~cm}$ in this variable. Cut 7 also enhances $\nu_{\mu}$ purity due to kinematic differences between $\nu_{\mu}$ and $\bar{\nu}_{\mu} \mathrm{CCQE}$, where the more forward-going nature of the $\mu^{+}$from $\bar{\nu}_{\mu}$ interactions preferentially stop at high radius in the downstream region of the detector. A summary of nucleon-level interactions contributing to the selected subevent samples is given in Table V.

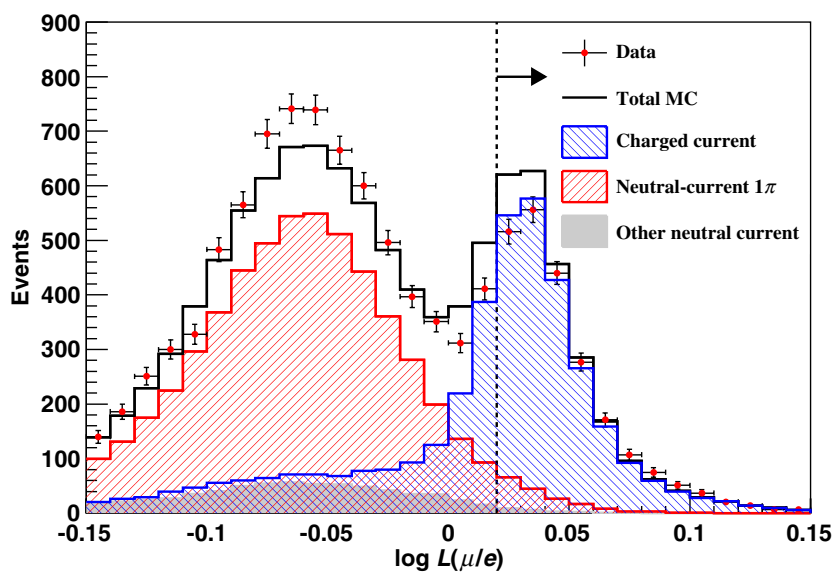

FIG. 10 (color online). The log-likelihood $\mu / e$ particle-ID variable in the single-subevent sample. Events with a muonlike score of 0.02 and higher are selected. Expectations are normalized to flux, and errors shown on data are statistical only.

\section{Calibrations using neutrino-mode data}

Charged-current $\nu_{\mu}$ and $\bar{\nu}_{\mu}$ events without final-state $\pi^{+}$typically have two subevents: one from the primary $\mu$ and another from its decay electron. Two effects determine the majority of the migration rate of these events from the two-subevent to the one-subevent sample: $\mu^{-}$nuclear capture and detection efficiency for Michel electrons. Since an appreciable number $(\sim 7 \%)$ of charged-current events enter the single-subevent sample due to Michel electron detection inefficiencies, the measurement of the $\nu_{\mu}$ content of the antineutrino-mode data is sensitive to the accuracy of both the Michel electron detection efficiency and the effective $\mu^{-}$capture rate. The rate of nondetection is mostly due to Michel electron production too close in time with the parent muon to be separated by the subevent definition. This effect can be seen at low values of the timing difference distribution between the two subevents shown in Fig. 6, Sec. IV B.

Fortunately, the neutrino-mode data offer an opportunity to calibrate the migration rate between the subevent samples for $\nu_{\mu}$ charged-current events. Due to a convolution of flux and cross-section effects [34], the neutrinomode subevent samples are mostly due to charged-current $\nu_{\mu}$ interactions. Table VI shows the predicted neutrino

TABLE V. Summary of predicted nucleon-level interactions in the antineutrino-mode subevent samples. The small contributions from neutral-current processes are presented as the sum of the $\nu_{\mu}$ and $\bar{\nu}_{\mu}$ interactions.

\begin{tabular}{lcc}
\hline \hline & \multicolumn{2}{c}{ Contribution $(\%)$ to } \\
\cline { 2 - 3 } Process & One subevent & Two subevents \\
\hline $\bar{\nu}_{\mu} p \rightarrow \mu^{+} n$ & 31 & 49 \\
$\nu_{\mu} n \rightarrow \mu^{-} p$ & 48 & 36 \\
$\bar{\nu}_{\mu} N \rightarrow \mu^{+} N \pi^{-}$ & 3 & 5 \\
$\nu_{\mu} N \rightarrow \mu^{-} N \pi^{+}$ & 7 & 7 \\
$\nu_{\mu}\left(\bar{\nu}_{\mu}\right) N \rightarrow \nu_{\mu}\left(\bar{\nu}_{\mu}\right) N$ & 1 & 0 \\
$\nu_{\mu}\left(\bar{\nu}_{\mu}\right) N \rightarrow \nu_{\mu}\left(\bar{\nu}_{\mu}\right) N \pi^{0}$ & 3 & 0 \\
$\nu_{\mu}\left(\bar{\nu}_{\mu}\right) N \rightarrow \nu_{\mu}\left(\bar{\nu}_{\mu}\right) N \pi^{ \pm}$ & 4 & 0 \\
Other & 3 & 3 \\
\hline \hline
\end{tabular}


TABLE VI. A brief description of the neutrino-mode subevent samples for the same selection described in the previous section.

\begin{tabular}{lcc}
\hline \hline & \multicolumn{2}{c}{ Contribution (\%) to } \\
\cline { 2 - 3 } Process & One subevent & Two subevents \\
\hline All $\nu_{\mu}$ charged current & 95.4 & 99.0 \\
All $\bar{\nu}_{\mu}$ & 0.4 & 0.7 \\
All neutral current & 4.3 & 0.3 \\
\hline \hline
\end{tabular}

species and interaction-type contributions to the neutrinomode subevent samples. With a high-purity $\nu_{\mu}$ chargedcurrent sample, the accuracy of Michel electron detection and effective $\mu^{-}$capture in simulation can be tested. For charged-current $\nu_{\mu}$ events without final-state $\pi^{+}\left(\nu_{\mu} \mathrm{CC}\right)$, the number of events in the neutrino-mode one-subevent $\left(1 \mathrm{SE}^{\nu}\right)$ and two-subevent $\left(2 \mathrm{SE}^{\nu}\right)$ samples are given by

$$
\begin{gathered}
1 \mathrm{SE}^{\nu}=\nu_{\mu} \mathrm{CC} \times(\delta+\beta(1-\delta))+\mathrm{N}_{1}^{\nu}, \\
2 \mathrm{SE}^{\nu}=\nu_{\mu} \mathrm{CC} \times(1-\delta-\beta(1-\delta))+\mathrm{N}_{2}^{\nu},
\end{gathered}
$$

where $\mathrm{N}_{1}^{\nu}\left(\mathrm{N}_{2}^{\nu}\right)$ is the neutral-current contribution to the 1SE (2SE) sample, $\delta$ is the Michel electron detection inefficiency, and $\beta$ is the effective $\mu^{-}$capture rate described previously. The rate for Michel electron nondetection can be solved in terms of the effective $\mu^{-}$capture rate and the small neutral-current contribution:

$$
\delta=\frac{\frac{1 \mathrm{SE}^{\nu}-\mathrm{N}_{1}^{\nu}}{1 \mathrm{SE}^{\nu}+2 \mathrm{SE}^{\nu}-\left(\mathrm{N}_{1}^{\nu}+\mathrm{N}_{2}^{\nu}\right)}-\beta}{1-\beta} .
$$

Noting the symmetry in Eqs. (A1) and (A2) between $\delta$ and $\beta$, Eq. (A3) can also express the effective $\mu^{-}$capture rate in terms of Michel electron detection with $\delta \leftrightarrow \beta$. Table VII gives values of $\delta$ and $\beta$ from simulation and data based on the observed or predicted event rates in the $1 \mathrm{SE}^{\nu}$ and $2 \mathrm{SE}^{\nu}$ samples.

As the $\nu_{\mu}$ charged-current migration rate to the singlesubevent sample is due to a convolution of Michel electron detection and effective $\mu^{-}$capture, the processes cannot be simultaneously calibrated with the neutrino-mode data; that is, for example, the calibration of $\delta$ assumes the MC valuation of $\beta$ is correct. Future experiments may have the ability to separate the two processes by examining the low-energy region of the Michel electron spectrum, where the contribution from events following $\mu^{-}$capture is enhanced. As the calibration results shown in Table VII

TABLE VII. Calibration summary for Michel electron detection inefficiency $(\delta)$ and the rate of effective $\mu^{-}$nuclear capture $(\beta)$. Note that both processes cannot be simultaneously constrained.

\begin{tabular}{lccc}
\hline \hline Process & Data & MC & Data/MC \\
\hline$\delta$ & 0.073 & 0.074 & 0.98 \\
$\beta$ & 0.071 & 0.073 & 0.98 \\
\hline \hline
\end{tabular}

are quite mild and within systematic uncertainties, this procedure gives confidence in the ability to unambiguously measure the $\nu_{\mu}$ content of the antineutrino-mode data using $\mu^{-}$capture.

The high-statistics neutrino-mode data also allow for a stability check of the ratio of samples one subevent/two subevents, and four sequential sample periods are consistent within one standard deviation.

\section{Measurement and systematic errors}

The $\nu_{\mu}$ flux is measured by adjusting the MC prediction of the $\nu_{\mu}$ and $\bar{\nu}_{\mu}$ content to match the data in regions of reconstructed energy for the subevent samples. Following the conventions of Eqs. (A1) and (A2) and introducing $\bar{\nu}_{\mu}$ CC for the $\bar{\nu}_{\mu}$ charged-current content, the predicted $\nu_{\mu}$ and $\bar{\nu}_{\mu}$ contributions to the subevent samples in antineutrino mode are defined as

$$
\begin{gathered}
\nu_{\mathrm{MC}}^{1 \mathrm{SE}}=\nu_{\mu} \mathrm{CC} \times(\delta+\beta(1-\delta)), \\
\nu_{\mathrm{MC}}^{2 \mathrm{SE}}=\nu_{\mu} \mathrm{CC} \times(1-\delta-\beta(1-\delta)), \\
\bar{\nu}_{\mathrm{MC}}^{1 \mathrm{SE}}=\bar{\nu}_{\mu} \mathrm{CC} \times \delta, \\
\bar{\nu}_{\mathrm{MC}}^{2 \mathrm{SE}}=\bar{\nu}_{\mu} \mathrm{CC} \times(1-\delta) .
\end{gathered}
$$

Then the $1 \mathrm{SE}^{\bar{\nu}}$ and $2 \mathrm{SE}^{\bar{\nu}}$ data samples in antineutrino mode are given by

$$
\begin{aligned}
& 1 \mathrm{SE}^{\bar{\nu}}=\alpha_{\nu} \times \nu_{\mathrm{MC}}^{1 \mathrm{SE}}+\alpha_{\bar{\nu}} \times \bar{\nu}_{\mathrm{MC}}^{1 \mathrm{SE}}+\mathrm{N}_{1}^{\bar{\nu}}, \\
& 2 \mathrm{SE}^{\bar{\nu}}=\alpha_{\nu} \times \nu_{\mathrm{MC}}^{2 \mathrm{SE}}+\alpha_{\bar{\nu}} \times \bar{\nu}_{\mathrm{MC}}^{2 \mathrm{SE}}+\mathrm{N}_{2}^{\bar{\nu}},
\end{aligned}
$$

where $\alpha_{\nu}$ and $\alpha_{\bar{\nu}}$ are scale factors for the $\nu_{\mu}$ and $\bar{\nu}_{\mu}$ charged-current content, respectively, to be measured in this analysis and the neutral-current content $\left(\mathrm{N}_{2}^{\bar{\nu}}\right.$ and $\left.\mathrm{N}_{1}^{\bar{\nu}}\right)$ include contributions from both $\nu_{\mu}$ and $\bar{\nu}_{\mu}$. Equations (A8) and (A9) can be solved for $\alpha_{\nu}$ and $\alpha_{\bar{\nu}}$ :

$$
\begin{aligned}
& \alpha_{\nu}=\frac{\left(1 \mathrm{SE}^{\bar{\nu}}-\mathrm{N}_{1}^{\bar{\nu}}\right) \bar{\nu}_{\mathrm{MC}}^{2 \mathrm{SE}}-\left(2 \mathrm{SE}^{\bar{\nu}}-\mathrm{N}_{2}^{\bar{\nu}}\right) \bar{\nu}_{\mathrm{MC}}^{1 \mathrm{SE}},}{\bar{\nu}_{\mathrm{MC}}^{2 \mathrm{SE}} \nu_{\mathrm{MC}}^{1 \mathrm{SE}}-\bar{\nu}_{\mathrm{MC}}^{1 \mathrm{SE}} \nu_{\mathrm{MC}}^{2 \mathrm{SE}}} \\
& \alpha_{\bar{\nu}}=\frac{\left(1 \mathrm{SE}^{\bar{\nu}}-\mathrm{N}_{1}^{\bar{\nu}}\right) \nu_{\mathrm{MC}}^{2 \mathrm{SE}}-\left(2 \mathrm{SE}^{\bar{\nu}}-\mathrm{N}_{2}^{\bar{\nu}}\right) \nu_{\mathrm{MC}}^{1 \mathrm{SE}}}{\nu_{\mathrm{MC}}^{2 \mathrm{SE}} \bar{\nu}_{\mathrm{MC}}^{1 \mathrm{SE}}-\nu_{\mathrm{MC}}^{1 \mathrm{SE}} \bar{\nu}_{\mathrm{MC}}^{2 \mathrm{SE}}}
\end{aligned}
$$

To check the modeling of the $\nu_{\mu}$ flux spectrum, this measurement is performed in three regions of reconstructed energy $E_{\nu}^{\mathrm{QE}}$ defined as

$$
E_{\nu}^{\mathrm{QE}}=\frac{2\left(M_{p}-E_{B}\right) E_{\mu}-\left(E_{B}^{2}-2 M_{p} E_{B}+m_{\mu}^{2}+\Delta M^{2}\right)}{2\left[\left(M_{p}-E_{B}\right)-E_{\mu}+p_{\mu} \cos \theta_{\mu}\right]},
$$

where, $E_{B}=30 \mathrm{MeV}$ is the binding energy, $m_{\mu}$ is the muon mass, $\Delta M^{2}=M_{p}^{2}-M_{n}^{2}$, where $M_{n}\left(M_{p}\right)$ is the neutron (proton) mass, $p_{\mu}$ is the muon momentum, and 
TABLE VIII. Central-value results for scale factors relative to MC expectation for the $\nu_{\mu}$ and $\bar{\nu}_{\mu}$ charged-current content of the antineutrino-mode data.

\begin{tabular}{lcccc}
\hline \hline & & \multicolumn{3}{c}{$E_{\nu}^{\mathrm{QE}}$ range $(\mathrm{GeV})$} \\
\cline { 3 - 5 } Parameter & Calibrated process & $<0.9$ & $\geq 0.9$ & All \\
\hline$\alpha_{\nu}$ & $\delta$ & 0.78 & 0.79 & 0.78 \\
& $\beta$ & 0.78 & 0.79 & 0.78 \\
$\alpha_{\bar{\nu}}$ & Average & $\mathbf{0 . 7 8}$ & $\mathbf{0 . 7 9}$ & $\mathbf{0 . 7 8}$ \\
& $\delta$ & 1.16 & 1.15 & 1.16 \\
& $\beta$ & 1.16 & 1.15 & 1.16 \\
\hline \hline & Average & $\mathbf{1 . 1 6}$ & $\mathbf{1 . 1 5}$ & $\mathbf{1 . 1 6}$ \\
\hline
\end{tabular}

$\theta_{\mu}$ is the outgoing muon angle relative to the incoming neutrino beam. This reconstruction assumes $\bar{\nu}_{\mu} \mathrm{CCQE}$ interactions with at-rest, independently acting nucleons. Though this is a model-dependent valuation of the neutrino energy complicated further by the significant non- $\bar{\nu}_{\mu}$ CCQE content, separating the samples into exclusive regions of $E_{\nu}^{\mathrm{QE}}$ nevertheless affords statistical sensitivity to the accuracy of the simulated flux spectrum. The three energy regions explored are $E_{\nu}^{\mathrm{QE}}<0.9 \mathrm{GeV}, E_{\nu}^{\mathrm{QE}} \geq$ $0.9 \mathrm{GeV}$, and an inclusive sample. The statistics of the single-subevent sample prohibit the analysis of more than two exclusive $E_{\nu}^{\mathrm{QE}}$ regions. As described in the previous section, the calibration from the neutrino-mode data is ambiguous between Michel electron detection and the effective $\mu^{-}$capture model. As these effects change the expectations for $\bar{\nu}_{\mathrm{MC}}^{1 \mathrm{SE}}, \bar{\nu}_{\mathrm{MC}}^{2 \mathrm{SE}}, \nu_{\mathrm{MC}}^{1 \mathrm{SE}}$, and $\nu_{\mathrm{MC}}^{2 \mathrm{SE}}$ in different ways, the measurement of $\alpha_{\nu}$ and $\alpha_{\bar{\nu}}$ is, in principle, sensitive to which rate is calibrated. In the absence of a compelling reason to choose one over the other, the final evaluations for $\alpha_{\nu}$ and $\alpha_{\bar{\nu}}$ are taken to be the average of the two calculations assuming each rate is calibrated. A calibration uncertainty spanning the difference in the two measurements is added to the systematic errors discussed next. The central values for $\alpha_{\nu}$ and $\alpha_{\bar{\nu}}$ are presented in Table VIII.

Systematic uncertainties on $\alpha_{\nu}$ and $\alpha_{\bar{\nu}}$ are evaluated by assigning relevant errors to the physics processes contributing to the subevent samples and observing how the measurement changes as the channels are varied within their uncertainty. These uncertainties are treated as uncorrelated, so the uncertainty on $\alpha_{\nu}$, for example, due to physics processes $P_{1}, \ldots, P_{N}$ is simply

$$
\delta \alpha_{\nu}^{2}=\sum_{i=1}^{N}\left(\frac{\partial \alpha_{\nu}}{\partial P_{i}} \delta P_{i}\right)^{2}
$$

Table IX shows the errors assigned to the various contributing processes and their propagated uncertainty onto $\alpha_{\nu}$ and $\alpha_{\bar{\nu}}$. The most important process for extracting the $\nu_{\mu}$ flux measurement is the $\nu_{\mu}$ CCQE interaction, and its cross section and assigned uncertainty reflect the measurement and accuracy of the MiniBooNE result [21]. The same is true for the $\nu_{\mu}$ and $\bar{\nu}_{\mu}$ neutral-current single $\pi^{0}$ channels [79]; however, the error is increased to recognize a possible rate difference in these interactions between the cross-section measurements and this analysis due to using the opposite side of the log-likelihood variable shown in Fig. 10. The $\nu_{\mu}$ and $\bar{\nu}_{\mu}$ charged-current single charged $\pi$ channels are adjusted to reflect the $\nu_{\mu}$ measurement [21], and their uncertainty is increased to recognize the extrapolation to the $\bar{\nu}_{\mu}$ processes. Treating the uncertainties on the $\nu_{\mu}$ processes constrained by MiniBooNE data as uncorrelated ignores a common dependence on the neutrino-mode flux uncertainties and a small cancellation of errors that could be propagated onto $\alpha_{\nu}$ and $\alpha_{\bar{\nu}}$ is ignored. The $\nu_{\mu}$ neutral-current elastic process is also constrained by MiniBooNE data [80], while the neutral-current chargedpion production processes are completely unconstrained

TABLE IX. Uncertainty summary for this analysis. Included are the assumed errors on physics processes and their contributions to the total errors in $\alpha_{\nu}$ and $\alpha_{\bar{\nu}}$ in the regions of reconstructed neutrino energy studied. The statistics of the $\nu$-mode data enter the uncertainty from the calibration procedure described in Sec. IV B.

\begin{tabular}{lccccccc}
\hline \hline & & \multicolumn{3}{c}{ Uncertainty contribution to $\alpha_{\nu}$} & \multicolumn{3}{c}{ Uncertainty contribution to $\alpha_{\bar{\nu}}$} \\
\cline { 2 - 8 } & $\begin{array}{c}\text { Fractional } \\
\text { uncertainty }(\%)\end{array}$ & $E_{\nu}^{\mathrm{QE}}<0.9 \mathrm{GeV}$ & $E_{\nu}^{\mathrm{QE}} \geq 0.9 \mathrm{GeV}$ & $\mathrm{All}$ & $E_{\nu}^{\mathrm{QE}}<0.9 \mathrm{GeV}$ & $E_{\nu}^{\mathrm{QE}} \geq 0.9 \mathrm{GeV}$ & $\mathrm{All}$ \\
\hline$\nu_{\mu} n \rightarrow \mu^{-} p$ & 10 & 0.07 & 0.08 & 0.07 & 0.00 & 0.00 & 0.00 \\
$\bar{\nu}_{\mu} p \rightarrow \mu^{+} n$ & 20 & 0.04 & 0.02 & 0.03 & 0.20 & 0.20 & 0.21 \\
$\nu_{\mu}\left(\bar{\nu}_{\mu}\right) N \rightarrow \mu^{-}\left(\mu^{+}\right) N \pi^{+}\left(\pi^{-}\right)$ & 20 & 0.04 & 0.05 & 0.04 & 0.02 & 0.02 & 0.01 \\
$\nu_{\mu}\left(\bar{\nu}_{\mu}\right) N \rightarrow \nu_{\mu}\left(\bar{\nu}_{\mu}\right) N$ & 30 & 0.00 & 0.00 & 0.00 & 0.00 & 0.00 & 0.00 \\
$\nu_{\mu}\left(\bar{\nu}_{\mu}\right) N \rightarrow \nu_{\mu}\left(\bar{\nu}_{\mu}\right) N \pi^{0}$ & 25 & 0.02 & 0.01 & 0.01 & 0.01 & 0.01 & 0.01 \\
$\nu_{\mu}\left(\bar{\nu}_{\mu}\right) N \rightarrow \nu_{\mu}\left(\bar{\nu}_{\mu}\right) N \pi^{ \pm}$ & 50 & 0.05 & 0.02 & 0.01 & 0.03 & 0.03 & 0.01 \\
$\mu^{-}$capture & 2.8 & 0.00 & 0.00 & 0.00 & 0.00 & 0.00 & 0.00 \\
$\bar{\nu}$-mode statistics & $\cdots$ & 0.10 & 0.11 & 0.08 & 0.08 & 0.08 & 0.06 \\
$\nu$-mode statistics & $\cdots$ & 0.04 & 0.05 & 0.04 & 0.03 & 0.03 & 0.03 \\
All & $\cdots$ & $\mathbf{0 . 1 4}$ & $\mathbf{0 . 1 6}$ & $\mathbf{0 . 1 2}$ & $\mathbf{0 . 2 2}$ & $\mathbf{0 . 2 2}$ & $\mathbf{0 . 2 2}$ \\
\hline \hline
\end{tabular}


TABLE X. Summary of measurements for the $\nu_{\mu}$ flux scale $\alpha_{\nu}$ and the $\bar{\nu}_{\mu}$ rate scale $\alpha_{\bar{\nu}}$.

\begin{tabular}{lccc}
\hline \hline & \multicolumn{3}{c}{$E_{\nu}^{\mathrm{QE}}$ range $(\mathrm{GeV})$} \\
\cline { 2 - 4 } Parameter & $<0.9$ & $\geq 0.9$ & All \\
\hline$\alpha_{\nu}$ & $0.78 \pm 0.14$ & $0.79 \pm 0.16$ & $0.78 \pm 0.12$ \\
$\alpha_{\bar{\nu}}$ & $1.16 \pm 0.22$ & $1.15 \pm 0.22$ & $1.16 \pm 0.22$ \\
\hline \hline
\end{tabular}

and so the assigned uncertainty is large. Preliminary results for the $\bar{\nu}_{\mu}$ CCQE process [38] informs the choice of a $20 \%$ uncertainty relative to the RFG model with $M_{A}=$ $1.35 \mathrm{GeV}$. With these systematic uncertainty assumptions, as seen in Table IX, the uncertainty on the main result of this work $\alpha_{\nu}$ is dominated by statistics and the $\nu_{\mu} \mathrm{CCQE}$ cross section. As the $\nu_{\mu} \mathrm{CCQE}$ process is directly constrained by MiniBooNE data, the measurement of the $\nu_{\mu}$ flux scale $\alpha_{\nu}$ features negligible model dependence. Table X summarizes the measurements of $\alpha_{\nu}$ and $\alpha_{\bar{\nu}}$.

As the cross sections for the dominant $\nu_{\mu}$ processes have been applied to simulation, the deviation from unity for $\alpha_{\nu}$ represents the accuracy of the highly uncertain $\nu_{\mu}$ flux prediction in antineutrino mode. As the bulk of the $\bar{\nu}_{\mu}$ flux prediction is constrained by the HARP data, the $\alpha_{\bar{\nu}}$ scale factor is representative of the level of cross-section agreement between the data and the RFG with $M_{A}=1.35 \mathrm{GeV}$ for the $\bar{\nu}_{\mu}$ CCQE process.

\section{Summary}

This appendix presents a measurement of the $\nu_{\mu}$ flux in antineutrino mode using a nonmagnetized detector. The results are consistent with and complementary to the two measurements in Ref. [34]. A summary of the results from all three analyses is shown in Fig. 3, Sec. IVA. As no energy dependence among the measurements is observed, the simulation of the $\nu_{\mu}$ flux in antineutrino mode, which is unconstrained by the HARP hadroproduction data, appears to be roughly $20 \%$ high in normalization, while the flux spectrum is well modeled.

These techniques could also aid future neutrino experiments that will test for $C P$ violation in the lepton sector using large unmagnetized detectors such as $\mathrm{NO} \nu \mathrm{A}[6]$, T2K [4], LBNE [8], LAGUNA [9], and Hyper-K [10]. In particular, the precision of $\sim 15 \%$ in the determination of the $\nu_{\mu}$ flux of the antineutrino-mode beam using $\mu^{-}$ capture obtained here could easily be surpassed and the flux spectrum more rigorously checked by future experiments housing heavier nuclei. As an example, the $\mu^{-}$ capture rate on ${ }^{40} \mathrm{Ar}$ exceeds $70 \%$ [72], almost affording event-by-event discrimination of the $\mu$ charge without a magnetic field. Detector-specific complications arising from $\pi / \mu$ identification and Michel electron detection should not reduce sensitivity to the $\mu$ charge dramatically.

\section{APPENDIX B: MODEL-DEPENDENT MEASUREMENTS FOR $\bar{\nu}_{\mu}$ INTERACTIONS ON $\mathrm{CH}_{2}$}

This appendix presents MiniBooNE $\bar{\nu}_{\mu}$ CCQE crosssection measurements that are explicitly dependent on CCQE interaction assumptions. These measurements include all $\bar{\nu}_{\mu}$ CCQE interactions as signal, while Appendix $\mathrm{C}$ gives cross sections treating the hydrogen CCQE component as background. All results are tabulated in Appendix D.

\section{Total cross section}

As the energy distribution of the incident $\bar{\nu}_{\mu}$ beam is quite broad (Fig. 1), the a priori knowledge of the neutrino energy is highly uncertain on an event-by-event basis. If hadronic reconstruction is unavailable, it is typical for neutrino experiments to reconstruct the neutrino energy of events in the CCQE sample assuming scattering off of at-rest and independently acting nucleons $\left(E_{\nu}^{\mathrm{QE}}\right)$ based solely on the outgoing lepton kinematics [Eq. (A12)]. Finding the neutrino energy in this way is often used to measure neutrino oscillation parameters, in particular the mass splitting, and it has been argued elsewhere that the assumptions implicit in this reconstruction significantly bias these measurements due to ignored nuclear effects [11,12].

Apart from the bias in the reconstructed energy distribution, a measurement of the absolute cross section over the observed energy range additionally suffers from model dependence through the unfolding procedure. The total cross section is typically computed by unfolding the reconstructed neutrino energy to the "true" energy distribution, and this correction is dependent on both the nuclear model used and detector resolution effects. This is the main reason MiniBooNE has generally opted to report cross sections in terms of observed kinematics. Due to these measurement biases, the MiniBooNE $\bar{\nu}_{\mu}$ CCQE absolute cross section is not the main result of the work but is provided here for historical comparisons.

A consequence of the unfolding bias is that one should exercise caution in comparing theoretical calculations to these results. A strict comparison with these data and an external model involves finding the total cross section as a function of $E_{\nu}^{\mathrm{QE}}$ using the generated muon kinematics, and subsequently unfolding this distribution according to the RFG. An example of this procedure can be found in Ref. [81].

The flux-unfolded $\bar{\nu}_{\mu} \mathrm{CCQE}$ cross section per nucleon is calculated assuming

$$
\sigma_{i}=\frac{\sum_{j} U_{i j}\left(d_{j}-b_{j}\right)}{\epsilon_{i} \Phi_{i} N}
$$

where the same conventions used in Eq. (3) apply here with a few exceptions: as mentioned, the unfolding matrix $U_{i j}$ 


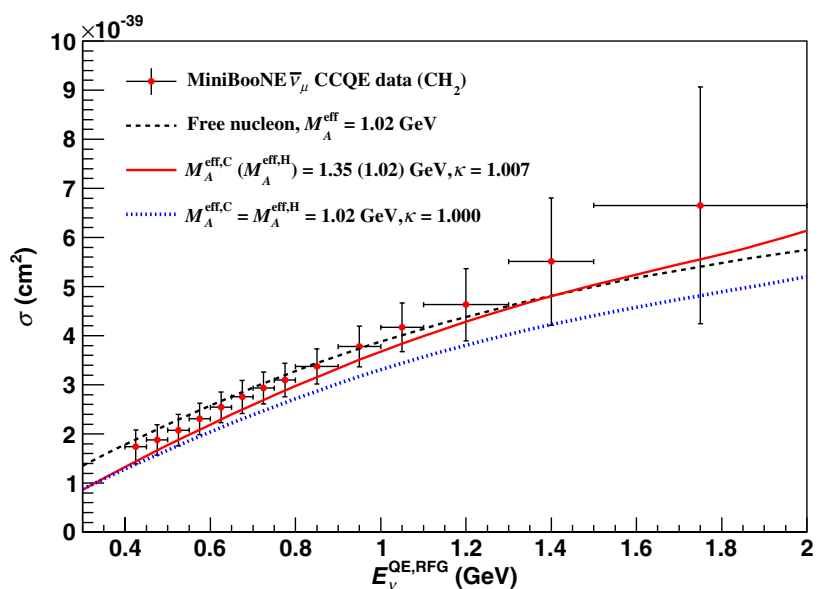

FIG. 11 (color online). Per-nucleon total cross section for MiniBooNE $\bar{\nu}_{\mu}$ CCQE data including the hydrogen scattering component. The distribution is labeled $E_{\bar{\nu}}^{\mathrm{QE}, \mathrm{RFG}}$ to recognize the dependence on the assumptions inherent in both the reconstruction and in the unfolding model. Total errors are shown with data. Numerical values are provided in Table XVIII.

here connects the reconstructed neutrino energy [inferred from the observed $\mu$ kinematics via Eq. (A12)] to the generated distribution, and the flux term $\Phi_{i}$ refers to the $\bar{\nu}_{\mu}$ flux exclusive to the $i$ th neutrino energy bin. Figure 11 compares the observed total cross section to a few predictions from the RFG.

\section{Momentum transfer}

Another important quantity for CCQE interactions is the squared four-momentum transfer $Q^{2}=\left(p_{\nu}-p_{\mu}\right)^{2}$. However, again ignorance of the incoming neutrino energy prevents a clean measurement of this variable. As in the case for $E_{\nu}^{\mathrm{QE}}$ [Eq. (A12)], if only lepton kinematics are available the distribution can be inferred by assuming CCQE scattering with an at-rest, independent nucleon:

$$
Q_{\mathrm{QE}}^{2}=-m_{\mu}^{2}+2 E_{\nu}^{\mathrm{QE}}\left(E_{\mu}-p_{\mu} \cos \theta_{\mu}\right),
$$

where $E_{\mu}, p_{\mu}$, and $m_{\mu}$ refer to the muon energy, momentum, and mass, respectively. The value of the axial mass is typically extracted from the shape of this distribution, so the differential cross section with respect to this variable is provided for historical comparisons despite the reconstruction assumptions. However, to minimize the model dependence of this cross-section configuration, the reconstructed distribution of $Q_{\mathrm{QE}}^{2}$ is corrected to true $Q_{\mathrm{QE}}^{2}$-that is, Eq. (B2) with the generated muon kinematics. In this way, the unfolding procedure only corrects for muon resolution effects and is not biased by the CCQE interaction model. Note that truth-level $Q_{\mathrm{QE}}^{2}$ is only the same as the squared four-momentum transfer up to the naive reconstruction assumptions. This choice is not typically made

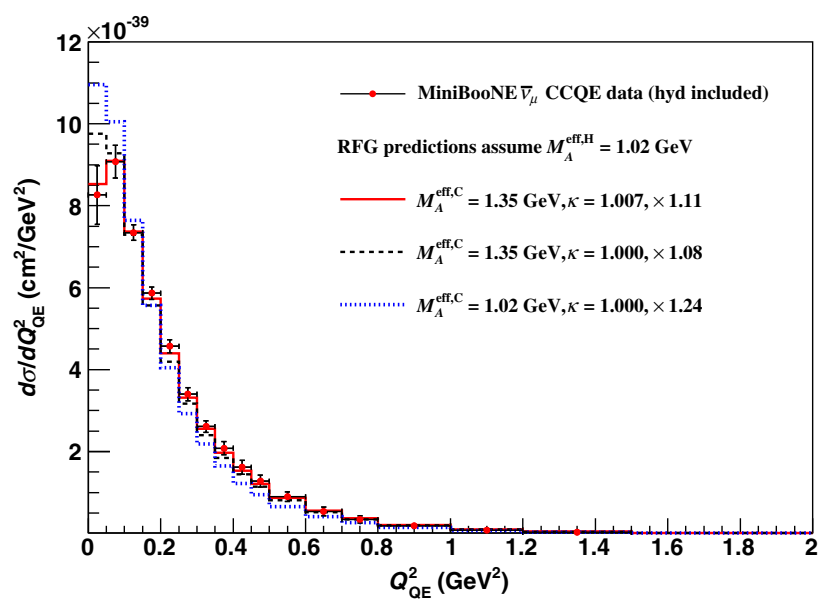

FIG. 12 (color online). Per-nucleon single-differential cross section for MiniBooNE $\bar{\nu}_{\mu}$ CCQE data including hydrogen CCQE events. The RFG predictions are normalized to the observed total cross section $\int \frac{d \sigma}{d Q_{\mathrm{QE}}^{2}} d Q_{\mathrm{QE}}^{2}$ and the relative scales are indicated. All predictions assume an effective axial mass of $1.026 \mathrm{GeV}$ for the hydrogen scattering component. Shape errors are shown with data. Numerical values are provided in Table XVII.

and so comparisons with similar cross sections from other experiments should be made with care.

The flux-folded, single-differential cross section $d \sigma / d Q_{\mathrm{QE}}^{2}$ calculated in the same manner as the double-differential cross section [Eq. (3)] but for a single dimension:

$$
\left(\frac{d \sigma}{d Q_{\mathrm{QE}}^{2}}\right)_{i}=\frac{\sum_{j} U_{i j}\left(d_{j}-b_{j}\right)}{\left(\Delta Q_{\mathrm{QE}}^{2}\right)_{i} \epsilon_{i} \Phi N}
$$

where the same conventions used in Eq. (3) apply. Figure 12 compares the results with shape uncertainty to predictions from the RFG normalized to data.

The conventions used to calculate $\sigma\left(E_{\nu}\right)$ and $\frac{d \sigma}{d Q_{\mathrm{OE}}^{2}}$ are the same used to calculate the corresponding $\nu_{\mu} \mathrm{CCQE}$ cross sections reported in Ref. [21].

\section{APPENDIX C: MODEL-DEPENDENT MEASUREMENTS FOR $\bar{\nu}_{\mu}$ CCQE INTERACTIONS WITH CARBON}

Following the same definitions for the total and singledifferential [Eqs. (B1) and (B3), respectively] cross sections, results for $\bar{\nu}_{\mu} \mathrm{CCQE}$ on carbon are obtained following the subtraction of $\bar{\nu}_{\mu} \mathrm{CCQE}$ events on quasifree protons assuming $M_{A}=1.026 \mathrm{GeV}$. In this configuration, the total per-nucleon cross section for CCQE interactions on carbon from both the MiniBooNE $\nu_{\mu}$ and $\bar{\nu}_{\mu}$ analyses may be compared to the corresponding NOMAD results, and this is shown in Fig. 13. 


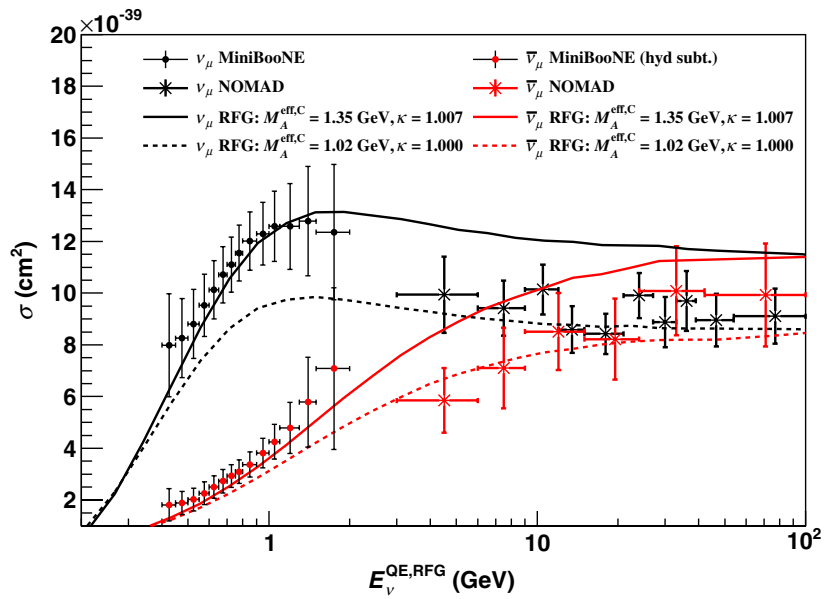

FIG. 13 (color online). Total $\nu_{\mu}$ and $\bar{\nu}_{\mu}$ CCQE cross sections for the MiniBooNE and NOMAD experiments, shown with two choices for the axial mass in the RFG for comparison. The hydrogen content has been subtracted from the MiniBooNE $\bar{\nu}_{\mu}$ data, and total uncertainties are shown. Note the two experiments use difference detector technologies and so naturally assume different topologies in defining CCQE events. Therefore, conclusions should be drawn with care. NOMAD data taken from Ref. [25], and MiniBooNE $\nu_{\mu}$ data taken from Ref. [21]. Numerical values for the MiniBooNE $\bar{\nu}_{\mu}$ cross section are provided in Table XXIII.

\section{APPENDIX D: TABULATION OF RESULTS}

This appendix provides numerical values for the observed $\bar{\nu}_{\mu}$ flux and all cross-section results presented in this work. In addition, each cross section is accompanied by both the $\bar{\nu}_{\mu}$ "CCQE-like" and the $\mathrm{CC} 1 \pi^{-}$backgrounds subtracted from the data in the procedure to obtain the $\bar{\nu}_{\mu}$ CCQE cross sections. The $\mathrm{CC} 1 \pi^{-}$background is a subset of the $\bar{\nu}_{\mu}$ CCQE-like background and is dominant in most regions. Note that in order to facilitate comparisons with the predictions of $\bar{\nu}_{\mu} \mathrm{CCQE}$ and CCQE-like processes, the CCQE-like measurements exclude the $\nu_{\mu}$ content of the subtracted data. The cross sections for these background processes are calculated for the various cross sections [Eqs. (3), (B1), and (B2)] by replacing $\left(d_{j}-b_{j}\right)$ with the appropriate subset of $b_{j}$ : in the case of the $\mathrm{CC} 1 \pi^{-}$, included are all resonance and coherent $\mathrm{CC} 1 \pi^{-}$as predicted by the Rein-Sehgal model [41], while the CCQE-like cross sections include all background $\bar{\nu}_{\mu}$ processes. Note also these measurements are normalized to the total number of proton targets in the detector, even though the dominant interaction of $\mathrm{CC} 1 \pi^{-}$has nucleon-level interactions with neutrons as well. This configuration is chosen for consistency with the $\nu_{\mu}$ CCQE-like background measurements, which were normalized to the number of neutron targets in the $\nu_{\mu} \mathrm{CCQE}$ analysis [21]. As the CCQE-like cross sections on mineral oil and carbon differ only by the inclusion of the hydrogen content, the amount of $\bar{\nu}_{\mu}$ hydrogen CCQE subtracted from the data (in the case of the latter calculation) can be found by taking the difference of these two cross sections. To find the calculated pernucleon $\bar{\nu}_{\mu}$ hydrogen CCQE cross section, this difference should also be scaled by the ratio of total protons targets to quasifree proton targets, $2.03 \times 10^{32} / 0.70 \times 10^{32}=2$.9.

\section{Antineutrino-mode fluxes}

Section II A describes the flux prediction, and Table XI (Table XII) lists the predicted $\bar{\nu}_{\mu}\left(\nu_{\mu}\right)$ flux in antineutrino mode running per POT in $50 \mathrm{MeV}$ wide bins of energy up to $3 \mathrm{GeV}$. These values normalized to the observed exposure of $10.1 \times 10^{20}$ POT are shown in Fig. 1 .

\section{Cross-section results on $\mathrm{CH}_{2}$}

All measurements in this section include the quasifree hydrogen CCQE scattering component and is therefore less model dependent compared to the results given in Appendix D, Sec. III, where the RFG model is relied on to subtract their contribution. Shape uncertainties are provided for the double- and single-differential cross-section measurements, and these values should be used along with the total normalization uncertainty of $13.0 \%$ in the context of a fit to these distributions. The total uncertainty, including errors affecting both shape and normalization, is provided for the total cross section.

Numerical values for the MiniBooNE $\bar{\nu}_{\mu} \mathrm{CCQE}$ cross section including the hydrogen content are given in Table XIII, while Table XIV provides the uncertainty on the shape of these data. These tables correspond to Figs. 8 and 9. The CCQE-like and $\mathrm{CC} 1 \pi^{-}$backgrounds are reported in Tables XV and XVI, respectively.

The single-differential cross-section $\frac{d \sigma}{d Q_{\mathrm{QE}}^{2}}$ measurement with shape uncertainty and CCQE-like background is given in Table XVII.

The total cross section including the hydrogen content is given in Table XVIII. As discussed in Appendix B, these results are dependent on both the $E_{\nu}^{\mathrm{QE}}$ reconstruction assumptions [Eq. (A12)] and the nuclear model used. To recognize these dependencies, the neutrino energy is labeled here as " $E_{\nu}^{\mathrm{QE}, \mathrm{RFG}}$."

\section{Cross-section results on ${ }^{12} \mathrm{C}$}

The tables in this section report the cross-section results reliant on the RFG subtract hydrogen CCQE events from the data. In addition to the shape errors provided with each measurement, a normalization uncertainty of $17.4 \%$ is applicable here.

The MiniBooNE $\bar{\nu}_{\mu}$ CCQE double-differential cross section, shape uncertainty, and CCQE-like cross section treating the hydrogen CCQE content as background are given in Tables XIX, XX, and XXI, respectively. Tables XXII and XXIII provide the same information for the single-differential and total cross section. 
TABLE XI. Predicted $\bar{\nu}_{\mu}$ flux at the MiniBooNE detector in antineutrino mode.

\begin{tabular}{|c|c|c|c|c|c|}
\hline$E_{\nu}$ bin $(\mathrm{GeV})$ & $\begin{array}{c}\bar{\nu}_{\mu} \text { flux } \\
\left(\bar{\nu}_{\mu} / \text { POT } / 50 \mathrm{MeV} / \mathrm{cm}^{2}\right)\end{array}$ & $\begin{array}{l}E_{\nu} \text { bin } \\
(\mathrm{GeV})\end{array}$ & $\begin{array}{c}\bar{\nu}_{\mu} \text { flux } \\
\left(\bar{\nu}_{\mu} / \text { POT } / 50 \mathrm{MeV} / \mathrm{cm}^{2}\right)\end{array}$ & $\begin{array}{l}E_{\nu} \text { bin } \\
(\mathrm{GeV})\end{array}$ & $\begin{array}{c}\bar{\nu}_{\mu} \text { flux } \\
\left(\bar{\nu}_{\mu} / \mathrm{POT} / 50 \mathrm{MeV} / \mathrm{cm}^{2}\right)\end{array}$ \\
\hline $0.00-0.05$ & $2.157 \times 10^{-12}$ & $1.00-1.05$ & $7.658 \times 10^{-12}$ & $2.00-2.05$ & $2.577 \times 10^{-13}$ \\
\hline $0.05-0.10$ & $7.840 \times 10^{-12}$ & $1.05-1.10$ & $6.907 \times 10^{-12}$ & $2.05-2.10$ & $2.066 \times 10^{-13}$ \\
\hline $0.10-0.15$ & $9.731 \times 10^{-12}$ & $1.10-1.15$ & $6.180 \times 10^{-12}$ & $2.10-2.15$ & $1.665 \times 10^{-13}$ \\
\hline $0.15-0.20$ & $1.141 \times 10^{-11}$ & $1.15-1.20$ & $5.505 \times 10^{-12}$ & $2.15-2.20$ & $1.346 \times 10^{-13}$ \\
\hline $0.20-0.25$ & $1.319 \times 10^{-11}$ & $1.20-1.25$ & $4.877 \times 10^{-12}$ & $2.20-2.25$ & $1.081 \times 10^{-13}$ \\
\hline $0.25-0.30$ & $1.438 \times 10^{-11}$ & $1.25-1.30$ & $4.269 \times 10^{-12}$ & $2.25-2.30$ & $8.837 \times 10^{-14}$ \\
\hline $0.30-0.35$ & $1.477 \times 10^{-11}$ & $1.30-1.35$ & $3.686 \times 10^{-12}$ & $2.30-2.35$ & $7.136 \times 10^{-14}$ \\
\hline $0.35-0.40$ & $1.479 \times 10^{-11}$ & $1.35-1.40$ & $3.151 \times 10^{-12}$ & $2.35-2.40$ & $5.707 \times 10^{-14}$ \\
\hline $0.40-0.45$ & $1.500 \times 10^{-11}$ & $1.40-1.45$ & $2.678 \times 10^{-12}$ & $2.40-2.45$ & $4.620 \times 10^{-14}$ \\
\hline $0.45-0.50$ & $1.485 \times 10^{-11}$ & $1.45-1.50$ & $2.262 \times 10^{-12}$ & $2.45-2.50$ & $3.778 \times 10^{-14}$ \\
\hline $0.50-0.55$ & $1.447 \times 10^{-11}$ & $1.50-1.55$ & $1.898 \times 10^{-12}$ & $2.50-2.55$ & $3.028 \times 10^{-14}$ \\
\hline $0.55-0.60$ & $1.406 \times 10^{-11}$ & $1.55-1.60$ & $1.580 \times 10^{-12}$ & $2.55-2.60$ & $2.412 \times 10^{-14}$ \\
\hline $0.60-0.65$ & $1.345 \times 10^{-11}$ & $1.60-1.65$ & $1.311 \times 10^{-12}$ & $2.60-2.65$ & $1.977 \times 10^{-14}$ \\
\hline $0.65-0.70$ & $1.287 \times 10^{-11}$ & $1.65-1.70$ & $1.083 \times 10^{-12}$ & $2.65-2.70$ & $1.638 \times 10^{-14}$ \\
\hline $0.70-0.75$ & $1.221 \times 10^{-11}$ & $1.70-1.75$ & $8.917 \times 10^{-13}$ & $2.70-2.75$ & $1.323 \times 10^{-14}$ \\
\hline $0.75-0.80$ & $1.152 \times 10^{-11}$ & $1.75-1.80$ & $7.285 \times 10^{-13}$ & $2.75-2.80$ & $1.038 \times 10^{-14}$ \\
\hline $0.80-0.85$ & $1.075 \times 10^{-11}$ & $1.80-1.85$ & $5.941 \times 10^{-13}$ & $2.80-2.85$ & $8.707 \times 10^{-15}$ \\
\hline $0.85-0.90$ & $9.980 \times 10^{-12}$ & $1.85-1.90$ & $4.834 \times 10^{-13}$ & $2.85-2.90$ & $6.981 \times 10^{-15}$ \\
\hline $0.90-0.95$ & $9.177 \times 10^{-12}$ & $1.90-1.95$ & $3.937 \times 10^{-13}$ & $2.90-2.95$ & $6.078 \times 10^{-15}$ \\
\hline $0.95-1.00$ & $8.411 \times 10^{-12}$ & $1.95-2.00$ & $3.180 \times 10^{-13}$ & $2.95-3.00$ & $5.111 \times 10^{-15}$ \\
\hline
\end{tabular}

TABLE XII. Predicted $\nu_{\mu}$ flux at the MiniBooNE detector in antineutrino mode. Note that, based on the results of Ref. [34] and Appendix A, the $\nu_{\mu}$ flux spectrum given here should be scaled by 0.77 to reflect the data-based constraints.

\begin{tabular}{lccccc}
\hline \hline & $\begin{array}{c}\nu_{\mu} \text { flux } \\
E_{\nu} \text { bin }(\mathrm{GeV})\end{array}$ & $\begin{array}{c}E_{\nu} \text { bin } \\
\left(\nu_{\mu} / \mathrm{POT} / 50 \mathrm{MeV} / \mathrm{cm}^{2}\right)\end{array}$ & $\begin{array}{c}\nu_{\mu} \text { flux } \\
\left(\nu_{\mu} / \mathrm{POT} / 50 \mathrm{MeV} / \mathrm{cm}^{2}\right)\end{array}$ & $\begin{array}{c}E_{\nu} \text { bin } \\
(\mathrm{GeV})\end{array}$ & $\begin{array}{c}\nu_{\mu} \text { flux } \\
\left(\nu_{\mu} / \mathrm{POT} / 50 \mathrm{MeV} / \mathrm{cm}^{2}\right)\end{array}$ \\
\hline $0.00-0.05$ & $2.298 \times 10^{-12}$ & $1.00-1.05$ & $1.087 \times 10^{-12}$ & $2.00-2.05$ & $1.886 \times 10^{-13}$ \\
$0.05-0.10$ & $5.903 \times 10^{-12}$ & $1.05-1.10$ & $1.044 \times 10^{-12}$ & $2.05-2.10$ & $1.669 \times 10^{-13}$ \\
$0.10-0.15$ & $3.726 \times 10^{-12}$ & $1.10-1.15$ & $9.967 \times 10^{-13}$ & $2.10-2.15$ & $1.486 \times 10^{-13}$ \\
$0.15-0.20$ & $2.338 \times 10^{-12}$ & $1.15-1.20$ & $9.435 \times 10^{-13}$ & $2.15-2.20$ & $1.310 \times 10^{-13}$ \\
$0.20-0.25$ & $2.570 \times 10^{-12}$ & $1.20-1.25$ & $8.826 \times 10^{-13}$ & $2.20-2.25$ & $1.171 \times 10^{-13}$ \\
$0.25-0.30$ & $1.797 \times 10^{-12}$ & $1.25-1.30$ & $8.320 \times 10^{-13}$ & $2.25-2.30$ & $1.030 \times 10^{-13}$ \\
$0.30-0.35$ & $1.776 \times 10^{-12}$ & $1.30-1.35$ & $7.736 \times 10^{-13}$ & $2.30-2.35$ & $9.279 \times 10^{-14}$ \\
$0.35-0.40$ & $1.855 \times 10^{-12}$ & $1.35-1.40$ & $7.180 \times 10^{-13}$ & $2.35-2.40$ & $8.199 \times 10^{-14}$ \\
$0.40-0.45$ & $1.834 \times 10^{-12}$ & $1.40-1.45$ & $6.609 \times 10^{-13}$ & $2.40-2.45$ & $7.353 \times 10^{-14}$ \\
$0.45-0.50$ & $1.770 \times 10^{-12}$ & $1.45-1.50$ & $6.053 \times 10^{-13}$ & $2.45-2.50$ & $6.577 \times 10^{-14}$ \\
$0.50-0.55$ & $1.701 \times 10^{-12}$ & $1.50-1.55$ & $5.533 \times 10^{-13}$ & $2.50-2.55$ & $5.830 \times 10^{-14}$ \\
$0.55-0.60$ & $1.618 \times 10^{-12}$ & $1.55-1.60$ & $5.058 \times 10^{-13}$ & $2.55-2.60$ & $5.318 \times 10^{-14}$ \\
$0.60-0.65$ & $1.555 \times 10^{-12}$ & $1.60-1.65$ & $4.577 \times 10^{-13}$ & $2.60-2.65$ & $4.822 \times 10^{-14}$ \\
$0.65-0.70$ & $1.493 \times 10^{-12}$ & $1.65-1.70$ & $4.134 \times 10^{-13}$ & $2.65-2.70$ & $4.317 \times 10^{-14}$ \\
$0.70-0.75$ & $1.425 \times 10^{-12}$ & $1.70-1.75$ & $3.725 \times 10^{-13}$ & $2.70-2.75$ & $3.997 \times 10^{-14}$ \\
$0.75-0.80$ & $1.357 \times 10^{-12}$ & $1.75-1.80$ & $3.336 \times 10^{-13}$ & $2.75-2.80$ & $3.619 \times 10^{-14}$ \\
$0.80-0.85$ & $1.302 \times 10^{-12}$ & $1.80-1.85$ & $3.003 \times 10^{-13}$ & $2.80-2.85$ & $3.375 \times 10^{-14}$ \\
$0.85-0.90$ & $1.236 \times 10^{-12}$ & $1.85-1.90$ & $2.663 \times 10^{-13}$ & $2.85-2.90$ & $3.050 \times 10^{-14}$ \\
$0.90-0.95$ & $1.192 \times 10^{-12}$ & $1.90-1.95$ & $2.375 \times 10^{-13}$ & $2.90-2.95$ & $2.926 \times 10^{-14}$ \\
$0.95-1.00$ & $1.141 \times 10^{-12}$ & $1.95-2.00$ & $2.126 \times 10^{-13}$ & $2.95-3.00$ & $2.705 \times 10^{-14}$ \\
\hline \hline
\end{tabular}




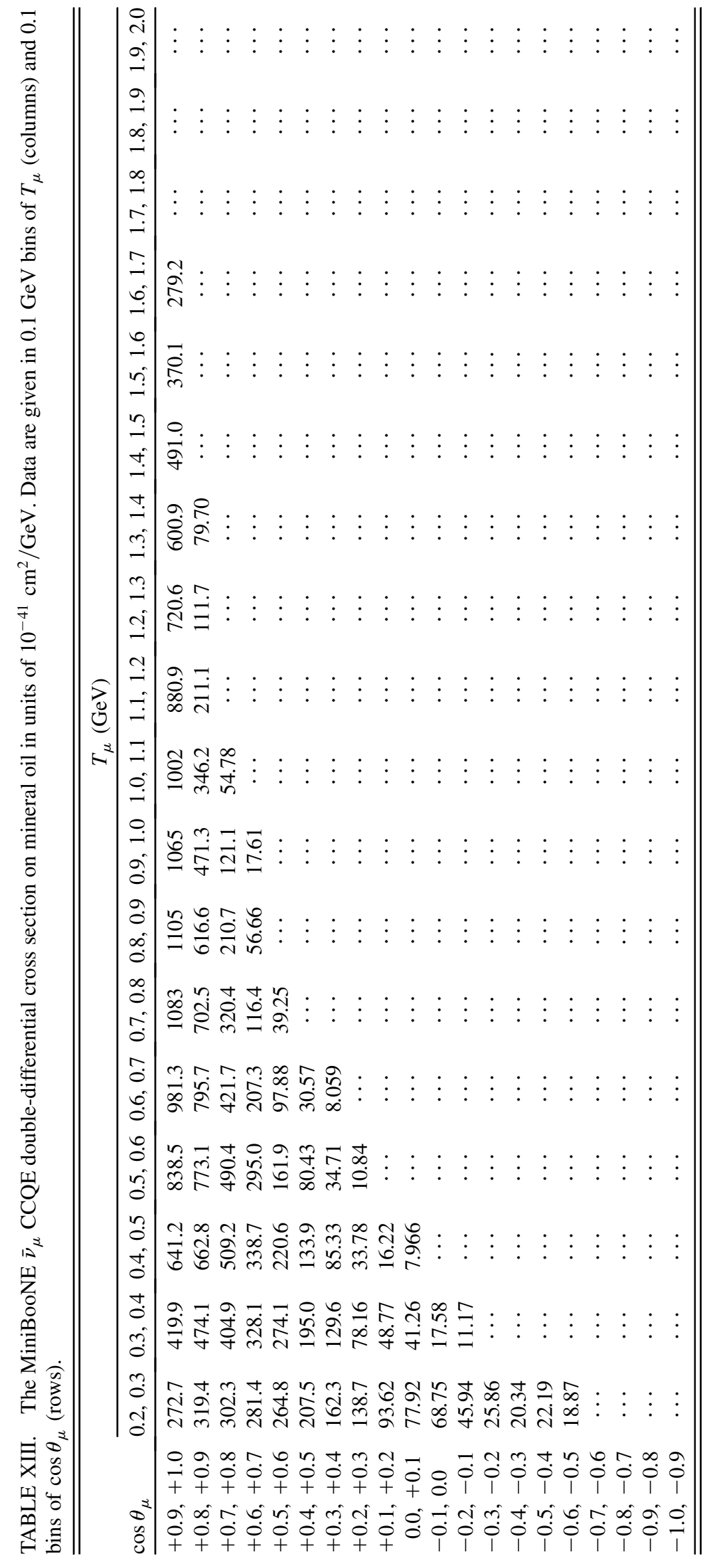




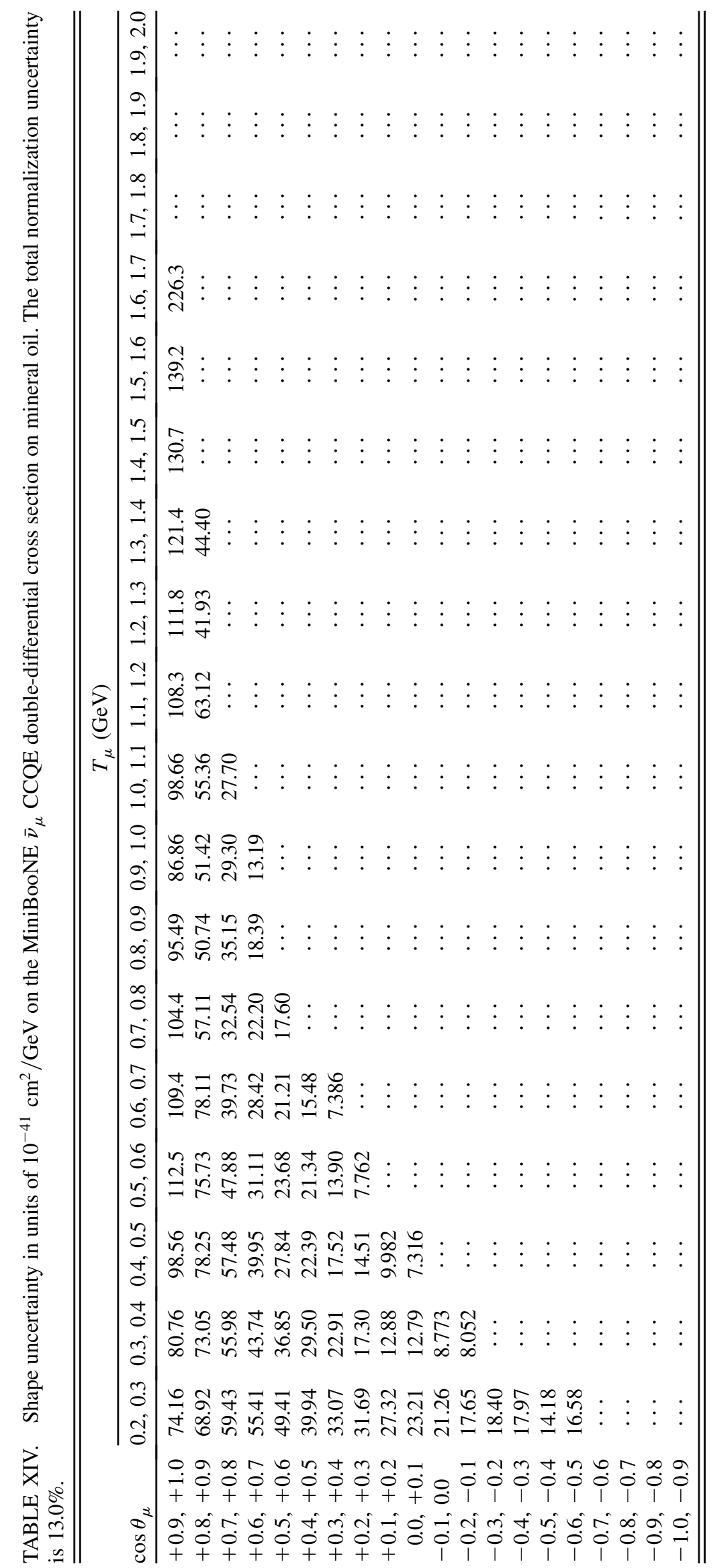




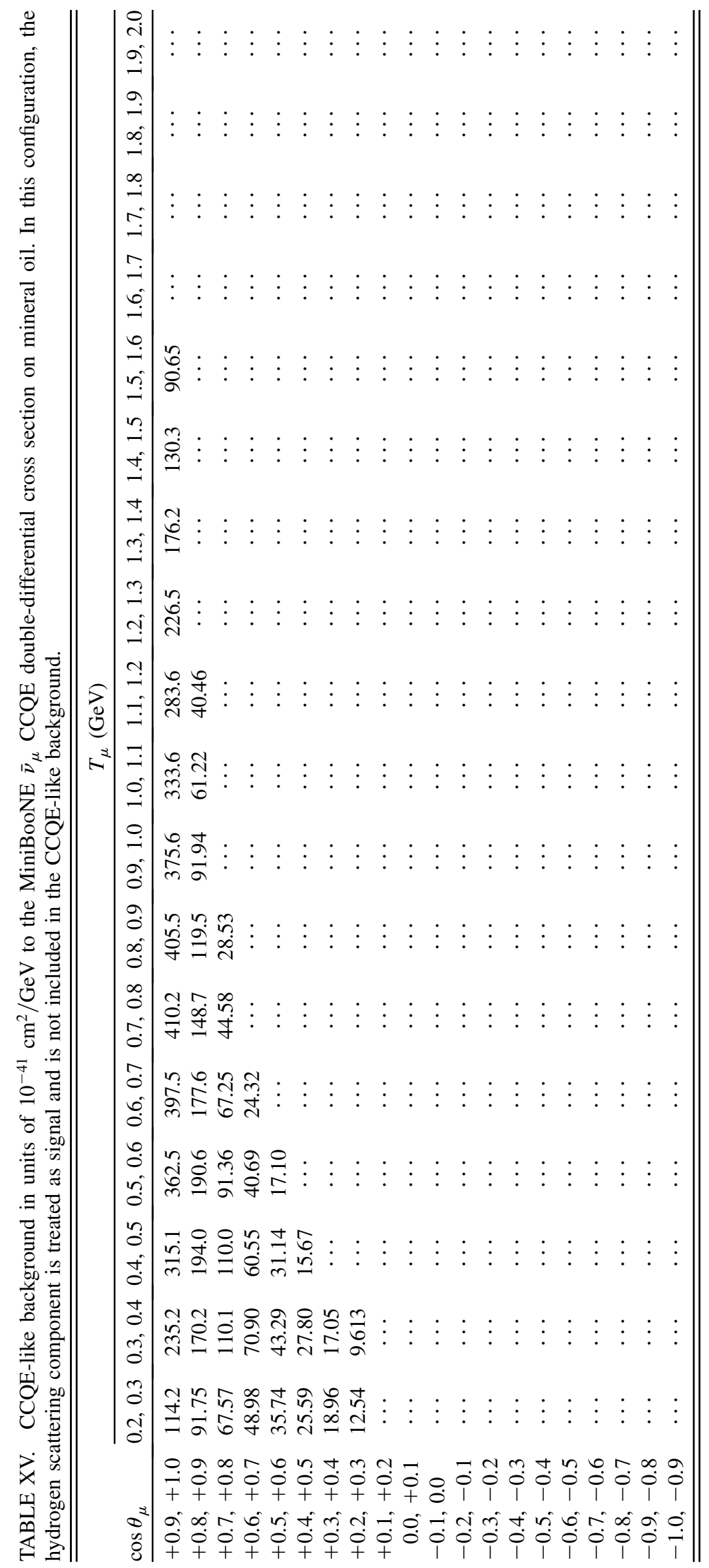




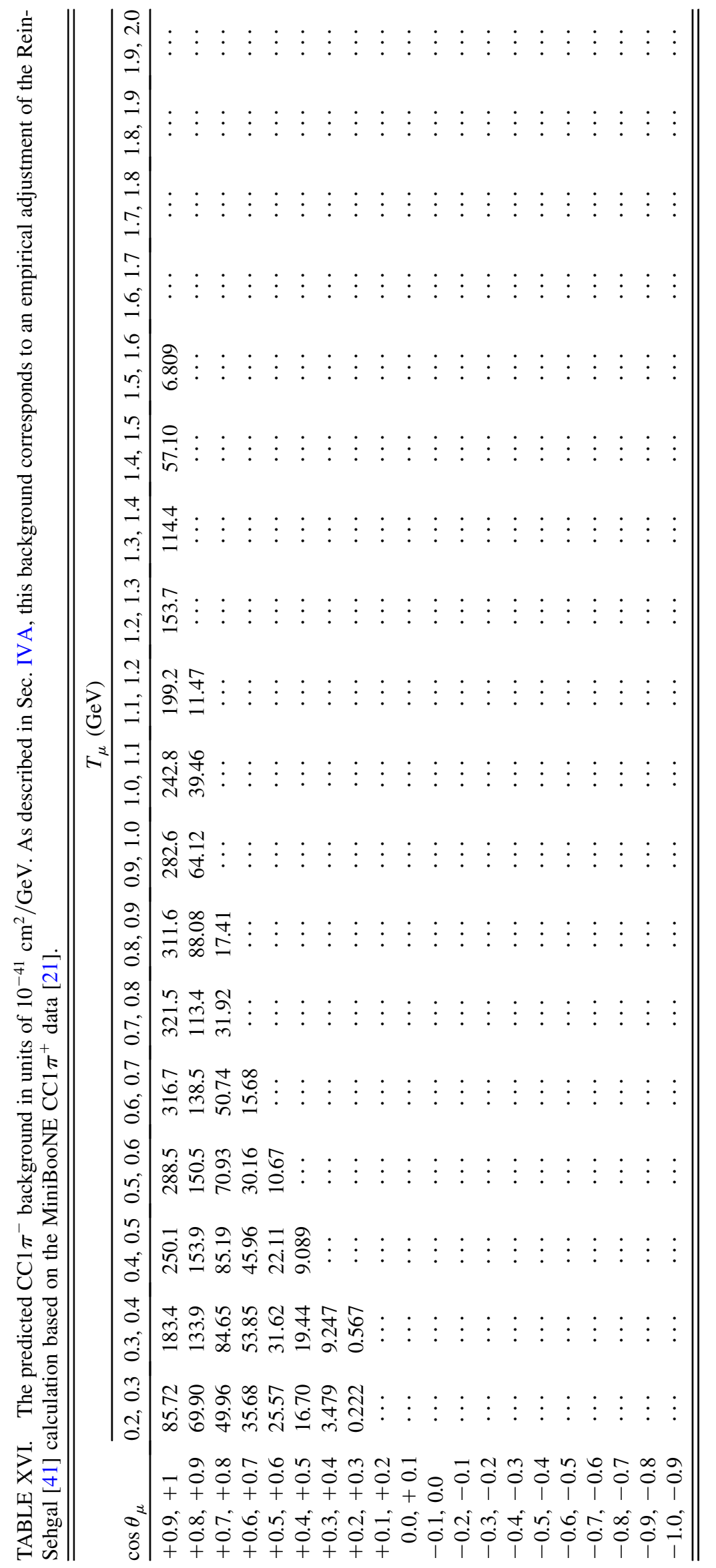




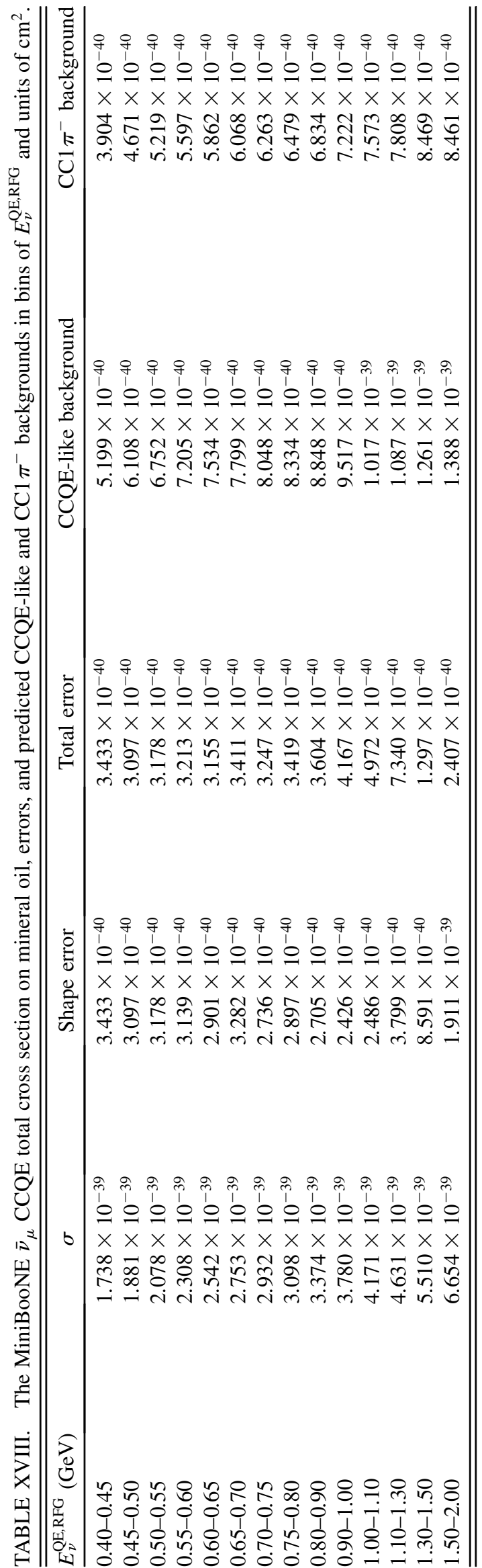




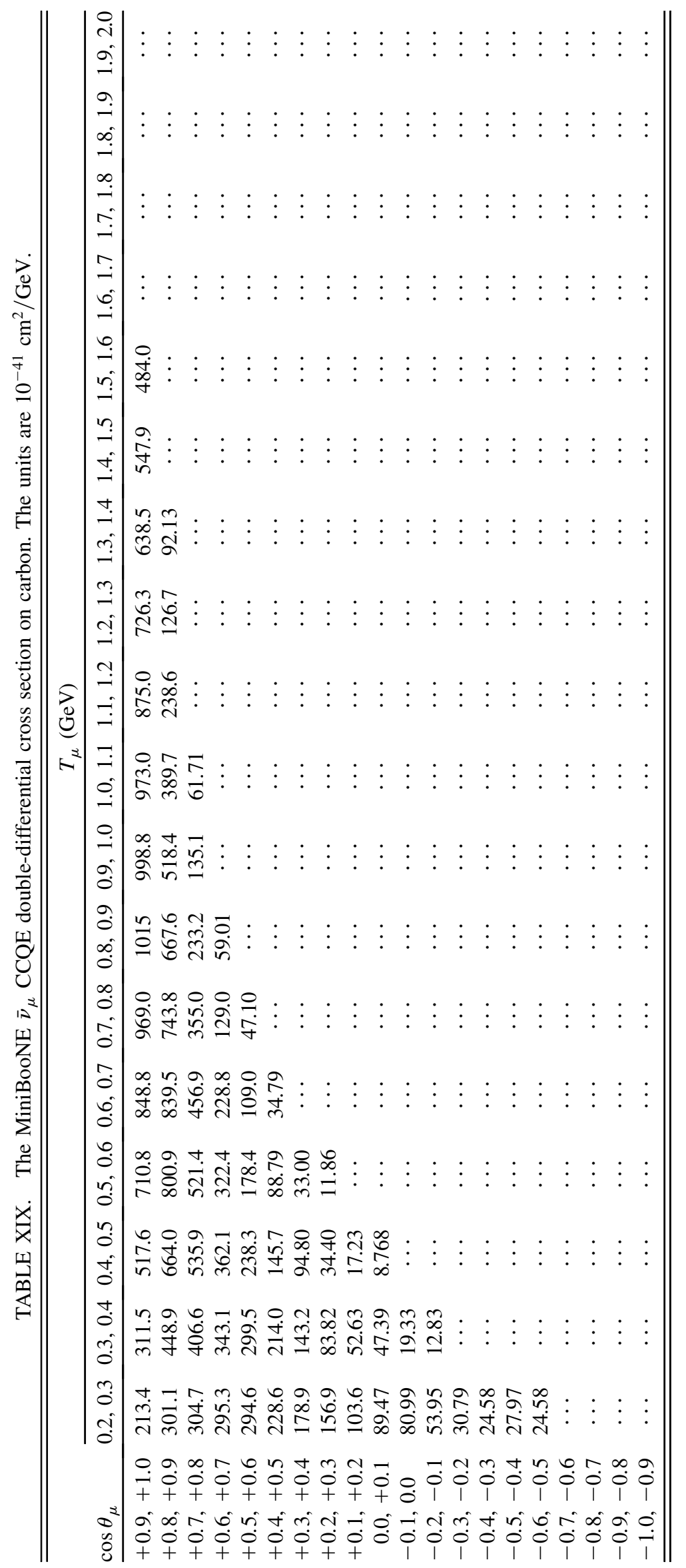




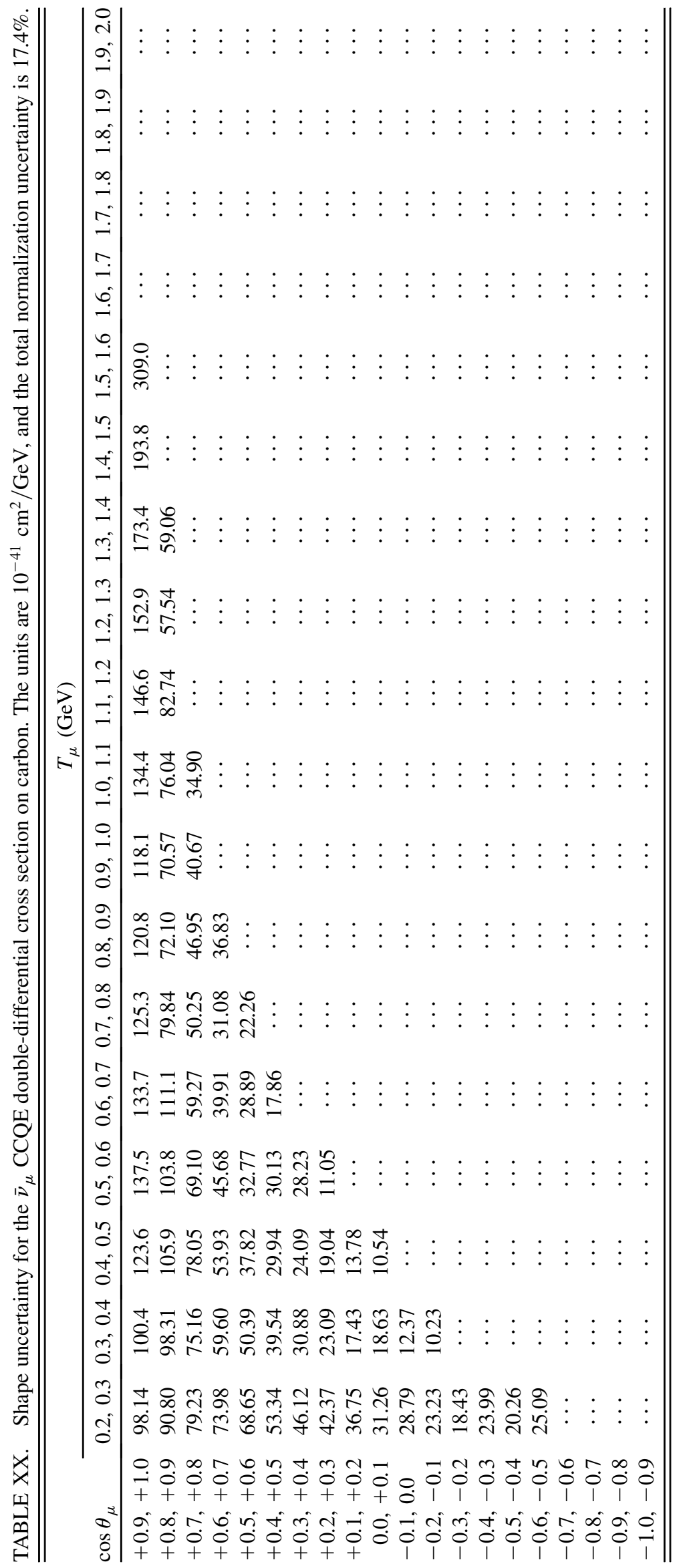




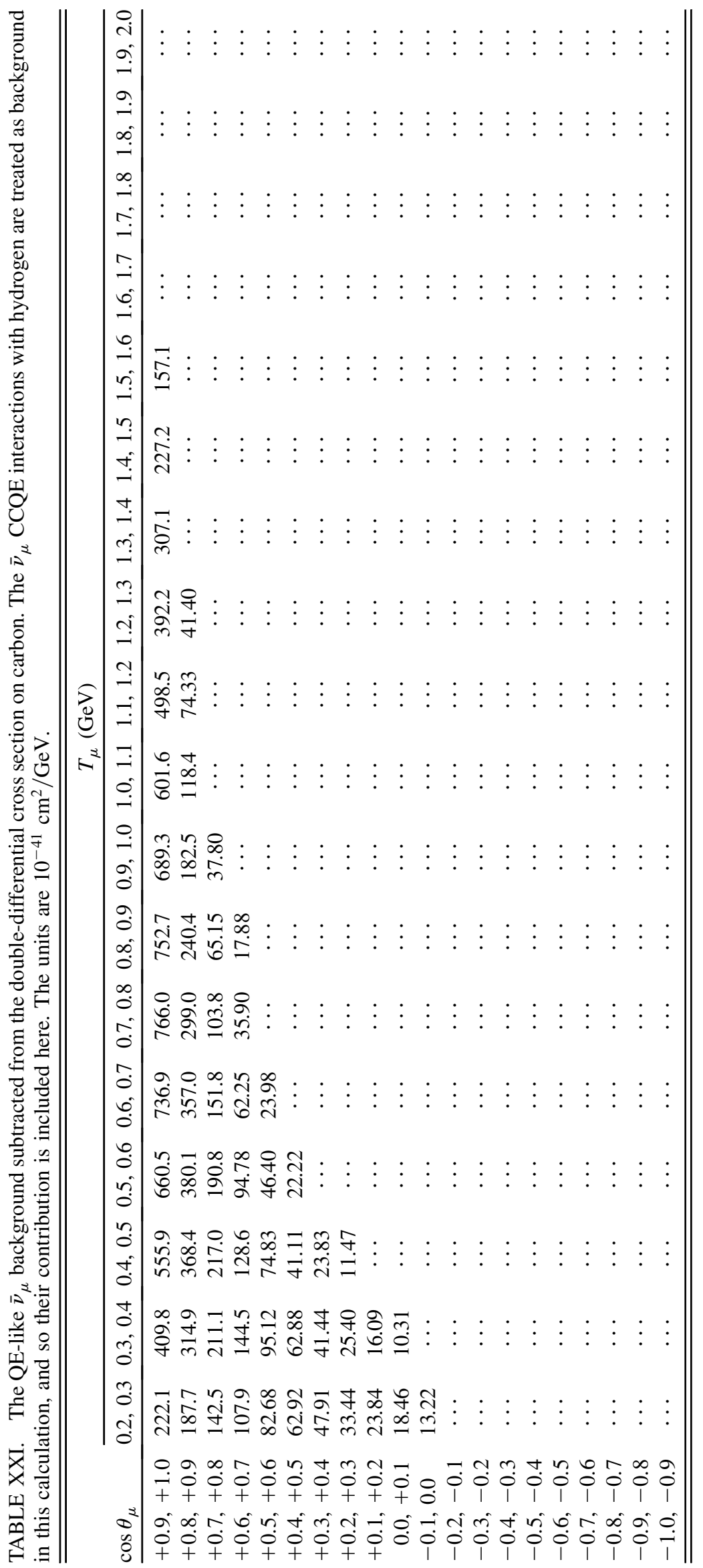



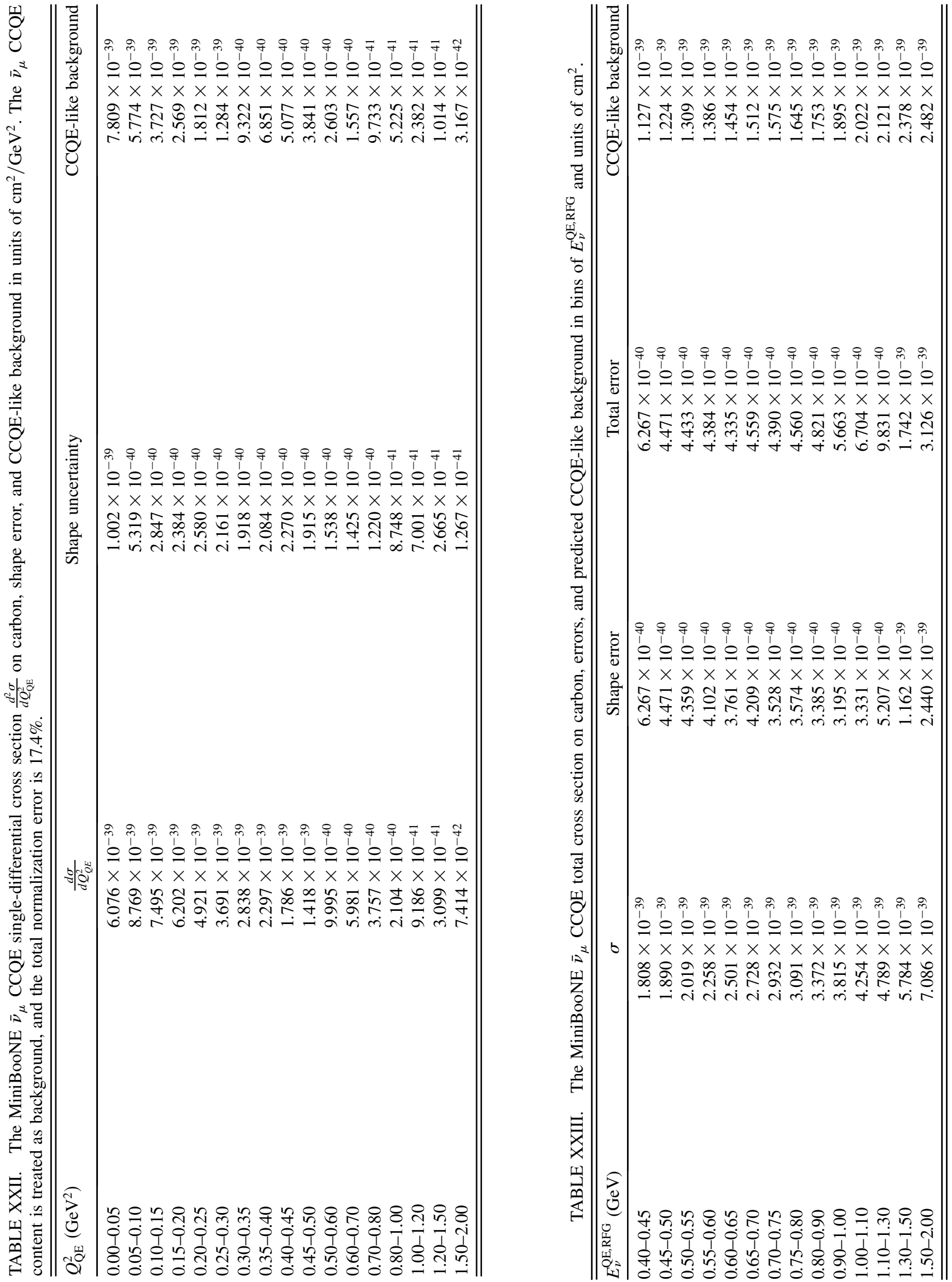
[1] F. P. An et al. (Daya Bay Collaboration), Phys. Rev. Lett. 108, 171803 (2012).

[2] J. K. Ahn et al. (RENO Collaboration), Phys. Rev. Lett. 108, 191802 (2012).

[3] Y. Abe et al. (Double Chooz Collaboration), Phys. Rev. D 86, 052008 (2012).

[4] K. Abe et al. (T2K Collaboration), Phys. Rev. Lett. 107, 041801 (2011).

[5] P. Adamson et al. (MINOS Collaboration), Phys. Rev. Lett. 107, 181802 (2011).

[6] D.S. Ayres et al. (NOvA Collaboration), Report No. FERMILAB-DESIGN-2007-01, 2007.

[7] Y. Itow et al., arXiv:hep-ex/0106019.

[8] V. Barger et al., arXiv:0705.4396.

[9] A. de Bellefon et al., arXiv:hep-ex/0607026.

[10] K. Nakamura, Int. J. Mod. Phys. A 18, 4053 (2003).

[11] M. Martini, M. Ericson, and G. Chanfray, Phys. Rev. D 85, 093012 (2012).

[12] O. Lalakulich and U. Mosel, Phys. Rev. C 86, 054606 (2012).

[13] G. P. Zeller and J. Formaggio, Rev. Mod. Phys. 84, 1307 (2012).

[14] R. A. Smith and E. J. Moniz, Nucl. Phys. B43, 605 (1972); B101, 547(E) (1975).

[15] B. Bhattacharya, R. J. Hill, and G. Paz, Phys. Rev. D 84, 073006 (2011).

[16] C. H. L. Smith, Phys. Rep. 3, 261 (1972).

[17] H. Budd, A. Bodek, and J. Arrington, arXiv:hep-ex/ 0308005.

[18] C. Amsler et al. (Particle Data Group), Phys. Lett. B 667, 1 (2008).

[19] V. Bernard, L. Elouadrhiri, and U.-G. Meißner, J. Phys. G 28, R1 (2002).

[20] A. Bodek, S. Avvakumov, R. Bradford, and H. Budd, J. Phys. Conf. Ser. 110, 082004 (2008).

[21] A. A. Aguilar-Arevalo et al. (MiniBooNE Collaboration), Phys. Rev. D 81, 092005 (2010).

[22] R. Gran et al., Phys. Rev. D 74, 052002 (2006).

[23] J.L. Alcaraz-Aunion and J. Walding, AIP Conf. Proc. 1189, 145 (2009).

[24] M. Dorman (MINOS Collaboration), AIP Conf. Proc. 1189, 133 (2009).

[25] K.S. Kuzmin, V. V. Lyubushkin, and V.A. Naumov, Eur. Phys. J. C 54, 517 (2008); V. V. Lyubushkin et al. (NOMAD Collaboration), Eur. Phys. J. C 63, 355 (2009).

[26] K. McFarland (MINER $\nu$ A Collaboration), AIP Conf. Proc. 1405, 95 (2011).

[27] H. Gallagher, G. Garvey, and G. P. Zeller, Annu. Rev. Nucl. Part. Sci. 61, 355 (2011).

[28] W. M. Alberico, A. Molinari, T. Donnelly, E. Kronenberg, and J. Van Orden, Phys. Rev. C 38, 1801 (1988).

[29] T. W. Donnelly and I. Sick, Phys. Rev. C 60, 065502 (1999).

[30] M. G. Catanesi et al. (HARP Collaboration), Eur. Phys. J. C 52, 29 (2007).

[31] A. A. Aguilar-Arevalo et al. (MiniBooNE Collaboration), Phys. Rev. D 79, 072002 (2009).

[32] S. Agostinelli et al., Nucl. Instrum. Methods Phys. Res., Sect. A 506, 250 (2003).

[33] S. Kopp, Phys. Rep. 439, 101 (2007).
[34] A. A. Aguilar-Arevalo et al. (MiniBooNE Collaboration), Phys. Rev. D 84, 072005 (2011).

[35] R. B. Patterson, E. M. Laird, Y. Liu, P. D. Meyers, I. Stancu, and H. A. Tanaka, Nucl. Instrum. Methods Phys. Res., Sect. A 608, 206 (2009).

[36] A. A. Aguilar-Arevalo et al. (MiniBooNE Collaboration), Nucl. Instrum. Methods Phys. Res., Sect. A 599, 28 (2009).

[37] D. Casper, Nucl. Phys. B, Proc. Suppl. 112, 161 (2002).

[38] J. Grange (MiniBooNE Collaboration), AIP Conf. Proc. 1405, 83 (2011).

[39] E. J. Moniz, I. Sick, R. Whitney, J. Ficenec, R. Kephart, and W. Trower, Phys. Rev. Lett. 26, 445 (1971).

[40] T. Katori, Report No. FERMILAB-THESIS-2008-64, 2008.

[41] D. Rein and L. M. Sehgal, Ann. Phys. (N.Y.) 133, 79 (1981).

[42] R. P. Feynman, M. Kislinger, and F. Ravndal, Phys. Rev. D 3, 2706 (1971).

[43] A. A. Aguilar-Arevalo et al. (MiniBooNE Collaboration), Phys. Rev. D 83, 052007 (2011).

[44] W. Reuter, G. Fricke, K. Merle, and H. Miska, Phys. Rev. C 26, 806 (1982).

[45] V. Flaminino et al., Report No. CERN-HERA-83-01, 1983; Report No. CERN-HERA-83-02, 1983; Report No. CERN-HERA-84-01, 1984.

[46] D. Casper (private communication).

[47] A. Shinohara, T. Muroyama, J. Shintai, J. Kurachi, M. Furukawa, T. Miura, Y. Yoshimura, T. Saito, T. Ohdaira, and N. Imanishi, Phys. Rev. A 53, 130 (1996).

[48] M. G. Cox, C. Eiø, G. Mana, and F. Pennecchi, Metrologia 43, S268 (2006).

[49] J. Nowak, AIP Conf. Proc. 1189, 243 (2009).

[50] K. S. Kuzmin, V. V. Lyubushkin, and V. A. Naumov, Mod. Phys. Lett. A 19, 2815 (2004).

[51] K. S. Kuzmin, V. V. Lyubushkin, and V. A. Naumov, Nucl. Phys. B, Proc. Suppl. 139, 158 (2005).

[52] Ch. Berger and L. M. Sehgal, Phys. Rev. D 76, 113004 (2007); 77, 059901(E) (2008).

[53] K. M. Graczyk and J. T. Sobczyk, Phys. Rev. D 77, 053001 (2008).

[54] A. A. Aguilar-Arevalo et al. (MiniBooNE Collaboration), Phys. Rev. D 83, 052009 (2011).

[55] G. D’Agostini, Nucl. Instrum. Methods Phys. Res., Sect. A 362, 487 (1995).

[56] B. P. Roe, Nucl. Instrum. Methods Phys. Res., Sect. A 570, 159 (2007).

[57] B. C. Brown et al., IEEE Nucl. Sci. Symp. Conf. Rec. 1, 652 (2004).

[58] D. Ashery, I. Navon, G. Azuelos, and F. W. Schlepütz, Phys. Rev. C 23, 2173 (1981); M. K. Jones et al., Phys. Rev. C 48, 2800 (1993); R.D. Ransome et al., Phys. Rev. C 45, R509 (1992).

[59] C. Juszczak, J. T. Sobczyk, and J. Żmuda, Phys. Rev. C 82, 045502 (2010).

[60] M. S. Athar, S. Ahmad, and S. K. Singh, Phys. Rev. D 75, 093003 (2007).

[61] M. Martini, M. Ericson, G. Chanfray, and J. Marteau, Phys. Rev. C 81, 045502 (2010).

[62] J. Nieves, I. R. Simo, and M. J. V. Vacas, Phys. Rev. C 83, 045501 (2011). 
[63] J. Amaro, M. B. Barbaro, J.A. Caballero, and T.W. Donnelly, Phys. Rev. Lett. 108, 152501 (2012).

[64] A. Meucci and C. Giusti, Phys. Rev. D 85, 093002 (2012).

[65] A. Bodek, H. S. Budd, and M. E. Christy, Eur. Phys. J. C 71, 1726 (2011).

[66] D. Drakoulakos et al. (MINERvA Collaboration), arXiv: hep-ex/0405002.

[67] A. A. Aguilar-Arevalo et al. (SciBooNE Collaboration), arXiv:hep-ex/0601022.

[68] M. Soderberg (MicroBooNE Collaboration), AIP Conf. Proc. 1189, 83 (2009).

[69] C. Anderson et al. (ArgoNeuT Collaboration), Phys. Rev. Lett. 108, 161802 (2012).

[70] S. Amoruso et al. (ICARUS Collaboration), Eur. Phys. J. C 33, 233 (2004).

[71] P. Huber and T. Schwetz, arXiv:0805.2019.

[72] T. Suzuki, D. Measday, and J. Roalsvig, Phys. Rev. C 35, 2212 (1987).
[73] E. J. Maier, R. M. Edelstein, and R. T. Siegel, Phys. Rev. 133, B663 (1964).

[74] L. Ph. Roesch, N. Schlumpf, D. Taqqu, V.L. Telegdi, P. Truttmann, and A. Zehnder, Phys. Lett. 107B, 31 (1981).

[75] Yu. G. Budyashov et al., Sov. Phys. JETP 31, 651 (1970).

[76] M.E. Plett and S.E. Sobottka, Phys. Rev. C 3, 1003 (1971).

[77] B. Macdonald, J. Diaz, S. Kaplan, and R. Pyle, Phys. Rev. 139, B1253 (1965).

[78] G. H. Miller, M. Eckhause, F. R. Kane, P. Martin, and R. E. Welsh, Phys. Lett. 41B, 50 (1972).

[79] A. A. Aguilar-Arevalo et al. (MiniBooNE Collaboration), Phys. Rev. D 81, 013005 (2010).

[80] A. A. Aguilar-Arevalo et al. (MiniBooNE Collaboration), Phys. Rev. D 82, 092005 (2010).

[81] J. Nieves, F. Sánchez, I. R. Simo, and M. J. V. Vacas, Phys. Rev. D 85, 113008 (2012). 